\title{
Palladium-Catalyzed Enantioselective Carbene Insertion into Strained Carbon-Silicon Bonds of Silacyclobutanes
}

Jingfeng Huo, Kangbao Zhong, Yazhen Xue, MyeeMay Lyu, Yifan Ping, Zhenxing Liu, Yu Lan,* and Jianbo Wang* email: wangjb@pku.edu.cn (J.W.); 1anyu@,cqu.edu.cn (Y.L)

\section{CONTENTS}

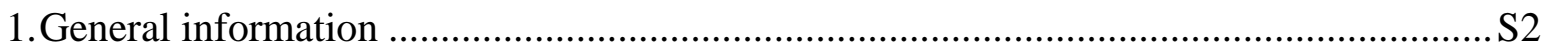

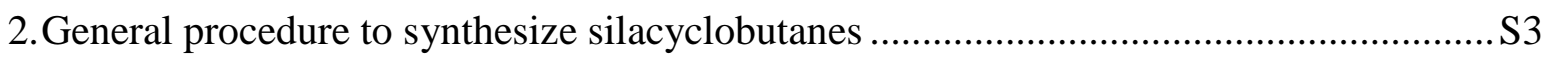

3. Optimization for the carbene insertion into strained $\mathrm{C}-\mathrm{Si}$ bonds .................................... 5

4.General procedure for palladium-catalyzed carbene insertion into strained C-Si bonds .. S7

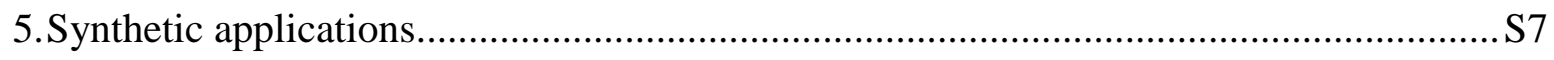

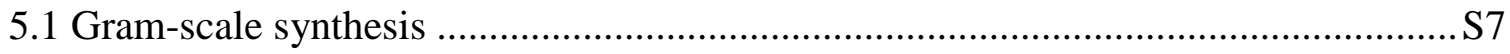

5.2 Oxidative hydrolysis of $\mathbf{3 a}$ to the corresponding chiral 1,4-diol.............................S8

5.3 The reaction with natural product and drug molecules..........................................S

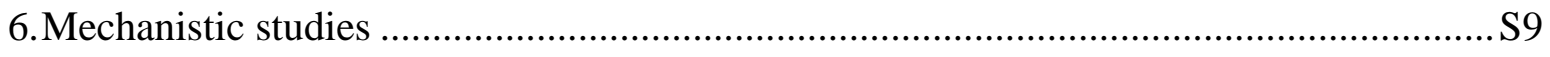

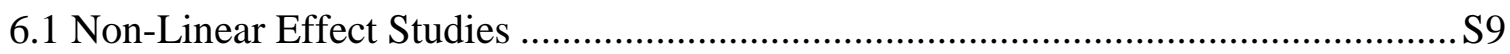

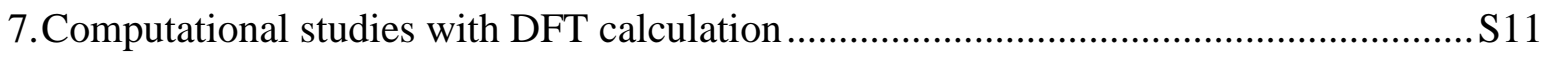

7.1 Calculation on the choice of starting point in the reaction ......................................S11

7.2 Calculation on palladium-catalyzed carbene insertion into $\mathrm{C}-\mathrm{Si}$ bonds of

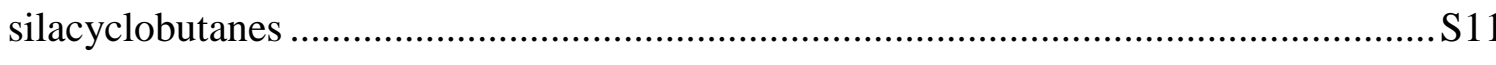

7.3 B3-LYP and M06-2X calculated absolute energies, enthalpies, and free energies for

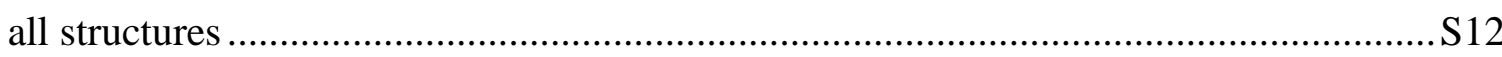

7.4 B3-LYP geometries for all the optimized compounds and transition states ............. S14

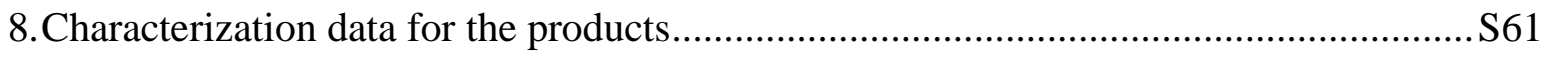

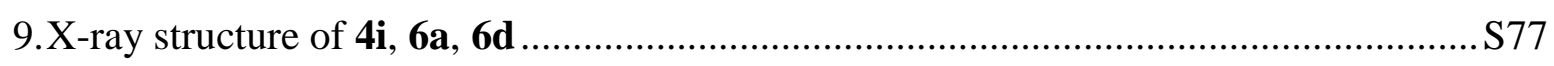

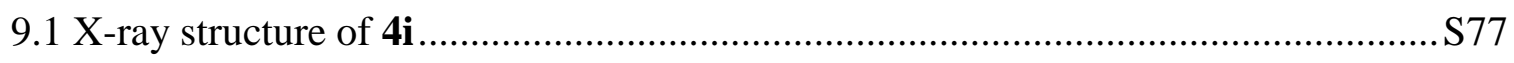

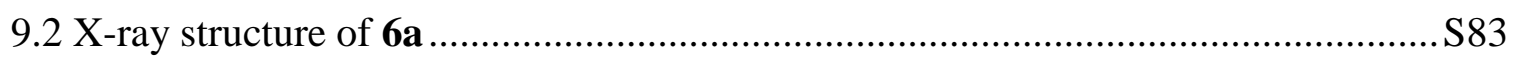

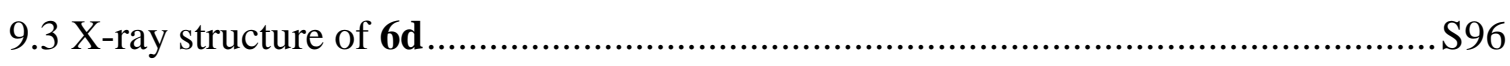

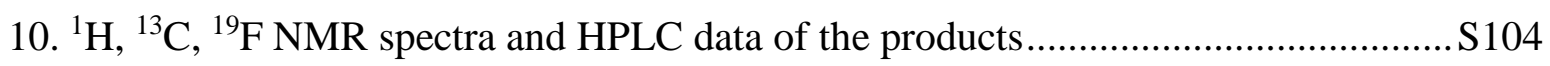

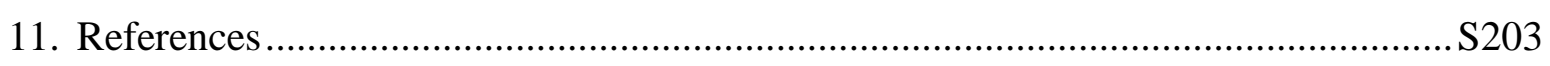




\section{General information}

\section{Materials:}

All the reactions of palladium-catalyzed enantioselective carbene insertion into strained C-Si bonds of silacyclobutanes were performed with a dried reaction tube. All solvents were distilled under nitrogen atmosphere prior to use. Toluene and dioxane were distilled over $\mathrm{Na}$ with a benzophenone-ketyl intermediate as an indicator. For chromatography, 200-300 mesh silica gel (Yantai, China) was employed. $\operatorname{PdCp}\left(\eta^{3}-\mathrm{C}_{3} \mathrm{H}_{5}\right)$ was prepared according to the literature procedures ${ }^{1}$, and 2,4,6-triisopropylbenzenesulfonyl hydrazones were also prepared according to previously reported procedures ${ }^{2}$. The chiral phosphoramidites were purchased from Daicel and TCI. Other starting materials were obtained from commercial suppliers and were used without further purification.

\section{Analytical Methods:}

${ }^{1} \mathrm{H}$ and ${ }^{13} \mathrm{C}$ NMR spectra were recorded at $400 \mathrm{MHz}$ and $100 \mathrm{MHz}$ with Brucker ARX 400 spectrometer. ${ }^{19}$ F NMR was recorded at $471 \mathrm{MHz}$ with Brucker ARX 500 spectrometer. Chemical shifts are reported in ppm using tetramethylsilane (0) as internal standard when using $\mathrm{CDCl}_{3}$ as solvent for ${ }^{1} \mathrm{H} \mathrm{NMR}$ spectra. For ${ }^{13} \mathrm{C}$ NMR spectra, $\mathrm{CDCl}_{3}$ was used as the internal standard with chemical shift at $77 \mathrm{ppm}$. For ${ }^{19} \mathrm{~F}$ NMR spectra, $\mathrm{CFCl}_{3}$ was used as the reference with chemical shift at $0 \mathrm{ppm}$. The data for NMR spectra were reported as following: chemical shifts $(\delta)$ were reported in ppm, and coupling constants $(\mathrm{J})$ were reported in Hertz $(\mathrm{Hz})$. The following abbreviations were used to symbolize the multiplicities: $\mathrm{s}=$ singlet, $\mathrm{d}=\mathrm{doublet}, \mathrm{t}$ $=$ triplet, $\mathrm{q}=$ quartet, $\mathrm{m}=$ multiplet, $\mathrm{br}=$ broad. Infrared spectra were recorded on a Nicolet Avatar 330 Fourier transform spectrometer (FT-IR) and are reported in terms of frequency of absorption $\left(\mathrm{cm}^{-1}\right)$. HRMS was detected through Thermo scientific Q executive. GC-MS, ESI-mass data were obtained on Bruker APEX IV FTMS. Enantiomeric excesses (ee) values were measured with HPLC (Agilent Technologies 1200 series) with Daicel chiral column, eluted with n-hexane and isopropanol. Optical rotations were measured with CANY JH-P300 Polarimeter and concentrations (c) were reported in $\mathrm{g} \times(100 \mathrm{~mL})^{-1}$. Single crystal X-ray diffraction data were collected at $180 \mathrm{~K}$ using graphite-monochromated $\mathrm{Cu}-\mathrm{K} \alpha$ radiation $(\lambda=1.54178 \mathrm{~A})$ on a SuperNova X-ray Diffraction System from Agilent Technologies. 


\section{General procedure to synthesize silacyclobutanes}

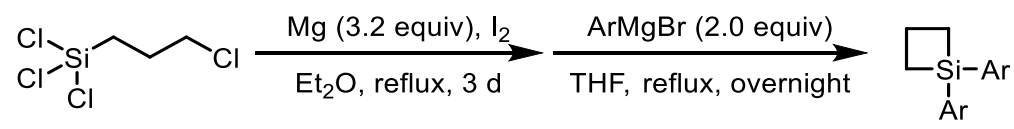

The silacyclobutanes was prepared according to the literature with modification ${ }^{3}$. Magnesium $(768 \mathrm{mg}$, $32 \mathrm{mmol}), 0.2 \mathrm{~mL}$ solution of the (3-chloropropyl)-trichlorosilane in $\mathrm{Et}_{2} \mathrm{O}$ ((3-chloropropyl)-trichlorosilane $(2.12 \mathrm{~g}, 10 \mathrm{mmol})$ was dissolved in $\left.3.0 \mathrm{mLEt}_{2} \mathrm{O}\right)$ and a grain of $\mathrm{I}_{2}$ in $\operatorname{dry}_{\mathrm{Et}} \mathrm{O}(2.0 \mathrm{~mL})$ were heated to reflux. The rest of the (3-chloropropyl)-trichlorosilane solution was dropwise over a period of $0.5 \mathrm{~h}$. The reaction began to thicken after $1-3 \mathrm{~h}$, and an additional $10 \mathrm{~mL}$ of $\mathrm{Et}_{2} \mathrm{O}$ was added. The reaction was stirred for 3 days, and $\mathrm{Et}_{2} \mathrm{O}$ was added (for a total of $40 \mathrm{~mL}$ ) periodically as the reaction became very thick. After 3 days the reaction was allowed to cool to room temperature, and the magnesium chloride/excess magnesium was filtered and washed several times with $\mathrm{Et}_{2} \mathrm{O}$. To the crude, the Grignard reagent of aromatic bromide $(1.0 \mathrm{M}$, $20 \mathrm{~mL}, 20 \mathrm{mmol}$, prepared from aromatic bromide and Magnesium in THF at gentle reflux) was added dropwise at $0{ }^{\circ} \mathrm{C}$. Then the solution was allowed to warm to room temperature and reflux overnight. After cooling to room temperature, the reaction was quenched with water and extracted with $\mathrm{Et}_{2} \mathrm{O}(3 \times 15 \mathrm{~mL})$. The combined organic layers were then dried over $\mathrm{Na}_{2} \mathrm{SO}_{4}$ and concentrated under reduced vacuum. The residue was purified by silica gel flash column chromatography to afford the corresponding silacyclobutanes.

\section{1,1-diphenylsiletane (2a)}

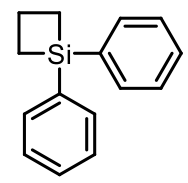

The title compound was prepared according to the general procedure; spectra data are in agreement with literature values. ${ }^{4}$

\section{1,1-bis(4-(trifluoromethyl)phenyl)siletane (2b)}

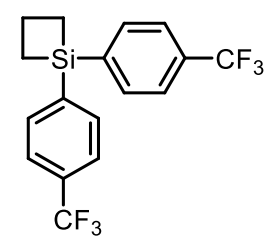

The title compound was prepared according to the general procedure; spectra data are in agreement with literature values. ${ }^{5}$

\section{1,1-di-p-tolylsiletane (2c)}

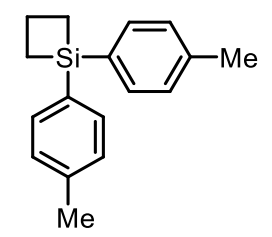

The title compound was prepared according to the general procedure; spectra data are in agreement with literature values..$^{5}$

\section{1,1-di-m-tolylsiletane (2d)}




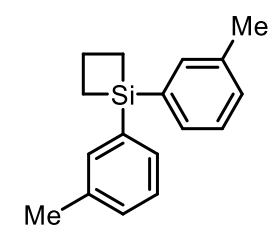

Yield: 54\% (1.36 g); colorless oil; ${ }^{1} \mathrm{H}$ NMR (400 MHz, $\left.\mathrm{CDCl}_{3}\right) \delta$ 7.44-7.40 (m, 4H), $7.28(\mathrm{t}, J=7.2 \mathrm{~Hz}, 2 \mathrm{H})$, 7.22-7.20 (m, 2H), 2.35 (s, 6H), 2.29-2.21 (m, 2H), $1.48(\mathrm{t}, J=8.2 \mathrm{~Hz}, 4 \mathrm{H}) ;{ }^{13} \mathrm{C} \mathrm{NMR}\left(100 \mathrm{MHz}, \mathrm{CDCl}_{3}\right) \delta$ 137.3, 136.4 135.0, 131.5, 130.4, 127.9, 21.5, 18.3, 13.8; HRMS (EI, m/z): calcd for $\mathrm{C}_{17} \mathrm{H}_{20} \mathrm{Si}\left[\mathrm{M}^{+}\right] 252.1329$, found 252.1328; IR (film): 2928, 1119, 873, 776, $702 \mathrm{~cm}^{-1}$.

\section{1,1-di-o-tolylsiletane (2e)}<smiles>Cc1ccccc1[Si]1(c2ccccc2C)CCC1</smiles>

Yield: 42\% (1.07 g); white solid; ${ }^{1} \mathrm{H}$ NMR (400 MHz, $\left.\mathrm{CDCl}_{3}\right) \delta 7.68(\mathrm{~d}, J=7.2 \mathrm{~Hz}, 2 \mathrm{H}), 7.31-7.27(\mathrm{~m}, 2 \mathrm{H})$, 7.25-7.21 (m, 2H), $7.11(\mathrm{~d}, J=7.4 \mathrm{~Hz}, 2 \mathrm{H}), 2.26(\mathrm{~s}, 6 \mathrm{H}), 2.26-2.19(\mathrm{~m}, 2 \mathrm{H}), 1.55(\mathrm{t}, J=8.2 \mathrm{~Hz}, 4 \mathrm{H}) ;{ }^{13} \mathrm{C}$ NMR $\left(100 \mathrm{MHz}, \mathrm{CDCl}_{3}\right) \delta 143.8,135.7,134.8,129.7,129.5,124.9,22.5,18.4,13.8 ; \mathrm{HRMS}(\mathrm{EI}, \mathrm{m} / \mathrm{z})$ : calcd for $\mathrm{C}_{17} \mathrm{H}_{20} \mathrm{Si}\left[\mathrm{M}^{+}\right]$252.1329, found 252.1328; IR (film): 3057, 2931, 1451, 1131, 860, 744, $695 \mathrm{~cm}^{-1}$.

\section{1,1-bis(4-methoxyphenyl)siletane (2f)}

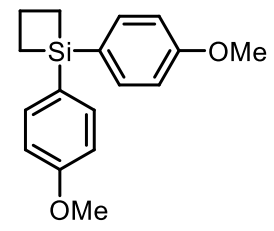

The title compound was prepared according to the general procedure; spectra data are in agreement with literature values. ${ }^{6}$

\section{1,1-bis(4-chlorophenyl)siletane (2g)}

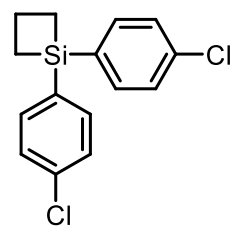

The title compound was prepared according to the general procedure; spectra data are in agreement with literature values. ${ }^{5}$

1,1-bis(4-fluorophenyl)siletane (2h)

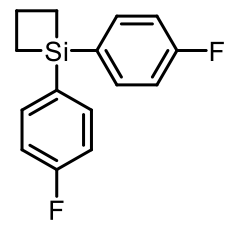

The title compound was prepared according to the general procedure; spectra data are in agreement with literature values. ${ }^{5}$ 


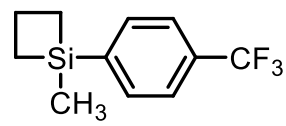

The title compound was prepared according to the literature ${ }^{21}$; spectra data are in agreement with literature values. $^{21}$

\section{Optimization for the carbene insertion into strained $\mathrm{C}-\mathrm{Si}$ bonds}

\section{Scheme S1. Optimization of the Reactions with Various SCBs ${ }^{a}$}

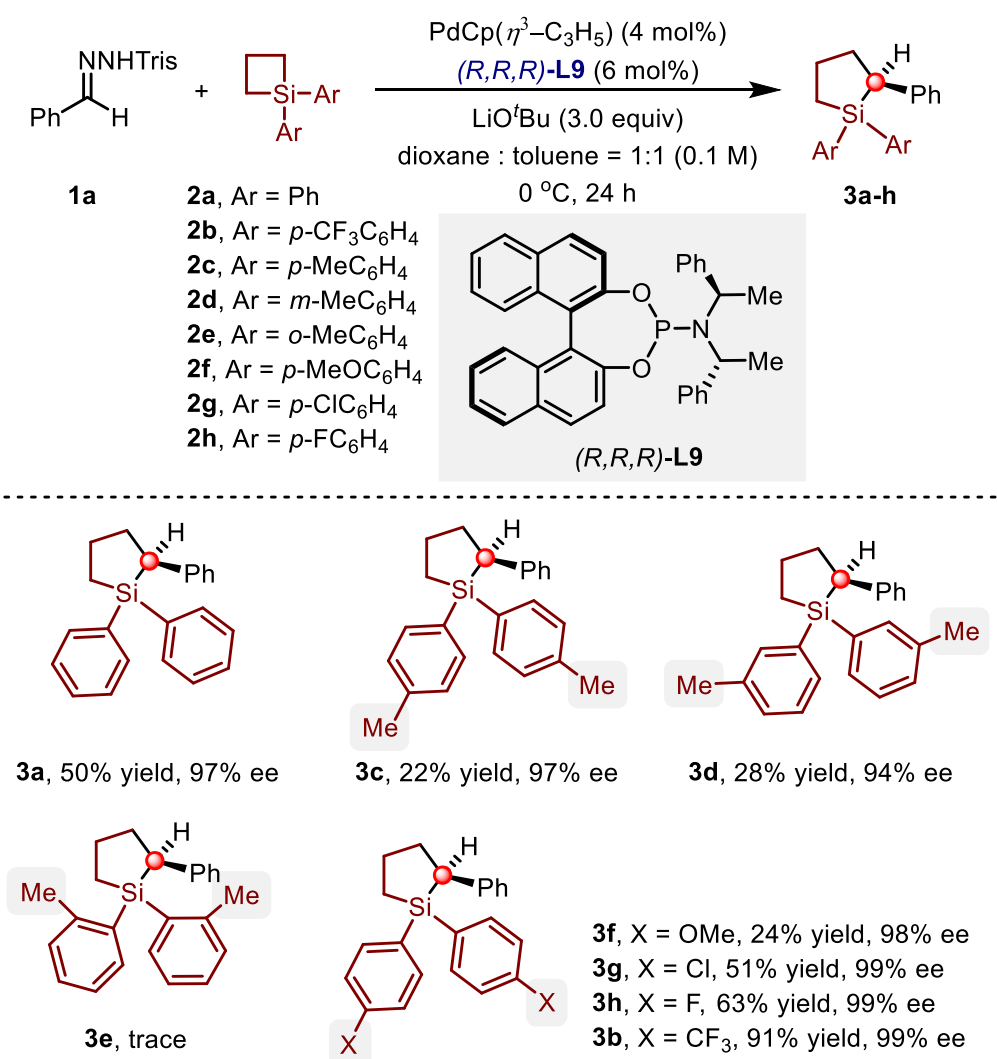

${ }^{a}$ Reaction conditions: $\mathbf{1 a}\left(0.10 \mathrm{mmol}, 1.0\right.$ equiv), $\mathbf{2 a - h}\left(0.15 \mathrm{mmol}, 1.5\right.$ equiv), $\mathrm{PdCp}\left(\eta^{3}-\mathrm{C}_{3} \mathrm{H}_{5}\right)(4 \mathrm{~mol} \%)$, $(R, R, R)-\mathbf{L 9}(6 \mathrm{~mol} \%), \mathrm{LiO}^{t} \mathrm{Bu}\left(3.0\right.$ equiv), dioxane : toluene $=1: 1(1.0 \mathrm{~mL}), 0{ }^{\circ} \mathrm{C}, 24 \mathrm{~h}$. Isolated yields are reported; ee values were determined by HPLC.

After a survey of chiral ligands, we found that with $\mathrm{PdCp}\left(\eta^{3}-\mathrm{C}_{3} \mathrm{H}_{5}\right)$ as the catalyst precursor and phosphoramidite $(R, R, R)$-L9 as the chiral ligand, the reaction could proceed at $0{ }^{\circ} \mathrm{C}$ when using trisylhydrazone 1a as the carbene precursor, affording $\mathbf{3 a}$ in $50 \%$ yield with $97 \%$ ee. To further improve the yield of the reaction, the 1,1-diarylsiletanes having substituent groups at the aromatic moiety were examined (Scheme S1). The 1,1-diarylsiletanes bearing methyl substituents at para and meta positions gave the corresponding products $\mathbf{3 c}$ and $\mathbf{3 d}$ with lower yields, whereas ortho methyl substituted SCB $\mathbf{2 d}$ failed to afford 3e, apparently due to steric effect. The electron-donating MeO-substituted SCB 2e also afforded product $\mathbf{3 f}$ in low yield. On the contrary, the reaction with electron-withdrawing group-substituted SCBs (2g, $\mathbf{2 h}$ ) proceeded effectively, affording the insertion products $(\mathbf{3 g}, \mathbf{3 h})$ with significantly improved yields. 
Finally, the more electron-withdrawing substrate $\mathbf{2 b}$ could afford the desired product $\mathbf{3 b}$ in $91 \%$ yield and $99 \%$ ee. Notably, while the yields of the insertion reactions were affected by the substituents, the reaction consistently gave high enantioselectivities for most of the reactions.

\section{Scheme S2. Optimization of the Reactions with Various alkyl-substituted silacyclobutanes}
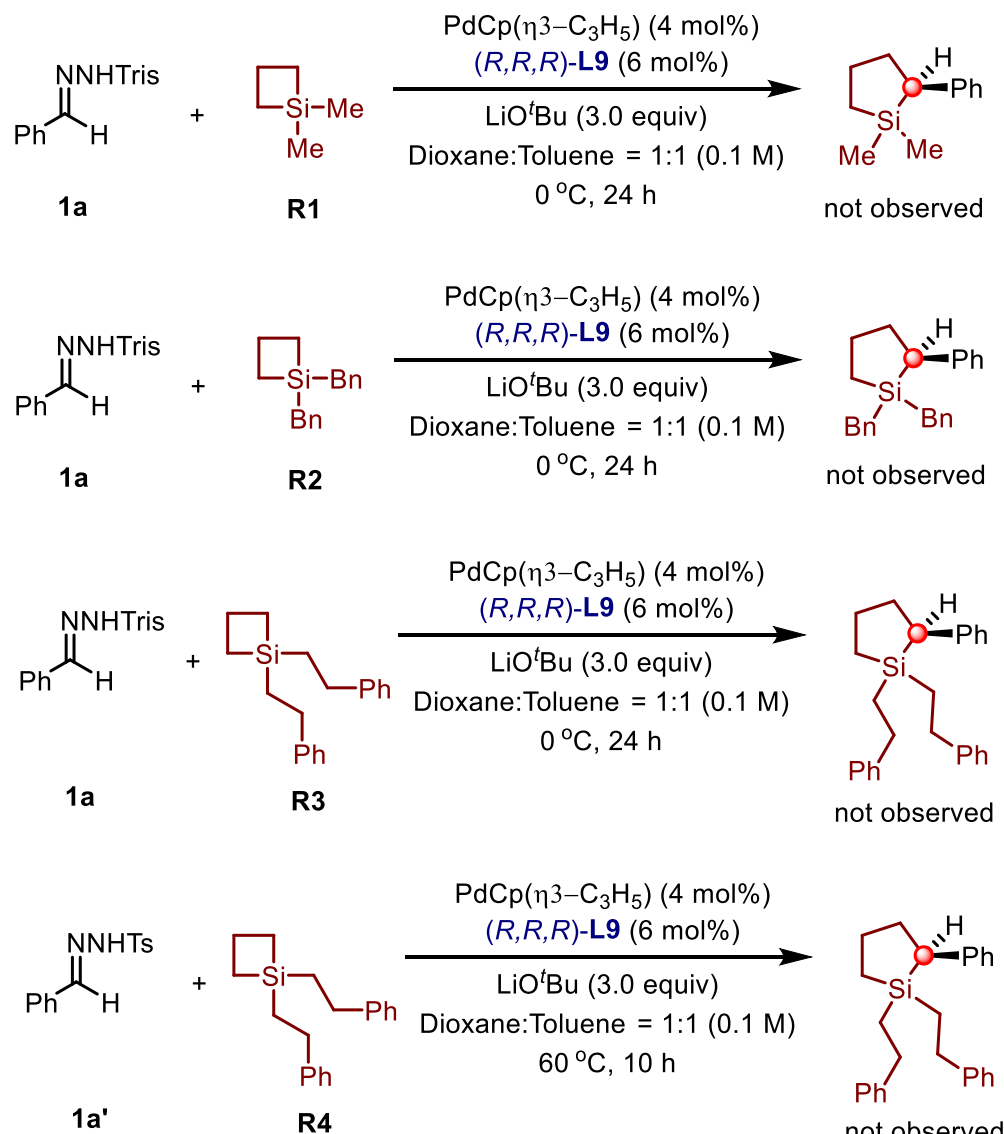

Table S1. Optimization for enantioselective carbene insertion into C-Si of silacyclobutane with trisylhydrazones derived from aromatic ketones

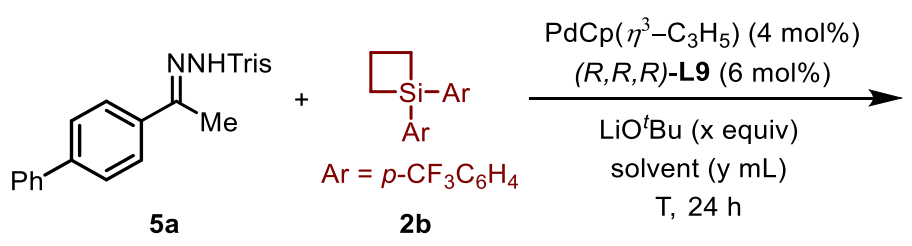

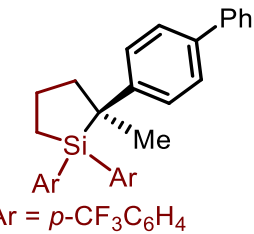

$6 a$ 


\begin{tabular}{cccccc}
\hline Entry & x equiv & Solvent $(\mathrm{y} \mathrm{mL})$ & $\mathrm{T}\left({ }^{\circ} \mathrm{C}\right)$ & Yield $(\%)^{a}$ & ee $(\%)$ \\
\hline 1 & 3.0 & dioxane : toluene $=1: 1(1.0 \mathrm{~mL})$ & 0 & trace & - \\
2 & 3.0 & dioxane : toluene $=1: 1(1.0 \mathrm{~mL})$ & $\mathrm{RT}$ & 32 & 99 \\
3 & 3.0 & dioxane : toluene $=1: 1(1.0 \mathrm{~mL})$ & 40 & 27 & 99 \\
4 & 3.0 & tolune $(1.0 \mathrm{~mL})$ & $\mathrm{RT}$ & 56 & 99 \\
5 & 3.0 & dioxane $(1.0 \mathrm{~mL})$ & $\mathrm{RT}$ & 23 & 99 \\
6 & 3.0 & tolune $(0.5 \mathrm{~mL})$ & $\mathrm{RT}$ & 62 & 99 \\
$7^{b}$ & 3.0 & tolune $(0.5 \mathrm{~mL})$ & $\mathrm{RT}$ & 75 & 99 \\
$\mathbf{8}^{b}$ & $\mathbf{6 . 0}$ & tolune $(0.5 \mathrm{~mL})$ & $\mathrm{RT}$ & $\mathbf{7 9}$ & 99 \\
\hline
\end{tabular}

Reaction conditions: $\mathbf{2 b}\left(0.15 \mathrm{mmol}, 1.5\right.$ equiv), 5a (0.10 mmol, 1.0 equiv), $\mathrm{PdCp}\left(\eta^{3}-\mathrm{C}_{3} \mathrm{H}_{5}\right)(4 \mathrm{~mol} \%)$, $(R, R, R)$-L9 (6 mol\%), $\mathrm{LiO}^{t} \mathrm{Bu}$ (x equiv), solvent (y mL), T, $24 \mathrm{~h} .{ }^{a}$ Isolated yields are reported. Enantiomeric excesses (ee) were determined by HPLC. ${ }^{b}$ The reaction time was $36 \mathrm{~h}$.

\section{General procedure for palladium-catalyzed carbene insertion into strained C-Si bonds}

In an oven dried $10 \mathrm{~mL}$ Schlenk tube equipped with a magnetic stir bar, $\operatorname{PdCp}\left(\eta^{3}-\mathrm{C}_{3} \mathrm{H}_{5}\right)(0.85 \mathrm{mg}, 0.004$ mmol, $4 \mathrm{~mol} \%)$ and ligand $(R, R, R)-\mathbf{L 9}(3.2 \mathrm{mg}, 0.006 \mathrm{mmol}, 6 \mathrm{~mol} \%)$ were added into toluene $(0.5 \mathrm{~mL})$ and dioxane $(0.5 \mathrm{~mL})$ under nitrogen atmosphere. The mixture was stirred for 30 minutes at room temperature. In another separate Schlenk tube, 2,4,6-triisopropylbenzenesulfonyl hydrazones derived from aldehydes $(0.1$ mmol, 1.0 equiv), silacyclobutanes ( $0.15 \mathrm{mmol}, 1.5$ equiv) (if solid) and $\mathrm{LiO}^{t} \mathrm{Bu}(24 \mathrm{mg}, 0.3 \mathrm{mmol}, 3.0$ equiv) were added, and the tube was evacuated and filled with nitrogen (three times). Then the solution containing the Pd complex was added to this tube by syringe. The reaction mixture was stirred for $24 \mathrm{~h}$ at $0{ }^{\circ} \mathrm{C}$. After completion of the reaction, the reaction mixture was filtered through a short plug of silica gel with ethyl acetate as eluents. The solvent was removed with rotary evaporator under reduced pressure to leave a crude mixture, which was purified by preparative thin-layer chromatography to afford pure product.

For 2,4,6-triisopropylbenzenesulfonyl hydrazones derived from aromatic ketones, 6.0 equiv $\mathrm{LiO}^{t} \mathrm{Bu}$ and $0.5 \mathrm{~mL}$ toluene were used, and the reaction was conducted at room temperature for $36 \mathrm{~h}$.

\section{Synthetic applications}

\subsection{Gram-scale synthesis}

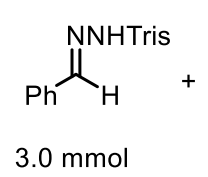

$1 \mathbf{a}$

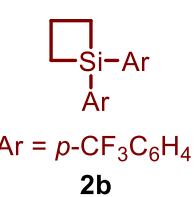

2b

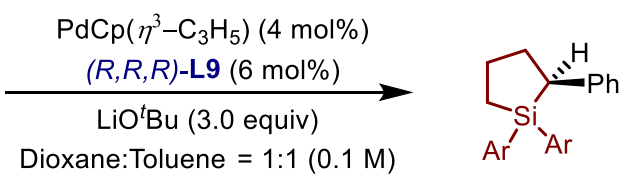

$0^{\circ} \mathrm{C}, 24 \mathrm{~h}$

3b, 1.265 g, $94 \%$ yield, $>99 \%$ ee

In an oven dried $100 \mathrm{~mL}$ Schlenk flask equipped with a magnetic stir bar, $\operatorname{PdCp}\left(\eta^{3}-\mathrm{C}_{3} \mathrm{H}_{5}\right)(25.5 \mathrm{mg}, 0.12$ mmol, $4 \mathrm{~mol} \%)$ and ligand $(R, R, R)-\mathbf{L 9}(112.4 \mathrm{mg}, 0.18 \mathrm{mmol}, 6 \mathrm{~mol} \%)$ were added into toluene $(15.0 \mathrm{~mL})$ and dioxane $(15.0 \mathrm{~mL})$ under nitrogen atmosphere. The mixture was stirred for 30 minutes at room temperature. In another separate oven dried $100 \mathrm{~mL}$ Schlenk flask, trisylhydrazone 1a (1.16 g, $3.0 \mathrm{mmol}, 1.0$ equiv), silacyclobutane $\mathbf{2 b}$ (1.62 g, $4.5 \mathrm{mmol}, 1.5$ equiv) and $\mathrm{LiO}^{t} \mathrm{Bu}(720 \mathrm{mg}, 9.0 \mathrm{mmol}, 3.0$ equiv) were 
added, and the tube was evacuated and filled with nitrogen (three times). Then the solution containing the Pd complex was added to this flask by syringe. The reaction mixture was stirred for $24 \mathrm{~h}$ at $0{ }^{\circ} \mathrm{C}$. After completion of the reaction, the reaction mixture was filtered through a short plug of silica gel with ethyl acetate as eluents. The solvent was removed with rotary evaporator under reduced pressure to leave a crude mixture, which was purified by silica gel column chromatography (PE) to afford pure product $\mathbf{3 b}(1.265 \mathrm{~g}$, $95 \%$ yield, $>99 \%$ ee).

\subsection{Oxidative hydrolysis of 3a to the corresponding chiral 1,4-diol}

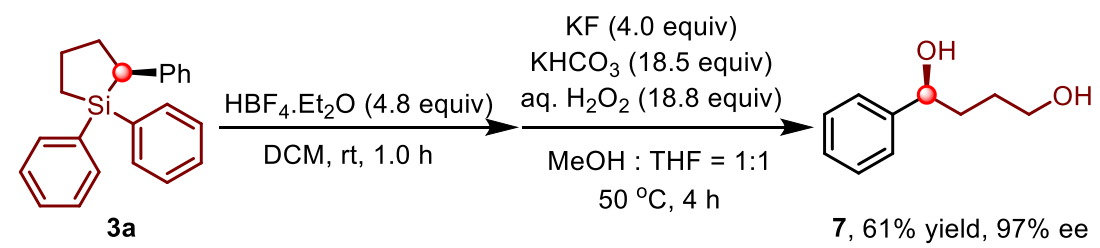

Tetrafluoroboric acid $\left(\mathrm{HBF}_{4}\right.$. $\left.\mathrm{OEt}_{2}, 148 \mathrm{mg}, 0.91 \mathrm{mmol}\right)$ was added to a solution of $\mathbf{3 a}(60.0 \mathrm{mg}, 0.19 \mathrm{mmol})$ in dichloromethane $(0.6 \mathrm{~mL})$ at $0{ }^{\circ} \mathrm{C}$ and the reaction mixture was warmed to room temperature and stirred for $4 \mathrm{~h}$. The mixture was concentrated in vacuo and the residual oil was dissolved in THF $(1.0 \mathrm{~mL})$ and $\mathrm{MeOH}(1.0 \mathrm{~mL}$ ). Then $\mathrm{KF}$ (44 mg, $0.76 \mathrm{mmol}), \mathrm{KHCO}_{3}(350 \mathrm{mg}, 3.5 \mathrm{mmol})$ and $\mathrm{H}_{2} \mathrm{O}_{2}(30 \%, 495 \mathrm{mg}, 4.37$ mmol) were added and the mixture was stirred at $50{ }^{\circ} \mathrm{C}$ overnight. After cooling to room temperature, the mixture was diluted with saturated aqueous $\mathrm{NH}_{4} \mathrm{Cl}$ and extracted with $\mathrm{CH}_{2} \mathrm{Cl}_{2}$. The organic extracts were dried over $\mathrm{Na}_{2} \mathrm{SO}_{4}$ and concentrated under reduced pressure. The residue was purified by flash column chromatography $(\mathrm{PE}: \mathrm{EtOAc}=1: 4)$ to afford the alcohol $7(61 \%$ yield, $97 \%$ ee $)$ as white solid.

\subsection{The reaction with natural product and drug molecules}<smiles>C=C(C)[C@H]1CC=C(C=O)CC1</smiles>

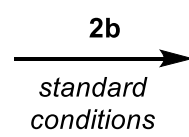

8

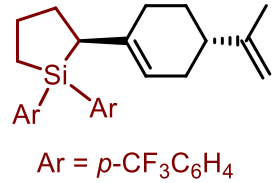

9, $70 \%$ yield, $94 \%$ de

The natural product (-)-perillaldehyde was transformed to the corresponding 2,4,6triisopropylbenzenesulfonyl hydrazones $\mathbf{8}$ according to the literature procedure ${ }^{2}$. Then the silacyclopentane $\mathbf{9}$ (34.6 mg, 70\% yield, $94 \%$ de) could be obtained under standard conditions using hydrazones 8 (0.1 mmol, 1.0 equiv) as substrate.

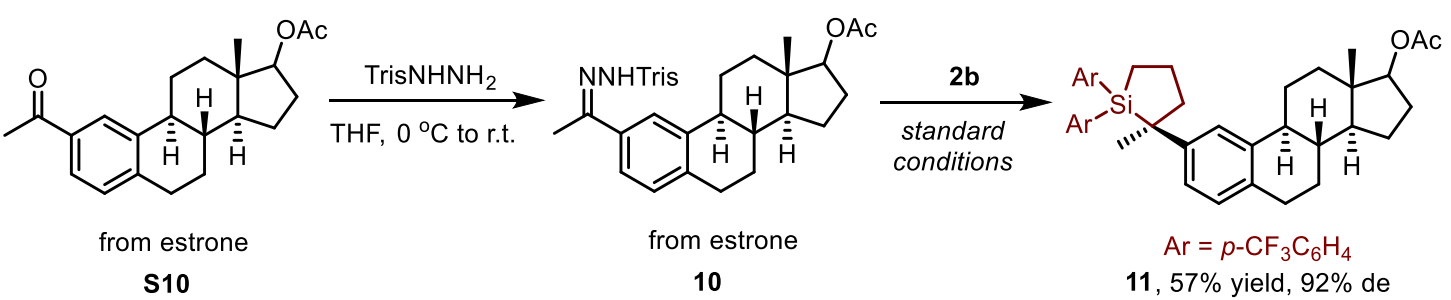

Compound S10 was synthesized by the same procedure of literature. ${ }^{7}$ Then $\mathbf{S 1 0}$ was transformed to the corresponding trisylhydrazone $\mathbf{1 0}$ according to the literature procedure. ${ }^{2}$ Under standard conditions, trisylhydrazone $\mathbf{1 0}$ could react smoothly with silacyclobutane $\mathbf{2 b}$ to give the product $\mathbf{1 1}$ ( $38.9 \mathrm{mg}, 57 \%$ yield, 
$92 \%$ de) in excellent stereoselectivity.

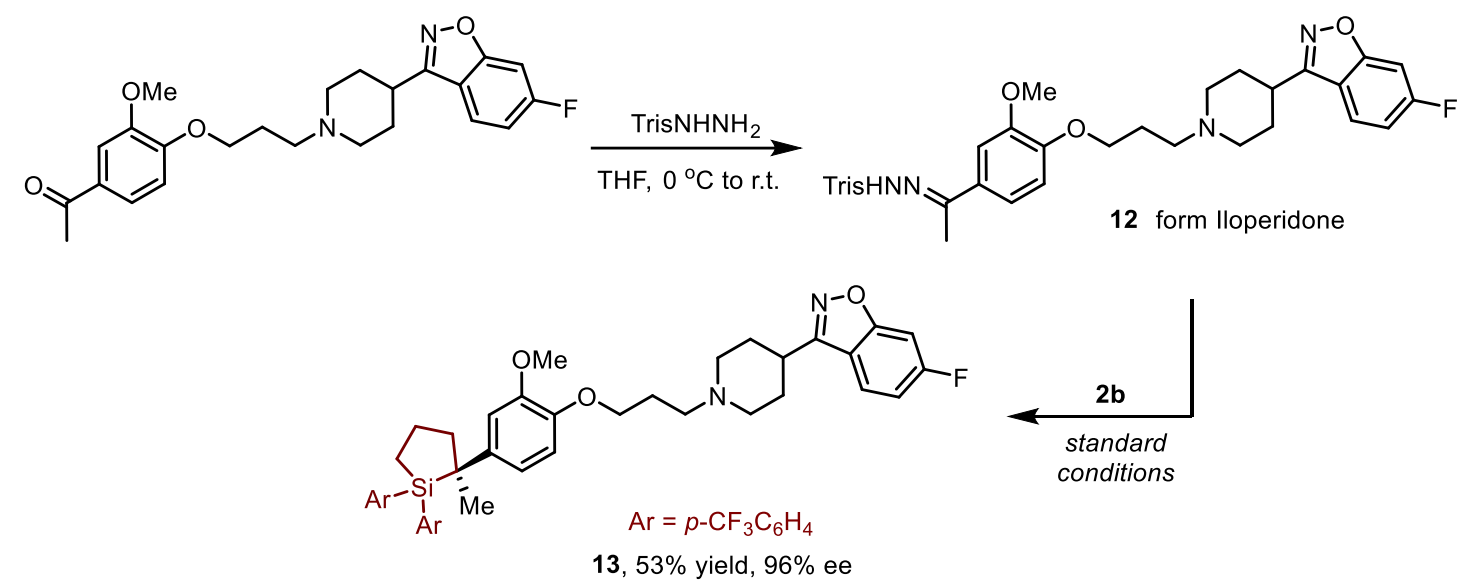

Drug molecules iloperidone was transformed to the corresponding trisylhydrazone $\mathbf{1 2}$ according to the literature procedure. ${ }^{2}$ Then the derivative products $\mathbf{1 3}(41.0 \mathrm{mg}, 53 \%$ yield, $96 \%$ ee) could be obtained as a white solid under standard conditions.

\section{Mechanistic studies}

\subsection{Non-Linear Effect Studies}

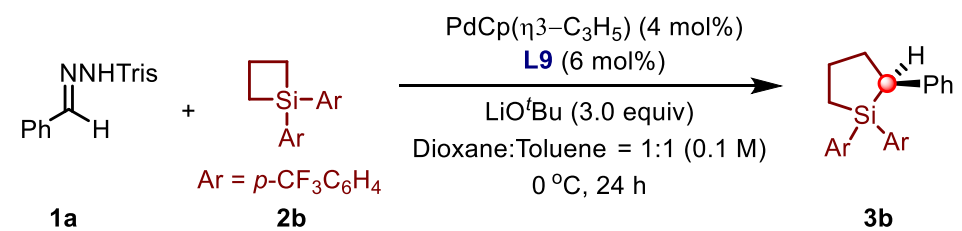

Combining the certain amounts of optically pure $(R, R, R)$-L9 with pure $(S, S, S)$-L9 leads to the formation of the specified $e e$ values of chiral ligand. Six reactions containing the mixed ligand of racemic, $20 \%, 40 \%$, $60 \%, 80 \%$ and $>99 \%$ ee optical purity were performed in parallel. A linear plot of ee prod $_{\text {(the enantiomeric }}$ excess of product) as a function of eeligand (the enantiomeric excess of ligand $\mathbf{L 9}$ ) was obtained $\left(\mathrm{R}_{2}=0.97\right)$ (Figure S2). The absence of an NLE indicated that most likely one chiral ligand L9 was involved in the catalytic cycle.

General procedure: In an oven dried $10 \mathrm{~mL}$ Schlenk tube equipped with a magnetic stir bar, $(R, R, R)-\mathbf{L 9}$ and (S,S,S)-L9 (both $c=10.0 \mathrm{mg} / \mathrm{mL}$ in toluene) (a total mass of $3.2 \mathrm{mg}, 0.006 \mathrm{mmol}, 6 \mathrm{~mol} \%$ ), $\mathrm{PdCp}\left(\eta^{3}-\right.$ $\left.\mathrm{C}_{3} \mathrm{H}_{5}\right)(0.85 \mathrm{mg}, 0.004 \mathrm{mmol}, 4 \mathrm{~mol} \%)$ were added into toluene $(0.5 \mathrm{~mL})$ and dioxane $(0.5 \mathrm{~mL})$ under nitrogen atmosphere. The mixture was stirred for 30 minutes at room temperature. In another separate Schlenk tube, 2,4,6-triisopropylbenzenesulfonyl hydrazones derived from aldehydes 1a (0.1 mmol, 1.0 equiv), silacyclobutanes $\mathbf{2 b}\left(0.15 \mathrm{mmol}, 1.5\right.$ equiv) and $\mathrm{LiO}^{t} \mathrm{Bu}(24 \mathrm{mg}, 0.3 \mathrm{mmol}, 3.0$ equiv) were added, and the tube was evacuated and filled with nitrogen (three times). Then the solution containing the Pd complex was added to this tube by syringe. The reaction mixture was stirred for $24 \mathrm{~h}$ at $0{ }^{\circ} \mathrm{C}$. Then it was filtered through a short plug of silica gel. The organic phase was concentrated in vacuo. The mixture was purified by flash column chromatography (silica gel, PE) to give the enantioenriched $\mathbf{3 b}$. A graph of ee of product versus ee of ligand was then plotted. 


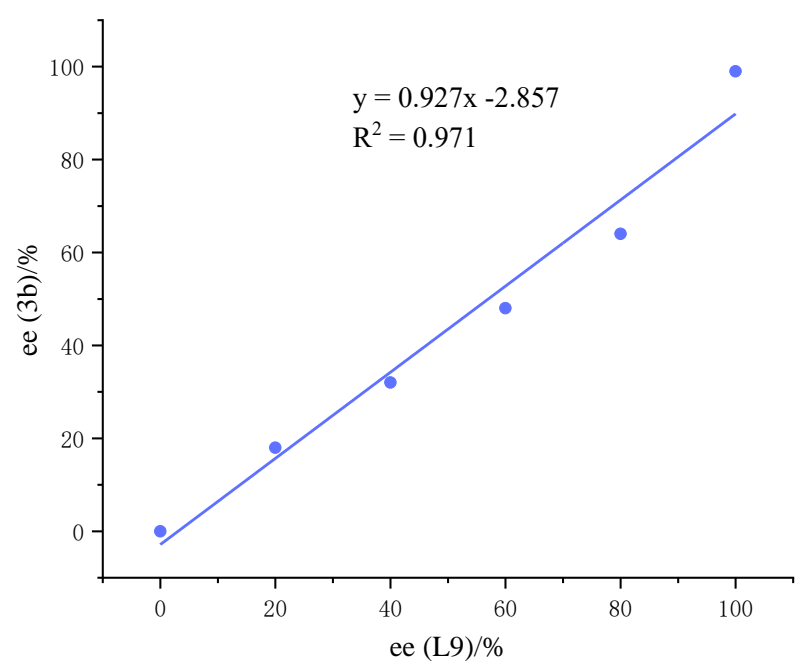

\begin{tabular}{ccc}
\hline Entry & ee (L9)\% & ee (3b)\% \\
\hline 1 & 0 & 0 \\
2 & 20 & 18 \\
3 & 40 & 32 \\
4 & 60 & 48 \\
5 & 80 & 64 \\
6 & 100 & 99 \\
\hline
\end{tabular}

Figure S2. Nonlinear effect studies with chiral ligand L9 


\section{Computational studies with DFT calculation}

Complete reference for Gaussian 09 M. J. Frisch, G. W. Trucks, H. B. Schlegel, G. E. Scuseria, M. A. Robb, J. R. Cheeseman, G. Scalmani, V. Barone, B. Men-nucci, G. A. Petersson, H. Nakatsuji, M. Caricato, X. Li, H. P. Hratchian, A. F. Izmaylov, J. Bloino, G. Zheng, J. L. Sonnenberg, M. Hada, M. Ehara, K. Toyota, R. Fukuda, J. Hasegawa, M. Ishida, T. Nakajima, Y. Honda, O. Kitao, H. Nakai, T. Vreven, J. A. Montgomery, Jr., J. E. Peralta, F. Ogliaro, M. Bearpark, J. J. Heyd, E. Brothers, K. N. Kudin, V. N. Staroverov, R. Kobayashi, J. Normand, K. Raghavachari, A. Rendell, J. C. Burant, S. S. Iyen-gar, J. T omasi, M. Cossi, N. Rega, J. M. Millam, M. Klene, J. E. Knox, J. B. Cross, V. Bakken, C. Adamo, J. Jaramillo, R. Gomperts, R. E. Stratmann, O. Yazyev, A. J. Austin, R. Cammi, C. Pomelli, J. W. Ochterski, R. L. Martin, K. Morokuma, V. G. Zakrzewski, G. A. Voth, P. Salvador, J. J. Dannenberg, S. Dapprich, A. D. Daniels, Ö. Farkas, J. B. Foresman, J. V. Ortiz, J. Cio-slowski, D. J. Fox, Gaussian 09, revision D.01; Gaussian, Inc.: Wallingford, CT, 2013.

Computational methods All of the density functional theory (DFT) calculations were performed with the Gaussian 09 series of programs. The B3-LYP functional ${ }^{8,9}$ with the standard 6-31G(d) basis set (LANL08(f) basis set for Pd) was used for the geometry optimizations in the gas phase. Harmonic vibrational frequency calculations were performed for all of the stationary points to determine whether they are local minima or transition structures and to derive the thermochemical corrections for the enthalpies and free energies. The M06-2X functiona $1{ }^{10}$ proposed by Truhlar et al. with the 6-311+G(d,p) basis set (LANL08(f) basis set for Pd) was used to calculate the single-point energies in chloroform solvent to provide more accurate energy information. The solvent effect was considered by single-point calculations based on the gas-phase stationary points with the $\mathrm{SMD}^{11,12}$ continuum solvation model. The Gibbs free energies of the stationary points calculated using the M06-2X functional are used to discuss the energies. The independent gradient model (IGM) was conducted with Multiwfn ${ }^{13,14}$ and VMD. ${ }^{15}$ The 3D images of the calculated structures were prepared using CYLview. ${ }^{16}$

\subsection{Calculation on the choice of starting point in the reaction}

It has been known that the catalytically active species in palladium-catalyzed $\mathrm{C}-\mathrm{C}$ couplings are often monophosphine palladium complexes (i.e., LPd), when bulky phosphine ligands are applied. ${ }^{17,18}$ The starting point of this reaction was examined in order to clarify the oxidative addition process. According to the concentration correction formula, ${ }^{19}$ the coordination of solvent with palladium and the coordination of substrate with palladium were analyzed. The analysis results are shown in Figure S3, which indicated that the coordination between the silacyclobutane and palladium has lower coordination energy. In a previous report, we have noted substrate-coordinated monophosphine palladium was selected as starting point in DFT calculation. ${ }^{20}$ So we selected complex $\mathbf{C} 4$ as the starting point of this reaction, and the whole reaction process was calculated. 


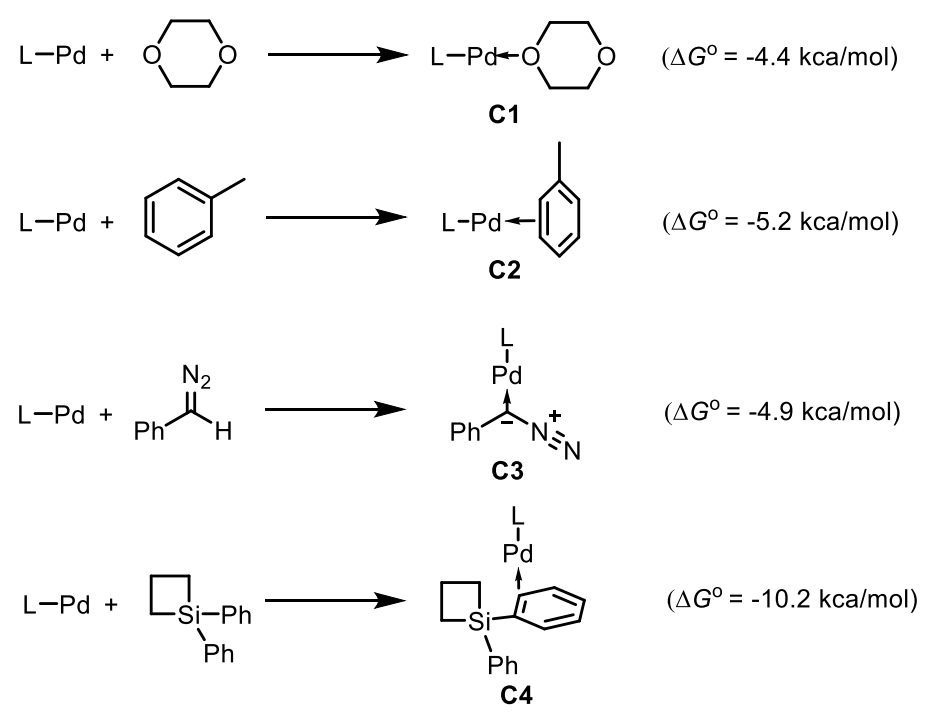

Figure S3. Calculation on the choice of starting point in the reaction. The energies are in $\mathrm{kcal} / \mathrm{mol}$ and represent the relative free energies, which were calculated using the M06-2X method in toluene solvent.

\subsection{Calculation on palladium-catalyzed carbene insertion into C-Si bonds of silacyclobutanes}

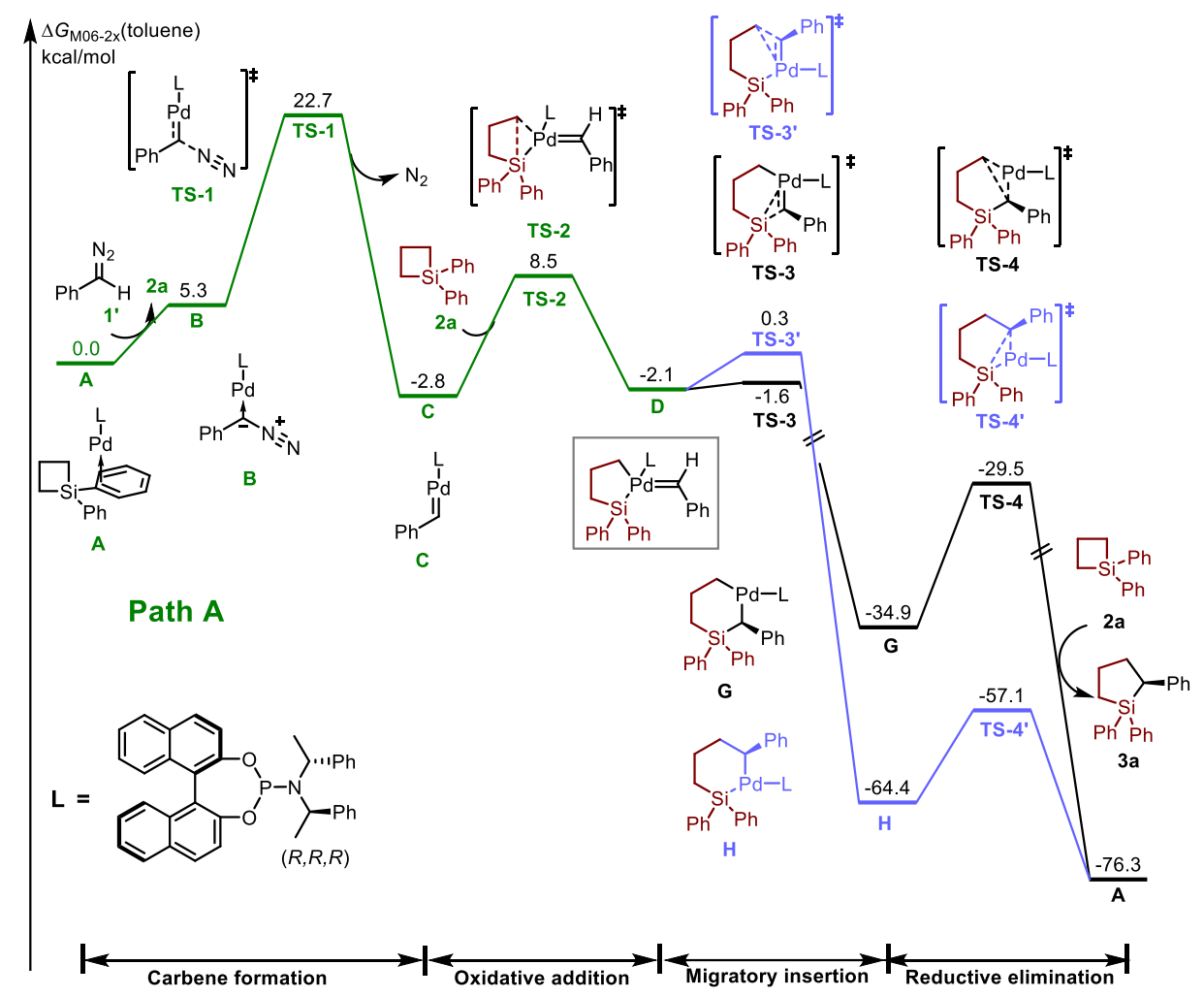

Figure S4. Free energy profiles for path A of catalytic carbene insertion into Si-C bond of silacyclobutane. The energies are in $\mathrm{kcal} / \mathrm{mol}$ and represent the relative free energies, which were calculated using the M06$2 \mathrm{X}$ method in toluene solvent. 
We initially focused on path $\mathbf{A}$, as in experimental work, we chose (diazomethyl)benzene $\mathbf{1}^{\prime}$ and 1,1diphenylsiletane 2a as the model reaction, in which the $(R, R, R)$ - $\mathbf{L}$ ligand is selected as the chiral ligand. The ligand and SCB-coordinated $\operatorname{Pd}(0)$ complex $\mathbf{A}$ was chosen as the starting point for the free energy profiles (Figure S3). Ligand exchange with diazo 1' generates complex B, which is endergonic by $5.3 \mathrm{kcal} / \mathrm{mol}$. Subsequent denitrogenation of intermediate $\mathbf{B}$ occurs via transition state TS-1 by cleavage of the $\mathrm{C}-\mathrm{N}$ bond with an energy barrier of $17.4 \mathrm{kcal} / \mathrm{mol}$ to give palladium-carbene intermediate $\mathbf{C}$, which releases 8.1 $\mathrm{kcal} / \mathrm{mol}$ free energy. Oxidative addition of intermediate $\mathbf{C}$ with 1,1-diphenylsiletane $\mathbf{2 a}$ generates carbenecoordinated palladacycle intermediate $\mathbf{D}$, which is endergonic by $0.7 \mathrm{kcal} / \mathrm{mol}$. The energy barrier for this step is $11.3 \mathrm{kcal} / \mathrm{mol}$. Next, migratory insertion of the carbene moiety into Pd-Si bond or Pd-C bond via sixmembered transition state TS-3 or TS-3' releases $32.8 \mathrm{kcal} / \mathrm{mol}$ or $62.3 \mathrm{kcal} / \mathrm{mol}$ free energy, and sixmembered palladium complex $\mathbf{G}$ or $\mathbf{H}$ is generated. Finally, reductive elimination occurs via transition state TS-4 or TS-4' to release final product silacyclobutane 3a, which is exergonic by 41.4 or $11.9 \mathrm{kcal} / \mathrm{mol}$, and active catalyst palladium species $\mathbf{A}$ is regenerated.

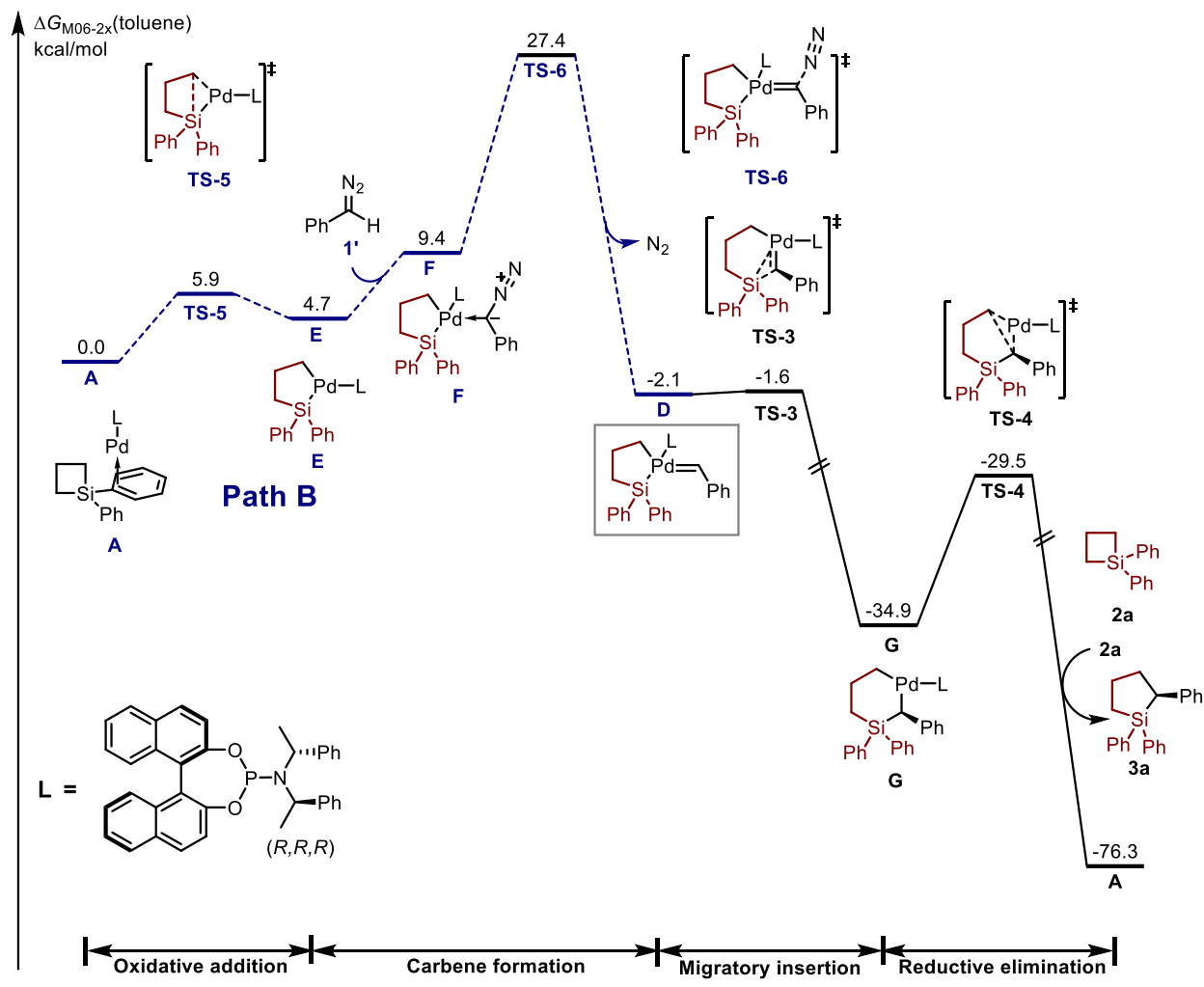

Figure S5. Free energy profiles for path $\mathbf{B}$ of catalytic carbene insertion into Si-C bond of silacyclobutane. The energies are in $\mathrm{kcal} / \mathrm{mol}$ and represent the relative free energies, which were calculated using the M06$2 \mathrm{X}$ method in toluene solvent.

Certainly, an alternative pathway is taken into account (path B) (Figure S4), in which oxidative addition of active catalyst palladium species A firstly occurs with 1,1-diphenylsiletane 2a to generate five-membered ring palladium intermediate $\mathbf{E}$ via transition state $\mathbf{T S}-\mathbf{5}$, which is endergonic by $4.7 \mathrm{kcal} / \mathrm{mol}$. Subsequent coordination of intermediate $\mathbf{E}$ with diazo 1' generates complex $\mathbf{F}$, which is endergonic by $4.7 \mathrm{kcal} / \mathrm{mol}$. 
Carbene formation occurs via transition state TS-6 by dissociation of nitrogen gas with an energy barrier of $22.7 \mathrm{kcal} / \mathrm{mol}$ to give carbene-coordinated palladacycle intermediate $\mathbf{D}$. Next, migratory insertion of the carbene moiety into Pd-Si bond and reductive elimination are the same as path A. The rate-determining step in both two pathways is carbene formation, and the total activation energy of path A is $22.7 \mathrm{kcal} / \mathrm{mol}$ and path $\mathrm{B}$ is $27.4 \mathrm{kcal} / \mathrm{mol}$, which reveals path $\mathrm{A}$ is more favorable.
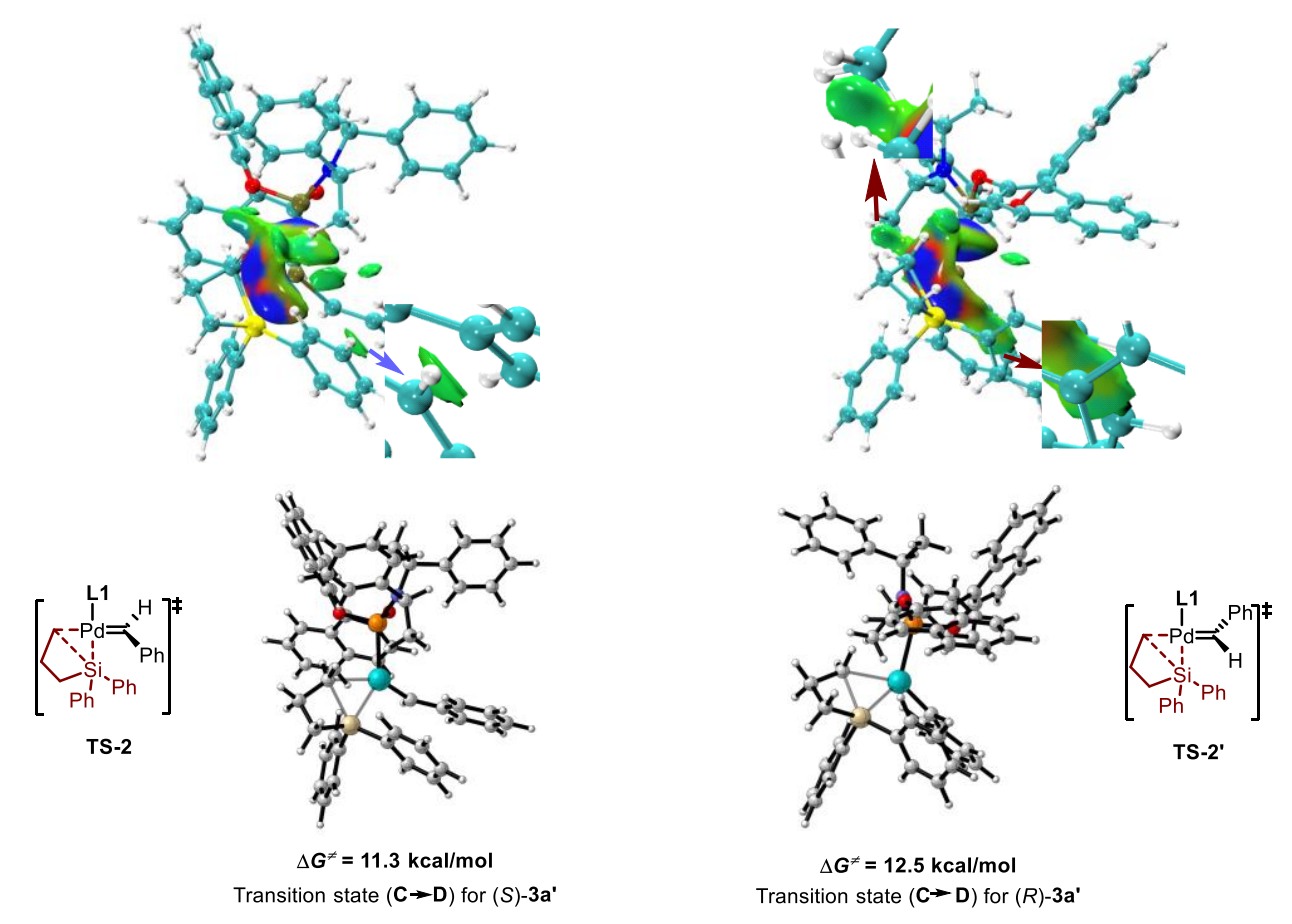

Figure S6. Optimized structures and independent gradient model (IGM) of the transition states for forming $(S)$-3a and $(R)-3 \mathbf{a}$. For IGM analysis, blue, attraction; green, weak interaction; red: steric effect.

To investigate the origin of the enantioselectivity, IGM analysis of the oxidative addition transition states TS-2 and TS-2' were performed (Figure S5). There is a clear steric repulsion between the phenyl group of the carbene moiety and the phenyl group of reacting 1,1-diphenylsiletane in the transition state TS-2' for generating of $(R)$-3a. Furthermore, there is a weak repulsion between 1,1-diphenylsiletane reactant and $(R, R, R)$-L ligand in this transition state. On the contrary, there is a weak attraction between the phenyl group of the carbene moiety and the phenyl group of 1,1-diphenylsiletane in the transition state TS-2 for generating of $(S)$-3a. Therefore, the enantioselectivity is mainly controlled by the steric effect of the oxidative addition transition state, which means that chiral phosphoramidite with large substituent groups is important for the enantioselectivity.

7.3 B3-LYP and M06-2X calculated absolute energies, enthalpies, and free energies for all structures

\begin{tabular}{cccccc}
\hline Geometry & $\mathrm{E}_{(\text {(elec-B3-LYP) }}{ }^{1}$ & $\mathrm{H}_{(\text {corr-B3-LYP) }}$ & $\mathrm{G}_{(\text {corr-B3-LYP) }}{ }^{3}$ & $\mathrm{E}_{(\text {(solv-M06-2X) }}{ }^{4}$ & IF $^{5}$ \\
\hline $\mathbf{A}$ & -2933.980363 & 0.742562 & 0.887099 & -2933.596871 & \\
\hline
\end{tabular}




\begin{tabular}{|c|c|c|c|c|c|}
\hline $1^{\prime}$ & -379.792980 & 0.123186 & 0.082719 & -379.741239 & \\
\hline $\mathbf{S}$ & -307.653142 & 0.129413 & 0.094919 & -307.624693 & \\
\hline B & -2443.054484 & 0.728106 & 0.599489 & -2442.711095 & \\
\hline TS-1 & -2443.036529 & 0.725420 & 0.599068 & -2442.682860 & -495.5 \\
\hline $\mathbf{N}_{2}$ & -109.514600 & 0.008905 & -0.012849 & -109.515843 & \\
\hline C & -2333.550056 & 0.716696 & 0.594916 & -2333.190737 & \\
\hline $2 a$ & -870.713219 & 0.280538 & 0.222693 & -870.615476 & \\
\hline TS-2 & -3204.247444 & 0.999395 & 0.841641 & -3203.812169 & -122.7 \\
\hline TS-2' & -3204.247830 & 0.999393 & 0.842411 & -3203.809547 & -97.2 \\
\hline D & -3204.274657 & 1.000883 & 0.843800 & -3203.831200 & \\
\hline TS-3 & -3204.272828 & 1.000314 & 0.847239 & -3203.833825 & -98.4 \\
\hline TS-3' & -3204.272065 & 0.999974 & 0.847192 & -3203.830801 & -18.2 \\
\hline $\mathbf{G}$ & -3204.307121 & 1.000070 & 0.843952 & -3203.883707 & \\
\hline H & -3204.356193 & 1.003953 & 0.851031 & -3203.937855 & \\
\hline TS-4 & -3204.293000 & 0.999560 & 0.844220 & -3203.875326 & -327.2 \\
\hline TS-4' & -3204.332642 & 1.002760 & 0.850303 & -3203.925393 & -93.2 \\
\hline $3 \mathbf{a}$ & -1141.096731 & 0.398254 & 0.325512 & -1140.969686 & \\
\hline TS-5 & -2933.967682 & 0.886158 & 0.744948 & -2933.589798 & -19.1 \\
\hline $\mathbf{E}$ & -2933.987263 & 0.887780 & 0.748060 & -2933.594824 & \\
\hline $\mathbf{F}$ & -3313.776929 & 1.012712 & 0.850699 & -3313.348559 & \\
\hline TS-6 & -3313.748972 & 1.010453 & 0.849047 & -3313.318138 & -520.2 \\
\hline
\end{tabular}

The electronic energy calculated by B3-LYP in gas phase. ${ }^{2}$ The thermal correction to enthalpy calculated by B3-LYP in gas phase. ${ }^{3}$ The thermal correction to Gibbs free energy calculated by B3-LYP in gas phase. ${ }^{4}$ The 
electronic energy calculated by M06-2X in toluene solvent. ${ }^{5}$ The B3-LYP calculated imaginary frequencies for the transition states.

\subsection{B3-LYP geometries for all the optimized compounds and transition states}

\begin{tabular}{|c|c|c|c|}
\hline $\mathbf{A}$ & & & \\
\hline $\mathrm{C}$ & -3.17185000 & 6.53769600 & -0.90810600 \\
\hline $\mathrm{C}$ & -3.41724200 & 5.50050100 & -1.77832100 \\
\hline $\mathrm{C}$ & -2.73628700 & 4.26004300 & -1.65240200 \\
\hline $\mathrm{C}$ & -1.75054000 & 4.09377200 & -0.62200200 \\
\hline $\mathrm{C}$ & -1.54369100 & 5.18034300 & 0.27447500 \\
\hline $\mathrm{C}$ & -2.23387600 & 6.36502100 & 0.13622500 \\
\hline $\mathrm{H}$ & -3.77020100 & 3.30456000 & -3.30068000 \\
\hline $\mathrm{H}$ & -3.70419700 & 7.47928600 & -1.01070600 \\
\hline $\mathrm{H}$ & -4.15119900 & 5.60835700 & -2.57371300 \\
\hline $\mathrm{C}$ & -3.03386800 & 3.16893100 & -2.51225200 \\
\hline $\mathrm{C}$ & -1.04836600 & 2.83990200 & -0.50284000 \\
\hline $\mathrm{H}$ & -0.83872100 & 5.06535700 & 1.08944900 \\
\hline $\mathrm{H}$ & -2.05948500 & 7.17259600 & 0.84225400 \\
\hline $\mathrm{C}$ & -1.46413400 & 1.78446000 & -1.30680300 \\
\hline $\mathrm{C}$ & -2.43152500 & 1.95040200 & -2.32844200 \\
\hline $\mathrm{H}$ & -2.67518900 & 1.09082700 & -2.94462500 \\
\hline $\mathrm{C}$ & 0.06431300 & 2.64374900 & 0.46908800 \\
\hline $\mathrm{C}$ & 1.22705200 & 3.49639900 & 0.49112000 \\
\hline $\mathrm{C}$ & 0.02094500 & 1.59215200 & 1.37405100 \\
\hline $\mathrm{C}$ & 1.46931100 & 4.49175100 & -0.49759800 \\
\hline $\mathrm{C}$ & 2.21421700 & 3.32163700 & 1.51842400 \\
\hline $\mathrm{C}$ & 1.00091500 & 1.40575100 & 2.37559600 \\
\hline $\mathrm{C}$ & 2.58898000 & 5.29326600 & -0.44803500 \\
\hline $\mathrm{H}$ & 0.76289600 & 4.60982200 & -1.31066600 \\
\hline $\mathrm{C}$ & 3.34543100 & 4.18182700 & 1.55496500 \\
\hline $\mathrm{C}$ & 2.06029900 & 2.27326500 & 2.46401800 \\
\hline $\mathrm{H}$ & 0.88204000 & 0.57416000 & 3.06192400 \\
\hline $\mathrm{C}$ & 3.53196000 & 5.15228200 & 0.59708400 \\
\hline $\mathrm{H}$ & 2.75026800 & 6.03865900 & -1.22213200 \\
\hline $\mathrm{H}$ & 4.07018200 & 4.04740300 & 2.35458400 \\
\hline $\mathrm{H}$ & 2.81135000 & 2.14772000 & 3.23947000 \\
\hline $\mathrm{H}$ & 4.40364500 & 5.80003500 & 0.63323000 \\
\hline $\mathrm{O}$ & -1.03537100 & 0.69259200 & 1.34953000 \\
\hline $\mathrm{O}$ & -0.90322700 & 0.53281100 & -1.19433700 \\
\hline $\mathrm{P}$ & -0.96808600 & -0.49436900 & 0.16138100 \\
\hline $\mathrm{N}$ & -2.53027600 & -1.10666600 & 0.37645400 \\
\hline $\mathrm{C}$ & -2.87415600 & -2.48753700 & -0.11341700 \\
\hline $\mathrm{H}$ & -3.70747200 & -2.78596200 & 0.52972800 \\
\hline
\end{tabular}




\begin{tabular}{|c|c|c|c|}
\hline $\mathrm{C}$ & -1.81227600 & -3.57607600 & 0.09843700 \\
\hline $\mathrm{H}$ & -0.94259100 & -3.46638700 & -0.55410500 \\
\hline $\mathrm{H}$ & -1.46306100 & -3.57771300 & 1.13522000 \\
\hline $\mathrm{H}$ & -2.27887900 & -4.54480200 & -0.11713400 \\
\hline $\mathrm{C}$ & -3.78139600 & -0.46746600 & 0.91280400 \\
\hline $\mathrm{H}$ & -4.56910100 & -0.78578700 & 0.21808500 \\
\hline $\mathrm{C}$ & -3.40283600 & -2.48545600 & -1.54827600 \\
\hline $\mathrm{C}$ & -2.59953600 & -2.09580500 & -2.63090700 \\
\hline $\mathrm{C}$ & -4.71036300 & -2.91361800 & -1.81000400 \\
\hline $\mathrm{C}$ & -3.09549900 & -2.12986600 & -3.93436600 \\
\hline $\mathrm{H}$ & -1.58309800 & -1.75521300 & -2.45592400 \\
\hline $\mathrm{C}$ & -5.21104800 & -2.94953600 & -3.11458100 \\
\hline $\mathrm{H}$ & -5.34412400 & -3.22895200 & -0.98331700 \\
\hline $\mathrm{C}$ & -4.40415000 & -2.55586400 & -4.18130900 \\
\hline $\mathrm{H}$ & -2.45690600 & -1.82689800 & -4.76036000 \\
\hline $\mathrm{H}$ & -6.23006300 & -3.28305700 & -3.29334600 \\
\hline $\mathrm{H}$ & -4.78842100 & -2.58128800 & -5.19760900 \\
\hline $\mathrm{C}$ & -4.12969800 & -1.04543600 & 2.28646100 \\
\hline $\mathrm{C}$ & -3.18998900 & -1.08224400 & 3.32611600 \\
\hline $\mathrm{C}$ & -5.42734400 & -1.50766300 & 2.53945100 \\
\hline $\mathrm{C}$ & -3.54385800 & -1.56820100 & 4.58486400 \\
\hline $\mathrm{H}$ & -2.18144700 & -0.72474700 & 3.14400000 \\
\hline $\mathrm{C}$ & -5.78509200 & -1.99357700 & 3.79966800 \\
\hline $\mathrm{H}$ & -6.16787800 & -1.48561300 & 1.74172500 \\
\hline $\mathrm{C}$ & -4.84206600 & -2.02599800 & 4.82703800 \\
\hline $\mathrm{H}$ & -2.80349400 & -1.58926100 & 5.38072000 \\
\hline $\mathrm{H}$ & -6.79674000 & -2.35110900 & 3.97399600 \\
\hline $\mathrm{H}$ & -5.11400600 & -2.40659600 & 5.80805500 \\
\hline $\mathrm{C}$ & -3.87388900 & 1.06394700 & 0.94599000 \\
\hline $\mathrm{H}$ & -3.18779100 & 1.51233800 & 1.66284900 \\
\hline $\mathrm{H}$ & -3.69831500 & 1.49767100 & -0.03972800 \\
\hline $\mathrm{H}$ & -4.89678600 & 1.31658900 & 1.24729900 \\
\hline $\mathrm{C}$ & 2.84772600 & -0.27333600 & -2.46211700 \\
\hline $\mathrm{H}$ & 3.35665700 & 0.69187000 & -2.58093800 \\
\hline $\mathrm{H}$ & 1.78175400 & -0.08333200 & -2.31977700 \\
\hline $\mathrm{Pd}$ & 0.89954300 & -1.74901700 & 0.04530000 \\
\hline $\mathrm{C}$ & 4.43786400 & -2.01655300 & -2.98494200 \\
\hline $\mathrm{C}$ & 3.22622300 & -1.25530200 & -3.62006800 \\
\hline $\mathrm{Si}$ & 3.81617800 & -1.41340100 & -1.27643800 \\
\hline $\mathrm{C}$ & 2.88701900 & -2.78160600 & -0.32523200 \\
\hline $\mathrm{C}$ & 2.43835600 & -3.95910200 & -0.99550100 \\
\hline $\mathrm{C}$ & 2.80322000 & -2.78857800 & 1.09852800 \\
\hline $\mathrm{C}$ & 2.01139100 & -5.08406100 & -0.29489800 \\
\hline $\mathrm{H}$ & 2.47863400 & -3.99995700 & -2.08088500 \\
\hline
\end{tabular}




\begin{tabular}{|c|c|c|c|}
\hline $\mathrm{C}$ & 2.35310900 & -3.92611200 & 1.79793900 \\
\hline $\mathrm{H}$ & 3.20701500 & -1.95321300 & 1.66352900 \\
\hline $\mathrm{C}$ & 1.97996200 & -5.07467500 & 1.10716200 \\
\hline $\mathrm{H}$ & 1.70914700 & -5.97522700 & -0.83903300 \\
\hline $\mathrm{H}$ & 2.32513100 & -3.90535300 & 2.88427500 \\
\hline $\mathrm{H}$ & 1.65792900 & -5.95893300 & 1.65058700 \\
\hline $\mathrm{C}$ & 5.05331300 & -0.59785900 & -0.10750200 \\
\hline $\mathrm{C}$ & 5.06562300 & 0.79568500 & 0.08189800 \\
\hline $\mathrm{C}$ & 6.02749000 & -1.35934200 & 0.56815600 \\
\hline $\mathrm{C}$ & 6.01170100 & 1.40616600 & 0.90926200 \\
\hline $\mathrm{H}$ & 4.32603400 & 1.41613100 & -0.41763700 \\
\hline $\mathrm{C}$ & 6.97727300 & -0.75486200 & 1.39241000 \\
\hline $\mathrm{H}$ & 6.04706500 & -2.44133600 & 0.45028300 \\
\hline $\mathrm{C}$ & 6.97049200 & 0.63206000 & 1.56436500 \\
\hline $\mathrm{H}$ & 5.99588100 & 2.48527100 & 1.03795300 \\
\hline $\mathrm{H}$ & 7.72149500 & -1.36338600 & 1.90031500 \\
\hline $\mathrm{H}$ & 7.70999000 & 1.10516600 & 2.20576500 \\
\hline $\mathrm{H}$ & 2.39799600 & -1.95094200 & -3.80297300 \\
\hline $\mathrm{H}$ & 3.45144500 & -0.76376600 & -4.57484700 \\
\hline $\mathrm{H}$ & 5.39048000 & -1.54398400 & -3.25290300 \\
\hline $\mathrm{H}$ & 4.51098300 & -3.08128000 & -3.22741100 \\
\hline \multicolumn{4}{|c|}{ 1' } \\
\hline $\mathrm{C}$ & 2.55515200 & 0.49616700 & -0.00004700 \\
\hline $\mathrm{C}$ & 2.25638000 & -0.86838900 & -0.00012200 \\
\hline $\mathrm{C}$ & 0.93281400 & -1.30138400 & -0.00000500 \\
\hline $\mathrm{C}$ & -0.12641300 & -0.37376800 & 0.00014800 \\
\hline $\mathrm{C}$ & 0.18380500 & 0.99970500 & 0.00009400 \\
\hline $\mathrm{C}$ & 1.51009900 & 1.42397600 & 0.00003300 \\
\hline $\mathrm{H}$ & 3.05818700 & -1.60219500 & -0.00023500 \\
\hline $\mathrm{H}$ & 0.71022200 & -2.36570500 & -0.00008400 \\
\hline $\mathrm{H}$ & -0.61554400 & 1.73631600 & 0.00016600 \\
\hline $\mathrm{H}$ & 1.72790700 & 2.48883400 & 0.00003400 \\
\hline $\mathrm{C}$ & -1.49499500 & -0.87623400 & 0.00014600 \\
\hline $\mathrm{H}$ & -1.72255700 & -1.93512900 & 0.00021000 \\
\hline $\mathrm{H}$ & 3.58788900 & 0.83254900 & -0.00007900 \\
\hline $\mathrm{N}$ & -2.52739000 & -0.08438400 & -0.00003800 \\
\hline $\mathrm{N}$ & -3.42220400 & 0.63365400 & -0.00017600 \\
\hline
\end{tabular}

\section{B}

$\begin{array}{llll}\mathrm{C} & -6.75264800 & -1.56200700 & -1.63841600 \\ \mathrm{C} & -5.67400400 & -2.28898900 & -2.08733100 \\ \mathrm{C} & -4.34409000 & -1.83695600 & -1.87399700 \\ \mathrm{C} & -4.11981000 & -0.58506400 & -1.20853800\end{array}$




\begin{tabular}{|c|c|c|c|}
\hline $\mathrm{C}$ & -5.25964900 & 0.12571300 & -0.73625500 \\
\hline $\mathrm{C}$ & -6.53653500 & -0.34898200 & -0.94378700 \\
\hline $\mathrm{H}$ & -3.40330900 & -3.55102900 & -2.80753100 \\
\hline $\mathrm{H}$ & -7.76479700 & -1.92162000 & -1.80244700 \\
\hline $\mathrm{H}$ & -5.82152300 & -3.23424700 & -2.60492100 \\
\hline $\mathrm{C}$ & -3.22815700 & -2.61282900 & -2.28631000 \\
\hline $\mathrm{C}$ & -2.77111000 & -0.11742300 & -1.00536700 \\
\hline $\mathrm{H}$ & -5.11530100 & 1.05154200 & -0.19185800 \\
\hline $\mathrm{H}$ & -7.38587100 & 0.21286900 & -0.56418700 \\
\hline $\mathrm{C}$ & -1.72690500 & -0.97364900 & -1.34113900 \\
\hline $\mathrm{C}$ & -1.94920300 & -2.20400800 & -2.00566700 \\
\hline $\mathrm{H}$ & -1.08264100 & -2.79275900 & -2.28629500 \\
\hline $\mathrm{C}$ & -2.47777800 & 1.22079400 & -0.41861500 \\
\hline $\mathrm{C}$ & -2.98485100 & 2.44816500 & -0.97711900 \\
\hline $\mathrm{C}$ & -1.65324700 & 1.31059200 & 0.69272100 \\
\hline $\mathrm{C}$ & -3.69610300 & 2.49714700 & -2.20900000 \\
\hline $\mathrm{C}$ & -2.73050100 & 3.68896200 & -0.30059200 \\
\hline $\mathrm{C}$ & -1.39705600 & 2.52817400 & 1.36233700 \\
\hline $\mathrm{C}$ & -4.16181300 & 3.68940000 & -2.71896500 \\
\hline $\mathrm{H}$ & -3.86086000 & 1.57786800 & -2.75862900 \\
\hline $\mathrm{C}$ & -3.24218200 & 4.89800600 & -0.84447100 \\
\hline $\mathrm{C}$ & -1.94968000 & 3.69102800 & 0.88644800 \\
\hline $\mathrm{H}$ & -0.77397400 & 2.50449800 & 2.24990500 \\
\hline $\mathrm{C}$ & -3.94742800 & 4.90378900 & -2.02590900 \\
\hline $\mathrm{H}$ & -4.69481800 & 3.69663500 & -3.66601400 \\
\hline $\mathrm{H}$ & -3.05083400 & 5.82634400 & -0.31097200 \\
\hline $\mathrm{H}$ & -1.77466800 & 4.63338300 & 1.39968800 \\
\hline $\mathrm{H}$ & -4.32702300 & 5.83644100 & -2.43433100 \\
\hline $\mathrm{O}$ & -1.07474800 & 0.16139600 & 1.20809700 \\
\hline $\mathrm{O}$ & -0.41257700 & -0.62487000 & -1.11453900 \\
\hline $\mathrm{P}$ & 0.28348600 & -0.39327500 & 0.41082200 \\
\hline $\mathrm{N}$ & 0.38073300 & -1.87263800 & 1.24578700 \\
\hline $\mathrm{C}$ & 1.56813200 & -2.75768000 & 0.98417200 \\
\hline $\mathrm{H}$ & 1.25597000 & -3.73435900 & 1.36769100 \\
\hline $\mathrm{C}$ & 2.80699500 & -2.33594200 & 1.79048300 \\
\hline $\mathrm{H}$ & 3.12180200 & -1.31892700 & 1.53054500 \\
\hline $\mathrm{H}$ & 2.58516700 & -2.35871500 & 2.86233100 \\
\hline $\mathrm{H}$ & 3.63971100 & -3.02233500 & 1.59896000 \\
\hline $\mathrm{C}$ & -0.59571900 & -2.39118000 & 2.25301300 \\
\hline $\mathrm{H}$ & -0.08924400 & -3.27463600 & 2.66311700 \\
\hline $\mathrm{C}$ & 1.86586800 & -3.00201400 & -0.49801700 \\
\hline $\mathrm{C}$ & 2.80632900 & -2.25456000 & -1.21860100 \\
\hline $\mathrm{C}$ & 1.21273200 & -4.05475800 & -1.15558800 \\
\hline $\mathrm{C}$ & 3.06860700 & -2.53593400 & -2.56130500 \\
\hline
\end{tabular}




$\begin{array}{lrrr}\mathrm{H} & 3.33328400 & -1.43631800 & -0.73755900 \\ \mathrm{C} & 1.47286200 & -4.34253500 & -2.49612200 \\ \mathrm{H} & 0.49474700 & -4.66185200 & -0.60748400 \\ \mathrm{C} & 2.40235000 & -3.57843800 & -3.20602400 \\ \mathrm{H} & 3.79815600 & -1.93510700 & -3.09769000 \\ \mathrm{H} & 0.95891400 & -5.16840900 & -2.98173500 \\ \mathrm{H} & 2.60986100 & -3.80012600 & -4.24958600 \\ \mathrm{C} & -0.80990900 & -1.47006600 & 3.46557300 \\ \mathrm{C} & 0.25180500 & -0.71616200 & 3.98726900 \\ \mathrm{C} & -2.03607200 & -1.42618200 & 4.14139900 \\ \mathrm{C} & 0.09574600 & 0.05230100 & 5.13986700 \\ \mathrm{H} & 1.20487200 & -0.70910700 & 3.47004900 \\ \mathrm{C} & -2.19698300 & -0.65726600 & 5.29757700 \\ \mathrm{H} & -2.88666800 & -1.98578900 & 3.76820400 \\ \mathrm{C} & -1.13240700 & 0.08536700 & 5.80482400 \\ \mathrm{H} & 0.93665200 & 0.62920400 & 5.51717600 \\ \mathrm{H} & -3.16273700 & -0.63910600 & 5.79649600 \\ \mathrm{H} & -1.25681400 & 0.68462900 & 6.70293700 \\ \mathrm{C} & -1.89119200 & -2.90615800 & 1.60800000 \\ \mathrm{H} & -2.52607400 & -2.08635100 & 1.26440200 \\ \mathrm{H} & -1.64782400 & -3.53414100 & 0.74645300 \\ \mathrm{H} & -2.46237300 & -3.51789400 & 2.31484700 \\ \mathrm{Pd} & 2.06712600 & 0.93953300 & 0.20968300 \\ \mathrm{C} & 3.70248000 & 2.38643500 & -0.02376900 \\ \mathrm{H} & 3.07424300 & 3.15892300 & -0.47404100 \\ \mathrm{C} & 4.74523000 & 1.73426600 & -0.86323900 \\ \mathrm{C} & 5.85551600 & 1.06706800 & -0.31963600 \\ \mathrm{C} & 4.59968600 & 1.78198100 & -2.26065800 \\ \mathrm{C} & 6.79458900 & 0.46502800 & -1.15577900 \\ \mathrm{H} & 5.98645100 & 1.01239100 & 0.75812100 \\ \mathrm{C} & 5.54157800 & 1.17598600 & -3.08959700 \\ \mathrm{H} & 3.73902000 & 2.28552800 & -2.69241800 \\ \mathrm{C} & 6.64418400 & 0.51372300 & -2.54350400 \\ \mathrm{H} & & & \\ \mathrm{H} & & & \end{array}$

TS-1

$\begin{array}{lrrr}\mathrm{C} & -7.15738400 & 0.71416100 & 1.33815100 \\ \mathrm{C} & -6.17873200 & 1.14372100 & 2.20474700 \\ \mathrm{C} & -4.81460300 & 0.80520400 & 1.99762400 \\ \mathrm{C} & -4.45254600 & -0.02708700 & 0.88507400\end{array}$




\begin{tabular}{|c|c|c|c|}
\hline $\mathrm{C}$ & -5.48948800 & -0.43059900 & -0.00311800 \\
\hline $\mathrm{C}$ & -6.80119600 & -0.06981800 & 0.21598400 \\
\hline $\mathrm{H}$ & -4.07989800 & 1.91092500 & 3.71061400 \\
\hline $\mathrm{H}$ & -8.19635100 & 0.98552500 & 1.50391800 \\
\hline $\mathrm{H}$ & -6.43270600 & 1.76479600 & 3.06080100 \\
\hline $\mathrm{C}$ & -3.79718400 & 1.29684500 & 2.85895000 \\
\hline $\mathrm{C}$ & -3.07065600 & -0.38283900 & 0.68164300 \\
\hline $\mathrm{H}$ & -5.23561100 & -1.02248800 & -0.87470700 \\
\hline $\mathrm{H}$ & -7.56889800 & -0.38680100 & -0.48491100 \\
\hline $\mathrm{C}$ & -2.11913700 & 0.21769100 & 1.49871600 \\
\hline $\mathrm{C}$ & -2.47614800 & 1.03002300 & 2.60267300 \\
\hline $\mathrm{H}$ & -1.67595100 & 1.41979500 & 3.22374500 \\
\hline $\mathrm{C}$ & -2.64160600 & -1.33389900 & -0.38345500 \\
\hline $\mathrm{C}$ & -3.16684900 & -2.67342100 & -0.47998100 \\
\hline $\mathrm{C}$ & -1.67031100 & -0.95622500 & -1.29949200 \\
\hline $\mathrm{C}$ & -4.03623300 & -3.23133600 & 0.49915100 \\
\hline $\mathrm{C}$ & -2.77338400 & -3.51060300 & -1.57816700 \\
\hline $\mathrm{C}$ & -1.27270400 & -1.78092100 & -2.37614900 \\
\hline $\mathrm{C}$ & -4.51998400 & -4.51585600 & 0.37834500 \\
\hline $\mathrm{H}$ & -4.30993200 & -2.63551700 & 1.36162700 \\
\hline $\mathrm{C}$ & -3.30567100 & -4.82394800 & -1.68251600 \\
\hline $\mathrm{C}$ & -1.83642500 & -3.02262400 & -2.52748200 \\
\hline $\mathrm{H}$ & -0.52578400 & -1.40185500 & -3.06549700 \\
\hline $\mathrm{C}$ & -4.16564900 & -5.31991400 & -0.73005400 \\
\hline $\mathrm{H}$ & -5.17579000 & -4.91660800 & 1.14665100 \\
\hline $\mathrm{H}$ & -3.00545300 & -5.43745900 & -2.52892700 \\
\hline $\mathrm{H}$ & -1.55016100 & -3.65913200 & -3.36106800 \\
\hline $\mathrm{H}$ & -4.56021900 & -6.32851500 & -0.81789400 \\
\hline $\mathrm{O}$ & -1.07089300 & 0.29307200 & -1.20453600 \\
\hline $\mathrm{O}$ & -0.77460700 & -0.00524800 & 1.31433200 \\
\hline $\mathrm{P}$ & 0.13153900 & 0.44070900 & -0.03884000 \\
\hline $\mathrm{N}$ & 0.31475400 & 2.12194600 & -0.09732300 \\
\hline $\mathrm{C}$ & 1.64574400 & 2.65476700 & 0.37914700 \\
\hline $\mathrm{H}$ & 1.51015400 & 3.74046600 & 0.35803500 \\
\hline $\mathrm{C}$ & 2.79870900 & 2.35690600 & -0.58851300 \\
\hline $\mathrm{H}$ & 2.98465200 & 1.28052900 & -0.67994700 \\
\hline $\mathrm{H}$ & 2.55150200 & 2.74443500 & -1.58037300 \\
\hline $\mathrm{H}$ & 3.71619900 & 2.84602200 & -0.24041800 \\
\hline $\mathrm{C}$ & -0.59698300 & 3.21845200 & -0.55919800 \\
\hline $\mathrm{H}$ & -0.47736100 & 4.01350600 & 0.18819700 \\
\hline $\mathrm{C}$ & 1.92551800 & 2.30607200 & 1.84433500 \\
\hline $\mathrm{C}$ & 2.92699800 & 1.41107500 & 2.23833500 \\
\hline $\mathrm{C}$ & 1.16368500 & 2.93152200 & 2.84462000 \\
\hline $\mathrm{C}$ & 3.15081700 & 1.13865900 & 3.59163100 \\
\hline
\end{tabular}




\begin{tabular}{|c|c|c|c|}
\hline $\mathrm{H}$ & 3.53363800 & 0.91058200 & 1.49137000 \\
\hline $\mathrm{C}$ & 1.38198200 & 2.66155700 & 4.19450200 \\
\hline $\mathrm{H}$ & 0.38892800 & 3.64089700 & 2.56061300 \\
\hline $\mathrm{C}$ & 2.37983800 & 1.75883900 & 4.57343200 \\
\hline $\mathrm{H}$ & 3.93215800 & 0.43747700 & 3.8730780 \\
\hline $\mathrm{H}$ & 0.78242700 & 3.16226000 & 4.9507520 \\
\hline $\mathrm{H}$ & 2.55607400 & 1.54734800 & 5.6248300 \\
\hline $\mathrm{C}$ & -0.13771900 & 3.80036400 & -1.89737400 \\
\hline $\mathrm{C}$ & -0.02625400 & 2.99634100 & -3.04157000 \\
\hline $\mathrm{C}$ & 0.13836300 & 5.16774100 & -2.00982800 \\
\hline $\mathrm{C}$ & 0.35320500 & 3.55089800 & -4.26329100 \\
\hline $\mathrm{H}$ & -0.23626900 & 1.93358300 & -2.96666400 \\
\hline $\mathrm{C}$ & 0.51738500 & 5.72700500 & -3.23359600 \\
\hline $\mathrm{H}$ & 0.05478100 & 5.80480500 & -1.13113500 \\
\hline $\mathrm{C}$ & 0.62663200 & 4.91868200 & -4.36454000 \\
\hline $\mathrm{H}$ & 0.43559500 & 2.91442000 & -5.14077500 \\
\hline $\mathrm{H}$ & 0.73046500 & 6.79095400 & -3.29868900 \\
\hline $\mathrm{H}$ & 0.92399100 & 5.34784500 & -5.31773100 \\
\hline $\mathrm{C}$ & -2.10332400 & 2.92510200 & -0.59617800 \\
\hline $\mathrm{H}$ & -2.37375400 & 2.21173500 & -1.37311000 \\
\hline $\mathrm{H}$ & -2.46224600 & 2.55563700 & 0.36633100 \\
\hline $\mathrm{H}$ & -2.61284800 & 3.87186100 & -0.80830300 \\
\hline $\mathrm{Pd}$ & 1.87141000 & -1.03210400 & -0.18971200 \\
\hline $\mathrm{C}$ & 3.27788200 & -2.51437800 & -0.07700600 \\
\hline $\mathrm{H}$ & 2.91268900 & -3.41193200 & 0.43450300 \\
\hline $\mathrm{C}$ & 4.72439800 & -2.29240300 & 0.18605300 \\
\hline $\mathrm{C}$ & 5.42477700 & -1.24490700 & -0.44239900 \\
\hline $\mathrm{C}$ & 5.40248600 & -3.08085800 & 1.13311800 \\
\hline $\mathrm{C}$ & 6.76184300 & -1.00322600 & -0.14025700 \\
\hline $\mathrm{H}$ & 4.89851400 & -0.62214200 & -1.16068900 \\
\hline $\mathrm{C}$ & 6.73124300 & -2.81474400 & 1.46360400 \\
\hline $\mathrm{H}$ & 4.87689000 & -3.89983000 & 1.61877100 \\
\hline $\mathrm{C}$ & 7.41649000 & -1.78176200 & 0.82065400 \\
\hline $\mathrm{H}$ & 7.29149700 & -0.19607100 & -0.63902500 \\
\hline $\mathrm{H}$ & 7.23537600 & -3.42243700 & 2.21020200 \\
\hline $\mathrm{H}$ & 8.45533400 & -1.58073800 & 1.06812200 \\
\hline $\mathrm{N}$ & 3.24374200 & -3.25686800 & -1.67481100 \\
\hline $\mathrm{N}$ & 3.09685700 & -3.41820200 & -2.76918700 \\
\hline \\
\hline $\mathrm{N}$ & 0.00000000 & 0.00000000 & 0.55265200 \\
\hline $\mathrm{N}$ & 0.00000000 & 0.00000000 & -0.55265200 \\
\hline
\end{tabular}

C 


\begin{tabular}{|c|c|c|c|}
\hline $\mathrm{C}$ & 0.73870800 & -6.55554100 & 1.42923900 \\
\hline $\mathrm{C}$ & 0.11707700 & -5.66906300 & 2.27828300 \\
\hline $\mathrm{C}$ & 0.13493900 & -4.27195500 & 2.02109100 \\
\hline $\mathrm{C}$ & 0.84008600 & -3.77069700 & 0.87587500 \\
\hline $\mathrm{C}$ & 1.44959000 & -4.72064900 & 0.00813200 \\
\hline $\mathrm{C}$ & 1.39890700 & -6.07135300 & 0.27598600 \\
\hline $\mathrm{H}$ & -1.07729800 & -3.74337300 & 3.73851300 \\
\hline $\mathrm{H}$ & 0.71218500 & -7.62247900 & 1.63275500 \\
\hline $\mathrm{H}$ & -0.41340400 & -6.02591300 & 3.15816200 \\
\hline $\mathrm{C}$ & -0.55739400 & -3.36155400 & 2.86331400 \\
\hline $\mathrm{C}$ & 0.87020100 & -2.35159100 & 0.62018700 \\
\hline $\mathrm{H}$ & 1.95089400 & -4.37195100 & -0.88690200 \\
\hline $\mathrm{H}$ & 1.86653900 & -6.77198900 & -0.41061900 \\
\hline $\mathrm{C}$ & 0.08432000 & -1.53237300 & 1.42203900 \\
\hline $\mathrm{C}$ & -0.60393700 & -2.02518000 & 2.55762100 \\
\hline $\mathrm{H}$ & -1.16466300 & -1.31978100 & 3.16224900 \\
\hline $\mathrm{C}$ & 1.67954200 & -1.76594900 & -0.48593600 \\
\hline $\mathrm{C}$ & 3.09884100 & -1.99084700 & -0.60736200 \\
\hline $\mathrm{C}$ & 1.07712100 & -0.94444900 & -1.42672800 \\
\hline $\mathrm{C}$ & 3.85865300 & -2.66651700 & 0.38836500 \\
\hline $\mathrm{C}$ & 3.79963400 & -1.48064600 & -1.75151200 \\
\hline $\mathrm{C}$ & 1.76386400 & -0.43118600 & -2.54927100 \\
\hline $\mathrm{C}$ & 5.21454300 & -2.86346400 & 0.24228200 \\
\hline $\mathrm{H}$ & 3.35974900 & -3.01912600 & 1.28314100 \\
\hline $\mathrm{C}$ & 5.19460400 & -1.71826100 & -1.88041400 \\
\hline $\mathrm{C}$ & 3.09357200 & -0.72113000 & -2.72206300 \\
\hline $\mathrm{H}$ & 1.21423000 & 0.18391600 & -3.25349500 \\
\hline $\mathrm{C}$ & 5.89173200 & -2.39945900 & -0.90950000 \\
\hline $\mathrm{H}$ & 5.76967700 & -3.37548200 & 1.02368000 \\
\hline $\mathrm{H}$ & 5.70511200 & -1.33423600 & -2.76059400 \\
\hline $\mathrm{H}$ & 3.63019500 & -0.34481400 & -3.58930600 \\
\hline $\mathrm{H}$ & 6.95978300 & -2.56811200 & -1.01639100 \\
\hline $\mathrm{O}$ & -0.27155200 & -0.62166600 & -1.30635700 \\
\hline $\mathrm{O}$ & -0.01465400 & -0.17635600 & 1.19058600 \\
\hline $\mathrm{P}$ & -0.63915800 & 0.57039000 & -0.19096600 \\
\hline $\mathrm{N}$ & -2.32425300 & 0.45512400 & -0.24234300 \\
\hline $\mathrm{C}$ & -3.13883400 & 1.64978200 & 0.14977800 \\
\hline $\mathrm{H}$ & -4.01187200 & 1.61176200 & -0.51121300 \\
\hline $\mathrm{C}$ & -2.48921900 & 3.01698200 & -0.11543000 \\
\hline $\mathrm{H}$ & -1.65280800 & 3.23837700 & 0.56482600 \\
\hline $\mathrm{H}$ & -2.14287200 & 3.09825300 & -1.15238900 \\
\hline $\mathrm{H}$ & -3.24047600 & 3.79664100 & 0.05434200 \\
\hline $\mathrm{C}$ & -3.24509400 & -0.68588000 & -0.56654400 \\
\hline $\mathrm{H}$ & -4.03313400 & -0.62868600 & 0.19514100 \\
\hline
\end{tabular}




\begin{tabular}{|c|c|c|c|}
\hline $\mathrm{C}$ & -3.65595800 & 1.56102300 & 1.58644200 \\
\hline $\mathrm{C}$ & -2.78014900 & 1.43933100 & 2.67549200 \\
\hline $\mathrm{C}$ & -5.03024900 & 1.64755300 & 1.84028300 \\
\hline $\mathrm{C}$ & -3.27028000 & 1.40721800 & 3.98116300 \\
\hline $\mathrm{H}$ & -1.71038200 & 1.36028700 & 2.50426300 \\
\hline $\mathrm{C}$ & -5.52464700 & 1.61505700 & 3.14713000 \\
\hline $\mathrm{H}$ & -5.72234800 & 1.74466300 & 1.00591500 \\
\hline $\mathrm{C}$ & -4.64458500 & 1.49432600 & 4.22215700 \\
\hline $\mathrm{H}$ & -2.57700500 & 1.31683500 & 4.81363800 \\
\hline $\mathrm{H}$ & -6.59548600 & 1.68088900 & 3.32147200 \\
\hline $\mathrm{H}$ & -5.02390900 & 1.46779900 & 5.24020400 \\
\hline $\mathrm{C}$ & -3.91594000 & -0.47180100 & -1.92446400 \\
\hline $\mathrm{C}$ & -3.16365600 & -0.25674200 & -3.08808300 \\
\hline $\mathrm{C}$ & -5.31103200 & -0.53571400 & -2.03007200 \\
\hline $\mathrm{C}$ & -3.79505500 & -0.10859400 & -4.32300300 \\
\hline $\mathrm{H}$ & -2.08117700 & -0.21001900 & -3.02156400 \\
\hline $\mathrm{C}$ & -5.94589500 & -0.39059000 & -3.26638300 \\
\hline $\mathrm{H}$ & -5.90760900 & -0.70342800 & -1.13515100 \\
\hline $\mathrm{C}$ & -5.18807800 & -0.17456800 & -4.41732900 \\
\hline $\mathrm{H}$ & -3.19770000 & 0.05793800 & -5.21585000 \\
\hline $\mathrm{H}$ & -7.03010500 & -0.44046800 & -3.32650300 \\
\hline $\mathrm{H}$ & -5.67727700 & -0.05668300 & -5.38061800 \\
\hline $\mathrm{C}$ & -2.67575100 & -2.10638000 & -0.46677100 \\
\hline $\mathrm{H}$ & -1.90793500 & -2.30330000 & -1.21433500 \\
\hline $\mathrm{H}$ & -2.26803300 & -2.30525600 & 0.52635900 \\
\hline $\mathrm{H}$ & -3.50426700 & -2.80293700 & -0.63610500 \\
\hline $\mathrm{Pd}$ & 0.42720900 & 2.60308900 & -0.45780000 \\
\hline $\mathrm{C}$ & 2.14827200 & 3.41583300 & -0.86873900 \\
\hline $\mathrm{H}$ & 2.45795100 & 3.46236700 & -1.92475700 \\
\hline $\mathrm{C}$ & 3.26972400 & 3.77270700 & -0.02297300 \\
\hline $\mathrm{C}$ & 3.15203700 & 3.75398800 & 1.38901000 \\
\hline $\mathrm{C}$ & 4.51157600 & 4.17275700 & -0.57581300 \\
\hline $\mathrm{C}$ & 4.21901400 & 4.11446900 & 2.20239400 \\
\hline $\mathrm{H}$ & 2.20174900 & 3.44175400 & 1.81284400 \\
\hline $\mathrm{C}$ & 5.57751100 & 4.54432200 & 0.23708800 \\
\hline $\mathrm{H}$ & 4.62126000 & 4.18937500 & -1.65769600 \\
\hline $\mathrm{C}$ & 5.43260100 & 4.51298000 & 1.62799800 \\
\hline $\mathrm{H}$ & 4.11314900 & 4.09003800 & 3.28358200 \\
\hline $\mathrm{H}$ & 6.52119500 & 4.85254900 & -0.20502500 \\
\hline $\mathrm{H}$ & 6.26578400 & 4.79776200 & 2.265529 \\
\hline
\end{tabular}

$2 a$

$\begin{array}{llll}\text { C } & -0.00726100 & 2.41996400 & -1.20604000\end{array}$

$\begin{array}{llll}\text { C } & 0.06818400 & 3.39034100 & 0.02746200\end{array}$ 


$\begin{array}{lccc}\mathrm{C} & 0.08943400 & 2.39686500 & 1.24412700 \\ \mathrm{H} & -0.77942800 & 2.49714000 & 1.90268700 \\ \mathrm{H} & 0.98469900 & 2.48358000 & 1.86881400 \\ \mathrm{Si} & 0.01337900 & 0.94252400 & 0.00620000 \\ \mathrm{C} & 1.53661300 & -0.17439100 & -0.04166000 \\ \mathrm{C} & 1.51231800 & -1.41627600 & -0.70552000 \\ \mathrm{C} & 2.75313200 & 0.22838500 & 0.54073700 \\ \mathrm{C} & 2.65255500 & -2.21736300 & -0.78868000 \\ \mathrm{H} & 0.58736400 & -1.76806200 & -1.15641900 \\ \mathrm{C} & 3.89661800 & -0.56872800 & 0.46281300 \\ \mathrm{H} & 2.81192200 & 1.17783800 & 1.06815000 \\ \mathrm{C} & 3.84811300 & -1.79457700 & -0.20390900 \\ \mathrm{H} & 2.60732800 & -3.17248900 & -1.30609100 \\ \mathrm{H} & 4.82287500 & -0.23547600 & 0.92432300 \\ \mathrm{H} & 4.73643200 & -2.41817600 & -0.26520900 \\ \mathrm{C} & -1.54605900 & -0.12308200 & 0.04005900 \\ \mathrm{C} & -1.64024700 & -1.23655400 & 0.89838900 \\ \mathrm{C} & -2.66843700 & 0.18678800 & -0.74935600 \\ \mathrm{C} & -2.80475100 & -2.00211600 & 0.96913500 \\ \mathrm{H} & -0.78864800 & -1.51610600 & 1.51510600 \\ \mathrm{C} & -3.83575600 & -0.57760000 & -0.68629200 \\ \mathrm{H} & -2.63350400 & 1.03604900 & -1.42762500 \\ \mathrm{C} & -3.90627800 & -1.67347500 & 0.17517800 \\ \mathrm{H} & -2.85235400 & -2.85653100 & 1.63973500 \\ \mathrm{H} & -4.68782000 & -0.31879200 & -1.30997900 \\ \mathrm{H} & -4.81350000 & -2.27013000 & 0.22674400 \\ \mathrm{H} & -0.90425500 & 2.55942300 & -1.81850500 \\ \mathrm{H} & 0.85824300 & 2.49364200 & -1.87250300 \\ \mathrm{H} & -0.79230800 & 4.06706000 & 0.06774700 \\ \mathrm{H} & 0.96658200 & 4.01660600 & -0.00244300 \\ & & & \\ & & & \\ \mathrm{H} & & \end{array}$

TS-2

$\begin{array}{lrrr}\mathrm{C} & -7.74170000 & 2.02825300 & -0.14393200 \\ \mathrm{C} & -7.18495300 & 1.28006300 & -1.15543400 \\ \mathrm{C} & -5.78624600 & 1.03560100 & -1.20241600 \\ \mathrm{C} & -4.93246500 & 1.60375100 & -0.19797100 \\ \mathrm{C} & -5.54867800 & 2.35041100 & 0.84587700 \\ \mathrm{C} & -6.91118600 & 2.55485600 & 0.87241300 \\ \mathrm{H} & -5.86895300 & -0.20391300 & -2.97843100 \\ \mathrm{H} & -8.81359400 & 2.20319800 & -0.11522400 \\ \mathrm{H} & -7.81112300 & 0.84922700 & -1.93331000 \\ \mathrm{C} & -5.21980100 & 0.21330500 & -2.21259400 \\ \mathrm{C} & -3.51080000 & 1.36589800 & -0.25446200 \\ \mathrm{H} & -4.93356600 & 2.75435000 & 1.64106600\end{array}$




\begin{tabular}{|c|c|c|c|}
\hline $\mathrm{H}$ & -7.35199900 & 3.12246700 & 1.68756000 \\
\hline $\mathrm{C}$ & -3.04011500 & 0.47384200 & -1.20911900 \\
\hline $\mathrm{C}$ & -3.88025200 & -0.08181300 & -2.20425600 \\
\hline $\mathrm{H}$ & -3.43677500 & -0.74278100 & -2.94149900 \\
\hline $\mathrm{C}$ & -2.56744800 & 2.00633500 & 0.70530200 \\
\hline $\mathrm{C}$ & -2.50290500 & 3.43628400 & 0.87959300 \\
\hline $\mathrm{C}$ & -1.70445700 & 1.22709800 & 1.46391000 \\
\hline $\mathrm{C}$ & -3.19031400 & 4.34422000 & 0.02580500 \\
\hline $\mathrm{C}$ & -1.68256300 & 3.98744200 & 1.92062300 \\
\hline $\mathrm{C}$ & -0.90849000 & 1.76401200 & 2.50103800 \\
\hline $\mathrm{C}$ & -3.10042200 & 5.70653400 & 0.21078000 \\
\hline $\mathrm{H}$ & -3.78094300 & 3.95222200 & -0.79384500 \\
\hline $\mathrm{C}$ & -1.62928700 & 5.39647700 & 2.09761700 \\
\hline $\mathrm{C}$ & -0.92089900 & 3.11487400 & 2.74210400 \\
\hline $\mathrm{H}$ & -0.30484800 & 1.08354600 & 3.09126300 \\
\hline $\mathrm{C}$ & -2.32436600 & 6.24287400 & 1.26493700 \\
\hline $\mathrm{H}$ & -3.62683400 & 6.37570600 & -0.46436900 \\
\hline $\mathrm{H}$ & -1.01210600 & 5.79396400 & 2.90002900 \\
\hline $\mathrm{H}$ & -0.32254500 & 3.53574300 & 3.54622500 \\
\hline $\mathrm{H}$ & -2.26849700 & 7.31888100 & 1.40469300 \\
\hline $\mathrm{O}$ & -1.63302700 & -0.14564700 & 1.26354400 \\
\hline $\mathrm{O}$ & -1.70483800 & 0.13600500 & -1.27098300 \\
\hline $\mathrm{P}$ & -0.80444500 & -0.68868300 & -0.09059400 \\
\hline $\mathrm{N}$ & -1.26844900 & -2.30966500 & 0.01018000 \\
\hline $\mathrm{C}$ & -0.45364800 & -3.37362500 & -0.66444500 \\
\hline $\mathrm{H}$ & -0.53202200 & -4.23414300 & 0.00878400 \\
\hline $\mathrm{C}$ & 1.04941300 & -3.09622100 & -0.78901300 \\
\hline $\mathrm{H}$ & 1.28357500 & -2.31179700 & -1.51123500 \\
\hline $\mathrm{H}$ & 1.47590800 & -2.81293600 & 0.17682300 \\
\hline $\mathrm{H}$ & 1.53499900 & -4.01716500 & -1.13062700 \\
\hline $\mathrm{C}$ & -2.46112900 & -2.96225400 & 0.65832000 \\
\hline $\mathrm{H}$ & -2.76497100 & -3.73215300 & -0.06147000 \\
\hline $\mathrm{C}$ & -1.04483100 & -3.80051200 & -2.00872000 \\
\hline $\mathrm{C}$ & -1.21903700 & -2.88792500 & -3.05971600 \\
\hline $\mathrm{C}$ & -1.37602700 & -5.14310800 & -2.22920300 \\
\hline $\mathrm{C}$ & -1.70917100 & -3.31082400 & -4.29562300 \\
\hline $\mathrm{H}$ & -0.97751500 & -1.83905300 & -2.91293500 \\
\hline $\mathrm{C}$ & -1.86886500 & -5.57029700 & -3.46530600 \\
\hline $\mathrm{H}$ & -1.24286700 & -5.86440700 & -1.42512500 \\
\hline $\mathrm{C}$ & -2.03693300 & -4.65393400 & -4.50294600 \\
\hline $\mathrm{H}$ & -1.83187200 & -2.59061500 & -5.10065500 \\
\hline $\mathrm{H}$ & -2.12234600 & -6.61682200 & -3.61341100 \\
\hline $\mathrm{H}$ & -2.41936300 & -4.98099500 & -5.46612400 \\
\hline $\mathrm{C}$ & -2.05342900 & -3.68614300 & 1.94258600 \\
\hline
\end{tabular}




\begin{tabular}{|c|c|c|c|}
\hline $\mathrm{C}$ & -1.33743300 & -3.03941500 & 2.95963900 \\
\hline $\mathrm{C}$ & -2.43940100 & -5.01860200 & 2.13794200 \\
\hline $\mathrm{C}$ & -1.01762500 & -3.71169500 & 4.13951800 \\
\hline $\mathrm{H}$ & -1.03375700 & -2.00659700 & 2.82346400 \\
\hline $\mathrm{C}$ & -2.12394000 & -5.69294700 & 3.32008700 \\
\hline $\mathrm{H}$ & -2.99502800 & -5.53419200 & 1.35659800 \\
\hline $\mathrm{C}$ & -1.40955900 & -5.04023100 & 4.32493200 \\
\hline $\mathrm{H}$ & -0.45995400 & -3.19598100 & 4.91713400 \\
\hline $\mathrm{H}$ & -2.43065300 & -6.72762000 & 3.45047300 \\
\hline $\mathrm{H}$ & -1.15719700 & -5.56190900 & 5.24434500 \\
\hline $\mathrm{C}$ & -3.71870300 & -2.11474400 & 0.89138400 \\
\hline $\mathrm{H}$ & -3.58186400 & -1.35810400 & 1.66237300 \\
\hline $\mathrm{H}$ & -4.05309200 & -1.63291900 & -0.02946300 \\
\hline $\mathrm{H}$ & -4.51042800 & -2.79720200 & 1.22049600 \\
\hline $\mathrm{C}$ & 2.24923800 & 0.62252400 & 1.53320900 \\
\hline $\mathrm{H}$ & 1.94451600 & 1.58313400 & 1.97569700 \\
\hline $\mathrm{C}$ & 3.01826500 & -0.12980600 & 2.50574100 \\
\hline $\mathrm{C}$ & 3.52321800 & -1.41895700 & 2.20792600 \\
\hline $\mathrm{C}$ & 3.29759800 & 0.40607600 & 3.78849100 \\
\hline $\mathrm{C}$ & 4.24812000 & -2.14063900 & 3.14905700 \\
\hline $\mathrm{H}$ & 3.34309600 & -1.82269700 & 1.21751800 \\
\hline $\mathrm{C}$ & 4.03122300 & -0.31090000 & 4.72796900 \\
\hline $\mathrm{H}$ & 2.92504600 & 1.39833900 & 4.03277600 \\
\hline $\mathrm{C}$ & 4.50298100 & -1.58920800 & 4.41019100 \\
\hline $\mathrm{H}$ & 4.62739300 & -3.12811700 & 2.90141000 \\
\hline $\mathrm{H}$ & 4.23442000 & 0.11716100 & 5.70603600 \\
\hline $\mathrm{H}$ & 5.07374200 & -2.15351600 & 5.14339500 \\
\hline $\mathrm{C}$ & 1.25625000 & 0.56286400 & -2.65869100 \\
\hline $\mathrm{H}$ & 0.28591100 & 0.94526400 & -2.33689700 \\
\hline $\mathrm{H}$ & 1.13909400 & -0.48948700 & -2.94155500 \\
\hline $\mathrm{Pd}$ & 1.43985400 & 0.21171200 & -0.19216500 \\
\hline $\mathrm{C}$ & 3.32246800 & 0.97325700 & -3.81727100 \\
\hline $\mathrm{C}$ & 1.82663500 & 1.33533800 & -3.87584800 \\
\hline $\mathrm{Si}$ & 3.22516800 & 0.92531900 & -1.90397300 \\
\hline $\mathrm{C}$ & 4.57760300 & -0.25216200 & -1.25983200 \\
\hline $\mathrm{C}$ & 4.76394800 & -1.51490700 & -1.85444500 \\
\hline $\mathrm{C}$ & 5.46861100 & 0.11984500 & -0.23703300 \\
\hline $\mathrm{C}$ & 5.78807200 & -2.37128500 & -1.44400500 \\
\hline $\mathrm{H}$ & 4.10667400 & -1.83733800 & -2.65992500 \\
\hline $\mathrm{C}$ & 6.50708800 & -0.72258100 & 0.16470500 \\
\hline $\mathrm{H}$ & 5.35030000 & 1.08392600 & 0.25065100 \\
\hline $\mathrm{C}$ & 6.66777000 & -1.97400800 & -0.43447600 \\
\hline $\mathrm{H}$ & 5.90682400 & -3.34176500 & -1.92053500 \\
\hline $\mathrm{H}$ & 7.18740200 & -0.40427000 & 0.95104600 \\
\hline
\end{tabular}




$\begin{array}{lrrr}\mathrm{H} & 7.47374100 & -2.63265300 & -0.12016700 \\ \mathrm{C} & 3.53186800 & 2.72516200 & -1.37021500 \\ \mathrm{C} & 2.58054200 & 3.51583100 & -0.70208300 \\ \mathrm{C} & 4.75734800 & 3.33254300 & -1.70753900 \\ \mathrm{C} & 2.83647600 & 4.85221700 & -0.38516500 \\ \mathrm{H} & 1.62622800 & 3.07632900 & -0.42192100 \\ \mathrm{C} & 5.02763800 & 4.66245400 & -1.37845600 \\ \mathrm{H} & 5.51835900 & 2.75906000 & -2.23353800 \\ \mathrm{C} & 4.06412100 & 5.42782100 & -0.71796700 \\ \mathrm{H} & 2.07628400 & 5.44146800 & 0.12247700 \\ \mathrm{H} & 5.98677400 & 5.10190400 & -1.64227700 \\ \mathrm{H} & 4.26835000 & 6.46595900 & -0.46712400 \\ \mathrm{H} & 1.31085900 & 1.06789600 & -4.80979800 \\ \mathrm{H} & 1.69082600 & 2.41357300 & -3.73034500 \\ \mathrm{H} & 3.49348400 & -0.02265000 & -4.24663000 \\ \mathrm{H} & 4.00752300 & 1.67635400 & -4.30259800\end{array}$

\section{TS-2'}

C

C

C

C

C

C

$\mathrm{H}$

$\mathrm{H}$

$\mathrm{H}$

C

C

$\mathrm{H}$

$\mathrm{H}$

C

C

$\mathrm{H}$

C

C

C

C

C

C

C

$\mathrm{H}$

C

C

$\begin{array}{ccc}7.58913300 & -1.99227700 & -1.00627800 \\ 6.96008400 & -1.04692700 & -1.78297400 \\ 5.56185800 & -0.81993300 & -1.67390700 \\ 4.78374200 & -1.61126800 & -0.76302700 \\ 5.47466000 & -2.56069800 & 0.04197900 \\ 6.83528400 & -2.74439800 & -0.07543800 \\ 5.51703000 & 0.78816000 & -3.12591600 \\ 8.66009600 & -2.15249600 & -1.09442700 \\ 7.52771000 & -0.44408800 & -2.48807800 \\ 4.92413800 & 0.19898400 & -2.43066700 \\ 3.36206700 & -1.38998100 & -0.66495500 \\ 4.91890600 & -3.14052200 & 0.76921500 \\ 7.33451100 & -3.47183500 & 0.55904400 \\ 2.82273900 & -0.31660400 & -1.36283600 \\ 3.58869100 & 0.46337100 & -2.26217700 \\ 3.09251300 & 1.26713000 & -2.79585800 \\ 2.48627100 & -2.24227500 & 0.18921500 \\ 2.43025400 & -3.67596600 & 0.03963100 \\ 1.67114100 & -1.66425800 & 1.15366900 \\ 3.06995700 & -4.35970800 & -1.03227600 \\ 1.66603200 & -4.45749100 & 0.96981900 \\ 0.92583400 & -2.43141200 & 2.07848300 \\ 2.99170800 & -5.72989700 & -1.15389700 \\ 3.61466400 & -3.78597700 & -1.77263000 \\ 1.62284500 & -5.87057200 & 0.82742400 \\ 0.94672000 & -3.80154300 & 2.00382700\end{array}$


H

C

$\mathrm{H}$

$\mathrm{H}$

$\mathrm{H}$

$\mathrm{H}$

$\mathrm{O}$

$\mathrm{O}$

$\mathrm{P}$

N

C

$\mathrm{H}$

C

$\mathrm{H}$

$\mathrm{H}$

$\mathrm{H}$

C

$\mathrm{H}$

C

C

C

C

$\mathrm{H}$

C

$\mathrm{H}$

C

$\mathrm{H}$

$\mathrm{H}$

$\mathrm{H}$

C

C

C

C

$\mathrm{H}$

C

$\mathrm{H}$

C

$\mathrm{H}$

$\mathrm{H}$

$\mathrm{H}$

C

$\mathrm{H}$

$\mathrm{H}$

$\mathrm{H}$

$\begin{array}{ccc}0.35717000 & -1.90773200 & 2.83880900 \\ 2.27409400 & -6.49934500 & -0.20856700 \\ 3.48162800 & -6.22335400 & -1.98890900 \\ 1.04789500 & -6.44591400 & 1.54928600 \\ 0.38894700 & -4.40028900 & 2.71952000 \\ 2.22647500 & -7.57984300 & -0.31185900 \\ 1.59647300 & -0.28330300 & 1.28295100 \\ 1.48553700 & 0.00115300 & -1.25227300 \\ 0.69598400 & 0.56092600 & 0.14557300 \\ 1.23326400 & 2.10710800 & 0.56317200 \\ 0.45783100 & 3.32995900 & 0.15325400 \\ 0.68424100 & 4.05445900 & 0.94185700 \\ -1.06799600 & 3.19723800 & 0.16172400 \\ -1.45411400 & 2.52988200 & -0.60951900 \\ -1.41551900 & 2.83825600 & 1.13397600 \\ -1.49225500 & 4.19068400 & -0.02036200 \\ 2.49261600 & 2.56079500 & 1.25702300 \\ 2.80860500 & 3.43986300 & 0.68225100 \\ 0.96630200 & 3.93065100 & -1.15810500 \\ 0.88262000 & 3.23344900 & -2.37265700 \\ 1.49425400 & 5.22773400 & -1.17178000 \\ 1.31720700 & 3.81820700 & -3.56248200 \\ 0.47949100 & 2.22562200 & -2.39130900 \\ 1.93236500 & 5.81732700 & -2.36113700 \\ 1.55743300 & 5.78729800 & -0.24047900 \\ 1.84585800 & 5.11267500 & -3.56109300 \\ 1.24040300 & 3.26344700 & -4.49441200 \\ 2.34083700 & 6.82450500 & -2.34647000 \\ 2.18475200 & 5.56604300 & -4.48882200 \\ 2.17796800 & 3.03766400 & 2.67622500 \\ 1.45308300 & 2.24361700 & 3.57551100 \\ 2.66282100 & 4.27625600 & 3.11542900 \\ 1.22100000 & 2.68099400 & 4.87975800 \\ 1.07346500 & 1.28042500 & 3.25004300 \\ 2.43474800 & 4.71542700 & 4.42188900 \\ 3.22688900 & 4.90412900 & 2.42783000 \\ 1.71031600 & 3.91799700 & 5.30821600 \\ 0.65551400 & 2.05344900 & 5.56394300 \\ 2.81682300 & 5.68149500 & 4.74181100 \\ 1.52565000 & 4.25742200 & 6.32401900 \\ 3.71633100 & 1.63455900 & 1.27058000 \\ 3.57406100 & 0.75647400 & 1.89832300 \\ 3.99320800 & 1.31801200 & 0.26401300 \\ 4.55008400 & 2.21544700 & 1.68110600 \\ & & \end{array}$




\begin{tabular}{|c|c|c|c|}
\hline $\mathrm{C}$ & -2.89387200 & 0.29875600 & 1.53553100 \\
\hline $\mathrm{H}$ & -3.49094000 & 1.20873000 & 1.36613800 \\
\hline $\mathrm{C}$ & -3.16479100 & -0.20809500 & 2.87145900 \\
\hline $\mathrm{C}$ & -2.61845300 & -1.43832800 & 3.31172400 \\
\hline $\mathrm{C}$ & -3.98752200 & 0.50470400 & 3.77729600 \\
\hline $\mathrm{C}$ & -2.87401500 & -1.92448000 & 4.58846900 \\
\hline $\mathrm{H}$ & -2.00076800 & -1.99558800 & 2.61338600 \\
\hline $\mathrm{C}$ & -4.23749100 & 0.02429800 & 5.06002500 \\
\hline $\mathrm{H}$ & -4.42396400 & 1.44765500 & 3.45648900 \\
\hline $\mathrm{C}$ & -3.68124800 & -1.19164800 & 5.46794800 \\
\hline $\mathrm{H}$ & -2.45094900 & -2.87408900 & 4.90582600 \\
\hline $\mathrm{H}$ & -4.86765100 & 0.59007100 & 5.74114700 \\
\hline $\mathrm{H}$ & -3.87980700 & -1.57155800 & 6.46681000 \\
\hline $\mathrm{C}$ & -1.25045700 & -1.91665300 & -1.53295600 \\
\hline $\mathrm{H}$ & -1.84404500 & -2.82176800 & -1.34700300 \\
\hline $\mathrm{H}$ & -0.37378700 & -1.98469300 & -0.88583200 \\
\hline $\mathrm{Pd}$ & -1.62432400 & -0.20493200 & 0.13107100 \\
\hline $\mathrm{C}$ & -2.30481000 & -1.37158400 & -3.62196300 \\
\hline $\mathrm{C}$ & -0.93227400 & -1.77077400 & -3.03074800 \\
\hline $\mathrm{Si}$ & -2.97819200 & -0.73472300 & -1.93373700 \\
\hline $\mathrm{C}$ & -3.40952200 & 1.11375700 & -2.14170900 \\
\hline $\mathrm{C}$ & -2.71400500 & 1.91973300 & -3.06304700 \\
\hline $\mathrm{C}$ & -4.47017300 & 1.70908600 & -1.43178500 \\
\hline $\mathrm{C}$ & -3.04796300 & 3.26237100 & -3.25722600 \\
\hline $\mathrm{H}$ & -1.89929900 & 1.49511400 & -3.64520400 \\
\hline $\mathrm{C}$ & -4.81769500 & 3.04576900 & -1.62985600 \\
\hline $\mathrm{H}$ & -5.03843400 & 1.11327500 & -0.72184900 \\
\hline $\mathrm{C}$ & -4.10250900 & 3.82946300 & -2.54010600 \\
\hline $\mathrm{H}$ & -2.48571800 & 3.86276800 & -3.96808900 \\
\hline $\mathrm{H}$ & -5.64858100 & 3.47698800 & -1.07618800 \\
\hline $\mathrm{H}$ & -4.36995500 & 4.87205400 & -2.69301000 \\
\hline $\mathrm{C}$ & -4.55743000 & -1.68443500 & -1.47433300 \\
\hline $\mathrm{C}$ & -4.74965600 & -2.35187500 & -0.25293700 \\
\hline $\mathrm{C}$ & -5.59635400 & -1.75116400 & -2.42316500 \\
\hline $\mathrm{C}$ & -5.92811500 & -3.05353400 & 0.01394600 \\
\hline $\mathrm{H}$ & -3.96815300 & -2.31281800 & 0.50098200 \\
\hline $\mathrm{C}$ & -6.78005900 & -2.44282000 & -2.16018000 \\
\hline $\mathrm{H}$ & -5.48305400 & -1.25341300 & -3.38474000 \\
\hline $\mathrm{C}$ & -6.94812600 & -3.09790900 & -0.93803700 \\
\hline $\mathrm{H}$ & -6.05040500 & -3.56272400 & 0.96711800 \\
\hline $\mathrm{H}$ & -7.56873200 & -2.47255500 & -2.90826400 \\
\hline $\mathrm{H}$ & -7.86735600 & -3.64035700 & -0.73064800 \\
\hline $\mathrm{H}$ & -0.20008200 & -0.96774000 & -3.16800000 \\
\hline $\mathrm{H}$ & -0.49859800 & -2.68443300 & -3.46284700 \\
\hline
\end{tabular}


H

H

D

C

C

C

C

C

C

$\mathrm{H}$

$\mathrm{H}$

$\mathrm{H}$

C

C

$\mathrm{H}$

$\mathrm{H}$

C

C

$\mathrm{H}$

C

C

C

C

C

C

C

$\mathrm{H}$

C

C

$\mathrm{H}$

C

$\mathrm{H}$

$\mathrm{H}$

$\mathrm{H}$

$\mathrm{H}$

O

O

P

$\mathrm{N}$

C

$\mathrm{H}$

C

$\mathrm{H}$ $\begin{array}{lll}-2.25959800 & -0.67794900 & -4.46800600\end{array}$

$\begin{array}{lll}-2.87055900 & -2.25247800 & -3.94891700\end{array}$

$\begin{array}{lll}-7.56380000 & 2.71812800 & 0.14178600\end{array}$

$\begin{array}{lll}-7.18395300 & 1.82831600 & -0.83628600\end{array}$

$\begin{array}{lll}-5.82595200 & 1.43890300 & -0.98616200\end{array}$

$\begin{array}{lll}-4.82577200 & 2.00653100 & -0.12702000\end{array}$

$\begin{array}{lll}-5.26157900 & 2.90339700 & 0.88905200\end{array}$

$\begin{array}{lll}-6.58961600 & 3.24699400 & 1.02005200\end{array}$

$\begin{array}{lll}-6.20313900 & 0.05710400 & -2.61261400\end{array}$

$\begin{array}{lll}-8.60639900 & 3.00348900 & 0.25093500\end{array}$

$\begin{array}{lll}-7.92265100 & 1.39474400 & -1.50641700\end{array}$

$\begin{array}{lll}-5.44408500 & 0.47296600 & -1.95479600\end{array}$

$\begin{array}{lll}-3.44506800 & 1.61974000 & -0.28892900\end{array}$

$\begin{array}{lll}-4.53452900 & 3.31211800 & 1.58043000\end{array}$

$\begin{array}{lll}-6.89135700 & 3.92769700 & 1.81158100\end{array}$

$\begin{array}{lll}-3.16066300 & 0.59704300 & -1.18443200\end{array}$

$\begin{array}{lll}-4.14464300 & 0.04184800 & -2.03741600\end{array}$

$\begin{array}{lll}-3.84242900 & -0.72636600 & -2.74098500\end{array}$

$\begin{array}{lll}-2.35384800 & 2.24990800 & 0.50690200\end{array}$

$\begin{array}{lll}-2.14779600 & 3.67713000 & 0.53293700\end{array}$

$\begin{array}{lll}-1.48650500 & 1.46366600 & 1.25306100\end{array}$

$\begin{array}{lll}-2.83846900 & 4.56725100 & -0.33682200\end{array}$

$\begin{array}{lll}-1.18094500 & 4.23771100 & 1.43370000\end{array}$

$\begin{array}{lll}-0.54133200 & 2.01178200 & 2.14905500\end{array}$

$\begin{array}{lll}-2.61540100 & 5.92613400 & -0.29291200\end{array}$

$\begin{array}{lll}-3.53981900 & 4.16185900 & -1.05645800\end{array}$

$\begin{array}{lll}-0.99279600 & 5.64618500 & 1.47057600\end{array}$

$\begin{array}{lll}-0.41241500 & 3.37351000 & 2.25782500\end{array}$

$\begin{array}{lll}0.05947000 & 1.33027800 & 2.74052400\end{array}$

$\begin{array}{lll}-1.69502400 & 6.47777300 & 0.62883700\end{array}$

$\begin{array}{lll}-3.14829500 & 6.57988200 & -0.97791300\end{array}$

$\begin{array}{lll}-0.26913200 & 6.05311200 & 2.17316800\end{array}$

$\begin{array}{lll}0.30298900 & 3.80528800 & 2.95278500\end{array}$

$\begin{array}{lll}-1.53582100 & 7.55203400 & 0.65981600\end{array}$

$\begin{array}{lll}-1.55903900 & 0.07720700 & 1.19231600\end{array}$

$\begin{array}{lll}-1.87596000 & 0.11854600 & -1.33600600\end{array}$

$\begin{array}{lll}-0.95390400 & -0.67658300 & -0.17504300\end{array}$

$\begin{array}{lll}-1.55646000 & -2.22165300 & 0.13633400\end{array}$

$\begin{array}{lll}-0.95018400 & -3.43867000 & -0.50233600\end{array}$

$\begin{array}{lll}-1.03190300 & -4.20911400 & 0.27198000\end{array}$

$\begin{array}{lll}0.54473800 & -3.35576600 & -0.83148500\end{array}$

$\begin{array}{lll}0.77300500 & -2.65985600 & -1.64101600\end{array}$ 
$\mathrm{H}$

$\mathrm{H}$

C

$\mathrm{H}$

C

C

C

C

$\mathrm{H}$

C

$\mathrm{H}$

C

$\mathrm{H}$

$\mathrm{H}$

$\mathrm{H}$

C

C

C

C

$\mathrm{H}$

C

$\mathrm{H}$

C

$\mathrm{H}$

$\mathrm{H}$

$\mathrm{H}$

C

$\mathrm{H}$

$\mathrm{H}$

$\mathrm{H}$

C

$\mathrm{H}$

C

C

C

C

$\mathrm{H}$

C

$\mathrm{H}$

C

$\mathrm{H}$

$\mathrm{H}$

$\mathrm{H}$

C

$\begin{array}{rrr}1.11551000 & -3.06112600 & 0.05326900 \\ 0.87508600 & -4.35326500 & -1.14253800 \\ -2.72923600 & -2.66433900 & 0.97373700 \\ -3.19004800 & -3.46059300 & 0.37720200 \\ -1.75579700 & -3.93065600 & -1.70582700 \\ -1.97731200 & -3.11962600 & -2.82825200 \\ -2.24813100 & -5.24164500 & -1.72222900 \\ -2.67076300 & -3.60997200 & -3.93507500 \\ -1.60850600 & -2.09856100 & -2.83864200 \\ -2.94401200 & -5.73627800 & -2.82869300 \\ -2.08117700 & -5.88542800 & -0.86066300 \\ -3.15795600 & -4.92017300 & -3.93914800 \\ -2.82587400 & -2.96896900 & -4.79923900 \\ -3.31928700 & -6.75632800 & -2.81905300 \\ -3.69806300 & -5.30009100 & -4.80222400 \\ -2.25556800 & -3.30159800 & 2.28107000 \\ -1.36650500 & -2.64625800 & 3.14429500 \\ -2.75499000 & -4.55482900 & 2.65911600 \\ -0.98918500 & -3.23280300 & 4.35255400 \\ -0.97409600 & -1.67324400 & 2.86765700 \\ -2.38210700 & -5.14253400 & 3.87036600 \\ -3.44521800 & -5.07673200 & 1.99861300 \\ -1.49520400 & -4.48225600 & 4.72099400 \\ -0.29707700 & -2.71179100 & 5.00921400 \\ -2.77953200 & -6.11678000 & 4.14323600 \\ -1.19808500 & -4.93719900 & 5.66219900 \\ -3.86018800 & -1.66256900 & 1.24032800 \\ -3.56196800 & -0.85633100 & 1.90856600 \\ -4.24435700 & -1.23778800 & 0.31111100 \\ -4.67494400 & -2.21784400 & 1.71845200 \\ 2.24889100 & 0.16897400 & 1.09554500 \\ 2.19767700 & 1.18679700 & 1.50580300 \\ 3.07969500 & -0.66285200 & 1.93834800 \\ 3.45127900 & -1.97704900 & 1.56052300 \\ 3.49918800 & -0.19868200 & 3.21104400 \\ 4.18243900 & -2.78871900 & 2.41807500 \\ 3.17186900 & -2.32698900 & 0.57261100 \\ 4.21310100 & -1.01910700 & 4.07834800 \\ 3.24459000 & 0.81577600 & 3.50862600 \\ 0.879379600 & -2.31566700 & 3.68177800 \\ -12540700 & -2.95457200 & 4.35290000 \\ -0.06625500 & -2.74882700\end{array}$


$\mathrm{H}$

$\mathrm{H}$

Pd

C

C

$\mathrm{Si}$

C

C

C

C

$\mathrm{H}$

C

$\mathrm{H}$

$\mathrm{C}$

$\mathrm{H}$

$\mathrm{H}$

$\mathrm{H}$

C

C

C

C

$\mathrm{H}$

C

$\mathrm{H}$

C

$\mathrm{H}$

$\mathrm{H}$

$\mathrm{H}$

$\mathrm{H}$

$\mathrm{H}$

$\mathrm{H}$

$\mathrm{H}$

TS-3

$\mathrm{C}$

C

C

C

C

C

$\mathrm{H}$

$\mathrm{H}$

$\mathrm{H}$

C

$\begin{array}{ccc}0.28669800 & 0.85257800 & -2.86138900 \\ 0.21587200 & -0.89956200 & -3.01195600 \\ 1.33464900 & -0.11205700 & -0.63175400 \\ 3.05170100 & 1.06915000 & -3.30636300 \\ 2.07175400 & -0.03753000 & -3.70940800 \\ 3.42818000 & 0.88476000 & -1.44094400 \\ 4.99541000 & -0.18562100 & -1.25553900 \\ 5.37331300 & -1.08438700 & -2.27321700 \\ 5.82628000 & -0.12396600 & -0.11896500 \\ 6.51576200 & -1.88006300 & -2.16424400 \\ 4.77029500 & -1.16348000 & -3.17385600 \\ 6.97498300 & -0.91000000 & -0.00556100 \\ 5.58101000 & 0.55762700 & 0.69134800 \\ 7.32300200 & -1.79408600 & -1.02835900 \\ 6.77926400 & -2.56109400 & -2.97020500 \\ 7.59755700 & -0.83128100 & 0.88263100 \\ 8.21650100 & -2.40771800 & -0.94269800 \\ 3.79062600 & 2.60933400 & -0.71234700 \\ 2.73177000 & 3.43490500 & -0.28593500 \\ 5.08807900 & 3.15527900 & -0.67625200 \\ 2.95305900 & 4.74186800 & 0.15299500 \\ 1.71450700 & 3.04901300 & -0.29916900 \\ 5.31816400 & 4.45953200 & -0.23103800 \\ 5.93499100 & 2.55476700 & -0.99875000 \\ 4.25064700 & 5.25733200 & 0.18502600 \\ 2.11115500 & 5.35509100 & 0.46598700 \\ 6.33187000 & 4.85330300 & -0.21213300 \\ 4.42870800 & 6.27351400 & 0.52880100 \\ 2.58186600 & -1.01193100 & -3.69339500 \\ 1.73310300 & 0.10627700 & -4.75045800 \\ 3.97074900 & 1.07064300 & -3.90877600 \\ 2.57580700 & 2.04908500 & -3.45049300 \\ & & \end{array}$

$-7.54257100$

2.81360600

0.21731900

$-7.19311600$

1.91497800

$-0.76402000$

$-5.84365200$

1.50571600

$-0.93663500$

$-4.82029200$

2.06283300

$-0.09823900$

$-5.22495600$

2.96911300

0.92232800

$-6.54546100$

3.33167500

1.07610600

$-6.26940200$

0.12152500

$-2.54908200$

$-8.57894800$

3.11387700

0.34427100

$-7.94993500$

1.48925100

$-1.41878000$

$-5.49302100$

0.52964900

$-1.90685100$ 


\begin{tabular}{|c|c|c|c|}
\hline $\mathrm{C}$ & -3.44795900 & 1.65651000 & -0.28264100 \\
\hline $\mathrm{H}$ & -4.47969900 & 3.36929700 & 1.59916700 \\
\hline $\mathrm{H}$ & -6.82341700 & 4.01885400 & 1.87072000 \\
\hline $\mathrm{C}$ & -3.19480700 & 0.62355800 & -1.17627600 \\
\hline $\mathrm{C}$ & -4.20182100 & 0.07842000 & -2.00865900 \\
\hline $\mathrm{H}$ & -3.92338200 & -0.69918700 & -2.71177600 \\
\hline $\mathrm{C}$ & -2.33334400 & 2.27709200 & 0.48759100 \\
\hline $\mathrm{C}$ & -2.10557300 & 3.70119500 & 0.50116800 \\
\hline $\mathrm{C}$ & -1.46027700 & 1.48182800 & 1.21673500 \\
\hline $\mathrm{C}$ & -2.80892800 & 4.59961100 & -0.34989000 \\
\hline $\mathrm{C}$ & -1.10311700 & 4.24926300 & 1.37026900 \\
\hline $\mathrm{C}$ & -0.47232900 & 2.01612300 & 2.07380200 \\
\hline $\mathrm{C}$ & -2.56610200 & 5.95538700 & -0.31549300 \\
\hline $\mathrm{H}$ & -3.53765500 & 4.20324100 & -1.04695300 \\
\hline $\mathrm{C}$ & -0.89454500 & 5.65525800 & 1.39832100 \\
\hline $\mathrm{C}$ & -0.31875200 & 3.37630500 & 2.17010200 \\
\hline $\mathrm{H}$ & 0.14265500 & 1.32740700 & 2.64160300 \\
\hline $\mathrm{C}$ & -1.61071000 & 6.49555100 & 0.57703500 \\
\hline $\mathrm{H}$ & -3.11084800 & 6.61542000 & -0.98498900 \\
\hline $\mathrm{H}$ & -0.14498400 & 6.05298600 & 2.07859900 \\
\hline $\mathrm{H}$ & 0.42939700 & 3.79960400 & 2.83500700 \\
\hline $\mathrm{H}$ & -1.43677000 & 7.56770800 & 0.60225600 \\
\hline $\mathrm{O}$ & -1.56684600 & 0.09678500 & 1.17051500 \\
\hline $\mathrm{O}$ & -1.92062100 & 0.12147700 & -1.34900200 \\
\hline $\mathrm{P}$ & -0.99326400 & -0.67750700 & -0.19428300 \\
\hline $\mathrm{N}$ & -1.60376300 & -2.21247600 & 0.13855400 \\
\hline $\mathrm{C}$ & -1.02258100 & -3.45477000 & -0.47513800 \\
\hline $\mathrm{H}$ & -1.14022200 & -4.20844600 & 0.31039300 \\
\hline $\mathrm{C}$ & 0.48108300 & -3.43224300 & -0.77545300 \\
\hline $\mathrm{H}$ & 0.74673500 & -2.76631200 & -1.59826000 \\
\hline $\mathrm{H}$ & 1.04598500 & -3.13833100 & 0.11359200 \\
\hline $\mathrm{H}$ & 0.77944200 & -4.44889500 & -1.05598900 \\
\hline $\mathrm{C}$ & -2.77633100 & -2.62373700 & 0.99512500 \\
\hline $\mathrm{H}$ & -3.25697000 & -3.41648700 & 0.40941300 \\
\hline $\mathrm{C}$ & -1.82536300 & -3.94468500 & -1.68131900 \\
\hline $\mathrm{C}$ & -2.06821700 & -3.12732700 & -2.79442200 \\
\hline $\mathrm{C}$ & -2.29356900 & -5.26459900 & -1.70834700 \\
\hline $\mathrm{C}$ & -2.75681200 & -3.62049200 & -3.90337600 \\
\hline $\mathrm{H}$ & -1.72192000 & -2.09872600 & -2.79537000 \\
\hline $\mathrm{C}$ & -2.98371400 & -5.76200700 & -2.81682500 \\
\hline $\mathrm{H}$ & -2.11256300 & -5.91294000 & -0.85303100 \\
\hline $\mathrm{C}$ & -3.21786200 & -4.93972000 & -3.91886100 \\
\hline $\mathrm{H}$ & -2.92936900 & -2.97361800 & -4.75987800 \\
\hline $\mathrm{H}$ & -3.33996400 & -6.78890400 & -2.81510400 \\
\hline
\end{tabular}


H

C

C

C

C

$\mathrm{H}$

C

H

C

H

H

H

C

H

H

$\mathrm{H}$

C

C

H

$\mathrm{H}$

$\mathrm{Pd}$

C

C

$\mathrm{Si}$

C

C

C

C

H

C

H

C

H

H

H

C

C

C

C

H

C

$\mathrm{H}$

C

$\mathrm{H}$

\begin{tabular}{|c|c|c|}
\hline 00 & 68400 & -4 \\
\hline 30120300 & -3.25782900 & 2.30346600 \\
\hline .37447800 & -2.62280200 & 3.14158900 \\
\hline 400 & -4 & 500 \\
\hline 774500 & -3.2 & 8200 \\
\hline 700 & -1.6 & 00 \\
\hline 0 & -5.0 & 00 \\
\hline 824000 & -4.9 & 2.06 \\
\hline 500 & -4 & \\
\hline .27480200 & -2.7 & 4.98 \\
\hline 430600 & -6.02567100 & \\
\hline 00 & -4 & 5 \\
\hline 700 & -1.6 & 00 \\
\hline & -0.7 & \\
\hline 631400 & -1.17763200 & 59600 \\
\hline 0900 & -2.14214200 & \\
\hline & & \\
\hline 0 & -0.2459 & 00 \\
\hline & 400 & \\
\hline 500 & -1.0 & 00 \\
\hline 1.20559300 & -0.22748600 & -0.6 \\
\hline & & \\
\hline 2.11737900 & -0.1 & 00 \\
\hline & 00 & -1.2 \\
\hline & 00 & -1 \\
\hline 51228100 & -1.0043 & -2.1 \\
\hline & 00 & \\
\hline 00 & 0 & -2 \\
\hline 00 & -1.1 & \\
\hline 000 & -0.77 & \\
\hline 800 & 00 & 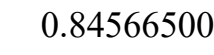 \\
\hline 54657700 & -1.6201 & 300 \\
\hline 97161000 & -2.38517300 & -2.9281 \\
\hline 863700 & 83500 & \\
\hline 00 & -2.1 & -0.5 \\
\hline 79630900 & 2.62176200 & -0.6 \\
\hline 9092000 & 3.451 & -0.3 \\
\hline 5.07679700 & 3.19918200 & -0.53604400 \\
\hline 2.85403100 & 0 & -0 . \\
\hline 8412400 & 3.04051200 & -0.39 \\
\hline 5.24682500 & 4.54351800 & -0.19385900 \\
\hline 5.95586300 & 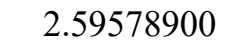 & -0.74602 \\
\hline 4.13614500 & 5.34561900 & 0.07169 \\
\hline 1.97988200 & 5.41169900 & 0.19132 \\
\hline
\end{tabular}




$\begin{array}{lrrr}\mathrm{H} & 6.24828400 & 4.96377600 & -0.13831400 \\ \mathrm{H} & 4.26807700 & 6.39223300 & 0.33523700 \\ \mathrm{C} & 3.20348700 & -0.71517400 & 1.71499800 \\ \mathrm{C} & 3.62524000 & -0.28085000 & 2.99491700 \\ \mathrm{C} & 3.53334900 & -2.03836900 & 1.33655600 \\ \mathrm{C} & 4.29358200 & -1.13632900 & 3.86795900 \\ \mathrm{H} & 3.40679500 & 0.73993000 & 3.30081700 \\ \mathrm{C} & 4.21992700 & -2.88459400 & 2.19839100 \\ \mathrm{H} & 3.25614300 & -2.37803200 & 0.34344200 \\ \mathrm{C} & 4.59622100 & -2.44184200 & 3.47258400 \\ \mathrm{H} & 4.59184700 & -0.78134800 & 4.85125200 \\ \mathrm{H} & 4.46812100 & -3.89352700 & 1.87959400 \\ \mathrm{H} & 5.12978600 & -3.10720200 & 4.14611800 \\ \mathrm{H} & 2.38132700 & 1.17823500 & 1.29839300 \\ \mathrm{H} & 3.93471200 & 1.03579700 & -3.65572200 \\ \mathrm{H} & 2.47643600 & 1.90364400 & -3.18694000 \\ \mathrm{H} & 2.64714200 & -1.15427100 & -3.45014400 \\ \mathrm{H} & 1.91856000 & -0.07673300 & -4.63010700\end{array}$

TS-3'

$\begin{array}{lrrr}\mathrm{C} & -7.71691000 & 2.35142800 & -0.27931500 \\ \mathrm{C} & -7.23198900 & 1.51406600 & -1.25742100 \\ \mathrm{C} & -5.85772300 & 1.15765100 & -1.30216500 \\ \mathrm{C} & -4.95037700 & 1.70372300 & -0.33338400 \\ \mathrm{C} & -5.49371700 & 2.54771200 & 0.67588800 \\ \mathrm{C} & -6.83590900 & 2.85942600 & 0.70326400 \\ \mathrm{H} & -6.04933500 & -0.13419000 & -3.03259700 \\ \mathrm{H} & -8.77134100 & 2.61156000 & -0.25103100 \\ \mathrm{H} & -7.89853500 & 1.09770600 & -2.00913500 \\ \mathrm{C} & -5.36663400 & 0.25189600 & -2.27981500 \\ \mathrm{C} & -3.55147200 & 1.35062400 & -0.38461600 \\ \mathrm{H} & -4.83950200 & 2.93944700 & 1.44561900 \\ \mathrm{H} & -7.22136400 & 3.49858800 & 1.49307800 \\ \mathrm{C} & -3.16868100 & 0.36226600 & -1.28246600 \\ \mathrm{C} & -4.05729100 & -0.15469100 & -2.25732000 \\ \mathrm{H} & -3.66361500 & -0.86018700 & -2.98153400 \\ \mathrm{C} & -2.54953400 & 1.99637400 & 0.50921400 \\ \mathrm{C} & -2.39241800 & 3.42916900 & 0.57202700 \\ \mathrm{C} & -1.71693900 & 1.22528000 & 1.30778000 \\ \mathrm{C} & -3.03767100 & 4.31285000 & -0.33821900 \\ \mathrm{C} & -1.51704800 & 4.00289300 & 1.55441200 \\ \mathrm{C} & -0.86384500 & 1.78528900 & 2.28551000 \\ \mathrm{C} & -2.85672400 & 5.67643200 & -0.26054200 \\ \mathrm{H} & -3.66747800 & 3.90009800 & -1.11727100\end{array}$




\begin{tabular}{|c|c|c|c|}
\hline $\mathrm{C}$ & -1.37049900 & 5.41509100 & 1.62208500 \\
\hline $\mathrm{C}$ & -0.79066400 & 3.14845000 & 2.42553000 \\
\hline $\mathrm{H}$ & -0.28467500 & 1.11266600 & 2.90864300 \\
\hline $\mathrm{C}$ & -2.02690200 & 6.23960900 & 0.73722800 \\
\hline $\mathrm{H}$ & -3.35190200 & 6.32506700 & -0.97794000 \\
\hline $\mathrm{H}$ & -0.71313400 & 5.83101800 & 2.38197300 \\
\hline $\mathrm{H}$ & -0.14862300 & 3.59065100 & 3.18302400 \\
\hline $\mathrm{H}$ & -1.90028700 & 7.31720000 & 0.79341000 \\
\hline $\mathrm{O}$ & -1.73246200 & -0.16009200 & 1.20758300 \\
\hline $\mathrm{O}$ & -1.87761000 & -0.11282100 & -1.33755400 \\
\hline $\mathrm{P}$ & -0.97327800 & -0.84345600 & -0.12514100 \\
\hline $\mathrm{N}$ & -1.44744400 & -2.44360300 & 0.13783200 \\
\hline $\mathrm{C}$ & -0.56036900 & -3.56120000 & -0.32679400 \\
\hline $\mathrm{H}$ & -0.92356100 & -4.42110200 & 0.24732900 \\
\hline $\mathrm{C}$ & 0.90697500 & -3.41112600 & 0.10339700 \\
\hline $\mathrm{H}$ & 1.43948900 & -2.65178300 & -0.47761900 \\
\hline $\mathrm{H}$ & 0.94862400 & -3.14244800 & 1.16257400 \\
\hline $\mathrm{H}$ & 1.43010400 & -4.36523200 & -0.01542400 \\
\hline $\mathrm{C}$ & -2.68080900 & -3.00604600 & 0.79505200 \\
\hline $\mathrm{H}$ & -2.97827000 & -3.83556400 & 0.14103600 \\
\hline $\mathrm{C}$ & -0.75265300 & -3.92573500 & -1.80616300 \\
\hline $\mathrm{C}$ & -1.86003400 & -3.47401800 & -2.53629100 \\
\hline $\mathrm{C}$ & 0.13826500 & -4.80420900 & -2.44381800 \\
\hline $\mathrm{C}$ & -2.06693100 & -3.87403500 & -3.85807800 \\
\hline $\mathrm{H}$ & -2.56020200 & -2.79005400 & -2.07136900 \\
\hline $\mathrm{C}$ & -0.06382900 & -5.20277600 & -3.76556800 \\
\hline $\mathrm{H}$ & 1.00399700 & -5.18561700 & -1.91219800 \\
\hline $\mathrm{C}$ & -1.16847900 & -4.73915700 & -4.48145700 \\
\hline $\mathrm{H}$ & -2.93279300 & -3.50360100 & -4.40134600 \\
\hline $\mathrm{H}$ & 0.64762900 & -5.87666900 & -4.23582700 \\
\hline $\mathrm{H}$ & -1.32484500 & -5.04722300 & -5.51163200 \\
\hline $\mathrm{C}$ & -2.35197600 & -3.61202500 & 2.16021700 \\
\hline $\mathrm{C}$ & -1.71929600 & -2.86634600 & 3.16529400 \\
\hline $\mathrm{C}$ & -2.72782700 & -4.93192700 & 2.44042100 \\
\hline $\mathrm{C}$ & -1.47177500 & -3.43110500 & 4.41646900 \\
\hline $\mathrm{H}$ & -1.42477500 & -1.84175000 & 2.96261100 \\
\hline $\mathrm{C}$ & -2.48358900 & -5.49874000 & 3.69394800 \\
\hline $\mathrm{H}$ & -3.21874300 & -5.52375300 & 1.66990500 \\
\hline $\mathrm{C}$ & -1.85270000 & -4.74863300 & 4.68627500 \\
\hline $\mathrm{H}$ & -0.97962700 & -2.84003800 & 5.18469000 \\
\hline $\mathrm{H}$ & -2.78113100 & -6.52564900 & 3.88967200 \\
\hline $\mathrm{H}$ & -1.65652500 & -5.18587800 & 5.66166500 \\
\hline $\mathrm{C}$ & -3.91364800 & -2.09908700 & 0.88971600 \\
\hline $\mathrm{H}$ & -3.76920500 & -1.26198000 & 1.57140700 \\
\hline
\end{tabular}




\begin{tabular}{|c|c|c|c|}
\hline $\mathrm{H}$ & -4.20786600 & -1.71410100 & -0.08921000 \\
\hline $\mathrm{H}$ & -4.73858100 & -2.71081700 & 1.27093300 \\
\hline $\mathrm{C}$ & 2.04901500 & 0.56445700 & 1.15094700 \\
\hline $\mathrm{H}$ & 1.89362100 & 1.63243000 & 1.35164100 \\
\hline $\mathrm{C}$ & 2.90214000 & -0.01104000 & 2.16933500 \\
\hline $\mathrm{C}$ & 3.38476500 & -1.33984700 & 2.07821700 \\
\hline $\mathrm{C}$ & 3.23421200 & 0.73363400 & 3.32948600 \\
\hline $\mathrm{C}$ & 4.14310000 & -1.89723300 & 3.09968200 \\
\hline $\mathrm{H}$ & 3.16358700 & -1.90718300 & 1.18080600 \\
\hline $\mathrm{C}$ & 3.97622500 & 0.16843400 & 4.36176900 \\
\hline $\mathrm{H}$ & 2.89166000 & 1.76285500 & 3.40625100 \\
\hline $\mathrm{C}$ & 4.43414000 & -1.14811500 & 4.24669900 \\
\hline $\mathrm{H}$ & 4.51383300 & -2.91427000 & 3.00783300 \\
\hline $\mathrm{H}$ & 4.21090400 & 0.75156100 & 5.24822100 \\
\hline $\mathrm{H}$ & 5.02434900 & -1.58840300 & 5.04620800 \\
\hline $\mathrm{C}$ & 0.93600800 & -0.53520100 & -2.62931100 \\
\hline $\mathrm{H}$ & 0.27195100 & 0.28465400 & -2.93696100 \\
\hline $\mathrm{H}$ & 0.37198000 & -1.46568600 & -2.75478000 \\
\hline $\mathrm{Pd}$ & 1.26425500 & -0.12904600 & -0.52308400 \\
\hline $\mathrm{C}$ & 3.01542200 & 0.70935400 & -3.30207100 \\
\hline $\mathrm{C}$ & 2.18015100 & -0.55677800 & -3.52106700 \\
\hline $\mathrm{Si}$ & 3.29135400 & 0.92592200 & -1.42267400 \\
\hline $\mathrm{C}$ & 4.94754500 & 0.09781000 & -0.96635500 \\
\hline $\mathrm{C}$ & 5.48221900 & -0.92776000 & -1.77163500 \\
\hline $\mathrm{C}$ & 5.69141700 & 0.46770500 & 0.17175800 \\
\hline $\mathrm{C}$ & 6.69137400 & -1.55270800 & -1.45915600 \\
\hline $\mathrm{H}$ & 4.95074200 & -1.24402000 & -2.66535500 \\
\hline $\mathrm{C}$ & 6.90492200 & -0.14683400 & 0.48754400 \\
\hline $\mathrm{H}$ & 5.32414600 & 1.25831200 & 0.82086900 \\
\hline $\mathrm{C}$ & 7.40900300 & -1.16251500 & -0.32695300 \\
\hline $\mathrm{H}$ & 7.07649300 & -2.33879700 & -2.10467400 \\
\hline $\mathrm{H}$ & 7.45618500 & 0.16840100 & 1.37041000 \\
\hline $\mathrm{H}$ & 8.35364200 & -1.64289100 & -0.08367100 \\
\hline $\mathrm{C}$ & 3.42145500 & 2.78522100 & -1.01780700 \\
\hline $\mathrm{C}$ & 2.26244200 & 3.53812500 & -0.74570200 \\
\hline $\mathrm{C}$ & 4.64463700 & 3.48073100 & -1.06963200 \\
\hline $\mathrm{C}$ & 2.31722100 & 4.91730900 & -0.53328500 \\
\hline $\mathrm{H}$ & 1.29879800 & 3.03449800 & -0.69955600 \\
\hline $\mathrm{C}$ & 4.70834200 & 4.86000800 & -0.85591900 \\
\hline $\mathrm{H}$ & 5.56431300 & 2.93869200 & -1.27606200 \\
\hline $\mathrm{C}$ & 3.54432500 & 5.58225700 & -0.58558200 \\
\hline . & 1.40317500 & 5.46821400 & -0.32395300 \\
\hline $\mathrm{H}$ & 5.66779400 & 5.37041400 & -0.90062600 \\
\hline $\mathrm{H}$ & 3.59338300 & 6.65564600 & -0.41824500 \\
\hline
\end{tabular}




$\begin{array}{lrrr}\mathrm{H} & 2.78307100 & -1.44857500 & -3.29549400 \\ \mathrm{H} & 1.89358500 & -0.65150200 & -4.58314600 \\ \mathrm{H} & 3.96495300 & 0.70089200 & -3.85522900 \\ \mathrm{H} & 2.45033500 & 1.58367600 & -3.65407500\end{array}$

G

$\mathrm{C}$

$\begin{array}{lll}3.42051500 & -1.06594600 & 3.33500600\end{array}$

$\mathrm{C}$

C

$\begin{array}{lll}4.55602300 & -1.33699100 & 2.30876600\end{array}$

$\begin{array}{lll}2.40746100 & -0.00536000 & 2.91087000\end{array}$

$\mathrm{C}$

C

$\mathrm{C}$

$-6.22292200$

$4.14181000 \quad-2.22488700$

$\begin{array}{lll}-5.72437900 & 3.07107200 & -2.93072000\end{array}$

$-4.57662300$

$2.36578200 \quad-2.47962800$

$-3.90495200$

$2.79535700 \quad-1.28609800$

$\mathrm{C}$

$-4.46406700$

$3.89177500-0.57083800$

$-5.58976300$

4.54350900

$-1.02562900$

$\mathrm{H}$

$-4.60015000$

0.91114000

$-4.08593500$

$-7.10558100$

4.66888100

$-2.57630400$

$-6.21139500$

2.73549100

$-3.84345300$

$-4.09650800$

1.22355600

$-3.17448400$

$-2.73362600$

2.08628900

$-0.83451800$

$-4.00000200$

4.20839400

0.35576000

$-5.99808200$

5.37178000

$-0.45276200$

$-2.38148700$

0.92107500

$-1.50373400$

$-3.03723100$

0.50154700

$-2.68620200$

$-2.68166300$

$-0.39688900$

$-3.17906100$

$-1.93142600$

2.54989100

0.33278500

$-1.35466100$

3.86999500

0.39438200

$-1.68563900$

1.69107800

1.39353300

$-1.38714200$

4.77322800

$-0.70508400$

$-0.67302500$

4.29051200

1.58572400

$-1.01931300$

2.10212000

2.57063900

$-0.81779600$

6.02503400

$-0.61801300$

$-1.85737400$

4.46154200

$-1.63014800$

$-0.11350900$

5.59520700

1.64875600

$-0.54536700$

3.38639300

2.67307800

$-0.90991000$

1.38877800

3.38182000

$-0.18565200$

6.45047400

0.57371800

$-0.85039900$

6.69010600

$-1.47678800$

0.38997500

5.89831300

2.56362300

$-0.04117000$

3.71551100

3.57783800

0.25318400

7.44268200

0.63101900

$-2.12412500$

0.36984000

1.34292900

$-1.32423800$

0.14485300

$-1.07660800$

$-1.19903000$

$-0.66599700$

0.39309800 
$-1.66782000$

$-2.00839800$

0.44851100

$\mathrm{H}$

$-2.19481100 \quad-4.02412600$

0.18485700

$\mathrm{C}$

$-0.17349400 \quad-3.56779200$

0.89619500

$\mathrm{H}$

$0.46089200 \quad-3.05130300$

0.47173400

$\mathrm{H}$

0.09291300

$-3.21960600$

$-0.25176900$

$\mathrm{H}$

$0.04730800 \quad-4.63864100$

1.47426600

$\mathrm{C}$

$\mathrm{H}$

$-3.70530800$

$-2.11852400$

0.40063700

$-4.02672100$

$-2.88242400$

0.66959200

$-2.00603900$

$-3.87611600$

$-0.04816900$

$-1.55255700$

$-3.19935500$

$-1.22162700$

C

C

$-2.73908100$

$-5.05703800$

$-2.36404100$

$-1.82954600$

$-3.69486800$

$-1.38961100$

$-0.97883600 \quad-2.28211500$

$-3.63834600$

$\mathrm{H}$

$-3.01916500$

$-5.55490300$

$-2.26239900$

$-3.09175400$

$-5.59608800$

$-2.66533800$

$-2.56522600$

$-4.87345900$

$-0.51234000$

$-1.46526600$

$-3.16130400$

$-3.79414300$

$-3.59222300$

$-6.47211900$

$-4.51269400$

$-2.78015800$

$-5.25664400$

$-2.77363500$

$-4.00637500$

$-2.66285700$

$-4.78811200$

$-3.52931000 \quad-2.02859000$

2.06699700

$-4.81585200$

$-3.79676600$

3.22302500

$-3.85375500 \quad-2.51964800$

2.21137400

4.48757600

$-2.90828700 \quad-1.14385200$

3.12625100

$-5.14493000 \quad-4.28941800$

3.47699800

$-5.19603500 \quad-4.29859100$

1.32346300

$-4.66204700 \quad-3.65229800$

4.61990400

$-3.47648100 \quad-2.01532300$

5.37368800

$-5.77250900 \quad-5.17236000$

3.56686200

$-4.91181200$

$-4.03385700$

5.60634300

$-4.57014800$

$-0.88859800$

0.36591900

$-4.39866100$

$-0.06897900$

1.06240400

$-4.41075300$

$-0.53207500$

$-0.65377400$

$-5.61737200$

$-1.19847400$

0.45431200

1.66127700

0.15709800

3.71464900

2.88835700

0.95828900

2.70727900

4.93168300

1.22676800

0.55284200

5.02605900

1.95434900

$-0.65130700$

5.34777300

1.87930800

1.72875600

5.49847200

3.26794900

$-0.67714400$

4.72713200

1.49057400

$-1.58720000$

5.82093300

3.19386100

1.71149800

5.30812600

1.35598900

2.68052700 


\begin{tabular}{|c|c|c|c|}
\hline $\mathrm{C}$ & 5.89546900 & 3.89339800 & 0.50660600 \\
\hline $\mathrm{H}$ & 5.55710800 & 3.80219000 & -1.62234400 \\
\hline $\mathrm{H}$ & 6.13417300 & 3.66845300 & 2.63835400 \\
\hline $\mathrm{H}$ & 6.26404100 & 4.91607900 & 0.48869700 \\
\hline $\mathrm{C}$ & 5.45896100 & -1.57674700 & -0.59371000 \\
\hline $\mathrm{C}$ & 6.64186100 & -1.05330300 & -1.14658900 \\
\hline $\mathrm{C}$ & 5.14971500 & -2.91830800 & -0.89532700 \\
\hline $\mathrm{C}$ & 7.47287300 & -1.82630600 & -1.96215900 \\
\hline $\mathrm{H}$ & 6.92141900 & -0.02407200 & -0.93942300 \\
\hline $\mathrm{C}$ & 5.97324000 & -3.69754000 & -1.70857100 \\
\hline $\mathrm{H}$ & 4.24832900 & -3.37180800 & -0.48631000 \\
\hline $\mathrm{C}$ & 7.14041100 & -3.15083600 & -2.24715200 \\
\hline $\mathrm{H}$ & 8.38053700 & -1.39178700 & -2.37448100 \\
\hline $\mathrm{H}$ & 5.70514300 & -4.72953700 & -1.92258800 \\
\hline $\mathrm{H}$ & 7.78491700 & -3.75376400 & -2.88208400 \\
\hline $\mathrm{Si}$ & 4.33379800 & -0.57596900 & 0.57960900 \\
\hline $\mathrm{Pd}$ & 1.04710300 & -0.44719200 & 1.45688700 \\
\hline $\mathrm{C}$ & 2.53702500 & -0.85650600 & 0.02921700 \\
\hline $\mathrm{H}$ & 2.43829400 & -1.95176200 & -0.01412300 \\
\hline $\mathrm{C}$ & 2.09644900 & -0.27645000 & -1.27594800 \\
\hline $\mathrm{C}$ & 1.87569800 & -1.11594600 & -2.38684600 \\
\hline $\mathrm{C}$ & 1.90806200 & 1.10836300 & -1.46355200 \\
\hline $\mathrm{C}$ & 1.49908300 & -0.59864200 & -3.62727500 \\
\hline $\mathrm{H}$ & 2.02529400 & -2.18781400 & -2.27849400 \\
\hline $\mathrm{C}$ & 1.52522500 & 1.62519200 & -2.69891000 \\
\hline $\mathrm{H}$ & 2.04773300 & 1.77573900 & -0.61884400 \\
\hline $\mathrm{C}$ & 1.31938100 & 0.77586900 & -3.79046400 \\
\hline $\mathrm{H}$ & 1.35164100 & -1.27281900 & -4.46799200 \\
\hline $\mathrm{H}$ & 1.37988200 & 2.69712900 & -2.80782200 \\
\hline $\mathrm{H}$ & 1.02091800 & 1.18126400 & -4.75373100 \\
\hline $\mathrm{H}$ & 2.88980500 & -2.00205600 & 3.54659800 \\
\hline $\mathrm{H}$ & 3.87231800 & -0.76248200 & 4.29451200 \\
\hline $\mathrm{H}$ & 5.52498500 & -0.99975300 & 2.69984900 \\
\hline $\mathrm{H}$ & 4.67833500 & -2.41815100 & 2.16237800 \\
\hline
\end{tabular}

H

$\begin{array}{lrrr}\mathrm{C} & 4.34125800 & -3.82704700 & -0.30277600 \\ \mathrm{C} & 3.32052000 & -3.61270500 & -1.44661200 \\ \mathrm{C} & 4.01586100 & -3.08905000 & 1.01450600 \\ \mathrm{C} & 2.53872300 & -3.14096100 & 1.38177300 \\ \mathrm{C} & -2.84109600 & 6.64436100 & -0.91141500 \\ \mathrm{C} & -2.98728300 & 5.61860100 & -1.81652000 \\ \mathrm{C} & -2.29577200 & 4.38865700 & -1.64872100 \\ \mathrm{C} & -1.40271200 & 4.22439200 & -0.53715700\end{array}$




\begin{tabular}{|c|c|c|c|}
\hline $\mathrm{C}$ & -1.29790800 & 5.29838600 & 0.39162900 \\
\hline $\mathrm{C}$ & -1.99724000 & 6.47161400 & 0.21058500 \\
\hline $\mathrm{H}$ & -3.15895600 & 3.44137700 & -3.39656100 \\
\hline $\mathrm{H}$ & -3.38068400 & 7.57762400 & -1.04663500 \\
\hline $\mathrm{H}$ & -3.64923800 & 5.72668700 & -2.67261100 \\
\hline $\mathrm{C}$ & -2.49399100 & 3.30657500 & -2.54716100 \\
\hline $\mathrm{C}$ & -0.69198900 & 2.98070900 & -0.37325800 \\
\hline $\mathrm{H}$ & -0.66526700 & 5.18193800 & 1.26357000 \\
\hline $\mathrm{H}$ & -1.90361500 & 7.27016500 & 0.94163000 \\
\hline $\mathrm{C}$ & -1.01675400 & 1.93284000 & -1.22548300 \\
\hline $\mathrm{C}$ & -1.88841700 & 2.09488500 & -2.32779100 \\
\hline $\mathrm{H}$ & -2.06001300 & 1.24423600 & -2.97769800 \\
\hline $\mathrm{C}$ & 0.33432600 & 2.78748300 & 0.68952200 \\
\hline $\mathrm{C}$ & 1.47408000 & 3.66156700 & 0.82216400 \\
\hline $\mathrm{C}$ & 0.23578900 & 1.71980500 & 1.57136000 \\
\hline $\mathrm{C}$ & 1.77415000 & 4.68385000 & -0.12226200 \\
\hline $\mathrm{C}$ & 2.37989600 & 3.48069200 & 1.92111400 \\
\hline $\mathrm{C}$ & 1.13214500 & 1.52958600 & 2.64675500 \\
\hline $\mathrm{C}$ & 2.86995500 & 5.50429100 & 0.03543400 \\
\hline $\mathrm{H}$ & 1.13433300 & 4.80799000 & -0.98751600 \\
\hline $\mathrm{C}$ & 3.49137700 & 4.35496800 & 2.06354200 \\
\hline $\mathrm{C}$ & 2.16751700 & 2.41127700 & 2.83049700 \\
\hline $\mathrm{H}$ & 0.97961700 & 0.68589200 & 3.31067300 \\
\hline $\mathrm{C}$ & 3.73261800 & 5.35219400 & 1.14660100 \\
\hline $\mathrm{H}$ & 3.07691100 & 6.27123300 & -0.70598100 \\
\hline $\mathrm{H}$ & 4.15909700 & 4.20812800 & 2.90916900 \\
\hline $\mathrm{H}$ & 2.85550300 & 2.28066700 & 3.66168600 \\
\hline $\mathrm{H}$ & 4.58900700 & 6.01049400 & 1.26355200 \\
\hline $\mathrm{O}$ & -0.81118800 & 0.81140900 & 1.45797800 \\
\hline $\mathrm{O}$ & -0.44986200 & 0.68153100 & -1.06103600 \\
\hline $\mathrm{P}$ & -0.73419200 & -0.33819000 & 0.24518200 \\
\hline $\mathrm{N}$ & -2.34563600 & -0.83996800 & 0.31995000 \\
\hline $\mathrm{C}$ & -2.77838900 & -2.17154600 & -0.23146400 \\
\hline $\mathrm{H}$ & -3.50462600 & -2.53726900 & 0.50308100 \\
\hline $\mathrm{C}$ & -1.70421600 & -3.26030900 & -0.32582400 \\
\hline $\mathrm{H}$ & -0.95039000 & -3.05609500 & -1.08896800 \\
\hline $\mathrm{H}$ & -1.19944300 & -3.40404600 & 0.63462000 \\
\hline $\mathrm{H}$ & -2.20554900 & -4.19682200 & -0.59576900 \\
\hline $\mathrm{C}$ & -3.56366000 & -0.14713300 & 0.87797700 \\
\hline $\mathrm{H}$ & -4.36026500 & -0.42074900 & 0.17778300 \\
\hline $\mathrm{C}$ & -3.51690400 & -2.02800500 & -1.56271400 \\
\hline $\mathrm{C}$ & -2.86139900 & -1.58267500 & -2.71964900 \\
\hline $\mathrm{C}$ & -4.86802500 & -2.38580700 & -1.65388600 \\
\hline $\mathrm{C}$ & -3.54656400 & -1.49123100 & -3.93146600 \\
\hline
\end{tabular}


H

C

$\mathrm{H}$

C

$\mathrm{H}$

$\mathrm{H}$

$\mathrm{H}$

C

C

C

C

$\mathrm{H}$

C

$\mathrm{H}$

C

$\mathrm{H}$

$\mathrm{H}$

$\mathrm{H}$

C

$\mathrm{H}$

$\mathrm{H}$

$\mathrm{H}$

Pd

$\mathrm{H}$

$\mathrm{H}$

$\mathrm{H}$

C

C

C

C

$\mathrm{H}$

C

$\mathrm{H}$

C

$\mathrm{H}$

$\mathrm{H}$

$\mathrm{H}$

C

C

C

C

$\mathrm{H}$

C

$\mathrm{H}$

$\begin{array}{ccc}-1.80876000 & -1.31610900 & -2.68378700 \\ -5.55777600 & -2.29250600 & -2.86578700 \\ -5.38552200 & -2.74670100 & -0.76697700 \\ -4.89772000 & -1.84146400 & -4.00833200 \\ -3.01592100 & -1.15698400 & -4.81847300 \\ -6.60696600 & -2.57252000 & -2.91372200 \\ -5.42849400 & -1.76854400 & -4.95396300 \\ -3.95435700 & -0.72755800 & 2.23871900 \\ -3.04956400 & -0.79642600 & 3.30747400 \\ -5.27158600 & -1.15687700 & 2.44912600 \\ -3.45791200 & -1.27925600 & 4.55136900 \\ -2.02607400 & -0.46614300 & 3.16523900 \\ -5.68263100 & -1.64069600 & 3.69358600 \\ -5.98638700 & -1.10802200 & 1.62960100 \\ -4.77446800 & -1.70356600 & 4.75038500 \\ -2.74501200 & -1.31788100 & 5.37125100 \\ -6.70869700 & -1.97120300 & 3.83303000 \\ -5.08788800 & -2.08006000 & 5.72050000 \\ -3.59005700 & 1.38681000 & 0.91579700 \\ -2.89958800 & 1.80728700 & 1.64493200 \\ -3.38193500 & 1.81228800 & -0.06763800 \\ -4.60652500 & 1.68153200 & 1.20027400 \\ 1.07650500 & -1.83115300 & 0.58817200 \\ 2.11407900 & -4.13052700 & 1.17052400 \\ 4.61906700 & -3.54352800 & 1.81727200 \\ 4.35101000 & -2.04723700 & 0.93759000 \\ 2.11776800 & -2.65618500 & 2.71379200 \\ 0.83880800 & -3.01095200 & 3.21689400 \\ 2.90841300 & -1.79301900 & 3.51423700 \\ 0.37669800 & -2.52777600 & 4.44533200 \\ 0.23313100 & -3.72214000 & 2.65926900 \\ 2.44962100 & -1.32962400 & 4.74132700 \\ 3.89503900 & -1.50421100 & 3.16655100 \\ 1.17821700 & -1.68631500 & 5.21499300 \\ -0.60474000 & -2.82881100 & 4.80215400 \\ 3.08884200 & -0.68787800 & 5.34345200 \\ 0.83010100 & -1.32231600 & 6.17780600 \\ 3.65064200 & -0.54424300 & -1.49685700 \\ 3.44618000 & 0.72637800 & -0.92864900 \\ 4.85790000 & -0.75318800 & -2.19368300 \\ 5.05784800 & -1.71850800 & -2.65327300\end{array}$




$\begin{array}{lccc}\mathrm{C} & 5.58589700 & 1.50861300 & -1.74506700 \\ \mathrm{H} & 4.20958100 & 2.71520800 & -0.60391900 \\ \mathrm{H} & 6.73705700 & 0.06422900 & -2.86144200 \\ \mathrm{H} & 6.32918500 & 2.29680900 & -1.83872900 \\ \mathrm{C} & 1.34350800 & -1.89898300 & -2.99832700 \\ \mathrm{C} & 1.18257300 & -0.70451100 & -3.72711200 \\ \mathrm{C} & 0.76237000 & -3.06480400 & -3.53640700 \\ \mathrm{C} & 0.48754300 & -0.67709400 & -4.93843900 \\ \mathrm{H} & 1.62191900 & 0.21470900 & -3.34984400 \\ \mathrm{C} & 0.06639600 & -3.04586900 & -4.74700700 \\ \mathrm{H} & 0.86443800 & -4.01232300 & -3.01137600 \\ \mathrm{C} & -0.06955100 & -1.84977700 & -5.45541400 \\ \mathrm{H} & 0.39252700 & 0.25818900 & -5.48553400 \\ \mathrm{H} & -0.36305700 & -3.96429400 & -5.14002100 \\ \mathrm{H} & -0.59818800 & -1.83344800 & -6.40561600 \\ \mathrm{Si} & 2.37168500 & -1.95451600 & -1.39036900 \\ \mathrm{H} & 3.81455900 & -3.74243800 & -2.41900000 \\ \mathrm{H} & 2.55527500 & -4.39962300 & -1.40477500 \\ \mathrm{H} & 4.42893700 & -4.90285600 & -0.09540200 \\ \mathrm{H} & 5.33503100 & -3.50132900 & -0.63320400\end{array}$

\section{TS-4}

$\begin{array}{lrrr}\mathrm{C} & -3.66100700 & -0.91647000 & -2.94627000 \\ \mathrm{C} & -4.98172600 & -0.71430700 & -2.17887100 \\ \mathrm{C} & -2.62461500 & 0.14576100 & -2.60011100 \\ \mathrm{C} & 6.54006400 & 3.21963300 & 2.91615800 \\ \mathrm{C} & 5.76501800 & 2.25896700 & 3.52396400 \\ \mathrm{C} & 4.58087500 & 1.77369600 & 2.90694500 \\ \mathrm{C} & 4.16508300 & 2.31657100 & 1.64483000 \\ \mathrm{C} & 5.00631300 & 3.29059100 & 1.03592600 \\ \mathrm{C} & 6.15880600 & 3.72721300 & 1.65211700 \\ \mathrm{H} & 4.12520800 & 0.33825500 & 4.46540700 \\ \mathrm{H} & 7.44756700 & 3.57760000 & 3.39444600 \\ \mathrm{H} & 6.05480800 & 1.84162100 & 4.48561200 \\ \mathrm{C} & 3.81403800 & 0.73753500 & 3.50319000 \\ \mathrm{C} & 2.95669700 & 1.83343900 & 1.02446000 \\ \mathrm{H} & 4.73872500 & 3.68396500 & 0.06235600 \\ \mathrm{H} & 6.78454300 & 4.46537600 & 1.15757600 \\ \mathrm{C} & 2.31265900 & 0.74936700 & 1.61043000 \\ \mathrm{C} & 2.71976000 & 0.21836100 & 2.85886300 \\ \mathrm{H} & 2.15063100 & -0.60541700 & 3.27640400 \\ \mathrm{C} & 2.41654500 & 2.43220200 & -0.22854200 \\ \mathrm{C} & 2.10868900 & 3.83580400 & -0.34419300 \\ \mathrm{C} & 2.15795900 & 1.62250700 & -1.32511000\end{array}$




\begin{tabular}{|c|c|c|c|}
\hline $\mathrm{C}$ & 2.17388300 & 4.73136700 & 0.76065000 \\
\hline $\mathrm{C}$ & 1.67376800 & 4.36252100 & -1.60659600 \\
\hline $\mathrm{C}$ & 1.72172000 & 2.13584300 & -2.56755700 \\
\hline $\mathrm{C}$ & 1.87311900 & 6.06882400 & 0.61616100 \\
\hline $\mathrm{H}$ & 2.45710700 & 4.34867200 & 1.73400900 \\
\hline $\mathrm{C}$ & 1.38817500 & 5.74935800 & -1.72518300 \\
\hline $\mathrm{C}$ & 1.51004400 & 3.48422300 & -2.71049400 \\
\hline $\mathrm{H}$ & 1.56924700 & 1.44285000 & -3.38820200 \\
\hline $\mathrm{C}$ & 1.48994400 & 6.59113000 & -0.64170200 \\
\hline $\mathrm{H}$ & 1.92836800 & 6.72746000 & 1.47886400 \\
\hline $\mathrm{H}$ & 1.07043400 & 6.13042500 & -2.69278100 \\
\hline $\mathrm{H}$ & 1.18636100 & 3.89171400 & -3.66472900 \\
\hline $\mathrm{H}$ & 1.26339500 & 7.64879800 & -0.74434100 \\
\hline $\mathrm{O}$ & 2.36515700 & 0.25142200 & -1.23931100 \\
\hline $\mathrm{O}$ & 1.19937700 & 0.17840700 & 1.02939900 \\
\hline $\mathrm{P}$ & 1.15593600 & -0.62865500 & -0.47333700 \\
\hline $\mathrm{N}$ & 1.97355500 & -2.10532700 & -0.38936800 \\
\hline $\mathrm{C}$ & 1.21339400 & -3.38902600 & -0.20976600 \\
\hline $\mathrm{H}$ & 1.77635400 & -4.11419200 & -0.80758700 \\
\hline $\mathrm{C}$ & -0.21679800 & -3.43205000 & -0.76472000 \\
\hline $\mathrm{H}$ & -0.92105800 & -2.81848100 & -0.19840400 \\
\hline $\mathrm{H}$ & -0.24002500 & -3.11341400 & -1.81179800 \\
\hline $\mathrm{H}$ & -0.56485300 & -4.46958000 & -0.70762900 \\
\hline $\mathrm{C}$ & 3.44353600 & -2.42802900 & -0.42056500 \\
\hline $\mathrm{H}$ & 3.55872000 & -3.22053800 & 0.32861400 \\
\hline $\mathrm{C}$ & 1.24837900 & -3.87942100 & 1.23876500 \\
\hline $\mathrm{C}$ & 0.72807400 & -3.10670800 & 2.28719200 \\
\hline $\mathrm{C}$ & 1.76889800 & -5.14452900 & 1.53718100 \\
\hline $\mathrm{C}$ & 0.73449600 & -3.58775100 & 3.59690100 \\
\hline $\mathrm{H}$ & 0.32061400 & -2.12185200 & 2.07894700 \\
\hline $\mathrm{C}$ & 1.77534500 & -5.63091600 & 2.84755600 \\
\hline $\mathrm{H}$ & 2.17190700 & -5.75864400 & 0.73404900 \\
\hline $\mathrm{C}$ & 1.25927100 & -4.85183600 & 3.88256600 \\
\hline $\mathrm{H}$ & 0.32716300 & -2.97513200 & 4.39765000 \\
\hline $\mathrm{H}$ & 2.18728300 & -6.61483100 & 3.05672500 \\
\hline $\mathrm{H}$ & 1.26493600 & -5.22391300 & 4.90366500 \\
\hline $\mathrm{C}$ & 3.83652200 & -3.02839100 & -1.77192100 \\
\hline $\mathrm{C}$ & 3.57678300 & -2.36073600 & -2.97727800 \\
\hline $\mathrm{C}$ & 4.51406800 & -4.25347900 & -1.81798500 \\
\hline $\mathrm{C}$ & 3.98546800 & -2.90757400 & -4.19368500 \\
\hline $\mathrm{H}$ & 3.05719400 & -1.40807300 & -2.95640900 \\
\hline $\mathrm{C}$ & 4.92593100 & -4.80302500 & -3.03492400 \\
\hline $\mathrm{H}$ & 4.72429700 & -4.78298400 & -0.89040500 \\
\hline $\mathrm{C}$ & 4.66087400 & -4.13091700 & -4.22795900 \\
\hline
\end{tabular}


$\mathrm{H}$

$\mathrm{H}$

$\mathrm{H}$

C

$\mathrm{H}$

$\mathrm{H}$

$\mathrm{H}$

$\mathrm{H}$

$\mathrm{H}$

C

C

C

$\mathrm{C}$

$\mathrm{H}$

C

$\mathrm{H}$

C

$\mathrm{H}$

$\mathrm{H}$

$\mathrm{H}$

C

C

C

C

$\mathrm{H}$

C

$\mathrm{H}$

C

$\mathrm{H}$

$\mathrm{H}$

$\mathrm{H}$

$\mathrm{Si}$

$\mathrm{Pd}$

C

$\mathrm{H}$

C

C

C

C

$\mathrm{H}$

C

$\mathrm{H}$

C

$\mathrm{H}$

$$
3.77709600
$$

$-2.37636900$

$-5.75662800$

5.44758300

$-4.55561700$

4.97595600

4.44202300

$-1.33959400$

4.49475400

$-0.51937400$

4.20846300

$-0.94010000$

$-1.81156600$

5.42970500

$-1.69483800$

0.00342900

$-2.97252000$

1.15935900

0.40899400

1.26033300

0.14059400

1.81418700

1.50992500

0.69283100

$-0.51132500$

1.53106400

2.47160100

0.47000700

1.96373300

$-2.07494800$

$-3.31074300$

$-2.10470600$

$-4.52151400$

$-3.33734200$

$-3.30906600$

$-1.17142000$

$-4.52303900$

$-5.46141200$

$-3.30344600$

$-5.46221700$

$-0.42290100$

$-0.30459600$

0.61613300

0.26573200

2.10830500

2.81974000

2.85938600

4.21175900

2.26473400

4.25399600

2.34816400

4.93787900

4.73052200
$-5.11906900$

$-3.04842000$

$-5.17744500$

$-0.00542000$

$-0.71939800$

0.98303900

0.04653000

$-3.21758700$

$-2.79041100$

0.62336200

1.71138600

0.32517500

2.47151600

1.96211900

1.08024300

$-0.50955400$

2.15753000

3.30485900

0.82746500

2.74719500

0.44577000

$-0.15131400$

1.71119200

0.47937100

$-1.12557300$

2.34694500

2.21484800

1.73017600

$-0.00689600$

3.32020800

2.22284100

$-0.37616700$

$-1.34342500$

$-0.47972400$

0.31859300

$-0.47138600$

0.16501200

$-1.06155600$

0.21639800

0.60561300

$-1.01245300$

$-1.55261700$

$-0.37352600$

0.70673100 


$\begin{array}{lrrr}\mathrm{H} & -4.76561500 & 4.80493100 & -1.47164600 \\ \mathrm{H} & -2.91300700 & 6.02409100 & -0.33754600 \\ \mathrm{H} & -3.25224700 & -1.91264100 & -2.73810100 \\ \mathrm{H} & -3.84155800 & -0.88187300 & -4.03340100 \\ \mathrm{H} & -5.49527400 & 0.18171900 & -2.55395800 \\ \mathrm{H} & -5.66931600 & -1.55384000 & -2.33637800\end{array}$

TS-4'

C

C

C

C

C

C

C

C

C

C

$\mathrm{H}$

$\mathrm{H}$

$\mathrm{H}$

C

C

$\mathrm{H}$

$\mathrm{H}$

C

C

$\mathrm{H}$

C

C

C

C

C

C

C

$\mathrm{H}$

C

C

$\mathrm{H}$

C

$\mathrm{H}$

$\mathrm{H}$

$\mathrm{H}$

$\mathrm{H}$

$\begin{array}{rcc}6.04150100 & -1.88017700 & -0.52493300 \\ 5.10247400 & -1.68382400 & -1.73783800 \\ 5.37465400 & -1.31666400 & 0.73804800 \\ 3.87038500 & -1.69609000 & 0.76793800 \\ -5.66281400 & 4.70586400 & -1.09473200 \\ -5.35543700 & 3.67217300 & -1.94897400 \\ -4.18748800 & 2.88585300 & -1.75862700 \\ -3.29282500 & 3.19445500 & -0.67936000 \\ -3.65926600 & 4.25355700 & 0.19889600 \\ -4.80931700 & 4.98544400 & -0.00203800 \\ -4.57783600 & 1.55617400 & -3.42476800 \\ -6.56261500 & 5.29565300 & -1.24631700 \\ -6.01243200 & 3.42827200 & -2.78067400 \\ -3.90383200 & 1.77810600 & -2.60102200 \\ -2.10029300 & 2.40442200 & -0.49458700 \\ -3.02604300 & 4.47636300 & 1.04936500 \\ -5.06581700 & 5.78198000 & 0.69131900 \\ -1.93918100 & 1.27590700 & -1.29018600 \\ -2.81884200 & 0.97478700 & -2.35778600 \\ -2.61226700 & 0.09834800 & -2.96177100 \\ -1.08264600 & 2.73830400 & 0.54137500 \\ -0.45121100 & 4.03297100 & 0.61137800 \\ -0.69020600 & 1.77894100 & 1.46560100 \\ -0.63776900 & 5.03476200 & -0.38329300 \\ 0.44254200 & 4.32740200 & 1.69553000 \\ 0.19602400 & 2.06283800 & 2.52969900 \\ -0.01658300 & 6.26169600 & -0.28942100 \\ -1.27115100 & 4.82012900 & -1.23576000 \\ 1.05210000 & 5.60879100 & 1.77209200 \\ 0.72763500 & 3.32136300 & 2.65594000 \\ 0.43707100 & 1.26907400 & 3.22790500 \\ 0.82725200 & 6.56205500 & 0.80579800 \\ -0.17314800 & 7.00318100 & -1.06827300 \\ 1.71744000 & 5.81513100 & 2.60713900 \\ 1.39946100 & 3.55368900 & 3.47840000 \\ 1.30505000 & 7.53558800 & 0.87243800 \\ & & \end{array}$




\begin{tabular}{|c|c|c|c|}
\hline $\mathrm{O}$ & -1.21661200 & 0.49795800 & 1.41076800 \\
\hline $\mathrm{O}$ & -0.87017700 & 0.42117700 & -1.10961700 \\
\hline $\mathrm{P}$ & -0.61546900 & -0.55922900 & 0.25115500 \\
\hline $\mathrm{N}$ & -1.83426500 & -1.72451600 & 0.40660200 \\
\hline $\mathrm{C}$ & -1.63855100 & -3.13928700 & -0.06273300 \\
\hline $\mathrm{H}$ & -2.12348600 & -3.74463300 & 0.71121800 \\
\hline $\mathrm{C}$ & -0.19773100 & -3.65742000 & -0.14047000 \\
\hline $\mathrm{H}$ & 0.38031200 & -3.19599000 & -0.94378200 \\
\hline $\mathrm{H}$ & 0.32901300 & -3.50317600 & 0.80652200 \\
\hline $\mathrm{H}$ & -0.24742600 & -4.73434600 & -0.34113400 \\
\hline $\mathrm{C}$ & -3.21815800 & -1.62341000 & 0.99634200 \\
\hline $\mathrm{H}$ & -3.82345100 & -2.26286900 & 0.34409900 \\
\hline $\mathrm{C}$ & -2.37557400 & -3.41317800 & -1.37412100 \\
\hline $\mathrm{C}$ & -2.01853400 & -2.75725100 & -2.56062400 \\
\hline $\mathrm{C}$ & -3.39843300 & -4.36863700 & -1.41885200 \\
\hline $\mathrm{C}$ & -2.67520100 & -3.04722800 & -3.75662100 \\
\hline $\mathrm{H}$ & -1.21888700 & -2.02212500 & -2.55459300 \\
\hline $\mathrm{C}$ & -4.06022300 & -4.65978100 & -2.61513300 \\
\hline $\mathrm{H}$ & -3.67826600 & -4.89403900 & -0.50758200 \\
\hline $\mathrm{C}$ & -3.70093600 & -3.99672900 & -3.78816400 \\
\hline $\mathrm{H}$ & -2.37622600 & -2.53627500 & -4.66800200 \\
\hline $\mathrm{H}$ & -4.85415200 & -5.40215800 & -2.62702000 \\
\hline $\mathrm{H}$ & -4.21160900 & -4.21958300 & -4.72135500 \\
\hline $\mathrm{C}$ & -3.26806000 & -2.24560300 & 2.39410400 \\
\hline $\mathrm{C}$ & -2.37708000 & -1.87037400 & 3.40941100 \\
\hline $\mathrm{C}$ & -4.25991800 & -3.19000300 & 2.69082000 \\
\hline $\mathrm{C}$ & -2.47851500 & -2.42858600 & 4.68422900 \\
\hline $\mathrm{H}$ & -1.60680700 & -1.13609100 & 3.19946800 \\
\hline $\mathrm{C}$ & -4.36607100 & -3.74774200 & 3.96720100 \\
\hline $\mathrm{H}$ & -4.95953100 & -3.49147800 & 1.91319300 \\
\hline $\mathrm{C}$ & -3.47233200 & -3.36890600 & 4.96901100 \\
\hline $\mathrm{H}$ & -1.77867100 & -2.12552700 & 5.45902700 \\
\hline $\mathrm{H}$ & -5.14146300 & -4.48100700 & 4.17406200 \\
\hline $\mathrm{H}$ & -3.54737900 & -3.80269400 & 5.96274100 \\
\hline $\mathrm{C}$ & -3.93872100 & -0.26801000 & 0.98205900 \\
\hline $\mathrm{H}$ & -3.50960500 & 0.44976000 & 1.67862900 \\
\hline $\mathrm{H}$ & -3.95244300 & 0.16498400 & -0.01964000 \\
\hline $\mathrm{H}$ & -4.97639700 & -0.45496200 & 1.28155700 \\
\hline $\mathrm{Pd}$ & 1.58750100 & -1.04561700 & 0.54677200 \\
\hline $\mathrm{H}$ & 3.79614400 & -2.77365300 & 0.57714000 \\
\hline $\mathrm{H}$ & 5.84816800 & -1.70925000 & 1.64853700 \\
\hline $\mathrm{H}$ & 5.49213100 & -0.22690100 & 0.77510400 \\
\hline $\mathrm{C}$ & 3.26665800 & -1.41285100 & 2.12576800 \\
\hline $\mathrm{C}$ & 2.64920200 & -2.44934100 & 2.86715000 \\
\hline
\end{tabular}




\begin{tabular}{|c|c|c|c|}
\hline $\mathrm{C}$ & 3.40318200 & -0.15315300 & 2.75937400 \\
\hline $\mathrm{C}$ & 2.19463300 & -2.23870700 & 4.17046300 \\
\hline $\mathrm{H}$ & 2.56196000 & -3.43697500 & 2.42042500 \\
\hline $\mathrm{C}$ & 2.95762900 & 0.04586500 & 4.06485600 \\
\hline $\mathrm{H}$ & 3.87291700 & 0.66527500 & 2.22233100 \\
\hline $\mathrm{C}$ & 2.35243700 & -0.99266300 & 4.77985900 \\
\hline $\mathrm{H}$ & 1.73173600 & -3.05847500 & 4.71382400 \\
\hline $\mathrm{H}$ & 3.09031400 & 1.01992300 & 4.52967600 \\
\hline $\mathrm{H}$ & 2.01588100 & -0.83352700 & 5.80070100 \\
\hline $\mathrm{C}$ & 3.75427400 & 1.03515800 & -1.21236000 \\
\hline $\mathrm{C}$ & 2.70807900 & 1.95946700 & -1.01768700 \\
\hline $\mathrm{C}$ & 5.03908600 & 1.56239900 & -1.45233000 \\
\hline $\mathrm{C}$ & 2.92986900 & 3.33716900 & -1.07263500 \\
\hline $\mathrm{H}$ & 1.70279500 & 1.59459200 & -0.82630300 \\
\hline $\mathrm{C}$ & 5.27100200 & 2.93926900 & -1.49509300 \\
\hline $\mathrm{H}$ & 5.88000200 & 0.89454800 & -1.61639700 \\
\hline $\mathrm{C}$ & 4.21470500 & 3.83095600 & -1.30811700 \\
\hline $\mathrm{H}$ & 2.10032500 & 4.02325600 & -0.92988100 \\
\hline $\mathrm{H}$ & 6.27566100 & 3.31221400 & -1.68008800 \\
\hline $\mathrm{H}$ & 4.38926900 & 4.90341600 & -1.34591500 \\
\hline $\mathrm{C}$ & 2.29977000 & -1.35588300 & -2.63358300 \\
\hline $\mathrm{C}$ & 1.64875500 & -0.40373700 & -3.43997900 \\
\hline $\mathrm{C}$ & 2.18168500 & -2.70922700 & -3.01078000 \\
\hline $\mathrm{C}$ & 0.93344600 & -0.78272500 & -4.57899500 \\
\hline $\mathrm{H}$ & 1.71263900 & 0.65021600 & -3.18845300 \\
\hline $\mathrm{C}$ & 1.45962800 & -3.09503000 & -4.14087800 \\
\hline $\mathrm{H}$ & 2.67062800 & -3.48249600 & -2.42074400 \\
\hline $\mathrm{C}$ & 0.83815400 & -2.12906300 & -4.93617600 \\
\hline $\mathrm{H}$ & 0.45737000 & -0.02091900 & -5.19202700 \\
\hline $\mathrm{H}$ & 1.38952200 & -4.14719800 & -4.40578500 \\
\hline $\mathrm{H}$ & 0.28987300 & -2.42443200 & -5.82729100 \\
\hline $\mathrm{Si}$ & 3.45741200 & -0.84534100 & -1.19879300 \\
\hline $\mathrm{H}$ & 5.56517100 & -1.08821800 & -2.53357100 \\
\hline $\mathrm{H}$ & 4.86206700 & -2.64516400 & -2.20499200 \\
\hline $\mathrm{H}$ & 6.24530500 & -2.94800300 & -0.37277500 \\
\hline $\mathrm{H}$ & 7.01618400 & -1.40166300 & -0.67996600 \\
\hline \multicolumn{4}{|l|}{$\mathbf{3 a}$} \\
\hline $\mathrm{C}$ & 0.57255800 & -1.20745100 & 3.24302200 \\
\hline $\mathrm{C}$ & 1.06885900 & 0.16153800 & 2.71185400 \\
\hline $\mathrm{C}$ & -0.70623200 & -1.59958100 & 2.48023100 \\
\hline $\mathrm{H}$ & -1.04095500 & -2.61187300 & 2.74245800 \\
\hline $\mathrm{H}$ & -1.51592900 & -0.91688800 & 2.77066500 \\
\hline $\mathrm{C}$ & -0.26570400 & 1.67689900 & 0.27651700 \\
\hline
\end{tabular}




\begin{tabular}{|c|c|c|c|}
\hline $\mathrm{C}$ & -0.97887400 & 1.70701500 & -0.93928500 \\
\hline $\mathrm{C}$ & -0.22711900 & 2.86184400 & 1.03543000 \\
\hline $\mathrm{C}$ & -1.62249500 & 2.86691700 & -1.37401300 \\
\hline $\mathrm{H}$ & -1.04190100 & 0.81299700 & -1.55413200 \\
\hline $\mathrm{C}$ & -0.86875500 & 4.02534700 & 0.60517700 \\
\hline $\mathrm{H}$ & 0.31499400 & 2.88411300 & 1.97785200 \\
\hline $\mathrm{C}$ & -1.56900800 & 4.02956700 & -0.60199000 \\
\hline $\mathrm{H}$ & -2.16874000 & 2.86134900 & -2.31383800 \\
\hline $\mathrm{H}$ & -0.82120500 & 4.92629600 & 1.21180100 \\
\hline $\mathrm{H}$ & -2.07090800 & 4.93290100 & -0.93939100 \\
\hline $\mathrm{C}$ & 2.16776900 & -0.16114900 & -0.24678600 \\
\hline $\mathrm{C}$ & 2.49043200 & 0.67661900 & -1.32969500 \\
\hline $\mathrm{C}$ & 3.03451800 & -1.24016900 & 0.01986700 \\
\hline $\mathrm{C}$ & 3.62384900 & 0.44650800 & -2.11369300 \\
\hline $\mathrm{H}$ & 1.84863900 & 1.52109000 & -1.56645100 \\
\hline $\mathrm{C}$ & 4.16907200 & -1.47410900 & -0.75717400 \\
\hline $\mathrm{H}$ & 2.82561000 & -1.91254500 & 0.85038300 \\
\hline $\mathrm{C}$ & 4.46584100 & -0.62917800 & -1.82949400 \\
\hline $\mathrm{H}$ & 3.84858500 & 1.10908600 & -2.94587300 \\
\hline $\mathrm{H}$ & 4.82072700 & -2.31360800 & -0.52768600 \\
\hline $\mathrm{H}$ & 5.34844900 & -0.80879500 & -2.43812000 \\
\hline $\mathrm{Si}$ & 0.64964300 & 0.12642000 & 0.84603500 \\
\hline $\mathrm{C}$ & -0.42665000 & -1.48087600 & 0.95774800 \\
\hline $\mathrm{H}$ & 0.27891700 & -2.28477300 & 0.69846900 \\
\hline $\mathrm{C}$ & -1.62180600 & -1.61933000 & 0.04483800 \\
\hline $\mathrm{C}$ & -1.52975900 & -2.39824400 & -1.12137400 \\
\hline $\mathrm{C}$ & -2.84394100 & -0.97483700 & 0.30156200 \\
\hline $\mathrm{C}$ & -2.60841700 & -2.52750400 & -1.99742400 \\
\hline $\mathrm{H}$ & -0.59636600 & -2.91220400 & -1.34181200 \\
\hline $\mathrm{C}$ & -3.92749900 & -1.10873600 & -0.56740100 \\
\hline $\mathrm{H}$ & -2.95166000 & -0.35422400 & 1.18653500 \\
\hline $\mathrm{C}$ & -3.81666800 & -1.88420400 & -1.72321900 \\
\hline $\mathrm{H}$ & -2.50517400 & -3.13869000 & -2.89066700 \\
\hline $\mathrm{H}$ & -4.86133600 & -0.60022500 & -0.34059700 \\
\hline $\mathrm{H}$ & -4.66117900 & -1.98745100 & -2.39932000 \\
\hline $\mathrm{H}$ & 2.12782400 & 0.33266200 & 2.93243300 \\
\hline $\mathrm{H}$ & 0.50772600 & 0.96876200 & 3.20081300 \\
\hline $\mathrm{H}$ & 1.34227200 & -1.97249300 & 3.06771500 \\
\hline $\mathrm{H}$ & 0.39677500 & -1.18668100 & 4.32608100 \\
\hline
\end{tabular}

TS-5

$\begin{array}{lrrr}\mathrm{C} & 7.71504000 & -0.14317100 & 0.34500900 \\ \mathrm{C} & 7.07517800 & 0.72143200 & -0.51316400 \\ \mathrm{C} & 5.68002700 & 0.61356400 & -0.75797400\end{array}$




\begin{tabular}{|c|c|c|c|}
\hline $\mathrm{C}$ & 4.92799100 & -0.43388600 & -0.12787300 \\
\hline $\mathrm{C}$ & 5.62025000 & -1.29245300 & 0.77256400 \\
\hline $\mathrm{C}$ & 6.97147200 & -1.15033800 & 1.00289100 \\
\hline $\mathrm{H}$ & 5.58332300 & 2.32629000 & -2.08273500 \\
\hline $\mathrm{H}$ & 8.78138100 & -0.04625300 & 0.52960300 \\
\hline $\mathrm{H}$ & 7.62707800 & 1.51587900 & -1.01045300 \\
\hline $\mathrm{C}$ & 5.00752100 & 1.54322100 & -1.59544700 \\
\hline $\mathrm{C}$ & 3.51408400 & -0.55362200 & -0.38713500 \\
\hline $\mathrm{H}$ & 5.06757900 & -2.06383100 & 1.29567300 \\
\hline $\mathrm{H}$ & 7.46894400 & -1.81651800 & 1.70276800 \\
\hline $\mathrm{C}$ & 2.90200000 & 0.45056900 & -1.12966300 \\
\hline $\mathrm{C}$ & 3.64724700 & 1.47981100 & -1.75767800 \\
\hline $\mathrm{H}$ & 3.10852400 & 2.20669700 & -2.35690900 \\
\hline $\mathrm{C}$ & 2.71141300 & -1.68883700 & 0.14965700 \\
\hline $\mathrm{C}$ & 3.05256000 & -3.06599900 & -0.10927900 \\
\hline $\mathrm{C}$ & 1.57473100 & -1.43880400 & 0.90462900 \\
\hline $\mathrm{C}$ & 4.08373000 & -3.44499100 & -1.01414900 \\
\hline $\mathrm{C}$ & 2.30339200 & -4.11237900 & 0.52700900 \\
\hline $\mathrm{C}$ & 0.83146900 & -2.46587100 & 1.53084500 \\
\hline $\mathrm{C}$ & 4.38214600 & -4.77041800 & -1.24421500 \\
\hline $\mathrm{H}$ & 4.63269300 & -2.67277700 & -1.53983900 \\
\hline $\mathrm{C}$ & 2.65063700 & -5.46859700 & 0.28332800 \\
\hline $\mathrm{C}$ & 1.20935100 & -3.77527100 & 1.36818900 \\
\hline $\mathrm{H}$ & -0.02683400 & -2.18951700 & 2.13431600 \\
\hline $\mathrm{C}$ & 3.67077000 & -5.79660100 & -0.57967200 \\
\hline $\mathrm{H}$ & 5.16893500 & -5.02916500 & -1.94773300 \\
\hline $\mathrm{H}$ & 2.07950800 & -6.24658900 & 0.78480300 \\
\hline $\mathrm{H}$ & 0.65651200 & -4.57223200 & 1.85939700 \\
\hline $\mathrm{H}$ & 3.92139200 & -6.83781800 & -0.76326800 \\
\hline $\mathrm{O}$ & 1.14302700 & -0.13324300 & 1.09666900 \\
\hline $\mathrm{O}$ & 1.54531300 & 0.45730100 & -1.35837800 \\
\hline $\mathrm{P}$ & 0.29996400 & 0.53947800 & -0.19863200 \\
\hline $\mathrm{N}$ & 0.18268000 & 2.11036800 & 0.42369700 \\
\hline $\mathrm{C}$ & -0.84980100 & 3.06593700 & -0.10241000 \\
\hline $\mathrm{H}$ & -1.09366300 & 3.69147400 & 0.76341100 \\
\hline $\mathrm{C}$ & -2.18755800 & 2.46178800 & -0.54547100 \\
\hline $\mathrm{H}$ & -2.11297300 & 1.86366500 & -1.45571900 \\
\hline $\mathrm{H}$ & -2.61561700 & 1.83610500 & 0.24214600 \\
\hline $\mathrm{H}$ & -2.87840700 & 3.28899900 & -0.74347900 \\
\hline $\mathrm{C}$ & 1.04204600 & 2.83960500 & 1.42351300 \\
\hline $\mathrm{H}$ & 1.10116400 & 3.86065800 & 1.02849200 \\
\hline $\mathrm{C}$ & -0.28700100 & 3.99614100 & -1.17885500 \\
\hline $\mathrm{C}$ & 0.22566000 & 3.49952800 & -2.38670800 \\
\hline $\mathrm{C}$ & -0.31938600 & 5.38319300 & -0.98911000 \\
\hline
\end{tabular}




\begin{tabular}{|c|c|c|c|}
\hline $\mathrm{C}$ & 0.69278900 & 4.36949500 & -3.37227300 \\
\hline $\mathrm{H}$ & 0.26849700 & 2.42770400 & -2.55702900 \\
\hline $\mathrm{C}$ & 0.14876300 & 6.25800400 & -1.97360900 \\
\hline $\mathrm{H}$ & -0.72193100 & 5.78530000 & -0.06118200 \\
\hline $\mathrm{C}$ & 0.65761500 & 5.75240400 & -3.16928700 \\
\hline $\mathrm{H}$ & 1.08234800 & 3.96659600 & -4.30390900 \\
\hline $\mathrm{H}$ & 0.11684100 & 7.33103300 & -1.80269400 \\
\hline $\mathrm{H}$ & 1.02352600 & 6.42773500 & -3.93818100 \\
\hline $\mathrm{C}$ & 0.34221100 & 2.92834100 & 2.78085500 \\
\hline $\mathrm{C}$ & -0.00634000 & 1.77906400 & 3.50638900 \\
\hline $\mathrm{C}$ & 0.07835800 & 4.18112700 & 3.34892200 \\
\hline $\mathrm{C}$ & -0.59915500 & 1.88644200 & 4.76452900 \\
\hline $\mathrm{H}$ & 0.19811900 & 0.80142400 & 3.08247400 \\
\hline $\mathrm{C}$ & -0.51961800 & 4.29185800 & 4.60769400 \\
\hline $\mathrm{H}$ & 0.34918500 & 5.08288500 & 2.80261400 \\
\hline $\mathrm{C}$ & -0.86077100 & 3.14292600 & 5.31994500 \\
\hline $\mathrm{H}$ & -0.84998300 & 0.98503500 & 5.31875900 \\
\hline $\mathrm{H}$ & -0.71793900 & 5.27489100 & 5.02713100 \\
\hline $\mathrm{H}$ & -1.32405900 & 3.22292100 & 6.29982500 \\
\hline $\mathrm{C}$ & 2.50130900 & 2.39616200 & 1.58782100 \\
\hline $\mathrm{H}$ & 2.59739500 & 1.40639000 & 2.03105100 \\
\hline $\mathrm{H}$ & 3.02924700 & 2.41149600 & 0.63210300 \\
\hline $\mathrm{H}$ & 2.98780900 & 3.11861400 & 2.25296400 \\
\hline $\mathrm{C}$ & -2.10688700 & -3.10649900 & -1.25943000 \\
\hline $\mathrm{H}$ & -1.70376400 & -3.69211700 & -0.42650800 \\
\hline $\mathrm{H}$ & -1.23249800 & -2.74339100 & -1.83453600 \\
\hline $\mathrm{Pd}$ & -1.33183200 & -0.76412800 & -0.99948900 \\
\hline $\mathrm{C}$ & -4.42362200 & -3.77633600 & -1.29218300 \\
\hline $\mathrm{C}$ & -3.10505100 & -3.95740400 & -2.10250100 \\
\hline $\mathrm{Si}$ & -3.69922000 & -2.15675800 & -0.58214000 \\
\hline $\mathrm{C}$ & -4.26087100 & -0.65306200 & -1.58699000 \\
\hline $\mathrm{C}$ & -3.85786200 & -0.46248100 & -2.92560800 \\
\hline $\mathrm{C}$ & -5.16086100 & 0.28197000 & -1.03869900 \\
\hline $\mathrm{C}$ & -4.32929900 & 0.61380000 & -3.68038300 \\
\hline $\mathrm{H}$ & -3.16735900 & -1.16197700 & -3.39171700 \\
\hline $\mathrm{C}$ & -5.63779100 & 1.35616700 & -1.79157800 \\
\hline $\mathrm{H}$ & -5.49071000 & 0.17160300 & -0.00870000 \\
\hline $\mathrm{C}$ & -5.22127500 & 1.52648400 & -3.11396600 \\
\hline $\mathrm{H}$ & -3.99964500 & 0.73849700 & -4.70870400 \\
\hline $\mathrm{H}$ & -6.33362200 & 2.06225200 & -1.34507800 \\
\hline $\mathrm{H}$ & -5.59014600 & 2.36463200 & -3.69949000 \\
\hline $\mathrm{C}$ & -3.98418400 & -1.90370900 & 1.26605000 \\
\hline $\mathrm{C}$ & -3.27870600 & -0.93973300 & 2.01232900 \\
\hline $\mathrm{C}$ & -4.95015100 & -2.67381900 & 1.93982300 \\
\hline
\end{tabular}




$\begin{array}{lrrr}\mathrm{C} & -3.52808300 & -0.75040100 & 3.37216200 \\ \mathrm{H} & -2.52128900 & -0.33538300 & 1.51617900 \\ \mathrm{C} & -5.20398400 & -2.48936200 & 3.30144000 \\ \mathrm{H} & -5.51650800 & -3.42720000 & 1.39716100 \\ \mathrm{C} & -4.49271600 & -1.52704000 & 4.01979500 \\ \mathrm{H} & -2.97310000 & 0.00614700 & 3.92124300 \\ \mathrm{H} & -5.95596400 & -3.09655200 & 3.79926800 \\ \mathrm{H} & -4.68885600 & -1.38233400 & 5.07929300 \\ \mathrm{H} & -3.21415600 & -3.52714700 & -3.10455800 \\ \mathrm{H} & -2.78227200 & -4.99843000 & -2.23159900 \\ \mathrm{H} & -4.52055900 & -4.55706200 & -0.52806000 \\ \mathrm{H} & -5.34244600 & -3.75903800 & -1.88536200\end{array}$

\section{E}

7.30191400

6.66905500

1.11552600

1.29010600

5.38350500

1.88647900

0.34256900

4.74694800

1.53394600

$-0.14901200$

5.42263100

0.33812900

0.32378600

6.66117200

$-0.42075400$

1.32092200

5.19409000

$-0.04182100$

1.79097700

8.28196700

3.25481200

$-1.45250800$

7.13802900

1.39861600

1.66395100

4.70229700

2.79105800

$-0.03766000$

3.44711200

2.35853700

$-1.08274900$

$-0.02579400$

$-0.18339200$

4.94448000

$-1.30534900$

1.72442000

7.14801800

$-0.63649900$

2.55923100

2.79367800

0.87974300

$-1.00944500$

3.42694800

2.05257500

$-1.48574800$

2.88025800

2.69642300

$-2.16453300$

2.79404200

$-1.31406300$

0.17830600

3.43342300

$-2.58596900$

$-0.04869200$

1.51969500

$-1.32239500$

0.72568200

4.65874300

$-2.71550800$

$-0.76153500$

2.80123200

$-3.78592300$

0.41997500

0.88798700

$-2.50003600$

1.17903600

5.24192500

$-3.94766700$

$-0.96115800$

5.13186600

$-1.82810400$

$-1.16494300$

3.43993600

$-5.03887500$

0.21435800

1.53408300

$-3.70458800$

1.05429400

$-0.10227200$

$-2.43088100$

1.61384900

4.63766200

$-5.12324800$

$-0.45690900$

6.17298800

$-4.01695000$

$-1.51718400$

2.95087900

$-5.93551000$

0.58775900 


\begin{tabular}{|c|c|c|c|}
\hline $\mathrm{H}$ & 1.05677300 & -4.61363800 & 1.40793500 \\
\hline $\mathrm{H}$ & 5.11233200 & -6.08783800 & -0.61439100 \\
\hline $\mathrm{O}$ & 0.82690500 & -0.12176800 & 0.89684600 \\
\hline $\mathrm{O}$ & 1.50491600 & 0.64142800 & -1.45479300 \\
\hline $\mathrm{P}$ & 0.12533000 & 0.53074400 & -0.46865700 \\
\hline $\mathrm{N}$ & -0.27160400 & 2.04836300 & 0.14985700 \\
\hline $\mathrm{C}$ & -1.27913900 & 2.92255700 & -0.54741600 \\
\hline $\mathrm{H}$ & -1.97860700 & 3.23028800 & 0.23961300 \\
\hline $\mathrm{C}$ & -2.12745300 & 2.26157200 & -1.64061200 \\
\hline $\mathrm{H}$ & -1.53338700 & 1.94487600 & -2.50367400 \\
\hline $\mathrm{H}$ & -2.68620800 & 1.40467100 & -1.26557700 \\
\hline $\mathrm{H}$ & -2.84421400 & 3.01076100 & -1.99431200 \\
\hline $\mathrm{C}$ & 0.22289200 & 2.74563800 & 1.39470600 \\
\hline $\mathrm{H}$ & 0.17501000 & 3.80457900 & 1.12515200 \\
\hline $\mathrm{C}$ & -0.63500200 & 4.18867200 & -1.11696000 \\
\hline $\mathrm{C}$ & 0.33341600 & 4.11538400 & -2.12798100 \\
\hline $\mathrm{C}$ & -1.05988900 & 5.45228800 & -0.68949000 \\
\hline $\mathrm{C}$ & 0.86772000 & 5.27504700 & -2.68873000 \\
\hline $\mathrm{H}$ & 0.66299100 & 3.14252800 & -2.48145100 \\
\hline $\mathrm{C}$ & -0.52658000 & 6.61740800 & -1.24777400 \\
\hline $\mathrm{H}$ & -1.82194200 & 5.52535400 & 0.08399600 \\
\hline $\mathrm{C}$ & 0.44127400 & 6.53146200 & -2.24820400 \\
\hline $\mathrm{H}$ & 1.61360200 & 5.20012800 & -3.47613100 \\
\hline $\mathrm{H}$ & -0.86855600 & 7.58870600 & -0.90001900 \\
\hline $\mathrm{H}$ & 0.85823600 & 7.43451500 & -2.68575200 \\
\hline $\mathrm{C}$ & -0.73222600 & 2.56035000 & 2.57444400 \\
\hline $\mathrm{C}$ & -0.92460900 & 1.31689200 & 3.19655300 \\
\hline $\mathrm{C}$ & -1.40653200 & 3.67411600 & 3.09257900 \\
\hline $\mathrm{C}$ & -1.77225300 & 1.19589500 & 4.29798900 \\
\hline $\mathrm{H}$ & -0.40474500 & 0.44400800 & 2.81690700 \\
\hline $\mathrm{C}$ & -2.25615900 & 3.55690600 & 4.19640300 \\
\hline $\mathrm{H}$ & -1.25823700 & 4.64911800 & 2.63270600 \\
\hline $\mathrm{C}$ & -2.44298200 & 2.31450700 & 4.80212900 \\
\hline $\mathrm{H}$ & -1.90722300 & 0.22475000 & 4.76724800 \\
\hline $\mathrm{H}$ & -2.76777200 & 4.43576500 & 4.58035000 \\
\hline $\mathrm{H}$ & -3.10082700 & 2.21689700 & 5.66164700 \\
\hline $\mathrm{C}$ & 1.68447500 & 2.51478700 & 1.80119000 \\
\hline $\mathrm{H}$ & 1.87284600 & 1.51116000 & 2.17861100 \\
\hline $\mathrm{H}$ & 2.35841100 & 2.71168900 & 0.96398400 \\
\hline $\mathrm{H}$ & 1.91874500 & 3.22874000 & 2.59889000 \\
\hline $\mathrm{C}$ & -1.81678600 & -2.22903200 & -3.36396100 \\
\hline $\mathrm{H}$ & -1.37575600 & -3.22346300 & -3.20563300 \\
\hline $\mathrm{H}$ & -1.28251000 & -1.75651600 & -4.20703300 \\
\hline $\mathrm{Pd}$ & -1.15855200 & -0.97390800 & -1.85098700 \\
\hline
\end{tabular}




\begin{tabular}{|c|c|c|c|}
\hline $\mathrm{C}$ & -3.98989400 & -2.84656600 & -2.33239100 \\
\hline $\mathrm{C}$ & -3.32341700 & -2.34162400 & -3.61874300 \\
\hline $\mathrm{Si}$ & -3.10942100 & -1.90847000 & -0.90701400 \\
\hline $\mathrm{C}$ & -4.21263900 & -0.52054600 & -0.21775300 \\
\hline $\mathrm{C}$ & -5.44489300 & -0.20616000 & -0.82173200 \\
\hline $\mathrm{C}$ & -3.82693200 & 0.25336000 & 0.89636300 \\
\hline $\mathrm{C}$ & -6.25661600 & 0.82164200 & -0.33485300 \\
\hline $\mathrm{H}$ & -5.78265300 & -0.77079300 & -1.68643300 \\
\hline $\mathrm{C}$ & -4.63503800 & 1.27713900 & 1.39395000 \\
\hline $\mathrm{H}$ & -2.87560400 & 0.06232600 & $1.3866040 c$ \\
\hline $\mathrm{C}$ & -5.85515400 & 1.56475800 & 0.77609100 \\
\hline $\mathrm{H}$ & -7.20368700 & 1.03920200 & -0.82295700 \\
\hline $\mathrm{H}$ & -4.30910200 & 1.84736400 & $2.2599630 \mathrm{C}$ \\
\hline $\mathrm{H}$ & -6.48728600 & 2.36257700 & 1.15811500 \\
\hline $\mathrm{C}$ & -2.76016700 & -3.15769200 & 0.49169200 \\
\hline $\mathrm{C}$ & -2.07716600 & -4.35362800 & 0.19046300 \\
\hline $\mathrm{C}$ & -3.21524900 & -2.99361100 & 1.81371300 \\
\hline $\mathrm{C}$ & -1.86613000 & -5.33967300 & 1.15473300 \\
\hline $\mathrm{H}$ & -1.70263500 & -4.51984000 & -0.81715900 \\
\hline $\mathrm{C}$ & -2.99553600 & -3.97149600 & 2.78877300 \\
\hline $\mathrm{H}$ & -3.76441000 & -2.09758800 & 2.08770400 \\
\hline $\mathrm{C}$ & -2.32110900 & -5.14866500 & 2.46268000 \\
\hline $\mathrm{H}$ & -1.34819200 & -6.25768800 & 0.88630600 \\
\hline $\mathrm{H}$ & -3.36197900 & -3.81622700 & 3.80084100 \\
\hline $\mathrm{H}$ & -2.15682000 & -5.91374600 & 3.21741800 \\
\hline $\mathrm{H}$ & -3.72524600 & -1.35426500 & -3.88182300 \\
\hline $\mathrm{H}$ & -3.53650100 & -3.00432800 & -4.47415500 \\
\hline $\mathrm{H}$ & -3.77506800 & -3.91564900 & -2.19779600 \\
\hline $\mathrm{H}$ & -5.08335600 & -2.75456200 & -2.33939500 \\
\hline \multicolumn{4}{|c|}{$\mathbf{F}$} \\
\hline $\mathrm{C}$ & 7.93153100 & -1.75824400 & -0.18775900 \\
\hline $\mathrm{C}$ & 7.38565900 & -0.94091200 & -1.15055700 \\
\hline $\mathrm{C}$ & 5.97800200 & -0.79500800 & -1.27575800 \\
\hline $\mathrm{C}$ & 5.10889600 & -1.53650800 & -0.40670400 \\
\hline $\mathrm{C}$ & 5.71097800 & -2.35481400 & 0.59041400 \\
\hline $\mathrm{C}$ & 7.08060700 & -2.45984700 & 0.69798600 \\
\hline $\mathrm{H}$ & 6.07259300 & 0.62056500 & -2.91435000 \\
\hline $\mathrm{H}$ & 9.00972600 & -1.85632300 & -0.09703700 \\
\hline $\mathrm{H}$ & 8.02611500 & -0.37946300 & -1.82687900 \\
\hline $\mathrm{C}$ & 5.41274400 & 0.08523900 & -2.23622400 \\
\hline $\mathrm{C}$ & 3.68049400 & -1.39773800 & -0.54086500 \\
\hline $\mathrm{H}$ & 5.07667900 & -2.89268400 & 1.28517600 \\
\hline $\mathrm{H}$ & 7.51106700 & -3.08522800 & 1.47546400 \\
\hline
\end{tabular}




\begin{tabular}{|c|c|c|c|}
\hline $\mathrm{C}$ & 3.20016400 & -0.43089300 & -1.41496500 \\
\hline $\mathrm{C}$ & 4.05547200 & 0.27981200 & -2.29088200 \\
\hline $\mathrm{H}$ & 3.60521200 & 0.95603200 & -3.00985200 \\
\hline $\mathrm{C}$ & 2.72762700 & -2.23849400 & 0.23714100 \\
\hline $\mathrm{C}$ & 2.76639500 & -3.67942800 & 0.19424000 \\
\hline $\mathrm{C}$ & 1.74163600 & -1.64397900 & 1.00973700 \\
\hline $\mathrm{C}$ & 3.60912300 & -4.39948600 & -0.69816100 \\
\hline $\mathrm{C}$ & 1.89563800 & -4.43566100 & 1.04869800 \\
\hline $\mathrm{C}$ & 0.88786200 & -2.38240300 & 1.85884700 \\
\hline $\mathrm{C}$ & 3.61692300 & -5.77719200 & -0.71812000 \\
\hline $\mathrm{H}$ & 4.24346700 & -3.85080600 & -1.38406900 \\
\hline $\mathrm{C}$ & 1.94245900 & -5.85536400 & 1.01635200 \\
\hline $\mathrm{C}$ & 0.98497000 & -3.75113000 & 1.89604400 \\
\hline $\mathrm{H}$ & 0.17838300 & -1.84245500 & 2.47675400 \\
\hline $\mathrm{C}$ & 2.78685100 & -6.51764000 & 0.15562600 \\
\hline $\mathrm{H}$ & 4.26332000 & -6.29965500 & -1.41811800 \\
\hline $\mathrm{H}$ & 1.28107500 & -6.40978200 & 1.67779600 \\
\hline $\mathrm{H}$ & 0.34195400 & -4.32912400 & 2.55469500 \\
\hline $\mathrm{H}$ & 2.80718800 & -7.60364600 & 0.13352800 \\
\hline $\mathrm{O}$ & 1.60435000 & -0.25781400 & 1.02182100 \\
\hline $\mathrm{O}$ & 1.84948300 & -0.17648000 & -1.52342600 \\
\hline $\mathrm{P}$ & 0.86675300 & 0.45403800 & -0.30042300 \\
\hline $\mathrm{N}$ & 1.33724300 & 2.06030800 & -0.00173900 \\
\hline $\mathrm{C}$ & 0.54566800 & 3.15607700 & -0.65923000 \\
\hline $\mathrm{H}$ & -0.07128400 & 3.62713900 & 0.11557300 \\
\hline $\mathrm{C}$ & -0.40338400 & 2.68878300 & -1.77936100 \\
\hline $\mathrm{H}$ & 0.14345500 & 2.18608100 & -2.58528400 \\
\hline $\mathrm{H}$ & -1.19328400 & 2.02277800 & -1.42318300 \\
\hline $\mathrm{H}$ & -0.88287600 & 3.57634800 & -2.20409000 \\
\hline $\mathrm{C}$ & 2.28840200 & 2.60247700 & 1.03636200 \\
\hline $\mathrm{H}$ & 2.57036500 & 3.57377500 & 0.62506500 \\
\hline $\mathrm{C}$ & 1.44559000 & 4.24465300 & -1.24919000 \\
\hline $\mathrm{C}$ & 2.54586500 & 3.93908900 & -2.06053300 \\
\hline $\mathrm{C}$ & 1.11650500 & 5.59114400 & -1.04595600 \\
\hline $\mathrm{C}$ & 3.29964500 & 4.95405200 & -2.65060800 \\
\hline $\mathrm{H}$ & 2.82027000 & 2.89987000 & -2.21206000 \\
\hline $\mathrm{C}$ & 1.86480700 & 6.60968700 & -1.64066200 \\
\hline $\mathrm{H}$ & 0.26117300 & 5.84128400 & -0.42156600 \\
\hline $\mathrm{C}$ & 2.96081800 & 6.29385900 & -2.44477200 \\
\hline $\mathrm{H}$ & 4.15357500 & 4.69881800 & -3.27302000 \\
\hline $\mathrm{H}$ & 1.59395700 & 7.64851800 & -1.47039100 \\
\hline $\mathrm{H}$ & 3.54845300 & 7.08382200 & -2.90462800 \\
\hline $\mathrm{C}$ & 1.62177200 & 2.90646400 & 2.38036700 \\
\hline $\mathrm{C}$ & 1.26936500 & 1.90773900 & 3.30176300 \\
\hline
\end{tabular}




\begin{tabular}{|c|c|c|c|}
\hline $\mathrm{C}$ & 1.40059200 & 4.24414000 & 2.73801900 \\
\hline $\mathrm{C}$ & 0.70347400 & 2.24023200 & 4.53397700 \\
\hline $\mathrm{H}$ & 1.43995100 & 0.86684400 & 3.05353300 \\
\hline $\mathrm{C}$ & 0.83625600 & 4.58152000 & 3.97139900 \\
\hline $\mathrm{H}$ & 1.68118400 & 5.03363600 & 2.04410100 \\
\hline $\mathrm{C}$ & 0.48214700 & 3.57820700 & 4.87389000 \\
\hline $\mathrm{H}$ & 0.44015500 & 1.45079000 & 5.23315000 \\
\hline $\mathrm{H}$ & 0.68132300 & 5.62682400 & 4.22706900 \\
\hline $\mathrm{H}$ & 0.04569800 & 3.83406600 & 5.83575800 \\
\hline $\mathrm{C}$ & 3.61708500 & 1.85047300 & 1.19638100 \\
\hline $\mathrm{H}$ & 3.51146600 & 0.88442200 & 1.68706800 \\
\hline $\mathrm{H}$ & 4.09302400 & 1.69963700 & 0.22362600 \\
\hline $\mathrm{H}$ & 4.28124000 & 2.47272200 & 1.80699900 \\
\hline $\mathrm{C}$ & -2.73703000 & 0.73464500 & 1.23111100 \\
\hline $\mathrm{C}$ & -2.87822400 & 2.20051000 & 1.07725600 \\
\hline $\mathrm{C}$ & -3.73697500 & 2.67970600 & 0.07103600 \\
\hline $\mathrm{C}$ & -2.22398600 & 3.12257500 & 1.91050200 \\
\hline $\mathrm{C}$ & -3.92825000 & 4.04971900 & -0.09535100 \\
\hline $\mathrm{H}$ & -4.25513300 & 1.97762500 & -0.57444700 \\
\hline $\mathrm{C}$ & -2.42583900 & 4.49246900 & 1.73767400 \\
\hline $\mathrm{H}$ & -1.56230400 & 2.78095700 & 2.70119400 \\
\hline $\mathrm{C}$ & -3.27547700 & 4.96485600 & 0.73501800 \\
\hline $\mathrm{H}$ & -4.59833500 & 4.40050200 & -0.87564200 \\
\hline $\mathrm{H}$ & -1.91397100 & 5.18695100 & 2.39790500 \\
\hline $\mathrm{H}$ & -3.43338200 & 6.03220500 & 0.60680200 \\
\hline $\mathrm{C}$ & -0.83629000 & -1.51170200 & -2.40654100 \\
\hline $\mathrm{H}$ & -0.28647900 & -2.37548300 & -2.00968800 \\
\hline $\mathrm{H}$ & -0.10831900 & -0.83139300 & -2.85773300 \\
\hline $\mathrm{Pd}$ & -1.47835800 & -0.48958600 & -0.69347100 \\
\hline $\mathrm{C}$ & -2.99081000 & -2.77891400 & -2.74099200 \\
\hline $\mathrm{C}$ & -1.89032500 & -1.95696700 & -3.42430900 \\
\hline $\mathrm{Si}$ & -3.39916100 & -1.82031300 & -1.13667000 \\
\hline $\mathrm{C}$ & -4.94655400 & -0.72745300 & -1.42515000 \\
\hline $\mathrm{C}$ & -5.14065500 & -0.07937400 & -2.66310400 \\
\hline $\mathrm{C}$ & -5.89802500 & -0.46945400 & -0.41754700 \\
\hline $\mathrm{C}$ & -6.21931000 & 0.77853100 & -2.88330300 \\
\hline $\mathrm{H}$ & -4.43594200 & -0.24745900 & -3.47424500 \\
\hline $\mathrm{C}$ & -6.98018600 & 0.39055500 & -0.62829100 \\
\hline $\mathrm{H}$ & -5.80650300 & -0.96269500 & 0.54791500 \\
\hline $\mathrm{C}$ & -7.14385000 & 1.01987100 & -1.86286400 \\
\hline $\mathrm{H}$ & -6.34099800 & 1.25740500 & -3.85222800 \\
\hline $\mathrm{H}$ & -7.69653200 & 0.56434000 & 0.17132200 \\
\hline $\mathrm{H}$ & -7.98503500 & 1.68756800 & -2.03143000 \\
\hline $\mathrm{C}$ & -3.86934700 & -3.03863500 & 0.26073400 \\
\hline
\end{tabular}




$\begin{array}{lrrr}\mathrm{C} & -2.95489000 & -3.38621300 & 1.27215700 \\ \mathrm{C} & -5.12383900 & -3.68195500 & 0.27984300 \\ \mathrm{C} & -3.27471200 & -4.32219800 & 2.25976600 \\ \mathrm{H} & -1.96890100 & -2.92707600 & 1.27586700 \\ \mathrm{C} & -5.44949900 & -4.62123300 & 1.26023000 \\ \mathrm{H} & -5.86312100 & -3.44295500 & -0.48157800 \\ \mathrm{C} & -4.52518100 & -4.94181000 & 2.25739200 \\ \mathrm{H} & -2.54803300 & -4.56820400 & 3.03123000 \\ \mathrm{H} & -6.42502400 & -5.10190300 & 1.24742300 \\ \mathrm{H} & -4.77811300 & -5.66993400 & 3.02421800 \\ \mathrm{H} & -3.54613300 & 0.09533300 & 0.89545000 \\ \mathrm{~N} & -2.14098700 & 0.25640500 & 2.31114000 \\ \mathrm{~N} & -1.58740200 & -0.14733700 & 3.22023200 \\ \mathrm{H} & -2.32878300 & -1.07154100 & -3.90481200 \\ \mathrm{H} & -1.40314700 & -2.53007500 & -4.23247800 \\ \mathrm{H} & -3.85540300 & -2.96240000 & -3.39170300 \\ \mathrm{H} & -2.59254500 & -3.76169800 & -2.45255100\end{array}$

TS-6

C

C

C

C

C

C

$\mathrm{H}$

$\mathrm{H}$

$\mathrm{H}$

C

C

$\mathrm{H}$

$\mathrm{H}$

C

C

$\mathrm{H}$

C

C

C

C

C

C

C

$\mathrm{H}$

C

$\begin{array}{rrr}7.13221600 & 3.41300200 & -0.14339400 \\ 6.74272200 & 2.64742800 & 0.93131000 \\ 5.42538700 & 2.12222400 & 1.01824300 \\ 4.47213700 & 2.42468000 & -0.01124500 \\ 4.92037300 & 3.19750700 & -1.11972200 \\ 6.21145000 & 3.67451500 & -1.18468000 \\ 5.76168100 & 1.06497400 & 2.88035400 \\ 8.14454800 & 3.80295800 & -0.20242400 \\ 7.44469000 & 2.41707200 & 1.72942300 \\ 5.04196900 & 1.27736900 & 2.09375600 \\ 3.13009800 & 1.90468100 & 0.08573300 \\ 4.23418900 & 3.40075300 & -1.93326500 \\ 6.52626400 & 4.25351000 & -2.04871400 \\ 2.86213800 & 0.99195300 & 1.09741300 \\ 3.79701700 & 0.70180200 & 2.12009100 \\ 3.50435000 & 0.01380800 & 2.90562000 \\ 2.05824400 & 2.29701000 & -0.87145000 \\ 1.72639400 & 3.68249100 & -1.10626900 \\ 1.32469500 & 1.33351200 & -1.55206700 \\ 2.28356000 & 4.74638300 & -0.34105400 \\ 0.77310600 & 4.02320400 & -2.12291700 \\ 0.38184300 & 1.67036100 & -2.55099600 \\ 1.95784900 & 6.06070900 & -0.59902000 \\ 2.96698200 & 4.51495500 & 0.46669600 \\ 0.47051700 & 5.38845200 & -2.37385800\end{array}$




\begin{tabular}{|c|c|c|c|}
\hline $\mathrm{C}$ & 0.13167000 & 2.98573500 & -2.84821300 \\
\hline $\mathrm{H}$ & -0.11325100 & 0.86721900 & -3.08352800 \\
\hline $\mathrm{C}$ & 1.05417300 & 6.39174300 & -1.63569400 \\
\hline $\mathrm{H}$ & 2.39545100 & 6.84983600 & 0.00646800 \\
\hline $\mathrm{H}$ & -0.24814500 & 5.62351900 & -3.15519700 \\
\hline $\mathrm{H}$ & -0.57869600 & 3.24756300 & -3.62806700 \\
\hline $\mathrm{H}$ & 0.81053100 & 7.43198400 & -1.83259300 \\
\hline $\mathrm{O}$ & 1.54578000 & -0.02206600 & -1.33706600 \\
\hline $\mathrm{O}$ & 1.63708100 & 0.36472200 & 1.18454100 \\
\hline $\mathrm{P}$ & 0.98716300 & -0.73634800 & 0.08155600 \\
\hline $\mathrm{N}$ & 1.91854500 & -2.15092500 & -0.01006400 \\
\hline $\mathrm{C}$ & 1.44325600 & -3.39383500 & 0.68259100 \\
\hline $\mathrm{H}$ & 1.74619900 & -4.20581500 & 0.01304400 \\
\hline $\mathrm{C}$ & -0.07669400 & -3.54067100 & 0.82562100 \\
\hline $\mathrm{H}$ & -0.52005100 & -2.81843900 & 1.51623600 \\
\hline $\mathrm{H}$ & -0.56619700 & -3.44313100 & -0.14799500 \\
\hline $\mathrm{H}$ & -0.28315500 & -4.54418100 & 1.21416500 \\
\hline $\mathrm{C}$ & 3.27033200 & -2.44265900 & -0.61956800 \\
\hline $\mathrm{H}$ & 3.78721800 & -3.03027000 & 0.14889700 \\
\hline $\mathrm{C}$ & 2.14479100 & -3.63698500 & 2.02016700 \\
\hline $\mathrm{C}$ & 2.13725700 & -2.68102900 & 3.04537600 \\
\hline $\mathrm{C}$ & 2.76912500 & -4.86781300 & 2.25988100 \\
\hline $\mathrm{C}$ & 2.73449900 & -2.95266600 & 4.27694600 \\
\hline $\mathrm{H}$ & 1.66613200 & -1.71677000 & 2.88129400 \\
\hline $\mathrm{C}$ & 3.37003600 & -5.14301900 & 3.49086700 \\
\hline $\mathrm{H}$ & 2.78206900 & -5.62182800 & 1.47508300 \\
\hline $\mathrm{C}$ & 3.35436900 & -4.18442300 & 4.50403000 \\
\hline $\mathrm{H}$ & 2.71146700 & -2.20189900 & 5.06274600 \\
\hline $\mathrm{H}$ & 3.85152100 & -6.10369000 & 3.65432700 \\
\hline $\mathrm{H}$ & 3.81987800 & -4.39379300 & 5.46338500 \\
\hline $\mathrm{C}$ & 3.13278200 & -3.33940700 & -1.85026000 \\
\hline $\mathrm{C}$ & 2.42701700 & -2.91828100 & -2.98559000 \\
\hline $\mathrm{C}$ & 3.77087600 & -4.58605500 & -1.88224800 \\
\hline $\mathrm{C}$ & 2.35750700 & -3.72777700 & -4.11921100 \\
\hline $\mathrm{H}$ & 1.93244400 & -1.95294500 & -2.97640100 \\
\hline $\mathrm{C}$ & 3.70634200 & -5.39803900 & -3.01781600 \\
\hline $\mathrm{H}$ & 4.32870900 & -4.92319700 & -1.01050700 \\
\hline $\mathrm{C}$ & 2.99675900 & -4.97070600 & -4.14006700 \\
\hline $\mathrm{H}$ & 1.80384100 & -3.38660700 & -4.99018800 \\
\hline $\mathrm{H}$ & 4.20726800 & -6.36272000 & -3.02174300 \\
\hline $\mathrm{H}$ & 2.94123300 & -5.59953600 & -5.02473200 \\
\hline $\mathrm{C}$ & 4.20268800 & -1.26717500 & -0.93180600 \\
\hline $\mathrm{H}$ & 3.81461800 & -0.61495600 & -1.71329400 \\
\hline $\mathrm{H}$ & 4.41736700 & -0.67540000 & -0.04187800 \\
\hline
\end{tabular}




\begin{tabular}{|c|c|c|c|}
\hline $\mathrm{H}$ & 5.14799500 & -1.69395800 & -1.28506300 \\
\hline $\mathrm{C}$ & -2.64418600 & -0.85548900 & -1.21350800 \\
\hline $\mathrm{C}$ & -3.67409100 & -1.95490200 & -1.25199700 \\
\hline $\mathrm{C}$ & -3.61281000 & -3.01311300 & -0.33142200 \\
\hline $\mathrm{C}$ & -4.71442100 & -1.94636100 & -2.19685800 \\
\hline $\mathrm{C}$ & -4.56213200 & -4.03440900 & -0.35495000 \\
\hline $\mathrm{H}$ & -2.83046100 & -3.00495600 & 0.41975600 \\
\hline $\mathrm{C}$ & -5.66276500 & -2.96688300 & -2.22457300 \\
\hline $\mathrm{H}$ & -4.78601200 & -1.12377700 & -2.90341100 \\
\hline $\mathrm{C}$ & -5.58593500 & -4.01594900 & -1.30501200 \\
\hline $\mathrm{H}$ & -4.50527000 & -4.84175100 & 0.37023200 \\
\hline $\mathrm{H}$ & -6.46463100 & -2.94040700 & -2.95722500 \\
\hline $\mathrm{H}$ & -6.32619600 & -4.81152700 & -1.32378200 \\
\hline $\mathrm{C}$ & -1.15821400 & -0.13374700 & 2.63263500 \\
\hline $\mathrm{H}$ & -0.83744600 & 0.89807100 & 2.82193400 \\
\hline $\mathrm{H}$ & -0.29719700 & -0.77728900 & 2.84735200 \\
\hline $\mathrm{Pd}$ & -1.53620300 & -0.41603800 & 0.54258700 \\
\hline $\mathrm{C}$ & -3.56954100 & 0.36526600 & 3.20041800 \\
\hline $\mathrm{C}$ & -2.34947400 & -0.51647700 & 3.52005400 \\
\hline $\mathrm{Si}$ & -3.39150000 & 0.84941000 & 1.34936800 \\
\hline $\mathrm{C}$ & -5.07611300 & 0.67298700 & 0.45966300 \\
\hline $\mathrm{C}$ & -6.04315500 & -0.25657600 & 0.88708600 \\
\hline $\mathrm{C}$ & -5.40116700 & 1.46199000 & -0.66245600 \\
\hline $\mathrm{C}$ & -7.27180400 & -0.39032600 & 0.23735600 \\
\hline $\mathrm{H}$ & -5.83745000 & -0.89028300 & 1.74658000 \\
\hline $\mathrm{C}$ & -6.62552200 & 1.33190400 & -1.32241300 \\
\hline $\mathrm{H}$ & -4.69456200 & 2.21223700 & -1.01156900 \\
\hline $\mathrm{C}$ & -7.56753000 & 0.40423700 & -0.87215200 \\
\hline $\mathrm{H}$ & -7.99951200 & -1.11334000 & 0.59860800 \\
\hline $\mathrm{H}$ & -6.84929800 & 1.96348900 & -2.17952000 \\
\hline $\mathrm{H}$ & -8.52514100 & 0.30550500 & -1.37778800 \\
\hline $\mathrm{C}$ & -3.00429300 & 2.72096200 & 1.26913500 \\
\hline $\mathrm{C}$ & -1.72008100 & 3.22721600 & 1.00413100 \\
\hline $\mathrm{C}$ & -4.01828100 & 3.65525300 & 1.56379600 \\
\hline $\mathrm{C}$ & -1.45448800 & 4.59947200 & 1.03348700 \\
\hline $\mathrm{H}$ & -0.91325300 & 2.53465700 & 0.77684800 \\
\hline $\mathrm{C}$ & -3.76158400 & 5.02679400 & 1.59816800 \\
\hline $\mathrm{H}$ & -5.02926200 & 3.30769200 & 1.76653900 \\
\hline $\mathrm{C}$ & -2.47553500 & 5.50269600 & 1.33191900 \\
\hline $\mathrm{H}$ & -0.45159000 & 4.96070800 & 0.82357000 \\
\hline $\mathrm{H}$ & -4.56426600 & 5.72310200 & 1.83114300 \\
\hline $\mathrm{H}$ & -2.27144700 & 6.57069500 & 1.35614000 \\
\hline $\mathrm{H}$ & -3.02533300 & 0.04427800 & -1.70505500 \\
\hline $\mathrm{N}$ & -1.64486100 & -1.33835500 & -2.51252000 \\
\hline
\end{tabular}




$\begin{array}{lrrr}\mathrm{N} & -0.80451100 & -1.85616000 & -3.03769800 \\ \mathrm{H} & -3.54612700 & 1.29059800 & 3.79107900 \\ \mathrm{H} & -4.51618500 & -0.13245900 & 3.44339500 \\ \mathrm{H} & -2.60884900 & -1.56900300 & 3.33950900 \\ \mathrm{H} & -2.07814100 & -0.44977300 & 4.58785200\end{array}$

\section{Characterization data for the products}

\section{(S)-1,1,2-triphenylsilolan (3a)}

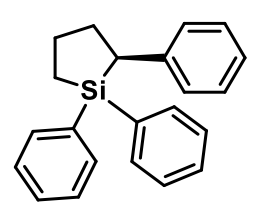

Yield: 50\% (15.7 mg); white solid; HPLC gave 97\% e.e., conditions: Daicel chiral column OD-H+OD-H, hexane: ${ }^{i} \mathrm{PrOH}=100: 0,0.5 \mathrm{~mL} / \mathrm{min}$, wavelength $=220 \mathrm{~nm} ;[\alpha]_{D}^{30}=-26.8^{\circ}\left(c 1.00, \mathrm{CHCl}_{3}\right) ;{ }^{1} \mathrm{H} \mathrm{NMR}(400$ $\left.\mathrm{MHz}, \mathrm{CDCl}_{3}\right) \delta$ 7.61-7.59 (m, 2H), 7.42-7.33 (m, 3H), 7.28-7.23 (m, 1H), 7.17-7.06 (m, 6H), 7.00-6.93 (m, $3 \mathrm{H}), 2.87(\mathrm{dd}, J=12.5,6.4 \mathrm{~Hz}, 1 \mathrm{H}), 2.35-2.25(\mathrm{~m}, 2 \mathrm{H}), 1.97(\mathrm{qd}, J=12.7,4.9 \mathrm{~Hz}, 1 \mathrm{H}), 1.78-1.65(\mathrm{~m}, 1 \mathrm{H})$, $1.40-1.36(\mathrm{~m}, 2 \mathrm{H}) ;{ }^{13} \mathrm{C} \mathrm{NMR}\left(100 \mathrm{MHz}, \mathrm{CDCl}_{3}\right) \delta 143.5,136.4,135.4,134.9,133.8,129.4,129.1,128.0$, 127.4, 127.2, 124.1, 37.0, 34.5, 25.6, 11.5; HRMS (EI, $m / z$ ): calcd for $\mathrm{C}_{22} \mathrm{H}_{22} \mathrm{Si}\left[\mathrm{M}^{+}\right]$314.1485, found 314.1484; IR (film): 2952, 1600,1427, 1111, 768, $696 \mathrm{~cm}^{-1}$.

\section{(S)-2-phenyl-1,1-bis(4-(trifluoromethyl)phenyl)silolane (3b)}

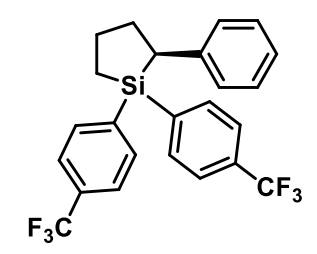

Yield: 94\% (42.7 mg); colorless oil; HPLC gave 99\% e.e., conditions: Daicel chiral column OD-H+OD-H, hexane: ${ }^{i} \mathrm{PrOH}=100: 0,0.5 \mathrm{~mL} / \mathrm{min}$, wavelength $=220 \mathrm{~nm} ;[\alpha]_{D}^{30}=-34.6^{\circ}\left(c 1.00, \mathrm{CHCl}_{3}\right) ;{ }^{1} \mathrm{H} \mathrm{NMR}(400$ $\left.\mathrm{MHz}, \mathrm{CDCl}_{3}\right) \delta 7.69(\mathrm{~d}, J=8.0 \mathrm{~Hz}, 2 \mathrm{H}), 7.63(\mathrm{~d}, J=8.0 \mathrm{~Hz}, 2 \mathrm{H}), 7.41(\mathrm{~d}, J=8.0 \mathrm{~Hz}, 2 \mathrm{H}), 7.19(\mathrm{~d}, J=8.0$ $\mathrm{Hz}, 2 \mathrm{H}), 7.13-7.10(\mathrm{~m}, 2 \mathrm{H}), 7.05-7.01(\mathrm{~m}, 1 \mathrm{H}), 6.93-6.92(\mathrm{~m}, 2 \mathrm{H}), 2.91(\mathrm{dd}, J=12.5,6.5 \mathrm{~Hz}, 1 \mathrm{H}), 2.40$ $-2.30(\mathrm{~m}, 2 \mathrm{H}), 1.98(\mathrm{qd}, J=12.7,5.0 \mathrm{~Hz}, 1 \mathrm{H}), 1.80-1.68(\mathrm{~m}, 1 \mathrm{H}), 1.44-1.40(\mathrm{~m}, 2 \mathrm{H}) ;{ }^{13} \mathrm{C} \mathrm{NMR}(101 \mathrm{MHz}$, $\left.\mathrm{CDCl}_{3}\right) \delta 142.3,140.6,138.0,135.6,135.0,131.7(\mathrm{q}, J=32.5 \mathrm{~Hz}, 1 \mathrm{C}), 131.4(\mathrm{q}, J=32.5 \mathrm{~Hz}, 1 \mathrm{C}), 128.3$, 127.0, 124.7, 124.7 (q, $J=3.3 \mathrm{~Hz}, 1 \mathrm{C}), 124.1$ (q, $J=3.7 \mathrm{~Hz}, 1 \mathrm{C}), 124.1$ (q, $J=272.3 \mathrm{~Hz}, 1 \mathrm{C}), 124.0$ (q, $J=$ $272.3 \mathrm{~Hz}, 1 \mathrm{C}$ ), 36.8, 34.2, 25.5, 11.4; ${ }^{19} \mathrm{~F}$ NMR $\left(471 \mathrm{MHz}, \mathrm{CDCl}_{3}\right) \delta$-63.2 (s, 3F), -63.3 (s, 3F); HRMS (EI, $m / z$ ): calcd for $\mathrm{C}_{24} \mathrm{H}_{20} \mathrm{~F}_{6} \mathrm{Si}\left[\mathrm{M}^{+}\right]$450.1233, found 450.1233; IR (film): 2943, 1602,1492, 1323, 1152 , $1124,1104,827 \mathrm{~cm}^{-1}$.

(S)-2-phenyl-1,1-di-p-tolylsilolane (3c)

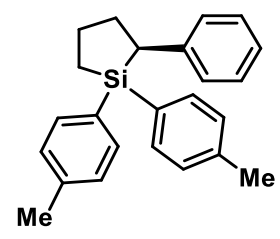


Yield: 22\% (7.5 mg); white solid; HPLC gave 97\% e.e., conditions: Daicel chiral column OD-H+OD-H, hexane: ${ }^{i} \mathrm{PrOH}=100: 0,0.5 \mathrm{~mL} / \mathrm{min}$, wavelength $=220 \mathrm{~nm} ;[\alpha]_{D}^{30}=-16.7^{\circ}\left(c 1.00, \mathrm{CHCl}_{3}\right) ;{ }^{1} \mathrm{H} \mathrm{NMR}(400$ $\left.\mathrm{MHz}, \mathrm{CDCl}_{3}\right) \delta 7.48(\mathrm{~d}, J=7.3 \mathrm{~Hz}, 2 \mathrm{H}), 7.20(\mathrm{~d}, J=7.4 \mathrm{~Hz}, 2 \mathrm{H}), 7.11-7.07(\mathrm{~m}, 2 \mathrm{H}), 7.01-6.95(\mathrm{~m}, 7 \mathrm{H})$, $2.83(\mathrm{dd}, J=12.5,6.4 \mathrm{~Hz}, 1 \mathrm{H}), 2.37(\mathrm{~s}, 3 \mathrm{H}), 2.32-2.22(\mathrm{~m}, 5 \mathrm{H}), 1.95(\mathrm{qd}, J=12.8,5.0 \mathrm{~Hz}, 1 \mathrm{H}), 1.77-1.63$ (m, $1 \mathrm{H}), 1.35-1.32(\mathrm{~m}, 2 \mathrm{H}) ;{ }^{13} \mathrm{C}$ NMR $\left(101 \mathrm{MHz}, \mathrm{CDCl}_{3}\right) \delta$ 143.7, 139.3, 138.9 135.5, 134.9, 133.0, 130.2, 128.8, 128.2, 127.9, 127.2, 124.0, 37.2, 34.4, 25.5, 21.5, 21.4, 11.7; HRMS (EI, $m / z)$ : calcd for $\mathrm{C}_{24} \mathrm{H}_{26} \mathrm{Si}_{[}\left[\mathrm{M}^{+}\right.$] 342.1798, found 342.1799; IR (film): 2925, 2853, 1602, 1450, 1109, 1014, $799 \mathrm{~cm}^{-1}$.

\section{(S)-2-phenyl-1,1-di-m-tolylsilolane (3d)}

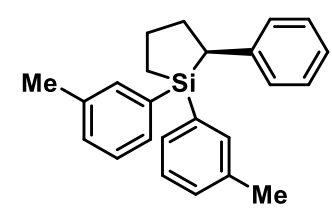

Yield: $28 \%$ (9.6 mg); white solid; HPLC gave 94\% e.e., conditions: Daicel chiral column OD-H+OD-H, hexane: ${ }^{i} \mathrm{PrOH}=100: 0,0.5 \mathrm{~mL} / \mathrm{min}$, wavelength $=220 \mathrm{~nm} ;[\alpha]_{D}^{30}=-20.9^{\circ}\left(c 1.00, \mathrm{CHCl}_{3}\right) ;{ }^{1} \mathrm{H}$ NMR $(400$ $\left.\mathrm{MHz}, \mathrm{CDCl}_{3}\right) \delta$ 7.40-7.38 (m, 2H), 7.29-7.27 (m, 1H), 7.22-7.21 (m, 1H), 7.11-7.03 (m, 4H), 7.01-6.99 (m, $1 \mathrm{H}), 6.96-6.91(\mathrm{~m}, 3 \mathrm{H}), 6.84(\mathrm{~s}, 1 \mathrm{H}), 2.86(\mathrm{dd}, J=12.5,6.3 \mathrm{~Hz}, 1 \mathrm{H}), 2.35(\mathrm{~s}, 3 \mathrm{H}), 2.31-2.26(\mathrm{~m}, 2 \mathrm{H}), 2.15$ $(\mathrm{s}, 3 \mathrm{H}), 1.95(\mathrm{qd}, J=12.6,4.9 \mathrm{~Hz}, 1 \mathrm{H}), 1.77-1.65(\mathrm{~m}, 1 \mathrm{H}), 1.37-1.34(\mathrm{~m}, 2 \mathrm{H}) ;{ }^{13} \mathrm{C} \mathrm{NMR}\left(101 \mathrm{MHz}, \mathrm{CDCl}_{3}\right)$ $\delta 143.7,137.3,136.6,136.5,136.2,135.4,133.6,132.5,132.0,130.2,129.9,127.9,127.8,127.2,124.1,37.1$, 34.3, 25.6, 21.6, 21.4, 11.6; HRMS (EI, $m / z$ ): calcd for $\mathrm{C}_{24} \mathrm{H}_{26} \mathrm{Si}\left[\mathrm{M}^{+}\right] 342.1798$, found 342.1798; IR (film): 2913, 2857, 1600,1226, 1117, 1041, $698 \mathrm{~cm}^{-1}$.

\section{(S)-1,1-bis(4-methoxyphenyl)-2-phenylsilolane (3f)}

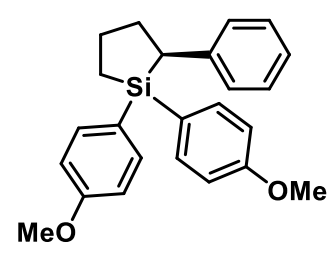

Yield: 24\% (9.0 mg); white solid; HPLC gave 98\% e.e., conditions: Daicel chiral column OD-H, hexane: ${ }^{i} \mathrm{PrOH}=100: 1,0.5 \mathrm{~mL} / \mathrm{min}$, wavelength $=220 \mathrm{~nm} ;[\alpha]_{D}^{30}=-31.8^{\circ}\left(c 1.00, \mathrm{CHCl}_{3}\right) ;{ }^{1} \mathrm{H} \mathrm{NMR}(400 \mathrm{MHz}$, $\left.\mathrm{CDCl}_{3}\right) \delta 7.50(\mathrm{~d}, J=8.3 \mathrm{~Hz}, 2 \mathrm{H}), 7.11-7.07(\mathrm{~m}, 2 \mathrm{H}), 7.03-6.97(\mathrm{~m}, 3 \mathrm{H}), 6.94-6.92(\mathrm{~m}, 4 \mathrm{H}), 6.71(\mathrm{~d}, J$ $=8.3 \mathrm{~Hz}, 2 \mathrm{H}), 3.82(\mathrm{~s}, 3 \mathrm{H}), 3.74(\mathrm{~s}, 3 \mathrm{H}), 2.80(\mathrm{dd}, J=12.6,6.3 \mathrm{~Hz}, 1 \mathrm{H}), 2.34-2.21(\mathrm{~m}, 2 \mathrm{H}), 1.93(\mathrm{qd}, J=$ 12.6, $4.9 \mathrm{~Hz}, 1 \mathrm{H}), 1.75-1.63(\mathrm{~m}, 1 \mathrm{H}), 1.34-1.30(\mathrm{~m}, J=8.2 \mathrm{~Hz}, 2 \mathrm{H}) ;{ }^{13} \mathrm{C} \mathrm{NMR}\left(101 \mathrm{MHz}, \mathrm{CDCl}_{3}\right) \delta 160.7$, 160.4, 143.8, 136.9, 136.3, 128.0, 127.5, 127.1, 124.8, 124.0, 113.7, 113.2, 55.0, 54.9, 37.3, 34.4, 25.5, 11.8; HRMS (EI, $m / z$ ): calcd for $\mathrm{C}_{24} \mathrm{H}_{27} \mathrm{O}_{2} \mathrm{~F}_{6} \mathrm{Si}^{+}\left[(\mathrm{M}+\mathrm{H})^{+}\right]$375.1775, found 375.1779; IR (film): 2933, 2849, $1593,1502,1278,1248,1112,1031,822 \mathrm{~cm}^{-1}$.

\section{(S)-1,1-bis(4-chlorophenyl)-2-phenylsilolane (3g)}

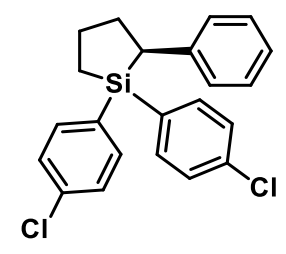

Yield: 51\% (19.5 mg); colorless oil; HPLC gave 99\% e.e., conditions: Daicel chiral column OD-3, hexane: ${ }^{i} \mathrm{PrOH}=100: 0,0.5 \mathrm{~mL} / \mathrm{min}$, wavelength $=220 \mathrm{~nm} ;[\alpha]_{D}^{30}=-45.2^{\circ}\left(c 1.00, \mathrm{CHCl}_{3}\right) ;{ }^{1} \mathrm{H} \mathrm{NMR}(400 \mathrm{MHz}$, 
$\left.\mathrm{CDCl}_{3}\right) \delta 7.48(\mathrm{~d}, J=8.1 \mathrm{~Hz}, 2 \mathrm{H}), 7.36(\mathrm{~d}, J=8.1 \mathrm{~Hz}, 2 \mathrm{H}), 7.15-7.08(\mathrm{~m}, 4 \mathrm{H}), 7.03-6.97(\mathrm{~m}, 3 \mathrm{H}), 6.91$ $(\mathrm{d}, J=7.5 \mathrm{~Hz}, 2 \mathrm{H}), \quad 2.82(\mathrm{dd}, J=12.5,6.4 \mathrm{~Hz}, 1 \mathrm{H}), 2.35-2.25(\mathrm{~m}, 2 \mathrm{H}), 1.93(\mathrm{qd}, J=12.7,5.0 \mathrm{~Hz}, 1 \mathrm{H})$, $1.76-1.64(\mathrm{~m}, 1 \mathrm{H}), 1.36-1.32(\mathrm{~m}, 2 \mathrm{H}) ;{ }^{13} \mathrm{C}$ NMR $\left(101 \mathrm{MHz}, \mathrm{CDCl}_{3}\right) \delta 142.8,136.7,136.1,134.3,131.6$, 128.7, 128.4, 128.2, 127.8, 127.0, 126.5, 124.5, 36.9, 34.2, 25.5, 11.6; HRMS (EI, $m / z)$ : calcd for $\mathrm{C}_{22} \mathrm{H}_{20} \mathrm{Cl}_{2} \mathrm{Si}$ $\left[\mathrm{M}^{+}\right]$382.0706, found 382.0706; IR (film): 2931, 2859, 1577, 1380, 1082,1014, $811 \mathrm{~cm}^{-1}$.

(S)-1,1-bis(4-fluorophenyl)-2-phenylsilolane (3h)

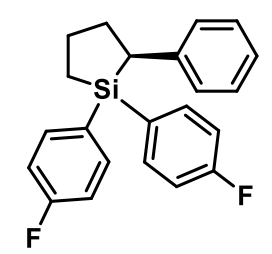

Yield: 63\% (22.1 mg); colorless oil; HPLC gave 99\% e.e., conditions: Daicel chiral column OD-H+OD-H, hexane: ${ }^{i} \mathrm{PrOH}=100: 0,0.5 \mathrm{~mL} / \mathrm{min}$, wavelength $=220 \mathrm{~nm} ;[\alpha]_{D}^{30}=-28.1^{\circ}\left(\mathrm{c} 1.00, \mathrm{CHCl}_{3}\right) ;{ }^{1} \mathrm{H} \mathrm{NMR}(400$ $\left.\mathrm{MHz}, \mathrm{CDCl}_{3}\right) \delta 7.56-7.52(\mathrm{~m}, 2 \mathrm{H}), 7.10-6.98(\mathrm{~m}, 7 \mathrm{H}), 6.91-6.84(\mathrm{~m}, 4 \mathrm{H}), 2.83(\mathrm{dd}, J=12.5,6.4 \mathrm{~Hz}$, $1 \mathrm{H}), 2.33-2.27(\mathrm{~m}, 2 \mathrm{H}), 1.94(\mathrm{qd}, J=12.7,4.9 \mathrm{~Hz}, 1 \mathrm{H}), 1.77-1.64(\mathrm{~m}, 1 \mathrm{H}), 1.37-1.33(\mathrm{~m}, 2 \mathrm{H}) ;{ }^{13} \mathrm{C} \mathrm{NMR}$ $\left(101 \mathrm{MHz}, \mathrm{CDCl}_{3}\right) \delta 164.0(\mathrm{~d}, J=249.3 \mathrm{~Hz}, 1 \mathrm{C}), 163.8(\mathrm{~d}, J=249.3 \mathrm{~Hz}, 1 \mathrm{C}), 143.1,137.3(\mathrm{~d}, J=7.5 \mathrm{~Hz}$, 1C), $136.8(\mathrm{~d}, J=7.5 \mathrm{~Hz}, 1 \mathrm{C}), 131.6(\mathrm{~d}, J=3.8 \mathrm{~Hz}, 1 \mathrm{C}), 129.0(\mathrm{~d}, J=3.9 \mathrm{~Hz}, 1 \mathrm{C}), 128.1,127.0,124.3,115.3$ (d, $J=19.8 \mathrm{~Hz}, 1 \mathrm{C}), 114.7$ (d, $J=19.7 \mathrm{~Hz}, 1 \mathrm{C}), 37.1,34.2,25.5,11.7 ;{ }^{19} \mathrm{~F} \mathrm{NMR}\left(471 \mathrm{MHz}, \mathrm{CDCl}_{3}\right) \delta-110.9$ - (-111.1) (m, 1F), -111.1 - (-111.2) (m, 1F); HRMS (EI, $m / z)$ : calcd for $\mathrm{C}_{22} \mathrm{H}_{20} \mathrm{~F}_{2} \mathrm{Si}\left[\mathrm{M}^{+}\right]$350.1297, found 350.1296; IR (film): 2931, 2850, 1587, 1498, 1235,1162, 1106, $824 \mathrm{~cm}^{-1}$.

(S)-2-(p-tolyl)-1,1-bis(4-(trifluoromethyl)phenyl)silolane (4a)

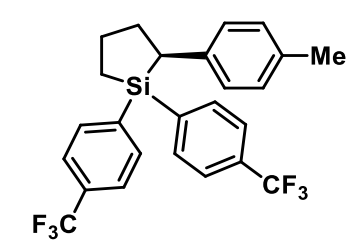

Yield: $91 \%$ (42.1 mg); colorless oil; HPLC gave >99\% e.e., conditions: Daicel chiral column OD-3+OD-H, hexane: ${ }^{i} \mathrm{PrOH}=100: 0,0.4 \mathrm{~mL} / \mathrm{min}$, wavelength $=220 \mathrm{~nm} ;[\alpha]_{D}^{30}=-23.7^{\circ}\left(c 1.00, \mathrm{CHCl}_{3}\right) ;{ }^{1} \mathrm{H} \mathrm{NMR}(400$ $\left.\mathrm{MHz}, \mathrm{CDCl}_{3}\right) \delta 7.68(\mathrm{~d}, J=8.0 \mathrm{~Hz}, 2 \mathrm{H}), 7.63(\mathrm{~d}, J=8.0 \mathrm{~Hz}, 2 \mathrm{H}), 7.42(\mathrm{~d}, J=7.9 \mathrm{~Hz}, 2 \mathrm{H}), 7.22(\mathrm{~d}, J=8.1$ $\mathrm{Hz}, 2 \mathrm{H}), 6.93(\mathrm{~d}, J=7.9 \mathrm{~Hz}, 2 \mathrm{H}), 6.82(\mathrm{~d}, J=8.0 \mathrm{~Hz}, 2 \mathrm{H}), 2.85(\mathrm{dd}, J=12.6,6.5 \mathrm{~Hz}, 1 \mathrm{H}), 2.36-2.29$ (m, $2 \mathrm{H}), 2.25(\mathrm{~s}, 3 \mathrm{H}), 1.95(\mathrm{qd}, J=12.6,4.9 \mathrm{~Hz}, 1 \mathrm{H}), 1.78-1.66(\mathrm{~m}, 1 \mathrm{H}), 1.43-1.39(\mathrm{~m}, 2 \mathrm{H}) ;{ }^{13} \mathrm{C}$ NMR $(101$ $\left.\mathrm{MHz}, \mathrm{CDCl}_{3}\right) \delta 140.8,139.0,138.2,135.7,135.0,134.1,131.7$ (q, $\left.J=32.3 \mathrm{~Hz}, 1 \mathrm{C}\right), 131.4$ (q, $J=32.3 \mathrm{~Hz}$, 1C), 129.0, 127.0, 124.7 (q, $J=3.6 \mathrm{~Hz}, 1 \mathrm{C}), 124.1$ (q, $J=3.7 \mathrm{~Hz}, 1 \mathrm{C}), 124.0$ (q, $J=273.1 \mathrm{~Hz}, 1 \mathrm{C}), 124.0$ (q, $J=273.1 \mathrm{~Hz}, 1 \mathrm{C}), 36.4,34.5,25.5,20.8,11.4 ;{ }^{19} \mathrm{~F} \mathrm{NMR}\left(471 \mathrm{MHz}, \mathrm{CDCl}_{3}\right) \delta-63.1(\mathrm{~s}, 3 \mathrm{~F}),-63.1(\mathrm{~s}, 3 \mathrm{~F})$; HRMS (EI, $m / z$ ): calcd for $\mathrm{C}_{25} \mathrm{H}_{22} \mathrm{~F}_{6} \mathrm{Si}\left[\mathrm{M}^{+}\right]$464.1390, found 464.1392; IR (film): 2946, 1608,1516, 1323 , $1165,1126,1104,827 \mathrm{~cm}^{-1}$.

(S)-2-(4-methoxyphenyl)-1,1-bis(4-(trifluoromethyl)phenyl)silolane (4b)<smiles>COc1ccc(C2CCC[Si]2(c2ccc(C(F)(F)F)cc2)c2ccc(C(F)(F)F)cc2)cc1</smiles> 
Yield: $82 \%$ (39.2 mg); colorless oil; HPLC gave $>99 \%$ e.e., conditions: Daicel chiral column OD-3*OD-H, hexane: ${ }^{i} \mathrm{PrOH}=200: 1,0.25 \mathrm{~mL} / \mathrm{min}$, wavelength $=210 \mathrm{~nm} ;[\alpha]_{D}^{30}=-30.0^{\circ}\left(c 1.00, \mathrm{CHCl}_{3}\right) ;{ }^{1} \mathrm{H} \mathrm{NMR}(400$ $\left.\mathrm{MHz}, \mathrm{CDCl}_{3}\right) \delta 7.68(\mathrm{~d}, J=8.0 \mathrm{~Hz}, 2 \mathrm{H}), 7.63(\mathrm{~d}, J=8.0 \mathrm{~Hz}, 2 \mathrm{H}), 7.44(\mathrm{~d}, J=8.0 \mathrm{~Hz}, 2 \mathrm{H}), 7.23(\mathrm{~d}, J=8.0$ $\mathrm{Hz}, 2 \mathrm{H}), 6.84(\mathrm{~d}, J=8.6 \mathrm{~Hz}, 2 \mathrm{H}), 6.68(\mathrm{~d}, J=8.6 \mathrm{~Hz}, 2 \mathrm{H}), 3.73(\mathrm{~s}, 3 \mathrm{H}), 2.83(\mathrm{dd}, J=12.6,6.5 \mathrm{~Hz}, 1 \mathrm{H}), 2.37$ $-2.30(\mathrm{~m}, 2 \mathrm{H}), 1.91(\mathrm{qd}, J=12.7,4.9 \mathrm{~Hz}, 1 \mathrm{H}), 1.78-1.66(\mathrm{~m}, 1 \mathrm{H}), 1.43-1.39(\mathrm{~m}, 2 \mathrm{H}) ;{ }^{13} \mathrm{C}$ NMR $(101$ $\left.\mathrm{MHz}, \mathrm{CDCl}_{3}\right) \delta 157.0,140.7,138.2,135.7,135.0,134.2,131.7$ (q, $\left.J=32.4 \mathrm{~Hz}, 1 \mathrm{C}\right), 131.4(\mathrm{q}, J=32.4 \mathrm{~Hz}$, 1C), 127.9, 124.7 (q, $J=3.7 \mathrm{~Hz}, 1 \mathrm{C}), 124.1$ (q, $J=3.6 \mathrm{~Hz}, 1 \mathrm{C}), 124.0$ (q, $J=272.2 \mathrm{~Hz}, 1 \mathrm{C}), 124.0$ (q, $J=$ $272.2 \mathrm{~Hz}, 1 \mathrm{C}$ ), 113.8, 55.2, 35.7, 34.7, 25.4, 11.4; ${ }^{19} \mathrm{~F}$ NMR (471 MHz, $\mathrm{CDCl}_{3}$ ) $\delta-63.1$ (s, 3F), -63.1 (s, 3F); HRMS (EI, $m / z$ ): calcd for $\mathrm{C}_{25} \mathrm{H}_{22} \mathrm{OF}_{6} \mathrm{Si}\left[\mathrm{M}^{+}\right]$480.1339, found 480.1343; IR (film): 2940, 1608,1394, $1322,1147,1125,1059,827 \mathrm{~cm}^{-1}$.

\section{(S)-2-([1,1'-biphenyl]-4-yl)-1,1-bis(4-(trifluoromethyl)phenyl)silolane (4c)}

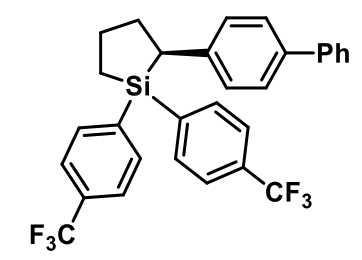

Yield: $81 \%$ (42.8 mg); white solid; HPLC gave >99\% e.e., conditions: Daicel chiral column AD-H, hexane: ${ }^{i} \mathrm{PrOH}=500: 1,0.3 \mathrm{~mL} / \mathrm{min}$, wavelength $=220 \mathrm{~nm} ;[\alpha]_{D}^{30}=-33.3^{\circ}\left(c 1.00, \mathrm{CHCl}_{3}\right) ;{ }^{1} \mathrm{H} \mathrm{NMR}(400 \mathrm{MHz}$, $\left.\mathrm{CDCl}_{3}\right) \delta 7.71(\mathrm{~d}, J=7.8 \mathrm{~Hz}, 2 \mathrm{H}), 7.65(\mathrm{~d}, J=7.9 \mathrm{~Hz}, 2 \mathrm{H}), 7.53(\mathrm{~d}, J=8.0 \mathrm{~Hz}, 2 \mathrm{H}), 7.43-7.36(\mathrm{~m}, 6 \mathrm{H})$, $7.32-7.28(\mathrm{~m}, 1 \mathrm{H}), 7.24(\mathrm{~d}, J=7.7 \mathrm{~Hz}, 2 \mathrm{H}), 6.99(\mathrm{~d}, J=7.8 \mathrm{~Hz}, 2 \mathrm{H}), 2.94(\mathrm{dd}, J=12.5,6.5 \mathrm{~Hz}, 1 \mathrm{H}), 2.41$ $-2.34(\mathrm{~m}, 2 \mathrm{H}), 2.01(\mathrm{qd}, J=12.8,5.0 \mathrm{~Hz}, 1 \mathrm{H}), 1.82-1.70(\mathrm{~m}, 1 \mathrm{H}), 1.46-1.42(\mathrm{~m}, 2 \mathrm{H}) ;{ }^{13} \mathrm{C}$ NMR $(101$ $\left.\mathrm{MHz}, \mathrm{CDCl}_{3}\right) \delta 141.6,140.9,140.5,137.9,137.6,135.7,135.1,131.8(\mathrm{q}, J=32.3 \mathrm{~Hz}, 1 \mathrm{C}), 131.5(\mathrm{q}, J=32.3$ $\mathrm{Hz}, 1 \mathrm{C}), 128.7,127.4,126.9,126.8,124.8$ (q, $J=3.7 \mathrm{~Hz}, 1 \mathrm{C}), 124.2$ (q, $J=3.7 \mathrm{~Hz}, 1 \mathrm{C}$ ), 124.0 (q, $J=272.5$ $\mathrm{Hz}, 1 \mathrm{C}), 124.0$ (q, $J=272.5 \mathrm{~Hz}, 1 \mathrm{C}), 36.7,34.4,25.5,11.4 ;{ }^{19} \mathrm{~F} \mathrm{NMR}\left(471 \mathrm{MHz}, \mathrm{CDCl}_{3}\right) \delta-63.1(\mathrm{~s}, 3 \mathrm{~F}),-$ 63.2 (s, 3F); HRMS (EI, $m / z$ ): calcd for $\mathrm{C}_{30} \mathrm{H}_{24} \mathrm{~F}_{6} \mathrm{Si}\left[\mathrm{M}^{+}\right]$526.1546, found 526.1560; IR (film): 3041, 2943, $1605,1323,1165,1125,1060,827 \mathrm{~cm}^{-1}$.

(S)-2-(4-fluorophenyl)-1,1-bis(4-(trifluoromethyl)phenyl)silolane (4d)

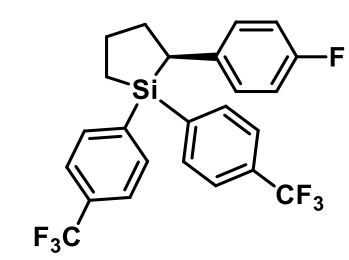

Yield: $72 \%$ (33.6 mg); colorless oil; HPLC gave >99\% e.e., conditions: Daicel chiral column OD-3, hexane: ${ }^{i} \mathrm{PrOH}=100: 0,0.5 \mathrm{~mL} / \mathrm{min}$, wavelength $=220 \mathrm{~nm} ;[\alpha]_{D}^{30}=-25.2^{\circ}\left(c 1.00, \mathrm{CHCl}_{3}\right) ;{ }^{1} \mathrm{H} \mathrm{NMR}(400 \mathrm{MHz}$, $\left.\mathrm{CDCl}_{3}\right) \delta 7.69-7.63(\mathrm{~m}, 4 \mathrm{H}), 7.45(\mathrm{~d}, J=7.9 \mathrm{~Hz}, 2 \mathrm{H}), 7.22(\mathrm{~d}, J=7.9 \mathrm{~Hz}, 2 \mathrm{H}), 6.87-6.79(\mathrm{~m}, 4 \mathrm{H}), 2.87$ $(\mathrm{dd}, J=12.5,6.5 \mathrm{~Hz}, 1 \mathrm{H}), 2.39-2.30(\mathrm{~m}, 2 \mathrm{H}), 1.91(\mathrm{qd}, J=12.5,4.9 \mathrm{~Hz}, 1 \mathrm{H}), 1.80-1.67(\mathrm{~m}, 1 \mathrm{H}), 1.44-$ $1.41(\mathrm{~m}, 2 \mathrm{H}) ;{ }^{13} \mathrm{C} \mathrm{NMR}\left(101 \mathrm{MHz}, \mathrm{CDCl}_{3}\right) \delta 160.4(\mathrm{~d}, J=242.9 \mathrm{~Hz}, 1 \mathrm{C}), 140.3,137.9$ (d, $\left.J=3.1 \mathrm{~Hz}, 1 \mathrm{C}\right)$, 137.7, 135.6, 135.0, 131.8 (q, $J=32.4 \mathrm{~Hz}, 1 \mathrm{C}), 131.6(\mathrm{q}, J=32.4 \mathrm{~Hz}, 1 \mathrm{C}), 128.2(\mathrm{~d}, J=7.5 \mathrm{~Hz}, 1 \mathrm{C}), 124.8$ (q, $J=3.6 \mathrm{~Hz}, 1 \mathrm{C}), 124.2$ (q, $J=3.6 \mathrm{~Hz}, 1 \mathrm{C}), 124.0$ (q, $J=272.2 \mathrm{~Hz}, 1 \mathrm{C}), 124.0(\mathrm{q}, J=272.2 \mathrm{~Hz}, 1 \mathrm{C}), 115.0$ (d, $J=21.1 \mathrm{~Hz}, 1 \mathrm{C}), 36.0,34.6,25.4,11.3 ;{ }^{19} \mathrm{~F} \mathrm{NMR}\left(471 \mathrm{MHz}, \mathrm{CDCl}_{3}\right) \delta-63.2$ (s, 3F), -63.2 (s, 3F), -119.2 - (-119.2) (m, 1F); HRMS (EI, $m / z)$ : calcd for $\mathrm{C}_{24} \mathrm{H}_{19} \mathrm{~F}_{7} \mathrm{Si}\left[\mathrm{M}^{+}\right]$468.1139, found 468.1139; IR (film): 2946, $1611,1392,1324,1165,1128,1060,827 \mathrm{~cm}^{-1}$. 


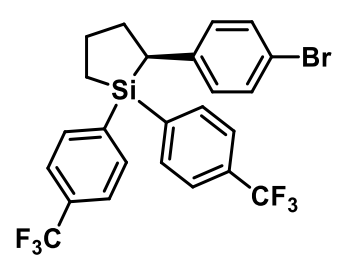

Yield: 77\% (40.9 mg); white solid; HPLC gave 97\% e.e., conditions: Daicel chiral column AD-3*AD-H, hexane: ${ }^{i} \mathrm{PrOH}=100: 0,0.15 \mathrm{~mL} / \mathrm{min}$, wavelength $=210 \mathrm{~nm} ;[\alpha]_{D}^{30}=-15.9^{\circ}\left(c 1.00, \mathrm{CHCl}_{3}\right) ;{ }^{1} \mathrm{H}$ NMR $(400$ $\left.\mathrm{MHz}, \mathrm{CDCl}_{3}\right) \delta 7.68-7.63(\mathrm{~m}, 4 \mathrm{H}), 7.46(\mathrm{~d}, J=7.7 \mathrm{~Hz}, 2 \mathrm{H}), 7.24-7.22(\mathrm{~m}, 4 \mathrm{H}), 6.78(\mathrm{~d}, J=8.4 \mathrm{~Hz}, 2 \mathrm{H})$, $2.84(\mathrm{dd}, J=12.6,6.4 \mathrm{~Hz}, 1 \mathrm{H}), 2.37-2.30(\mathrm{~m}, 2 \mathrm{H}), 1.91(\mathrm{qd}, J=12.2,4.6 \mathrm{~Hz}, 1 \mathrm{H}), 1.79-1.67(\mathrm{~m}, 1 \mathrm{H})$, 1.45-1.41 (m, 2H); ${ }^{13} \mathrm{C}$ NMR (101 MHz, $\left.\mathrm{CDCl}_{3}\right) \delta 141.5,140.1,137.5,135.6,135.0,131.9$ (q, $J=32.4 \mathrm{~Hz}$, 1C), $131.6(\mathrm{q}, J=32.4 \mathrm{~Hz}, 1 \mathrm{C}), 131.3,128.7,124.8(\mathrm{q}, J=3.7 \mathrm{~Hz}, 1 \mathrm{C}), 124.3(\mathrm{q}, J=3.7 \mathrm{~Hz}, 1 \mathrm{C}), 124.0(\mathrm{q}$, $J=272.5 \mathrm{~Hz}, 1 \mathrm{C}), 124.0$ (q, $J=272.5 \mathrm{~Hz}, 1 \mathrm{C}), 118.1,36.5,34.4,25.4,11.3 ;{ }^{19} \mathrm{~F} \mathrm{NMR}\left(471 \mathrm{MHz}, \mathrm{CDCl}_{3}\right) \delta$ -63.1 (s, 3F), -63.1 (s, 3F); HRMS (EI, $m / z$ ): calcd for $\mathrm{C}_{24} \mathrm{H}_{19} \mathrm{BrF}_{6} \mathrm{Si}\left[\mathrm{M}^{+}\right]$528.0338, found 528.0353; IR (film): 2968, 1611, 1392, 1325, 1166, 1131, 1061, $827 \mathrm{~cm}^{-1}$.

\section{Methyl (S)-4-(1,1-bis(4-(trifluoromethyl)phenyl)silolan-2-yl)benzoate (4f)}

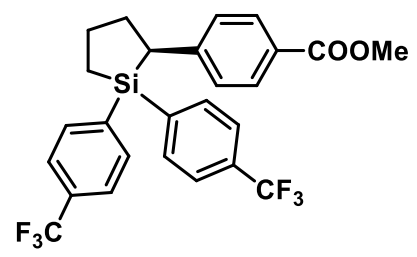

Yield: 65\% (32.9 mg); colorless oil; HPLC gave 98\% e.e., conditions: Daicel chiral column OD-H*OD-3, hexane: ${ }^{i} \mathrm{PrOH}=100: 1,0.5 \mathrm{~mL} / \mathrm{min}$, wavelength $=210 \mathrm{~nm} ;[\alpha]_{D}^{30}=-32.7^{\circ}\left(c 1.00, \mathrm{CHCl}_{3}\right) ;{ }^{1} \mathrm{H} \mathrm{NMR}(400$ $\left.\mathrm{MHz}, \mathrm{CDCl}_{3}\right) \delta 7.79(\mathrm{~d}, J=8.3 \mathrm{~Hz}, 2 \mathrm{H}), 7.70-7.64(\mathrm{~m}, 4 \mathrm{H}), 7.42(\mathrm{~d}, J=7.9 \mathrm{~Hz}, 2 \mathrm{H}), 7.19(\mathrm{~d}, J=7.9 \mathrm{~Hz}$, $2 \mathrm{H}), 6.98(\mathrm{~d}, J=8.3 \mathrm{~Hz}, 2 \mathrm{H}), 3.87(\mathrm{~s}, 3 \mathrm{H}), 2.98(\mathrm{dd}, J=12.5,6.3 \mathrm{~Hz}, 1 \mathrm{H}), 2.41-2.35(\mathrm{~m}, 2 \mathrm{H}), 2.01(\mathrm{qd}, J$ $=12.5,4.9 \mathrm{~Hz}, 1 \mathrm{H}), 1.83-1.70(\mathrm{~m}, 1 \mathrm{H}), 1.47-1.43(\mathrm{~m}, 2 \mathrm{H}) ;{ }^{13} \mathrm{C} \mathrm{NMR}\left(101 \mathrm{MHz}, \mathrm{CDCl}_{3}\right) \delta 167.1,148.6$, 139.9, 137.2, 135.5, 135.0, 132.0 (q, $J=32.5 \mathrm{~Hz}, 1 \mathrm{C}), 131.6$ (q, $J=32.5 \mathrm{~Hz}, 1 \mathrm{C}), 129.7,126.8,126.6,124.8$ (q, $J=3.6 \mathrm{~Hz}, 1 \mathrm{C}), 124.3$ (q, $J=3.6 \mathrm{~Hz}, 1 \mathrm{C}), 124.0$ (q, $J=272.5 \mathrm{~Hz}, 1 \mathrm{C}), 124.0$ (q, $J=272.5 \mathrm{~Hz}, 1 \mathrm{C}), 51.9$, 37.7, 34.0, 25.4, 11.4; ${ }^{19} \mathrm{~F}$ NMR (471 MHz, $\left.\mathrm{CDCl}_{3}\right) \delta$-63.2 (s, 3F), -63.3 (s, 3F); HRMS (ESI, $\mathrm{m} / \mathrm{z}$ ): calcd for $\mathrm{C}_{24} \mathrm{H}_{20} \mathrm{BrF}_{6} \mathrm{Si}^{+}\left[(\mathrm{M}+\mathrm{H})^{+}\right]$509.1366, found 509.1360; IR (film): 2952, 1720, 1607,1392, 1324,1166, $1127,1060,827 \mathrm{~cm}^{-1}$.

\section{(S)-2-(4-(4,4,5,5-tetramethyl-1,3,2-dioxaborolan-2-yl)phenyl)-1,1-bis(4-} (trifluoromethyl)phenyl)silolane (4g)

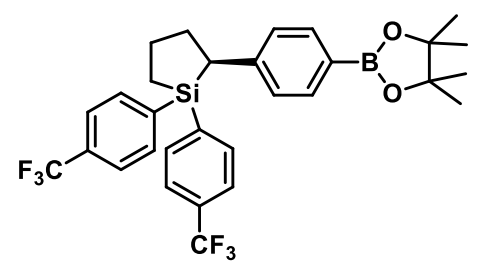

Yield: $57 \%$ (33.1 mg); white solid; HPLC gave >99\% e.e., conditions: Daicel chiral column AD-H, hexane: ${ }^{i} \mathrm{PrOH}=100: 0,0.5 \mathrm{~mL} / \mathrm{min}$, wavelength $=220 \mathrm{~nm} ;[\alpha]_{D}^{30}=-13.4^{\circ}\left(c 1.00, \mathrm{CHCl}_{3}\right) ;{ }^{1} \mathrm{H} \mathrm{NMR}(400 \mathrm{MHz}$, $\left.\mathrm{CDCl}_{3}\right) \delta 7.68(\mathrm{~d}, J=8.0 \mathrm{~Hz}, 2 \mathrm{H}), 7.63(\mathrm{~d}, J=8.0 \mathrm{~Hz}, 2 \mathrm{H}), 7.57(\mathrm{~d}, J=7.9 \mathrm{~Hz}, 2 \mathrm{H}), 7.41(\mathrm{~d}, J=8.0 \mathrm{~Hz}$, $2 \mathrm{H}), 7.21(\mathrm{~d}, J=7.9 \mathrm{~Hz}, 2 \mathrm{H}), 6.95(\mathrm{~d}, J=7.9 \mathrm{~Hz}, 2 \mathrm{H}), 2.90(\mathrm{dd}, \mathrm{J}=12.6,6.4 \mathrm{~Hz}, 1 \mathrm{H}), 2.37-2.33(\mathrm{~m}, 2 \mathrm{H})$, $2.06-1.95(\mathrm{~m}, 1 \mathrm{H}), 1.80-1.68(\mathrm{~m}, 1 \mathrm{H}), 1.49-1.40(\mathrm{~m}, 2 \mathrm{H}), 1.32(\mathrm{~s}, 12 \mathrm{H}) ;{ }^{13} \mathrm{C} \mathrm{NMR}\left(101 \mathrm{MHz}, \mathrm{CDCl}_{3}\right)$ $\delta$ 146.1, 140.5, 137.8, 135.6, 135.0, 134.9, 131.8 (q, $J=32.6 \mathrm{~Hz}, 1 \mathrm{C}), 131.5$ (q, $J=32.3 \mathrm{~Hz}, 1 \mathrm{C}), 126.5$, 
$124.7(\mathrm{q}, J=3.6 \mathrm{~Hz}, 1 \mathrm{C}), 124.2(\mathrm{q}, J=3.7 \mathrm{~Hz}, 1 \mathrm{C}), 124.0(\mathrm{q}, J=272.5 \mathrm{~Hz}, 1 \mathrm{C}), 124.0(\mathrm{q}, J=272.5 \mathrm{~Hz}, 1 \mathrm{C})$, 83.6, 37.6, 34.3, 25.5, 24.9, 24.8, 11.4; ${ }^{19} \mathrm{~F}$ NMR (471 MHz, $\left.\mathrm{CDCl}_{3}\right) \delta-63.1$ (s, 3F), -63.1 (s, 3F); HRMS (EI, $m / z$ ): calcd for $\mathrm{C}_{30} \mathrm{H}_{31} \mathrm{BO}_{2} \mathrm{~F}_{6} \mathrm{Si}\left[\mathrm{M}^{+}\right]$576.2091, found 576.2107; IR (film): 2984, 1607,1391, 1324,1166, $1129,1060,827 \mathrm{~cm}^{-1}$.

\section{(S)-1,1,2-tris(4-(trifluoromethyl)phenyl)silolane (4h)}

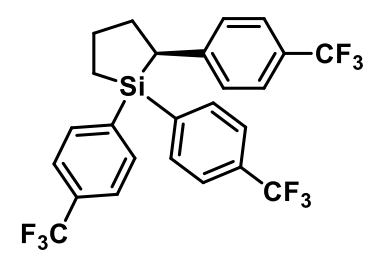

Yield: 73\% (37.6 mg); colorless oil; HPLC gave 99\% e.e., conditions: Daicel chiral column OD-3, hexane: ${ }^{i} \mathrm{PrOH}=100: 0,0.25 \mathrm{~mL} / \mathrm{min}$, wavelength $=220 \mathrm{~nm} ;[\alpha]_{D}^{30}=-33.8^{\circ}\left(c 1.00, \mathrm{CHCl}_{3}\right) ;{ }^{1} \mathrm{H} \mathrm{NMR}(400 \mathrm{MHz}$, $\left.\mathrm{CDCl}_{3}\right) \delta 7.70-7.63(\mathrm{~m}, 4 \mathrm{H}), 7.43(\mathrm{~d}, J=7.8 \mathrm{~Hz}, 2 \mathrm{H}), 7.35(\mathrm{~d}, J=8.1 \mathrm{~Hz}, 2 \mathrm{H}), 7.19(\mathrm{~d}, J=7.7 \mathrm{~Hz}, 2 \mathrm{H})$, $7.00(\mathrm{~d}, J=8.0 \mathrm{~Hz}, 2 \mathrm{H}), 2.97(\mathrm{dd}, J=12.4,6.4 \mathrm{~Hz}, 1 \mathrm{H}), 2.44-2.30(\mathrm{~m}, 2 \mathrm{H}), 1.99(\mathrm{qd}, J=12.3,4.7 \mathrm{~Hz}, 1 \mathrm{H})$, $1.85-1.69(\mathrm{~m}, 1 \mathrm{H}), 1.50-1.38(\mathrm{~m}, 2 \mathrm{H}) ;{ }^{13} \mathrm{C} \mathrm{NMR}\left(101 \mathrm{MHz}, \mathrm{CDCl}_{3}\right) \delta 147.1,139.8,137.2,135.5,135.0$, $131.7(\mathrm{q}, J=32.5 \mathrm{~Hz}, 1 \mathrm{C}), 131.5(\mathrm{q}, J=32.5 \mathrm{~Hz}, 1 \mathrm{C}), 127.1,127.0$ (q, $J=32.2 \mathrm{~Hz}, 1 \mathrm{C}), 125.2$ (q, $J=3.7$ $\mathrm{Hz}, 1 \mathrm{C}), 124.9$ (q, $J=3.6 \mathrm{~Hz}, 1 \mathrm{C}), 124.3$ (q, $J=3.7 \mathrm{~Hz}, 1 \mathrm{C}), 124.3$ (q, $J=271.5 \mathrm{~Hz}, 1 \mathrm{C}), 124.0$ (q, $J=272.5$ $\mathrm{Hz}, 1 \mathrm{C}), 123.9$ (q, $J=272.2 \mathrm{~Hz}, 1 \mathrm{C}), 37.2,34.1,25.4,11.2 ;{ }^{19} \mathrm{~F} \mathrm{NMR}\left(471 \mathrm{MHz}, \mathrm{CDCl}_{3}\right) \delta-62.3,(\mathrm{~s}, 3 \mathrm{~F}),-$ 63.1 (s, 3F), -63.2 (s, 3F); HRMS (EI, $\mathrm{m} / \mathrm{z}$ ): calcd for $\mathrm{C}_{25} \mathrm{H}_{19} \mathrm{~F}_{9} \mathrm{Si}\left[\mathrm{M}^{+}\right]$518.1107, found 518.1122; IR (film): $2930,1617,1392,1324,1164,1122,1060,827 \mathrm{~cm}^{-1}$.

\section{(S)-2-(4-(tert-butyl)phenyl)-1,1-bis(4-(trifluoromethyl)phenyl)silolane (4i)}

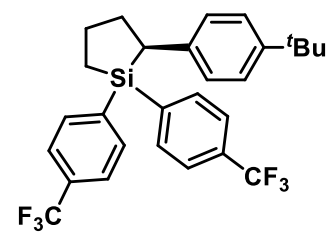

Yield: 75\% (37.7 mg); white solid; HPLC gave >99\% e.e., conditions: Daicel chiral column AD-H, hexane: ${ }^{i} \mathrm{PrOH}=100: 0,0.2 \mathrm{~mL} / \mathrm{min}$, wavelength $=220 \mathrm{~nm} ;[\alpha]_{D}^{30}=-47.2^{\circ}\left(c 1.00, \mathrm{CHCl}_{3}\right) ;{ }^{1} \mathrm{H} \mathrm{NMR}(400 \mathrm{MHz}$, $\left.\mathrm{CDCl}_{3}\right) \delta 7.69(\mathrm{~d}, J=7.9 \mathrm{~Hz}, 2 \mathrm{H}), 7.63(\mathrm{~d}, J=8.0 \mathrm{~Hz}, 2 \mathrm{H}), 7.36(\mathrm{~d}, J=7.9 \mathrm{~Hz}, 2 \mathrm{H}), 7.16-7.11(\mathrm{~m}, 4 \mathrm{H})$, $6.84(\mathrm{~d}, J=8.3 \mathrm{~Hz}, 2 \mathrm{H}), 2.90(\mathrm{dd}, J=12.2,6.6 \mathrm{~Hz}, 1 \mathrm{H}), 2.39-2.29(\mathrm{~m}, 2 \mathrm{H}), 1.97(\mathrm{qd}, J=12.7,5.0 \mathrm{~Hz}, 1 \mathrm{H})$, $1.80-1.68(\mathrm{~m}, 1 \mathrm{H}), 1.42-1.38(\mathrm{~m}, 2 \mathrm{H}), 1.25(\mathrm{~s}, 9 \mathrm{H}) ;{ }^{13} \mathrm{C} \mathrm{NMR}\left(100 \mathrm{MHz}, \mathrm{CDCl}_{3}\right) \delta$ 147.6, 140.7, 139.1, 138.2, 135.6, 135.1, 131.7 (q, $J=32.2 \mathrm{~Hz}, 1 \mathrm{C}), 131.3$ (q, $J=32.2 \mathrm{~Hz}, 1 \mathrm{C}), 126.5,125.1,124.7$ (q, $J=3.6$ $\mathrm{Hz}, 1 \mathrm{C}), 123.9$ (q, $J=3.7 \mathrm{~Hz}, 1 \mathrm{C}), 124.0$ (q, $J=272.3 \mathrm{~Hz}, 1 \mathrm{C}), 124.0$ (q, $J=272.3 \mathrm{~Hz}, 1 \mathrm{C}), 36.0,34.2,34.0$, 31.3, 25.7, 11.4; ${ }^{19} \mathrm{~F}$ NMR (471 MHz, $\left.\mathrm{CDCl}_{3}\right) \delta-63.1$ (s, 3F), -63.1 (s, 3F); HRMS (EI, $\mathrm{m} / \mathrm{z}$ ): calcd for $\mathrm{C}_{28} \mathrm{H}_{28} \mathrm{~F}_{6} \mathrm{Si}\left[\mathrm{M}^{+}\right]$506.1859, found 506.1868; IR (film): 2949, 1611,1391, 1323,1166, 1128,1060, $827 \mathrm{~cm}^{-1}$.

(S)-2-(m-tolyl)-1,1-bis(4-(trifluoromethyl)phenyl)silolane (4j)<smiles>Cc1cccc(C2CCC[Si]2(c2ccc(C(F)(F)F)cc2)c2ccc(C(F)(F)F)cc2)c1</smiles> 
Yield: 79\% (36.8 mg); colorless oil; HPLC gave 99\% e.e., conditions: Daicel chiral column OD-3, hexane: ${ }^{i} \mathrm{PrOH}=100: 0,0.5 \mathrm{~mL} / \mathrm{min}$, wavelength $=220 \mathrm{~nm} ;[\alpha]_{D}^{30}=-42.3^{\circ}\left(c 1.00, \mathrm{CHCl}_{3}\right) ;{ }^{1} \mathrm{H} \mathrm{NMR}(400 \mathrm{MHz}$, $\left.\mathrm{CDCl}_{3}\right) \delta 7.69(\mathrm{~d}, J=8.0 \mathrm{~Hz}, 2 \mathrm{H}), 7.63(\mathrm{~d}, J=8.0 \mathrm{~Hz}, 2 \mathrm{H}), 7.42(\mathrm{~d}, J=7.9 \mathrm{~Hz}, 2 \mathrm{H}), 7.20(\mathrm{~d}, J=7.8 \mathrm{~Hz}$, 2H), $7.01(\mathrm{t}, J=7.6 \mathrm{~Hz}, 1 \mathrm{H}), 6.84(\mathrm{~d}, J=7.5 \mathrm{~Hz}, 2 \mathrm{H}), 6.74(\mathrm{~d}, J=7.7 \mathrm{~Hz}, 2 \mathrm{H}), 6.66(\mathrm{~s}, 1 \mathrm{H}), 2.86(\mathrm{dd}, J=$ 12.5, 6.5 Hz, 1H), $2.38-2.30(\mathrm{~m}, 2 \mathrm{H}), 2.14(\mathrm{~s}, 3 \mathrm{H}), 2.00-1.90(\mathrm{~m}, 1 \mathrm{H}), 1.79-1.66(\mathrm{~m}, 1 \mathrm{H}), 1.44-1.40$ $(\mathrm{m}, 2 \mathrm{H}) ;{ }^{13} \mathrm{C} \mathrm{NMR}\left(101 \mathrm{MHz}, \mathrm{CDCl}_{3}\right) \delta 142.2,140.6,138.2,137.8,135.7,135.0,131.7$ (q, $\left.J=32.1 \mathrm{~Hz}, 1 \mathrm{C}\right)$, $131.4(\mathrm{q}, J=32.1 \mathrm{~Hz}, 1 \mathrm{C}), 128.2,127.9,125.4,124.7$ (q, $J=3.7 \mathrm{~Hz}, 1 \mathrm{C}), 124.2,124.0$ (q, $J=3.7 \mathrm{~Hz}, 1 \mathrm{C})$, 124.1 (q, $J=272.4 \mathrm{~Hz}, 1 \mathrm{C}), 124.1$ (q, $J=272.4 \mathrm{~Hz}, 1 \mathrm{C}), 36.7,34.5,25.5,21.3,11.4 ;{ }^{19} \mathrm{~F} \mathrm{NMR}(471 \mathrm{MHz}$, $\mathrm{CDCl}_{3}$ ) $\delta$-63.2 (s, 3F), -63.3 (s, 3F); HRMS (EI, $\mathrm{m} / \mathrm{z}$ ): calcd for $\mathrm{C}_{25} \mathrm{H}_{22} \mathrm{~F}_{6} \mathrm{Si}\left[\mathrm{M}^{+}\right]$464.1390, found 464.1390; IR (film): 2937, 1605,1391, 1324,1127,1060, $827 \mathrm{~cm}^{-1}$.

(S)-2-(3-methoxyphenyl)-1,1-bis(4-(trifluoromethyl)phenyl)silolane (4k)

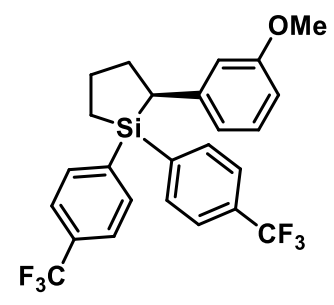

Yield: 72\% (34.4 mg); colorless oil; HPLC gave >99\% e.e., conditions: Daicel chiral column OD-3, hexane: ${ }^{i} \mathrm{PrOH}=200: 1,0.5 \mathrm{~mL} / \mathrm{min}$, wavelength $=220 \mathrm{~nm} ;[\alpha]_{D}^{30}=-25.0^{\circ}\left(c 1.00, \mathrm{CHCl}_{3}\right) ;{ }^{1} \mathrm{H} \mathrm{NMR}(400 \mathrm{MHz}$, $\left.\mathrm{CDCl}_{3}\right) \delta 7.70(\mathrm{~d}, J=7.9 \mathrm{~Hz}, 2 \mathrm{H}), 7.64(\mathrm{~d}, J=8.0 \mathrm{~Hz}, 2 \mathrm{H}), 7.43(\mathrm{~d}, J=7.9 \mathrm{~Hz}, 2 \mathrm{H}), 7.24(\mathrm{~d}, J=7.6 \mathrm{~Hz}$, 2H), $7.04(\mathrm{t}, J=7.9 \mathrm{~Hz}, 1 \mathrm{H}), 6.60-6.54(\mathrm{~m}, 2 \mathrm{H}), 6.41(\mathrm{~s}, 1 \mathrm{H}), 3.59(\mathrm{~s}, 3 \mathrm{H}), 2.88(\mathrm{dd}, J=12.5,6.5 \mathrm{~Hz}, 1 \mathrm{H})$, $2.39-2.32(\mathrm{~m}, 2 \mathrm{H}), 1.95(\mathrm{qd}, J=12.7,5.0 \mathrm{~Hz}, 1 \mathrm{H}), 1.79-1.67(\mathrm{~m}, 1 \mathrm{H}), 1.44-1.40(\mathrm{~m}, 2 \mathrm{H}) ;{ }^{13} \mathrm{C} \mathrm{NMR}(101$ $\left.\mathrm{MHz}, \mathrm{CDCl}_{3}\right) \delta 159.6,144.0,140.5,138.1,135.6,135.1,131.8(\mathrm{q}, J=32.2 \mathrm{~Hz}, 1 \mathrm{C}), 131.5(\mathrm{q}, J=32.2 \mathrm{~Hz}$, 1C), $129.2,124.0$ (q, $J=272.3 \mathrm{~Hz}, 1 \mathrm{C}), 124.0$ (q, $J=272.3 \mathrm{~Hz}, 1 \mathrm{C}), 124.7$ (q, $J=3.7 \mathrm{~Hz}, 1 \mathrm{C}), 124.1$ (q, $J=$ $3.7 \mathrm{~Hz}, 1 \mathrm{C}), 119.7,112.8,110.0,54.9,36.9,34.3,25.5,11.3 ;{ }^{19} \mathrm{~F} \mathrm{NMR}\left(471 \mathrm{MHz}, \mathrm{CDCl}_{3}\right) \delta-63.1(\mathrm{~s}, 3 \mathrm{~F}),-$ 63.1 (s, 3F); HRMS (EI, $m / z$ ): calcd for $\mathrm{C}_{25} \mathrm{H}_{22} \mathrm{OF}_{6} \mathrm{Si}\left[\mathrm{M}^{+}\right]$480.1339, found 480.1341; IR (film): 2939, $1602,1391,1323,1165,1126,1060,827 \mathrm{~cm}^{-1}$.

Methyl (S)-3-(1,1-bis(4-(trifluoromethyl)phenyl)silolan-2-yl)benzoate (4l)

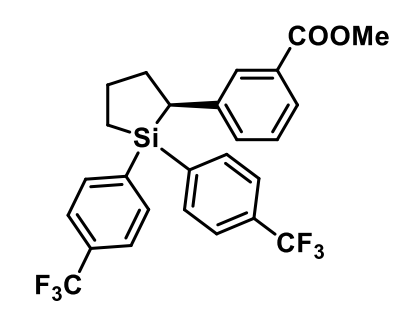

Yield: $71 \%$ (36.3 mg); colorless oil; HPLC gave >99\% e.e., conditions: Daicel chiral column AD-H, hexane: ${ }^{i} \mathrm{PrOH}=300: 1,0.5 \mathrm{~mL} / \mathrm{min}$, wavelength $=220 \mathrm{~nm} ;[\alpha]_{D}^{30}=-43.9^{\circ}\left(c 1.00, \mathrm{CHCl}_{3}\right) ;{ }^{1} \mathrm{H} \mathrm{NMR}(400 \mathrm{MHz}$, $\left.\mathrm{CDCl}_{3}\right) \delta 7.72-7.69(\mathrm{~m}, 3 \mathrm{H}), 7.67-7.65(\mathrm{~m}, 2 \mathrm{H}), 7.55(\mathrm{~s}, 1 \mathrm{H}), 7.41(\mathrm{~d}, J=8.0 \mathrm{~Hz}, 2 \mathrm{H}), 7.21(\mathrm{~d}, J=7.9 \mathrm{~Hz}$, $2 \mathrm{H}), 7.16(\mathrm{t}, J=7.7 \mathrm{~Hz}, 1 \mathrm{H}), 7.08(\mathrm{~d}, J=7.8 \mathrm{~Hz}, 1 \mathrm{H}), 3.83(\mathrm{~s}, 3 \mathrm{H}), 2.96(\mathrm{dd}, J=12.3,6.7 \mathrm{~Hz}, 1 \mathrm{H}), 2.46-$ $2.30(\mathrm{~m}, 2 \mathrm{H}), 2.00(\mathrm{qd}, J=12.8,5.1 \mathrm{~Hz}, 1 \mathrm{H}), 1.82-1.69(\mathrm{~m}, 1 \mathrm{H}), 1.52-1.40(\mathrm{~m}, 2 \mathrm{H}) ;{ }^{13} \mathrm{C} \mathrm{NMR}(101 \mathrm{MHz}$, $\left.\mathrm{CDCl}_{3}\right) \delta 167.1,143.0,140.0,137.5,135.6,135.1,131.9$ (q, $\left.J=32.4 \mathrm{~Hz}, 1 \mathrm{C}\right), 131.8,131.6$ (q, $J=32.4 \mathrm{~Hz}$, 1C), 130.0, 128.2, 128.0, 126.0, $124.8(\mathrm{q}, J=3.7 \mathrm{~Hz}, 1 \mathrm{C}), 124.2$ (q, $J=3.7 \mathrm{~Hz}, 1 \mathrm{C}), 124.0(\mathrm{q}, J=272.2 \mathrm{~Hz}$, 1C), 124.0 (q, $J=272.2 \mathrm{~Hz}, 1 \mathrm{C}), 51.9,36.6,34.6,25.5,11.3 ;{ }^{19} \mathrm{~F} \mathrm{NMR}\left(471 \mathrm{MHz}, \mathrm{CDCl}_{3}\right) \delta-63.1$ (s, 3F), - 
63.2 (s, 3F); HRMS (EI, $m / z$ ): calcd for $\mathrm{C}_{26} \mathrm{H}_{22} \mathrm{O}_{2} \mathrm{~F}_{6} \mathrm{Si}$ [M+] 508.1288, found 508.1294; IR (film): 2958, 1723 , $1608,1324,1166,1104,1060,827 \mathrm{~cm}^{-1}$.

(S)-2-(o-tolyl)-1,1-bis(4-(trifluoromethyl)phenyl)silolane (4m)

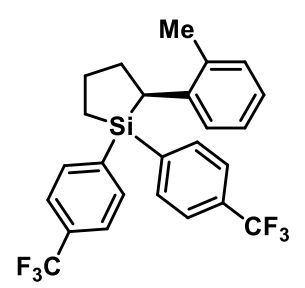

Yield: $87 \%$ (40.2 mg); colorless oil; HPLC gave >99\% e.e., conditions: Daicel chiral column OD-3, hexane: ${ }^{i} \mathrm{PrOH}=100: 0,0.5 \mathrm{~mL} / \mathrm{min}$, wavelength $=220 \mathrm{~nm} ;[\alpha]_{D}^{30}=+12.2^{\circ}\left(c 1.00, \mathrm{CHCl}_{3}\right) ;{ }^{1} \mathrm{H} \mathrm{NMR}(400 \mathrm{MHz}$, $\left.\mathrm{CDCl}_{3}\right) \delta 7.63-7.58(\mathrm{~m}, 4 \mathrm{H}), 7.41(\mathrm{~d}, J=7.7 \mathrm{~Hz}, 2 \mathrm{H}), 7.13(\mathrm{~d}, J=7.6 \mathrm{~Hz}, 2 \mathrm{H}), 7.05(\mathrm{~d}, J=6.9 \mathrm{~Hz}, 1 \mathrm{H})$, $6.97-6.90(\mathrm{~m}, 2 \mathrm{H}), 6.80(\mathrm{~d}, J=7.3 \mathrm{~Hz}, 1 \mathrm{H}), 3.05(\mathrm{dd}, J=11.0,6.8 \mathrm{~Hz}, 1 \mathrm{H}), 2.40-2.28(\mathrm{~m}, 2 \mathrm{H}), 2.12-$ $2.01(\mathrm{~m}, 4 \mathrm{H}), 1.92-1.81(\mathrm{~m}, 1 \mathrm{H}), 1.50-1.35(\mathrm{~m}, 2 \mathrm{H}) ;{ }^{13} \mathrm{C} \mathrm{NMR}\left(101 \mathrm{MHz}, \mathrm{CDCl}_{3}\right) \delta 141.5,141.0,137.5$, 135.6, 134.8, 134.7, 131.7 (q, $J=32.3 \mathrm{~Hz}, 1 \mathrm{C}), 131.4$ (q, $J=32.3 \mathrm{~Hz}, 1 \mathrm{C}), 130.1,126.3,125.9,124.6$ (q, $J$ $=3.7 \mathrm{~Hz}, 1 \mathrm{C}), 124.4,124.0(\mathrm{q}, J=271.4 \mathrm{~Hz}, 1 \mathrm{C}), 124.0(\mathrm{q}, J=271.4 \mathrm{~Hz}, 1 \mathrm{C}), 124.0(\mathrm{q}, J=3.7 \mathrm{~Hz}, 1 \mathrm{C})$, 34.83, 32.95, 25.96, 20.87, 12.45; ${ }^{19} \mathrm{~F}$ NMR (471 MHz, $\left.\mathrm{CDCl}_{3}\right) \delta-63.2(\mathrm{~s}, 3 \mathrm{~F}),-63.3$ (s, 3F); HRMS (EI, $\mathrm{m} / \mathrm{z}$ ): calcd for $\mathrm{C}_{25} \mathrm{H}_{22} \mathrm{~F}_{6} \mathrm{Si}\left[\mathrm{M}^{+}\right]$464.1390, found 464.1389; IR (film): 2946, 1602,1486, 1323, 1166, $1127,1060,827 \mathrm{~cm}^{-1}$.

(S)-2-(naphthalen-2-yl)-1,1-bis(4-(trifluoromethyl)phenyl)silolane (4n)

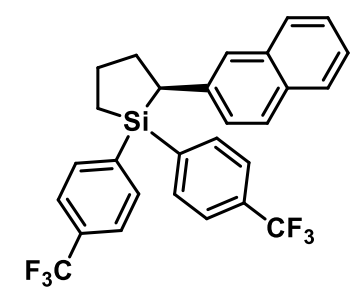

Yield: $88 \%$ (44.1 mg); colorless oil; HPLC gave >99\% e.e., conditions: Daicel chiral column OD-3, hexane: ${ }^{i} \mathrm{PrOH}=100: 1,0.5 \mathrm{~mL} / \mathrm{min}$, wavelength $=220 \mathrm{~nm} ;[\alpha]_{D}^{30}=-52.5^{\circ}\left(c 1.00, \mathrm{CHCl}_{3}\right) ;{ }^{1} \mathrm{H} \mathrm{NMR}(400 \mathrm{MHz}$, $\left.\mathrm{CDCl}_{3}\right)$ \& 7.73-7.71 (m, 3H), 7.66-7.64 (m, 2H), 7.61-7.55 (m, 2H), $7.41-7.32(\mathrm{~m}, 5 \mathrm{H}), 7.20(\mathrm{~d}, J=7.9 \mathrm{~Hz}$, $2 \mathrm{H}), 7.00(\mathrm{~d}, J=8.5 \mathrm{~Hz}, 1 \mathrm{H}), 3.05(\mathrm{dd}, J=12.6,6.4 \mathrm{~Hz}, 1 \mathrm{H}), 2.47-2.36(\mathrm{~m}, 2 \mathrm{H}), 2.10(\mathrm{qd}, J=12.9,5.0$ $\mathrm{Hz}, 1 \mathrm{H}), 1.85-1.73(\mathrm{~m}, 1 \mathrm{H}), 1.49-1.45(\mathrm{~m}, 2 \mathrm{H}),{ }^{13} \mathrm{C} \mathrm{NMR}\left(101 \mathrm{MHz}, \mathrm{CDCl}_{3}\right) \delta 140.5,140.0,137.9,135.6$, 135.1, 134.8, 133.7, 131.8 (q, $J=34.2 \mathrm{~Hz}, 1 \mathrm{C}), 131.5$ (q, $J=34.2 \mathrm{~Hz}, 1 \mathrm{C}), 131.3,127.7,127.5,127.1,126.7$, 126.0, 124.8 (q, $J=3.5 \mathrm{~Hz}, 1 \mathrm{C}), 124.3,124.2$ (q, $J=3.7 \mathrm{~Hz}, 1 \mathrm{C}), 124.1$ (q, $J=272.3 \mathrm{~Hz}, 1 \mathrm{C}), 124.0$ (q, $J=$ $272.3 \mathrm{~Hz}, 1 \mathrm{C}), 37.3,34.5,25.5,11.5 ;{ }^{19} \mathrm{~F}$ NMR $\left(471 \mathrm{MHz}, \mathrm{CDCl}_{3}\right) \delta-63.0$ (s, 3F), -63.1 (s, 3F); HRMS (EI, $\mathrm{m} / \mathrm{z}$ ): calcd for $\mathrm{C}_{28} \mathrm{H}_{22} \mathrm{~F}_{6} \mathrm{Si}\left[\mathrm{M}^{+}\right]$500.1390, found 500.1393; IR (film): 2936, 1608, 1391, 1323,1166, $1127,1060,827 \mathrm{~cm}^{-1}$.

(S)-2-(naphthalen-1-yl)-1,1-bis(4-(trifluoromethyl)phenyl)silolane (4o) 


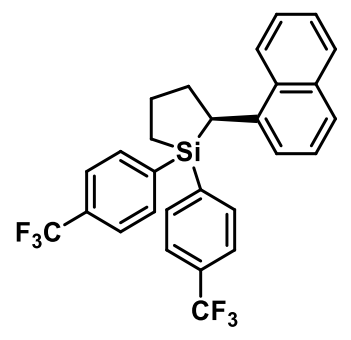

Yield: 92\% (45.8 mg); colorless oil; HPLC gave 97\% e.e., conditions: Daicel chiral column AD-3, hexane: ${ }^{i} \mathrm{PrOH}=100: 0,0.25 \mathrm{~mL} / \mathrm{min}$, wavelength $=210 \mathrm{~nm} ;[\alpha]_{D}^{30}=+185.6^{\circ}\left(c 1.00, \mathrm{CHCl}_{3}\right) ;{ }^{1} \mathrm{H} \mathrm{NMR}(400 \mathrm{MHz}$, $\left.\mathrm{CDCl}_{3}\right) \delta 7.79(\mathrm{~d}, J=8.1 \mathrm{~Hz}, 1 \mathrm{H}), 7.65(\mathrm{~d}, J=8.6 \mathrm{~Hz}, 1 \mathrm{H}), 7.62-7.60(\mathrm{~m}, 4 \mathrm{H}), 7.56(\mathrm{~d}, J=8.2 \mathrm{~Hz}, 1 \mathrm{H})$, $7.37(\mathrm{t}, J=7.5 \mathrm{~Hz}, 1 \mathrm{H}), 7.26-7.20(\mathrm{~m}, 3 \mathrm{H}), 7.15-7.11(\mathrm{~m}, 1 \mathrm{H}), 7.05(\mathrm{~d}, J=7.2 \mathrm{~Hz}, 1 \mathrm{H}), 6.90(\mathrm{~d}, J=8.0 \mathrm{~Hz}$, $2 \mathrm{H}), 3.57(\mathrm{dd}, J=11.5,6.3 \mathrm{~Hz}, 1 \mathrm{H}), 2.49-2.40(\mathrm{~m}, 2 \mathrm{H}), 2.24(\mathrm{qd}, J=12.5,5.1 \mathrm{~Hz}, 1 \mathrm{H}), 2.04-1.90(\mathrm{~m}$, 1H), 1.52-1.48 (m, 2H); ${ }^{13} \mathrm{C}$ NMR (101 MHz, $\left.\mathrm{CDCl}_{3}\right) \delta 141.4,138.9,137.1,135.4,135.0,133.7,131.6,131.8$ (q, $J=33.0 \mathrm{~Hz}, 1 \mathrm{C}), 131.5(\mathrm{q}, J=33.0 \mathrm{~Hz}, 1 \mathrm{C}), 128.9,125.5,125.4,125.3,125.2,124.7(\mathrm{q}, J=3.6 \mathrm{~Hz}, 1 \mathrm{C})$, 124.0 (q, $J=3.8 \mathrm{~Hz}, 1 \mathrm{C}), 124.1$ (q, $J=272.3 \mathrm{~Hz}, 1 \mathrm{C}), 124.0$ (q, $J=272.3 \mathrm{~Hz}, 1 \mathrm{C}), 123.8,122.7,34.4,33.0$, 26.0, 12.5; ${ }^{19} \mathrm{~F}$ NMR (471 MHz, CDCl $) \delta-63.1$ (s, 3F), -63.1 (s, 3F); HRMS (EI, $\left.m / z\right)$ : calcd for $\mathrm{C}_{28} \mathrm{H}_{22} \mathrm{~F}_{6} \mathrm{Si}$ $\left[\mathrm{M}^{+}\right]$500.1390, found 500.1393; IR (film): 2922, 1647,1391, 1324,1166, 1129,1060, $827 \mathrm{~cm}^{-1}$.

(S)-2-(benzo[d][1,3]dioxol-5-yl)-1,1-bis(4-(trifluoromethyl)phenyl)silolane (4p)

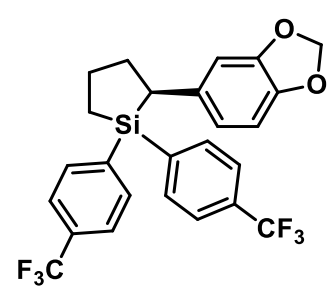

Yield: $81 \%$ (40.0 mg); colorless oil; HPLC gave >99\% e.e., conditions: Daicel chiral column OD-3, hexane: ${ }^{i} \mathrm{PrOH}=300: 1,0.5 \mathrm{~mL} / \mathrm{min}$, wavelength $=220 \mathrm{~nm} ;[\alpha]_{D}^{30}=-36.5^{\circ}\left(c 1.00, \mathrm{CHCl}_{3}\right) ;{ }^{1} \mathrm{H} \mathrm{NMR}(400 \mathrm{MHz}$, $\left.\mathrm{CDCl}_{3}\right) \delta 7.68(\mathrm{~d}, J=8.0 \mathrm{~Hz}, 2 \mathrm{H}), 7.64(\mathrm{~d}, J=8.0 \mathrm{~Hz}, 2 \mathrm{H}), 7.46(\mathrm{~d}, J=8.0 \mathrm{~Hz}, 2 \mathrm{H}), 7.27(\mathrm{~d}, J=7.9 \mathrm{~Hz}$, $2 \mathrm{H}), 6.60-6.58(\mathrm{~m}, 1 \mathrm{H}), 6.41-6.39(\mathrm{~m}, 2 \mathrm{H}), 5.84(\mathrm{dd}, J=7.5,1.3 \mathrm{~Hz}, 2 \mathrm{H}), 2.83(\mathrm{dd}, J=12.5,6.5 \mathrm{~Hz}, 1 \mathrm{H})$, 2.35-2.31 (m, 2H), $1.88(\mathrm{qd}, J=12.5,4.8 \mathrm{~Hz}, 1 \mathrm{H}), 1.77-1.65(\mathrm{~m}, 1 \mathrm{H}), 1.42-1.38(\mathrm{~m}, 2 \mathrm{H}) ;{ }^{13} \mathrm{C}$ NMR $(101$ $\left.\mathrm{MHz}, \mathrm{CDCl}_{3}\right) \delta 147.6,144.7,140.5,138.0,136.2,135.6,135.0,131.8$ (q, $\left.J=32.3 \mathrm{~Hz}, 1 \mathrm{C}\right), 131.5$ (q, $J=32.3$ $\mathrm{Hz}, 1 \mathrm{C}), 124.7$ (q, $J=3.7 \mathrm{~Hz}, 1 \mathrm{C}), 124.2$ (q, $J=3.6 \mathrm{~Hz}, 1 \mathrm{C}), 124.0$ (d, $J=272.2 \mathrm{~Hz}, 1 \mathrm{C}), 124.0$ (d, $J=272.2$ $\mathrm{Hz}, 1 \mathrm{C}), 119.5,108.1,107.6,100.6,36.4,34.6,25.4,11.4 ;{ }^{19} \mathrm{~F}$ NMR $\left(471 \mathrm{MHz}, \mathrm{CDCl}_{3}\right) \delta-63.1$ (s, 3F), 63.1 (s, 3F); HRMS (EI, $m / z$ ): calcd for $\mathrm{C}_{25} \mathrm{H}_{20} \mathrm{O}_{2} \mathrm{~F}_{6} \mathrm{Si}$ [M $\left.\mathrm{M}^{+}\right]$494.1131, found 494.1135; IR (film): 2951, $1611,1391,1324,1166,1127,1060,828 \mathrm{~cm}^{-1}$.

(S)-8-(1,1-bis(4-(trifluoromethyl)phenyl)silolan-2-yl)quinolone (4q)

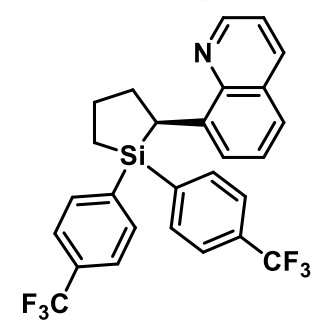

Yield: 65\% (32.5 mg); colorless oil; HPLC gave 97\% e.e., conditions: Daicel chiral column OD-3, hexane: ${ }^{i} \mathrm{PrOH}=200: 1,0.5 \mathrm{~mL} / \mathrm{min}$, wavelength $=220 \mathrm{~nm} ;[\alpha]_{D}^{30}=+18.2^{\circ}\left(c 1.00, \mathrm{CHCl}_{3}\right) ;{ }^{1} \mathrm{H} \mathrm{NMR}(400 \mathrm{MHz}$, 
$\left.\mathrm{CDCl}_{3}\right) \delta 8.52(\mathrm{dd}, J=4.2,1.7 \mathrm{~Hz}, 1 \mathrm{H}), 8.15(\mathrm{~d}, J=7.7 \mathrm{~Hz}, 2 \mathrm{H}), 7.95(\mathrm{dd}, J=8.2,1.7 \mathrm{~Hz}, 1 \mathrm{H}), 7.70(\mathrm{~d}, J=$ $7.8 \mathrm{~Hz}, 2 \mathrm{H}), 7.46-7.43(\mathrm{~m}, 2 \mathrm{H}), 7.37-7.33(\mathrm{~m}, 1 \mathrm{H}), 7.22(\mathrm{dd}, J=8.2,4.2 \mathrm{~Hz}, 1 \mathrm{H}), 7.07(\mathrm{~d}, J=7.9 \mathrm{~Hz}, 2 \mathrm{H})$, $6.91(\mathrm{~d}, J=7.8 \mathrm{~Hz}, 2 \mathrm{H}), 3.98(\mathrm{dd}, J=9.9,7.3 \mathrm{~Hz}, 1 \mathrm{H}), 2.50-2.43(\mathrm{~m}, 1 \mathrm{H}), 2.39-2.21(\mathrm{~m}, 2 \mathrm{H}), 1.93-1.82$ (m, 1H), $1.38-1.22(\mathrm{~m}, 2 \mathrm{H}) ;{ }^{13} \mathrm{C}$ NMR $\left(101 \mathrm{MHz}, \mathrm{CDCl}_{3}\right) \delta$ 147.6, 145.9, 142.4, 141.8, 140.2, 136.1, 136.0, 134.5, 130.8 (q, $J=32.2 \mathrm{~Hz}, 1 \mathrm{C}), 130.5(\mathrm{q}, J=32.2 \mathrm{~Hz}, 1 \mathrm{C}), 127.8,126.4,125.0,124.4$ (q, $J=272.3 \mathrm{~Hz}$, 1C), 124.2, 124.1 (q, $J=3.3 \mathrm{~Hz}, 1 \mathrm{C}$ ), 124.0 (q, $J=272.3 \mathrm{~Hz}, 1 \mathrm{C}), 123.2$ (q, $J=3.8 \mathrm{~Hz}, 1 \mathrm{C}$ ), 120.8, 32.7, 29.6, 25.8, 14.2; ${ }^{19} \mathrm{~F}$ NMR (471 MHz, $\left.\mathrm{CDCl}_{3}\right) \delta-62.8(\mathrm{~s}, 3 \mathrm{~F}),-63.2(\mathrm{~s}, 3 \mathrm{~F})$; HRMS (EI, $\mathrm{m} / \mathrm{z}$ ): calcd for $\mathrm{C}_{27} \mathrm{H}_{21} \mathrm{NF}_{6} \mathrm{Si}\left[\mathrm{M}^{+}\right]$501.1342, found 501.1333; IR (film): 2937, 1614,1391, 1323,1164, 1124,1060, $826 \mathrm{~cm}^{-1}$.

(S)-2-(thiophen-2-yl)-1,1-bis(4-(trifluoromethyl)phenyl)silolane (4r)

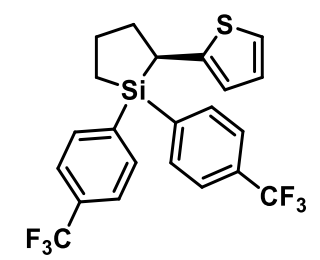

Yield: $81 \%$ (36.9 mg); colorless oil; HPLC gave 99\% e.e., conditions: Daicel chiral column OD-3, hexane: ${ }^{i} \mathrm{PrOH}=100: 0,0.5 \mathrm{~mL} / \mathrm{min}$, wavelength $=220 \mathrm{~nm} ;[\alpha]_{D}^{30}=-31.5^{\circ}\left(c 1.00, \mathrm{CHCl}_{3}\right) ;{ }^{1} \mathrm{H} \mathrm{NMR}(400 \mathrm{MHz}$, $\left.\mathrm{CDCl}_{3}\right) \delta 7.75(\mathrm{~d}, J=7.9 \mathrm{~Hz}, 2 \mathrm{H}), 7.66(\mathrm{~d}, J=8.0 \mathrm{~Hz}, 2 \mathrm{H}), 7.46(\mathrm{~d}, J=8.0 \mathrm{~Hz}, 2 \mathrm{H}), 7.30(\mathrm{~d}, J=8.0 \mathrm{~Hz}$, $2 \mathrm{H}), 6.92(\mathrm{~d}, J=5.1 \mathrm{~Hz}, 1 \mathrm{H}), 6.80-6.78(\mathrm{~m}, 1 \mathrm{H}), 6.52(\mathrm{~d}, J=3.0 \mathrm{~Hz}, 1 \mathrm{H}), 3.14(\mathrm{dd}, J=12.1,6.7 \mathrm{~Hz}, 1 \mathrm{H})$, $2.51-2.45(\mathrm{~m}, 1 \mathrm{H}), 2.36-2.28(\mathrm{~m}, 1 \mathrm{H}), 1.97(\mathrm{qd}, J=12.4,4.9 \mathrm{~Hz}, 1 \mathrm{H}), 1.78-1.66(\mathrm{~m}, 1 \mathrm{H}), 1.49-1.35$ $(\mathrm{m}, 2 \mathrm{H}) ;{ }^{13} \mathrm{C} \mathrm{NMR}\left(101 \mathrm{MHz}, \mathrm{CDCl}_{3}\right) \delta 146.1,139.7,138.0,135.5,135.1,131.8$ (q, $\left.J=32.6 \mathrm{~Hz}, 1 \mathrm{C}\right), 131.5$ (q, $J=32.6 \mathrm{~Hz}, 1 \mathrm{C}), 126.8,124.8(\mathrm{q}, J=3.7 \mathrm{~Hz}, 1 \mathrm{C}), 124.2(\mathrm{~d}, J=3.8 \mathrm{~Hz}, 1 \mathrm{C}), 124.0$ (q, $J=272 \mathrm{~Hz}, 1 \mathrm{C})$, 124.0 (q, $J=272 \mathrm{~Hz}, 1 \mathrm{C}), 122.0,121.4,36.1,31.5,25.3,11.4 ;{ }^{19} \mathrm{~F} \mathrm{NMR}\left(471 \mathrm{MHz}, \mathrm{CDCl}_{3}\right) \delta-63.1$ (s, 3F), -63.1 (s, 3F); HRMS (EI, $m / z$ ): calcd for $\mathrm{C}_{22} \mathrm{H}_{18} \mathrm{SF}_{6} \mathrm{Si}\left[\mathrm{M}^{+}\right]$456.0797, found 456.0801; IR (film): 2937, $1608,1391,1323,1166,1103,1060,827 \mathrm{~cm}^{-1}$.

(S)-2-(furan-2-yl)-1,1-bis(4-(trifluoromethyl)phenyl)silolane (4s)

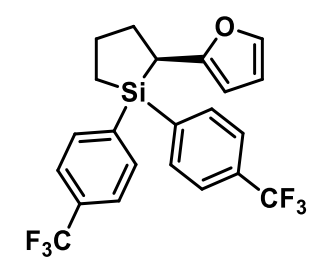

Yield: 59\% (25.9 mg); colorless oil; HPLC gave 97\% e.e., conditions: Daicel chiral column OD-3, hexane: ${ }^{i} \operatorname{PrOH}=100: 0,0.5 \mathrm{~mL} / \mathrm{min}$, wavelength $=220 \mathrm{~nm} ;[\alpha]_{D}^{30}=-38.5^{\circ}\left(\mathrm{c} 1.00, \mathrm{CHCl}_{3}\right) ;{ }^{1} \mathrm{H} \mathrm{NMR}(400 \mathrm{MHz}$, $\left.\mathrm{CDCl}_{3}\right) \delta 7.82(\mathrm{~d}, J=7.9 \mathrm{~Hz}, 2 \mathrm{H}), 7.67(\mathrm{~d}, J=7.9 \mathrm{~Hz}, 2 \mathrm{H}), 7.45(\mathrm{~d}, J=8.0 \mathrm{~Hz}, 2 \mathrm{H}), 7.28(\mathrm{~d}, J=8.0 \mathrm{~Hz}$, $2 \mathrm{H}), 7.14(\mathrm{~d}, J=1.1 \mathrm{~Hz}, 1 \mathrm{H}), 6.18-6.17(\mathrm{~m}, 1 \mathrm{H}), 5.79(\mathrm{~d}, J=2.8 \mathrm{~Hz}, 1 \mathrm{H}), 2.89(\mathrm{dd}, J=11.6,6.9 \mathrm{~Hz}, 1 \mathrm{H})$, $2.41-2.36(\mathrm{~m}, 1 \mathrm{H}), 2.33-2.25(\mathrm{~m}, 1 \mathrm{H}), 1.93(\mathrm{qd}, J=12.1,4.8 \mathrm{~Hz}, 1 \mathrm{H}), 1.76-1.64(\mathrm{~m}, 1 \mathrm{H}), 1.49-1.43$ $(\mathrm{m}, 1 \mathrm{H}), 1.35-1.29(\mathrm{~m}, 1 \mathrm{H}) ;{ }^{13} \mathrm{C}$ NMR $\left(101 \mathrm{MHz}, \mathrm{CDCl}_{3}\right) \delta 156.5,140.1,139.9,138.4,135.2,135.1,131.8$ (q, $J=32.3 \mathrm{~Hz}, 1 \mathrm{C}), 131.6$ (q, $J=32.3 \mathrm{~Hz}, 1 \mathrm{C}), 124.8$ (q, $J=3.7 \mathrm{~Hz}, 1 \mathrm{C}), 124.2$ (q, $J=3.7 \mathrm{~Hz}, 1 \mathrm{C}), 124.0$ (q, $J=272 \mathrm{~Hz}, 1 \mathrm{C}), 124.0$ (q, $J=272 \mathrm{~Hz}, 1 \mathrm{C}), 110.3,102.9,32.5,29.2,25.4,11.5 ;{ }^{19} \mathrm{~F}$ NMR $(471 \mathrm{MHz}$, $\mathrm{CDCl}_{3}$ ) $\delta$-63.2 (s, 3F), -63.3 (s, 3F); HRMS (EI, $\mathrm{m} / \mathrm{z}$ ): calcd for $\mathrm{C}_{22} \mathrm{H}_{18} \mathrm{OF}_{6} \mathrm{Si}\left[\mathrm{M}^{+}\right]$440.1026, found 440.1029; IR (film): 2943, 1510, 1392, 1324,1166, 1124,1060, $827 \mathrm{~cm}^{-1}$.

(S)-2-(benzo[b]thiophen-2-yl)-1,1-bis(4-(trifluoromethyl)phenyl)silolane (4t) 


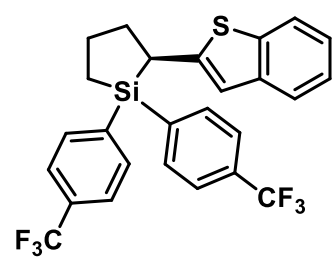

Yield: 57\% (28.7 mg); colorless oil; HPLC gave 99\% e.e., conditions: Daicel chiral column AD-H, hexane: ${ }^{i} \mathrm{PrOH}=500: 1,0.5 \mathrm{~mL} / \mathrm{min}$, wavelength $=220 \mathrm{~nm} ;[\alpha]_{D}^{30}=-43.2^{\circ}\left(c 1.00, \mathrm{CHCl}_{3}\right) ;{ }^{1} \mathrm{H} \mathrm{NMR}(400 \mathrm{MHz}$, $\left.\mathrm{CDCl}_{3}\right) \delta 7.79(\mathrm{~d}, J=7.8 \mathrm{~Hz}, 2 \mathrm{H}), 7.69(\mathrm{~d}, J=7.9 \mathrm{~Hz}, 2 \mathrm{H}), 7.62(\mathrm{~d}, J=7.9 \mathrm{~Hz}, 1 \mathrm{H}), 7.51(\mathrm{~d}, J=7.8 \mathrm{~Hz}$, 1H), $7.41-7.35(\mathrm{~m}, 4 \mathrm{H}), 7.24(\mathrm{~d}, J=7.5 \mathrm{~Hz}, 1 \mathrm{H}), 7.19-7.15(\mathrm{~m}, 1 \mathrm{H}), 6.74(\mathrm{~s}, 1 \mathrm{H}), 3.15$ (dd, $J=12.3,6.4$ $\mathrm{Hz}, 1 \mathrm{H}), 2.56-2.50(\mathrm{~m}, 1 \mathrm{H}), 2.39-2.33(\mathrm{~m}, 1 \mathrm{H}), 2.07(\mathrm{qd}, J=12.5,4.9 \mathrm{~Hz}, 1 \mathrm{H}), 1.81-1.69(\mathrm{~m}, 1 \mathrm{H}), 1.51$ $-1.37(\mathrm{~m}, 2 \mathrm{H}) ;{ }^{13} \mathrm{C}$ NMR $\left(101 \mathrm{MHz}, \mathrm{CDCl}_{3}\right) \delta 147.1,140.3,139.5,138.2,137.7,135.5,135.2,132.0(\mathrm{q}, J$ $=32.6 \mathrm{~Hz}, 1 \mathrm{C}), 131.9$ (q, $J=32.6 \mathrm{~Hz}, 1 \mathrm{C}), 124.9$ (q, $J=3.7 \mathrm{~Hz}, 1 \mathrm{C}), 124.3(\mathrm{q}, J=3.8 \mathrm{~Hz}, 1 \mathrm{C}), 124.1,124.0$ (q, $J=272 \mathrm{~Hz}, 1 \mathrm{C}), 124.0$ (q, $J=272 \mathrm{~Hz}, 1 \mathrm{C}), 123.1,122.2,121.8,118.2,35.6,32.8,25.3,11.5 ;{ }^{19} \mathrm{~F}$ NMR (471 MHz, $\mathrm{CDCl}_{3}$ ) $\delta-63.1$ (s, 3F), -63.2 (s, 3F); HRMS (EI, $m / z$ ): calcd for $\mathrm{C}_{26} \mathrm{H}_{20} \mathrm{SF}_{6} \mathrm{Si}\left[\mathrm{M}^{+}\right]$506.0954, found 506.0962; IR (film): 2943, 1611,1391, 1323,1166, 1103,1060, $826 \mathrm{~cm}^{-1}$.

\section{(S,E)-2-styryl-1,1-bis(4-(trifluoromethyl)phenyl)silolane (4u)}

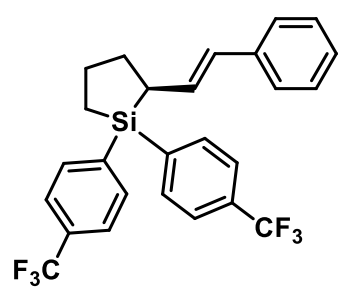

Yield: $52 \%$ (24.9 mg); colorless oil; HPLC gave 84\% e.e., conditions: Daicel chiral column OD-3, hexane: ${ }^{i} \mathrm{PrOH}=100: 0,0.5 \mathrm{~mL} / \mathrm{min}$, wavelength $=220 \mathrm{~nm} ;[\alpha]_{D}^{30}=-115.5^{\circ}\left(c 1.00, \mathrm{CHCl}_{3}\right) ;{ }^{1} \mathrm{H} \mathrm{NMR}(400 \mathrm{MHz}$, $\left.\mathrm{CDCl}_{3}\right) \delta 7.71(\mathrm{~d}, J=8.0 \mathrm{~Hz}, 2 \mathrm{H}), 7.65-7.57(\mathrm{~m}, 6 \mathrm{H}), 7.25-7.21(\mathrm{~m}, 2 \mathrm{H}), 7.16-7.12(\mathrm{~m}, 3 \mathrm{H}), 6.36(\mathrm{~d}, J$ $=15.7 \mathrm{~Hz}, 1 \mathrm{H}), 6.07(\mathrm{dd}, J=15.7,9.3 \mathrm{~Hz}, 1 \mathrm{H}), 2.54-2.48(\mathrm{~m}, 1 \mathrm{H}), 2.26-2.20(\mathrm{~m}, 2 \mathrm{H}), 1.78-1.63(\mathrm{~m}$, 2H), $1.44-1.33(\mathrm{~m}, 2 \mathrm{H}) ;{ }^{13} \mathrm{C}$ NMR $\left(101 \mathrm{MHz}, \mathrm{CDCl}_{3}\right) \delta 140.1,138.4,137.9,135.7,134.9,132.3,131.8$ (q, $J=32.3,1 \mathrm{C}), 131.6$ (q, $J=32.3,1 \mathrm{C}), 128.5,126.9,126.6,125.6,124.8$ (q, $J=3.8 \mathrm{~Hz}, 1 \mathrm{C}), 124.5$ (q, $J=3.8$ $\mathrm{Hz}, 1 \mathrm{C}), 124.0$ (q, $J=272 \mathrm{~Hz}, 1 \mathrm{C}), 124.0$ (q, $J=272 \mathrm{~Hz}, 1 \mathrm{C}), 34.9,34.4,25.4,11.2 ;{ }^{19} \mathrm{~F}$ NMR $(471 \mathrm{MHz}$, $\left.\mathrm{CDCl}_{3}\right) \delta-63.1$ (s, 3F), -63.1 (s, 3F); HRMS (EI, $\mathrm{m} / \mathrm{z}$ ): calcd for $\mathrm{C}_{26} \mathrm{H}_{22} \mathrm{~F}_{6} \mathrm{Si}\left[\mathrm{M}^{+}\right]$476.1390, found 476.1390; IR (film): 2934, 1608,1392, 1324, 1166, 1126, 1060, $827 \mathrm{~cm}^{-1}$.

\section{(S,E)-2-(1-phenylprop-1-en-2-yl)-1,1-bis(4-(trifluoromethyl)phenyl)silolane (4v)}

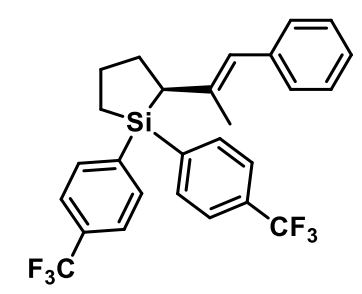

Yield: 16\% (7.9 mg); colorless oil; HPLC gave 98\% e.e., conditions: Daicel chiral column AD-3, hexane: ${ }^{i} \mathrm{PrOH}=100: 0,0.2 \mathrm{~mL} / \mathrm{min}$, wavelength $=210 \mathrm{~nm} ;[\alpha]_{D}^{30}=+5.8^{\circ}\left(c 1.00, \mathrm{CHCl}_{3}\right) ;{ }^{1} \mathrm{H} \mathrm{NMR}(400 \mathrm{MHz}$, $\left.\mathrm{CDCl}_{3}\right) \delta 7.77(\mathrm{~d}, J=7.8 \mathrm{~Hz}, 2 \mathrm{H}), 7.67(\mathrm{~d}, J=7.9 \mathrm{~Hz}, 2 \mathrm{H}), 7.62(\mathrm{~d}, J=8.0 \mathrm{~Hz}, 2 \mathrm{H}), 7.57(\mathrm{~d}, J=8.0 \mathrm{~Hz}$, $2 \mathrm{H}), 7.27-7.23(\mathrm{~m}, 2 \mathrm{H}), 7.15-7.12(\mathrm{~m}, 1 \mathrm{H}), 6.96(\mathrm{~d}, J=7.5 \mathrm{~Hz}, 2 \mathrm{H}), 6.22(\mathrm{~s}, 1 \mathrm{H}), 2.44(\mathrm{dd}, J=11.4,6.3$ $\mathrm{Hz}, 1 \mathrm{H}), 2.33-2.20(\mathrm{~m}, 2 \mathrm{H}), 2.04-1.93(\mathrm{~m}, 1 \mathrm{H}), 1.74-1.65(\mathrm{~m}, 1 \mathrm{H}), 1.62(\mathrm{~s}, 3 \mathrm{H}), 1.40-1.24(\mathrm{~m}, 2 \mathrm{H})$; 
${ }^{13} \mathrm{C}$ NMR (101 MHz, $\left.\mathrm{CDCl}_{3}\right) \delta 140.4,139.3,138.8,138.7,135.5,135.2,131.8$ (q, $\left.J=32.5 \mathrm{~Hz}, 1 \mathrm{C}\right), 131.5$ (q, $J=32.5 \mathrm{~Hz}, 1 \mathrm{C}), 128.7,128.0,125.6,124.8(\mathrm{q}, J=3.7 \mathrm{~Hz}, 1 \mathrm{C}), 124.4(\mathrm{~d}, J=3.8 \mathrm{~Hz}, 1 \mathrm{C}), 124.0$ (q, $J=$ $272 \mathrm{~Hz}, 1 \mathrm{C}), 124.1(\mathrm{q}, J=272 \mathrm{~Hz}, 1 \mathrm{C}), 121.9,40.4,32.5,25.4,20.3,12.6 ;{ }^{19} \mathrm{~F} \mathrm{NMR}\left(471 \mathrm{MHz}, \mathrm{CDCl}_{3}\right) \delta-$ 63.0 (s, 3F), -63.1 (s, 3F); HRMS (EI, $m / z$ ): calcd for $\mathrm{C}_{27} \mathrm{H}_{24} \mathrm{~F}_{6} \mathrm{Si}\left[\mathrm{M}^{+}\right]$490.1546, found 490.1549; IR (film): 2928, 1324, 1166, 1126, 1103, 1060, $827 \mathrm{~cm}^{-1}$.

1-methyl-2-phenyl-1-(4-(trifluoromethyl)phenyl)silolane (4w)

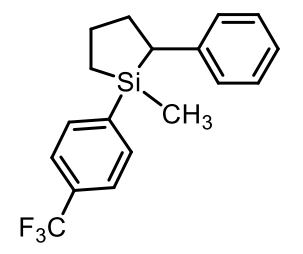

Yield: $24 \%$ (7.7 mg); colorless oil; HPLC gave major isomer $74 \%$ e.e., minor isomer $97 \%$ e.e., conditions: Daicel chiral column OD-3 $*$ OD-H, hexane: ${ }^{i} \mathrm{PrOH}=100: 0,0.5 \mathrm{~mL} / \mathrm{min}$, wavelength $=210 \mathrm{~nm} ;{ }^{1} \mathrm{H}$ NMR $\left(400 \mathrm{MHz}, \mathrm{CDCl}_{3}\right) \delta 7.68(\mathrm{~d}, J=7.9 \mathrm{~Hz}, 1.82 \mathrm{H}), 7.62(\mathrm{~d}, J=8.1 \mathrm{~Hz}, 2.11 \mathrm{H}), 7.37(\mathrm{~d}, J=7.8 \mathrm{~Hz}, 2.69 \mathrm{H})$, 7.26-7.18 (m, 6.01H), 7.10-7.02 (m, 5.47H), 6.98-6.93 (m, 1.37H), 6.87-6.85 (m, 2.76H), 2.57 (dd, $J=12.1$, $6.9 \mathrm{~Hz}, 1.00 \mathrm{H}), 2.45(\mathrm{dd}, J=12.6,6.2 \mathrm{~Hz}, 1.46 \mathrm{H}), 2.30-2.18(\mathrm{~m}, 4.95 \mathrm{H}), 1.98-1.81(\mathrm{~m}, 2.66 \mathrm{H}), 1.71-1.57(\mathrm{~m}$, $2.56 \mathrm{H}), 1.25-1.06(\mathrm{~m}, 4.55 \mathrm{H}), 0.93-0.84(\mathrm{~m}, 1.18 \mathrm{H}), 0.50(\mathrm{~s}, 4.33 \mathrm{H}), 0.09(\mathrm{~s}, 2.95 \mathrm{H}) ;{ }^{13} \mathrm{C} \mathrm{NMR}\left(101 \mathrm{MHz}, \mathrm{CDCl}_{3}\right)$ $\delta 143.8,143.2,134.5,134.1,131.2(\mathrm{~d}, J=31.9 \mathrm{~Hz}), 130.4(\mathrm{q}, J=32.1 \mathrm{~Hz}), 128.9,128.3,128.2,128.0,126.6$, 126.5, $124.5(\mathrm{q}, J=3.6 \mathrm{~Hz}), 124.3,124.2(\mathrm{q}, J=272 \mathrm{~Hz}), 124.1,124.1(\mathrm{q}, J=272 \mathrm{~Hz}), 123.8(\mathrm{q}, J=3.3 \mathrm{~Hz})$, 37.8, 36.5, 33.6, 33.5, 26.0, 25.1, 12.7, 11.2, -3.2, -5.3; HRMS (EI, $m / z)$ : calcd for $\mathrm{C}_{12} \mathrm{H}_{19} \mathrm{~F}_{3} \mathrm{Si}\left[\mathrm{M}^{+}\right]$320.1208, found 32.1203 .

(S)-2-([1,1'-biphenyl]-4-yl)-2-methyl-1,1-bis(4-(trifluoromethyl)phenyl)silolane (6a)

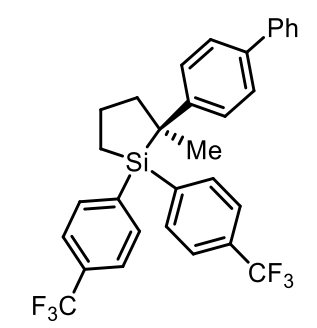

Yield: 77\% (41.5 mg); white soild; HPLC gave >99\% e.e., conditions: Daicel chiral column AD-H, hexane: ${ }^{i} \mathrm{PrOH}=100: 0,0.5 \mathrm{~mL} / \mathrm{min}$, wavelength $=220 \mathrm{~nm} ;[\alpha]_{D}^{30}=-20.1^{\circ}\left(c 1.00, \mathrm{CHCl}_{3}\right) ;{ }^{1} \mathrm{H} \mathrm{NMR}(400 \mathrm{MHz}$, $\left.\mathrm{CDCl}_{3}\right) \delta 7.76(\mathrm{~d}, J=7.8 \mathrm{~Hz}, 2 \mathrm{H}), 7.68(\mathrm{~d}, J=7.8 \mathrm{~Hz}, 2 \mathrm{H}), 7.59-7.57(\mathrm{~m}, 2 \mathrm{H}), 7.48(\mathrm{~d}, J=8.3 \mathrm{~Hz}, 2 \mathrm{H}), 7.43$ (t, $J=7.6 \mathrm{~Hz}, 2 \mathrm{H}), 7.36-7.31(\mathrm{~m}, 3 \mathrm{H}), 7.23(\mathrm{~d}, J=8.5 \mathrm{~Hz}, 2 \mathrm{H}), 7.10(\mathrm{~d}, J=7.9 \mathrm{~Hz}, 2 \mathrm{H}), 2.43-2.35(\mathrm{~m}, 1 \mathrm{H})$, 2.31-2.23 (m, 1H), 2.08-1.97 (m, 2H), 1.65-1.59 (m, 1H), $1.32(\mathrm{~s}, 3 \mathrm{H}), 1.27-1.19(\mathrm{~m}, 1 \mathrm{H}) ;{ }^{13} \mathrm{C} \mathrm{NMR}(100$ $\left.\mathrm{MHz}, \mathrm{CDCl}_{3}\right) \delta 147.0,140.7,139.6,138.9,137.8,135.7,135.5,131.8(\mathrm{q}, J=32.4 \mathrm{~Hz}), 131.1(\mathrm{q}, J=32.2$ $\mathrm{Hz}), 128.8,127.1,127.1,126.9,126.8,124.6(\mathrm{q}, J=3.7 \mathrm{~Hz}), 124.1(\mathrm{q}, J=272.6 \mathrm{~Hz}), 124.0(\mathrm{q}, J=272.3$ $\mathrm{Hz}), 124.0$ (q, $J=3.7 \mathrm{~Hz}), 40.6,34.9,25.9,22.2,10.1 ;{ }^{19} \mathrm{~F} \mathrm{NMR}\left(471 \mathrm{MHz}, \mathrm{CDCl}_{3}\right) \delta-63.0$ (s, 3F), -63.1 (s, 3F); HRMS (EI, $m / z)$ : calcd for $\mathrm{C}_{31} \mathrm{H}_{26} \mathrm{~F}_{6} \mathrm{Si}\left[\mathrm{M}^{+}\right]$540.1708, found 540.1712; IR (film): 2957, 1392, 1323, $1166,1126,1060,828,706 \mathrm{~cm}^{-1}$.

(S)-2-(3-methoxyphenyl)-2-methyl-1,1-bis(4-(trifluoromethyl)phenyl)silolane (6b) 


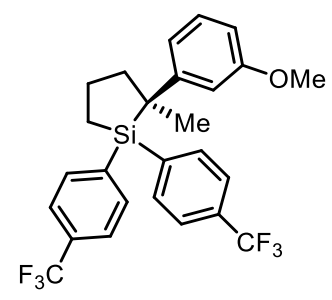

Yield: 78\% (38 mg); colorless oil; HPLC gave 91\% e.e., conditions: Daicel chiral column OD-3, hexane: ${ }^{i} \mathrm{PrOH}=100: 0,0.8 \mathrm{~mL} / \mathrm{min}$, wavelength $=220 \mathrm{~nm} ;[\alpha]_{D}^{30}=+17.2^{\circ}\left(c 1.00, \mathrm{CHCl}_{3}\right) ;{ }^{1} \mathrm{H} \mathrm{NMR}(400 \mathrm{MHz}$, $\left.\mathrm{CDCl}_{3}\right) \delta 7.74(\mathrm{~d}, J=7.9 \mathrm{~Hz}, 2 \mathrm{H}), 7.66(\mathrm{~d}, J=7.9 \mathrm{~Hz}, 2 \mathrm{H}), 7.37(\mathrm{~d}, J=7.9 \mathrm{~Hz}, 2 \mathrm{H}), 7.14(\mathrm{t}, J=7.9 \mathrm{~Hz}, 1 \mathrm{H})$, $7.09(\mathrm{~d}, J=7.8 \mathrm{~Hz}, 2 \mathrm{H}), 6.77-6.75(\mathrm{~m}, 1 \mathrm{H}), 6.70-6.66(\mathrm{~m}, 2 \mathrm{H}), 3.68(\mathrm{~s}, 3 \mathrm{H}), 2.37-2.22(\mathrm{~m}, 2 \mathrm{H}), 2.05-1.92$ $(\mathrm{m}, 2 \mathrm{H}), 1.62-1.56(\mathrm{~m}, 1 \mathrm{H}), 1.30-1.21(\mathrm{~m}, 1 \mathrm{H}), 1.27(\mathrm{~s}, 3 \mathrm{H}) ;{ }^{13} \mathrm{C} \mathrm{NMR}\left(100 \mathrm{MHz}, \mathrm{CDCl}_{3}\right) \delta 159.6,149.6$, 139.7, 138.9, 135.7, 135.4, 131.7 (q, $J=32.4 \mathrm{~Hz}), 131.0$ (q, $J=32.3 \mathrm{~Hz}), 129.2,124.6$ (q, $J=3.7 \mathrm{~Hz}), 124.1$ $(\mathrm{q}, J=272.4 \mathrm{~Hz}), 124.0(\mathrm{q}, J=272.3 \mathrm{~Hz}), 124.0(\mathrm{q}, J=3.7 \mathrm{~Hz}), 119.4,113.1,109.8,55.0,40.5,35.2,25.9$, 22.1, 10.0; ${ }^{19} \mathrm{~F}$ NMR (471 MHz, $\left.\mathrm{CDCl}_{3}\right) \delta-63.0$ (s, 3F), -63.1 (s, 3F); HRMS (EI, $\left.m / z\right)$ : calcd for $\mathrm{C}_{26} \mathrm{H}_{24} \mathrm{~F}_{6} \mathrm{OSi}$ $\left[\mathrm{M}^{+}\right]$494.1500, found 494.1493; IR (film): 2928, 1606, 1394, 1324, 1166, 1126, 1060, 1018, 829, $706 \mathrm{~cm}^{-1}$.

(R)-1',1'-bis(4-(trifluoromethyl)phenyl)spiro[bicyclo[4.2.0]octane-7,2'-silolane]-1(6),2,4-triene (6c)

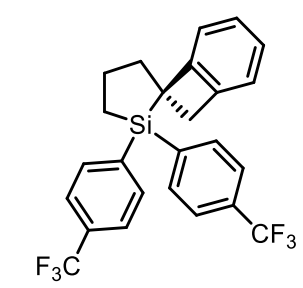

Yield: $91 \%$ (42.1 mg); white solid; HPLC gave 36\% e.e., conditions: Daicel chiral column OD-3, hexane: ${ }^{i}$ PrOH: Trifluoroacetic acid $=95: 5: 0.1,0.7 \mathrm{~mL} / \mathrm{min}$, wavelength $=220 \mathrm{~nm} ;[\alpha]_{D}^{30}=-43.6^{\circ}\left(c 1.00, \mathrm{CHCl}_{3}\right)$; ${ }^{1} \mathrm{H}$ NMR $\left(400 \mathrm{MHz}, \mathrm{CDCl}_{3}\right) \delta 7.59(\mathrm{~d}, J=7.8 \mathrm{~Hz}, 2 \mathrm{H}), 7.55-7.46(\mathrm{~m}, 6 \mathrm{H}), 7.12(\mathrm{t}, J=7.5 \mathrm{~Hz}, 1 \mathrm{H}), 7.05-$ $7.00(\mathrm{~m}, 2 \mathrm{H}), 6.53$ (d, $J=7.2 \mathrm{~Hz}, 1 \mathrm{H}), 3.23$ (d, $J=14.0 \mathrm{~Hz}, 1 \mathrm{H}), 2.85$ (d, $J=14.0 \mathrm{~Hz}, 1 \mathrm{H}), 2.25-2.09$ (m, $3 \mathrm{H}), 1.98-1.87$ (m, 1H), 1.53-1.45 (m, 2H); ${ }^{13} \mathrm{C} \mathrm{NMR}\left(100 \mathrm{MHz}, \mathrm{CDCl}_{3}\right) \delta 151.2,142.3,139.3,139.0,135.4$, 135.1, 131.7 (q, $J=32.4 \mathrm{~Hz}), 131.6(\mathrm{q}, J=32.4 \mathrm{~Hz}), 126.8,126.2,124.7$ (q, $J=3.7 \mathrm{~Hz}), 124.3$ (q, $J=3.7$ $\mathrm{Hz}), 124.1(\mathrm{q}, J=272.4 \mathrm{~Hz}), 124.0(\mathrm{q}, J=272.2 \mathrm{~Hz}), 122.8,121.2,44.1,41.8,40.4,24.2,10.7 ;{ }^{19} \mathrm{~F} \mathrm{NMR}$ (471 MHz, $\mathrm{CDCl}_{3}$ ) $\delta$-63.0 (s, 3F), -63.1 (s, 3F); HRMS (EI, $m / z$ ): calcd for $\mathrm{C}_{25} \mathrm{H}_{20} \mathrm{~F}_{6} \mathrm{Si}\left[\mathrm{M}^{+}\right]$462.1233, found 462.1235; IR (film): 2925, 1460, 1397, 1324, 1167, 1127, 1060, 1018, 827, $709 \mathrm{~cm}^{-1}$.

(S)-5-bromo-1',1'-bis(4-(trifluoromethyl)phenyl)-2,3-dihydrospiro[indene-1,2'-silolane] (6d)

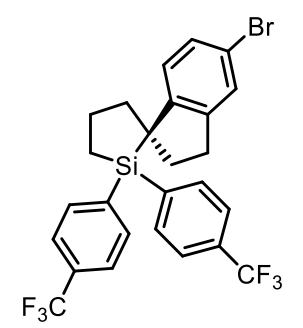

Yield: 41\% (22.7 mg); white solid; HPLC gave 97\% e.e., conditions: Daicel chiral column OD-3+OD-H, hexane: ${ }^{i} \mathrm{PrOH}=100: 0,0.25 \mathrm{~mL} / \mathrm{min}$, wavelength $=210 \mathrm{~nm} ;[\alpha]_{D}^{30}=-133.1^{\circ}\left(c 1.00, \mathrm{CHCl}_{3}\right) ;{ }^{1} \mathrm{H}$ NMR $(400$ $\left.\mathrm{MHz} \mathrm{CDCl}_{3}\right) \delta 7.60(\mathrm{q}, J=8.1 \mathrm{~Hz}, 4 \mathrm{H}), 7.51(\mathrm{~d}, J=7.8 \mathrm{~Hz}, 2 \mathrm{H}), 7.22(\mathrm{~d}, J=7.7 \mathrm{~Hz}, 2 \mathrm{H}), 7.16(\mathrm{dd}, J=7.9$, $1.8 \mathrm{~Hz}, 1 \mathrm{H}), 7.03(\mathrm{~d}, J=7.9 \mathrm{~Hz}, 1 \mathrm{H}), 6.53(\mathrm{~d}, J=1.7 \mathrm{~Hz}, 1 \mathrm{H}), 2.69(\mathrm{dd}, J=15.5,8.6 \mathrm{~Hz}, 1 \mathrm{H}), 2.42-2.36(\mathrm{~m}$, $1 \mathrm{H}), 2.33-2.21(\mathrm{~m}, 2 \mathrm{H}), 2.18-2.11(\mathrm{~m}, 1 \mathrm{H}), 2.01-1.93(\mathrm{~m}, 1 \mathrm{H}), 1.90-1.80(\mathrm{~m}, 2 \mathrm{H}), 1.58-1.54(\mathrm{~m}, 2 \mathrm{H}) ;{ }^{13} \mathrm{C}$ 
$\operatorname{NMR}\left(100 \mathrm{MHz}, \mathrm{CDCl}_{3}\right) \delta 151.5,141.7,139.2,137.7,135.5,135.1,131.8(\mathrm{q}, J=32.4 \mathrm{~Hz}), 131.6(\mathrm{q}, J=$ $32.4 \mathrm{~Hz}), 128.1,126.8,125.7,124.5$ (q, $J=3.6 \mathrm{~Hz}), 124.2(\mathrm{q}, J=3.6 \mathrm{~Hz}), 124.0(\mathrm{q}, J=272.7 \mathrm{~Hz}), 123.9(\mathrm{q}$, $J=272.2 \mathrm{~Hz}), 119.7,44.9,43.3,38.1,31.0,23.9,9.8 ;{ }^{19} \mathrm{~F} \mathrm{NMR}\left(471 \mathrm{MHz}, \mathrm{CDCl}_{3}\right) \delta-63.1(\mathrm{~s}, 3 \mathrm{~F}),-63.2(\mathrm{~s}$, 3F); HRMS (EI, $m / z$ ): calcd for $\mathrm{C}_{26} \mathrm{H}_{21} \mathrm{BrF}_{6} \mathrm{Si}\left[\mathrm{M}^{+}\right]$554.0500, found 554.0509; IR (film): 2937, 1392, 1324 , $1167,1127,1060,1018,829,709 \mathrm{~cm}^{-1}$.

(S)-1',1'-bis(4-(trifluoromethyl)phenyl)-3,4-dihydro-2H-spiro[naphthalene-1,2'-silolane] (6e)

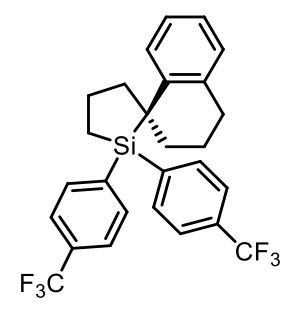

Yield: 74\% (36.3 mg); colorless oil; HPLC gave 99\% e.e., conditions: Daicel chiral column OD-3*OD-H, hexane: ${ }^{i} \mathrm{PrOH}=100: 0,0.3 \mathrm{~mL} / \mathrm{min}$, wavelength $=210 \mathrm{~nm} ;[\alpha]_{D}^{30}=+32.6^{\circ}\left(c 1.00, \mathrm{CHCl}_{3}\right) ;{ }^{1} \mathrm{H} \mathrm{NMR}(400$ $\left.\mathrm{MHz} \mathrm{CDCl}_{3}\right) \delta 7.55(\mathrm{~s}, 4 \mathrm{H}), 7.39(\mathrm{~d}, J=7.9 \mathrm{~Hz}, 2 \mathrm{H}), 7.12(\mathrm{~d}, J=7.8 \mathrm{~Hz}, 2 \mathrm{H}), 7.04(\mathrm{~d}, J=7.2 \mathrm{~Hz}, 1 \mathrm{H}), 6.96$ (td, $J=7.5,1.1 \mathrm{~Hz}, 1 \mathrm{H}), 6.77(\mathrm{t}, J=7.2 \mathrm{~Hz}, 1 \mathrm{H}), 6.67(\mathrm{~d}, J=7.8 \mathrm{~Hz}, 1 \mathrm{H}), 2.81-2.66(\mathrm{~m}, 2 \mathrm{H}), 2.33-2.24(\mathrm{~m}$, 2H), 2.15-2.00 (m, 3H), 1.62-1.46 (m, 4H), 1.40-1.30 (m, 1H); $\left.{ }^{13} \mathrm{C} \mathrm{NMR} \mathrm{(100} \mathrm{MHz,} \mathrm{CDCl}_{3}\right) \delta 142.2,140.3$, 139.0, 136.4, 135.7, 135.4, $131.3(\mathrm{q}, J=33.1 \mathrm{~Hz}), 131.0(\mathrm{q}, J=33.1 \mathrm{~Hz}), 129.2,129.0,125.2,124.5,124.3$ (q, $J=3.7 \mathrm{~Hz}), 124.1(\mathrm{q}, J=272.3 \mathrm{~Hz}), 124.0$ (q, $J=272.3 \mathrm{~Hz}), 124.0$ (q, $J=3.7 \mathrm{~Hz}), 45.9,36.6,34.0,30.2$, 23.9, 21.2, 11.9; ${ }^{19} \mathrm{~F}$ NMR (471 MHz, $\left.\mathrm{CDCl}_{3}\right) \delta-63.0$ (s, 3F), -63.0 (s, 3F); HRMS (EI, $\left.m / z\right)$ ) calcd for $\mathrm{C}_{27} \mathrm{H}_{24} \mathrm{~F}_{6} \mathrm{Si}\left[\mathrm{M}^{+}\right]$490.1540, found 490.1539; IR (film): 2934, 1392, 1324, 1166, 1126,1060, 1018, 827, 703 $\mathrm{cm}^{-1}$.

(R)-1',1'-bis(4-(trifluoromethyl)phenyl)spiro[chromane-4,2'-silolane] (6f)

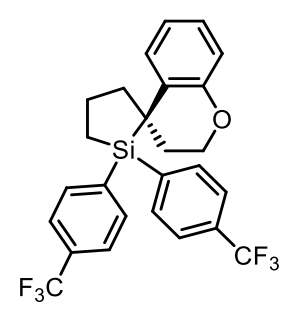

Yield: $88 \%$ (43.3 mg); white solid; HPLC gave 98\% e.e., conditions: Daicel chiral column OD-3, hexane: ${ }^{i} \operatorname{PrOH}=100: 0,0.5 \mathrm{~mL} / \mathrm{min}$, wavelength $=220 \mathrm{~nm} ;[\alpha]_{D}^{30}=+28.9^{\circ}\left(c 1.28, \mathrm{CHCl}_{3}\right) ;{ }^{1} \mathrm{H} \mathrm{NMR}(400 \mathrm{MHz}$, $\left.\mathrm{CDCl}_{3}\right) \delta$ 7.58-7.44 (m, 4H), $7.45(\mathrm{~d}, J=8.0 \mathrm{~Hz}, 2 \mathrm{H}), 7.23(\mathrm{~d}, J=8.0 \mathrm{~Hz}, 2 \mathrm{H}), 7.00-6.95(\mathrm{~m}, 1 \mathrm{H}), 6.81-6.79$ $(\mathrm{m}, 1 \mathrm{H}), 6.61-6.59(\mathrm{~m}, 1 \mathrm{H}), 6.56-6.52(\mathrm{~m}, 1 \mathrm{H}), 3.97-3.92(\mathrm{~m}, 1 \mathrm{H}), 3.76-3.70(\mathrm{~m}, 1 \mathrm{H}), 2.34-2.23(\mathrm{~m}, 2 \mathrm{H})$, 2.15-2.06 (m, 2H), 2.03-1.96 (m, 1H), 1.81-1.75 (m, 1H), 1.67-1.53 (m, 2H); ${ }^{13} \mathrm{C}$ NMR (100 MHz, $\left.\mathrm{CDCl}_{3}\right)$ $\delta 153.9,139.0,137.9,135.8,135.4,131.7(\mathrm{q}, J=32.6 \mathrm{~Hz}), 131.4(\mathrm{q}, J=32.4 \mathrm{~Hz}), 129.2,127.9,126.3,124.6$ (q, $J=3.6 \mathrm{~Hz}), 124.2$ (q, $J=3.6 \mathrm{~Hz}), 124.0$ (q, $J=272.3 \mathrm{~Hz}), 123.9$ (q, $J=272.3 \mathrm{~Hz}), 120.0,116.9,64.3$, 44.9, 32.6, 32.1, 23.4, 11.2; ${ }^{19} \mathrm{~F}$ NMR (471 MHz, $\left.\mathrm{CDCl}_{3}\right) \delta$-63.1 (s, 3F), -63.1 (s, 3F); HRMS (EI, $\left.m / z\right)$ : calcd for $\mathrm{C}_{26} \mathrm{H}_{22} \mathrm{~F}_{6} \mathrm{OSi}\left[\mathrm{M}^{+}\right]$492.1339, found 492.1334; IR (film): 2937, 1487, 1392, 1324, 1125,1060, 827, $705 \mathrm{~cm}^{-1}$.

(S)-1',1'-bis(4-(trifluoromethyl)phenyl)-6,7-dihydro-5H-spiro[benzo[b]thiophene-4,2'-silolane] (6g) 


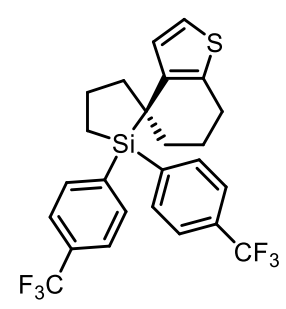

Yield: 67\% (32.5 mg); colorless oil; HPLC gave $>99 \%$ e.e., conditions: Daicel chiral column OD-3, hexane: ${ }^{i} \operatorname{PrOH}=100: 0,0.5 \mathrm{~mL} / \mathrm{min}$, wavelength $=220 \mathrm{~nm} ;[\alpha]_{D}^{30}=+71.2^{\circ}\left(c 1.00, \mathrm{CHCl}_{3}\right) ;{ }^{1} \mathrm{H} \mathrm{NMR}(400 \mathrm{MHz}$, $\left.\mathrm{CDCl}_{3}\right) \delta$ 7.57-7.52 (m, 4H), $7.45(\mathrm{~d}, J=7.9 \mathrm{~Hz}, 2 \mathrm{H}), 7.25(\mathrm{~d}, J=6.9 \mathrm{~Hz}, 2 \mathrm{H}), 6.76(\mathrm{~d}, J=5.2 \mathrm{~Hz}, 1 \mathrm{H}), 6.26$ $(\mathrm{d}, J=5.2 \mathrm{~Hz}, 1 \mathrm{H}), 2.78-2.74(\mathrm{~m}, 2 \mathrm{H}), 2.23-2.05(\mathrm{~m}, 3 \mathrm{H}), 1.99(\mathrm{ddd}, J=13.3,6.4,2.2 \mathrm{~Hz}, 1 \mathrm{H}), 1.92-1.87$ $(\mathrm{m}, 1 \mathrm{H}), 1.70-1.63(\mathrm{~m}, 1 \mathrm{H}), 1.57-1.50(\mathrm{~m}, 3 \mathrm{H}), 1.43-1.36(\mathrm{~m}, 1 \mathrm{H}) ;{ }^{13} \mathrm{C} \mathrm{NMR}\left(100 \mathrm{MHz}, \mathrm{CDCl}_{3}\right) \delta 140.3$, 139.9, 138.7, 135.6, 135.4, 133.4, 131.4 (q, $J=32.4 \mathrm{~Hz}), 131.2$ (q, $J=32.2 \mathrm{~Hz}), 127.5,124.3$ (q, $J=3.7 \mathrm{~Hz})$, 124.1 (q, $J=3.7 \mathrm{~Hz}), 124.1$ (q, $J=272.3 \mathrm{~Hz}), 124.0$ (q, $J=272.3 \mathrm{~Hz}), 120.9,44.6,35.0,33.3,24.9,23.7$, 22.1, 11.2; ${ }^{19} \mathrm{~F}$ NMR (471 MHz, $\mathrm{CDCl}_{3}$ ) $\delta$-63.0 (s, 3F), -63.0 (s, 3F); HRMS (EI, $m / z$ ): calcd for $\mathrm{C}_{25} \mathrm{H}_{22} \mathrm{~F}_{6} \mathrm{SSi}$ $\left[\mathrm{M}^{+}\right]$496.1110, found 496.1107; IR (film): 2934, 1397, 1324, 1166,1127, 1060, 828, $700 \mathrm{~cm}^{-1}$.

\section{(S)-1-phenylbutane-1,4-diol (7)}

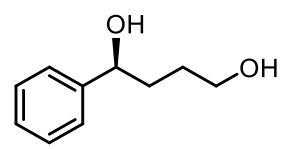

Yield: $61 \%$ (19.1 mg); white solid; HPLC gave 97\% e.e., conditions: Daicel chiral column OD-H, hexane: ${ }^{i} \mathrm{PrOH}=90: 10,0.5 \mathrm{ml} / \mathrm{min}$, wavelength $=220 \mathrm{~nm} ;[\alpha]_{D}^{20}=-26.0^{\circ}\left(c 0.50, \mathrm{CHCl}_{3}\right) ;{ }^{1} \mathrm{H} \mathrm{NMR}(400 \mathrm{MHz}$, $\left.\mathrm{CDCl}_{3}\right) \delta$ 7.35-7.34 (m, 4H), 7.30-7.24 (m, 1H), $4.72(\mathrm{t}, J=6.3 \mathrm{~Hz}, 1 \mathrm{H}), 3.72-3.62(\mathrm{~m}, 2 \mathrm{H}), 2.65(\mathrm{br}, 2 \mathrm{H})$, $1.85(\mathrm{q}, J=6.9 \mathrm{~Hz}, 2 \mathrm{H}), 1.75-1.61(\mathrm{~m}, 2 \mathrm{H}) ;{ }^{13} \mathrm{C} \mathrm{NMR}\left(100 \mathrm{MHz}, \mathrm{CDCl}_{3}\right) \delta 144.7,128.4,127.5,125.8,74.4$, 62.8, 36.2, 29.2; HRMS (ESI) calcd for $\mathrm{C}_{10} \mathrm{H}_{15} \mathrm{O}_{2}[\mathrm{M}+\mathrm{H}]^{+}$167.1067, found 167.1068; IR (film): 2937, 785, $734,702,667 \mathrm{~cm}^{-1}$.

(S)-2-((S)-4-(prop-1-en-2-yl)cyclohex-1-en-1-yl)-1,1-bis(4-(trifluoromethyl)phenyl)silolane (9)<smiles>C=C(C)[C@H]1CC=C(C2CCC[Si]2(c2ccc(C(F)(F)F)cc2)c2ccc(C(F)(F)F)cc2)CC1</smiles>

Yield: 70\% (34.6 mg); colorless oil; dr=20:1; HPLC gave 94\% e.e., conditions: Daicel chiral column AD-3, hexane: ${ }^{i} \mathrm{PrOH}=300: 1,0.5 \mathrm{ml} / \mathrm{min}$, wavelength $=220 \mathrm{~nm} ;[\alpha]_{D}^{30}=-28.4^{\circ}\left(c 1.00, \mathrm{CHCl}_{3}\right) ;{ }^{1} \mathrm{H} \mathrm{NMR}(400$ $\left.\mathrm{MHz}, \mathrm{CDCl}_{3}\right) \delta 7.74(\mathrm{~d}, J=8.1 \mathrm{~Hz}, 2 \mathrm{H}), 7.66(\mathrm{~d}, J=8.1 \mathrm{~Hz}, 2 \mathrm{H}), 7.58-7.52(\mathrm{~m}, 4 \mathrm{H}), 5.32(\mathrm{~d}, J=2.9 \mathrm{~Hz}$, $1 \mathrm{H}), 4.63(\mathrm{~s}, 1 \mathrm{H}), 4.57(\mathrm{~s}, 1 \mathrm{H}), 2.25-2.14(\mathrm{~m}, 2 \mathrm{H}), 2.10-1.97(\mathrm{~m}, 3 \mathrm{H}), 1.95-1.80(\mathrm{~m}, 2 \mathrm{H}), 1.77-1.65$ $(\mathrm{m}, 3 \mathrm{H}), 1.62(\mathrm{~s}, 3 \mathrm{H}), 1.55-1.50(\mathrm{~m}, 1 \mathrm{H}), 1.26-1.22(\mathrm{~m}, 2 \mathrm{H}), 0.89(\mathrm{qd}, J=11.7,5.1 \mathrm{~Hz}, 1 \mathrm{H}) ;{ }^{13} \mathrm{C} \mathrm{NMR}$ $\left(101 \mathrm{MHz}, \mathrm{CDCl}_{3}\right) \delta 149.9,140.7,139.1,136.9,135.6,135.3,131.8(\mathrm{q}, J=32.3 \mathrm{~Hz}), 131.5(\mathrm{q}, J=32.3 \mathrm{~Hz})$, 124.7 (q, $J=3.7 \mathrm{~Hz}), 124.0$ (q, $J=3.7 \mathrm{~Hz}), 124.0$ (q, $J=272 \mathrm{~Hz}), 124.0$ (q, $J=272 \mathrm{~Hz}), 117.3,108.4,41.0$, 36.6, 32.1, 31.5, 30.8, 27.6, 25.6, 20.5, 12.7; $\left.{ }^{19} \mathrm{~F} \mathrm{NMR} \mathrm{(471} \mathrm{MHz,} \mathrm{CDCl}_{3}\right) \delta-63.0$ (s, 3F), -63.2 (s, 3F); HRMS (EI, $m / z$ ): calcd for $\mathrm{C}_{27} \mathrm{H}_{28} \mathrm{~F}_{6} \mathrm{Si}\left[\mathrm{M}^{+}\right]$494.1859, found 494.1863; IR (film): 2946, 1392, 1324,1166, $1126,1060,828 \mathrm{~cm}^{-1}$.

(8R,9S,13S,14S,17S)-13-methyl-2-((S)-2-methyl-1,1-bis(4-(trifluoromethyl)phenyl)silolan-2-yl)$7,8,9,11,12,13,14,15,16,17-d e c a h y d r o-6 H$-cyclopenta $[a]$ phenanthren-17-yl acetate (11) 


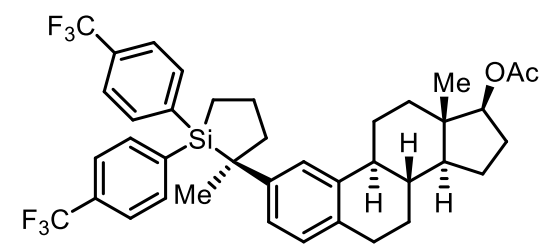

Yield: $57 \%$ (38.9 mg); colorless oil; ${ }^{1} \mathrm{H}$ NMR gave gave $92 \%$ d.e.; $[\alpha]_{D}^{20}=5.4^{\circ}\left(c 0.98, \mathrm{CHCl}_{3}\right)$; ${ }^{1} \mathrm{H}$ NMR $\left(400 \mathrm{MHz}, \mathrm{CDCl}_{3}\right) \delta 7.71(\mathrm{~d}, J=7.9 \mathrm{~Hz}, 2 \mathrm{H}), 7.64(\mathrm{~d}, J=7.9 \mathrm{~Hz}, 2 \mathrm{H}), 7.37(\mathrm{~d}, J=7.8 \mathrm{~Hz}, 2 \mathrm{H}), 7.11(\mathrm{~d}, J=$ $7.8 \mathrm{~Hz}, 2 \mathrm{H}), 6.97-6.96(\mathrm{~m}, 3 \mathrm{H}), 4.66(\mathrm{t}, J=8.4 \mathrm{~Hz}, 1 \mathrm{H}), 2.82-2.80(\mathrm{~m}, 2 \mathrm{H}), 2.38-2.30(\mathrm{~m}, 1 \mathrm{H}), 2.27-2.16(\mathrm{~m}$, $2 \mathrm{H}), 2.14-2.08(\mathrm{~m}, 1 \mathrm{H}), 2.06(\mathrm{~s}, 3 \mathrm{H}), 2.03-1.95(\mathrm{~m}, 1 \mathrm{H}), 1.93-1.92(\mathrm{~m}, 1 \mathrm{H}), 1.90-1.83(\mathrm{~m}, 2 \mathrm{H}), 1.79-1.69(\mathrm{~m}$, 2H), 1.60-1.49 (m, 2H), 1.45-1.30 (m, 4H), $1.28(\mathrm{~s}, 3 \mathrm{H}), 1.26-1.18(\mathrm{~m}, 2 \mathrm{H}), 1.06(\mathrm{qd}, J=13.2,3.9 \mathrm{~Hz}, 1 \mathrm{H})$, 0.77 (s, 3H); ${ }^{13} \mathrm{C}$ NMR $\left(100 \mathrm{MHz}, \mathrm{CDCl}_{3}\right) \delta 171.3,144.4,140.1,139.7,139.1,135.6,135.5,133.1,131.6$ (q, $J=32.4 \mathrm{~Hz}), 130.9(\mathrm{q}, J=32.2 \mathrm{~Hz}), 128.9,124.5(\mathrm{q}, J=3.8 \mathrm{~Hz}), 124.2,124.1(\mathrm{q}, J=272.7 \mathrm{~Hz}), 124.0(\mathrm{q}, J$ $=272.4 \mathrm{~Hz}), 123.9(\mathrm{q}, J=3.7 \mathrm{~Hz}), 123.6,82.7,49.8,44.3,42.7,41.3,38.4,36.7,34.7,28.9,27.5$, 27.2, 25.7, 25.4, 23.2, 22.2, 21.2, 11.9, 9.8; ${ }^{19} \mathrm{~F}$ NMR (471 MHz, $\left.\mathrm{CDCl}_{3}\right) \delta-62.9$ (s, 3F), -63.0 (s, 3F); HRMS (ESI) calcd for $\mathrm{C}_{39} \mathrm{H}_{46} \mathrm{~F}_{6} \mathrm{NO}_{2} \mathrm{Si}\left[\mathrm{M}+\mathrm{NH}_{4}\right]^{+}$702.3197, found 702.3199; IR (film): 2916, 1729, 1324, 1166, 1128, $1060,732 \mathrm{~cm}^{-1}$.

\section{(S)-6-fluoro-3-(1-(3-(2-methoxy-4-(2-methyl-1,1-bis(4-(trifluoromethyl)phenyl)silolan-2-}

\section{yl)phenoxy)propyl)piperidin-4-yl)benzo[ $d]$ isoxazole (13)}

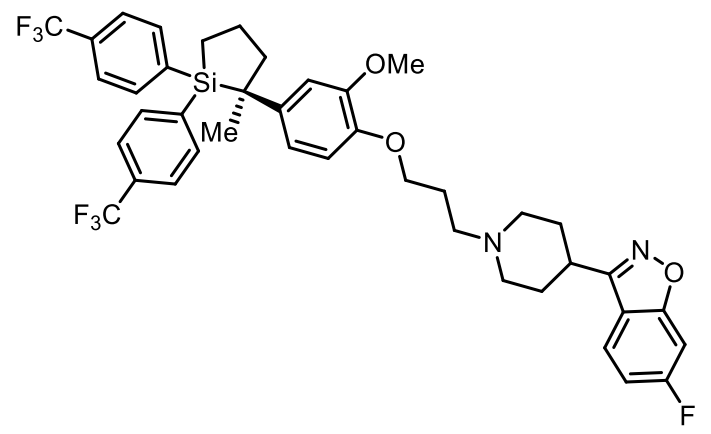

Yield: 53\% (41.0 mg); white solid; HPLC gave 92\% e.e., conditions: Daicel chiral column AD-H, hexane: ${ }^{i} \operatorname{PrOH}=90: 10,1.0 \mathrm{~mL} / \mathrm{min}$, wavelength $=210 \mathrm{~nm} ;[\alpha]_{D}^{30}=-5.2^{\circ}\left(c 1.00, \mathrm{CHCl}_{3}\right) ;{ }^{1} \mathrm{H} \mathrm{NMR}(400 \mathrm{MHz}$, $\left.\mathrm{CD}_{3} \mathrm{OD}\right) \delta 8.00(\mathrm{dd}, J=8.8,5.1 \mathrm{~Hz}, 1 \mathrm{H}), 7.89(\mathrm{~d}, J=7.8 \mathrm{~Hz}, 2 \mathrm{H}), 7.75(\mathrm{~d}, J=7.8 \mathrm{~Hz}, 2 \mathrm{H}), 7.43(\mathrm{~d}, J=7.9$ $\mathrm{Hz}, 2 \mathrm{H}), 7.39(\mathrm{dd}, J=9.0,2.1 \mathrm{~Hz}, 1 \mathrm{H}), 7.26(\mathrm{~d}, J=7.8 \mathrm{~Hz}, 2 \mathrm{H}), 7.19(\mathrm{td}, J=9.0,2.2 \mathrm{~Hz}, 1 \mathrm{H}), 6.90(\mathrm{~d}, J=$ $8.4 \mathrm{~Hz}, 1 \mathrm{H}), 6.80(\mathrm{dd}, J=8.4,2.2 \mathrm{~Hz}, 1 \mathrm{H}), 6.64(\mathrm{~d}, J=2.2 \mathrm{~Hz}, 1 \mathrm{H}), 4.12(\mathrm{t}, J=5.6 \mathrm{~Hz}, 2 \mathrm{H}), 3.70(\mathrm{~d}, J=$ $12.3 \mathrm{~Hz}, 2 \mathrm{H}), 3.62-3.54(\mathrm{~m}, 1 \mathrm{H}), 3.58(\mathrm{~s}, 3 \mathrm{H}), 3.32(\mathrm{p}, J=1.6 \mathrm{~Hz}, 1 \mathrm{H}), 3.26-3.17(\mathrm{~m}, 2 \mathrm{H}), 2.48-2.22(\mathrm{~m}$, 8H), $2.13-1.99(\mathrm{~m}, 1 \mathrm{H}), 1.92(\mathrm{dd}, J=12.4,5.1 \mathrm{~Hz}, 1 \mathrm{H}), 1.68(\mathrm{dd}, J=15.7,8.6 \mathrm{~Hz}, 1 \mathrm{H}), 1.40-1.31(\mathrm{~m}$, $1 \mathrm{H}), 1.26(\mathrm{~s}, 3 \mathrm{H}), 1.24-1.20(\mathrm{~m}, 1 \mathrm{H}) ;{ }^{13} \mathrm{C} \mathrm{NMR}\left(100 \mathrm{MHz}, \mathrm{CD}_{3} \mathrm{OD}\right) \delta 165.9(\mathrm{~d}, J=249.8 \mathrm{~Hz}), 165.2(\mathrm{~d}, J$ $=14.0 \mathrm{~Hz}), 161.2,150.3,146.7,143.0,141.9,140.4,137.2,136.9,132.8(\mathrm{q}, J=32.3 \mathrm{~Hz}), 132.0(\mathrm{q}, J=32.0$ $\mathrm{Hz}), 125.6(\mathrm{q}, J=271.6 \mathrm{~Hz}), 125.6(\mathrm{q}, J=3.8 \mathrm{~Hz}), 125.5(\mathrm{q}, J=271.6 \mathrm{~Hz}), 125.0(\mathrm{q}, J=3.8 \mathrm{~Hz}), 124.4(\mathrm{~d}$, $J=11.3 \mathrm{~Hz}), 119.8,118.3(\mathrm{~d}, J=1.0 \mathrm{~Hz}), 115.0,113.8(\mathrm{~d}, J=25.6 \mathrm{~Hz}), 112.9,98.1(\mathrm{~d}, J=27.4 \mathrm{~Hz}), 68.4$, 56.8, 56.2, 53.5, 42.4, 35.9, 32.6, 29.1, 26.6, 25.5, 23.2, 10.8; $\left.{ }^{19} \mathrm{~F} \mathrm{NMR} \mathrm{(471} \mathrm{MHz,} \mathrm{CD}_{3} \mathrm{OD}\right) \delta-64.4$ (s, 3F), -64.4 (s, 3F), -111.08 - (-111.13) (m, 1F); HRMS (ESI) calcd for $\mathrm{C}_{26} \mathrm{H}_{23} \mathrm{ClSiNa}[\mathrm{M}+\mathrm{H}]^{+}$771.2853, found 771.2865; IR (film): 2932, 1638, 1521, 1325, 1121, 1060, $656 \mathrm{~cm}^{-1}$. 


\section{X-ray structure of $4 i, 6 a, 6 d$}

\subsection{X-ray structure of $4 i$}

Single crystals of $\mathbf{4} \mathbf{i}$ were obtained by dissolving it with least amount of petroleum ether. The mixture was then evaporated at room temperature. A suitable crystal was selected and the sample was performed at 180 $\mathrm{K}$ on a Rigaku XtaLAB PRO 007HF(Mo) diffractometer, with Mo K $\alpha$ radiation $(\lambda=0.71073 \AA)$. Data reduction and empirical absorption correction were performed using the CrysAlisPro program. The structure was solved by a dual-space algorithm using SHELXT program or Olex2. All non-hydrogen atoms could be located directly from the difference Fourier maps. Framework hydrogen atoms were placed geometrically and constrained using the riding model to the parent atoms. Final structure refinement was done using the SHELXL program by minimizing the sum of squared deviations of $\mathrm{F}^{2}$ using a full-matrix technique. Refined structure and crystallographic parameters are summarized in Figure S6 and Tables S2-7. The Diamond diagram was drawn by Mercury.

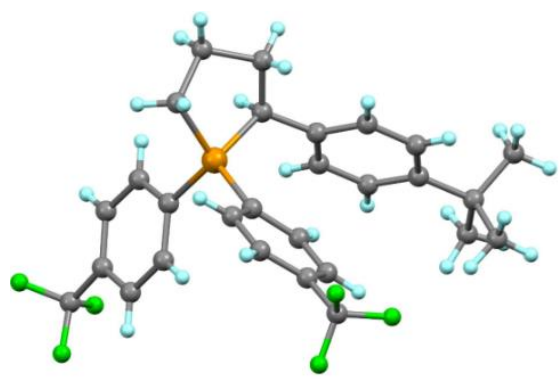

Figure S6 Diamond diagram of 4i. Ellipsoids are set at 50\% probability.

Table S2 Crystal data and structure refinement for $\mathbf{4 i}$.

Identification code

Empirical formula

Formula weight

Temperature/K

Crystal system

Space group

$\mathrm{a} / \AA ̊$

$\mathrm{b} / \AA$

c/Å

$\alpha /{ }^{\circ}$

$\beta /{ }^{\circ}$

$\gamma /{ }^{\circ}$

Volume/ $\AA^{3}$
3

$\mathrm{C}_{28} \mathrm{H}_{28} \mathrm{~F}_{6} \mathrm{Si}$

506.59

179.99(10)

monoclinic

$\mathrm{P} 21$

6.9228(2)

15.4359(4)

12.3863(3)

90

103.238(2)

90

1288.42(6) 


$\begin{array}{ll}\rho_{\text {calcg }} / \mathrm{cm}^{3} & 1.306 \\ \mu / \mathrm{mm}^{-1} & 0.149 \\ \mathrm{~F}(000) & 528.0 \\ \text { Crystal size } / \mathrm{mm}^{3} & 0.41 \times 0.18 \times 0.12 \\ \text { Radiation } & \mathrm{Mo} \mathrm{K \alpha}(\lambda=0.71073) \\ 2 \Theta \text { range for data collection } /{ }^{\circ} & 4.286 \text { to } 54.97 \\ \text { Index ranges } & -8 \leq \mathrm{h} \leq 8,-20 \leq \mathrm{k} \leq 20,-16 \leq 1 \leq 16 \\ \text { Reflections collected } & 29360 \\ \text { Independent reflections } & 5882\left[\mathrm{R}_{\text {int }}=0.0338, \mathrm{R}_{\text {sigma }}=0.0293\right] \\ \text { Data/restraints/parameters } & 5882 / 1 / 319 \\ \text { Goodness-of-fit on } \mathrm{F}^{2} & 1.010 \\ \text { Final R indexes [I }>=2 \sigma(\mathrm{I})] & \mathrm{R}_{1}=0.0456, \mathrm{wR}_{2}=0.1092 \\ \text { Final R indexes [all data] } & \mathrm{R}_{1}=0.0535, \mathrm{wR}_{2}=0.1139 \\ \text { Largest diff. peak/hole / e } \AA^{-3} & 0.45 /-0.37 \\ \text { Flack parameter } & -0.05(4)\end{array}$

Table S3 Fractional Atomic Coordinates $\left(\times 10^{4}\right)$ and Equivalent Isotropic Displacement Parameters $\left(\AA^{2} \times 10^{3}\right)$ for $4 \mathbf{i} . \mathrm{U}_{\text {eq }}$ is defined as $1 / 3$ of of the trace of the orthogonalised $U_{\text {IJ }}$ tensor.

\begin{tabular}{lrrrr} 
Atom & \multicolumn{1}{l}{$\boldsymbol{x}$} & \multicolumn{1}{l}{$\boldsymbol{l}$} \\
Si1 & $5330.7(12)$ & $4538.9(6)$ & $1233.3(7)$ & $33.40(19)$ \\
F1 & $11793(5)$ & $1226(2)$ & $3008(2)$ & $87.5(10)$ \\
F2 & $11404(5)$ & $1034(2)$ & $1286(3)$ & $88.4(10)$ \\
F3 & $13416(4)$ & $1994(2)$ & $2076(4)$ & $109.8(13)$ \\
F4 & $3568(7)$ & $4831(3)$ & $6419(2)$ & $138(2)$ \\
F5 & $3661(5)$ & $6165(3)$ & $6037(3)$ & $111.6(13)$ \\
F6 & $1027(4)$ & $5478(3)$ & $5478(2)$ & $91.3(11)$ \\
C1 & $3064(5)$ & $4326(3)$ & $87(3)$ & $45.3(9)$ \\
C2 & $3200(6)$ & $4999(3)$ & $-803(3)$ & $58.3(11)$ \\
C3 & $4226(6)$ & $5811(3)$ & $-232(3)$ & $53.5(10)$ \\
C4 & $6192(5)$ & $5513(2)$ & $526(3)$ & $37.9(7)$ \\
C5 & $7482(5)$ & $6186(2)$ & $1212(3)$ & $36.9(7)$
\end{tabular}




\begin{tabular}{|c|c|c|c|c|}
\hline C6 & $6782(5)$ & 6964(2) & 1531(3) & $47.7(9)$ \\
\hline $\mathrm{C} 7$ & $8032(6)$ & $7551(2)$ & $2185(3)$ & $48.2(9)$ \\
\hline $\mathrm{C} 8$ & $10061(5)$ & 7414(2) & 2551(3) & $41.2(7)$ \\
\hline C9 & $10761(5)$ & $6637(2)$ & $2217(3)$ & $43.8(8)$ \\
\hline $\mathrm{C} 10$ & $9532(5)$ & $6045(2)$ & $1568(3)$ & $42.1(8)$ \\
\hline $\mathrm{C} 11$ & $11394(6)$ & $8076(3)$ & $3280(3)$ & $47.5(8)$ \\
\hline $\mathrm{C} 12$ & $11286(12)$ & $8930(4)$ & $2686(5)$ & $108(3)$ \\
\hline $\mathrm{C} 13$ & $10684(12)$ & $8181(5)$ & $4349(5)$ & $108(2)$ \\
\hline $\mathrm{C} 14$ & 13529(9) & 7797(4) & $3625(8)$ & $128(3)$ \\
\hline $\mathrm{C} 15$ & $7202(5)$ & $3631(2)$ & 1473(3) & $34.4(7)$ \\
\hline C16 & $8088(5)$ & $3350(2)$ & $635(3)$ & $40.0(7)$ \\
\hline $\mathrm{C} 17$ & $9493(5)$ & $2695(2)$ & $812(3)$ & $42.7(8)$ \\
\hline $\mathrm{C} 18$ & $10047(5)$ & $2312(2)$ & $1842(3)$ & $41.4(8)$ \\
\hline C19 & $9176(6)$ & $2566(3)$ & $2686(3)$ & $47.6(9)$ \\
\hline $\mathrm{C} 20$ & $7757(5)$ & $3223(2)$ & $2502(3)$ & $44.0(8)$ \\
\hline $\mathrm{C} 21$ & 11666(6) & $1650(3)$ & $2058(4)$ & $58.0(11)$ \\
\hline $\mathrm{C} 22$ & 4681(5) & $4788.4(19)$ & 2593(3) & $33.7(7)$ \\
\hline $\mathrm{C} 23$ & $5895(5)$ & $5303(2)$ & $3404(3)$ & $40.5(7)$ \\
\hline $\mathrm{C} 24$ & $5361(5)$ & $5497(3)$ & $4392(3)$ & $45.8(8)$ \\
\hline $\mathrm{C} 25$ & $3596(5)$ & $5172(3)$ & $4580(3)$ & $45.4(8)$ \\
\hline $\mathrm{C} 26$ & $2416(5)$ & $4645(3)$ & $3814(3)$ & $45.1(8)$ \\
\hline $\mathrm{C} 27$ & 2953(5) & $4450(2)$ & $2829(3)$ & $40.5(7)$ \\
\hline $\mathrm{C} 28$ & $2960(7)$ & $5391(4)$ & $5622(3)$ & $64.2(12)$ \\
\hline
\end{tabular}

Table S4 Anisotropic Displacement Parameters $\left(\AA^{2} \times 10^{3}\right)$ for $\mathbf{4 i}$. The Anisotropic displacement factor exponent takes the form: $-2 \pi^{2}\left[h^{2} a * 2 U_{11}+2 h k a * b * U_{12}+\ldots\right]$.

\begin{tabular}{lrrrrrr} 
Atom & \multicolumn{1}{l}{$\mathbf{U}_{\mathbf{1 1}}$} & \multicolumn{1}{c}{$\mathbf{U}_{\mathbf{2 2}}$} & \multicolumn{1}{c}{$\mathbf{U}_{\mathbf{3 3}}$} & \multicolumn{1}{c}{$\mathbf{U}_{\mathbf{2 3}}$} & \multicolumn{1}{c}{$\mathbf{U}_{\mathbf{1 3}}$} & \multicolumn{1}{c}{$\mathbf{U}_{\mathbf{1 2}}$} \\
Si1 & \multicolumn{1}{c}{$33.5(4)$} & $38.3(4)$ & $29.9(4)$ & $0.0(4)$ & $10.5(3)$ & $-0.8(4)$ \\
F1 & $102(2)$ & $91(2)$ & $80(2)$ & $32.3(17)$ & $40.4(17)$ & $51.1(18)$ \\
F2 & $114(2)$ & $68.4(18)$ & $94(2)$ & $-6.3(16)$ & $46.4(18)$ & $38.0(17)$ \\
F3 & $49.3(16)$ & $94(2)$ & $191(4)$ & $38(2)$ & $38(2)$ & $19.9(16)$ \\
F4 & $173(4)$ & $209(5)$ & $46.9(15)$ & $45(2)$ & $56.6(19)$ & $107(4)$ \\
F5 & $109(3)$ & $154(3)$ & $84(2)$ & $-65(2)$ & $47.1(19)$ & $-18(2)$
\end{tabular}




\begin{tabular}{|c|c|c|c|c|c|c|}
\hline F6 & $63.8(16)$ & $152(3)$ & $68.1(17)$ & $-22.9(19)$ & $36.1(14)$ & $15.8(19)$ \\
\hline $\mathrm{C} 1$ & 41.1(18) & $57(2)$ & $37.7(17)$ & $-5.7(15)$ & $9.8(14)$ & $-6.0(15)$ \\
\hline $\mathrm{C} 2$ & $48(2)$ & $80(3)$ & $39.5(19)$ & $11.4(19)$ & $-4.2(16)$ & $-5(2)$ \\
\hline $\mathrm{C} 3$ & $47(2)$ & $60(2)$ & $50(2)$ & $18.7(18)$ & $3.7(17)$ & $1.6(18)$ \\
\hline $\mathrm{C} 4$ & $38.7(17)$ & $41.5(18)$ & $34.6(16)$ & $5.7(13)$ & $10.7(13)$ & $2.4(14)$ \\
\hline $\mathrm{C} 5$ & $37.0(16)$ & $38.6(17)$ & $37.0(16)$ & $7.7(14)$ & $12.3(13)$ & $1.2(13)$ \\
\hline C6 & $37.4(17)$ & $42.0(19)$ & $66(2)$ & $4.4(17)$ & $16.4(17)$ & $8.9(15)$ \\
\hline $\mathrm{C} 7$ & $47(2)$ & $37.9(18)$ & $63(2)$ & $0.5(17)$ & 19.6(17) & $8.9(16)$ \\
\hline $\mathrm{C} 8$ & 46.9(19) & $38.9(18)$ & $40.4(18)$ & $3.6(15)$ & $15.7(15)$ & $1.7(15)$ \\
\hline C9 & $37.6(18)$ & 45.7(19) & $47.0(19)$ & $-2.5(16)$ & $7.4(15)$ & $8.4(15)$ \\
\hline $\mathrm{C} 10$ & $40.4(18)$ & $40.5(18)$ & 46.1(18) & $-5.4(15)$ & $11.5(15)$ & $9.7(14)$ \\
\hline $\mathrm{C} 11$ & $59(2)$ & $43(2)$ & $40.7(18)$ & $-1.6(15)$ & $10.3(16)$ & $3.5(17)$ \\
\hline $\mathrm{C} 12$ & $169(7)$ & $70(3)$ & $65(3)$ & $12(3)$ & $-14(4)$ & $-51(4)$ \\
\hline $\mathrm{C} 13$ & $140(6)$ & $128(6)$ & $61(3)$ & $-32(4)$ & $32(4)$ & $-15(5)$ \\
\hline $\mathrm{C} 14$ & $70(4)$ & $79(4)$ & $204(8)$ & $-62(5)$ & $-32(4)$ & $13(3)$ \\
\hline $\mathrm{C} 15$ & $35.5(16)$ & $35.1(16)$ & $34.3(16)$ & $-1.1(13)$ & $11.3(13)$ & $-4.6(13)$ \\
\hline $\mathrm{C} 16$ & 46.1(19) & 43.1(19) & $33.3(16)$ & $-1.8(14)$ & $14.1(14)$ & $0.4(15)$ \\
\hline $\mathrm{C} 17$ & $49(2)$ & $42.7(19)$ & $43.0(18)$ & $-7.1(15)$ & $22.8(16)$ & $-1.4(15)$ \\
\hline $\mathrm{C} 18$ & $40.6(18)$ & $38.1(18)$ & 49.5(19) & $-0.7(15)$ & $18.5(15)$ & $-1.0(14)$ \\
\hline C19 & $54(2)$ & $50(2)$ & $42.3(19)$ & $9.3(16)$ & $18.0(16)$ & $8.8(17)$ \\
\hline $\mathrm{C} 20$ & $49(2)$ & $51(2)$ & $39.0(18)$ & $6.4(15)$ & $22.9(15)$ & $8.6(16)$ \\
\hline $\mathrm{C} 21$ & $57(2)$ & $56(2)$ & $69(3)$ & $10(2)$ & $31(2)$ & $13.3(19)$ \\
\hline $\mathrm{C} 22$ & $35.9(16)$ & $34.8(17)$ & $31.7(15)$ & $2.5(11)$ & $10.3(12)$ & $3.8(12)$ \\
\hline $\mathrm{C} 23$ & $36.7(17)$ & 49.2(19) & $36.7(16)$ & $-0.2(15)$ & $10.9(13)$ & $-3.3(15)$ \\
\hline $\mathrm{C} 24$ & 47.3(19) & $56(2)$ & $32.2(16)$ & $-5.2(15)$ & $4.9(14)$ & $1.8(17)$ \\
\hline $\mathrm{C} 25$ & $44.6(19)$ & 61(2) & $33.4(16)$ & $2.7(16)$ & $14.1(14)$ & $10.4(17)$ \\
\hline $\mathrm{C} 26$ & $41.8(17)$ & $56(2)$ & $41.6(17)$ & $2.3(17)$ & $17.4(13)$ & $-4.5(18)$ \\
\hline $\mathrm{C} 27$ & $40.4(16)$ & $42.7(19)$ & $40.0(16)$ & $-2.0(15)$ & $12.3(13)$ & $-4.6(15)$ \\
\hline $\mathrm{C} 28$ & $64(3)$ & $93(3)$ & $38(2)$ & $-9(2)$ & $16.3(18)$ & $10(3)$ \\
\hline
\end{tabular}

Table S5 Bond Lengths for $\mathbf{4 i}$. 


\begin{tabular}{|c|c|c|c|c|c|}
\hline Sil & $\mathrm{C} 22$ & $1.880(3)$ & $\mathrm{C} 8$ & $\mathrm{C} 11$ & $1.527(5)$ \\
\hline Sil & $\mathrm{C} 15$ & $1.885(3)$ & C9 & $\mathrm{C} 10$ & $1.375(5)$ \\
\hline Sil & $\mathrm{C} 1$ & $1.888(3)$ & $\mathrm{C} 11$ & $\mathrm{C} 12$ & $1.503(7)$ \\
\hline Si1 & $\mathrm{C} 4$ & $1.903(3)$ & $\mathrm{C} 11$ & $\mathrm{C} 14$ & $1.505(7)$ \\
\hline $\mathrm{F} 1$ & $\mathrm{C} 21$ & $1.331(5)$ & $\mathrm{C} 11$ & $\mathrm{C} 13$ & $1.522(7)$ \\
\hline $\mathrm{F} 2$ & $\mathrm{C} 21$ & $1.331(5)$ & $\mathrm{C} 15$ & $\mathrm{C} 16$ & $1.391(4)$ \\
\hline F3 & $\mathrm{C} 21$ & $1.319(5)$ & $\mathrm{C} 15$ & $\mathrm{C} 20$ & $1.395(5)$ \\
\hline $\mathrm{F} 4$ & $\mathrm{C} 28$ & $1.308(6)$ & C16 & $\mathrm{C} 17$ & $1.385(5)$ \\
\hline F5 & $\mathrm{C} 28$ & $1.346(7)$ & $\mathrm{C} 17$ & $\mathrm{C} 18$ & $1.378(5)$ \\
\hline F6 & $\mathrm{C} 28$ & $1.316(5)$ & $\mathrm{C} 18$ & C19 & $1.378(5)$ \\
\hline $\mathrm{C} 1$ & $\mathrm{C} 2$ & $1.534(5)$ & $\mathrm{C} 18$ & $\mathrm{C} 21$ & $1.495(5)$ \\
\hline $\mathrm{C} 2$ & $\mathrm{C} 3$ & $1.531(6)$ & $\mathrm{C} 19$ & $\mathrm{C} 20$ & $1.394(5)$ \\
\hline $\mathrm{C} 3$ & $\mathrm{C} 4$ & $1.537(5)$ & $\mathrm{C} 22$ & $\mathrm{C} 27$ & $1.396(4)$ \\
\hline $\mathrm{C} 4$ & $\mathrm{C} 5$ & $1.501(5)$ & $\mathrm{C} 22$ & $\mathrm{C} 23$ & $1.400(5)$ \\
\hline C5 & C6 & $1.385(5)$ & $\mathrm{C} 23$ & $\mathrm{C} 24$ & $1.389(5)$ \\
\hline $\mathrm{C} 5$ & $\mathrm{C} 10$ & $1.403(5)$ & $\mathrm{C} 24$ & $\mathrm{C} 25$ & $1.389(5)$ \\
\hline C6 & $\mathrm{C} 7$ & $1.381(6)$ & $\mathrm{C} 25$ & $\mathrm{C} 26$ & $1.370(5)$ \\
\hline $\mathrm{C} 7$ & $\mathrm{C} 8$ & $1.390(5)$ & $\mathrm{C} 25$ & $\mathrm{C} 28$ & $1.494(5)$ \\
\hline $\mathrm{C} 8$ & C9 & $1.392(5)$ & $\mathrm{C} 26$ & $\mathrm{C} 27$ & $1.388(5)$ \\
\hline
\end{tabular}

Table S6 Bond Angles for $4 \mathbf{i}$.

\begin{tabular}{|c|c|c|c|c|c|c|c|}
\hline \multicolumn{2}{|c|}{ Atom Atom } & \multirow{2}{*}{$\begin{array}{l}\text { Atom } \\
\text { C15 }\end{array}$} & \multirow{2}{*}{$\begin{array}{l}\text { Angle } /^{\circ} \\
\quad 107.93(14)\end{array}$} & \multicolumn{2}{|c|}{ Atom Atom } & \multirow[t]{2}{*}{ Atom } & \multirow{2}{*}{$\begin{array}{l}\text { Angle }{ }^{\circ} \\
121.0(2)\end{array}$} \\
\hline $\mathrm{C} 22$ & Si1 & & & $\mathrm{C} 20$ & $\mathrm{C} 15$ & & \\
\hline $\mathrm{C} 22$ & Si1 & $\mathrm{C} 1$ & $112.41(15)$ & $\mathrm{C} 17$ & $\mathrm{C} 16$ & $\mathrm{C} 15$ & $121.6(3)$ \\
\hline $\mathrm{C} 15$ & Sil & $\mathrm{C} 1$ & $114.31(16)$ & $\mathrm{C} 18$ & $\mathrm{C} 17$ & $\mathrm{C} 16$ & 119.7(3) \\
\hline $\mathrm{C} 22$ & Si1 & $\mathrm{C} 4$ & $114.52(15)$ & C19 & $\mathrm{C} 18$ & $\mathrm{C} 17$ & $120.2(3)$ \\
\hline $\mathrm{C} 15$ & Si1 & $\mathrm{C} 4$ & $112.52(14)$ & C19 & $\mathrm{C} 18$ & $\mathrm{C} 21$ & $119.9(3)$ \\
\hline $\mathrm{C} 1$ & Sil & $\mathrm{C} 4$ & $94.91(16)$ & $\mathrm{C} 17$ & $\mathrm{C} 18$ & $\mathrm{C} 21$ & $119.8(3)$ \\
\hline $\mathrm{C} 2$ & $\mathrm{C} 1$ & Si1 & $104.2(2)$ & $\mathrm{C} 18$ & C19 & $\mathrm{C} 20$ & $119.9(3)$ \\
\hline $\mathrm{C} 3$ & $\mathrm{C} 2$ & $\mathrm{C} 1$ & $108.7(3)$ & C19 & $\mathrm{C} 20$ & $\mathrm{C} 15$ & $120.9(3)$ \\
\hline $\mathrm{C} 2$ & $\mathrm{C} 3$ & $\mathrm{C} 4$ & $106.8(3)$ & F3 & $\mathrm{C} 21$ & $\mathrm{~F} 2$ & $105.8(4)$ \\
\hline C5 & $\mathrm{C} 4$ & $\mathrm{C} 3$ & $117.9(3)$ & F3 & $\mathrm{C} 21$ & $\mathrm{~F} 1$ & $108.0(4)$ \\
\hline $\mathrm{C} 5$ & $\mathrm{C} 4$ & Si1 & $119.7(2)$ & $\mathrm{F} 2$ & $\mathrm{C} 21$ & $\mathrm{~F} 1$ & $104.7(4)$ \\
\hline
\end{tabular}




$\begin{array}{llllllll}\text { C3 } & \text { C4 } & \text { Si1 } & 101.0(2) & \text { F3 } & \text { C21 } & \text { C18 } & 112.1(4) \\ \text { C6 } & \text { C5 } & \text { C10 } & 116.1(3) & \text { F2 } & \text { C21 } & \text { C18 } & 112.7(4) \\ \text { C6 } & \text { C5 } & \text { C4 } & 124.0(3) & \text { F1 } & \text { C21 } & \text { C18 } & 113.0(3) \\ \text { C10 } & \text { C5 } & \text { C4 } & 119.9(3) & \text { C27 } & \text { C22 } & \text { C23 } & 117.7(3) \\ \text { C7 } & \text { C6 } & \text { C5 } & 121.6(3) & \text { C27 } & \text { C22 } & \text { Si1 } & 120.2(2) \\ \text { C6 } & \text { C7 } & \text { C8 } & 122.8(3) & \text { C23 } & \text { C22 } & \text { Si1 } & 122.1(2) \\ \text { C7 } & \text { C8 } & \text { C9 } & 115.5(3) & \text { C24 } & \text { C23 } & \text { C22 } & 121.2(3) \\ \text { C7 } & \text { C8 } & \text { C11 } & 121.3(3) & \text { C25 } & \text { C24 } & \text { C23 } & 119.4(3) \\ \text { C9 } & \text { C8 } & \text { C11 } & 123.2(3) & \text { C26 } & \text { C25 } & \text { C24 } & 120.4(3) \\ \text { C10 } & \text { C9 } & \text { C8 } & 122.3(3) & \text { C26 } & \text { C25 } & \text { C28 } & 119.1(3) \\ \text { C9 } & \text { C10 } & \text { C5 } & 121.8(3) & \text { C24 } & \text { C25 } & \text { C28 } & 120.5(4) \\ \text { C12 } & \text { C11 } & \text { C14 } & 109.0(5) & \text { C25 } & \text { C26 } & \text { C27 } & 120.1(3) \\ \text { C12 } & \text { C11 } & \text { C13 } & 110.0(5) & \text { C26 } & \text { C27 } & \text { C22 } & 121.2(3) \\ \text { C14 } & \text { C11 } & \text { C13 } & 106.0(5) & \text { F4 } & \text { C28 } & \text { F6 } & 108.1(4) \\ \text { C12 } & \text { C11 } & \text { C8 } & 110.0(3) & \text { F4 } & \text { C28 } & \text { F5 } & 105.9(4) \\ \text { C14 } & \text { C11 } & \text { C8 } & 113.4(4) & \text { F6 } & \text { C28 } & \text { F5 } & 103.1(4) \\ \text { C13 } & \text { C11 } & \text { C8 } & 108.3(4) & \text { F4 } & \text { C28 } & \text { C25 } & 113.2(4) \\ \text { C16 } & \text { C15 } & \text { C20 } & 117.7(3) & \text { F6 } & \text { C28 } & \text { C25 } & 113.4(3) \\ \text { C16 } & \text { C15 } & \text { Si1 } & 121.3(3) & \text { F5 } & \text { C28 } & \text { C25 } & 112.3(4)\end{array}$

Table S7 Torsion Angles for 4i.

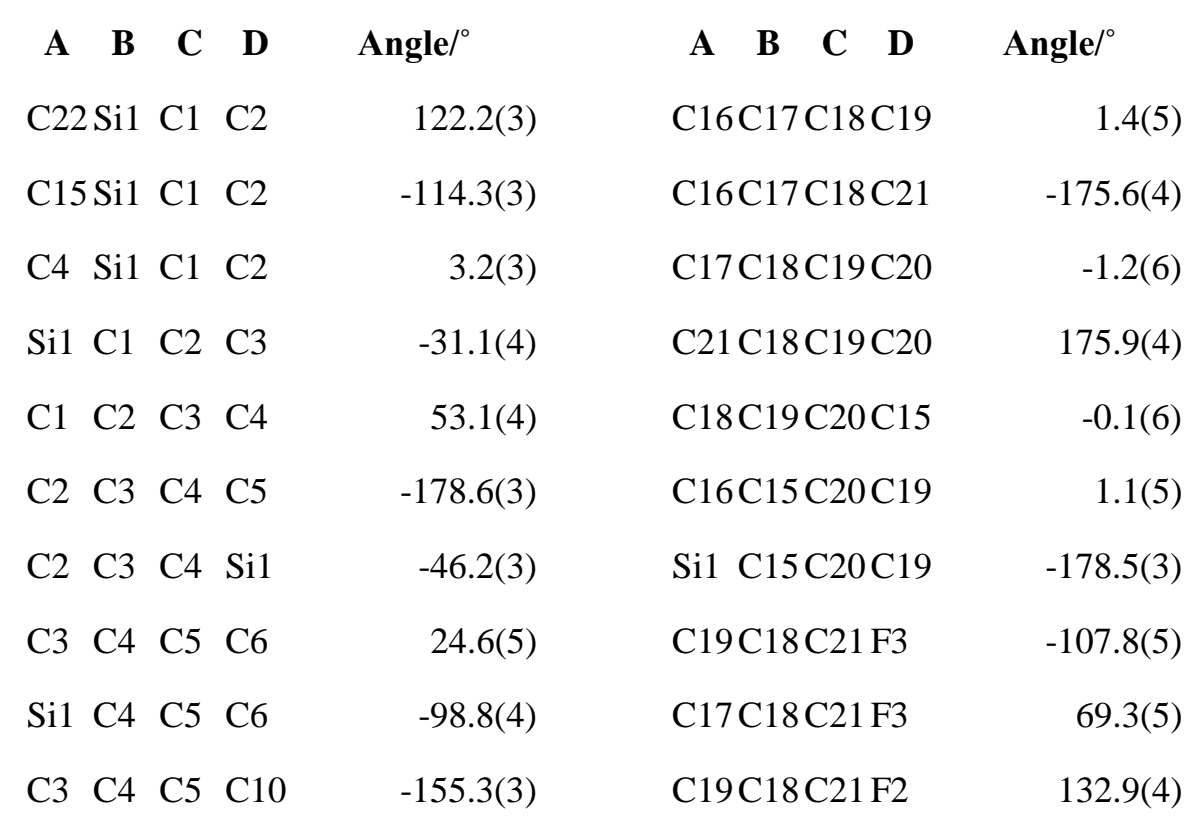




\begin{tabular}{|c|c|c|c|c|}
\hline $\mathrm{Si} 1 \mathrm{C} 4$ & C5 $\mathrm{C} 10$ & $81.4(4)$ & $\mathrm{C} 17 \mathrm{C} 18 \mathrm{C} 21 \mathrm{~F} 2$ & $-50.0(5)$ \\
\hline $\mathrm{C} 10 \mathrm{C} 5$ & C6 C7 & $-1.6(5)$ & C19C18C21 F1 & $14.5(6)$ \\
\hline $\mathrm{C} 4 \mathrm{C} 5$ & C6 C7 & $178.5(3)$ & $\mathrm{C} 17 \mathrm{C} 18 \mathrm{C} 21 \mathrm{~F} 1$ & $-168.5(4)$ \\
\hline C5 C6 & C7 $\mathrm{C} 8$ & $1.1(6)$ & C15Si1 C22 C27 & $-98.7(3)$ \\
\hline C6 C7 & $\mathrm{C} 8 \mathrm{C} 9$ & $-0.3(5)$ & C1 Si1 C22C27 & $28.3(3)$ \\
\hline C6 C7 & C8 $\quad$ C11 & $-179.6(4)$ & C4 Sil C22C27 & $135.1(3)$ \\
\hline $\mathrm{C} 7 \mathrm{C} 8$ & C9 $\mathrm{C} 10$ & $0.1(5)$ & C15Si1 C22C23 & $80.5(3)$ \\
\hline $\mathrm{C} 11 \mathrm{C} 8$ & C9 C10 & $179.4(3)$ & C1 Si1 C22C23 & $-152.5(3)$ \\
\hline $\mathrm{C} 8 \mathrm{C} 9$ & $\mathrm{C} 10 \mathrm{C} 5$ & $-0.8(6)$ & C4 Sil C22C23 & $-45.7(3)$ \\
\hline C6 C5 & $\mathrm{C} 10 \mathrm{C} 9$ & $1.5(5)$ & C27 C22 C23 C24 & $-2.4(5)$ \\
\hline $\mathrm{C} 4 \mathrm{C} 5$ & $\mathrm{C} 10 \mathrm{C} 9$ & $-178.6(3)$ & Si1 C22 C23 C24 & $178.4(3)$ \\
\hline $\mathrm{C} 7 \mathrm{C} 8$ & $\mathrm{C} 11 \mathrm{C} 12$ & $-59.8(6)$ & $\mathrm{C} 22 \mathrm{C} 23 \mathrm{C} 24 \mathrm{C} 25$ & $0.2(6)$ \\
\hline $\mathrm{C} 9 \mathrm{C} 8$ & $\mathrm{C} 11 \mathrm{C} 12$ & $121.0(5)$ & $\mathrm{C} 23 \mathrm{C} 24 \mathrm{C} 25 \mathrm{C} 26$ & $1.9(6)$ \\
\hline $\mathrm{C} 7 \mathrm{C} 8$ & $\mathrm{C} 11 \mathrm{C} 14$ & $177.9(5)$ & $\mathrm{C} 23 \mathrm{C} 24 \mathrm{C} 25 \mathrm{C} 28$ & $-178.3(4)$ \\
\hline $\mathrm{C} 9 \mathrm{C} 8$ & $\mathrm{C} 11 \mathrm{C} 14$ & $-1.3(6)$ & $\mathrm{C} 24 \mathrm{C} 25 \mathrm{C} 26 \mathrm{C} 27$ & $-1.7(6)$ \\
\hline $\mathrm{C} 7 \mathrm{C} 8$ & $\mathrm{C} 11 \mathrm{C} 13$ & $60.5(5)$ & $\mathrm{C} 28 \mathrm{C} 25 \mathrm{C} 26 \mathrm{C} 27$ & $178.5(4)$ \\
\hline $\mathrm{C} 9 \mathrm{C} 8$ & $\mathrm{C} 11 \mathrm{C} 13$ & $-118.7(5)$ & $\mathrm{C} 25 \mathrm{C} 26 \mathrm{C} 27 \mathrm{C} 22$ & $-0.6(6)$ \\
\hline C22 Si1 & $\mathrm{C} 15 \mathrm{C} 16$ & $-174.8(3)$ & $\mathrm{C} 23 \mathrm{C} 22 \mathrm{C} 27 \mathrm{C} 26$ & $2.6(5)$ \\
\hline C1 Si1 & $\mathrm{C} 15 \mathrm{C} 16$ & $59.3(3)$ & Si1 C22 C27 C26 & $-178.2(3)$ \\
\hline C4 Si1 & $\mathrm{C} 15 \mathrm{C} 16$ & $-47.5(3)$ & $\mathrm{C} 26 \mathrm{C} 25 \mathrm{C} 28 \mathrm{~F} 4$ & $88.3(5)$ \\
\hline C22 Si1 & $\mathrm{C} 15 \mathrm{C} 20$ & $4.7(3)$ & $\mathrm{C} 24 \mathrm{C} 25 \mathrm{C} 28 \mathrm{~F} 4$ & $-91.5(5)$ \\
\hline C1 Si1 & $\mathrm{C} 15 \mathrm{C} 20$ & $-121.1(3)$ & $\mathrm{C} 26 \mathrm{C} 25 \mathrm{C} 28 \mathrm{~F} 6$ & $-35.4(6)$ \\
\hline C4 Si1 & $\mathrm{C} 15 \mathrm{C} 20$ & 132.1(3) & $\mathrm{C} 24 \mathrm{C} 25 \mathrm{C} 28 \mathrm{~F} 6$ & $144.7(4)$ \\
\hline $\mathrm{C} 20 \mathrm{C} 15$ & $5 \mathrm{C} 16 \mathrm{C} 17$ & $-0.8(5)$ & $\mathrm{C} 26 \mathrm{C} 25 \mathrm{C} 28 \mathrm{~F} 5$ & $-151.9(4)$ \\
\hline Si1 C15 & $5 \mathrm{C} 16 \mathrm{C} 17$ & $178.7(3)$ & $\mathrm{C} 24 \mathrm{C} 25 \mathrm{C} 28 \mathrm{~F} 5$ & $28.3(6)$ \\
\hline $\mathrm{C} 15 \mathrm{C} 16$ & $6 \mathrm{C} 17 \mathrm{C} 18$ & $-0.4(5)$ & & \\
\hline
\end{tabular}

\subsection{X-ray structure of $6 a$}

Single crystals of $6 \mathbf{a}$ were obtained by dissolving it with least amount of petroleum ether. The mixture was then evaporated at room temperature. Single crystal X-ray diffraction data of $\mathbf{6 a}$ was collected at $180 \mathrm{~K}$ using graphite-monochromated $\mathrm{Cu}-\mathrm{K} \alpha$ radiation $(\lambda=1.54178 \mathrm{~A})$ on a SuperNova X-ray Diffraction System from Agilent Technologies. Data reduction and empirical absorption correction were performed using the CrysAlisPro program. The structure was solved by using SHELXT program or Olex2. All non-hydrogen 
atoms could be located directly from the difference Fourier maps. Framework hydrogen atoms were placed geometrically and constrained using the riding model to the parent atoms. Final structure refinement was done using the SHELXL program by minimizing the sum of squared deviations of $\mathrm{F}^{2}$ using a full-matrix technique. Refined structure and crystallographic parameters are summarized in Figure S7 and Tables S813. The Diamond diagram was drawn by Mercury.

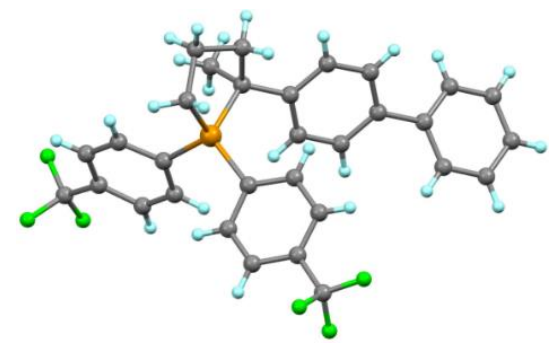

Figure S7 Diamond diagram of 6a. Ellipsoids are set at 50\% probability.

Table S8 Crystal data and structure refinement for $\mathbf{6 a}$.

Identification code

Empirical formula

Formula weight

Temperature/K

Crystal system

Space group

$\mathrm{a} / \AA$

$\mathrm{b} / \AA$

c/Å

$\alpha /{ }^{\circ}$

$\beta /{ }^{\circ}$

$\gamma /{ }^{\circ}$

Volume/ $/ \AA^{3}$

Z

$\rho_{\text {calc }} \mathrm{g} / \mathrm{cm}^{3}$

$\mu / \mathrm{mm}^{-1}$

$\mathrm{F}(000)$

Crystal size $/ \mathrm{mm}^{3}$

Radiation

$2 \Theta$ range for data collection/ ${ }^{\circ}$
3

$\mathrm{C}_{31} \mathrm{H}_{26} \mathrm{~F}_{6} \mathrm{Si}$

540.61

180.00(10)

orthorhombic

$\mathrm{P} 2{ }_{1} 2_{1} 2_{1}$

$9.77140(10)$

13.1624(2)

40.9693(6)

90

90

90

$5269.27(12)$

8

1.363

1.330

2240.0

$0.26 \times 0.1 \times 0.06$

$\mathrm{Cu} \mathrm{Ka}(\lambda=1.54184)$

7.054 to 150.312 
Index ranges

Reflections collected

Independent reflections

Data/restraints/parameters

Goodness-of-fit on $\mathrm{F}^{2}$

Final $\mathrm{R}$ indexes $[\mathrm{I}>=2 \sigma(\mathrm{I})]$

Final $\mathrm{R}$ indexes [all data]

Largest diff. peak/hole / e $\AA^{-3}$

Flack parameter
$-12 \leq \mathrm{h} \leq 12,-16 \leq \mathrm{k} \leq 16,-50 \leq 1 \leq 51$

86904

$10801\left[R_{\text {int }}=0.0369, R_{\text {sigma }}=0.0186\right]$

$10801 / 0 / 687$

1.028

$\mathrm{R}_{1}=0.0332, \mathrm{wR}_{2}=0.0892$

$\mathrm{R}_{1}=0.0348, \mathrm{wR}_{2}=0.0911$

$0.34 /-0.31$

$-0.008(6)$

Table S9 Fractional Atomic Coordinates $\left(\times 10^{4}\right)$ and Equivalent Isotropic

Displacement Parameters $\left(\AA^{2} \times 10^{3}\right)$ for 6 a. $U_{\text {eq }}$ is defined as $1 / 3$ of of the trace of the orthogonalised $\mathrm{U}_{\mathrm{IJ}}$ tensor.

\begin{tabular}{|c|c|c|c|c|}
\hline Atom & $x$ & $y$ & $z$ & $\mathbf{U}(\mathbf{e q})$ \\
\hline Si1 & $12277.6(6)$ & $3509.3(5)$ & $5377.9(2)$ & $25.23(12)$ \\
\hline $\mathrm{F} 1$ & 10769(3) & $-756.7(15)$ & 6177.3(4) & $69.0(6)$ \\
\hline $\mathrm{F} 2$ & 11351(2) & $-1534.3(13)$ & $5741.4(5)$ & $60.2(5)$ \\
\hline F3 & $9322(2)$ & $-965.5(14)$ & $5793.7(5)$ & $59.1(5)$ \\
\hline $\mathrm{F} 4$ & 12631(2) & $4175(2)$ & $3757.6(5)$ & $72.5(6)$ \\
\hline F5 & 10519(3) & 3962(4) & $3771.4(5)$ & $127.7(16)$ \\
\hline F6 & 11385(4) & $5430(2)$ & $3849.9(5)$ & $112.4(13)$ \\
\hline $\mathrm{C} 1$ & $11898(2)$ & 2137.1(18) & $5467.8(5)$ & $28.0(4)$ \\
\hline $\mathrm{C} 2$ & $12747(3)$ & $1571(2)$ & $5671.3(7)$ & $40.5(6)$ \\
\hline $\mathrm{C} 3$ & $12350(3)$ & $632(2)$ & $5790.4(7)$ & $42.4(6)$ \\
\hline $\mathrm{C} 4$ & 11085(3) & $242.6(18)$ & $5707.7(6)$ & $31.7(5)$ \\
\hline $\mathrm{C} 5$ & 10237(2) & $765.6(18)$ & $5495.5(6)$ & $30.2(5)$ \\
\hline C6 & $10648(2)$ & $1702.6(18)$ & $5376.2(6)$ & $28.5(4)$ \\
\hline $\mathrm{C} 7$ & $10635(3)$ & $-744(2)$ & $5853.6(6)$ & $40.0(6)$ \\
\hline $\mathrm{C} 8$ & $11820(2)$ & $3792.5(18)$ & $4939.6(5)$ & $27.0(4)$ \\
\hline C9 & 11520(3) & $4773(2)$ & $4832.7(6)$ & $35.0(5)$ \\
\hline $\mathrm{C} 10$ & $11400(3)$ & 4991(2) & 4501.1(7) & $39.5(6)$ \\
\hline $\mathrm{C} 11$ & $11598(2)$ & $4231(2)$ & $4273.9(6)$ & $36.8(5)$ \\
\hline $\mathrm{C} 12$ & $11879(3)$ & $3247(2)$ & $4373.4(6)$ & $34.7(5)$ \\
\hline
\end{tabular}




\begin{tabular}{|c|c|c|c|c|}
\hline $\mathrm{C} 13$ & 11983(2) & $3037.6(18)$ & $4703.4(6)$ & $31.1(5)$ \\
\hline $\mathrm{C} 14$ & $11528(3)$ & $4455(3)$ & $3913.6(7)$ & $51.6(8)$ \\
\hline $\mathrm{C} 15$ & $14113(2)$ & $3869(2)$ & $5454.0(6)$ & $34.4(5)$ \\
\hline $\mathrm{C} 16$ & 14067(3) & $4464(2)$ & $5776.7(6)$ & $39.8(6)$ \\
\hline $\mathrm{C} 17$ & 12731(3) & $5076(2)$ & $5782.0(6)$ & $38.0(5)$ \\
\hline $\mathrm{C} 18$ & $11500(2)$ & 4371.9(19) & $5708.9(5)$ & $30.4(5)$ \\
\hline C19 & 11199(3) & $3727(2)$ & $6017.0(6)$ & $42.4(6)$ \\
\hline $\mathrm{C} 20$ & 10192(2) & 4930.1(19) & $5619.8(6)$ & $30.5(5)$ \\
\hline $\mathrm{C} 21$ & $9979(3)$ & $5941(2)$ & $5702.4(7)$ & $39.0(6)$ \\
\hline $\mathrm{C} 22$ & $8736(3)$ & $6417(2)$ & $5639.0(7)$ & $40.0(6)$ \\
\hline $\mathrm{C} 23$ & 7671(3) & 5918.3(18) & $5483.8(6)$ & $32.3(5)$ \\
\hline $\mathrm{C} 24$ & 7889(3) & $4905(2)$ & $5398.7(8)$ & $42.9(6)$ \\
\hline $\mathrm{C} 25$ & $9118(3)$ & $4430(2)$ & $5467.6(8)$ & $44.0(7)$ \\
\hline $\mathrm{C} 26$ & $6363(3)$ & $6435.7(18)$ & $5408.7(6)$ & $33.3(5)$ \\
\hline $\mathrm{C} 27$ & $5740(3)$ & $7093(2)$ & $5631.1(7)$ & $42.9(6)$ \\
\hline $\mathrm{C} 28$ & $4520(3)$ & $7576(2)$ & $5556.6(8)$ & $48.8(7)$ \\
\hline $\mathrm{C} 29$ & 3893(3) & $7416(2)$ & $5259.2(8)$ & $49.7(7)$ \\
\hline $\mathrm{C} 30$ & $4486(3)$ & $6759(3)$ & $5036.8(7)$ & $49.9(7)$ \\
\hline C31 & $5705(3)$ & $6278(2)$ & $5110.9(7)$ & $42.6(6)$ \\
\hline $\mathrm{Si} 2$ & $8228.7(6)$ & $6050.7(5)$ & 2097.0(2) & $25.79(12)$ \\
\hline F7 & $8046(2)$ & $3247(2)$ & $3469.4(5)$ & $79.7(8)$ \\
\hline F8 & 5931(3) & $3573(2)$ & $3458.2(6)$ & $82.5(8)$ \\
\hline F9 & $6723(3)$ & $2329.5(15)$ & $3193.5(5)$ & $79.0(8)$ \\
\hline F10 & $7960(2)$ & $11195.3(14)$ & 2293.1(6) & $62.3(5)$ \\
\hline F11 & $5870(2)$ & $10871.8(14)$ & $2395.8(6)$ & $71.7(7)$ \\
\hline F12 & $6579(3)$ & 10974.2(16) & $1904.8(5)$ & $75.9(7)$ \\
\hline C32 & 7681(2) & $5244.7(17)$ & $2454.0(5)$ & $27.4(4)$ \\
\hline $\mathrm{C} 33$ & $7436(3)$ & $5637.9(19)$ & $2764.7(6)$ & $35.5(5)$ \\
\hline C34 & 7192(3) & $5005(2)$ & $3029.0(6)$ & $38.5(6)$ \\
\hline C35 & $7198(2)$ & $3964.3(19)$ & $2988.3(5)$ & $31.0(5)$ \\
\hline C36 & $7423(3)$ & $3548.9(18)$ & $2682.2(6)$ & $31.5(5)$ \\
\hline C37 & 7654(3) & $4187.5(18)$ & $2418.5(5)$ & $31.1(5)$ \\
\hline
\end{tabular}




\begin{tabular}{|c|c|c|c|c|}
\hline C38 & $6969(3)$ & $3285(2)$ & $3276.1(6)$ & $36.7(5)$ \\
\hline C39 & $7809(2)$ & $7435.9(18)$ & $2160.0(5)$ & $28.7(4)$ \\
\hline $\mathrm{C} 40$ & $6554(2)$ & 7784.8(19) & $2276.7(6)$ & $31.6(5)$ \\
\hline $\mathrm{C} 41$ & $6252(2)$ & $8812.8(19)$ & $2295.1(6)$ & $32.7(5)$ \\
\hline $\mathrm{C} 42$ & $7222(3)$ & $9520.3(18)$ & 2195.1(6) & $31.3(5)$ \\
\hline $\mathrm{C} 43$ & $8488(3)$ & 9197.9(19) & 2082.7(7) & $36.7(5)$ \\
\hline $\mathrm{C} 44$ & 8771(3) & $8169.8(19)$ & $2065.3(6)$ & $34.7(5)$ \\
\hline $\mathrm{C} 45$ & 6892(3) & $10627(2)$ & 2198.4(7) & $38.1(5)$ \\
\hline $\mathrm{C} 46$ & $10104(2)$ & $5828(2)$ & 2011.3(6) & $33.2(5)$ \\
\hline $\mathrm{C} 47$ & 10154(3) & $5596(2)$ & $1643.8(6)$ & $37.0(5)$ \\
\hline $\mathrm{C} 48$ & $8870(3)$ & 4989(2) & $1556.8(6)$ & $33.9(5)$ \\
\hline C49 & $7580(2)$ & $5553.8(18)$ & $1682.0(5)$ & $28.7(4)$ \\
\hline C50 & $7287(3)$ & 6463(2) & $1451.2(6)$ & $37.2(5)$ \\
\hline C51 & $6305(2)$ & 4901.6(18) & $1700.3(5)$ & $28.0(4)$ \\
\hline C52 & 6209(3) & $3936(2)$ & $1565.5(6)$ & $37.1(5)$ \\
\hline C53 & 4998(3) & $3385(2)$ & $1576.8(7)$ & $38.3(5)$ \\
\hline C54 & $3825(2)$ & $3776.0(17)$ & $1722.0(5)$ & 29.3(4) \\
\hline C55 & $3918(2)$ & 4744.6(18) & $1861.5(6)$ & $30.0(5)$ \\
\hline C56 & $5130(2)$ & $5286.5(17)$ & $1850.6(6)$ & 29.1(4) \\
\hline C57 & 2519(3) & $3204.0(18)$ & $1724.6(6)$ & $30.9(5)$ \\
\hline C58 & 2141(3) & 2601(2) & $1457.4(7)$ & $42.8(6)$ \\
\hline C59 & $929(4)$ & $2056(2)$ & 1460.1(7) & $49.0(7)$ \\
\hline $\mathrm{C} 60$ & $56(3)$ & 2094(2) & $1726.9(7)$ & $43.9(6)$ \\
\hline C61 & 411(3) & 2694(2) & 1992.0(7) & $37.7(5)$ \\
\hline C62 & $1625(2)$ & $3241.8(18)$ & $1989.5(6)$ & $31.4(5)$ \\
\hline
\end{tabular}

Table S10 Anisotropic Displacement Parameters $\left(\AA^{2} \times 10^{3}\right)$ for 6a. The Anisotropic displacement factor exponent takes the form: $-2 \pi^{2}\left[h^{2} a^{* 2} U_{11}+2 h k a * b * U_{12}+\ldots\right]$.

\begin{tabular}{lrrrrrr} 
Atom & \multicolumn{1}{l}{$\mathbf{U}_{\mathbf{1 1}}$} & \multicolumn{1}{c}{$\mathbf{U}_{\mathbf{2 2}}$} & \multicolumn{1}{c}{$\mathbf{U}_{\mathbf{3 3}}$} & \multicolumn{1}{c}{$\mathbf{U}_{\mathbf{2 3}}$} & \multicolumn{1}{c}{$\mathbf{U}_{\mathbf{1 3}}$} & \multicolumn{1}{c}{$\mathbf{U}_{\mathbf{1 2}}$} \\
Si1 & $22.3(3)$ & $27.2(3)$ & $26.2(3)$ & $0.3(2)$ & $0.7(2)$ & $-2.1(2)$ \\
F1 & $120.8(19)$ & $49.7(11)$ & $36.5(8)$ & $10.4(8)$ & $-6.3(10)$ & $-18.7(12)$ \\
F2 & $77.9(13)$ & $28.8(8)$ & $73.8(12)$ & $3.8(8)$ & $9.3(10)$ & $12.0(8)$
\end{tabular}




\begin{tabular}{|c|c|c|c|c|c|c|}
\hline F3 & $57.0(11)$ & 40.1(9) & $80.4(13)$ & 18.1(9) & $-4.6(9)$ & $-11.9(8)$ \\
\hline $\mathrm{F} 4$ & $70.6(13)$ & $107.3(18)$ & $39.7(9)$ & $15.4(10)$ & $15.8(9)$ & $14.4(13)$ \\
\hline F5 & $78.7(17)$ & $264(5)$ & $40.2(11)$ & $16.5(18)$ & $-23.8(11)$ & $-67(2)$ \\
\hline F6 & $192(3)$ & $100(2)$ & $44.4(11)$ & $26.0(12)$ & $-0.8(16)$ & $70(2)$ \\
\hline $\mathrm{C} 1$ & $26.9(10)$ & $27.0(10)$ & $30.2(10)$ & $-0.1(8)$ & $-1.2(8)$ & $0.1(9)$ \\
\hline $\mathrm{C} 2$ & $30.8(11)$ & $36.4(13)$ & $54.4(15)$ & $4.6(11)$ & $-13.1(11)$ & $-0.8(11)$ \\
\hline $\mathrm{C} 3$ & $42.0(14)$ & $34.8(13)$ & $50.3(15)$ & 7.6(11) & $-18.0(12)$ & $4.1(11)$ \\
\hline $\mathrm{C} 4$ & $39.1(12)$ & $24.6(11)$ & $31.4(11)$ & $1.0(9)$ & $-1.8(10)$ & $3.6(9)$ \\
\hline $\mathrm{C} 5$ & $27.9(10)$ & $29.4(11)$ & $33.2(11)$ & $0.6(9)$ & $-3.0(8)$ & $0.8(9)$ \\
\hline C6 & $26.8(10)$ & $28.1(11)$ & $30.4(10)$ & $1.9(9)$ & $-3.5(9)$ & $1.7(8)$ \\
\hline $\mathrm{C} 7$ & $51.8(15)$ & $29.9(13)$ & $38.3(13)$ & $3.7(10)$ & $-4.7(11)$ & $-0.5(11)$ \\
\hline $\mathrm{C} 8$ & 20.6(9) & $31.4(11)$ & $29.0(10)$ & $-1.4(8)$ & $2.6(8)$ & $-1.2(8)$ \\
\hline C9 & $38.8(13)$ & $32.1(12)$ & $34.3(12)$ & $-2.5(9)$ & $2.0(10)$ & $6.0(10)$ \\
\hline $\mathrm{C} 10$ & $40.0(13)$ & $40.5(14)$ & $38.0(13)$ & $8.3(11)$ & $-0.7(10)$ & $10.0(11)$ \\
\hline $\mathrm{C} 11$ & $30.1(11)$ & $50.2(15)$ & $30.1(11)$ & $3.0(10)$ & $-2.4(9)$ & $2.2(11)$ \\
\hline $\mathrm{C} 12$ & $31.3(11)$ & $41.2(13)$ & $31.8(11)$ & $-7.1(10)$ & $-0.1(9)$ & $-2.7(10)$ \\
\hline $\mathrm{C} 13$ & $29.0(11)$ & $30.4(11)$ & $33.9(11)$ & $-2.7(9)$ & $1.8(9)$ & $-2.5(9)$ \\
\hline C14 & $41.8(15)$ & $78(2)$ & $35.0(13)$ & $6.3(14)$ & $-5.5(11)$ & $9.6(15)$ \\
\hline $\mathrm{C} 15$ & $26.4(10)$ & $42.5(13)$ & $34.3(11)$ & $1.9(10)$ & $-0.4(9)$ & $-4.0(10)$ \\
\hline $\mathrm{C} 16$ & $32.9(12)$ & $49.8(15)$ & $36.6(12)$ & $-0.8(11)$ & $-8.3(10)$ & $-7.7(11)$ \\
\hline $\mathrm{C} 17$ & $41.5(13)$ & $39.2(13)$ & $33.2(11)$ & $-6.4(10)$ & $-2.7(10)$ & $-6.6(11)$ \\
\hline C18 & $33.4(12)$ & $31.2(11)$ & $26.6(10)$ & $-1.3(9)$ & $3.5(8)$ & $-3.3(9)$ \\
\hline C19 & $49.7(15)$ & $45.5(15)$ & $32.0(12)$ & $5.0(11)$ & $13.5(11)$ & $3.6(12)$ \\
\hline $\mathrm{C} 20$ & $32.5(11)$ & 29.1(11) & $29.8(10)$ & $-1.0(9)$ & $5.9(9)$ & $-3.5(9)$ \\
\hline $\mathrm{C} 21$ & $45.6(14)$ & $29.6(12)$ & $41.7(13)$ & $-7.3(10)$ & $-10.6(11)$ & $-1.0(11)$ \\
\hline $\mathrm{C} 22$ & $50.5(15)$ & $25.1(11)$ & $44.4(13)$ & $-6.9(10)$ & $-9.8(11)$ & $1.3(11)$ \\
\hline $\mathrm{C} 23$ & $36.9(12)$ & $26.6(11)$ & $33.4(10)$ & $-0.7(9)$ & $5.7(9)$ & $-2.5(9)$ \\
\hline $\mathrm{C} 24$ & $28.0(11)$ & $31.6(13)$ & $69.3(18)$ & $-15.3(12)$ & $2.9(12)$ & $-4.2(10)$ \\
\hline $\mathrm{C} 25$ & $29.9(12)$ & 28.1(12) & 74.1(19) & $-17.3(12)$ & $7.0(12)$ & $-2.8(10)$ \\
\hline $\mathrm{C} 26$ & $38.7(12)$ & $24.8(11)$ & $36.5(12)$ & $1.3(9)$ & $2.7(10)$ & $-1.0(9)$ \\
\hline $\mathrm{C} 27$ & $51.6(16)$ & $35.6(13)$ & $41.7(13)$ & $-5.4(11)$ & $-2.7(12)$ & $9.5(12)$ \\
\hline $\mathrm{C} 28$ & $52.7(17)$ & $37.8(15)$ & $56.0(17)$ & $-6.5(13)$ & $-0.1(14)$ & $11.2(13)$ \\
\hline
\end{tabular}




\begin{tabular}{|c|c|c|c|c|c|c|}
\hline $\mathrm{C} 29$ & $48.7(16)$ & $44.6(15)$ & $55.7(17)$ & $8.6(13)$ & $-3.5(13)$ & $9.0(13)$ \\
\hline $\mathrm{C} 30$ & 49.7(16) & $59.9(19)$ & $40.0(14)$ & $3.5(13)$ & $-6.5(12)$ & $-3.0(14)$ \\
\hline $\mathrm{C} 31$ & $46.7(15)$ & $45.0(15)$ & $36.0(12)$ & $-4.2(11)$ & $4.2(11)$ & $-2.4(12)$ \\
\hline $\mathrm{Si} 2$ & $25.4(3)$ & $26.7(3)$ & 25.3(3) & $1.9(2)$ & $0.2(2)$ & $-1.3(2)$ \\
\hline F7 & $71.9(14)$ & $107.7(19)$ & $59.4(11)$ & $45.2(12)$ & $-27.9(10)$ & $-30.5(13)$ \\
\hline F8 & $88.6(16)$ & $86.5(16)$ & $72.5(13)$ & $38.3(12)$ & 45.1(13) & $23.8(14)$ \\
\hline F9 & $143(2)$ & $43.5(11)$ & $50.6(10)$ & $10.5(8)$ & $4.2(13)$ & $-32.6(13)$ \\
\hline F10 & $60.3(11)$ & $34.9(9)$ & $91.8(14)$ & $-13.0(9)$ & $-3.3(10)$ & $-5.8(8)$ \\
\hline F11 & $73.8(13)$ & $37.6(10)$ & $103.6(16)$ & $7.7(10)$ & $45.3(13)$ & $14.5(9)$ \\
\hline $\mathrm{F} 12$ & $125(2)$ & $44.5(11)$ & $58.6(11)$ & $12.8(9)$ & $-18.8(12)$ & $17.7(12)$ \\
\hline $\mathrm{C} 32$ & 27.1(10) & 28.1(11) & $27.0(10)$ & $1.4(8)$ & $-0.3(8)$ & $0.1(9)$ \\
\hline $\mathrm{C} 33$ & 49.7(14) & $26.3(11)$ & $30.5(11)$ & $-1.7(9)$ & $2.1(10)$ & $-7.8(11)$ \\
\hline C34 & $55.4(16)$ & $34.6(12)$ & $25.4(10)$ & $-3.4(9)$ & $3.9(10)$ & $-8.1(12)$ \\
\hline C35 & $30.5(10)$ & $33.1(11)$ & $29.5(10)$ & $3.6(9)$ & $-1.0(9)$ & $-4.4(10)$ \\
\hline $\mathrm{C} 36$ & $36.8(12)$ & $24.6(10)$ & $33.0(11)$ & $0.8(9)$ & $-0.4(9)$ & $2.7(9)$ \\
\hline $\mathrm{C} 37$ & $36.2(11)$ & 31.1(11) & $26.0(10)$ & $-1.9(9)$ & $2.5(9)$ & $3.8(9)$ \\
\hline C38 & $40.8(13)$ & $37.3(13)$ & $32.1(11)$ & $5.1(10)$ & $1.5(10)$ & $-6.0(11)$ \\
\hline C39 & $29.6(11)$ & $29.6(11)$ & $26.8(10)$ & $1.0(8)$ & $-1.0(8)$ & $-2.4(9)$ \\
\hline $\mathrm{C} 40$ & $26.3(11)$ & $31.8(12)$ & $36.7(12)$ & $0.3(9)$ & $-0.4(9)$ & $-5.6(9)$ \\
\hline $\mathrm{C} 41$ & $26.6(10)$ & $34.9(12)$ & $36.8(12)$ & $-1.9(10)$ & $1.9(9)$ & $-2.0(9)$ \\
\hline $\mathrm{C} 42$ & $34.3(11)$ & $29.8(11)$ & $29.9(10)$ & $1.9(8)$ & $-0.9(9)$ & $-1.4(10)$ \\
\hline $\mathrm{C} 43$ & $35.5(12)$ & $31.0(12)$ & $43.5(13)$ & $3.5(10)$ & $9.0(10)$ & $-6.5(10)$ \\
\hline $\mathrm{C} 44$ & $30.4(11)$ & $34.4(12)$ & $39.2(12)$ & $0.6(10)$ & $8.0(10)$ & $-2.3(10)$ \\
\hline $\mathrm{C} 45$ & 41.7(13) & $31.3(12)$ & $41.4(12)$ & $2.2(10)$ & $3.6(11)$ & $-1.0(11)$ \\
\hline $\mathrm{C} 46$ & $27.4(11)$ & $36.8(13)$ & $35.5(12)$ & $1.8(9)$ & $-0.6(9)$ & $-1.1(9)$ \\
\hline $\mathrm{C} 47$ & $28.4(11)$ & $46.4(14)$ & $36.2(12)$ & $0.5(11)$ & $5.9(9)$ & $1.0(10)$ \\
\hline $\mathrm{C} 48$ & $32.8(11)$ & $41.2(13)$ & $27.8(10)$ & $-1.0(10)$ & $3.9(9)$ & $0.6(10)$ \\
\hline $\mathrm{C} 49$ & $27.7(11)$ & $32.1(11)$ & $26.3(10)$ & $2.3(9)$ & $-0.2(8)$ & $-2.0(9)$ \\
\hline C50 & $39.9(13)$ & $39.0(13)$ & $32.7(11)$ & $7.8(10)$ & $-6.0(10)$ & $-2.7(11)$ \\
\hline C51 & $29.5(10)$ & $29.7(11)$ & $24.9(10)$ & $1.4(8)$ & $-1.2(8)$ & $-0.4(9)$ \\
\hline C52 & $35.5(12)$ & $36.6(13)$ & $39.2(12)$ & $-10.1(10)$ & $7.2(10)$ & $0.3(11)$ \\
\hline C53 & $39.6(13)$ & $29.6(12)$ & $45.8(13)$ & $-12.3(10)$ & $4.9(11)$ & $-2.0(10)$ \\
\hline
\end{tabular}




$\begin{array}{llllrrr}\text { C54 } & 32.9(11) & 26.6(11) & 28.3(10) & -0.3(8) & -0.7(9) & -2.0(9) \\ \text { C55 } & 27.6(10) & 28.4(11) & 33.9(11) & -3.7(9) & -0.2(9) & 2.6(9) \\ \text { C56 } & 30.2(11) & 24.0(10) & 33.0(11) & -2.7(8) & -3.4(9) & 1.6(9) \\ \text { C57 } & 34.5(12) & 25.8(10) & 32.5(11) & 1.0(9) & -1.6(9) & -3.0(9) \\ \text { C58 } & 51.1(16) & 41.5(14) & 36.0(12) & -6.8(11) & 3.9(11) & -15.2(12) \\ \text { C59 } & 58.0(18) & 45.7(16) & 43.3(14) & -6.8(12) & -1.3(13) & -20.3(14) \\ \text { C60 } & 40.1(14) & 39.3(14) & 52.4(15) & 4.8(12) & -3.8(12) & -11.3(12) \\ \text { C61 } & 33.1(12) & 36.1(13) & 43.8(13) & 2.4(11) & 3.1(10) & -0.4(10) \\ \text { C62 } & 31.5(11) & 29.2(11) & 33.7(11) & -0.5(9) & -1.8(9) & 2.7(9)\end{array}$

Table S11 Bond Lengths for $6 \mathbf{a}$.

\begin{tabular}{|c|c|c|c|c|c|}
\hline \multicolumn{2}{|c|}{ Atom Atom } & \multirow{2}{*}{$\begin{array}{r}\text { Length/Å } \\
1.880(2)\end{array}$} & \multicolumn{2}{|c|}{ Atom Atom } & \multirow{2}{*}{ 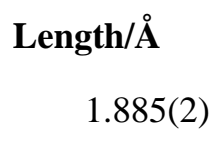 } \\
\hline Si1 & $\mathrm{C} 1$ & & $\mathrm{Si} 2$ & $\mathrm{C} 32$ & \\
\hline Si1 & $\mathrm{C} 15$ & $1.881(2)$ & $\mathrm{Si} 2$ & C39 & $1.887(2)$ \\
\hline Si1 & $\mathrm{C} 8$ & $1.888(2)$ & $\mathrm{Si} 2$ & $\mathrm{C} 46$ & $1.888(2)$ \\
\hline Si1 & $\mathrm{C} 18$ & $1.925(2)$ & $\mathrm{Si} 2$ & $\mathrm{C} 49$ & $1.929(2)$ \\
\hline $\mathrm{F} 1$ & $\mathrm{C} 7$ & $1.333(3)$ & F7 & $\mathrm{C} 38$ & $1.319(3)$ \\
\hline $\mathrm{F} 2$ & $\mathrm{C} 7$ & $1.336(3)$ & F8 & $\mathrm{C} 38$ & $1.314(3)$ \\
\hline F3 & $\mathrm{C} 7$ & $1.338(4)$ & F9 & $\mathrm{C} 38$ & $1.325(3)$ \\
\hline $\mathrm{F} 4$ & $\mathrm{C} 14$ & $1.306(4)$ & F10 & $\mathrm{C} 45$ & $1.341(3)$ \\
\hline F5 & $\mathrm{C} 14$ & $1.316(4)$ & F11 & $\mathrm{C} 45$ & $1.325(3)$ \\
\hline F6 & $\mathrm{C} 14$ & $1.318(5)$ & $\mathrm{F} 12$ & $\mathrm{C} 45$ & $1.323(3)$ \\
\hline $\mathrm{C} 1$ & $\mathrm{C} 2$ & $1.393(3)$ & C32 & $\mathrm{C} 33$ & $1.395(3)$ \\
\hline $\mathrm{C} 1$ & C6 & $1.400(3)$ & C32 & $\mathrm{C} 37$ & $1.399(3)$ \\
\hline $\mathrm{C} 2$ & $\mathrm{C} 3$ & $1.384(4)$ & C33 & $\mathrm{C} 34$ & $1.387(3)$ \\
\hline $\mathrm{C} 3$ & $\mathrm{C} 4$ & $1.380(4)$ & C34 & $\mathrm{C} 35$ & $1.380(4)$ \\
\hline $\mathrm{C} 4$ & $\mathrm{C} 5$ & $1.385(3)$ & C35 & $\mathrm{C} 36$ & $1.386(3)$ \\
\hline $\mathrm{C} 4$ & $\mathrm{C} 7$ & $1.495(3)$ & C35 & $\mathrm{C} 38$ & $1.497(3)$ \\
\hline $\mathrm{C} 5$ & C6 & $1.386(3)$ & C36 & $\mathrm{C} 37$ & $1.387(3)$ \\
\hline $\mathrm{C} 8$ & $\mathrm{C} 9$ & $1.394(3)$ & C39 & $\mathrm{C} 40$ & $1.394(3)$ \\
\hline $\mathrm{C} 8$ & $\mathrm{C} 13$ & $1.396(3)$ & C39 & $\mathrm{C} 44$ & $1.403(3)$ \\
\hline C9 & $\mathrm{C} 10$ & $1.393(4)$ & $\mathrm{C} 40$ & $\mathrm{C} 41$ & $1.387(4)$ \\
\hline
\end{tabular}




\begin{tabular}{|c|c|c|c|c|c|}
\hline $\mathrm{C} 10$ & $\mathrm{C} 11$ & $1.380(4)$ & $\mathrm{C} 41$ & $\mathrm{C} 42$ & $1.391(3)$ \\
\hline C11 & $\mathrm{C} 12$ & $1.385(4)$ & $\mathrm{C} 42$ & $\mathrm{C} 43$ & $1.387(4)$ \\
\hline C11 & $\mathrm{C} 14$ & $1.507(4)$ & $\mathrm{C} 42$ & $\mathrm{C} 45$ & $1.492(3)$ \\
\hline C12 & $\mathrm{C} 13$ & $1.384(3)$ & $\mathrm{C} 43$ & $\mathrm{C} 44$ & $1.383(4)$ \\
\hline $\mathrm{C} 15$ & $\mathrm{C} 16$ & $1.537(4)$ & $\mathrm{C} 46$ & $\mathrm{C} 47$ & $1.537(3)$ \\
\hline C16 & $\mathrm{C} 17$ & $1.534(4)$ & $\mathrm{C} 47$ & $\mathrm{C} 48$ & $1.531(3)$ \\
\hline C17 & $\mathrm{C} 18$ & $1.547(3)$ & $\mathrm{C} 48$ & $\mathrm{C} 49$ & $1.551(3)$ \\
\hline $\mathrm{C} 18$ & $\mathrm{C} 20$ & $1.519(3)$ & $\mathrm{C} 49$ & C51 & $1.514(3)$ \\
\hline $\mathrm{C} 18$ & C19 & $1.549(3)$ & $\mathrm{C} 49$ & $\mathrm{C} 50$ & $1.552(3)$ \\
\hline $\mathrm{C} 20$ & $\mathrm{C} 25$ & $1.387(4)$ & $\mathrm{C} 51$ & $\mathrm{C} 52$ & $1.389(3)$ \\
\hline $\mathrm{C} 20$ & $\mathrm{C} 21$ & $1.388(4)$ & $\mathrm{C} 51$ & C56 & $1.398(3)$ \\
\hline $\mathrm{C} 21$ & $\mathrm{C} 22$ & $1.391(4)$ & $\mathrm{C} 52$ & $\mathrm{C} 53$ & $1.389(4)$ \\
\hline $\mathrm{C} 22$ & $\mathrm{C} 23$ & $1.385(4)$ & $\mathrm{C} 53$ & C54 & $1.390(4)$ \\
\hline $\mathrm{C} 23$ & $\mathrm{C} 24$ & $1.394(3)$ & $\mathrm{C} 54$ & C55 & $1.400(3)$ \\
\hline $\mathrm{C} 23$ & $\mathrm{C} 26$ & $1.481(4)$ & $\mathrm{C} 54$ & C57 & $1.482(3)$ \\
\hline $\mathrm{C} 24$ & $\mathrm{C} 25$ & $1.384(4)$ & $\mathrm{C} 55$ & C56 & $1.383(3)$ \\
\hline $\mathrm{C} 26$ & $\mathrm{C} 27$ & $1.396(4)$ & $\mathrm{C} 57$ & C62 & $1.394(3)$ \\
\hline C26 & $\mathrm{C} 31$ & $1.395(4)$ & $\mathrm{C} 57$ & $\mathrm{C} 58$ & $1.402(3)$ \\
\hline $\mathrm{C} 27$ & $\mathrm{C} 28$ & $1.385(4)$ & $\mathrm{C} 58$ & C59 & $1.384(4)$ \\
\hline $\mathrm{C} 28$ & $\mathrm{C} 29$ & $1.380(5)$ & $\mathrm{C} 59$ & C60 & $1.387(4)$ \\
\hline $\mathrm{C} 29$ & $\mathrm{C} 30$ & $1.384(5)$ & C60 & C61 & $1.387(4)$ \\
\hline C30 & $\mathrm{C} 31$ & $1.383(4)$ & C61 & C62 & $1.388(4)$ \\
\hline
\end{tabular}

Table S12 Bond Angles for $6 \mathbf{a}$.

\begin{tabular}{|c|c|c|c|c|c|c|c|}
\hline Atom & Ator & Atom & Angle $/^{\circ}$ & Atom & Aton & Atom & Angle $/^{\circ}$ \\
\hline $\mathrm{C} 1$ & Sil & $\mathrm{C} 15$ & $113.40(11)$ & $\mathrm{C} 32$ & $\mathrm{Si} 2$ & C39 & $112.08(10)$ \\
\hline $\mathrm{C} 1$ & Sil & $\mathrm{C} 8$ & $109.22(10)$ & $\mathrm{C} 32$ & $\mathrm{Si} 2$ & $\mathrm{C} 46$ & $109.44(11)$ \\
\hline $\mathrm{C} 15$ & Si1 & $\mathrm{C} 8$ & $109.51(10)$ & C39 & $\mathrm{Si} 2$ & $\mathrm{C} 46$ & $112.70(11)$ \\
\hline $\mathrm{C} 1$ & Si1 & $\mathrm{C} 18$ & $110.54(10)$ & C32 & $\mathrm{Si} 2$ & $\mathrm{C} 49$ & $113.56(10)$ \\
\hline $\mathrm{C} 15$ & Sil & $\mathrm{C} 18$ & $96.39(11)$ & C39 & $\mathrm{Si} 2$ & $\mathrm{C} 49$ & $112.15(10)$ \\
\hline $\mathrm{C} 8$ & Si1 & $\mathrm{C} 18$ & $117.40(10)$ & $\mathrm{C} 46$ & $\mathrm{Si} 2$ & $\mathrm{C} 49$ & $95.90(11)$ \\
\hline $\mathrm{C} 2$ & $\mathrm{C} 1$ & C6 & $117.5(2)$ & C33 & C32 & C37 & $117.4(2)$ \\
\hline
\end{tabular}




\begin{tabular}{|c|c|c|c|c|c|c|c|}
\hline $\mathrm{C} 2$ & $\mathrm{C} 1$ & Si1 & $120.93(19)$ & $\mathrm{C} 33$ & $\mathrm{C} 32$ & $\mathrm{Si} 2$ & $123.23(17)$ \\
\hline C6 & $\mathrm{C} 1$ & Si1 & $120.80(17)$ & C37 & $\mathrm{C} 32$ & $\mathrm{Si} 2$ & $118.97(17)$ \\
\hline $\mathrm{C} 3$ & $\mathrm{C} 2$ & $\mathrm{C} 1$ & $121.5(2)$ & C34 & $\mathrm{C} 33$ & $\mathrm{C} 32$ & $121.3(2)$ \\
\hline $\mathrm{C} 4$ & $\mathrm{C} 3$ & $\mathrm{C} 2$ & $119.7(2)$ & $\mathrm{C} 35$ & $\mathrm{C} 34$ & C33 & $120.1(2)$ \\
\hline $\mathrm{C} 3$ & $\mathrm{C} 4$ & $\mathrm{C} 5$ & $120.4(2)$ & C34 & $\mathrm{C} 35$ & $\mathrm{C} 36$ & $120.1(2)$ \\
\hline $\mathrm{C} 3$ & $\mathrm{C} 4$ & $\mathrm{C} 7$ & $119.2(2)$ & C34 & $\mathrm{C} 35$ & C38 & $119.8(2)$ \\
\hline $\mathrm{C} 5$ & $\mathrm{C} 4$ & $\mathrm{C} 7$ & $120.4(2)$ & C36 & $\mathrm{C} 35$ & C38 & $120.1(2)$ \\
\hline $\mathrm{C} 4$ & $\mathrm{C} 5$ & C6 & $119.4(2)$ & C35 & $\mathrm{C} 36$ & C37 & $119.4(2)$ \\
\hline $\mathrm{C} 5$ & C6 & $\mathrm{C} 1$ & $121.4(2)$ & $\mathrm{C} 36$ & $\mathrm{C} 37$ & C32 & $121.6(2)$ \\
\hline $\mathrm{F} 1$ & $\mathrm{C} 7$ & $\mathrm{~F} 2$ & $106.3(2)$ & F8 & $\mathrm{C} 38$ & F7 & $106.6(2)$ \\
\hline $\mathrm{F} 1$ & $\mathrm{C} 7$ & F3 & $105.9(3)$ & F8 & $\mathrm{C} 38$ & F9 & $106.2(3)$ \\
\hline $\mathrm{F} 2$ & $\mathrm{C} 7$ & F3 & $105.6(2)$ & F7 & $\mathrm{C} 38$ & F9 & $105.2(3)$ \\
\hline $\mathrm{F} 1$ & $\mathrm{C} 7$ & $\mathrm{C} 4$ & $112.3(2)$ & F8 & $\mathrm{C} 38$ & $\mathrm{C} 35$ & $113.0(2)$ \\
\hline $\mathrm{F} 2$ & $\mathrm{C} 7$ & $\mathrm{C} 4$ & $112.6(2)$ & F7 & $\mathrm{C} 38$ & $\mathrm{C} 35$ & $112.1(2)$ \\
\hline F3 & $\mathrm{C} 7$ & $\mathrm{C} 4$ & $113.5(2)$ & F9 & $\mathrm{C} 38$ & C35 & $113.1(2)$ \\
\hline $\mathrm{C} 9$ & $\mathrm{C} 8$ & $\mathrm{C} 13$ & $117.7(2)$ & $\mathrm{C} 40$ & C39 & $\mathrm{C} 44$ & $117.2(2)$ \\
\hline C9 & $\mathrm{C} 8$ & Si1 & $122.10(18)$ & $\mathrm{C} 40$ & C39 & $\mathrm{Si} 2$ & $123.80(18)$ \\
\hline $\mathrm{C} 13$ & $\mathrm{C} 8$ & Si1 & $119.45(18)$ & $\mathrm{C} 44$ & C39 & $\mathrm{Si} 2$ & $118.83(18)$ \\
\hline $\mathrm{C} 8$ & C9 & $\mathrm{C} 10$ & $120.9(2)$ & $\mathrm{C} 41$ & $\mathrm{C} 40$ & C39 & $121.8(2)$ \\
\hline $\mathrm{C} 11$ & $\mathrm{C} 10$ & C9 & $119.8(2)$ & $\mathrm{C} 40$ & C41 & $\mathrm{C} 42$ & $119.5(2)$ \\
\hline $\mathrm{C} 10$ & $\mathrm{C} 11$ & $\mathrm{C} 12$ & $120.5(2)$ & $\mathrm{C} 43$ & $\mathrm{C} 42$ & $\mathrm{C} 41$ & $120.1(2)$ \\
\hline $\mathrm{C} 10$ & $\mathrm{C} 11$ & $\mathrm{C} 14$ & $120.8(3)$ & $\mathrm{C} 43$ & $\mathrm{C} 42$ & $\mathrm{C} 45$ & $119.7(2)$ \\
\hline $\mathrm{C} 12$ & $\mathrm{C} 11$ & $\mathrm{C} 14$ & $118.7(3)$ & $\mathrm{C} 41$ & $\mathrm{C} 42$ & $\mathrm{C} 45$ & $120.2(2)$ \\
\hline $\mathrm{C} 13$ & $\mathrm{C} 12$ & $\mathrm{C} 11$ & $119.3(2)$ & $\mathrm{C} 44$ & $\mathrm{C} 43$ & $\mathrm{C} 42$ & $119.7(2)$ \\
\hline $\mathrm{C} 12$ & $\mathrm{C} 13$ & $\mathrm{C} 8$ & $121.8(2)$ & $\mathrm{C} 43$ & $\mathrm{C} 44$ & C39 & $121.7(2)$ \\
\hline F4 & $\mathrm{C} 14$ & F5 & 105.2(3) & F12 & $\mathrm{C} 45$ & $\mathrm{~F} 11$ & $107.3(3)$ \\
\hline F4 & $\mathrm{C} 14$ & F6 & 105.4(3) & $\mathrm{F} 12$ & $\mathrm{C} 45$ & F10 & $104.5(2)$ \\
\hline F5 & $\mathrm{C} 14$ & F6 & $108.3(3)$ & F11 & $\mathrm{C} 45$ & F10 & $105.9(2)$ \\
\hline F4 & $\mathrm{C} 14$ & $\mathrm{C} 11$ & $112.7(2)$ & F12 & $\mathrm{C} 45$ & $\mathrm{C} 42$ & $112.3(2)$ \\
\hline F5 & $\mathrm{C} 14$ & $\mathrm{C} 11$ & $111.8(3)$ & F11 & $\mathrm{C} 45$ & $\mathrm{C} 42$ & $114.0(2)$ \\
\hline F6 & $\mathrm{C} 14$ & $\mathrm{C} 11$ & $112.9(3)$ & F10 & $\mathrm{C} 45$ & $\mathrm{C} 42$ & $112.3(2)$ \\
\hline C16 & $\mathrm{C} 15$ & Si1 & $104.02(16)$ & $\mathrm{C} 47$ & $\mathrm{C} 46$ & $\mathrm{Si} 2$ & $104.12(16)$ \\
\hline \multicolumn{8}{|c|}{ S92 } \\
\hline
\end{tabular}




\begin{tabular}{|c|c|c|c|c|c|c|c|}
\hline $\mathrm{C} 17$ & $\mathrm{C} 16$ & $\mathrm{C} 15$ & $107.8(2)$ & $\mathrm{C} 48$ & $\mathrm{C} 47$ & $\mathrm{C} 46$ & $107.79(19)$ \\
\hline $\mathrm{C} 16$ & $\mathrm{C} 17$ & $\mathrm{C} 18$ & $110.1(2)$ & $\mathrm{C} 47$ & $\mathrm{C} 48$ & $\mathrm{C} 49$ & $109.8(2)$ \\
\hline $\mathrm{C} 20$ & $\mathrm{C} 18$ & $\mathrm{C} 17$ & $114.3(2)$ & $\mathrm{C} 51$ & C49 & $\mathrm{C} 48$ & $114.4(2)$ \\
\hline $\mathrm{C} 20$ & $\mathrm{C} 18$ & $\mathrm{C} 19$ & $107.5(2)$ & C51 & C49 & $\mathrm{C} 50$ & $108.44(19)$ \\
\hline $\mathrm{C} 17$ & $\mathrm{C} 18$ & C19 & $108.5(2)$ & $\mathrm{C} 48$ & $\mathrm{C} 49$ & $\mathrm{C} 50$ & $108.5(2)$ \\
\hline $\mathrm{C} 20$ & $\mathrm{C} 18$ & Si1 & $116.63(16)$ & C51 & $\mathrm{C} 49$ & $\mathrm{Si} 2$ & $114.77(15)$ \\
\hline $\mathrm{C} 17$ & $\mathrm{C} 18$ & Si1 & $100.53(16)$ & $\mathrm{C} 48$ & $\mathrm{C} 49$ & $\mathrm{Si} 2$ & $100.77(15)$ \\
\hline C19 & $\mathrm{C} 18$ & Si1 & 109.02(17) & $\mathrm{C} 50$ & C49 & $\mathrm{Si} 2$ & $109.63(16)$ \\
\hline $\mathrm{C} 25$ & $\mathrm{C} 20$ & $\mathrm{C} 21$ & $116.8(2)$ & $\mathrm{C} 52$ & $\mathrm{C} 51$ & $\mathrm{C} 56$ & $116.8(2)$ \\
\hline $\mathrm{C} 25$ & $\mathrm{C} 20$ & $\mathrm{C} 18$ & $121.0(2)$ & C52 & $\mathrm{C} 51$ & C49 & $123.7(2)$ \\
\hline $\mathrm{C} 21$ & $\mathrm{C} 20$ & $\mathrm{C} 18$ & $122.1(2)$ & C56 & $\mathrm{C} 51$ & $\mathrm{C} 49$ & $119.5(2)$ \\
\hline $\mathrm{C} 20$ & $\mathrm{C} 21$ & $\mathrm{C} 22$ & $121.2(2)$ & C51 & $\mathrm{C} 52$ & $\mathrm{C} 53$ & $121.5(2)$ \\
\hline $\mathrm{C} 23$ & $\mathrm{C} 22$ & $\mathrm{C} 21$ & $121.9(2)$ & C54 & $\mathrm{C} 53$ & $\mathrm{C} 52$ & $121.6(2)$ \\
\hline $\mathrm{C} 22$ & $\mathrm{C} 23$ & $\mathrm{C} 24$ & $116.9(2)$ & $\mathrm{C} 53$ & $\mathrm{C} 54$ & C55 & $117.2(2)$ \\
\hline $\mathrm{C} 22$ & $\mathrm{C} 23$ & $\mathrm{C} 26$ & $121.7(2)$ & $\mathrm{C} 53$ & $\mathrm{C} 54$ & C57 & $121.7(2)$ \\
\hline $\mathrm{C} 24$ & $\mathrm{C} 23$ & $\mathrm{C} 26$ & $121.3(2)$ & C55 & $\mathrm{C} 54$ & $\mathrm{C} 57$ & $121.1(2)$ \\
\hline $\mathrm{C} 25$ & $\mathrm{C} 24$ & $\mathrm{C} 23$ & $120.9(2)$ & C56 & $\mathrm{C} 55$ & C54 & $120.8(2)$ \\
\hline $\mathrm{C} 24$ & $\mathrm{C} 25$ & $\mathrm{C} 20$ & $122.3(2)$ & C55 & $\mathrm{C} 56$ & C51 & $122.0(2)$ \\
\hline $\mathrm{C} 27$ & $\mathrm{C} 26$ & $\mathrm{C} 31$ & $117.5(3)$ & C62 & $\mathrm{C} 57$ & C58 & $117.6(2)$ \\
\hline $\mathrm{C} 27$ & $\mathrm{C} 26$ & $\mathrm{C} 23$ & $121.7(2)$ & C62 & $\mathrm{C} 57$ & C54 & $121.8(2)$ \\
\hline C31 & $\mathrm{C} 26$ & $\mathrm{C} 23$ & $120.7(2)$ & $\mathrm{C} 58$ & $\mathrm{C} 57$ & C54 & $120.6(2)$ \\
\hline $\mathrm{C} 28$ & $\mathrm{C} 27$ & $\mathrm{C} 26$ & $121.0(3)$ & C59 & $\mathrm{C} 58$ & C57 & $120.8(3)$ \\
\hline $\mathrm{C} 29$ & $\mathrm{C} 28$ & $\mathrm{C} 27$ & $120.5(3)$ & C58 & $\mathrm{C} 59$ & C60 & $120.9(3)$ \\
\hline $\mathrm{C} 28$ & $\mathrm{C} 29$ & $\mathrm{C} 30$ & $119.4(3)$ & C61 & C60 & C59 & $118.9(3)$ \\
\hline $\mathrm{C} 31$ & $\mathrm{C} 30$ & $\mathrm{C} 29$ & $120.2(3)$ & C60 & C61 & C62 & $120.3(3)$ \\
\hline $\mathrm{C} 30$ & $\mathrm{C} 31$ & $\mathrm{C} 26$ & $121.4(3)$ & C61 & C62 & C57 & $121.5(2)$ \\
\hline
\end{tabular}

Table S13 Torsion Angles for $6 \mathbf{a}$.

\begin{tabular}{|c|c|c|c|c|}
\hline A $\mathbf{B}$ & C D & Angle $/^{\circ}$ & $\begin{array}{llll}\mathbf{A} & \mathbf{B} & \mathbf{C} & \mathbf{D}\end{array}$ & Angle $/^{\circ}$ \\
\hline C15 Si1 & $\mathrm{C} 1 \mathrm{C} 2$ & $20.6(2)$ & C39Si2 C32 C33 & $-20.3(2)$ \\
\hline C8 Si1 & $\mathrm{C} 1 \mathrm{C} 2$ & $143.0(2)$ & $\mathrm{C} 46 \mathrm{Si} 2 \mathrm{C} 32 \mathrm{C} 33$ & $105.5(2)$ \\
\hline C18 Si1 & $\mathrm{C} 1 \mathrm{C} 2$ & $-86.3(2)$ & C49Si2 C32 C33 & $-148.7(2)$ \\
\hline
\end{tabular}




\begin{tabular}{|c|c|c|c|c|}
\hline C15 Si1 & $1 \mathrm{C} 1 \mathrm{C} 6$ & $-169.77(18)$ & C39Si2 C32 C37 & $166.77(19)$ \\
\hline C8 Sil & $1 \mathrm{C} 1 \mathrm{C} 6$ & $-47.4(2)$ & C46Si2 C32 C37 & $-67.4(2)$ \\
\hline C18 Si1 & $\mathrm{C} 1 \mathrm{C} 6$ & $83.3(2)$ & C49Si2 C32 C37 & $38.4(2)$ \\
\hline C6 C1 & $\mathrm{C} 2 \quad \mathrm{C} 3$ & $-2.7(4)$ & C37 C32 C33 C34 & $0.7(4)$ \\
\hline Sil C1 & $\mathrm{C} 2 \mathrm{C} 3$ & $167.3(2)$ & Si2 C32 C33 C34 & $-172.3(2)$ \\
\hline $\mathrm{C} 1 \mathrm{C} 2$ & $\mathrm{C} 3 \mathrm{C} 4$ & $-0.1(4)$ & C32C33 C34 C35 & $0.5(4)$ \\
\hline $\mathrm{C} 2 \mathrm{C} 3$ & $\mathrm{C} 4 \mathrm{C} 5$ & $2.7(4)$ & C33C34 C35 C36 & $-1.2(4)$ \\
\hline $\mathrm{C} 2 \mathrm{C} 3$ & $\mathrm{C} 4 \mathrm{C} 7$ & $-176.5(3)$ & C33C34 C35 C38 & $178.5(2)$ \\
\hline $\mathrm{C} 3 \mathrm{C} 4$ & $\mathrm{C} 5 \mathrm{C} 6$ & $-2.4(4)$ & $\mathrm{C} 34 \mathrm{C} 35 \mathrm{C} 36 \mathrm{C} 37$ & $0.6(4)$ \\
\hline $\mathrm{C} 7 \mathrm{C} 4$ & C5 C6 & $176.8(2)$ & C38C35 C36 C37 & $-179.1(2)$ \\
\hline $\mathrm{C} 4 \mathrm{C} 5$ & $\mathrm{C} 6 \mathrm{C} 1$ & $-0.6(4)$ & C35C36C37 C32 & $0.7(4)$ \\
\hline $\mathrm{C} 2 \mathrm{C} 1$ & $\mathrm{C} 6 \mathrm{C} 5$ & $3.1(4)$ & C33C32 C37 C36 & $-1.3(4)$ \\
\hline Sil C1 & C6 C5 & $-166.90(18)$ & Si2 C32 C37 C36 & $172.00(19)$ \\
\hline $\mathrm{C} 3 \mathrm{C} 4$ & C7 F1 & $50.6(4)$ & C34C35 C38 F8 & $46.3(4)$ \\
\hline $\mathrm{C} 5 \mathrm{C} 4$ & C7 F1 & $-128.6(3)$ & C36C35 C38 F8 & $-134.0(3)$ \\
\hline $\mathrm{C} 3 \mathrm{C} 4$ & $\mathrm{C} 7 \mathrm{~F} 2$ & $-69.4(3)$ & C34C35 C38 F7 & $-74.3(3)$ \\
\hline $\mathrm{C} 5 \mathrm{C} 4$ & $\mathrm{C} 7 \mathrm{~F} 2$ & 111.4(3) & $\mathrm{C} 36 \mathrm{C} 35 \mathrm{C} 38 \mathrm{~F} 7$ & $105.4(3)$ \\
\hline $\mathrm{C} 3 \mathrm{C} 4$ & C7 F3 & $170.7(3)$ & $\mathrm{C} 34 \mathrm{C} 35 \mathrm{C} 38 \mathrm{~F} 9$ & $167.0(3)$ \\
\hline $\mathrm{C} 5 \mathrm{C} 4$ & C7 $\mathrm{F} 3$ & $-8.5(4)$ & $\mathrm{C} 36 \mathrm{C} 35 \mathrm{C} 38 \mathrm{~F} 9$ & $-13.3(4)$ \\
\hline C1 Si1 & $1 \mathrm{C} 8 \mathrm{C} 9$ & 156.66(19) & C32Si2 C39 C40 & $-45.5(2)$ \\
\hline C15 Si1 & $1 \mathrm{C} 8 \mathrm{C} 9$ & $-78.6(2)$ & $\mathrm{C} 46 \mathrm{Si} 2 \mathrm{C} 39 \mathrm{C} 40$ & $-169.48(19)$ \\
\hline C18 Si1 & $1 \mathrm{C} 8 \mathrm{C} 9$ & $29.8(2)$ & C49Si2 C39C40 & $83.6(2)$ \\
\hline C1 Sil & $1 \mathrm{C} 8 \mathrm{C} 13$ & $-33.3(2)$ & C32Si2 C39C44 & $138.89(19)$ \\
\hline C15 Si1 & $1 \mathrm{C} 8 \mathrm{C} 13$ & $91.4(2)$ & C46Si2 C39 C44 & $14.9(2)$ \\
\hline C18 Si1 & $\mathrm{C} 8 \mathrm{C} 13$ & $-160.09(17)$ & C49Si2 C39C44 & $-92.0(2)$ \\
\hline $\mathrm{C} 13 \mathrm{C} 8$ & C9 $\mathrm{C} 10$ & $-0.6(4)$ & C44C39 C40 C41 & $0.8(4)$ \\
\hline Sil C8 & C9 $\mathrm{C} 10$ & $169.7(2)$ & Si2 C39C40 C41 & $-174.84(19)$ \\
\hline $\mathrm{C} 8 \mathrm{C} 9$ & $\mathrm{C} 10 \mathrm{C} 11$ & $-0.9(4)$ & C39C40C41 C42 & $0.0(4)$ \\
\hline $\mathrm{C} 9 \mathrm{C} 1 \mathrm{C}$ & $0 \mathrm{C} 11 \mathrm{C} 12$ & $1.8(4)$ & C40C41 C42 C43 & $-1.0(4)$ \\
\hline $\mathrm{C} 9 \mathrm{C} 1 \mathrm{C}$ & $0 \mathrm{C} 11 \mathrm{C} 14$ & $-177.7(3)$ & C40C41 C42 C45 & $177.1(2)$ \\
\hline $\mathrm{C} 10 \mathrm{C} 1$ & $1 \mathrm{C} 12 \mathrm{C} 13$ & $-1.2(4)$ & C41 C42 C43 C44 & $1.1(4)$ \\
\hline $\mathrm{C} 14 \mathrm{C} 1$ & $1 \mathrm{C} 12 \mathrm{C} 13$ & $178.2(2)$ & C45 C42 C43 C44 & $-177.0(2)$ \\
\hline
\end{tabular}




\begin{tabular}{|c|c|c|c|}
\hline $\mathrm{C} 11 \mathrm{C} 12 \mathrm{C} 13 \mathrm{C} 8$ & $-0.3(4)$ & C42C43 C44 C39 & $-0.3(4)$ \\
\hline C9 C8 C13C12 & $1.1(4)$ & $\mathrm{C} 40 \mathrm{C} 39 \mathrm{C} 44 \mathrm{C} 43$ & $-0.7(4)$ \\
\hline Si1 C8 C13C12 & $-169.34(18)$ & $\mathrm{Si} 2 \mathrm{C} 39 \mathrm{C} 44 \mathrm{C} 43$ & $175.2(2)$ \\
\hline C10C11 C14F4 & $124.8(3)$ & $\mathrm{C} 43 \mathrm{C} 42 \mathrm{C} 45 \mathrm{~F} 12$ & $77.2(3)$ \\
\hline $\mathrm{C} 12 \mathrm{C} 11 \mathrm{C} 14 \mathrm{~F} 4$ & $-54.6(4)$ & $\mathrm{C} 41 \mathrm{C} 42 \mathrm{C} 45 \mathrm{~F} 12$ & $-100.9(3)$ \\
\hline C10C11 C14F5 & $-116.9(4)$ & C43C42 C45 F11 & $-160.5(3)$ \\
\hline C12C11 C14F5 & $63.7(4)$ & $\mathrm{C} 41 \mathrm{C} 42 \mathrm{C} 45 \mathrm{~F} 11$ & $21.4(4)$ \\
\hline C10C11 C14F6 & $5.5(4)$ & $\mathrm{C} 43 \mathrm{C} 42 \mathrm{C} 45 \mathrm{~F} 10$ & $-40.2(3)$ \\
\hline C12C11 C14F6 & $-173.9(3)$ & $\mathrm{C} 41 \mathrm{C} 42 \mathrm{C} 45 \mathrm{~F} 10$ & $141.8(2)$ \\
\hline C1 Si1 C15C16 & $-104.24(19)$ & $\mathrm{C} 32 \mathrm{Si} 2 \mathrm{C} 46 \mathrm{C} 47$ & $129.06(17)$ \\
\hline C8 Si1 C15C16 & $133.50(17)$ & C39Si2 C46C47 & $-105.51(18)$ \\
\hline C18 Si1 C15C16 & $11.4(2)$ & $\mathrm{C} 49 \mathrm{Si} 2 \mathrm{C} 46 \mathrm{C} 47$ & $11.50(19)$ \\
\hline Si1 C15C16C17 & $-35.6(2)$ & $\mathrm{Si} 2 \mathrm{C} 46 \mathrm{C} 47 \mathrm{C} 48$ & $-36.1(2)$ \\
\hline $\mathrm{C} 15 \mathrm{C} 16 \mathrm{C} 17 \mathrm{C} 18$ & $51.8(3)$ & $\mathrm{C} 46 \mathrm{C} 47 \mathrm{C} 48 \mathrm{C} 49$ & $52.2(3)$ \\
\hline $\mathrm{C} 16 \mathrm{C} 17 \mathrm{C} 18 \mathrm{C} 20$ & $-164.6(2)$ & C47 C48 C49 C51 & $-163.0(2)$ \\
\hline $\mathrm{C} 16 \mathrm{C} 17 \mathrm{C} 18 \mathrm{C} 19$ & $75.4(3)$ & C47 C48 C49 C50 & $75.8(2)$ \\
\hline C16C17 C18 Si1 & $-38.9(2)$ & C47 C48 C49 Si2 & $-39.3(2)$ \\
\hline $\mathrm{C} 17 \mathrm{C} 18 \mathrm{C} 20 \mathrm{C} 25$ & 163.1(2) & C48 C49 C51 C52 & $-10.8(3)$ \\
\hline $\mathrm{C} 19 \mathrm{C} 18 \mathrm{C} 20 \mathrm{C} 25$ & $-76.4(3)$ & C50C49 C51 C52 & $110.4(3)$ \\
\hline Si1 C 18 C $20 \mathrm{C} 25$ & $46.4(3)$ & Si2 C49 C51 C52 & $-126.6(2)$ \\
\hline $\mathrm{C} 17 \mathrm{C} 18 \mathrm{C} 20 \mathrm{C} 21$ & $-20.7(3)$ & C48C49 C51 C56 & $171.4(2)$ \\
\hline C19C18 C20C21 & $99.8(3)$ & C50C49 C51 C56 & $-67.4(3)$ \\
\hline Si1 C 18 C $20 \mathrm{C} 21$ & $-137.5(2)$ & Si2 C49 C51 C56 & $55.6(3)$ \\
\hline $\mathrm{C} 25 \mathrm{C} 20 \mathrm{C} 21 \mathrm{C} 22$ & $1.0(4)$ & C56C51 C52 C53 & $0.6(4)$ \\
\hline $\mathrm{C} 18 \mathrm{C} 20 \mathrm{C} 21 \mathrm{C} 22$ & $-175.4(2)$ & C49C51 C52 C53 & $-177.2(2)$ \\
\hline $\mathrm{C} 20 \mathrm{C} 21 \mathrm{C} 22 \mathrm{C} 23$ & $-1.7(4)$ & C51 C52 C53 C54 & $0.3(4)$ \\
\hline $\mathrm{C} 21 \mathrm{C} 22 \mathrm{C} 23 \mathrm{C} 24$ & $1.2(4)$ & C52C53 C54 C55 & $-1.0(4)$ \\
\hline C21 C22 C23 C26 & $-178.3(2)$ & C52C53 C54 C57 & $177.9(2)$ \\
\hline $\mathrm{C} 22 \mathrm{C} 23 \mathrm{C} 24 \mathrm{C} 25$ & $0.0(4)$ & C53C54 C55 C56 & $0.7(4)$ \\
\hline $\mathrm{C} 26 \mathrm{C} 23 \mathrm{C} 24 \mathrm{C} 25$ & $179.5(3)$ & C57 C54 C55 C56 & $-178.2(2)$ \\
\hline $\mathrm{C} 23 \mathrm{C} 24 \mathrm{C} 25 \mathrm{C} 20$ & $-0.7(5)$ & C54C55 C56 C51 & $0.3(4)$ \\
\hline $\mathrm{C} 21 \mathrm{C} 20 \mathrm{C} 25 \mathrm{C} 24$ & $0.2(4)$ & C52C51 C56C55 & $-0.9(3)$ \\
\hline
\end{tabular}




$\begin{array}{lrlr}\mathrm{C} 18 \mathrm{C} 20 \mathrm{C} 25 \mathrm{C} 24 & 176.6(3) & \mathrm{C} 49 \mathrm{C} 51 \mathrm{C} 56 \mathrm{C} 55 & 177.0(2) \\ \mathrm{C} 22 \mathrm{C} 23 \mathrm{C} 26 \mathrm{C} 27 & -41.5(4) & \mathrm{C} 53 \mathrm{C} 54 \mathrm{C} 57 \mathrm{C} 62 & 144.2(3) \\ \mathrm{C} 24 \mathrm{C} 23 \mathrm{C} 26 \mathrm{C} 27 & 139.0(3) & \mathrm{C} 55 \mathrm{C} 54 \mathrm{C} 57 \mathrm{C} 62 & -37.0(3) \\ \mathrm{C} 22 \mathrm{C} 23 \mathrm{C} 26 \mathrm{C} 31 & 138.8(3) & \mathrm{C} 53 \mathrm{C} 54 \mathrm{C} 57 \mathrm{C} 58 & -35.8(4) \\ \mathrm{C} 24 \mathrm{C} 23 \mathrm{C} 26 \mathrm{C} 31 & -40.7(4) & \mathrm{C} 55 \mathrm{C} 54 \mathrm{C} 57 \mathrm{C} 58 & 143.0(3) \\ \mathrm{C} 31 \mathrm{C} 26 \mathrm{C} 27 \mathrm{C} 28 & -0.6(4) & \mathrm{C} 62 \mathrm{C} 57 \mathrm{C} 58 \mathrm{C} 59 & -0.7(4) \\ \mathrm{C} 23 \mathrm{C} 26 \mathrm{C} 27 \mathrm{C} 28 & 179.6(3) & \mathrm{C} 54 \mathrm{C} 57 \mathrm{C} 58 \mathrm{C} 59 & 179.3(3) \\ \mathrm{C} 26 \mathrm{C} 27 \mathrm{C} 28 \mathrm{C} 29 & 0.0(5) & \mathrm{C} 57 \mathrm{C} 58 \mathrm{C} 59 \mathrm{C} 60 & 0.1(5) \\ \mathrm{C} 27 \mathrm{C} 28 \mathrm{C} 29 \mathrm{C} 30 & 0.7(5) & \mathrm{C} 58 \mathrm{C} 59 \mathrm{C} 60 \mathrm{C} 61 & 0.4(5) \\ \mathrm{C} 28 \mathrm{C} 29 \mathrm{C} 30 \mathrm{C} 31 & -0.8(5) & \mathrm{C} 59 \mathrm{C} 60 \mathrm{C} 61 \mathrm{C} 62 & -0.3(4) \\ \mathrm{C} 29 \mathrm{C} 30 \mathrm{C} 31 \mathrm{C} 26 & 0.2(5) & \mathrm{C} 60 \mathrm{C} 61 \mathrm{C} 62 \mathrm{C} 57 & -0.3(4) \\ \mathrm{C} 27 \mathrm{C} 26 \mathrm{C} 31 \mathrm{C} 30 & 0.5(4) & \mathrm{C} 58 \mathrm{C} 57 \mathrm{C} 62 \mathrm{C} 61 & 0.8(4) \\ \mathrm{C} 23 \mathrm{C} 26 \mathrm{C} 31 \mathrm{C} 30 & -179.7(3) & \mathrm{C} 54 \mathrm{C} 57 \mathrm{C} 62 \mathrm{C} 61 & -179.2(2)\end{array}$

\subsection{X-ray structure of $6 \mathrm{~d}$}

Single crystals of $\mathbf{6 d}$ were obtained by dissolving it with least amount of petroleum ether. The mixture was then evaporated at room temperature. Single crystal X-ray diffraction data of $6 \mathbf{d}$ was collected at $180 \mathrm{~K}$ on a Rigaku XtaLAB PRO 007HF(Mo) diffractometer, with Mo K $\alpha$ radiation $(\lambda=0.71073 \AA$ ). Data reduction and empirical absorption correction were performed using the CrysAlisPro program. The structure was solved by a dual-space algorithm using SHELXT program or Olex. All non-hydrogen atoms could be located directly from the difference Fourier maps. Framework hydrogen atoms were placed geometrically and constrained using the riding model to the parent atoms. Final structure refinement was done using the SHELXL program by minimizing the sum of squared deviations of $\mathrm{F}^{2}$ using a full-matrix technique. Refined structure and crystallographic parameters are summarized in Figure S8 and Tables S14-20. The Diamond diagram was drawn by Mercury.

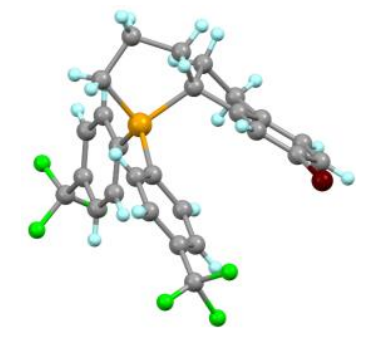

Figure S8 Diamond diagram of 6 d. Ellipsoids are set at 50\% probability.

Table 14 Crystal data and structure refinement for $\mathbf{6 d}$.

Identification code 3

Empirical formula $\mathrm{C}_{26} \mathrm{H}_{21} \mathrm{BrF}_{6} \mathrm{Si}$ 


\begin{tabular}{|c|c|}
\hline Formula weight & 555.43 \\
\hline Temperature/K & $179.99(10)$ \\
\hline Crystal system & orthorhombic \\
\hline Space group & $\mathrm{P} 2{ }_{1} 2{ }_{1}{ }_{1}$ \\
\hline $\mathrm{a} / \AA$ & $10.8869(2)$ \\
\hline $\mathrm{b} / \AA$ & $12.6893(2)$ \\
\hline $\mathrm{c} / \AA$ & $17.6200(3)$ \\
\hline$\alpha /^{\circ}$ & 90 \\
\hline$\beta /{ }^{\circ}$ & 90 \\
\hline$\gamma /{ }^{\circ}$ & 90 \\
\hline Volume $/ \AA^{3}$ & $2434.15(7)$ \\
\hline $\mathrm{Z}$ & 4 \\
\hline$\rho_{\text {calc }} \mathrm{g} / \mathrm{cm}^{3}$ & 1.516 \\
\hline$\mu / \mathrm{mm}^{-1}$ & 1.796 \\
\hline $\mathrm{F}(000)$ & 1120.0 \\
\hline Crystal size $/ \mathrm{mm}^{3}$ & $0.23 \times 0.19 \times 0.13$ \\
\hline Radiation & $\operatorname{Mo~K\alpha }(\lambda=0.71073)$ \\
\hline \multicolumn{2}{|c|}{$2 \Theta$ range for data collection $/{ }^{\circ} 3.956$ to 54.968} \\
\hline Index ranges & $-14 \leq \mathrm{h} \leq 14,-16 \leq \mathrm{k} \leq 16,-22 \leq 1 \leq 22$ \\
\hline Reflections collected & 58009 \\
\hline Independent reflections & $5578\left[\mathrm{R}_{\text {int }}=0.0347, \mathrm{R}_{\text {sigma }}=0.0194\right]$ \\
\hline Data/restraints/parameters & $5578 / 51 / 317$ \\
\hline Goodness-of-fit on $\mathrm{F}^{2}$ & 1.027 \\
\hline Final $\mathrm{R}$ indexes $[\mathrm{I}>=2 \sigma(\mathrm{I})]$ & $\mathrm{R}_{1}=0.0324, \mathrm{wR}_{2}=0.0788$ \\
\hline Final $\mathrm{R}$ indexes [all data] & $\mathrm{R}_{1}=0.0371, \mathrm{wR}_{2}=0.0808$ \\
\hline \multicolumn{2}{|c|}{ Largest diff. peak/hole / e $\AA^{-3} 0.38 /-0.26$} \\
\hline Flack parameter & $-0.004(2)$ \\
\hline
\end{tabular}

Table S15 Fractional Atomic Coordinates $\left(\times 10^{4}\right)$ and Equivalent Isotropic Displacement Parameters $\left(\AA^{2} \times 10^{3}\right)$ for $6 \mathbf{d}$. $\mathrm{U}_{\text {eq }}$ is defined as $1 / 3$ of of the trace of the orthogonalised $\mathrm{U}_{\mathrm{IJ}}$ tensor.
Atom
$x$
$y$
$z$
$\mathbf{U}(\mathbf{e q})$ 


\begin{tabular}{|c|c|c|c|c|}
\hline $\mathrm{Br} 1$ & $6729.0(4)$ & 10911.3(3) & $8415.4(2)$ & $48.58(12)$ \\
\hline Sil & $8404.4(8)$ & $7441.7(6)$ & $6117.3(5)$ & $26.72(17)$ \\
\hline $\mathrm{F} 1$ & $6720(4)$ & $2369(2)$ & $6779.2(14)$ & $83.4(10)$ \\
\hline $\mathrm{F} 2$ & $6051(3)$ & $2523.0(19)$ & $5648.2(16)$ & $64.6(7)$ \\
\hline F3 & $7905(3)$ & $2089.6(19)$ & $5841(2)$ & $78.6(9)$ \\
\hline $\mathrm{F} 4 \mathrm{~A}$ & $2870(4)$ & $9966(5)$ & $6100(6)$ & $100(2)$ \\
\hline F4B & $3023(6)$ & $9907(7)$ & $6875(7)$ & $100(2)$ \\
\hline F5A & $3838(9)$ & $10807(8)$ & 6948(3) & 133(4) \\
\hline F5B & $3298(14)$ & $10528(11)$ & $5778(5)$ & 133(4) \\
\hline F6A & $3961(5)$ & $11208(4)$ & $5783(4)$ & $83.9(16)$ \\
\hline F6B & $4113(8)$ & $11206(6)$ & $6722(7)$ & $83.9(16)$ \\
\hline $\mathrm{C} 1$ & $9514(3)$ & $7780(3)$ & $5335.9(19)$ & $36.9(8)$ \\
\hline $\mathrm{C} 2$ & 10678(3) & $8170(3)$ & $5751(2)$ & 46.1(9) \\
\hline $\mathrm{C} 3$ & 10258(4) & 8701(3) & $6488(2)$ & $42.9(8)$ \\
\hline $\mathrm{C} 4$ & $9437(3)$ & 7934(3) & 6927.9(19) & $30.4(7)$ \\
\hline $\mathrm{C} 5$ & 10177(3) & 7034(3) & $7324(2)$ & $41.5(8)$ \\
\hline C6 & $9377(4)$ & 6688(3) & 7993(2) & $45.8(9)$ \\
\hline $\mathrm{C} 7$ & $8696(3)$ & 7673(3) & 8198.2(19) & $36.9(8)$ \\
\hline $\mathrm{C} 8$ & $8069(4)$ & 7924(3) & $8856(2)$ & $44.8(9)$ \\
\hline $\mathrm{C} 9$ & $7487(4)$ & $8885(3)$ & $8922(2)$ & $44.4(9)$ \\
\hline $\mathrm{C} 10$ & $7549(3)$ & 9594(3) & $8325(2)$ & $35.4(7)$ \\
\hline $\mathrm{C} 11$ & $8174(3)$ & $9369(2)$ & $7659.2(18)$ & $31.5(6)$ \\
\hline $\mathrm{C} 12$ & $8749(3)$ & $8392(3)$ & 7598.6(18) & $31.4(7)$ \\
\hline $\mathrm{C} 13$ & $7972(3)$ & $6004(2)$ & $6132.7(17)$ & $29.4(6)$ \\
\hline $\mathrm{C} 14$ & $6753(3)$ & $5664(3)$ & $6159(2)$ & $38.4(7)$ \\
\hline $\mathrm{C} 15$ & $6455(3)$ & $4608(3)$ & $6143(2)$ & $43.6(8)$ \\
\hline $\mathrm{C} 16$ & $7369(3)$ & $3861(3)$ & 6094(2) & $36.7(7)$ \\
\hline $\mathrm{C} 17$ & $8578(3)$ & $4159(3)$ & $6060(2)$ & $41.9(8)$ \\
\hline $\mathrm{C} 18$ & $8879(3)$ & $5225(3)$ & $6068(2)$ & $38.3(8)$ \\
\hline C19 & $7015(4)$ & 2718(3) & 6092(2) & $46.3(9)$ \\
\hline $\mathrm{C} 20$ & 6974(3) & $8272(2)$ & $6125.1(18)$ & $27.2(6)$ \\
\hline $\mathrm{C} 21$ & $6797(3)$ & $9099(2)$ & $5614.4(17)$ & $32.0(6)$ \\
\hline
\end{tabular}




$\begin{array}{lrrrr}\mathrm{C} 22 & 5800(3) & 9781(3) & 5677(2) & 37.4(8) \\ \mathrm{C} 23 & 4961(3) & 9628(3) & 6250(2) & 40.1(8) \\ \mathrm{C} 24 & 8813(3) & 6760(2) & 43.6(9) \\ \mathrm{C} 25 & 5097(3) & 8147(3) & 6699(2) & 36.8(7) \\ \mathrm{C} 26 & 6094(3) & 10368(4) & 6328(3) & 57.6(12)\end{array}$

Table S16 Anisotropic Displacement Parameters $\left(\AA^{2} \times 10^{3}\right)$ for $\mathbf{6 d}$. The Anisotropic displacement factor exponent takes the form: $-2 \pi^{2}\left[h^{2} a^{* 2} U_{11}+2 h k a * b * U_{12}+\ldots\right]$.

\begin{tabular}{|c|c|c|c|c|c|c|}
\hline Atom & $\mathbf{U}_{11}$ & $\mathbf{U}_{22}$ & $\mathbf{U}_{33}$ & $\mathbf{U}_{23}$ & $\mathbf{U}_{13}$ & $\mathbf{U}_{12}$ \\
\hline Br1 & $42.48(19)$ & $50.5(2)$ & $52.7(2)$ & $-15.42(17)$ & 12.60(17) & $-1.09(18)$ \\
\hline Si1 & $24.4(4)$ & $27.2(4)$ & $28.6(4)$ & $0.4(3)$ & $2.6(3)$ & $1.4(3)$ \\
\hline $\mathrm{F} 1$ & $151(3)$ & $48.9(14)$ & $50.0(14)$ & $13.0(11)$ & $-4.1(18)$ & $-32.1(18)$ \\
\hline $\mathrm{F} 2$ & $79.1(18)$ & $44.0(13)$ & $70.8(17)$ & $-6.1(12)$ & $-21.4(15)$ & $-16.1(12)$ \\
\hline F3 & $86(2)$ & $34.0(12)$ & $116(2)$ & $-14.3(14)$ & $-5.0(18)$ & $6.4(13)$ \\
\hline F4A & $24.9(16)$ & $79(3)$ & 197(7) & $-4(5)$ & $-3(3)$ & $11.5(17)$ \\
\hline F4B & $24.9(16)$ & $79(3)$ & 197(7) & $-4(5)$ & $-3(3)$ & $11.5(17)$ \\
\hline F5A & $163(7)$ & $173(7)$ & $62(3)$ & $-52(4)$ & $-33(4)$ & $132(6)$ \\
\hline F5B & $163(7)$ & $173(7)$ & $62(3)$ & $-52(4)$ & $-33(4)$ & $132(6)$ \\
\hline F6A & $56(2)$ & $47(2)$ & $149(4)$ & $4(3)$ & 11(3) & $26.2(18)$ \\
\hline F6B & $56(2)$ & $47(2)$ & $149(4)$ & $4(3)$ & 11(3) & $26.2(18)$ \\
\hline $\mathrm{C} 1$ & $36.2(18)$ & $41.1(18)$ & $33.4(17)$ & $3.6(15)$ & $9.9(14)$ & $7.5(15)$ \\
\hline $\mathrm{C} 2$ & $37.4(19)$ & $48(2)$ & $53(2)$ & $-2.3(18)$ & $21.0(17)$ & $-7.1(16)$ \\
\hline $\mathrm{C} 3$ & $38.3(18)$ & $42.7(19)$ & $48(2)$ & $-4.3(16)$ & $15.4(17)$ & $-14.9(15)$ \\
\hline $\mathrm{C} 4$ & $24.8(15)$ & $31.8(16)$ & $34.6(16)$ & $-2.1(13)$ & $1.2(12)$ & $-4.0(12)$ \\
\hline C5 & $33.2(18)$ & $42.9(19)$ & $48(2)$ & $-4.9(16)$ & $-10.2(16)$ & $2.4(15)$ \\
\hline C6 & $52(2)$ & $41(2)$ & $44(2)$ & $5.3(16)$ & $-11.6(18)$ & $-4.2(17)$ \\
\hline $\mathrm{C} 7$ & $38.6(17)$ & $39.2(18)$ & $32.9(17)$ & $3.5(14)$ & $-8.5(13)$ & $-12.1(14)$ \\
\hline $\mathrm{C} 8$ & $61(2)$ & $47(2)$ & $27.3(16)$ & $4.4(15)$ & $-0.8(17)$ & $-20.5(18)$ \\
\hline C9 & $49(2)$ & $55(2)$ & $29.1(16)$ & $-8.3(16)$ & $8.4(15)$ & $-21.5(18)$ \\
\hline $\mathrm{C} 10$ & $29.6(15)$ & $39.3(17)$ & $37.4(18)$ & $-8.6(15)$ & $3.2(14)$ & $-8.0(13)$ \\
\hline C11 & $31.2(15)$ & $32.9(15)$ & $30.6(14)$ & $-1.7(11)$ & $0.9(13)$ & $-8.6(13)$ \\
\hline $\mathrm{C} 12$ & $28.5(15)$ & $36.2(17)$ & $29.4(16)$ & $-1.6(13)$ & $-2.2(12)$ & $-12.2(13)$ \\
\hline
\end{tabular}




$\begin{array}{rrrrrrr}\text { C13 } & 30.2(15) & 28.6(14) & 29.5(14) & 0.5(13) & -2.5(12) & 0.7(12) \\ \text { C14 } & 31.7(15) & 34.5(16) & 49.1(19) & -1.1(14) & -2.2(17) & 5.2(14) \\ \text { C15 } & 34.4(19) & 41.8(19) & 55(2) & 0.1(17) & -2.8(16) & -5.4(14) \\ \text { C16 } & 49(2) & 31.4(17) & 30.1(16) & 1.2(13) & -6.1(15) & -0.2(14) \\ \text { C17 } & 42.7(19) & 30.7(16) & 52(2) & -5.7(16) & -8.7(16) & 9.0(15) \\ \text { C18 } & 30.9(16) & 36.3(18) & 48(2) & -6.5(16) & -4.1(15) & 2.7(14) \\ \text { C19 } & 63(3) & 33.1(18) & 42.4(19) & -3.0(16) & -7.3(18) & -4.7(16) \\ \text { C20 } & 23.7(15) & 28.4(14) & 29.4(15) & -4.5(12) & 0.9(12) & 1.4(11) \\ \text { C21 } & 30.6(14) & 29.4(14) & 35.9(15) & -2.6(13) & 0.7(14) & -2.6(15) \\ \text { C22 } & 33.9(17) & 27.8(16) & 51(2) & -0.9(15) & -9.7(16) & 3.4(13) \\ \text { C23 } & 26.9(16) & 37.6(18) & 56(2) & -18.0(16) & -7.9(15) & 4.7(14) \\ \text { C24 } & 28.7(17) & 55(2) & 47(2) & -11.3(17) & 9.5(15) & -1.4(15) \\ \text { C25 } & 33.5(17) & 40.8(18) & 36.0(18) & 0.7(15) & 2.6(14) & -0.1(14) \\ \text { C26 } & 35(2) & 51(2) & 87(4) & -21(2) & -7(2) & 9.0(18)\end{array}$

Table S17 Bond Lengths for $6 \mathbf{d}$.

Atom Atom

Br1 C10

Si1 C20

Si1 C1

Si1 C13

Si1 C4

F1 C19

F2 C19

F3 $\quad$ C19

F4A C26

F4B C26

F5A $\mathrm{C} 26$

F5B C26

F6A C26

F6B C26

C1 $\mathrm{C} 2$

\section{Length/Å}

1.902(4)

$1.880(3)$

1.881(3)

1.884(3)

1.922(3)

1.329(5)

1.332(5)

1.331(5)

$1.290(7)$

1.473(10)

$1.228(7)$

1.185(9)

1.437(7)

1.292(10)

1.545(5)

\section{Atom Atom Length/Å}

C7 C8

$1.382(5)$

C7 $\mathrm{C} 12$

1.397(5)

C8 $\mathrm{C} 9$

1.379(6)

C9 $\mathrm{C} 10$

1.386(5)

C10 C11

1.386(5)

C11 C12

$1.392(5)$

C13 C14

1.396(5)

C13 C18

1.402(4)

C14 C15

1.379(5)

C15 C16

1.377(5)

C16 $\mathrm{C} 17$

$1.370(5)$

C16 C19

1.501(5)

1.392(5)

1.396(4)

C20 C21

1.402(5) 


$\begin{array}{llllll}\mathrm{C} 2 & \mathrm{C} 3 & 1.533(5) & \mathrm{C} 21 & \mathrm{C} 22 & 1.392(5) \\ \mathrm{C} 3 & \mathrm{C} 4 & 1.532(5) & \mathrm{C} 22 & \mathrm{C} 23 & 1.375(5) \\ \mathrm{C} 4 & \mathrm{C} 12 & 1.515(5) & \mathrm{C} 23 & \mathrm{C} 24 & 1.379(6) \\ \mathrm{C} 4 & \mathrm{C} 5 & 1.563(5) & \mathrm{C} 23 & \mathrm{C} 26 & 1.500(5) \\ \mathrm{C} 5 & \mathrm{C} 6 & 1.529(6) & \mathrm{C} 24 & \mathrm{C} 25 & 1.380(5) \\ \mathrm{C} 6 & \mathrm{C} 7 & 1.499(5) & & & \end{array}$

Table S18 Bond Angles for 6 d.

\begin{tabular}{|c|c|c|c|c|c|c|c|}
\hline Atom & Atom & n Atom & Angle $/^{\circ}$ & Aton & Aton & n Atom & Angle $/^{\circ}$ \\
\hline $\mathrm{C} 20$ & Si1 & $\mathrm{C} 1$ & $114.17(15)$ & $\mathrm{C} 17$ & $\mathrm{C} 16$ & $\mathrm{C} 15$ & $120.5(3)$ \\
\hline $\mathrm{C} 20$ & Si1 & $\mathrm{C} 13$ & $109.61(14)$ & $\mathrm{C} 17$ & $\mathrm{C} 16$ & C19 & $120.9(3)$ \\
\hline $\mathrm{C} 1$ & Sil & $\mathrm{C} 13$ & $113.06(15)$ & $\mathrm{C} 15$ & $\mathrm{C} 16$ & C19 & $118.6(3)$ \\
\hline $\mathrm{C} 20$ & Si1 & $\mathrm{C} 4$ & $107.26(14)$ & $\mathrm{C} 16$ & $\mathrm{C} 17$ & $\mathrm{C} 18$ & $119.6(3)$ \\
\hline $\mathrm{C} 1$ & Si1 & $\mathrm{C} 4$ & $95.40(15)$ & $\mathrm{C} 17$ & $\mathrm{C} 18$ & $\mathrm{C} 13$ & $121.3(3)$ \\
\hline $\mathrm{C} 13$ & Si1 & $\mathrm{C} 4$ & $116.76(14)$ & $\mathrm{F} 1$ & $\mathrm{C} 19$ & F3 & $106.2(3)$ \\
\hline $\mathrm{C} 2$ & $\mathrm{C} 1$ & Sil & $104.7(2)$ & $\mathrm{F} 1$ & $\mathrm{C} 19$ & $\mathrm{~F} 2$ & $106.4(4)$ \\
\hline $\mathrm{C} 3$ & $\mathrm{C} 2$ & $\mathrm{C} 1$ & 107.3(3) & F3 & C19 & $\mathrm{F} 2$ & $105.5(3)$ \\
\hline $\mathrm{C} 4$ & $\mathrm{C} 3$ & $\mathrm{C} 2$ & $108.9(3)$ & $\mathrm{F} 1$ & $\mathrm{C} 19$ & $\mathrm{C} 16$ & $112.4(3)$ \\
\hline $\mathrm{C} 12$ & $\mathrm{C} 4$ & $\mathrm{C} 3$ & $116.0(3)$ & F3 & C19 & $\mathrm{C} 16$ & $113.1(3)$ \\
\hline $\mathrm{C} 12$ & $\mathrm{C} 4$ & $\mathrm{C} 5$ & $100.8(3)$ & $\mathrm{F} 2$ & C19 & $\mathrm{C} 16$ & $112.6(3)$ \\
\hline $\mathrm{C} 3$ & $\mathrm{C} 4$ & $\mathrm{C} 5$ & $112.9(3)$ & $\mathrm{C} 21$ & $\mathrm{C} 20$ & $\mathrm{C} 25$ & $117.1(3)$ \\
\hline $\mathrm{C} 12$ & $\mathrm{C} 4$ & Sil & $114.5(2)$ & $\mathrm{C} 21$ & $\mathrm{C} 20$ & Si1 & $122.1(2)$ \\
\hline C3 & $\mathrm{C} 4$ & Sil & $99.9(2)$ & $\mathrm{C} 25$ & $\mathrm{C} 20$ & Si1 & $120.5(2)$ \\
\hline C5 & $\mathrm{C} 4$ & Sil & $113.3(2)$ & $\mathrm{C} 22$ & $\mathrm{C} 21$ & $\mathrm{C} 20$ & $121.6(3)$ \\
\hline C6 & $\mathrm{C} 5$ & $\mathrm{C} 4$ & 105.1(3) & $\mathrm{C} 23$ & $\mathrm{C} 22$ & $\mathrm{C} 21$ & $119.3(3)$ \\
\hline $\mathrm{C} 7$ & C6 & $\mathrm{C} 5$ & 103.2(3) & $\mathrm{C} 22$ & $\mathrm{C} 23$ & $\mathrm{C} 24$ & $120.9(3)$ \\
\hline $\mathrm{C} 8$ & $\mathrm{C} 7$ & $\mathrm{C} 12$ & $120.3(3)$ & $\mathrm{C} 22$ & $\mathrm{C} 23$ & $\mathrm{C} 26$ & $119.6(4)$ \\
\hline $\mathrm{C} 8$ & $\mathrm{C} 7$ & $\mathrm{C} 6$ & 129.7(3) & $\mathrm{C} 24$ & $\mathrm{C} 23$ & $\mathrm{C} 26$ & $119.5(4)$ \\
\hline $\mathrm{C} 12$ & $\mathrm{C} 7$ & C6 & $110.0(3)$ & $\mathrm{C} 23$ & $\mathrm{C} 24$ & $\mathrm{C} 25$ & $119.5(3)$ \\
\hline C9 & $\mathrm{C} 8$ & $\mathrm{C} 7$ & $120.1(3)$ & $\mathrm{C} 24$ & $\mathrm{C} 25$ & $\mathrm{C} 20$ & $121.6(3)$ \\
\hline $\mathrm{C} 8$ & $\mathrm{C} 9$ & $\mathrm{C} 10$ & $119.2(3)$ & F5A & $\mathrm{C} 26$ & F4A & $114.4(7)$ \\
\hline C9 & $\mathrm{C} 10$ & $\mathrm{C} 11$ & $122.2(3)$ & F5B & $\mathrm{C} 26$ & F6B & $113.5(8)$ \\
\hline
\end{tabular}




$\begin{array}{lllllllr}\text { C9 } & \text { C10 } & \text { Br1 } & 118.9(3) & \text { F5A } & \text { C26 } & \text { F6A } & 105.1(6) \\ \text { C11 } & \text { C10 } & \text { Br1 } & 118.9(3) & \text { F4A } & \text { C26 } & \text { F6A } & 97.3(5) \\ \text { C10 } & \text { C11 } & \text { C12 } & 118.0(3) & \text { F5B } & \text { C26 } & \text { F4B } & 104.5(9) \\ \text { C11 } & \text { C12 } & \text { C7 } & 120.3(3) & \text { F6B } & \text { C26 } & \text { F4B } & 95.4(6) \\ \text { C11 } & \text { C12 } & \text { C4 } & 128.6(3) & \text { F5B } & \text { C26 } & \text { C23 } & 117.2(5) \\ \text { C7 } & \text { C12 } & \text { C4 } & 111.1(3) & \text { F5A } & \text { C26 } & \text { C23 } & 113.9(5) \\ \text { C14 } & \text { C13 } & \text { C18 } & 117.0(3) & \text { F4A } & \text { C26 } & \text { C23 } & 113.2(4) \\ \text { C14 } & \text { C13 } & \text { Si1 } & 122.5(2) & \text { F6B } & \text { C26 } & \text { C23 } & 115.0(5) \\ \text { C18 } & \text { C13 } & \text { Si1 } & 120.3(2) & \text { F6A } & \text { C26 } & \text { C23 } & 111.3(4) \\ \text { C15 } & \text { C14 } & \text { C13 } & 121.5(3) & \text { F4B } & \text { C26 } & \text { C23 } & 108.1(5) \\ \text { C16 } & \text { C15 } & \text { C14 } & 120.0(3) & & & & \end{array}$

Table S19 Torsion Angles for $6 \mathbf{d}$.

\begin{tabular}{|c|c|c|c|c|c|c|c|c|}
\hline $\mathbf{A}$ & B & C & D & Angle $/^{\circ}$ & $\mathbf{A}$ & B & C D & Angle $/^{\circ}$ \\
\hline $\mathrm{C} 20$ & Sil & $\mathrm{C} 1$ & $\mathrm{C} 2$ & $116.4(2)$ & $\mathrm{C} 14$ & $\mathrm{C} 15$ & $\mathrm{C} 16 \mathrm{C} 17$ & $-0.2(6)$ \\
\hline $\mathrm{C} 13$ & Sil & $\mathrm{C} 1$ & $\mathrm{C} 2$ & $-117.4(2)$ & $\mathrm{C} 14$ & $\mathrm{C} 15$ & C16 C19 & $178.9(3)$ \\
\hline $\mathrm{C} 4$ & Sil & $\mathrm{C} 1$ & $\mathrm{C} 2$ & $4.7(3)$ & $\mathrm{C} 15$ & $\mathrm{C} 16$ & $\mathrm{C} 17 \mathrm{C} 18$ & $-0.6(6)$ \\
\hline Si1 & $\mathrm{C} 1$ & $\mathrm{C} 2$ & $\mathrm{C} 3$ & $-31.6(4)$ & C19 & $\mathrm{C} 16$ & $\mathrm{C} 17 \mathrm{C} 18$ & $-179.6(4)$ \\
\hline $\mathrm{C} 1$ & $\mathrm{C} 2$ & $\mathrm{C} 3$ & $\mathrm{C} 4$ & $53.4(4)$ & $\mathrm{C} 16$ & $\mathrm{C} 17$ & C18 C13 & $2.0(6)$ \\
\hline $\mathrm{C} 2$ & $\mathrm{C} 3$ & $\mathrm{C} 4$ & $\mathrm{C} 12$ & $-169.2(3)$ & $\mathrm{C} 14$ & $\mathrm{C} 13$ & C18 C17 & $-2.4(5)$ \\
\hline $\mathrm{C} 2$ & $\mathrm{C} 3$ & $\mathrm{C} 4$ & C5 & $75.2(4)$ & Si1 & $\mathrm{C} 13$ & $\mathrm{C} 18 \mathrm{C} 17$ & $-178.5(3)$ \\
\hline $\mathrm{C} 2$ & $\mathrm{C} 3$ & $\mathrm{C} 4$ & Sil & $-45.5(3)$ & $\mathrm{C} 17$ & $\mathrm{C} 16$ & $\mathrm{C} 19 \mathrm{~F} 1$ & $102.2(5)$ \\
\hline $\mathrm{C} 12$ & $\mathrm{C} 4$ & $\mathrm{C} 5$ & $\mathrm{C} 6$ & $30.1(3)$ & $\mathrm{C} 15$ & $\mathrm{C} 16$ & C19 F1 & $-76.9(5)$ \\
\hline $\mathrm{C} 3$ & $\mathrm{C} 4$ & $\mathrm{C} 5$ & C6 & $154.5(3)$ & $\mathrm{C} 17$ & $\mathrm{C} 16$ & C19 F3 & $-18.1(5)$ \\
\hline Si1 & $\mathrm{C} 4$ & $\mathrm{C} 5$ & C6 & $-92.7(3)$ & $\mathrm{C} 15$ & $\mathrm{C} 16$ & C19 F3 & $162.9(4)$ \\
\hline $\mathrm{C} 4$ & $\mathrm{C} 5$ & C6 & C7 & $-29.7(4)$ & $\mathrm{C} 17$ & $\mathrm{C} 16$ & $\mathrm{C} 19 \mathrm{~F} 2$ & $-137.6(4)$ \\
\hline $\mathrm{C} 5$ & C6 & $\mathrm{C} 7$ & $\mathrm{C} 8$ & $-162.7(4)$ & C15 & $\mathrm{C} 16$ & $\mathrm{C} 19 \mathrm{~F} 2$ & $43.4(5)$ \\
\hline $\mathrm{C} 5$ & C6 & $\mathrm{C} 7$ & $\mathrm{C} 12$ & $17.7(4)$ & $\mathrm{C} 1$ & Si1 & $\mathrm{C} 20 \mathrm{C} 21$ & $-3.1(3)$ \\
\hline $\mathrm{C} 12$ & $\mathrm{C} 7$ & $\mathrm{C} 8$ & C9 & $0.3(5)$ & $\mathrm{C} 13$ & Si1 & C20 C21 & $-131.1(2)$ \\
\hline C6 & $\mathrm{C} 7$ & $\mathrm{C} 8$ & C9 & $-179.2(4)$ & $\mathrm{C} 4$ & Si1 & C20 C21 & 101.3(3) \\
\hline $\mathrm{C} 7$ & $\mathrm{C} 8$ & $\mathrm{C} 9$ & $\mathrm{C} 10$ & $-0.5(5)$ & $\mathrm{C} 1$ & Si1 & $\mathrm{C} 20 \mathrm{C} 25$ & $-176.6(3)$ \\
\hline $\mathrm{C} 8$ & C9 & $\mathrm{C} 10$ & C11 & $0.3(5)$ & $\mathrm{C} 13$ & Si1 & $\mathrm{C} 20 \mathrm{C} 25$ & $55.4(3)$ \\
\hline
\end{tabular}




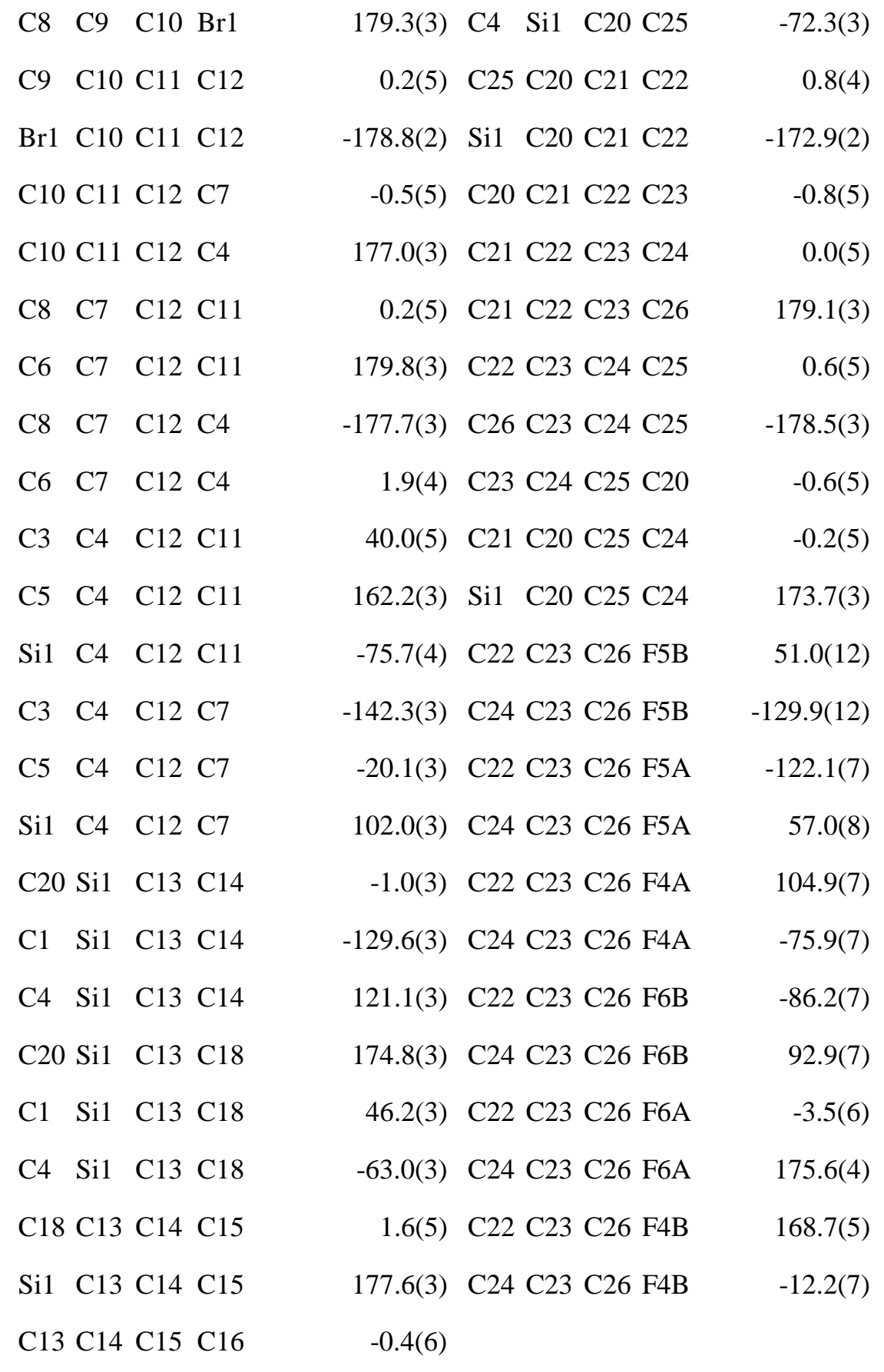

Table S20 Atomic Occupancy for $\mathbf{6 d}$.

$\begin{array}{lrlrcc}\text { Atom } & \text { Occupancy } & \text { Atom } & \text { Occupancy } & \text { Atom } & \text { Occupancy } \\ \text { F4A } & 0.583(4) & \text { F4B } & 0.417(4) & \text { F5A } & 0.583(4) \\ \text { F5B } & 0.417(4) & \text { F6A } & 0.583(4) & \text { F6B } & 0.417(4)\end{array}$


10. ${ }^{1} \mathrm{H},{ }^{13} \mathrm{C},{ }^{19} \mathrm{~F}$ NMR spectra and HPLC data of the products
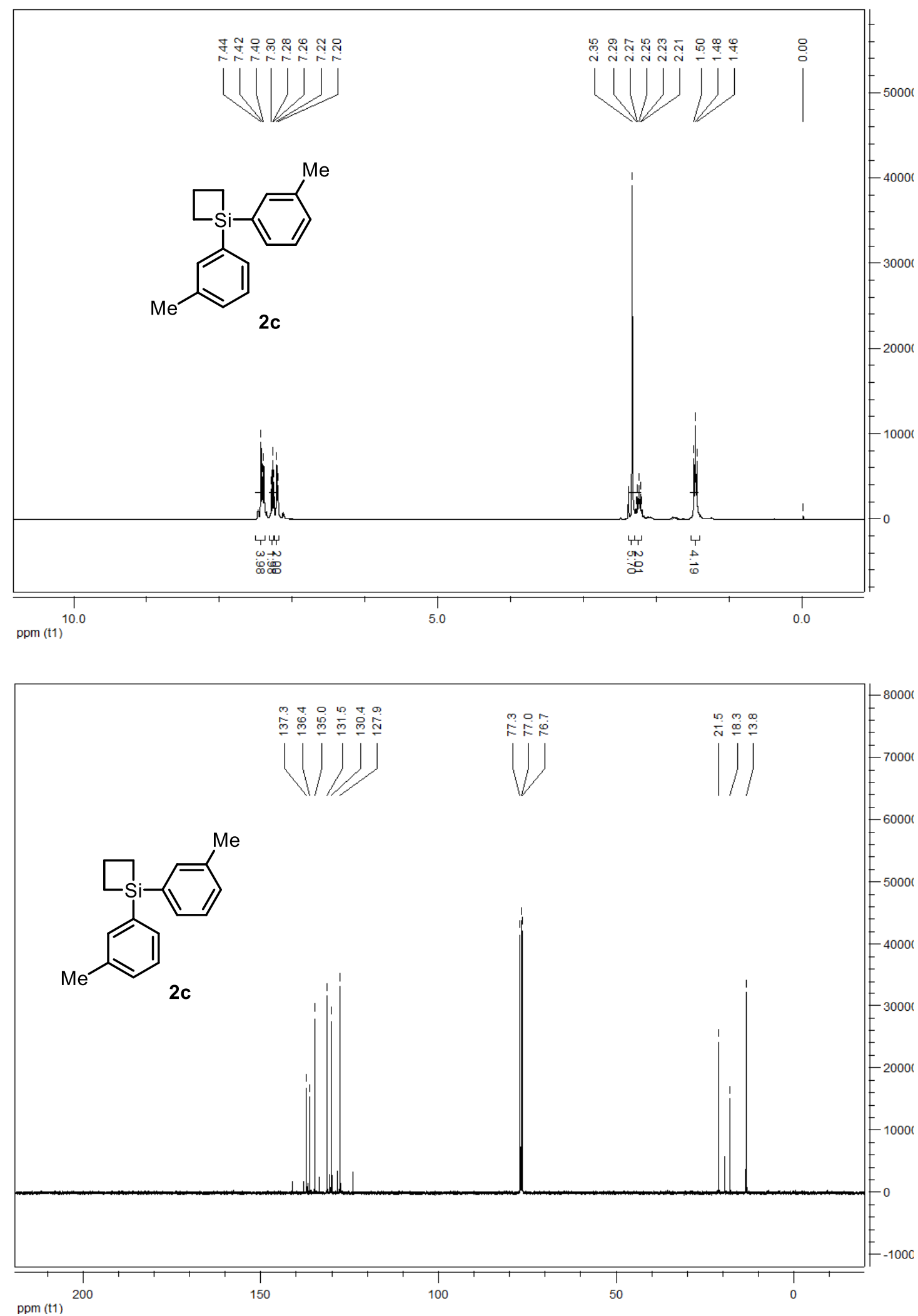

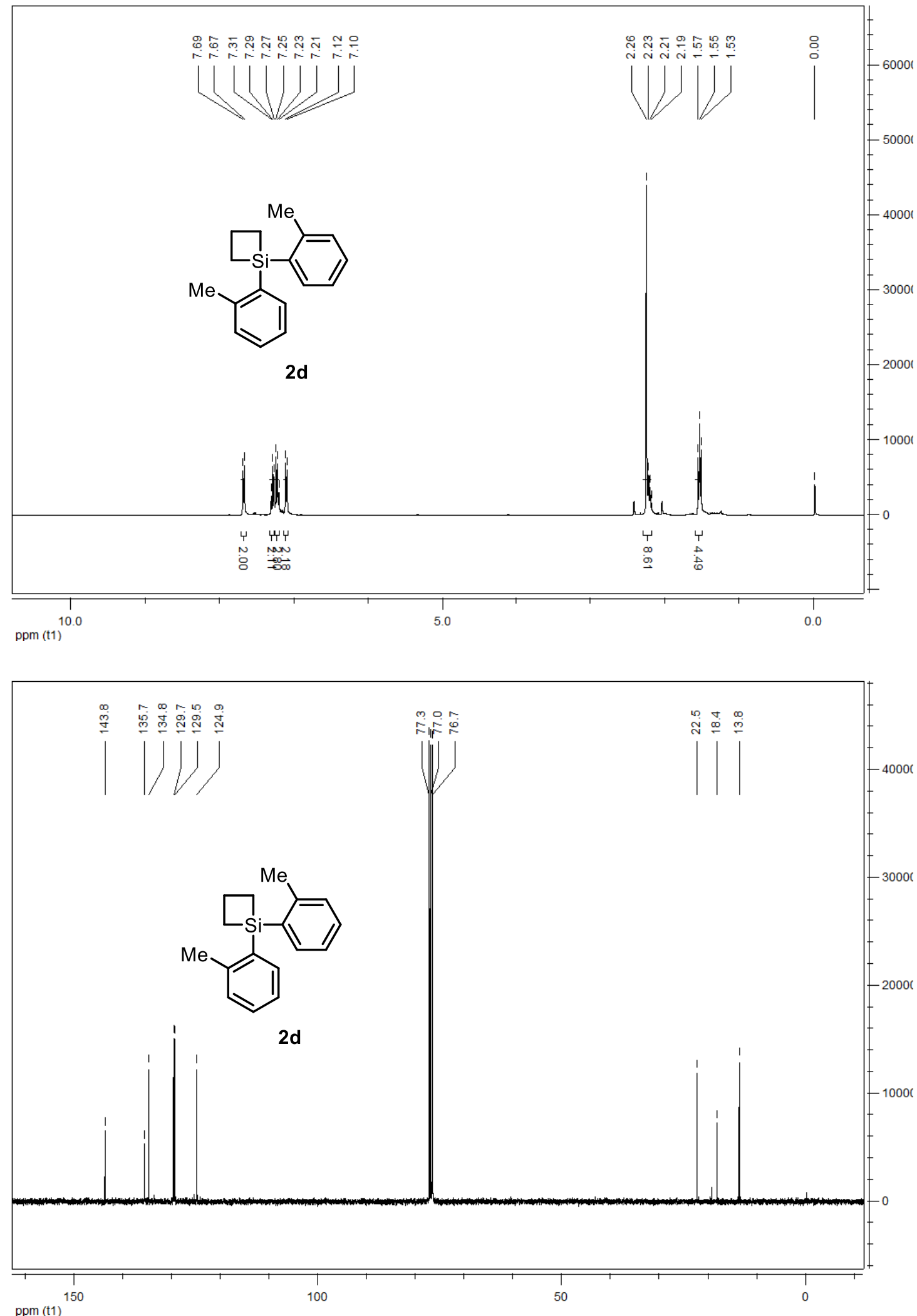

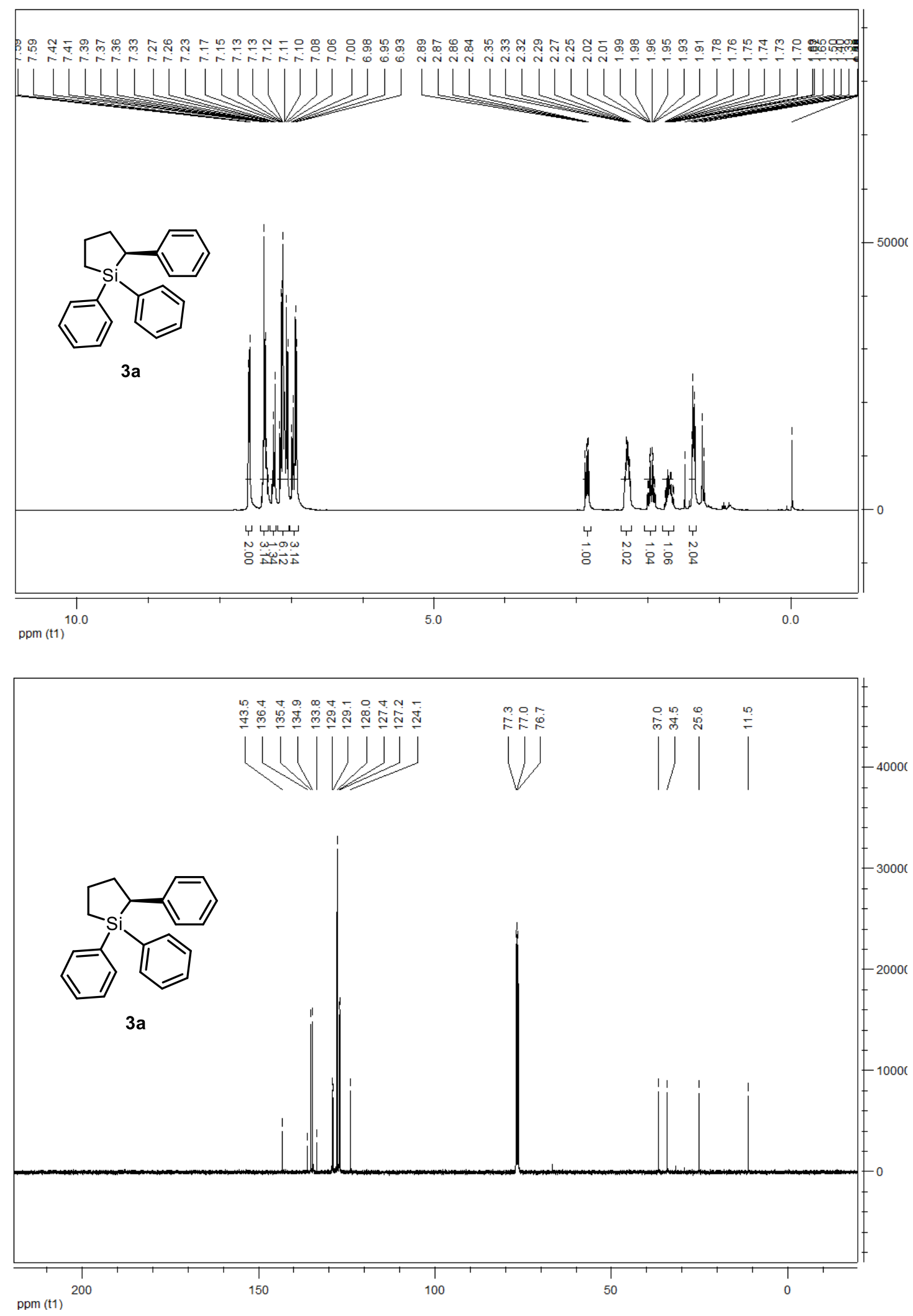
HPLC conditions: Daicel chiral column $O D-H+O D-H$, hexane $:{ }^{i} P r O H=100: 0,0.5 \mathrm{~mL} / \mathrm{min}, 25{ }^{\circ} \mathrm{C}$, wavelength $=220 \mathrm{~nm}$
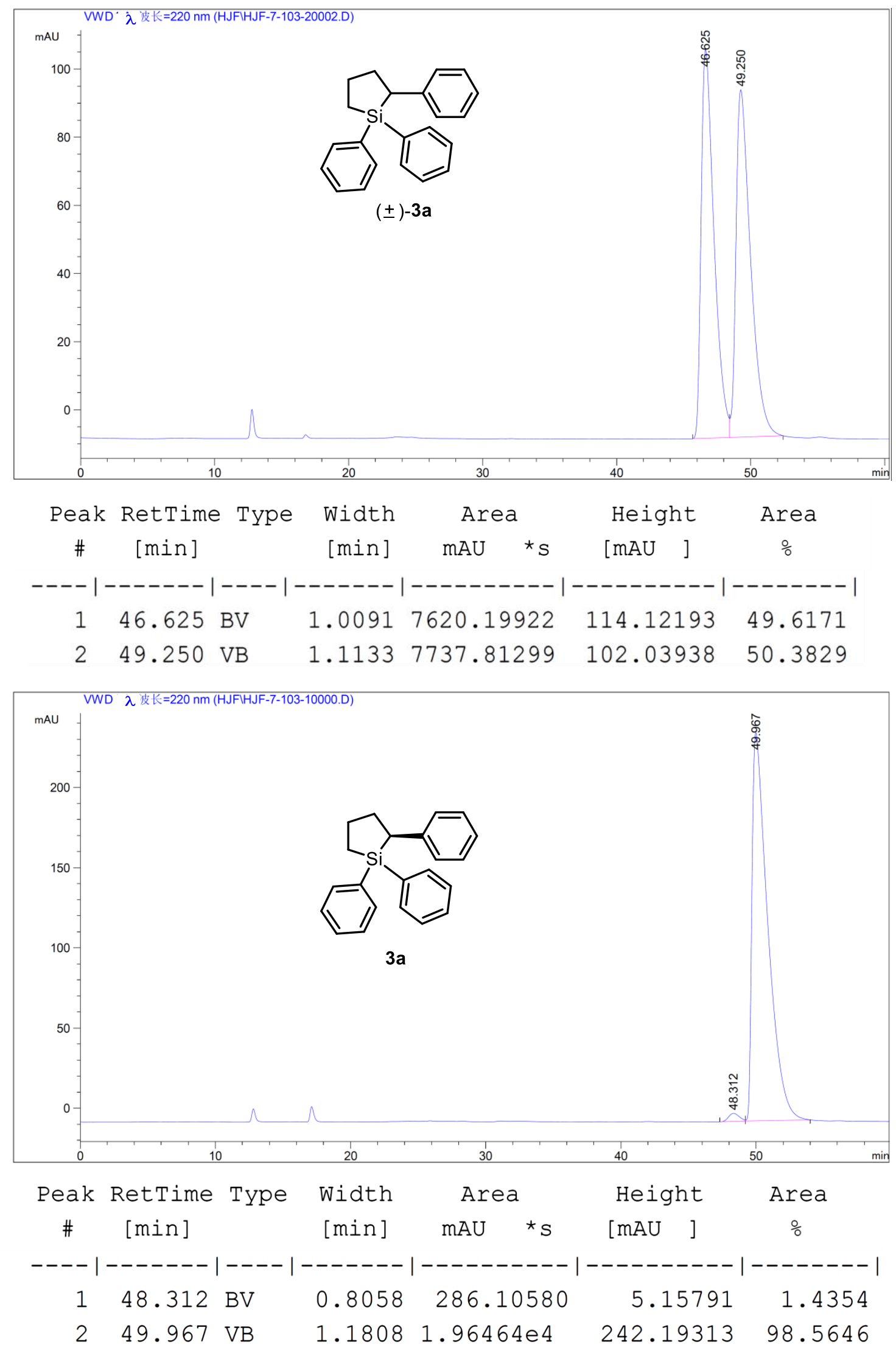

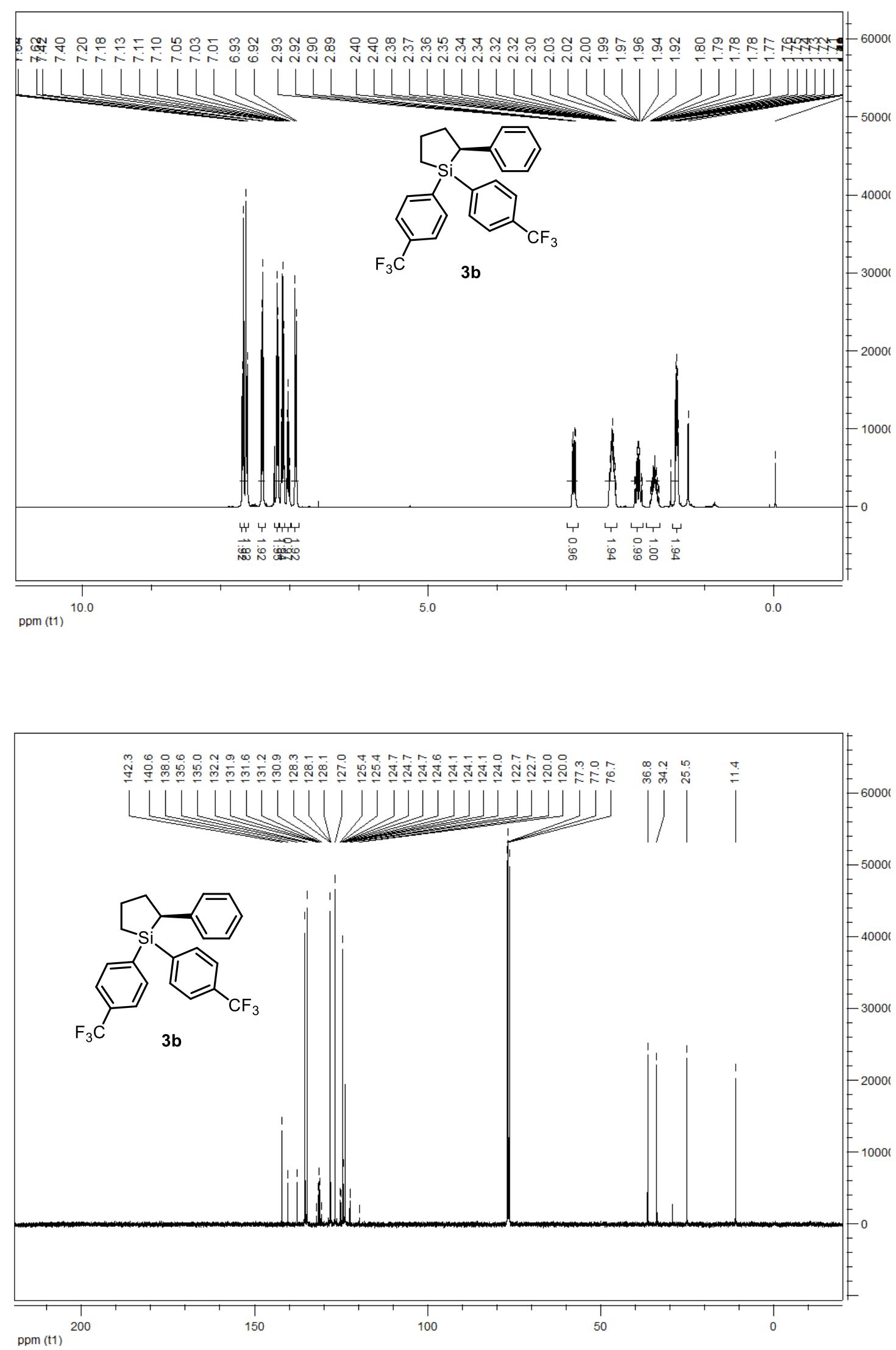


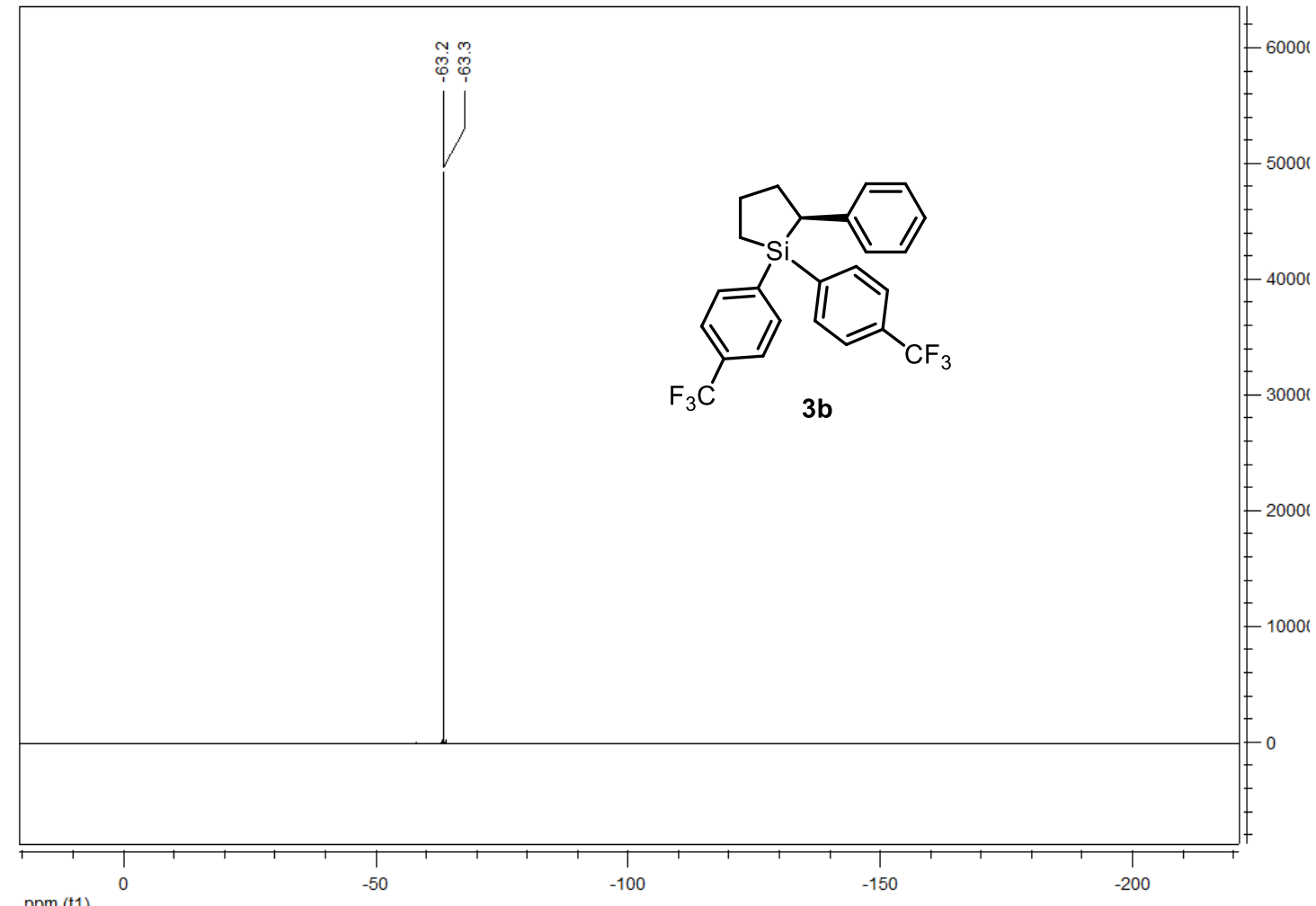

HPLC conditions: Daicel chiral column $O D-H+O D-H$, hexane: ${ }^{i} \mathrm{PrOH}=100: 0,0.5 \mathrm{~mL} / \mathrm{min}, 25{ }^{\circ} \mathrm{C}$, wavelength $=220 \mathrm{~nm}$

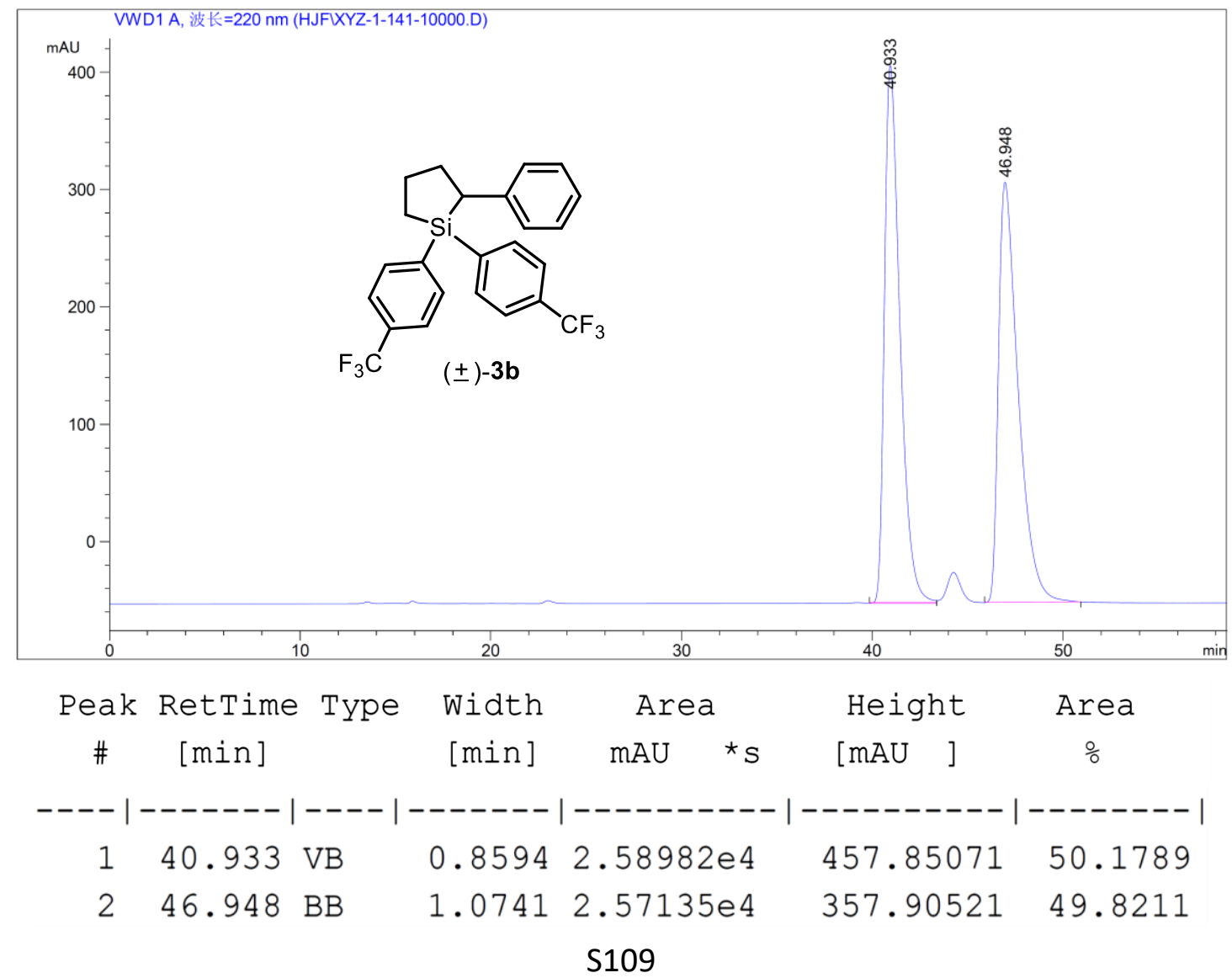



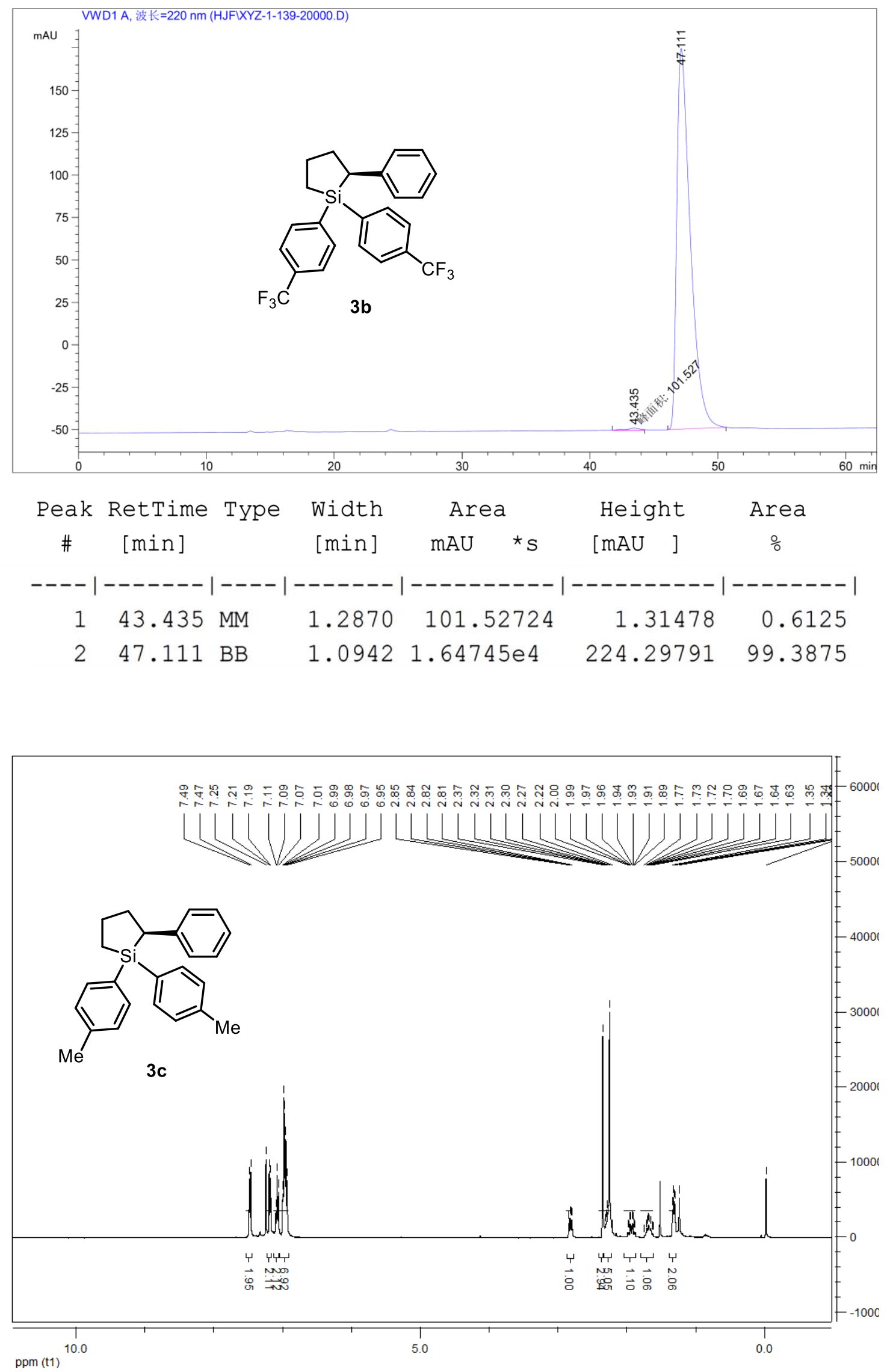


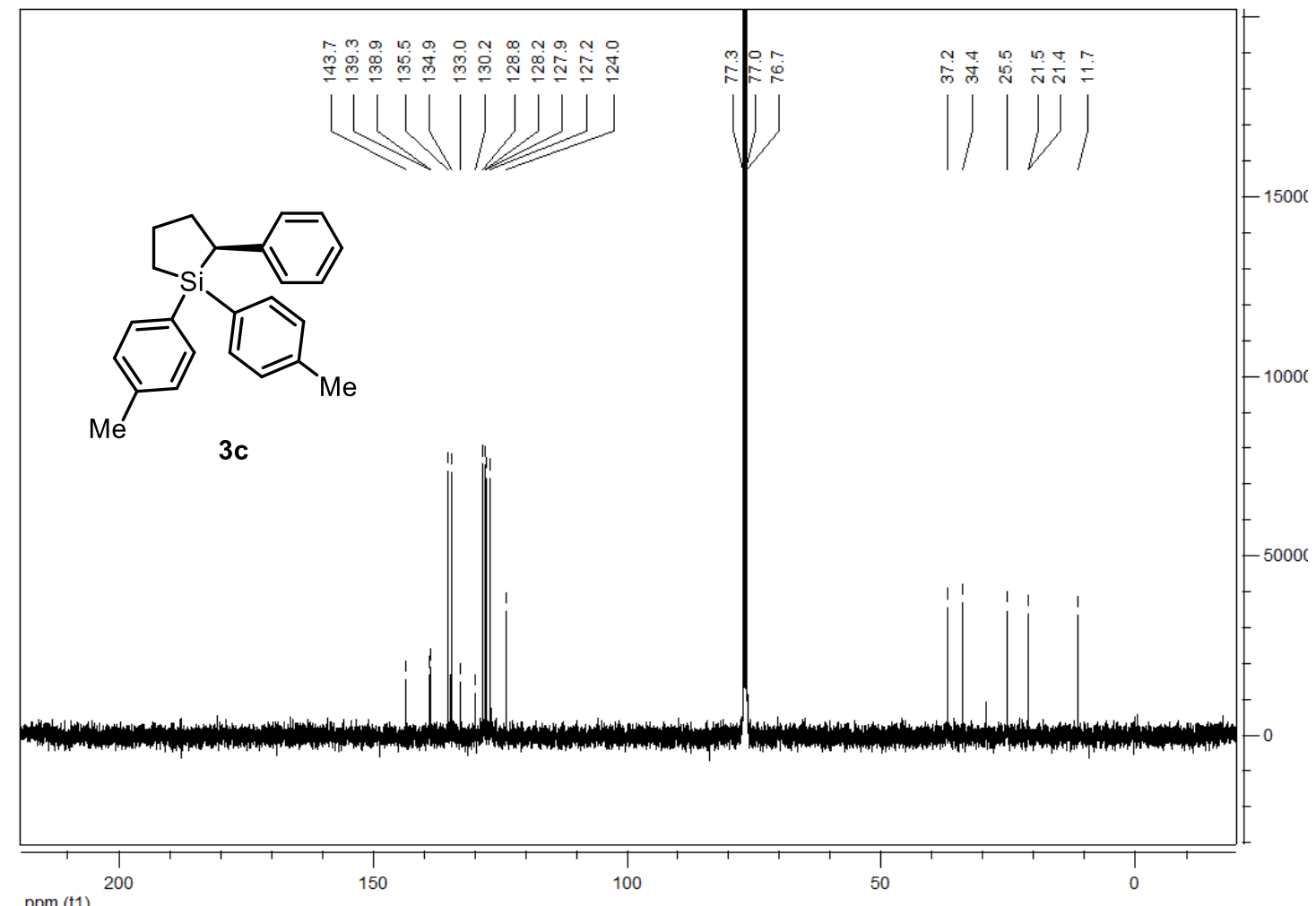

HPLC conditions: Daicel chiral column $O D-H+O D-H$, hexane $:{ }^{i} \mathrm{PrOH}=100: 0,0.5 \mathrm{~mL} / \mathrm{min}, 25^{\circ} \mathrm{C}$, wavelength $=220 \mathrm{~nm}$
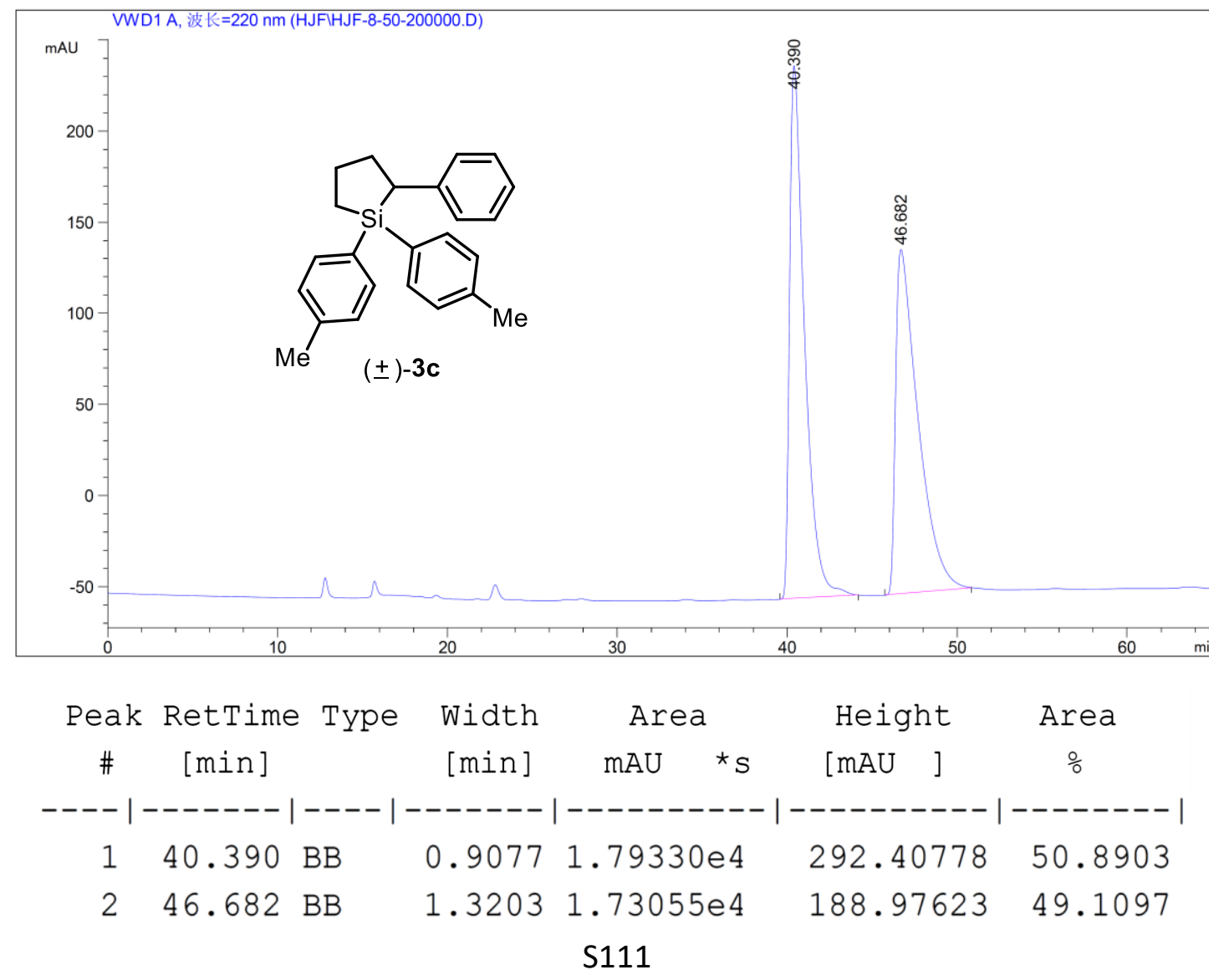

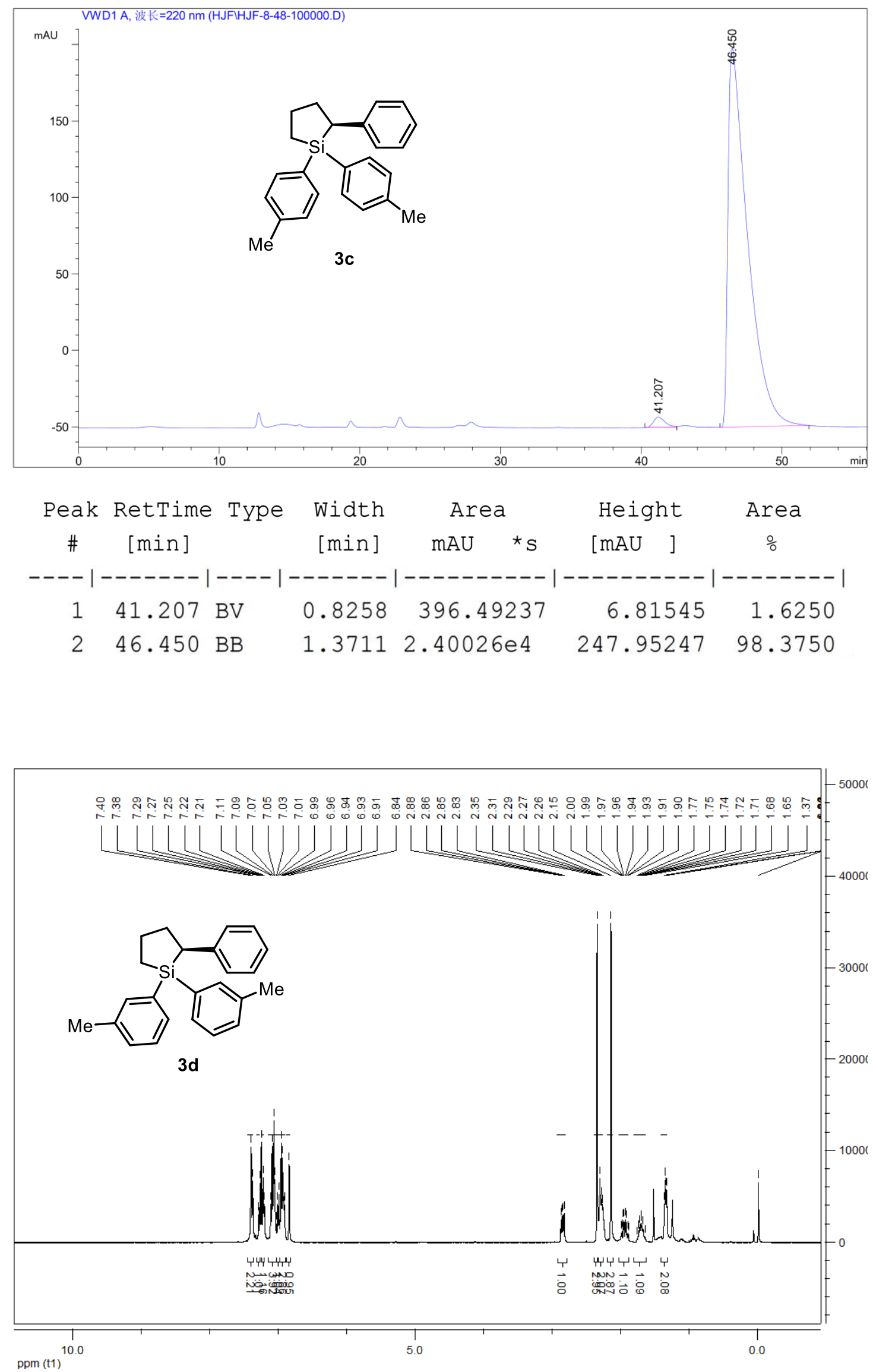


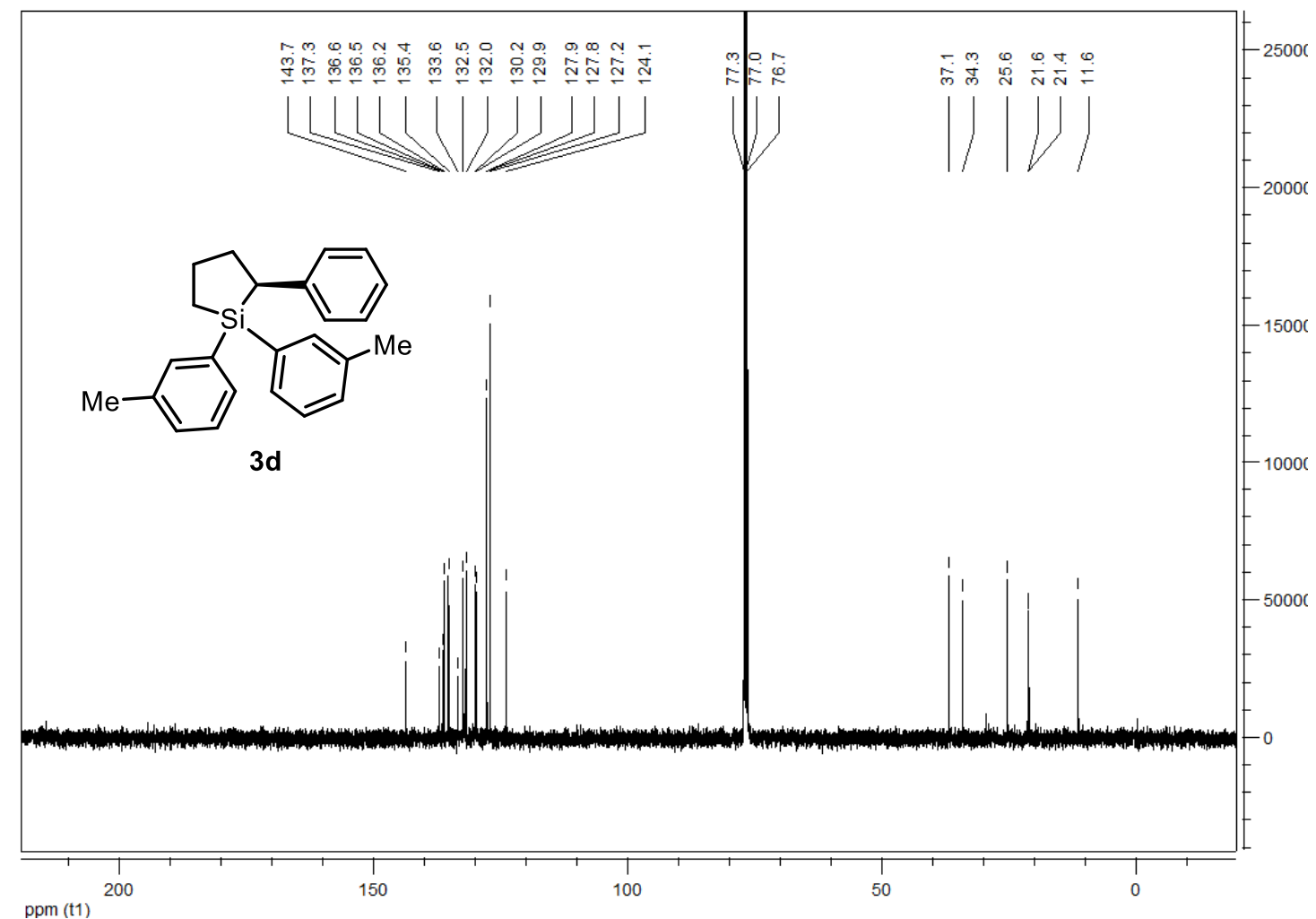

HPLC conditions: Daicel chiral column OD-H $+O D-H$, hexane $:{ }^{i} \mathrm{PrOH}=100: 0,0.5 \mathrm{~mL} / \mathrm{min}, 25{ }^{\circ} \mathrm{C}$, wavelength $=220 \mathrm{~nm}$

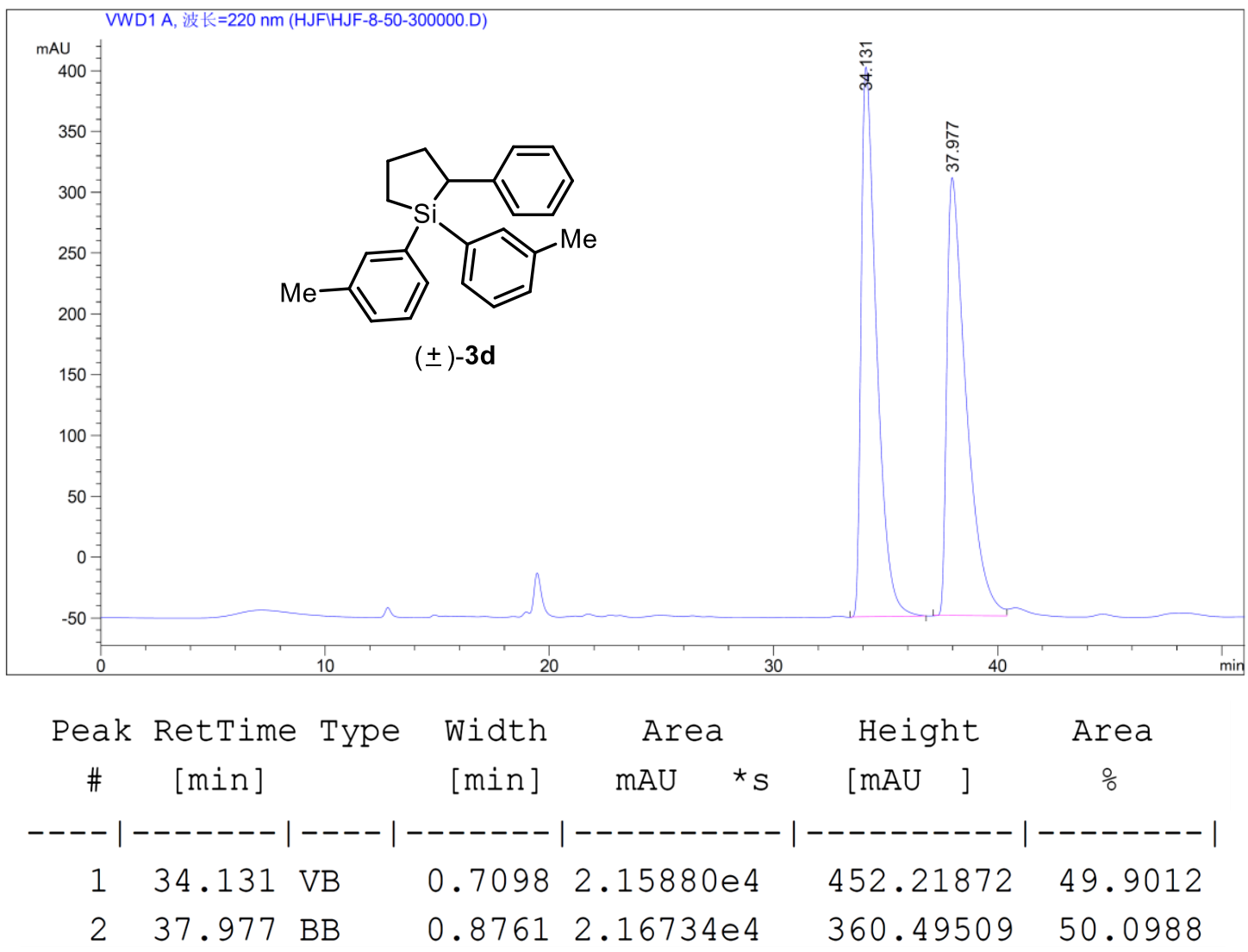




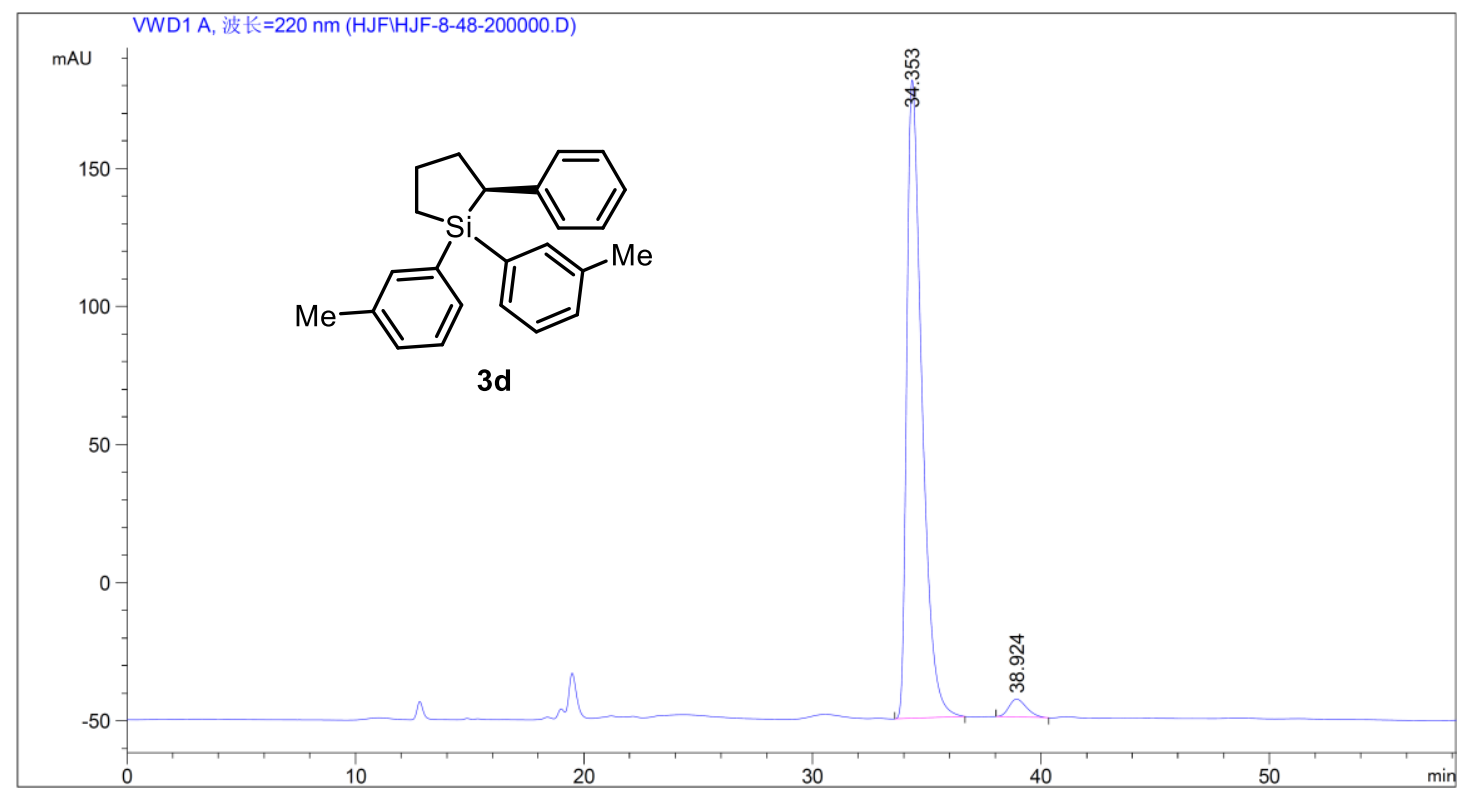

\begin{tabular}{|c|c|c|c|c|c|c|}
\hline \multirow{2}{*}{$\begin{array}{c}\text { Peak } \\
\quad \#\end{array}$} & \multirow{2}{*}{\multicolumn{2}{|c|}{$\begin{array}{l}\text { RetTime Type } \\
\text { [min] }\end{array}$}} & \multirow{2}{*}{$\begin{array}{l}\text { Width } \\
\text { [min] }\end{array}$} & Area & Height & \multirow{2}{*}{$\begin{array}{c}\text { Area } \\
\frac{\circ}{0}\end{array}$} \\
\hline & & & & $\mathrm{mAU}$ & {$[\mathrm{mAU} \quad]$} & \\
\hline & & & & & & \\
\hline & & & & 1.0 & 231 & 19 \\
\hline 7 & 24 & & 05 & 351.06839 & 6.49629 & .1781 \\
\hline
\end{tabular}

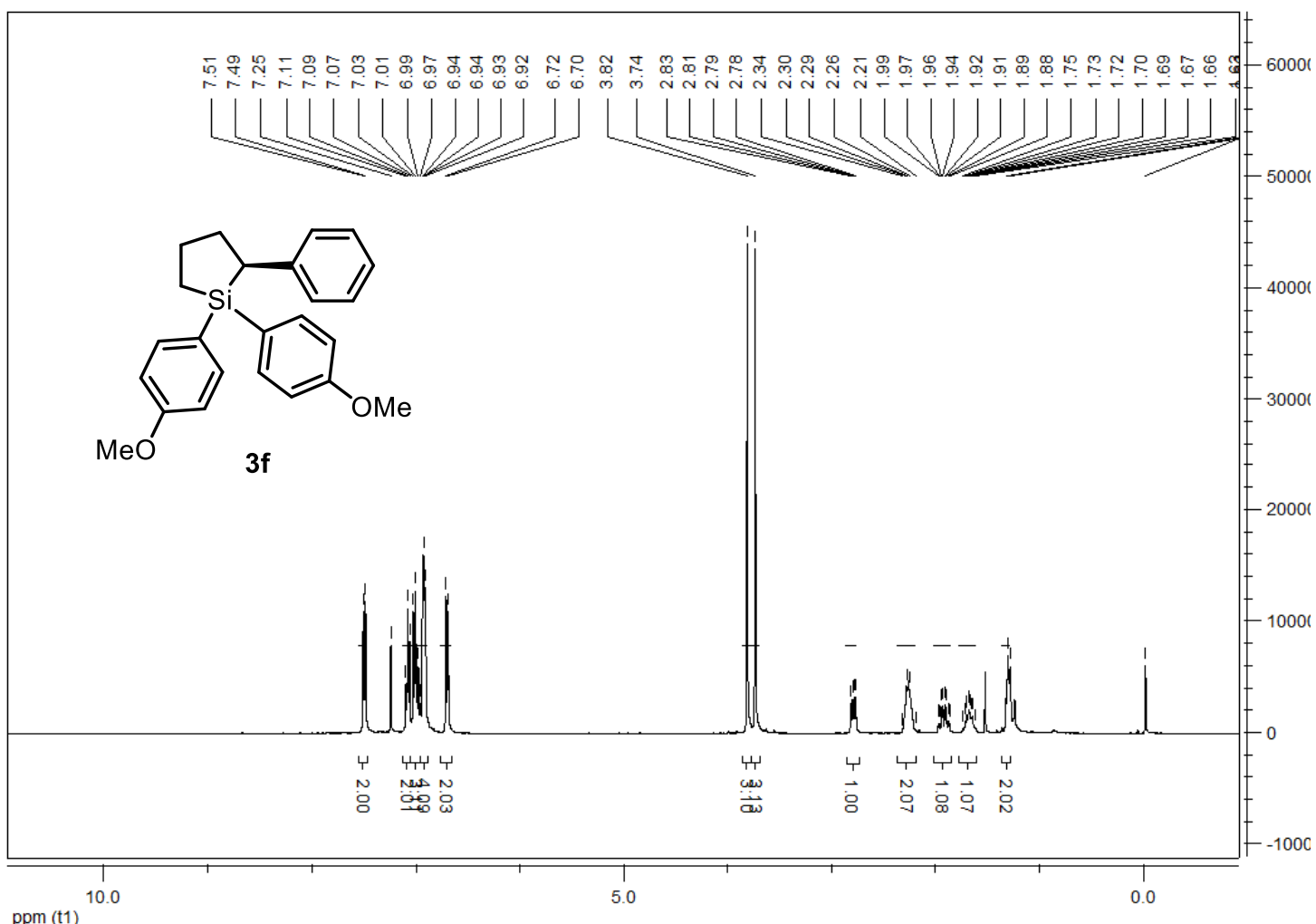




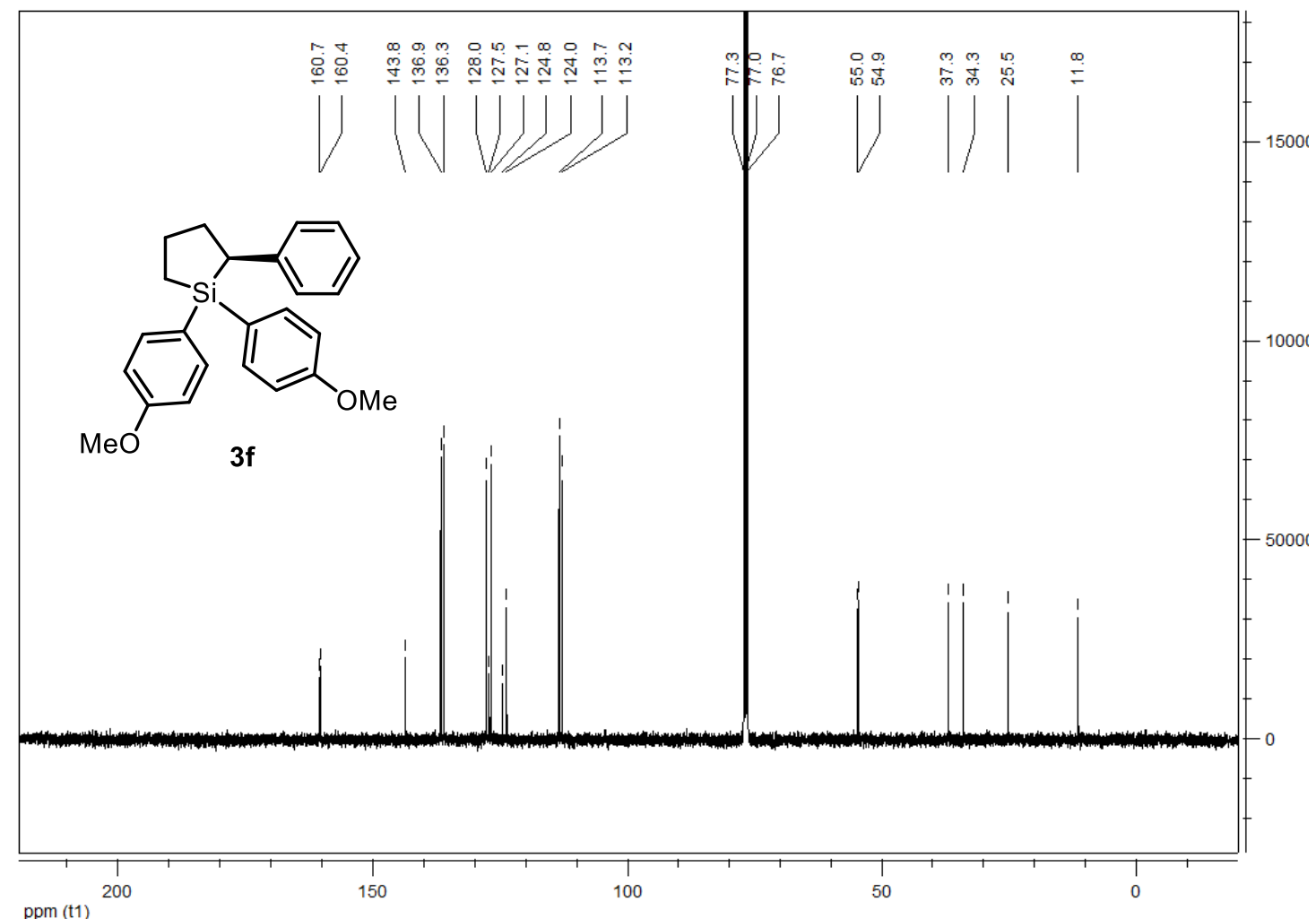

HPLC conditions: Daicel chiral column $O D-H+O D-H$, hexane: $:{ }^{i} \mathrm{PrOH}=100: 0,0.5 \mathrm{~mL} / \mathrm{min}, 25^{\circ} \mathrm{C}$, wavelength $=220 \mathrm{~nm}$

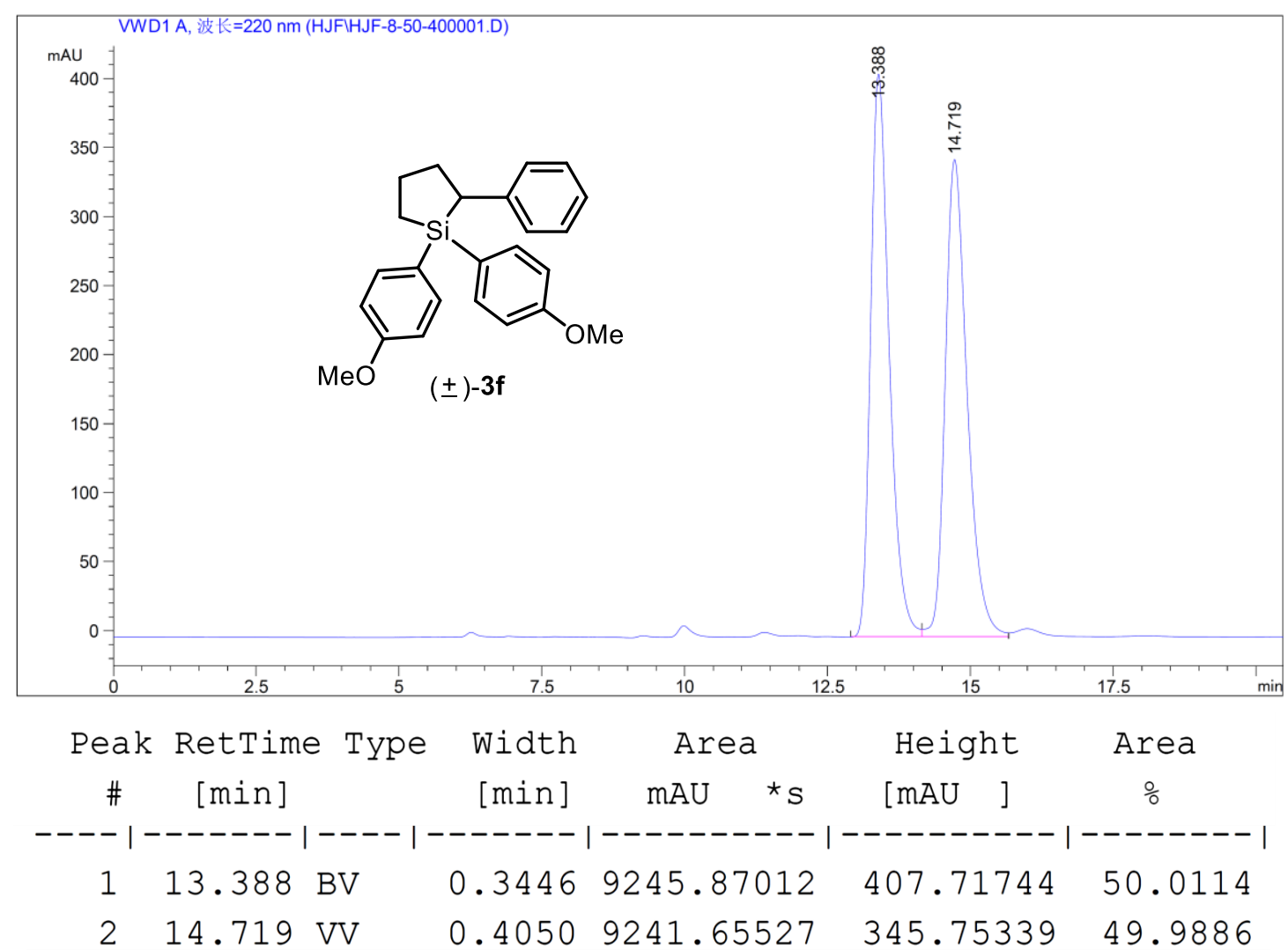



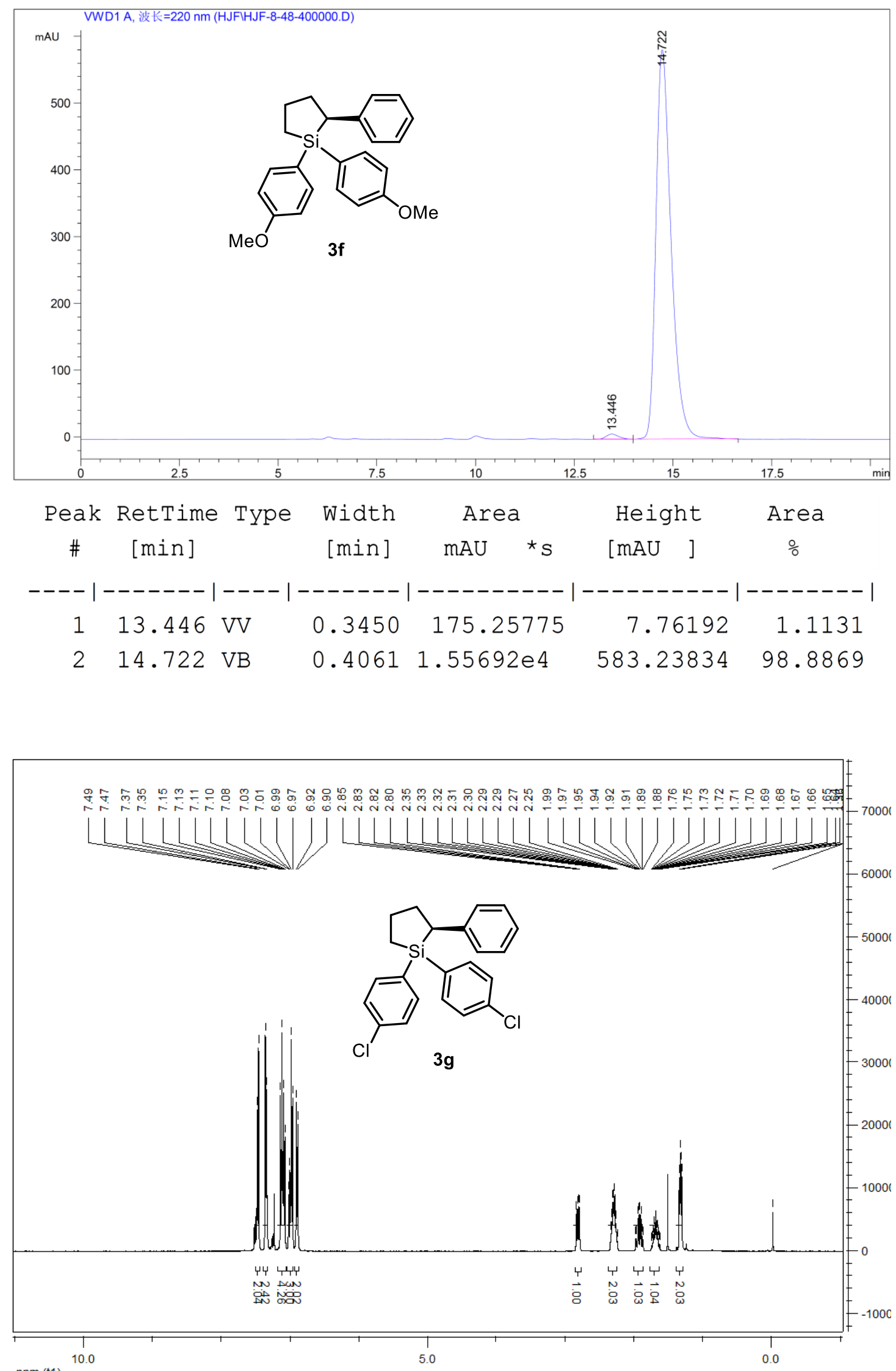


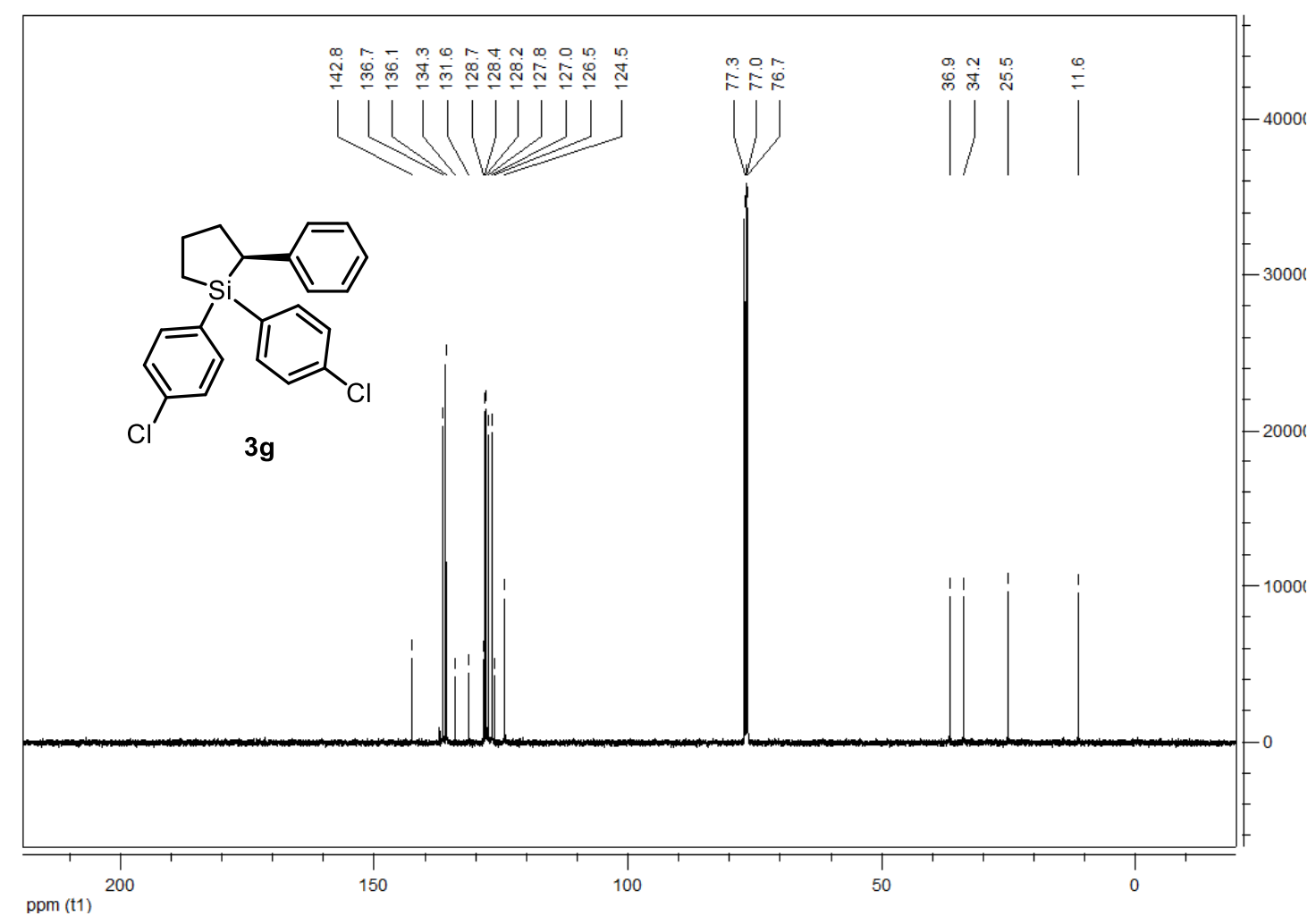

HPLC conditions: Daicel chiral column OD-3, hexane: ${ }^{i} \mathrm{PrOH}=100: 0,0.5 \mathrm{~mL} / \mathrm{min}, 25^{\circ} \mathrm{C}$, wavelength $=$ $220 \mathrm{~nm}$

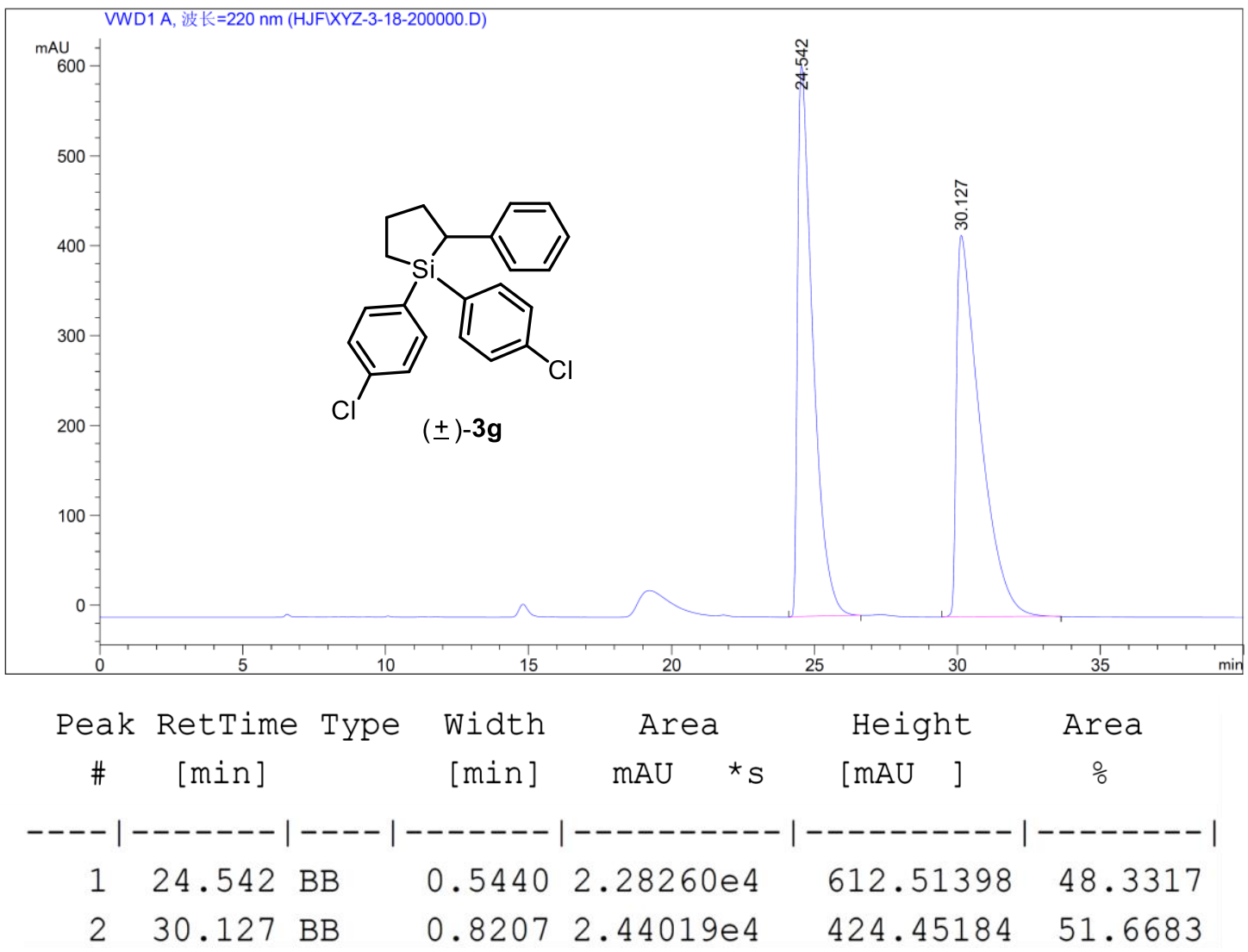



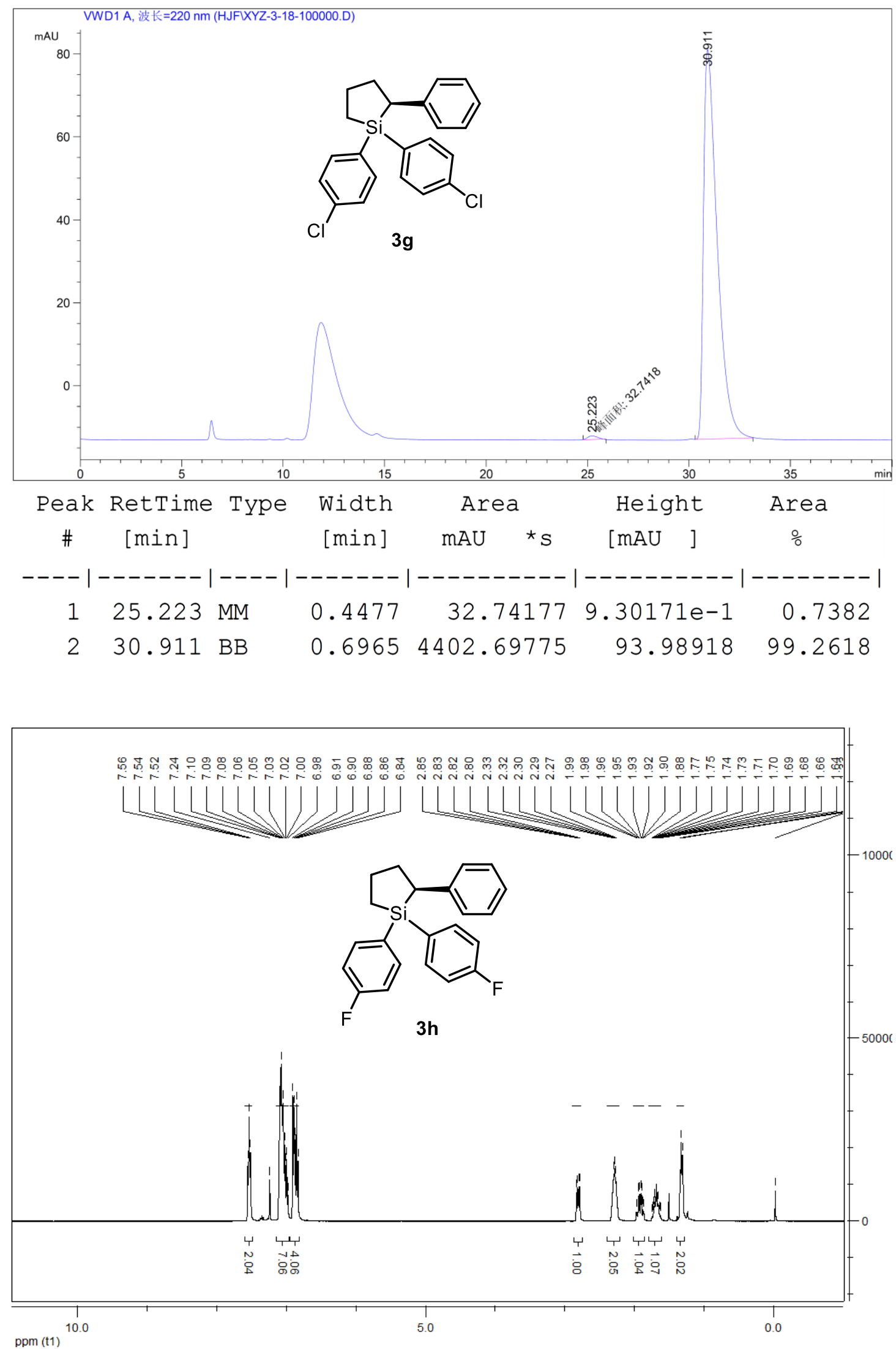

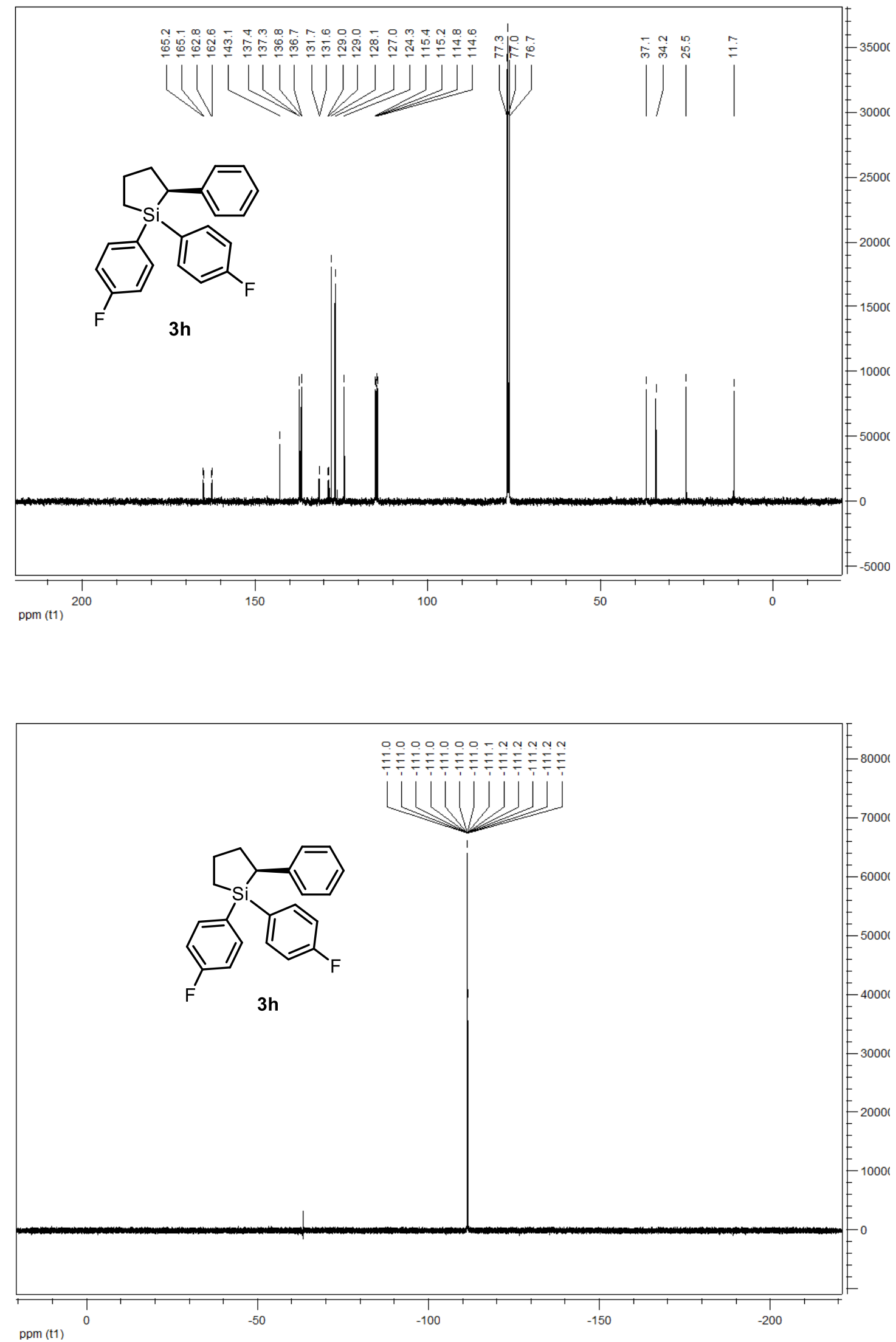
HPLC conditions: Daicel chiral column OD-H+OD-H, hexane: ${ }^{i} \mathrm{PrOH}=100: 0,0.5 \mathrm{~mL} / \mathrm{min}, 25{ }^{\circ} \mathrm{C}$, wavelength $=220 \mathrm{~nm}$
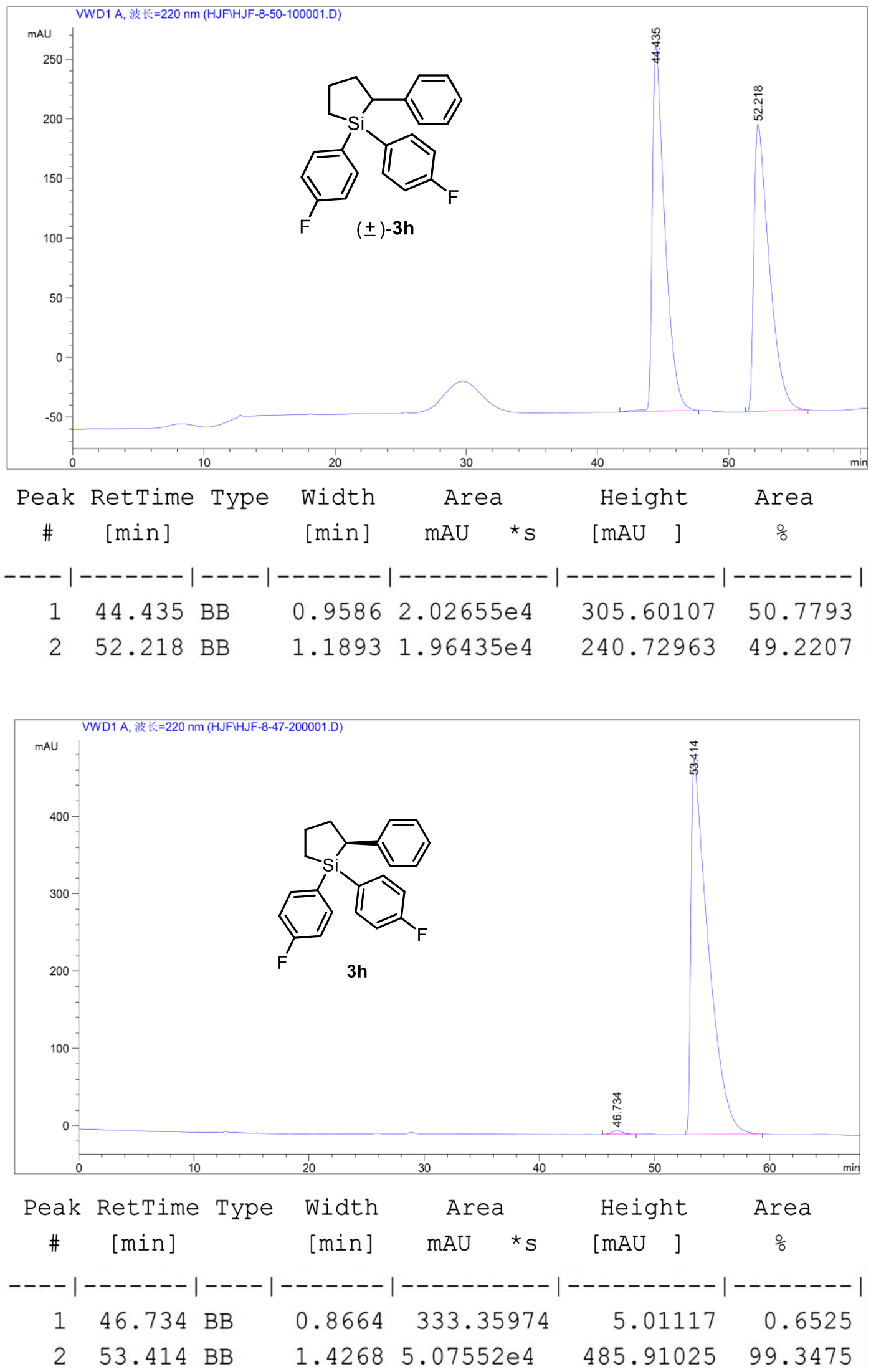

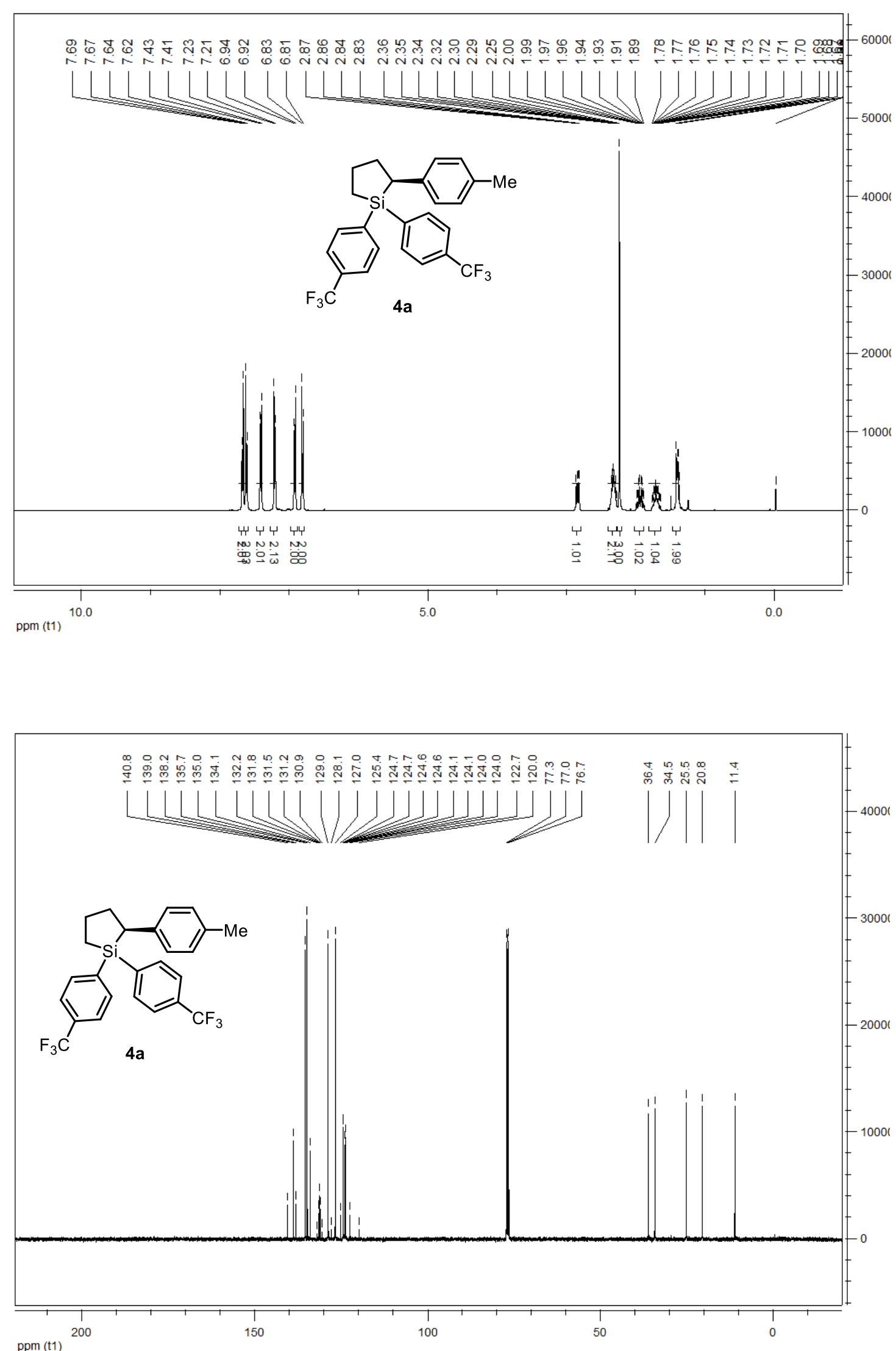


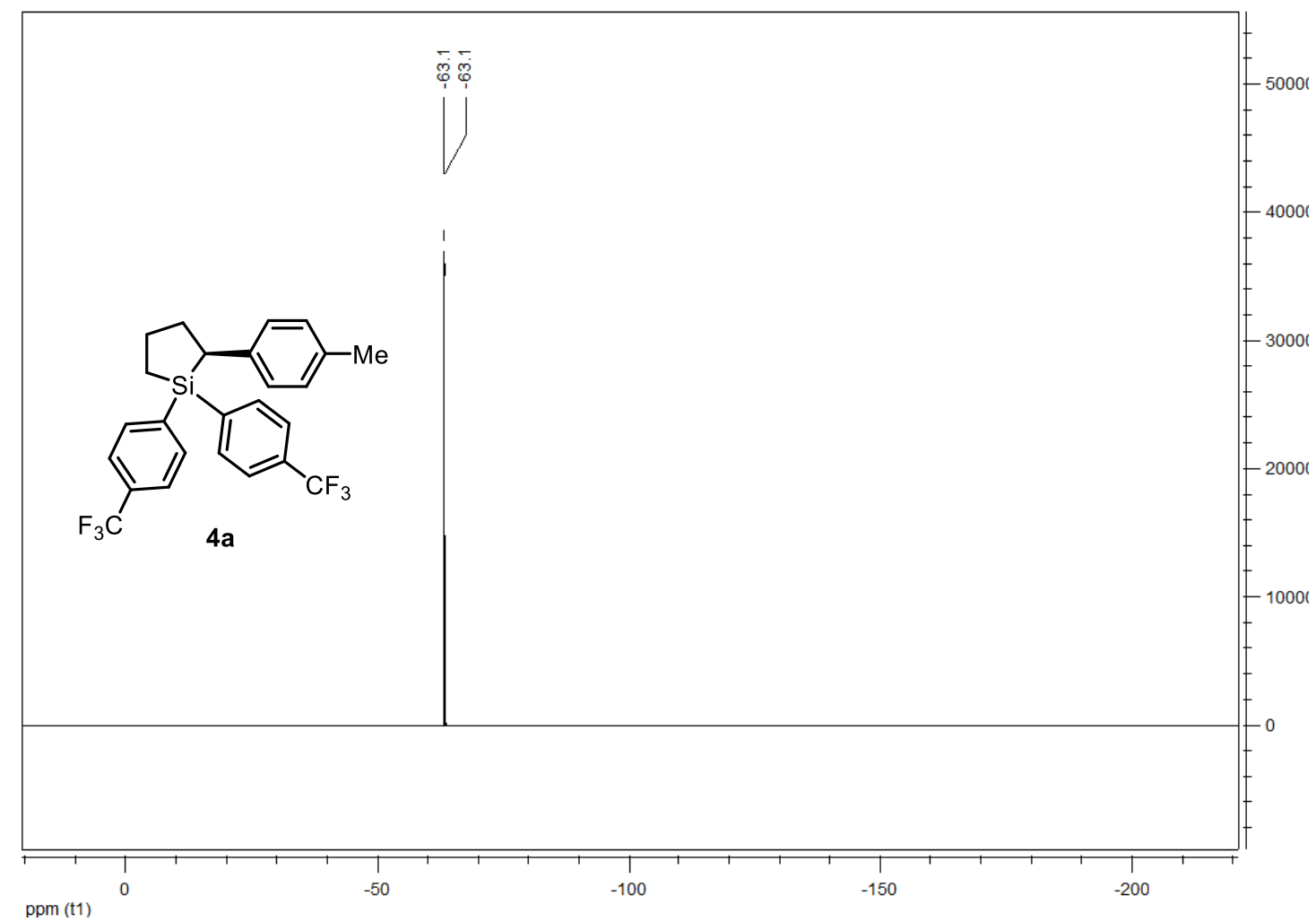

HPLC conditions: Daicel chiral column OD-3+OD-H, hexane $:{ }^{i} \mathrm{PrOH}=100: 0,0.4 \mathrm{~mL} / \mathrm{min}, 25{ }^{\circ} \mathrm{C}$, wavelength $=220 \mathrm{~nm}$
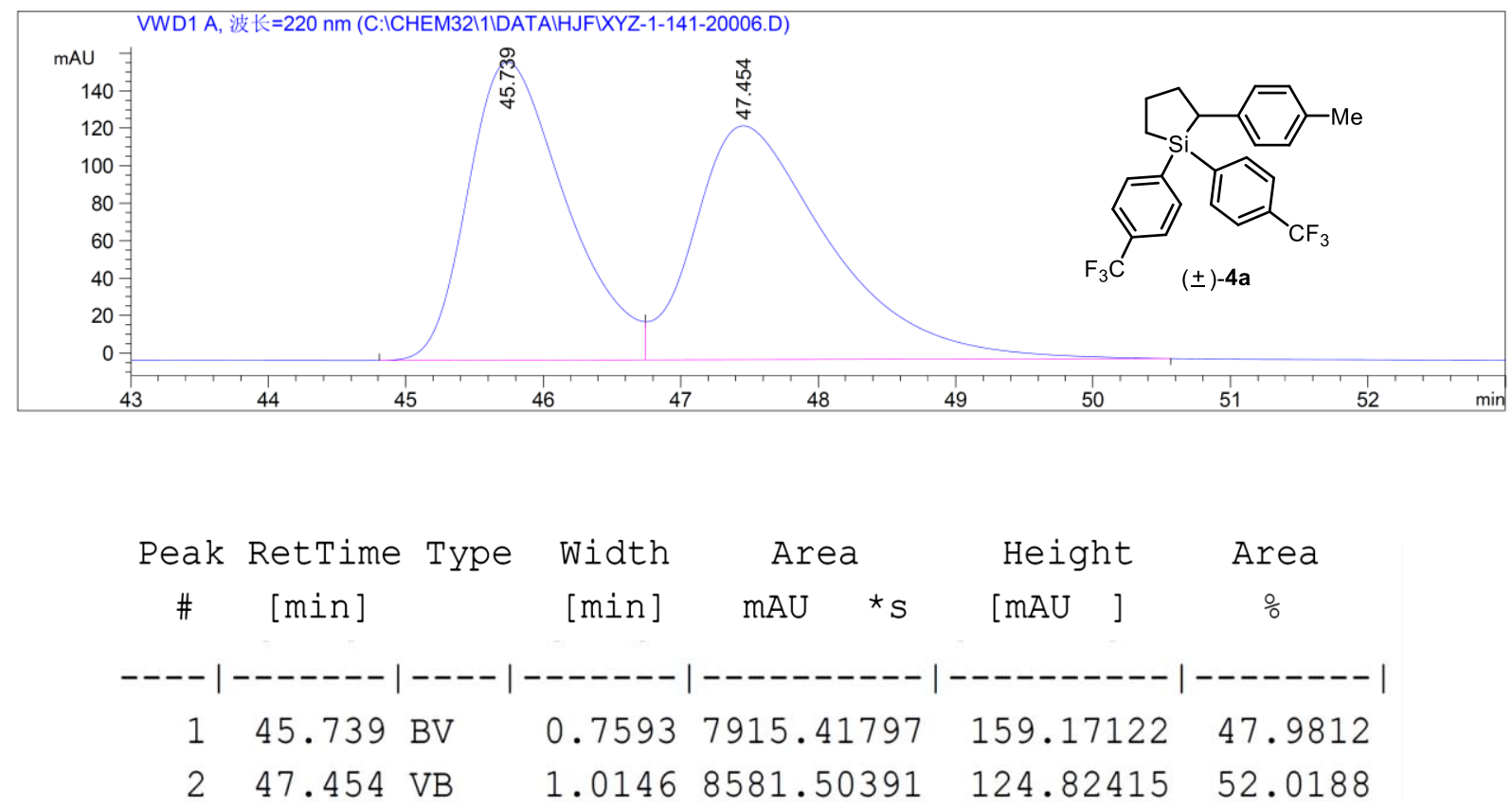


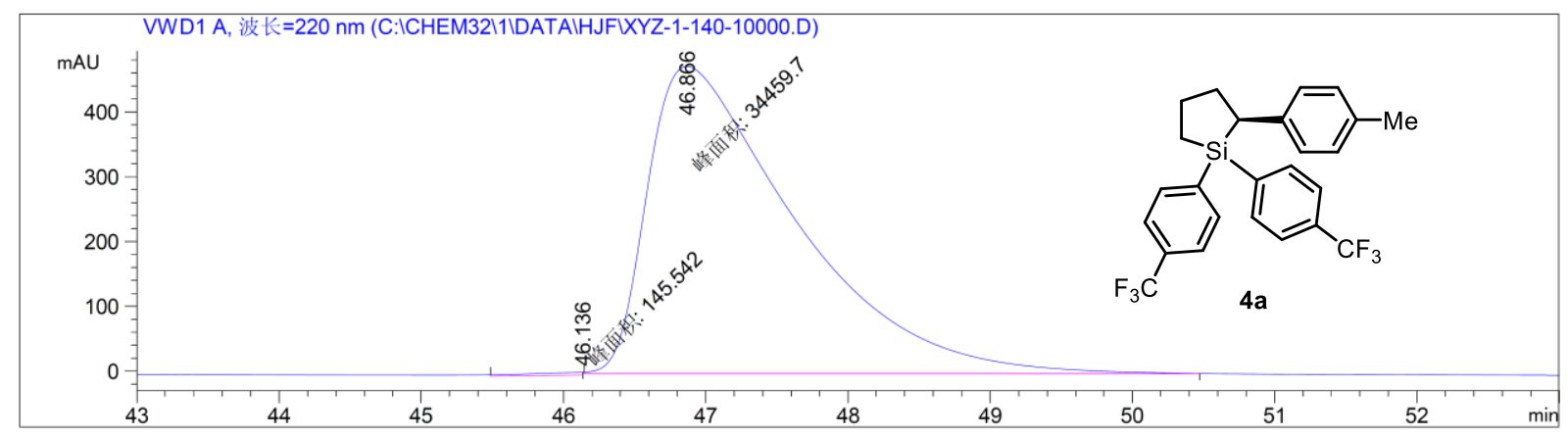

\begin{tabular}{|c|c|c|c|c|c|c|c|}
\hline \multirow{2}{*}{$\begin{array}{c}\text { Peak } \\
\#\end{array}$} & \multirow{2}{*}{$\begin{array}{c}\text { RetTime } \\
\text { [min] }\end{array}$} & \multirow{2}{*}{ Type } & \multirow{2}{*}{$\begin{array}{l}\text { Width } \\
\text { [min] }\end{array}$} & \multicolumn{2}{|c|}{ Area } & Height & \multirow{2}{*}{$\begin{array}{c}\text { Area } \\
\div\end{array}$} \\
\hline & & & & $\mathrm{mAU}$ & ${ }^{*} \mathrm{~S}$ & {$[\mathrm{mAU}$} & \\
\hline & & & 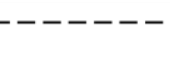 & -- & & 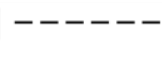 & \\
\hline 1 & 36 & M & 006 & 14 & 54228 & & 0.4206 \\
\hline 2 & 46.866 & $\mathrm{MM}$ & 1.2093 & 3.44 & $97 e 4$ & 474. & 99.5794 \\
\hline
\end{tabular}

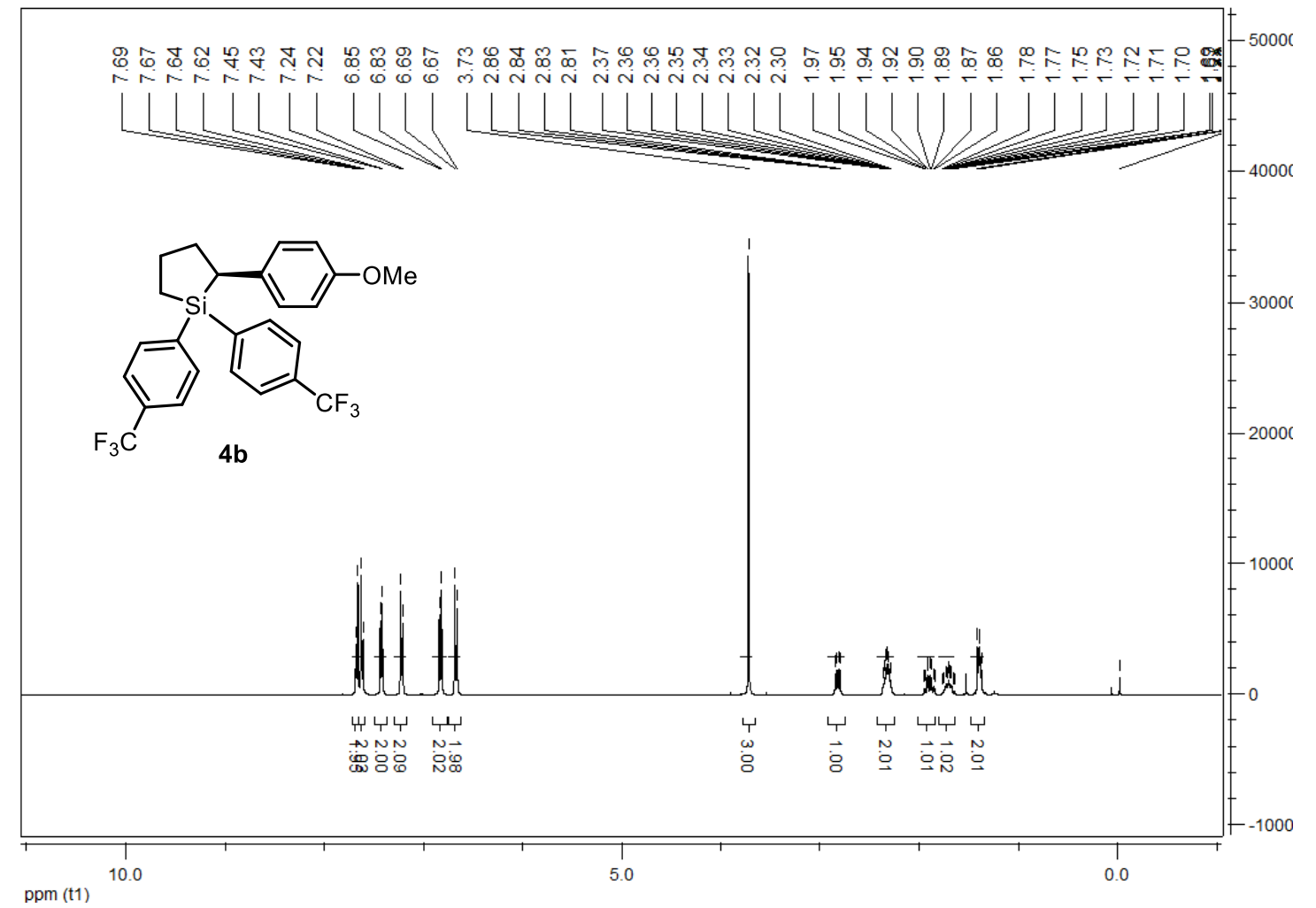



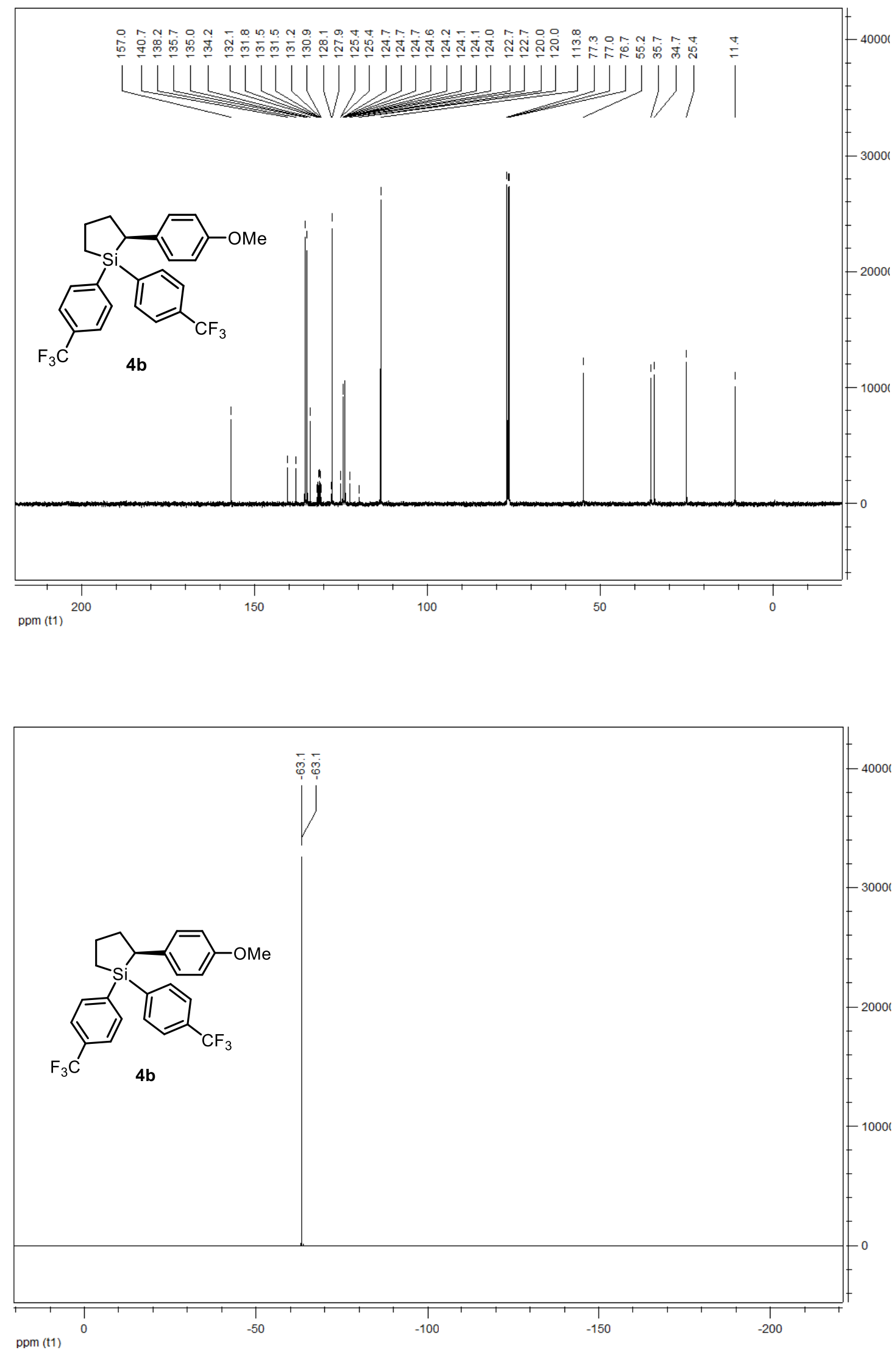
HPLC conditions: Daicel chiral column OD-3*OD-H, hexane: ${ }^{i} \mathrm{PrOH}=200: 1,0.25 \mathrm{~mL} / \mathrm{min}, 25{ }^{\circ} \mathrm{C}$, wavelength $=210 \mathrm{~nm}$

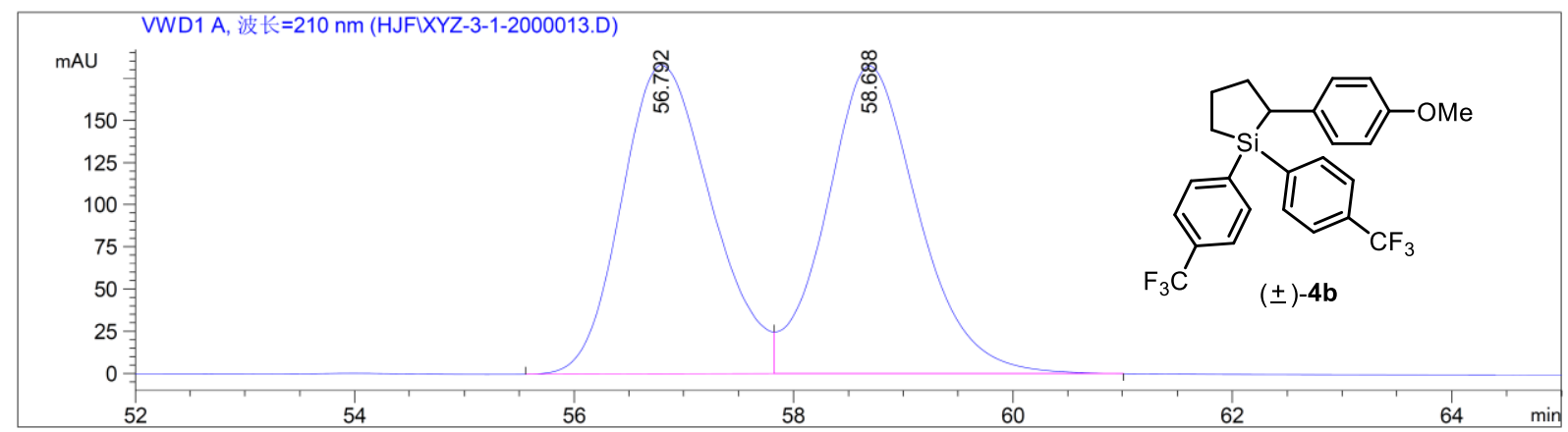

\begin{tabular}{|c|c|c|c|c|c|c|c|}
\hline \multirow{2}{*}{$\begin{array}{c}\text { Peak } \\
\#\end{array}$} & \multirow{2}{*}{$\begin{array}{c}\text { RetTime } \\
\text { [min] }\end{array}$} & \multirow{2}{*}{ Type } & \multirow{2}{*}{$\begin{array}{l}\text { Width } \\
\text { [min] }\end{array}$} & \multicolumn{2}{|c|}{ Area } & Height & \multirow{2}{*}{$\begin{array}{c}\text { Area } \\
\quad \circ\end{array}$} \\
\hline & & & & $\mathrm{mAU}$ & $\star_{S}$ & {$[\mathrm{mAU} \quad]$} & \\
\hline & & & & & & & \\
\hline 1 & & BV & & 1. & e 4 & 183.28604 & 54 \\
\hline 0 & 58.688 & VB & 0.8 & 1.07 & $6 e 4$ & 182.65469 & 50.5446 \\
\hline
\end{tabular}

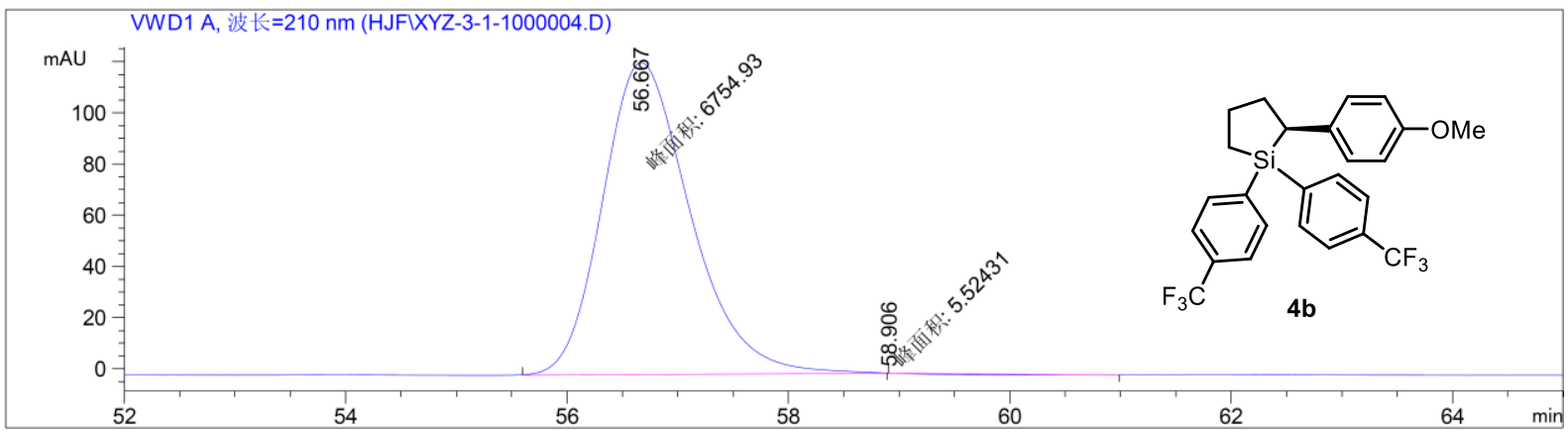

\begin{tabular}{|c|c|c|c|c|c|c|c|}
\hline Peak & RetTime & Type & Width & Are & & Height & Area \\
\hline \# & [min ] & & {$[\mathrm{min}]$} & mAU & ${ }^{\star} \mathrm{s}$ & {$[\mathrm{mAU} \quad]$} & $\%$ \\
\hline 1 & 56.667 & MM & 0.9231 & 6754.9 & 773 & 121.95987 & 99.9183 \\
\hline 2 & 58.906 & MM & 0.2337 & 5.52 & 431 & $2.85169 e-1$ & 0.0817 \\
\hline
\end{tabular}



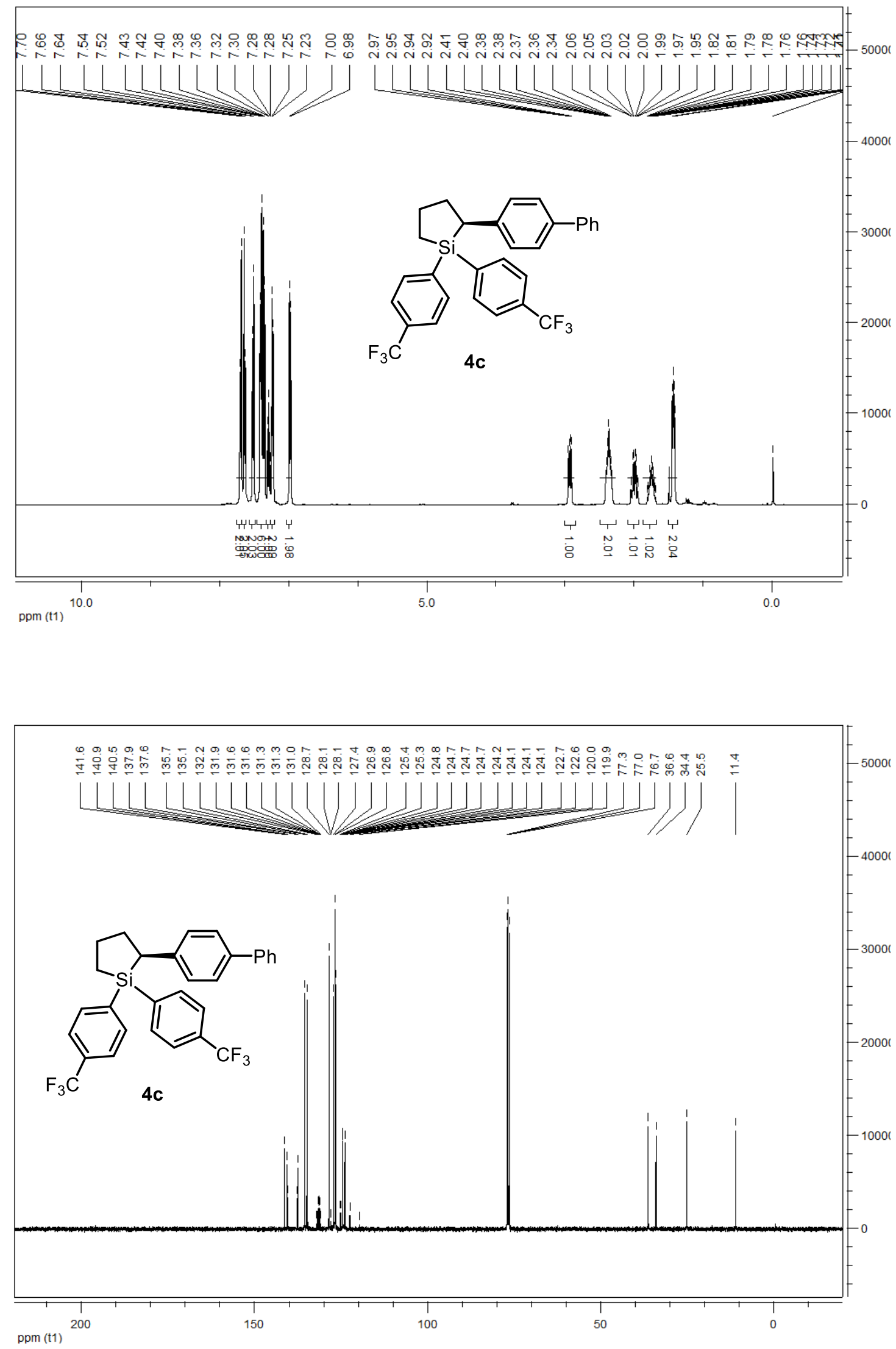


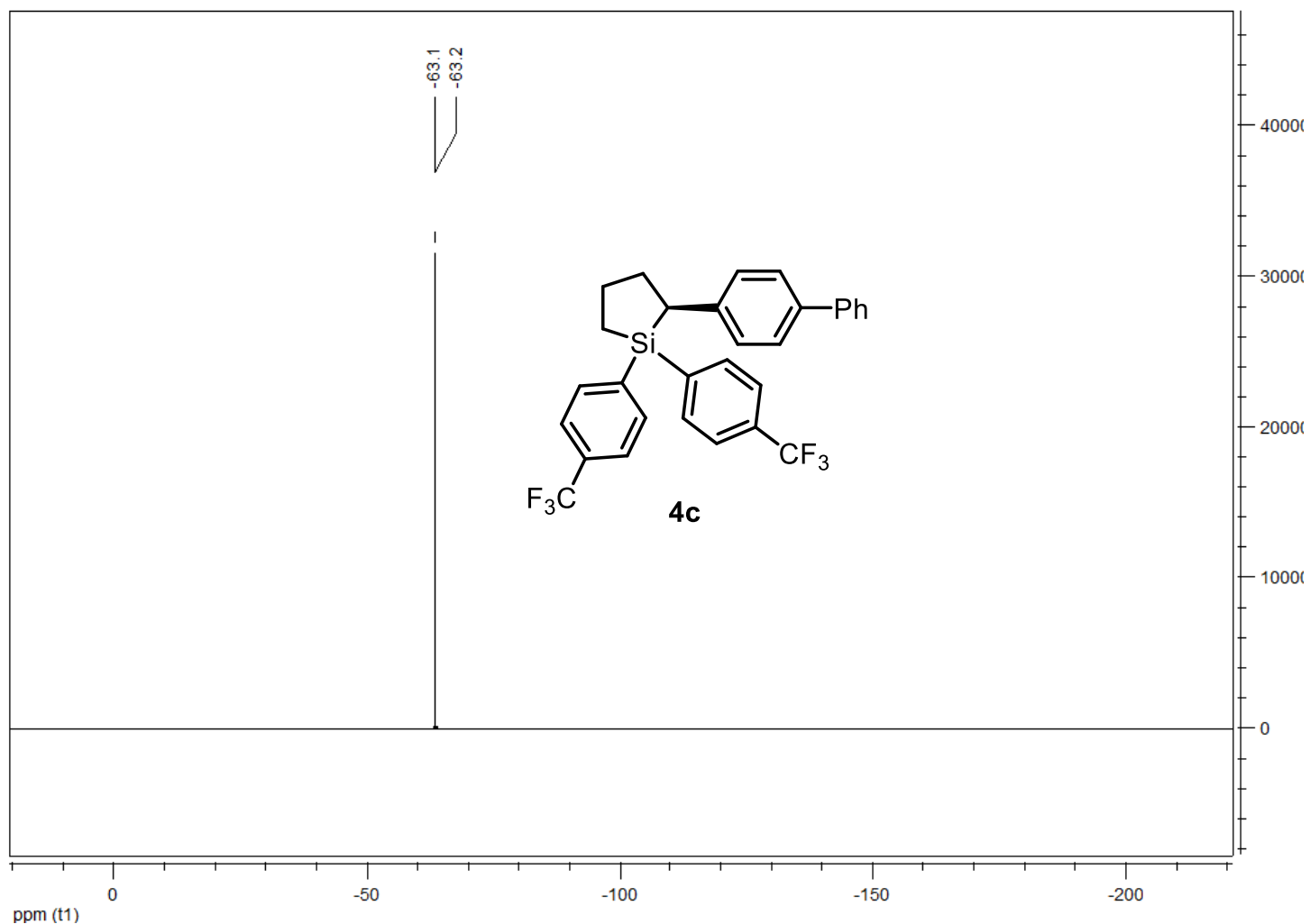

HPLC conditions: Daicel chiral column AD-H, hexane $:{ }^{i} \mathrm{PrOH}=500: 1,0.3 \mathrm{~mL} / \mathrm{min}, 25^{\circ} \mathrm{C}$, wavelength $=$ $220 \mathrm{~nm}$

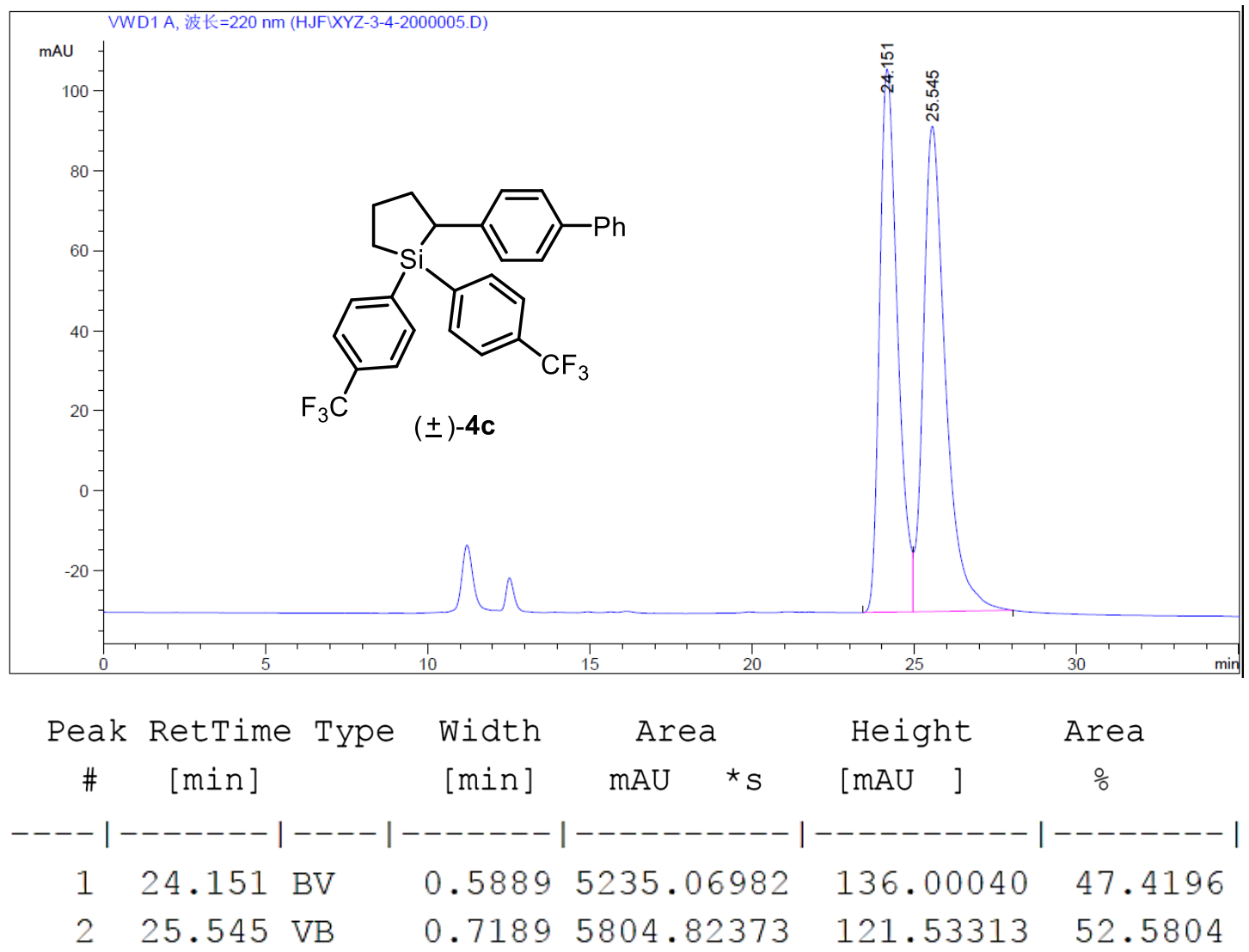



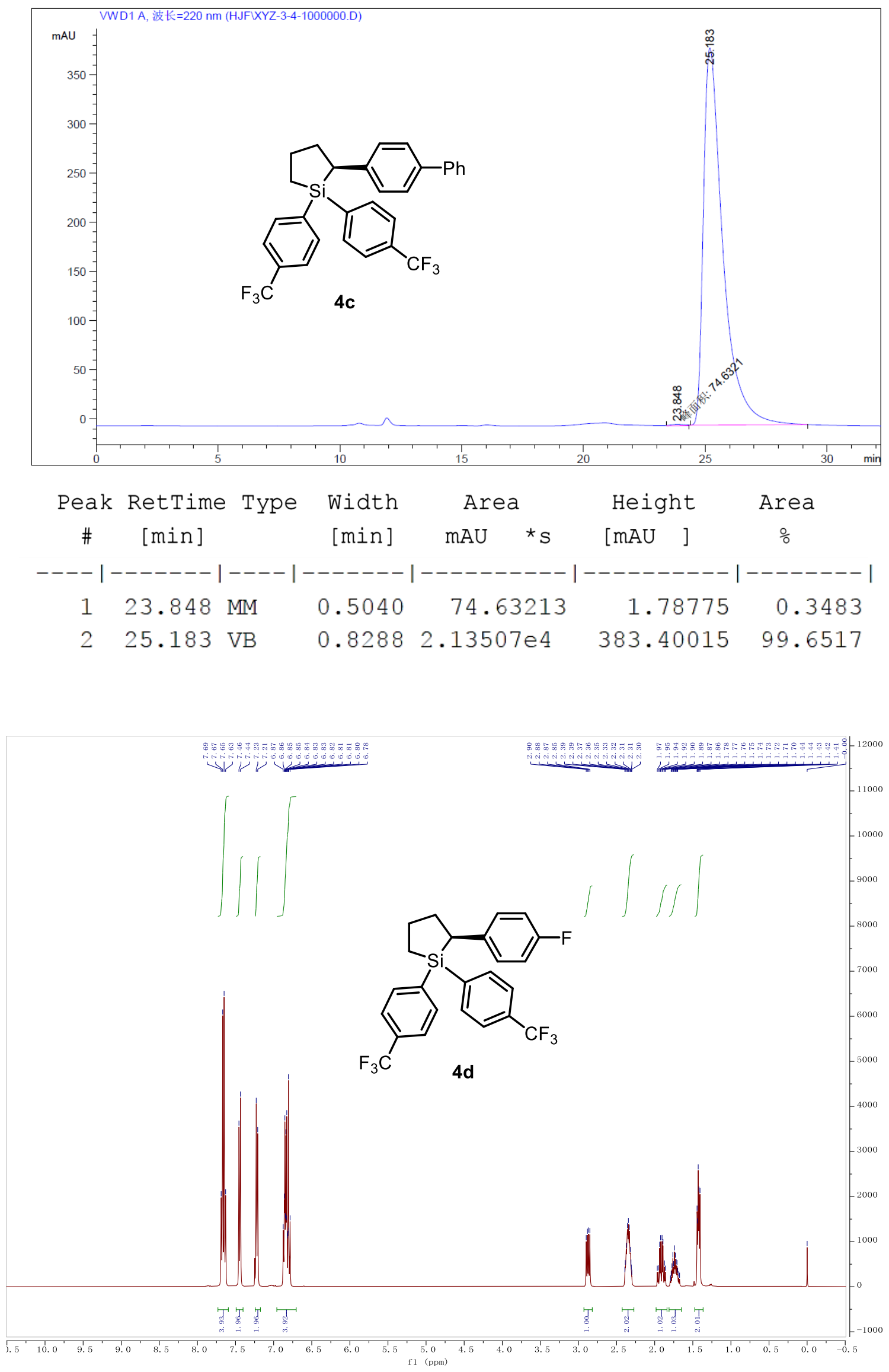

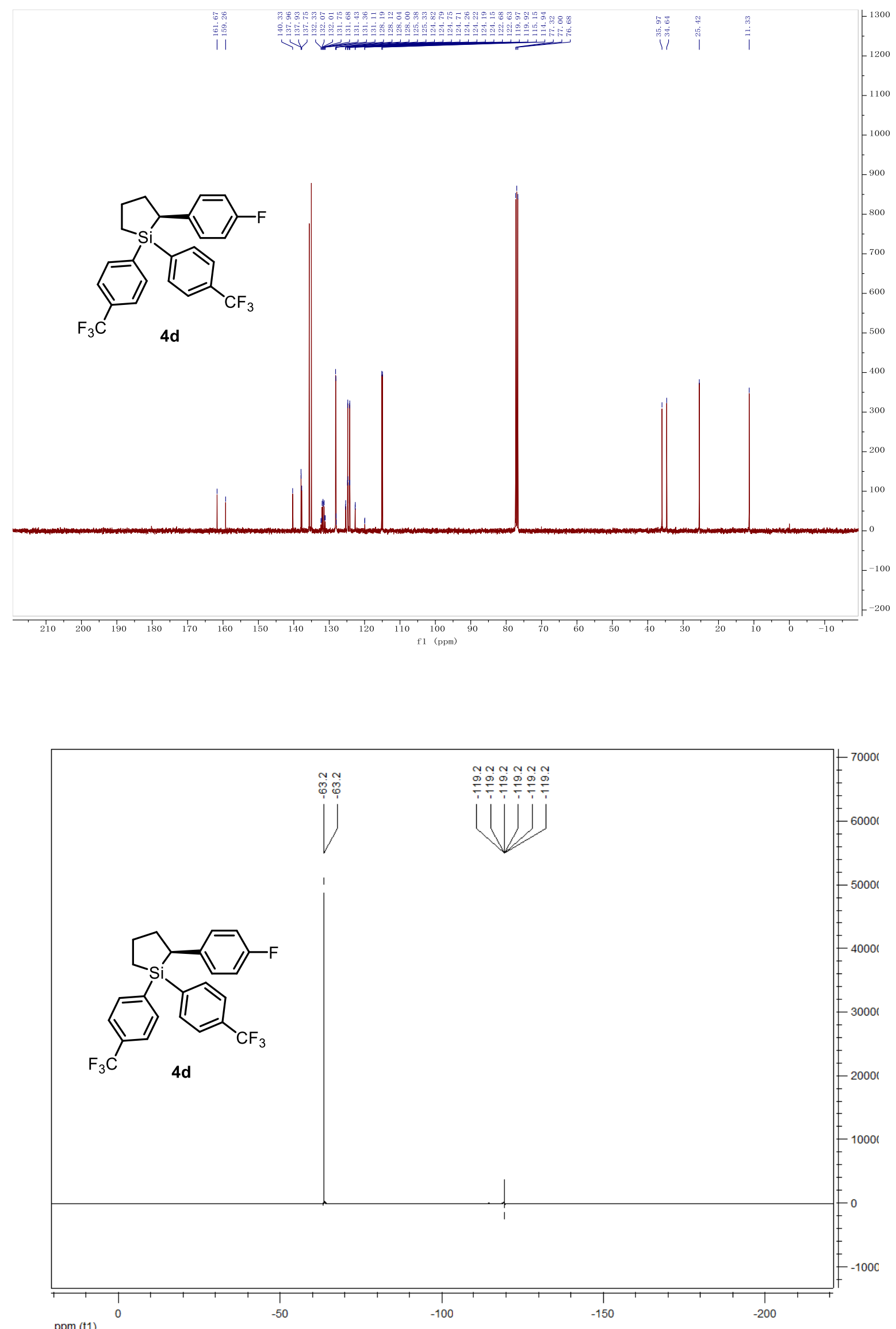
HPLC conditions: Daicel chiral column OD-3, hexane $:{ }^{i} \mathrm{PrOH}=100: 0,0.5 \mathrm{~mL} / \mathrm{min}, 25^{\circ} \mathrm{C}$, wavelength $=$ $220 \mathrm{~nm}$
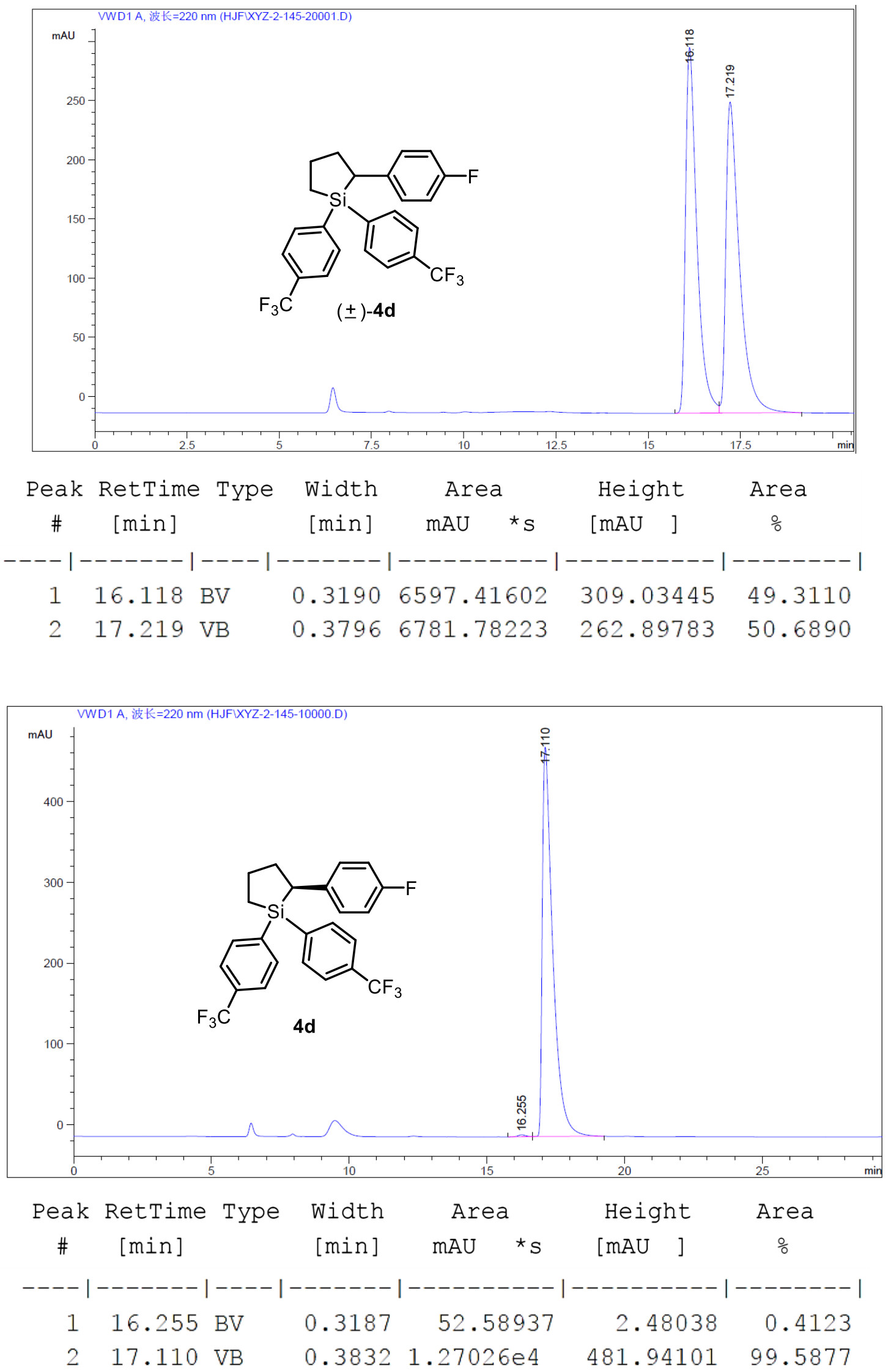

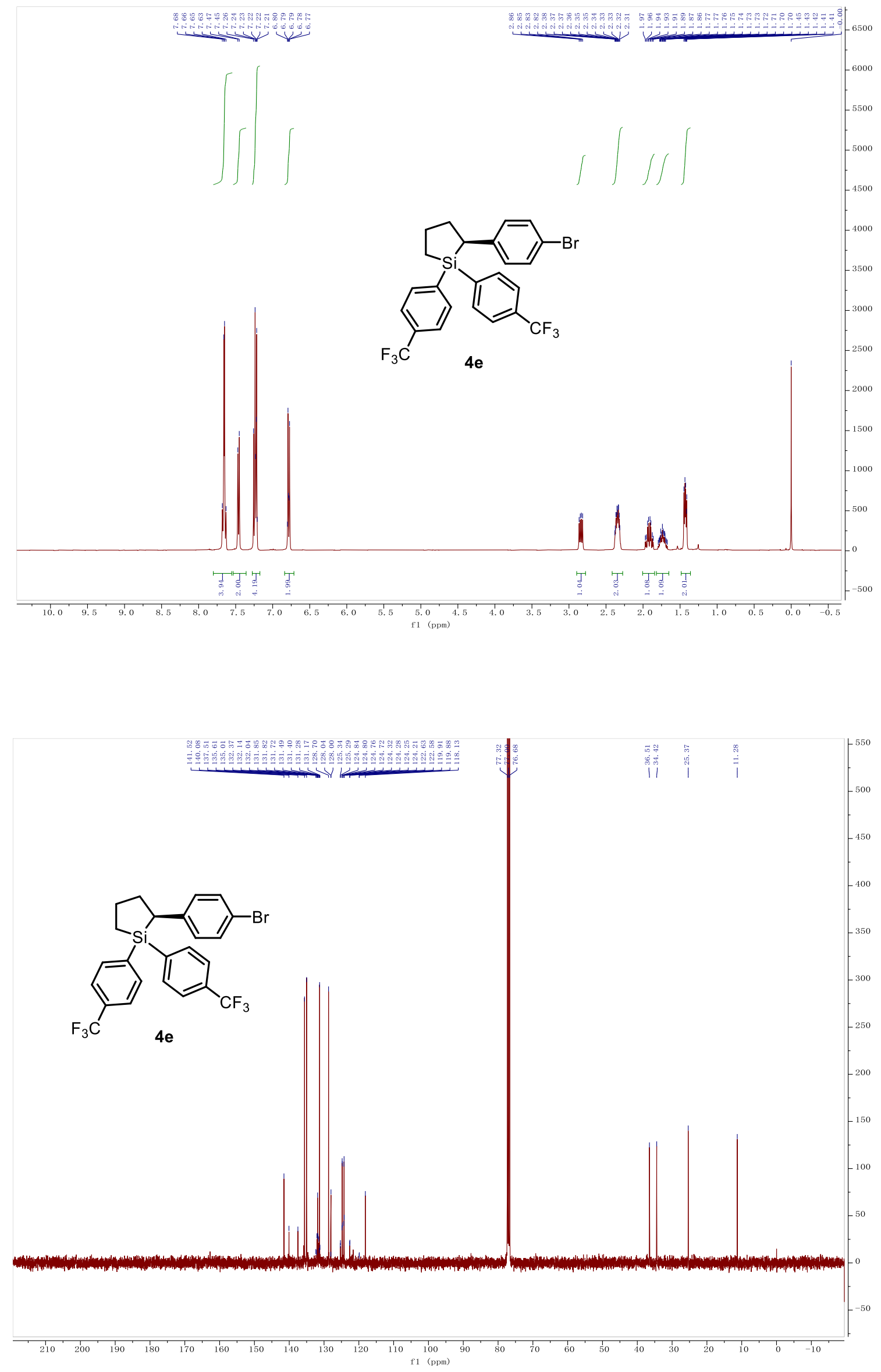


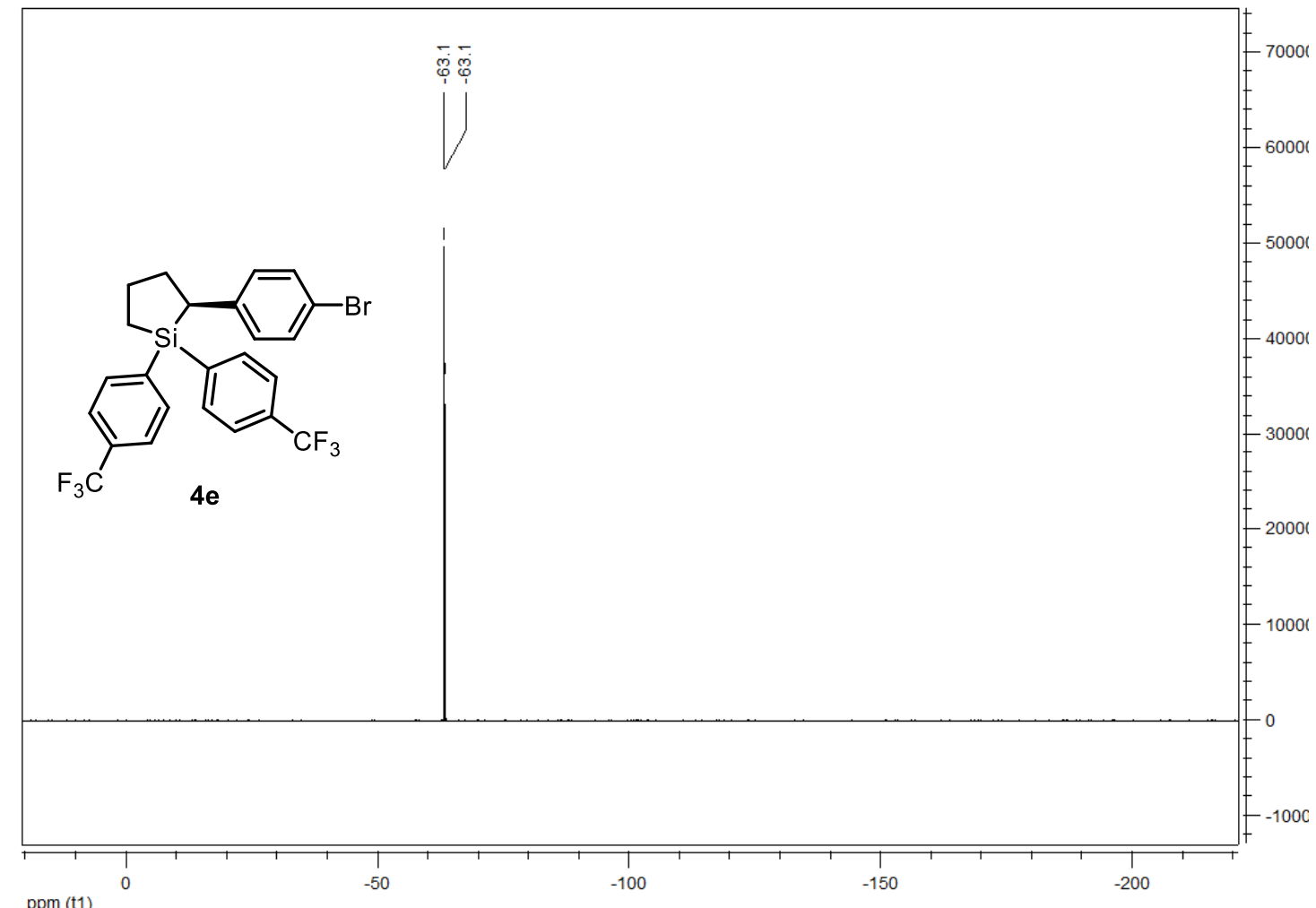

HPLC conditions: Daicel chiral column AD-3*AD-H, hexane: ${ }^{i} \mathrm{PrOH}=100: 0,0.15 \mathrm{~mL} / \mathrm{min}, 25{ }^{\circ} \mathrm{C}$, wavelength $=210 \mathrm{~nm}$
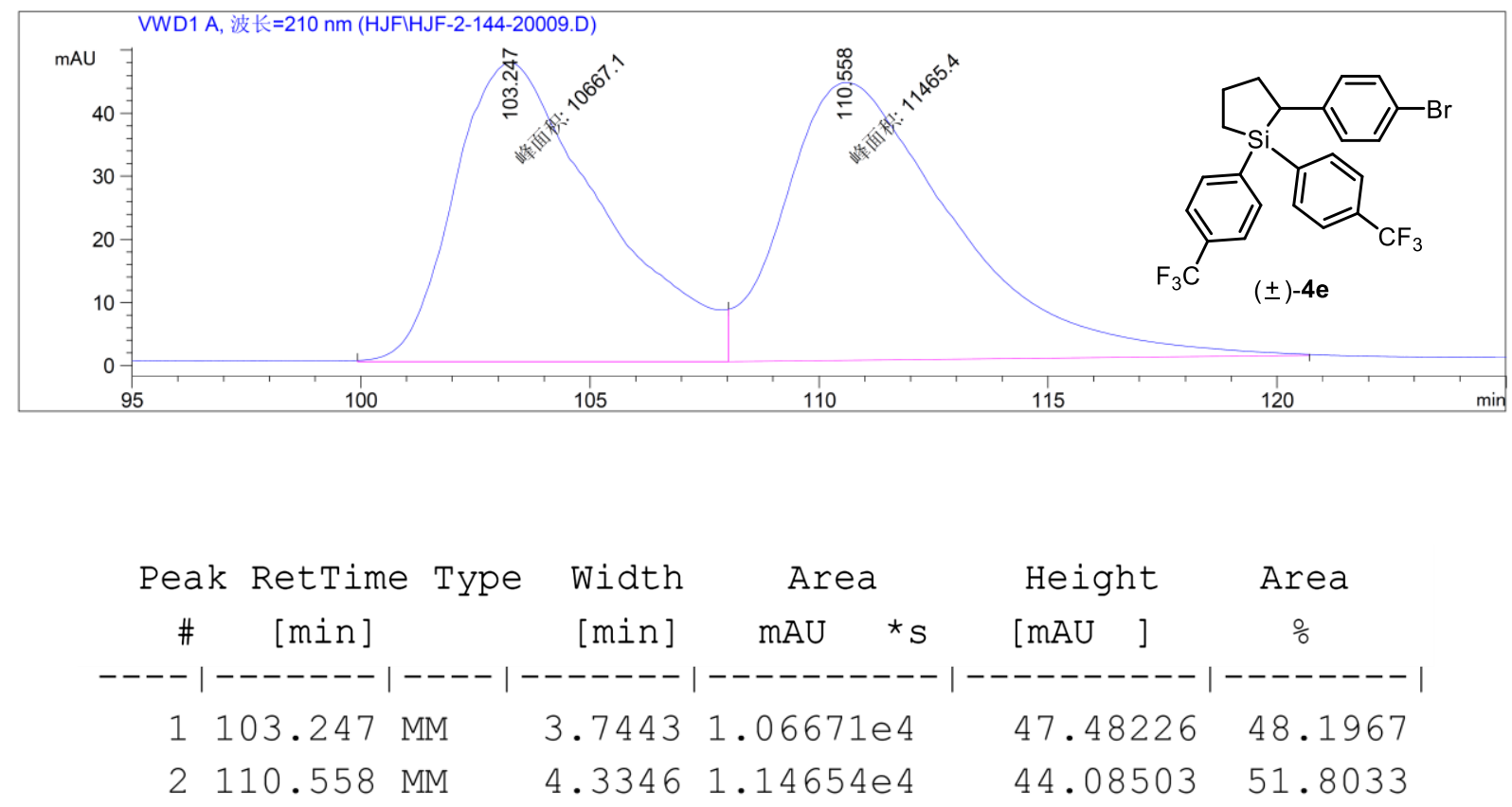


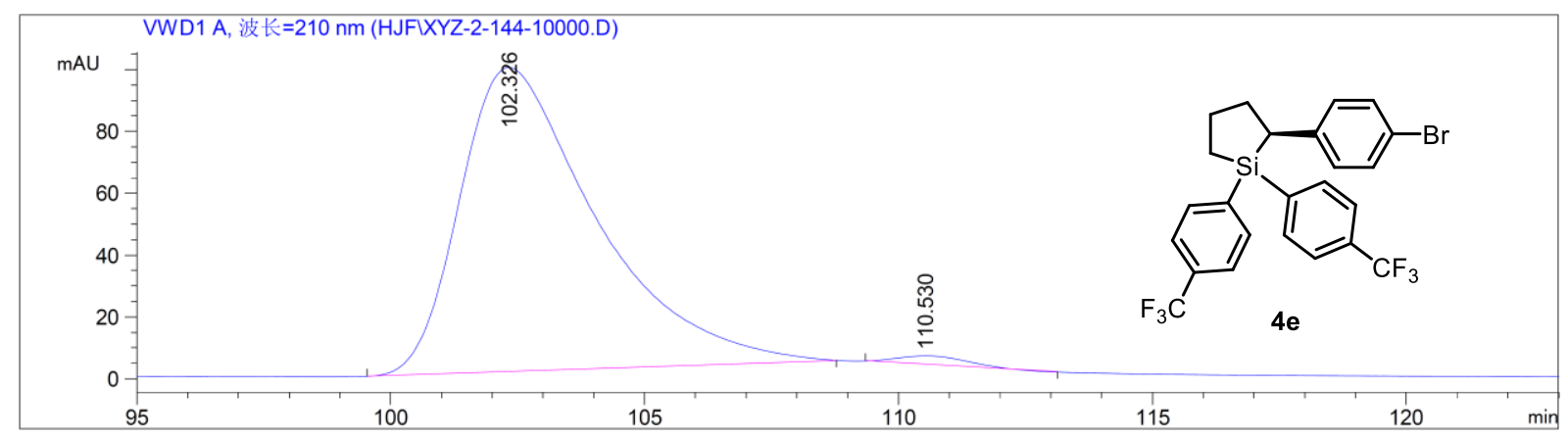

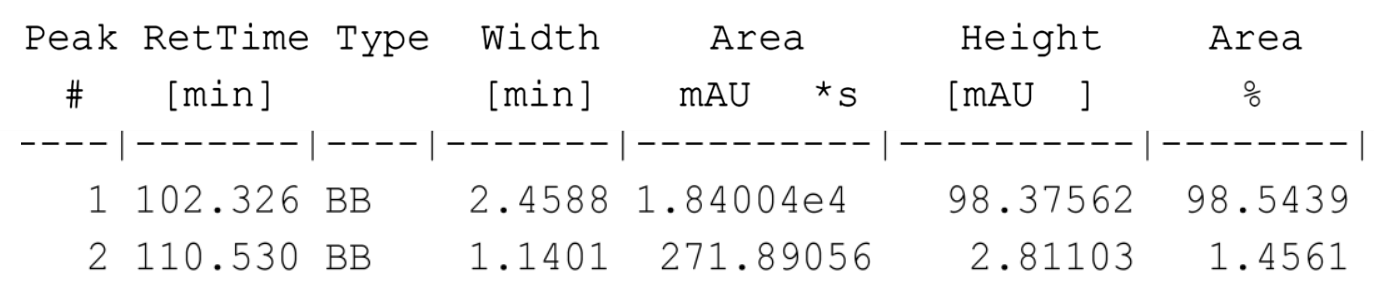

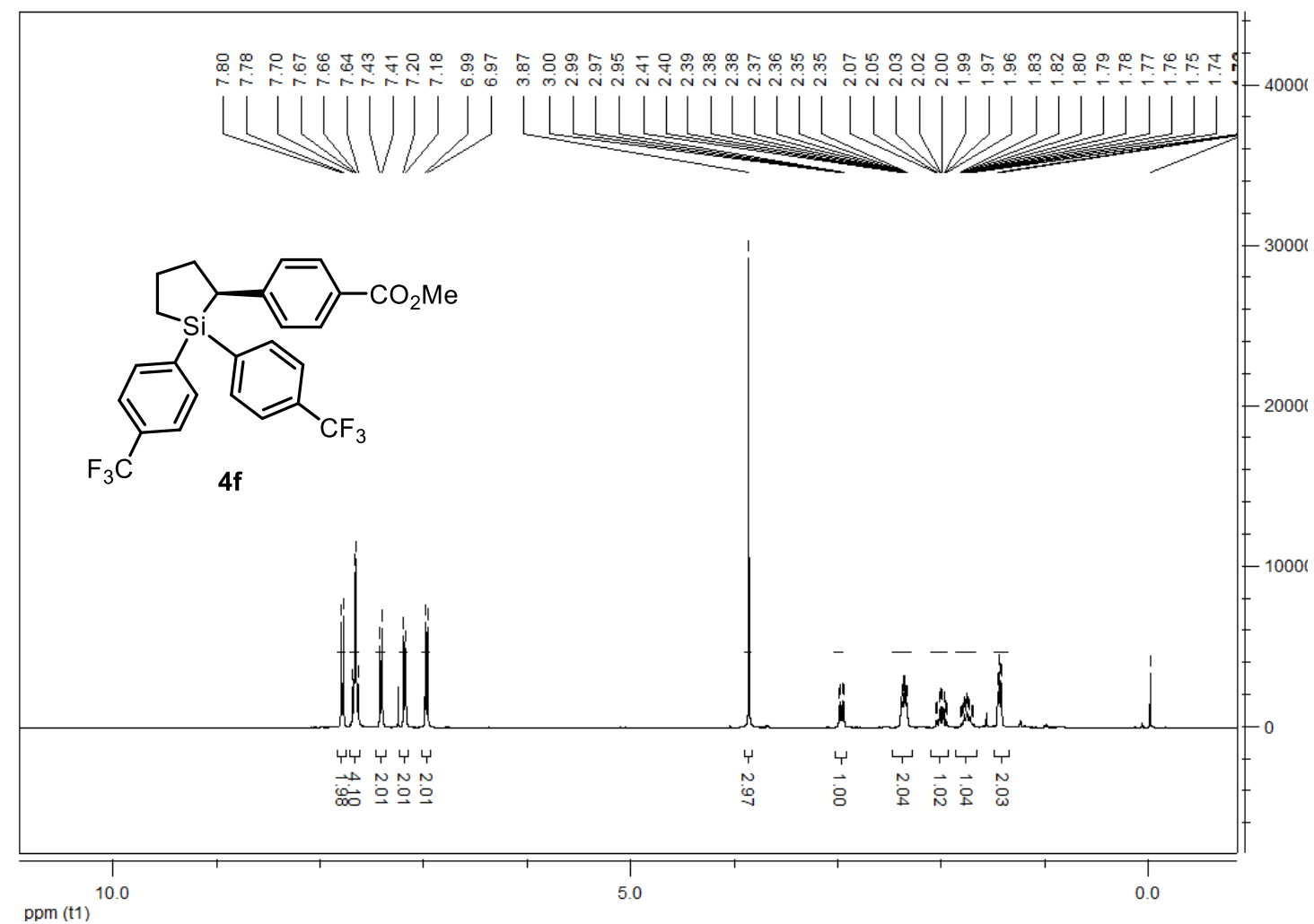



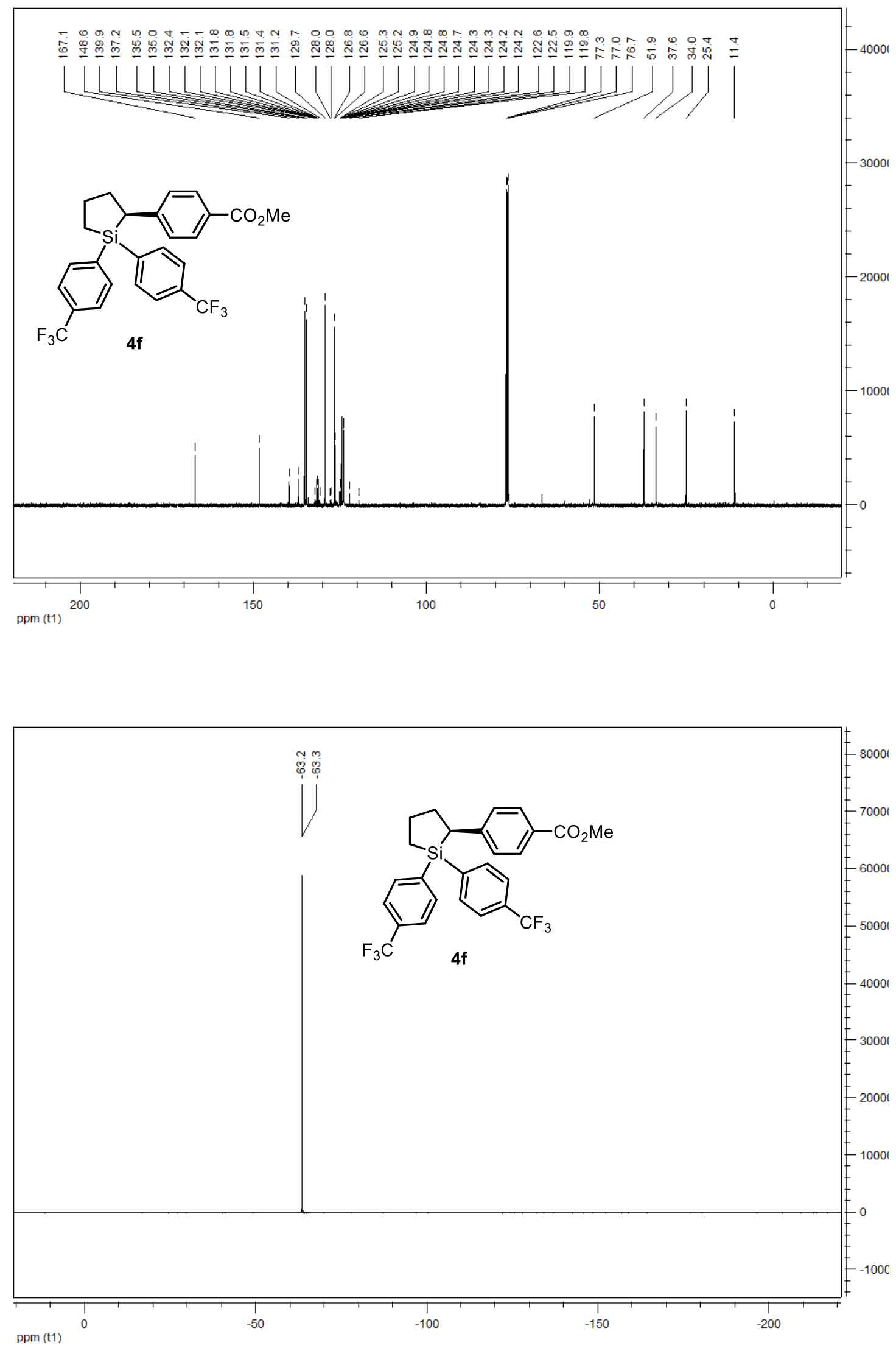
HPLC conditions: Daicel chiral column OD- $H^{*} O D-3$, hexane $:{ }^{i} \mathrm{PrOH}=100: 1,0.5 \mathrm{~mL} / \mathrm{min}, 25^{\circ} \mathrm{C}$, wavelength $=210 \mathrm{~nm}$
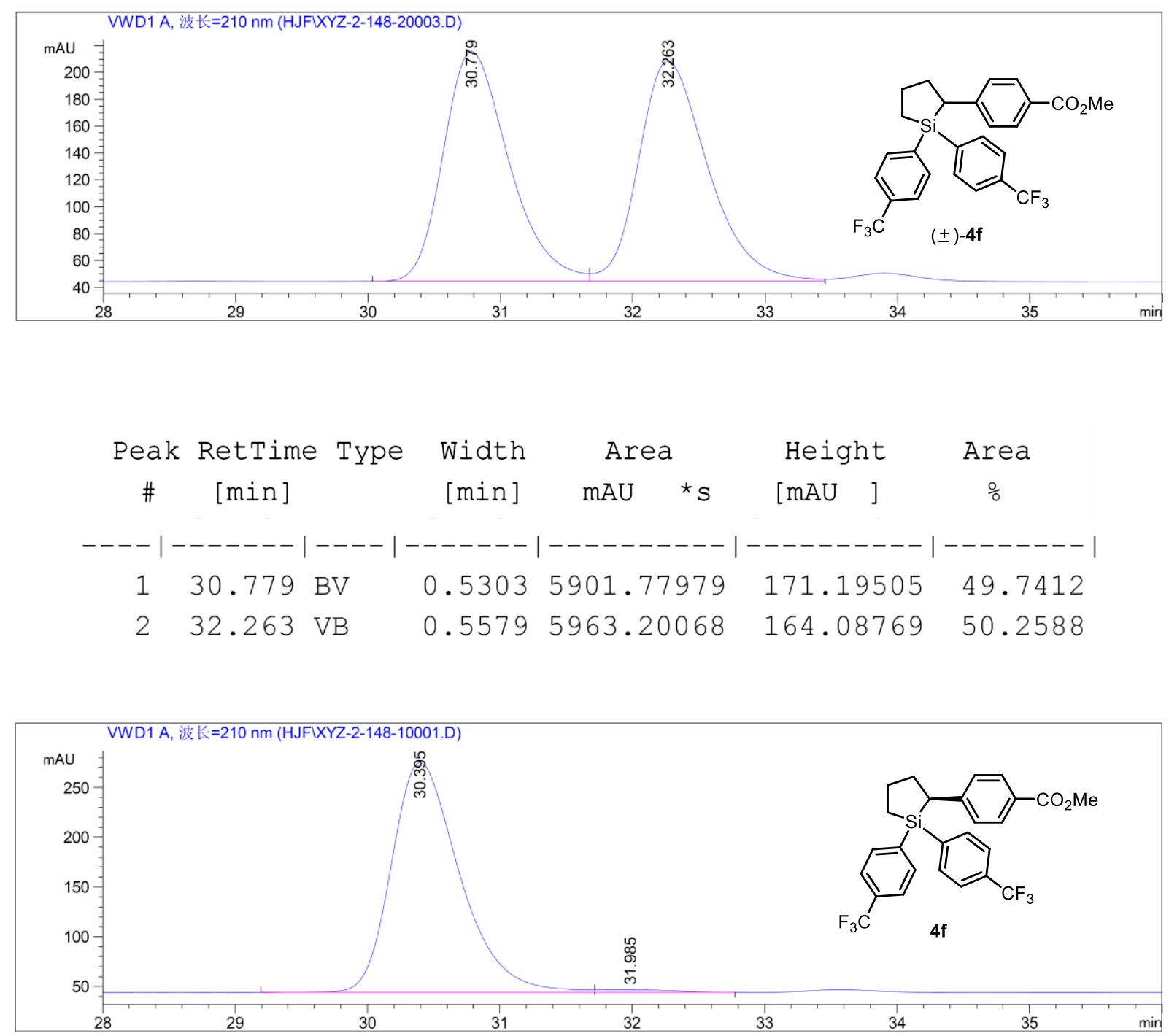

\begin{tabular}{|c|c|c|c|c|c|}
\hline \multirow{2}{*}{$\begin{array}{c}\text { Peak } \\
\quad \#\end{array}$} & \multirow{2}{*}{$\begin{array}{l}\mathrm{k} \text { RetTime Type } \\
\text { [min] }\end{array}$} & \multirow{2}{*}{$\begin{array}{l}\text { Width } \\
\text { [min] }\end{array}$} & Area & Height & \multirow{2}{*}{$\begin{array}{c}\text { Area } \\
\quad \%\end{array}$} \\
\hline & & & mAU & {$[\mathrm{mAU} \quad]$} & \\
\hline & & & ----------- & & \\
\hline 1 & $395 \mathrm{BB}$ & & 8294.41309 & 231.19691 & 8349 \\
\hline 2 & $31.985 \mathrm{BB}$ & 0.4731 & 97.77856 & 2.62948 & 1.1651 \\
\hline
\end{tabular}



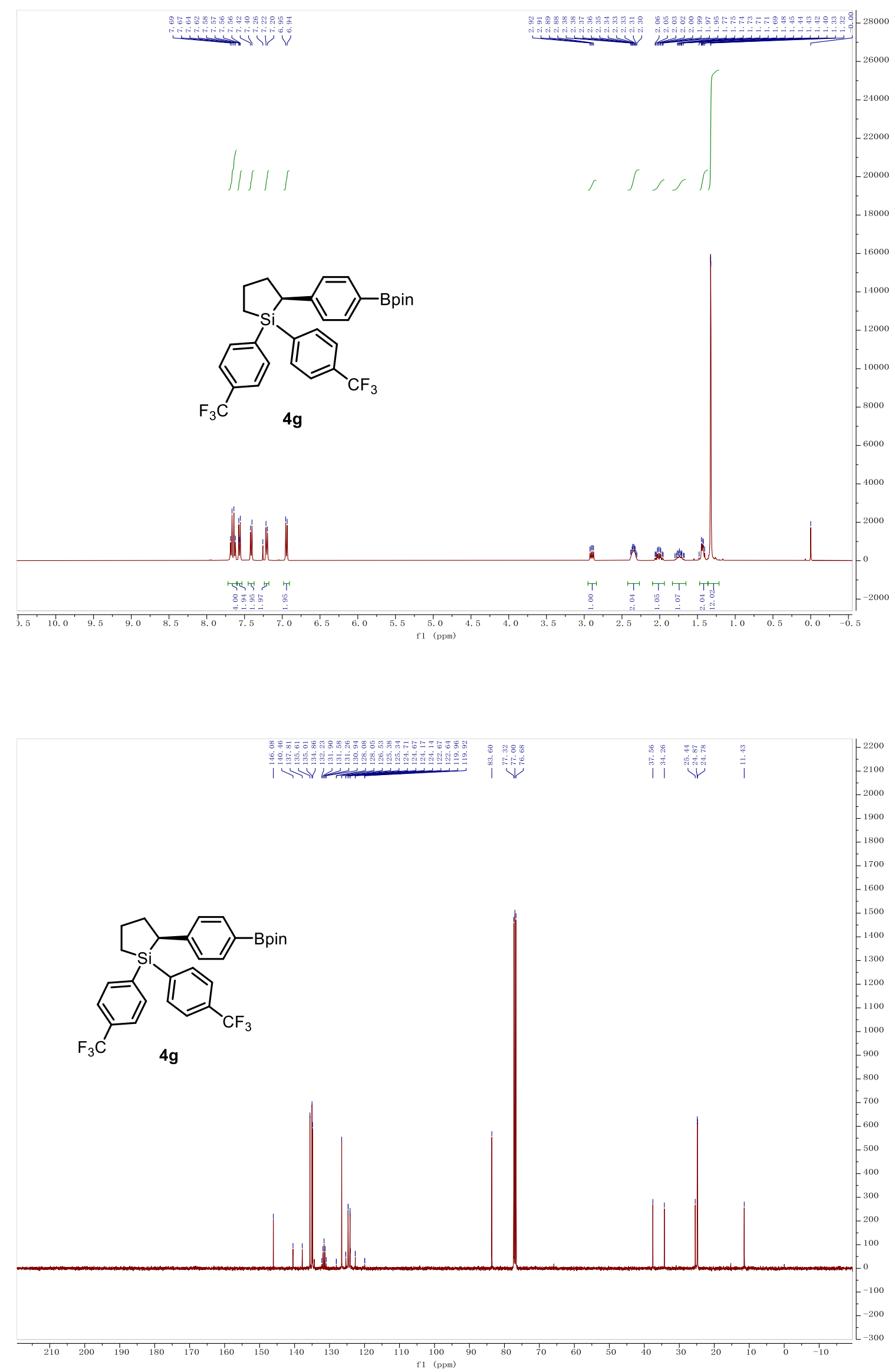


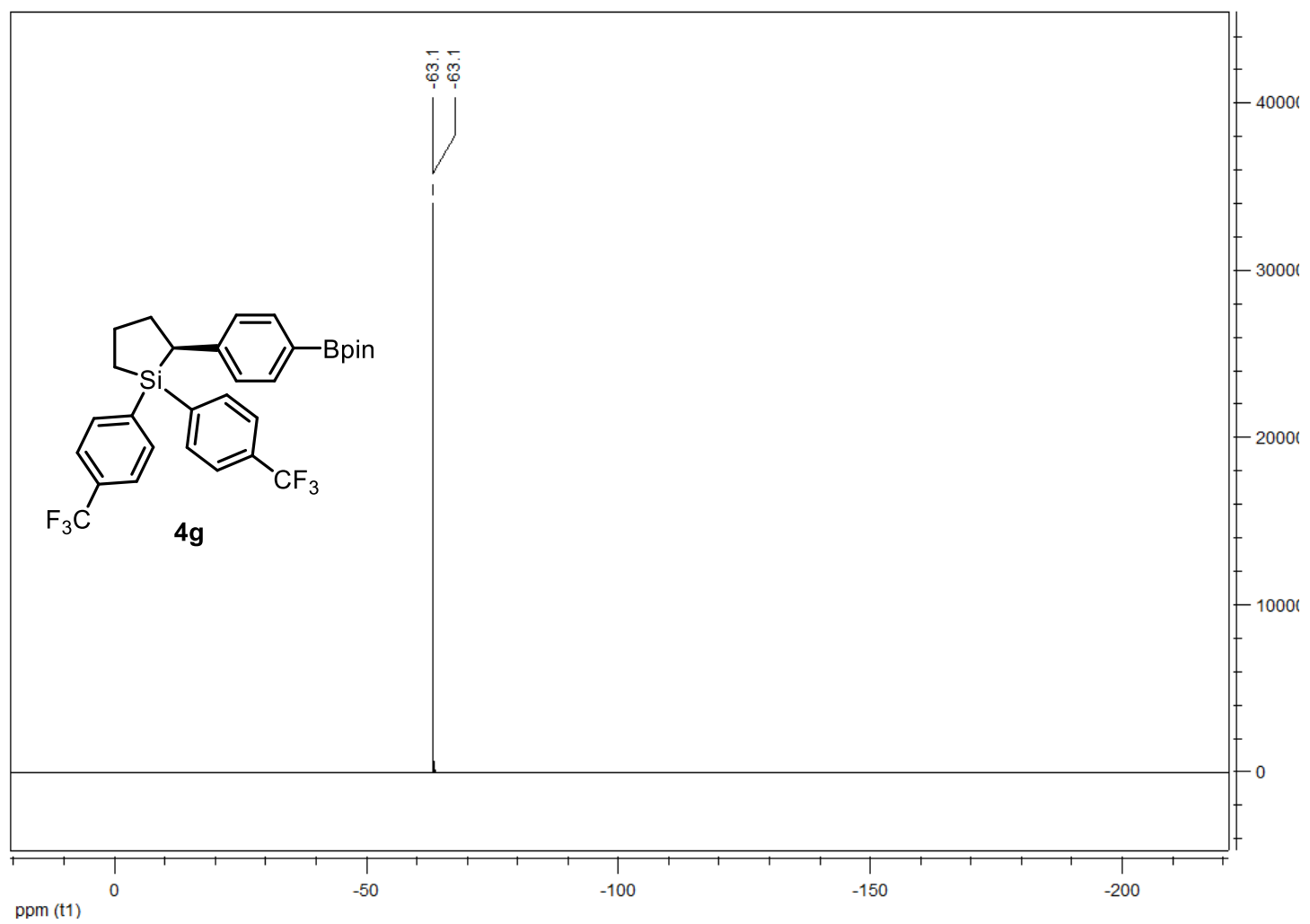

HPLC conditions: Daicel chiral column AD-H, hexane $:{ }^{i} \mathrm{PrOH}=100: 0,0.5 \mathrm{~mL} / \mathrm{min}, 25{ }^{\circ} \mathrm{C}$, wavelength $=$ $220 \mathrm{~nm}$

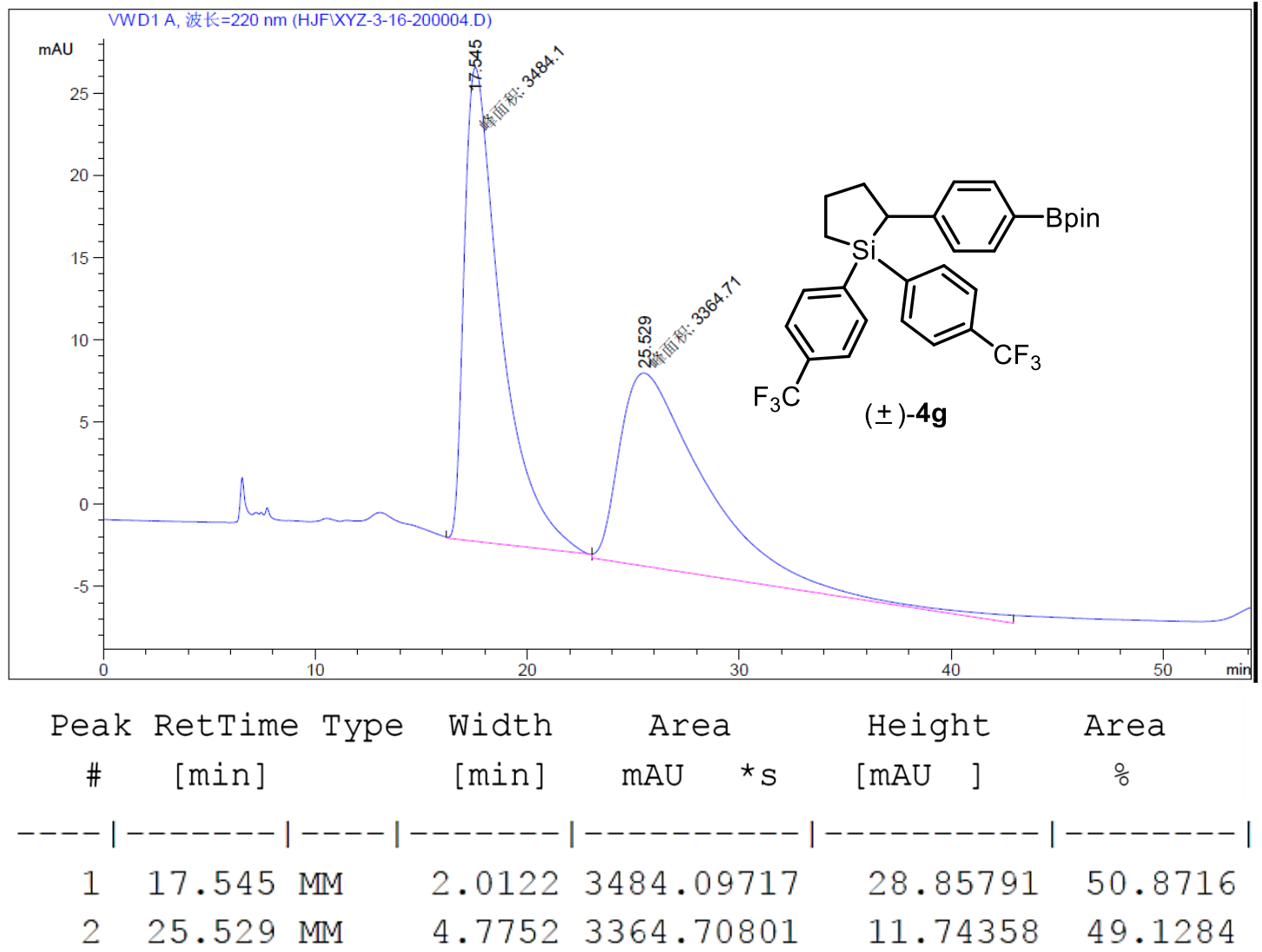



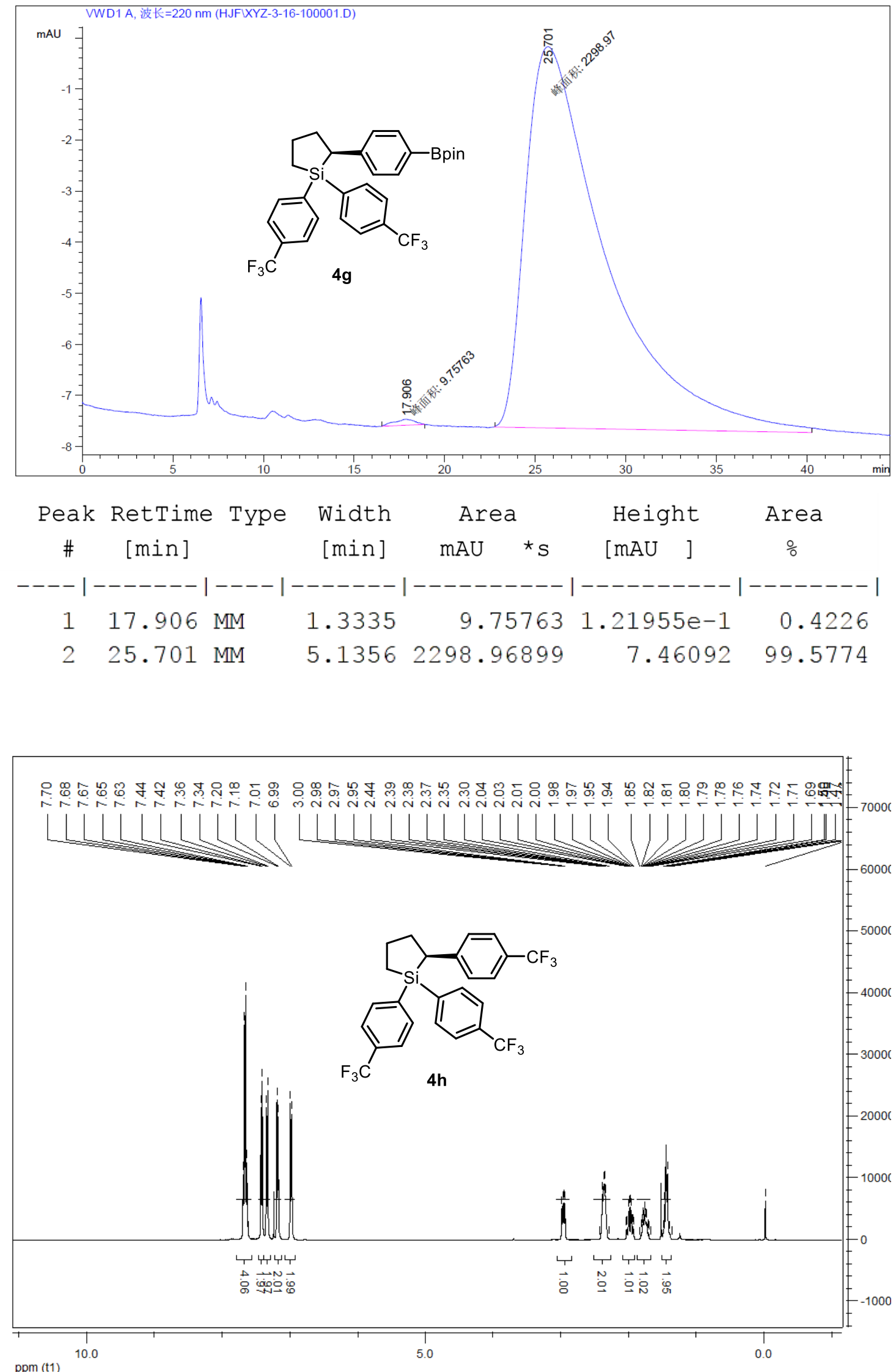

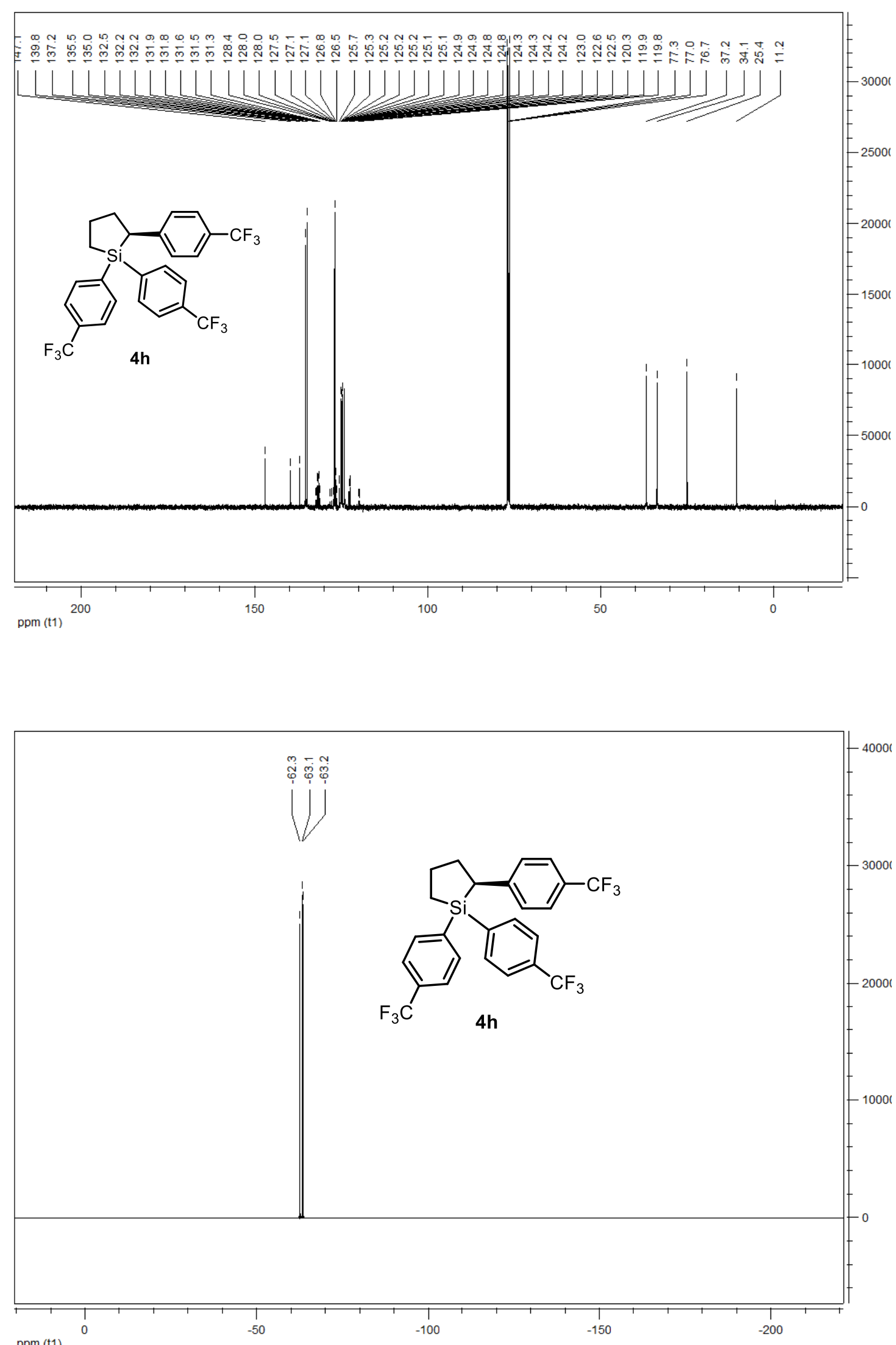
HPLC conditions: Daicel chiral column OD-3, hexane $:{ }^{i} \mathrm{PrOH}=100: 0,0.25 \mathrm{~mL} / \mathrm{min}, 25^{\circ} \mathrm{C}$, wavelength $=$ $220 \mathrm{~nm}$
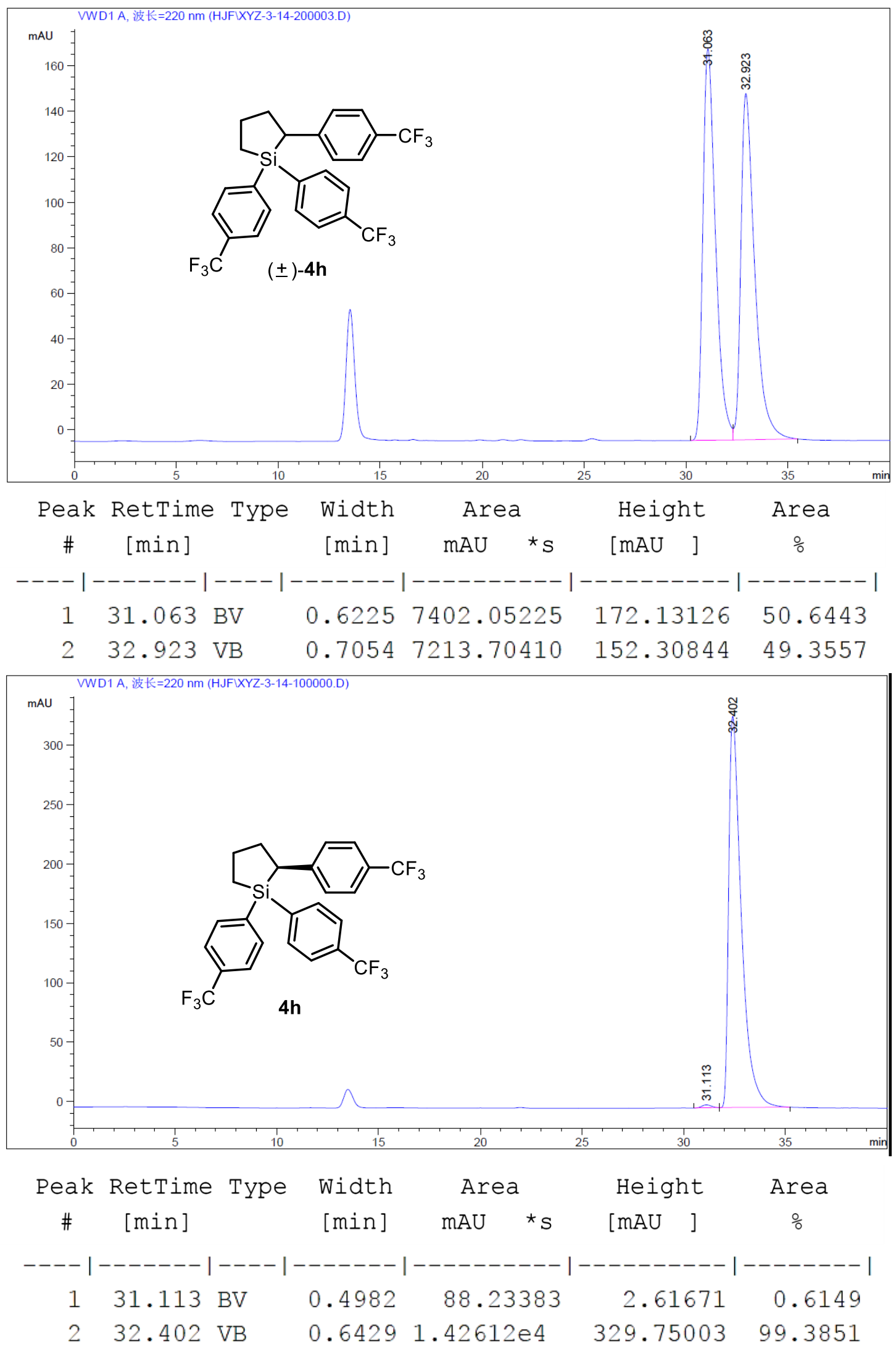

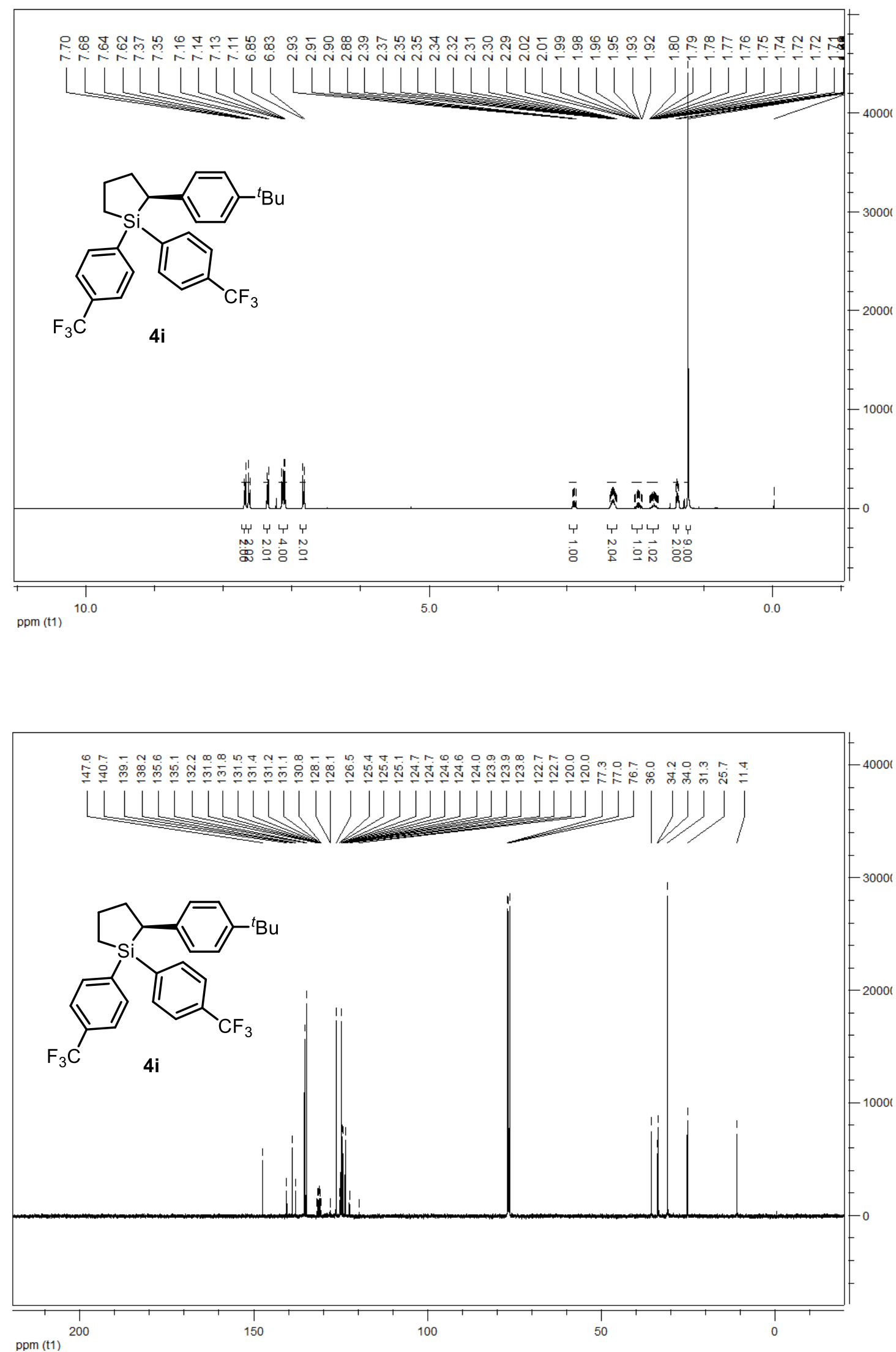


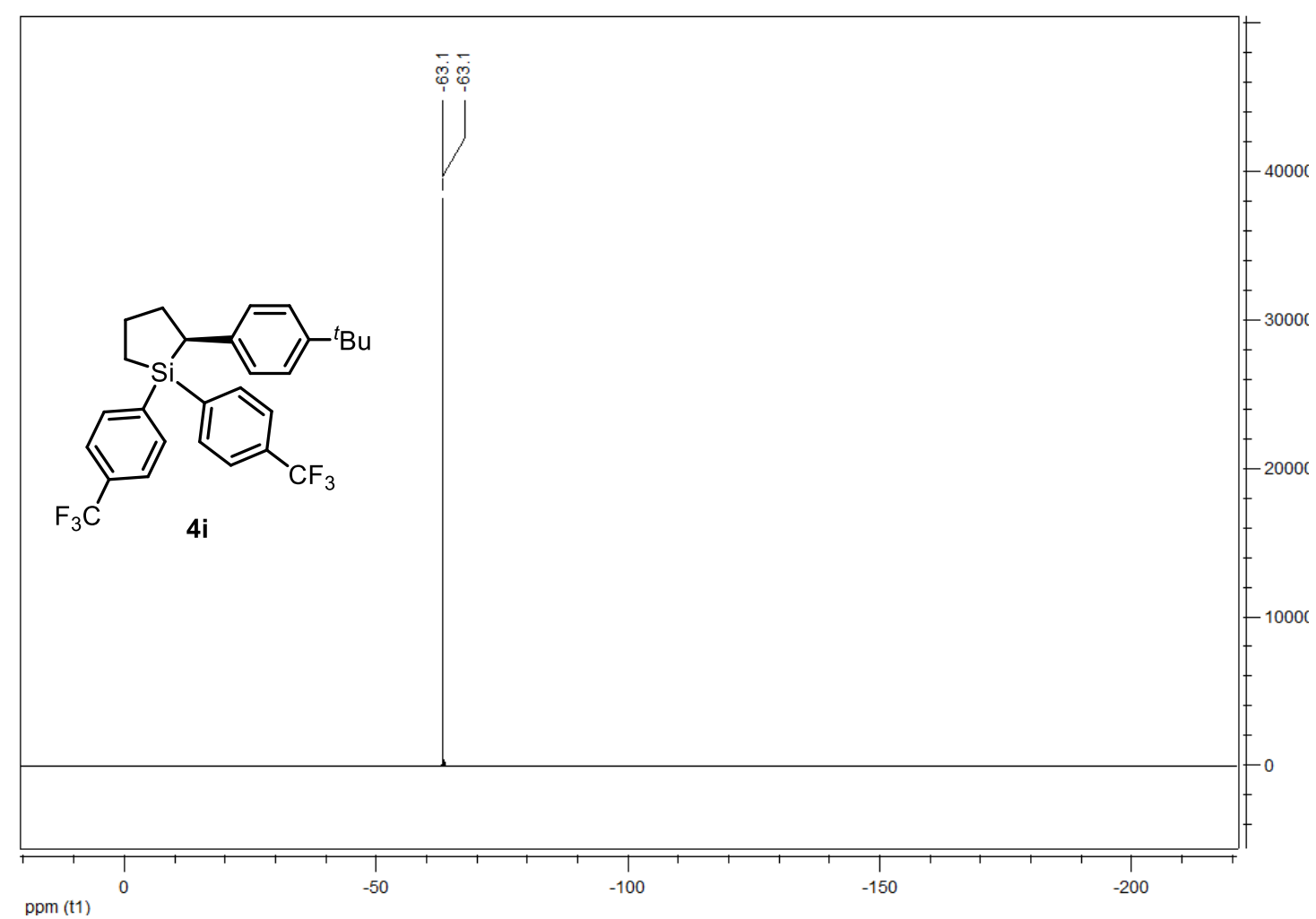

HPLC conditions: Daicel chiral column AD-H, hexane $:{ }^{i} \mathrm{PrOH}=100: 0,0.2 \mathrm{~mL} / \mathrm{min}, 25^{\circ} \mathrm{C}$, wavelength $=$ $220 \mathrm{~nm}$

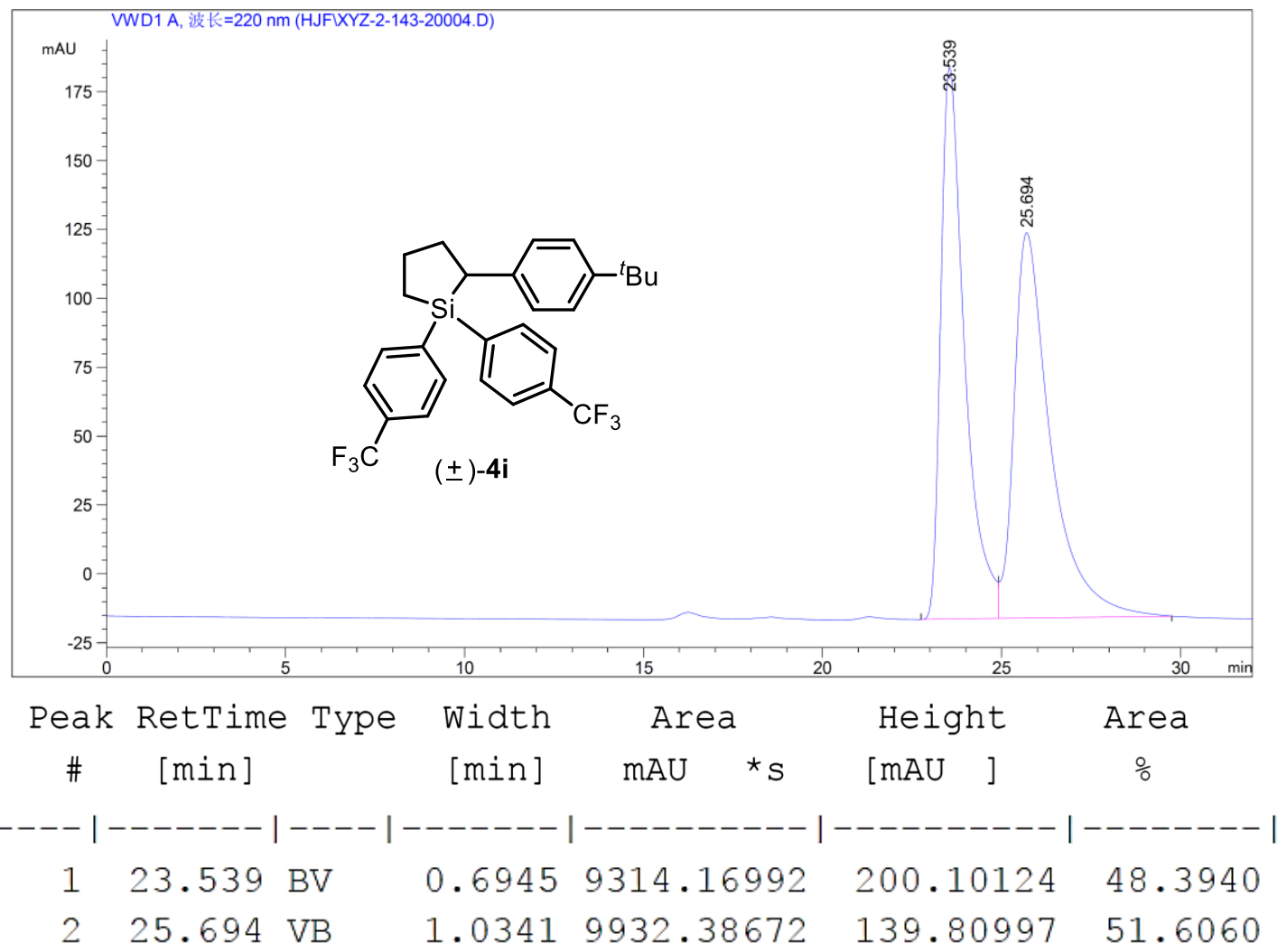



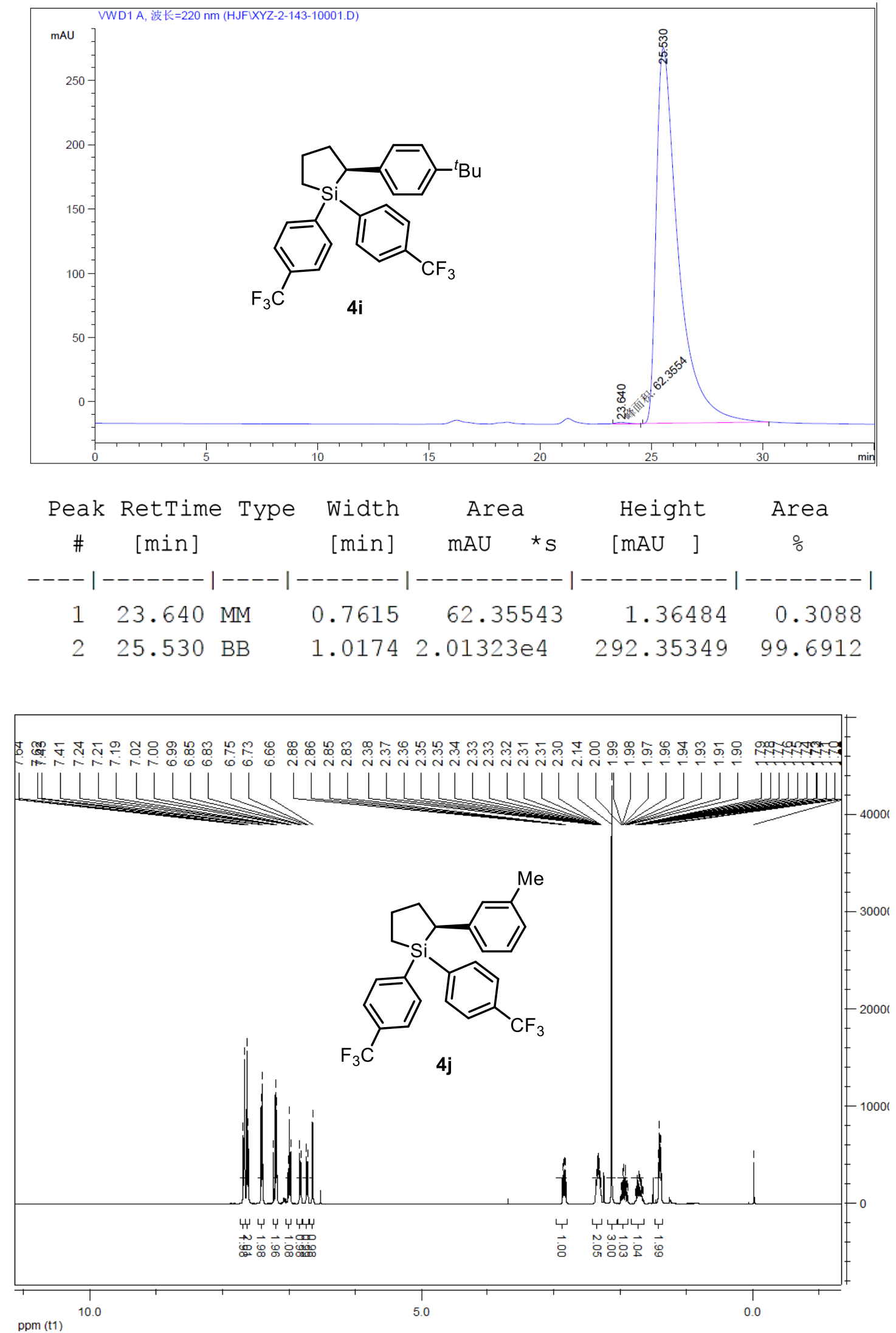

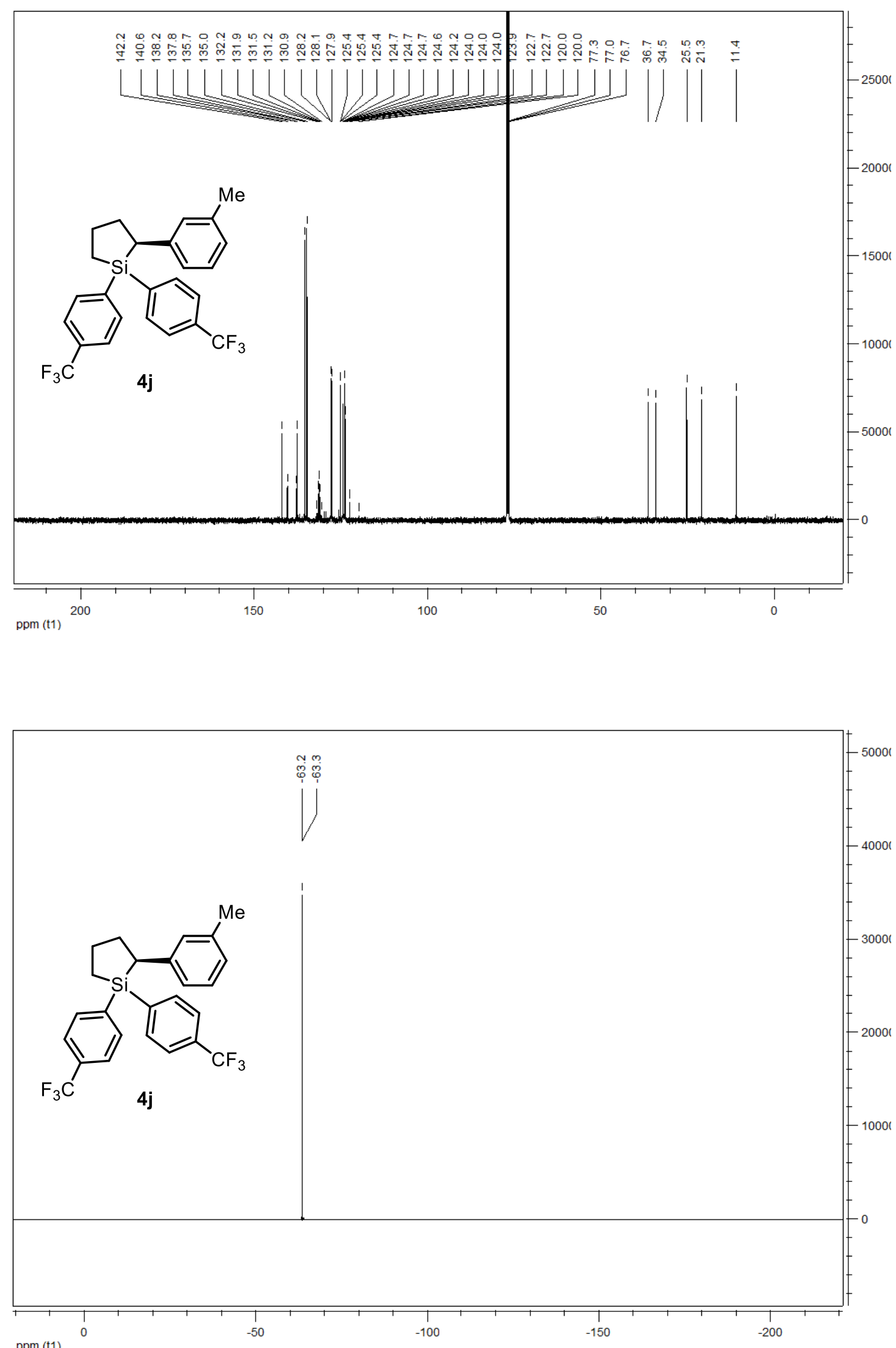
HPLC conditions: Daicel chiral column $O D-3$, hexane $:{ }^{i} \mathrm{PrOH}=100: 0,0.5 \mathrm{~mL} / \mathrm{min}, 25{ }^{\circ} \mathrm{C}$, wavelength $=$ $220 \mathrm{~nm}$

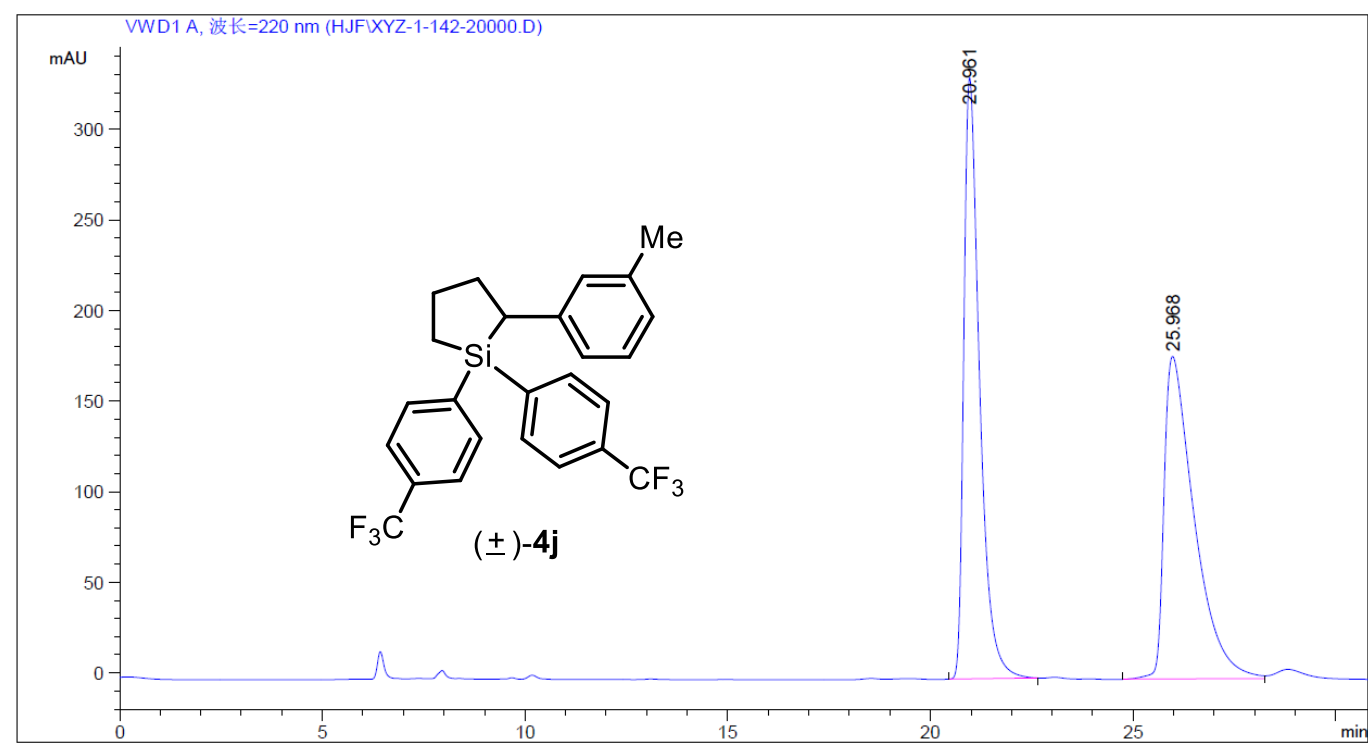

\begin{tabular}{|c|c|c|c|c|c|c|}
\hline Peak & RetTime Type & Width & Are & & Height & Area \\
\hline \# & {$[\min ]$} & {$[\min ]$} & mAU & ${ }^{*} \mathrm{~S}$ & {$[\mathrm{mAU} \quad]$} & $\%$ \\
\hline & 61 & 3 & 89 & 742 & 25 & \\
\hline 2 & $25.968 \mathrm{BB}$ & 0.7 & 9255 & 3887 & 00674 & 129 \\
\hline
\end{tabular}
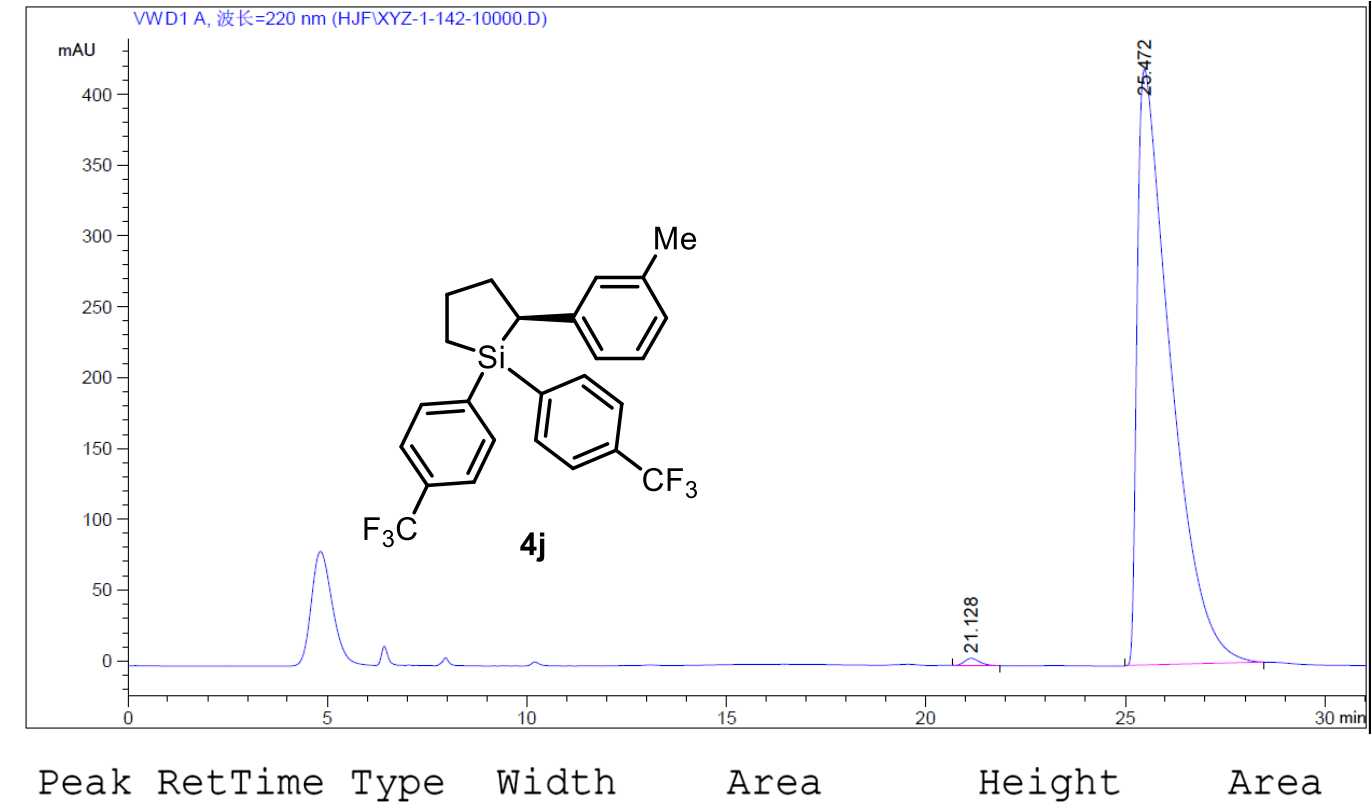

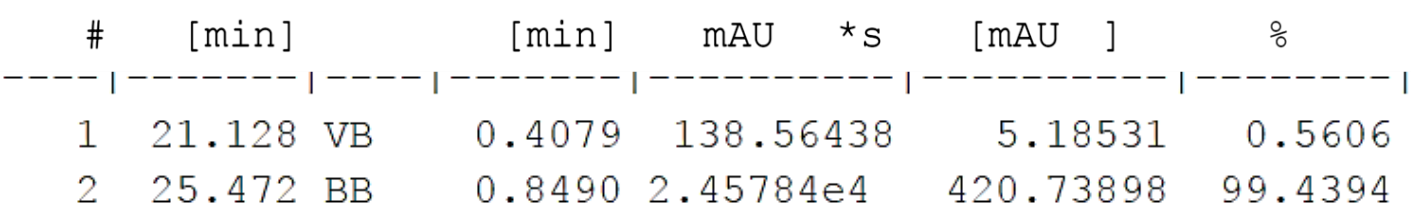



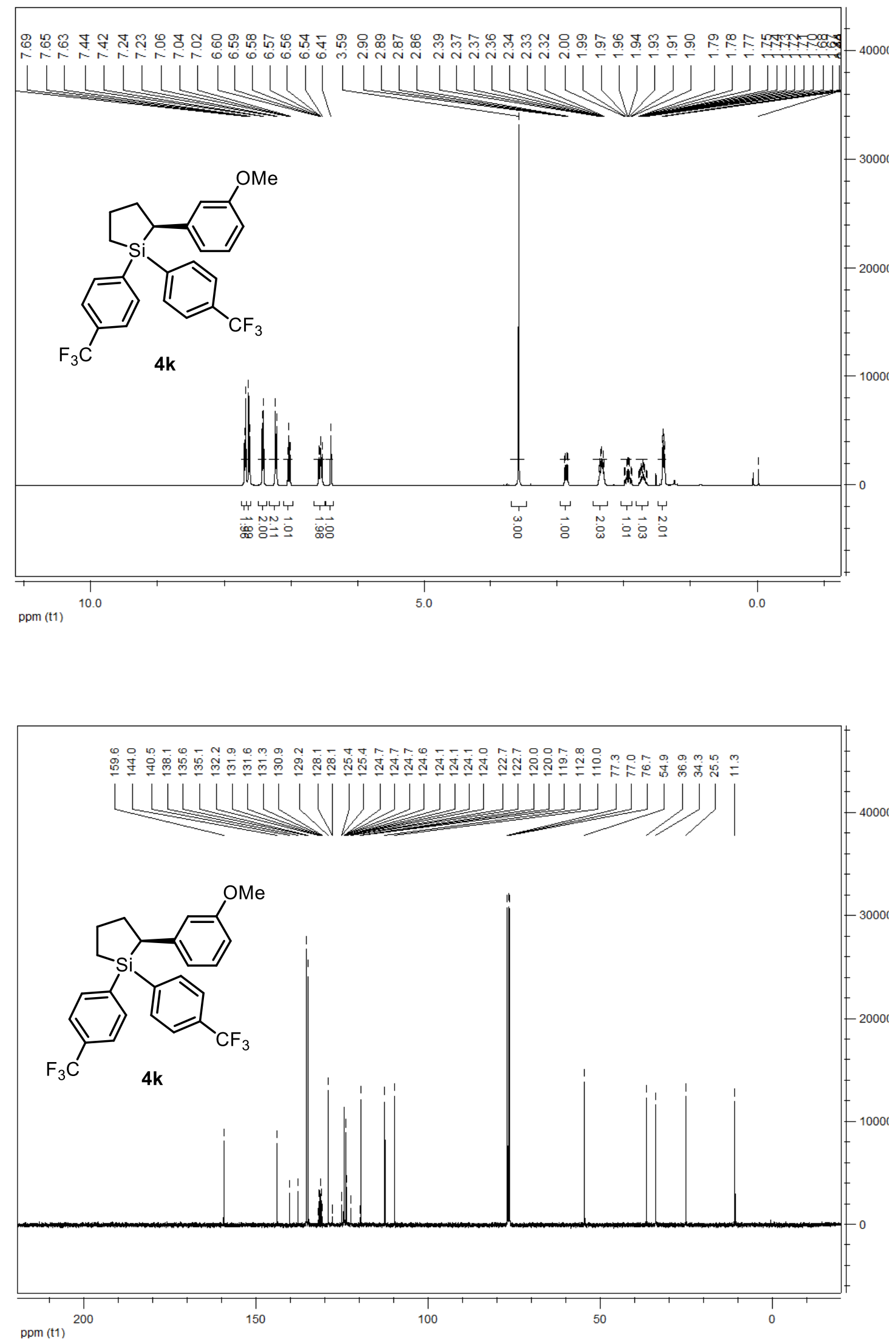


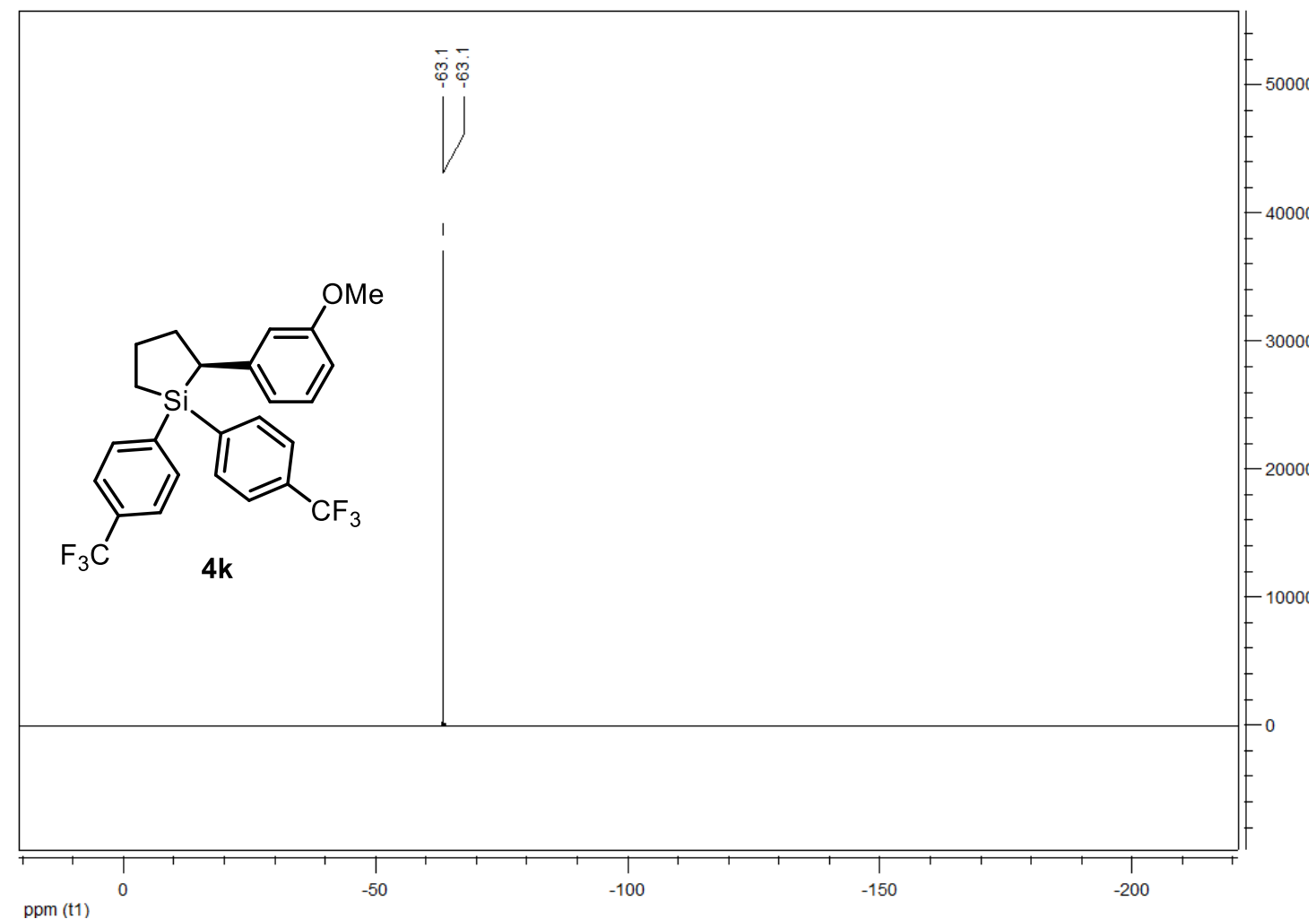

HPLC conditions: Daicel chiral column OD-3, hexane $:{ }^{i} \mathrm{PrOH}=200: 1,0.5 \mathrm{~mL} / \mathrm{min}, 25{ }^{\circ} \mathrm{C}$, wavelength $=$ $220 \mathrm{~nm}$

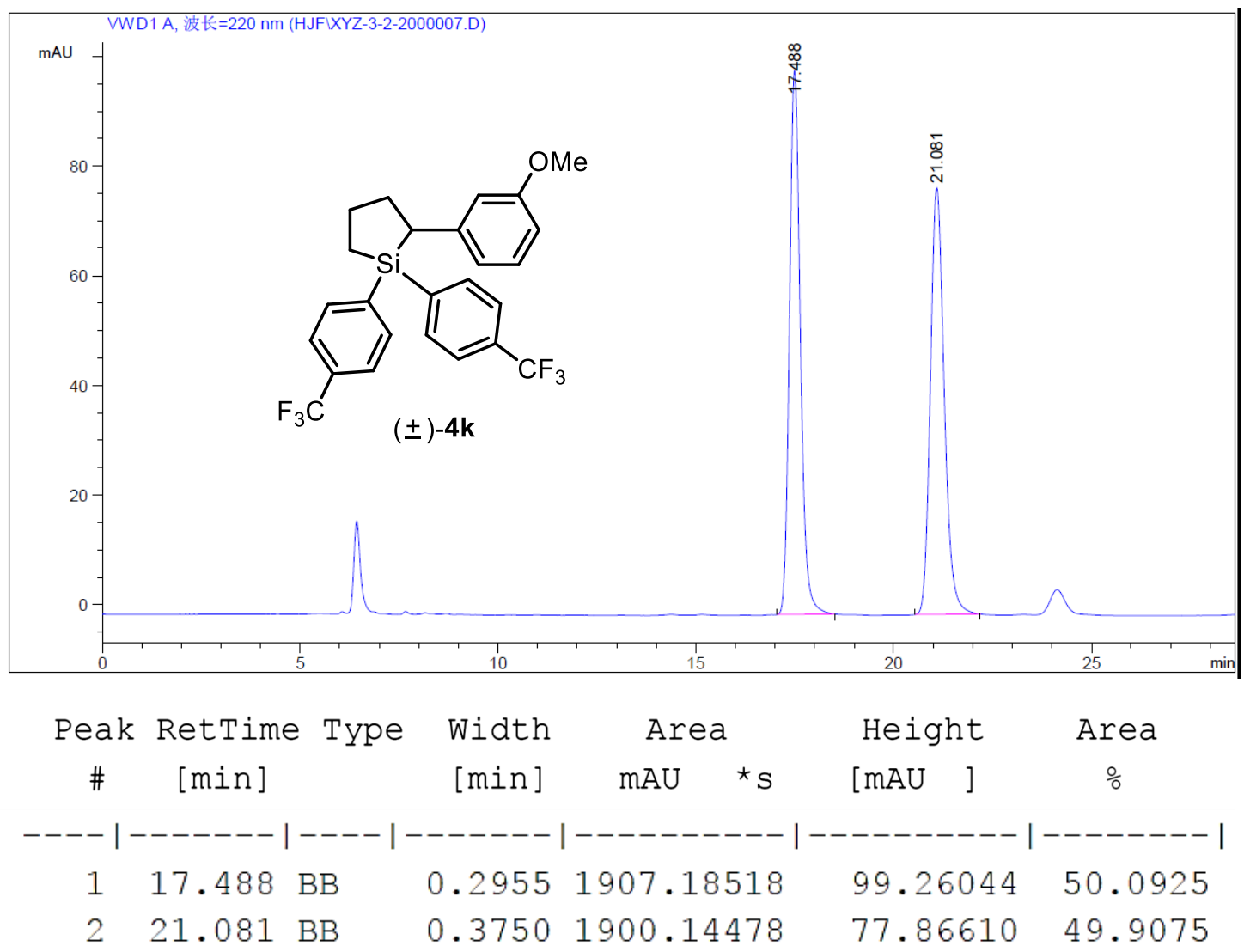




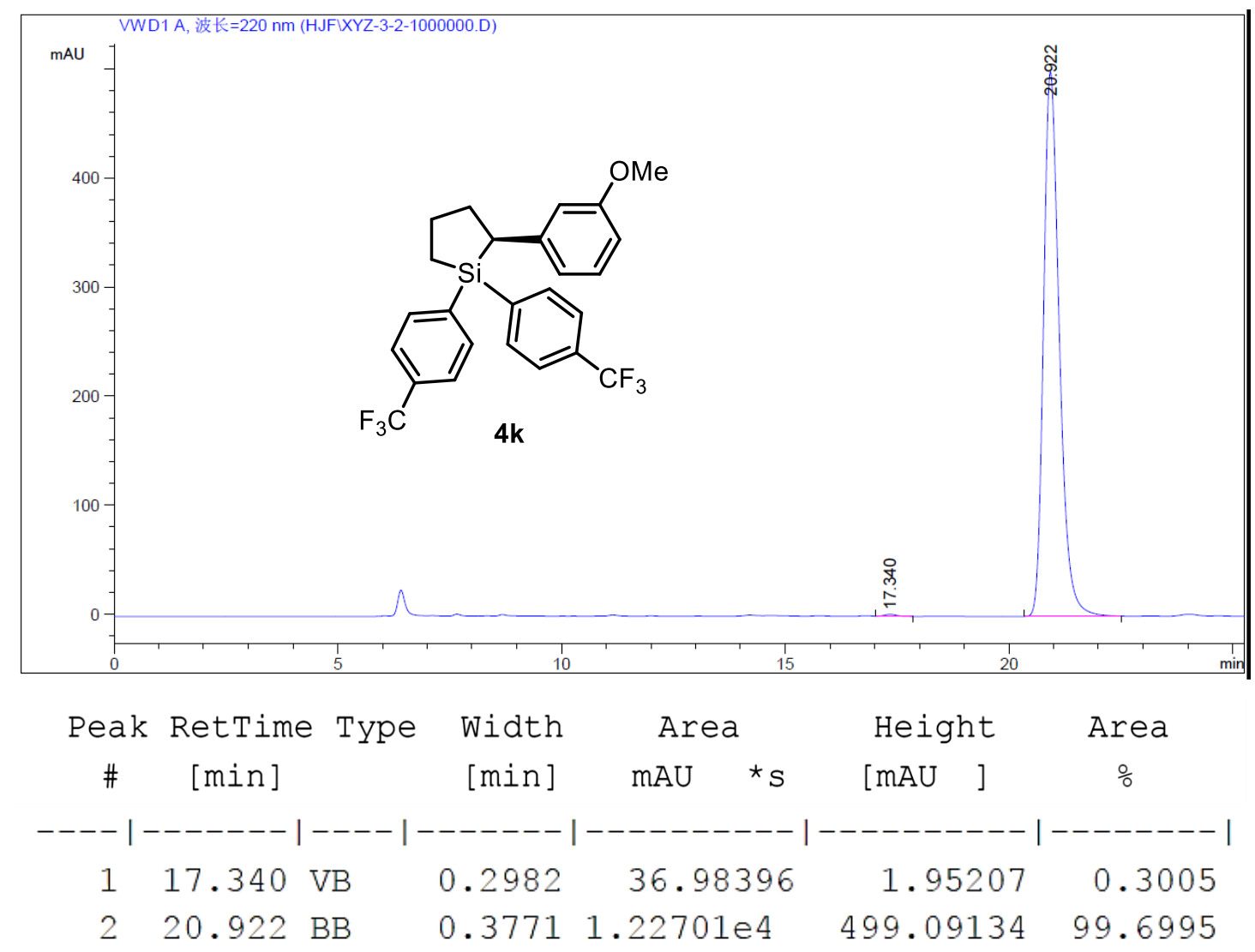

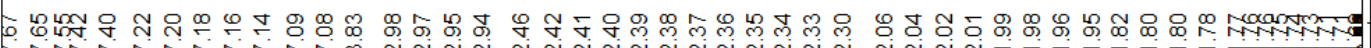

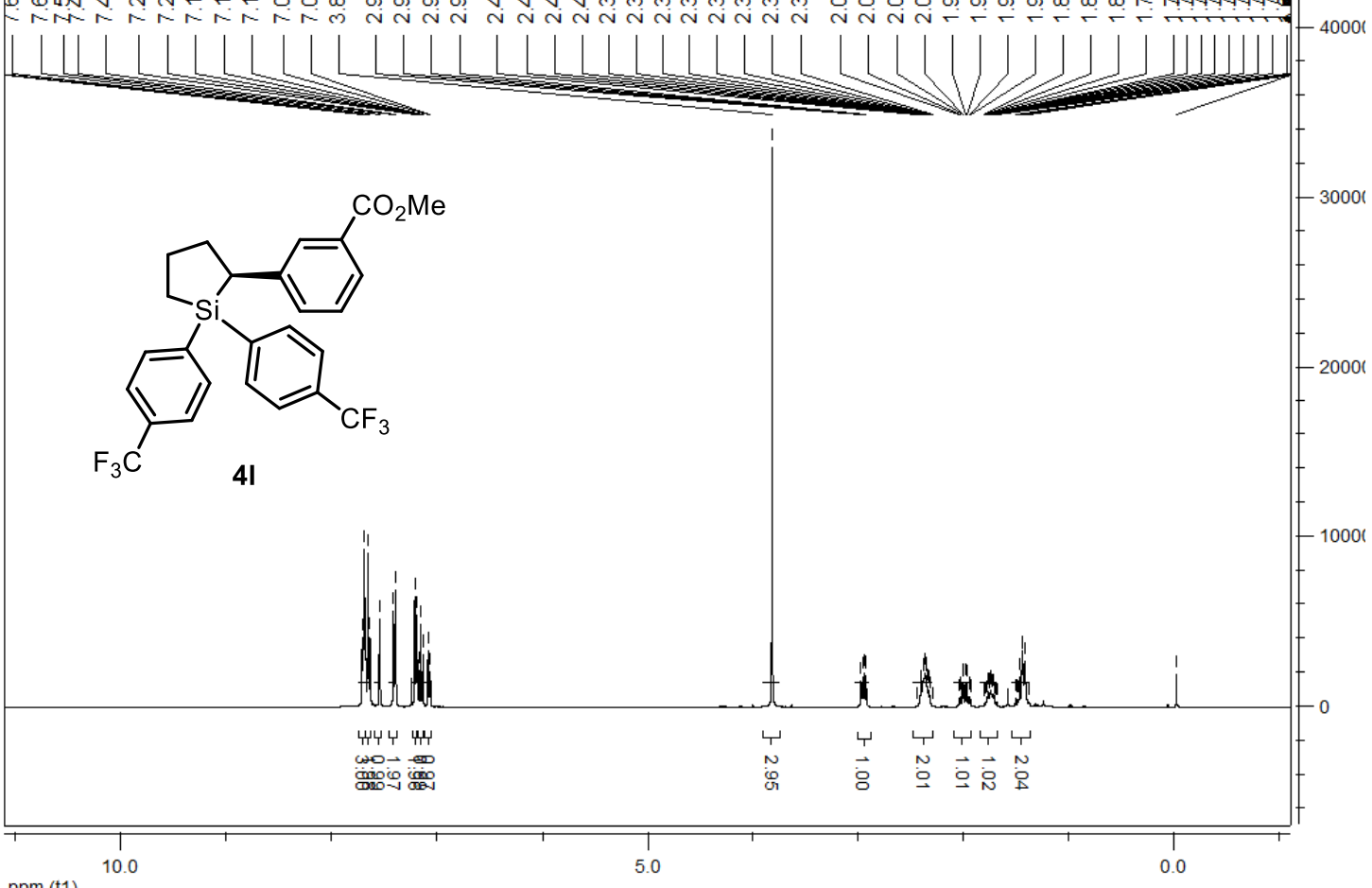



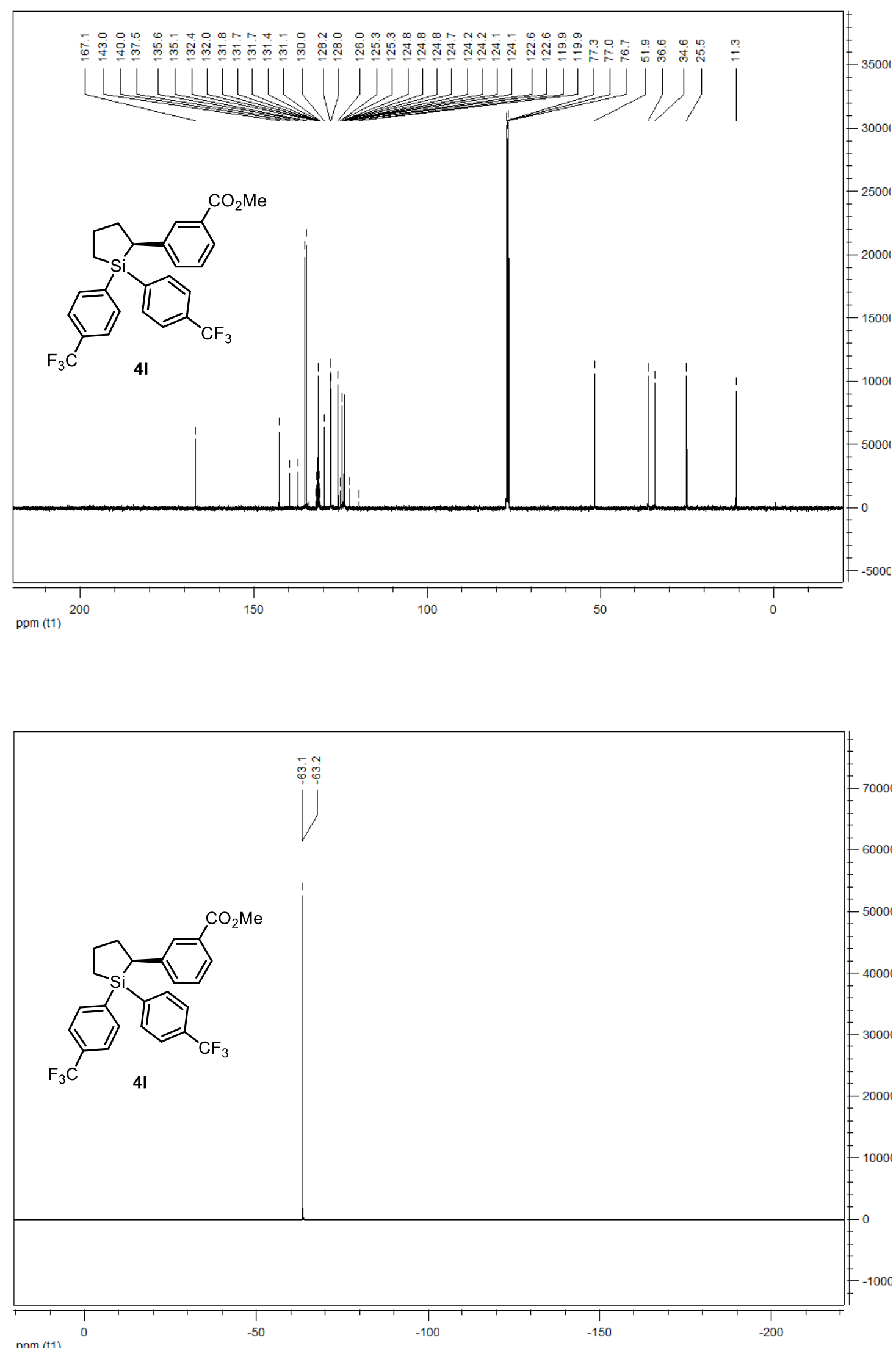
HPLC conditions: Daicel chiral column AD-H, hexane $:{ }^{i} \mathrm{PrOH}=300: 1,0.5 \mathrm{~mL} / \mathrm{min}, 25^{\circ} \mathrm{C}$, wavelength $=$ $220 \mathrm{~nm}$
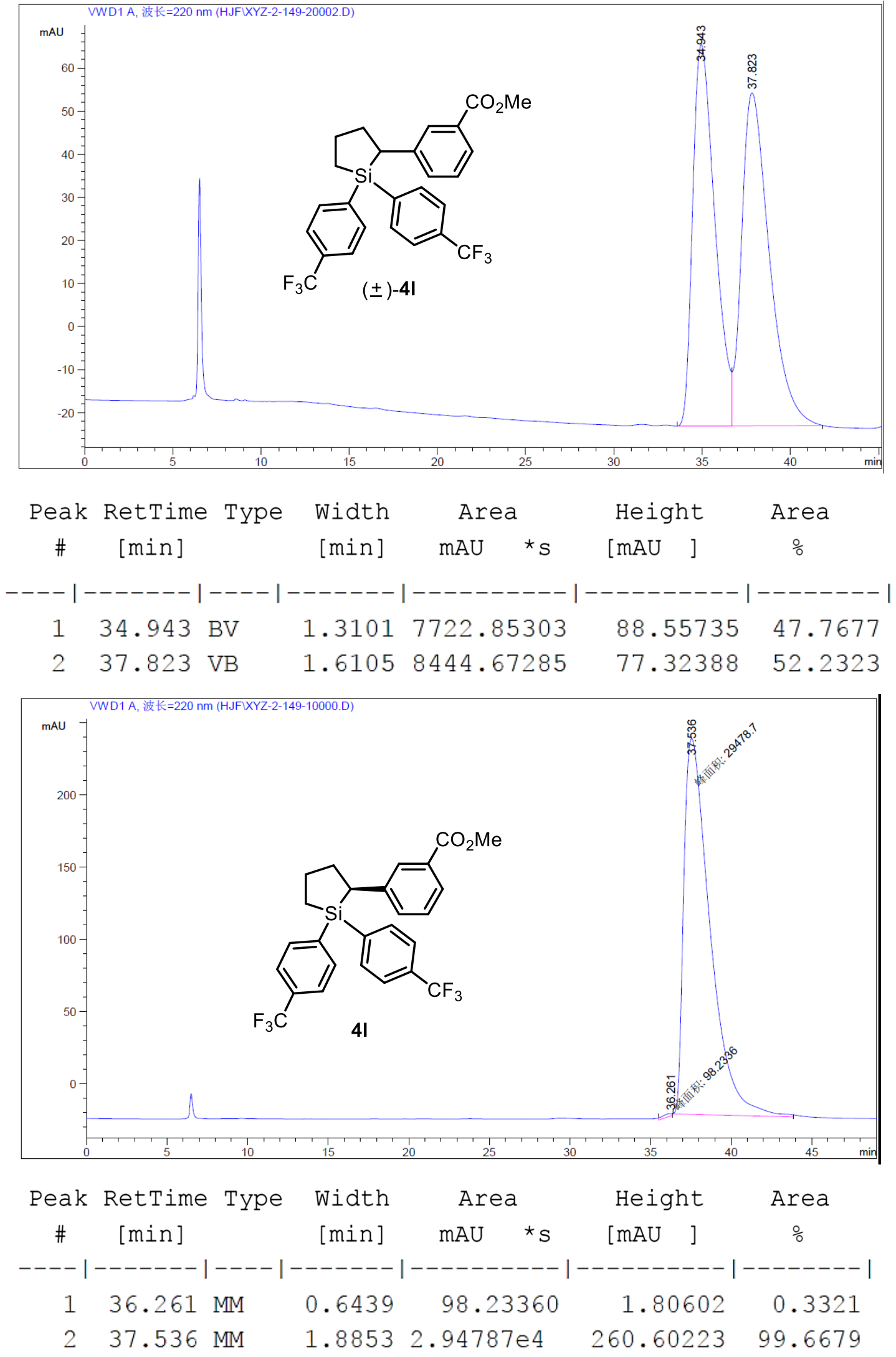


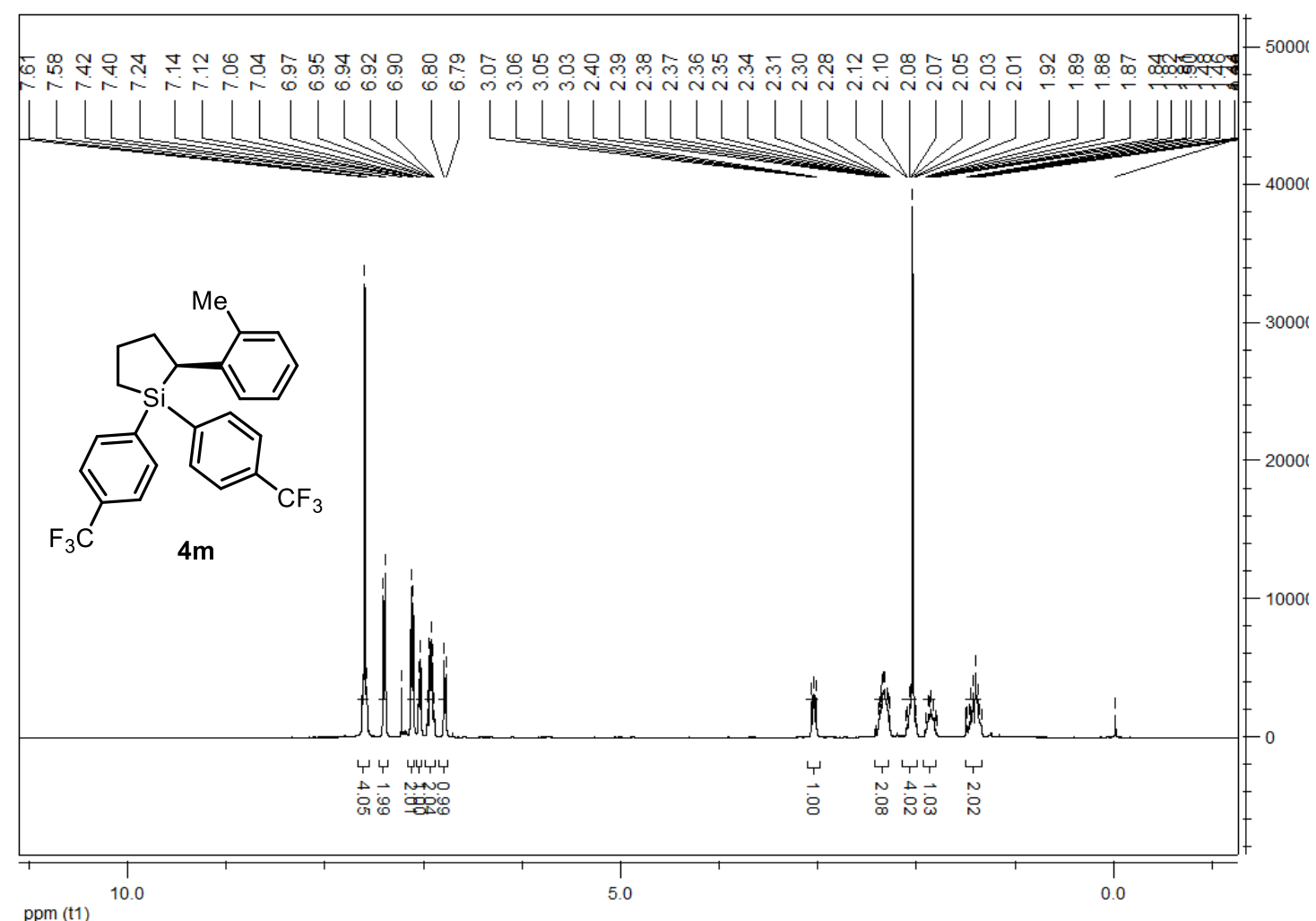

ppm (t1)

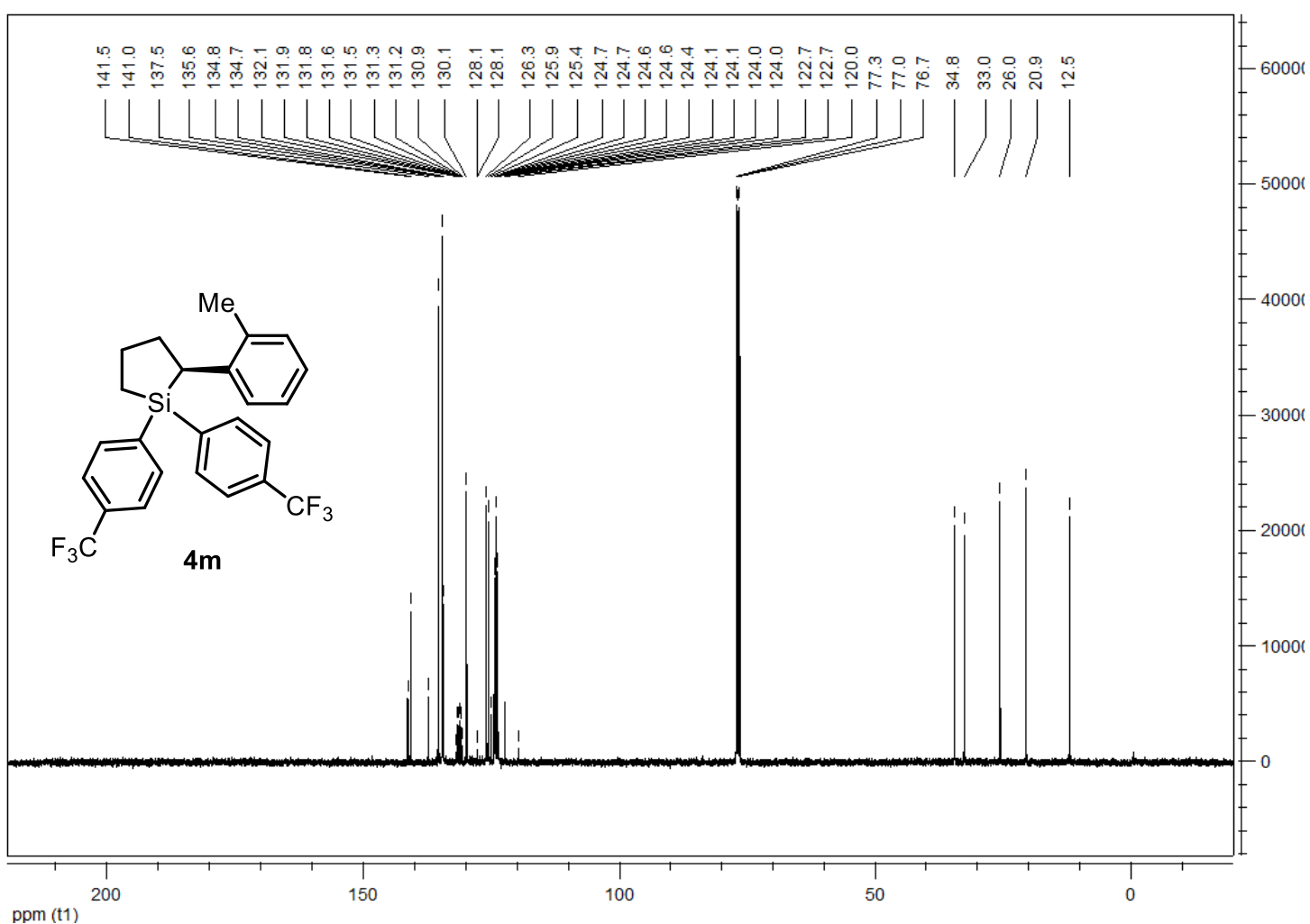




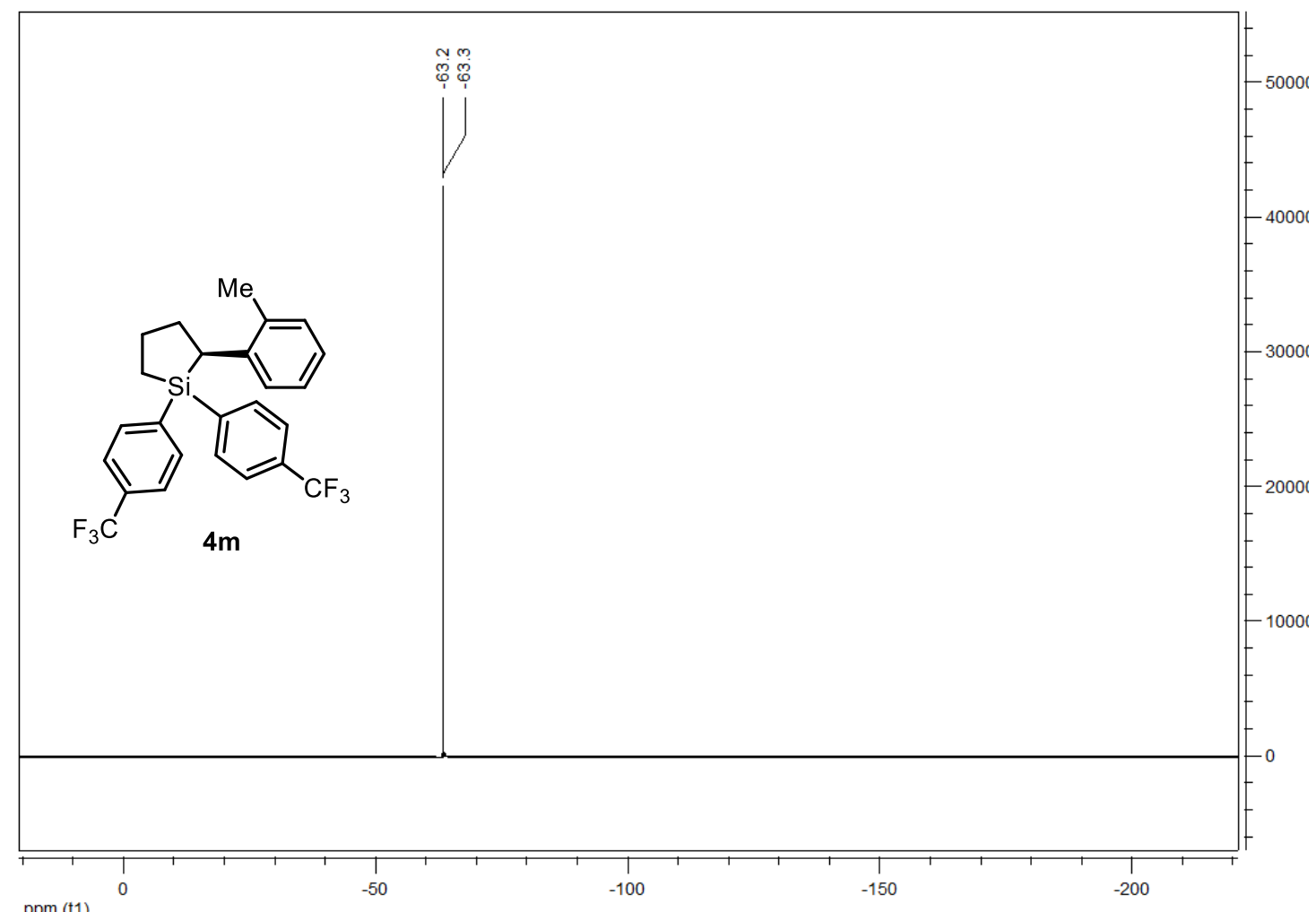

HPLC conditions: Daicel chiral column OD-3, hexane $:{ }^{i} \mathrm{PrOH}=100: 0,0.5 \mathrm{~mL} / \mathrm{min}, 25{ }^{\circ} \mathrm{C}$, wavelength $=$ $220 \mathrm{~nm}$

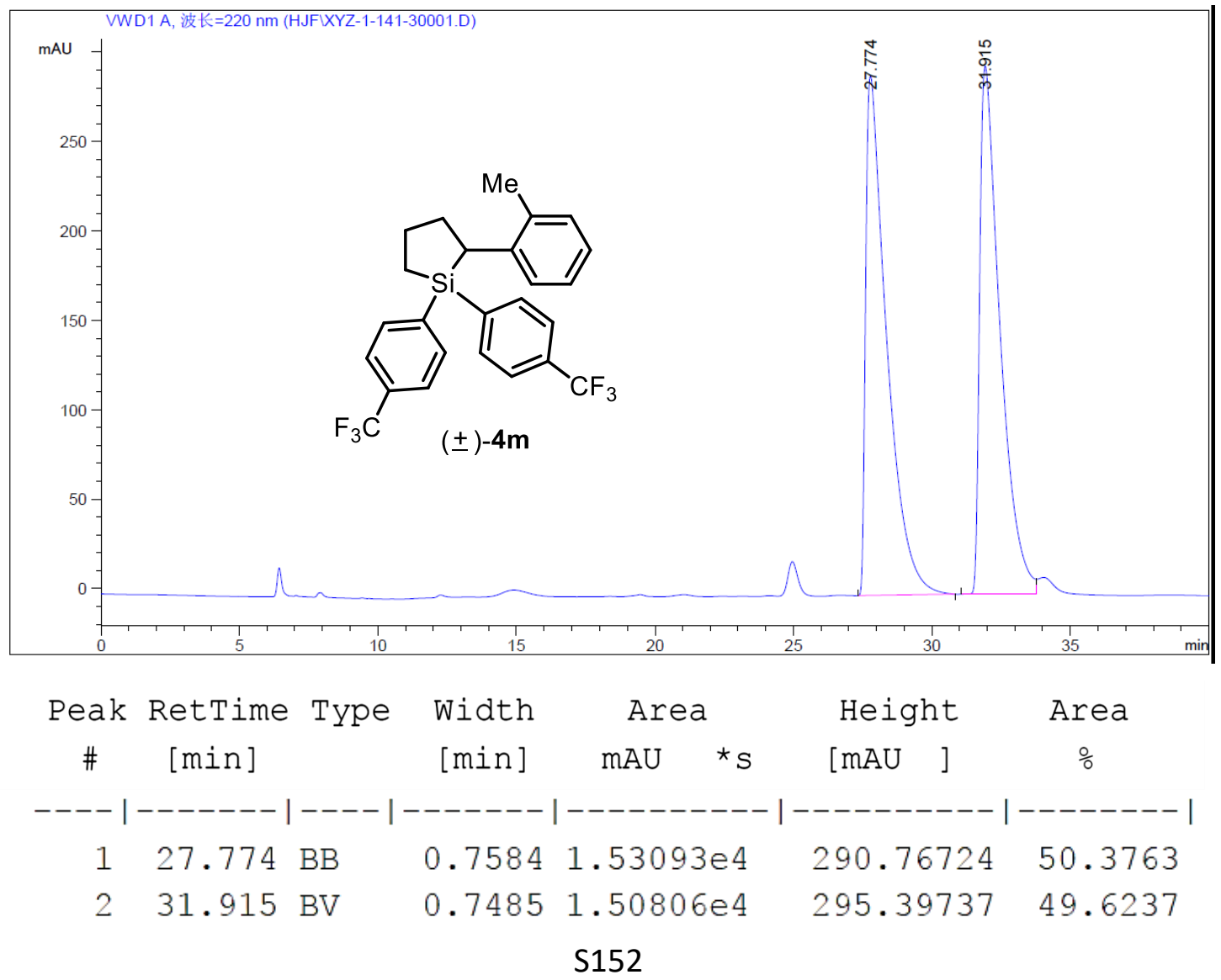




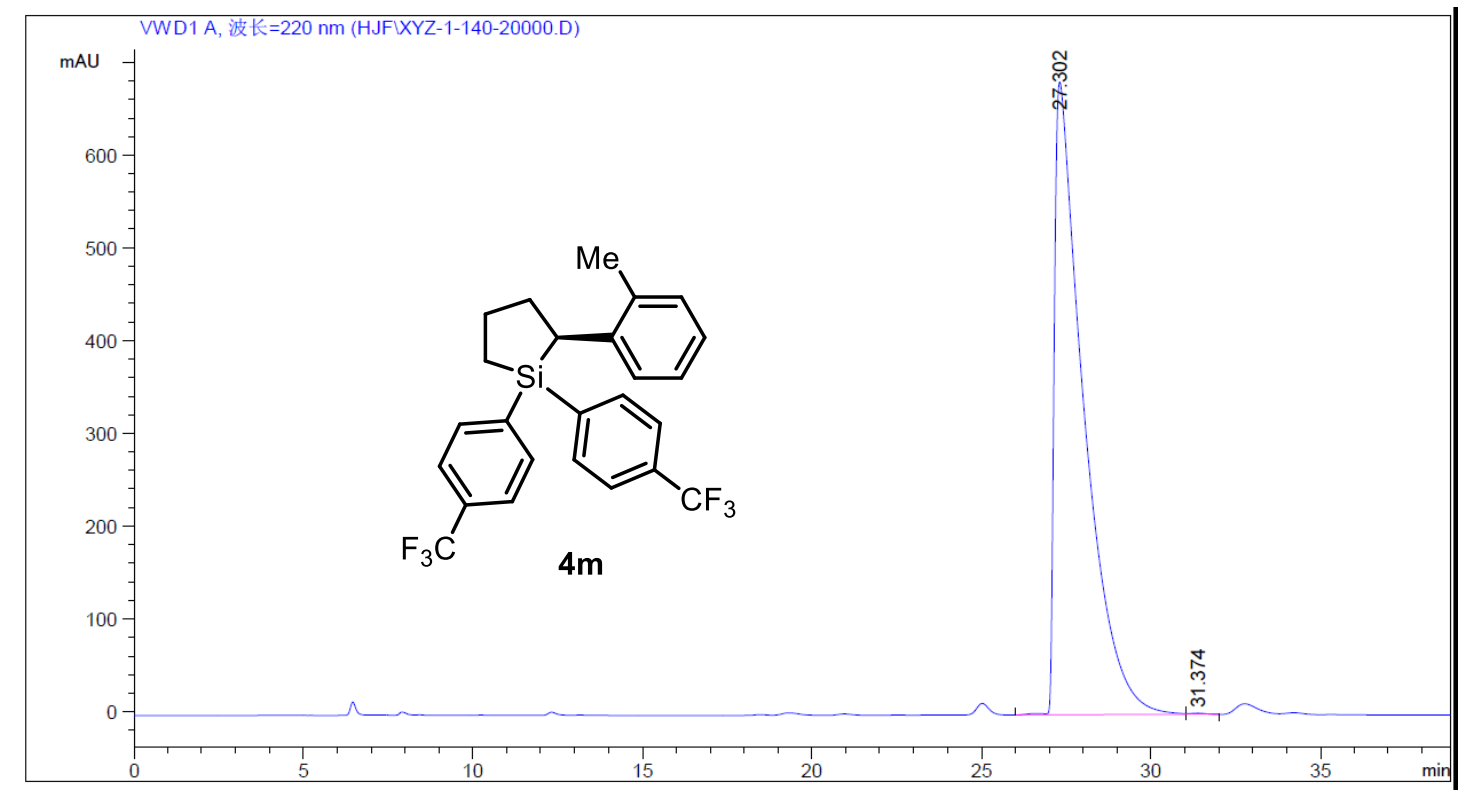

Peak RetTime Type Width Area Height Area \# [min] [min] $\mathrm{mAU}{ }^{*} \mathrm{~S} \quad[\mathrm{mAU}]$ $----|-------|----|-------|----------|----------|--------\mid$

$\begin{array}{lllllll}1 & 27.302 & \mathrm{BB} & 0.8395 & 4.11000 \mathrm{e} & 682.41541 & 99.8338\end{array}$

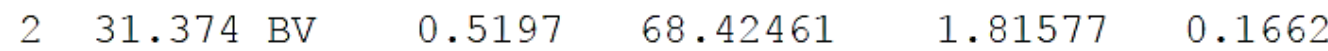

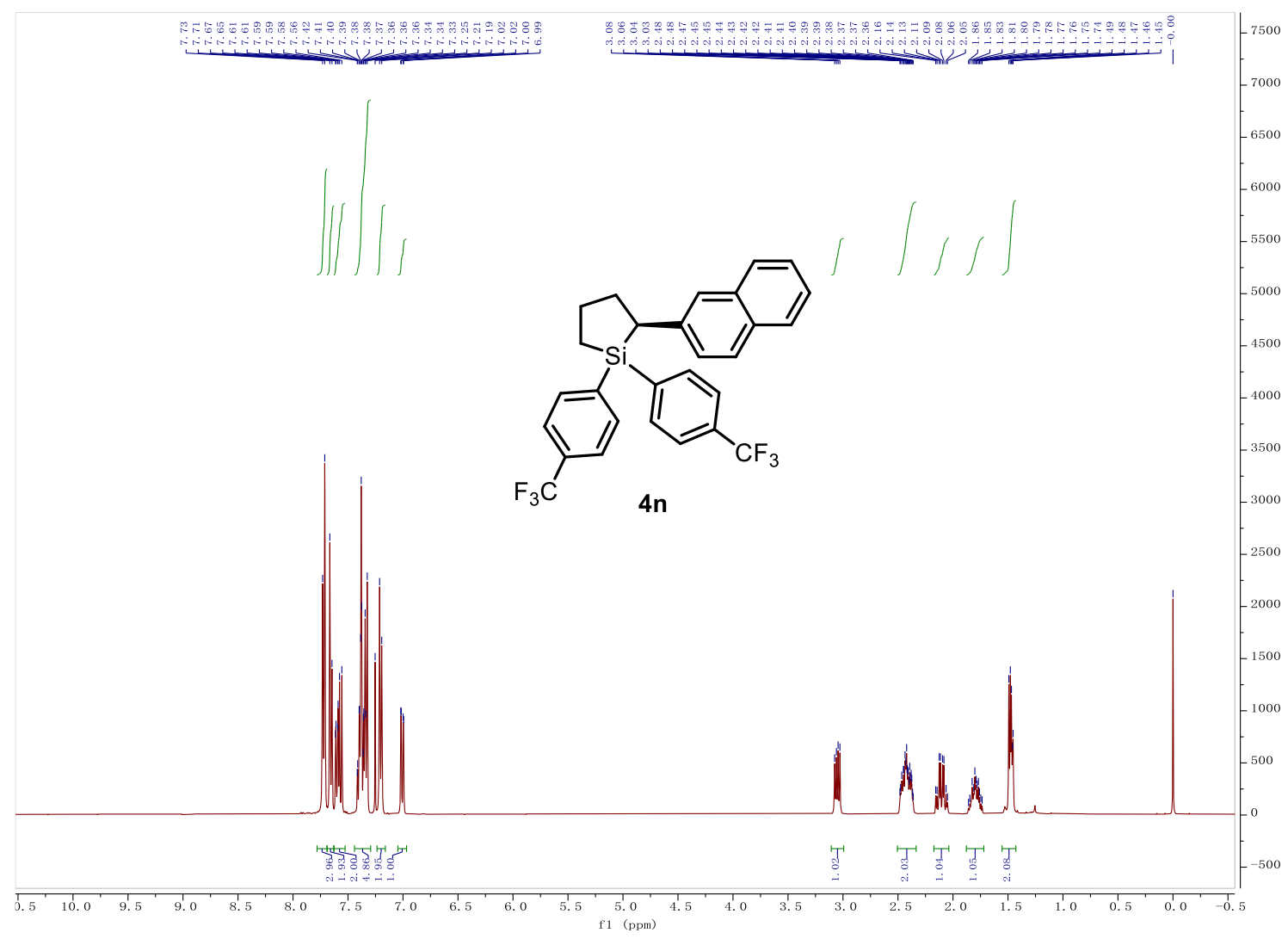



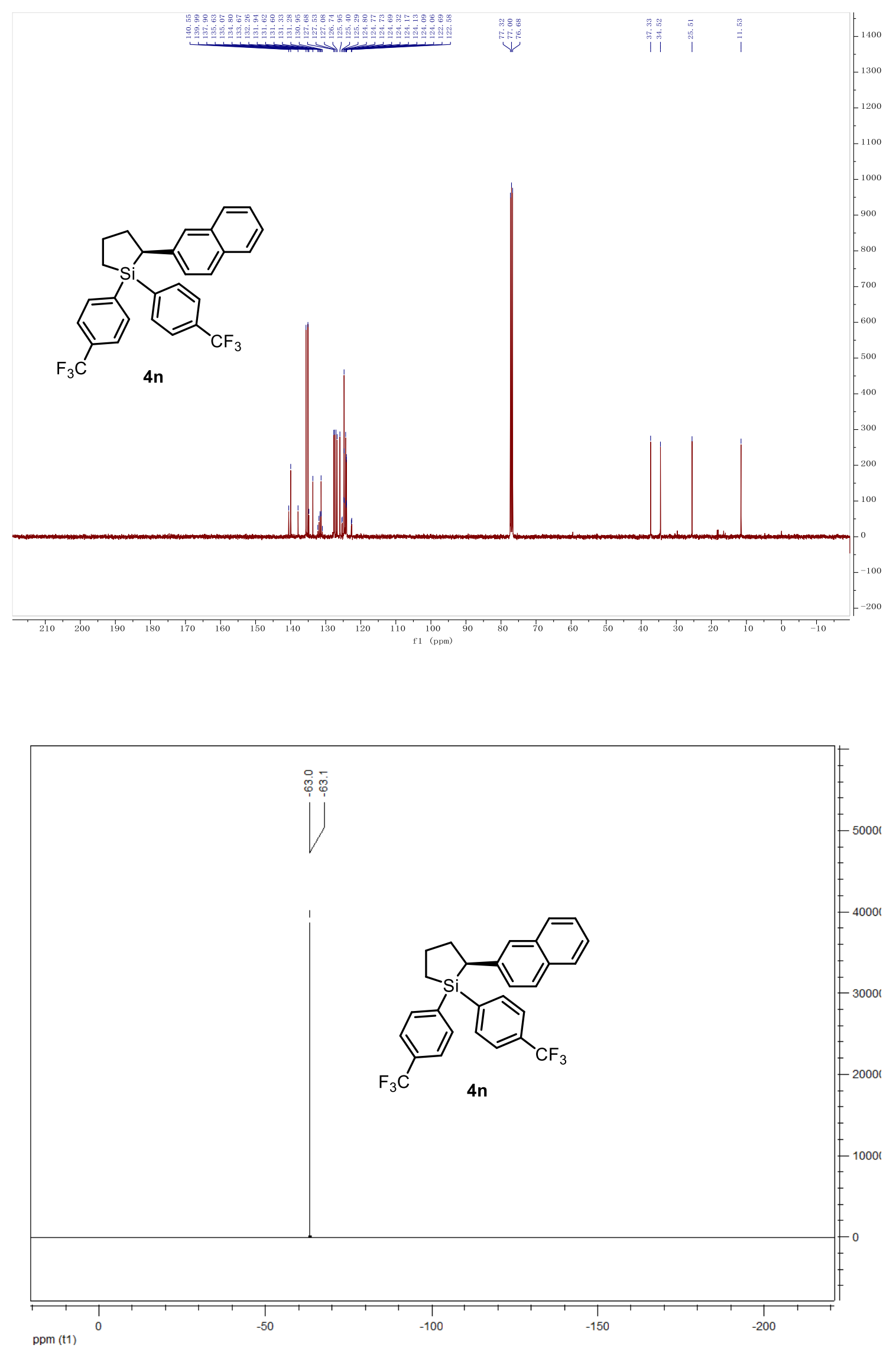
HPLC conditions: Daicel chiral column OD-3, hexane $:{ }^{i} \mathrm{PrOH}=100: 1,0.5 \mathrm{~mL} / \mathrm{min}, 2{ }^{\circ} \mathrm{C}$, wavelength $=$ $220 \mathrm{~nm}$
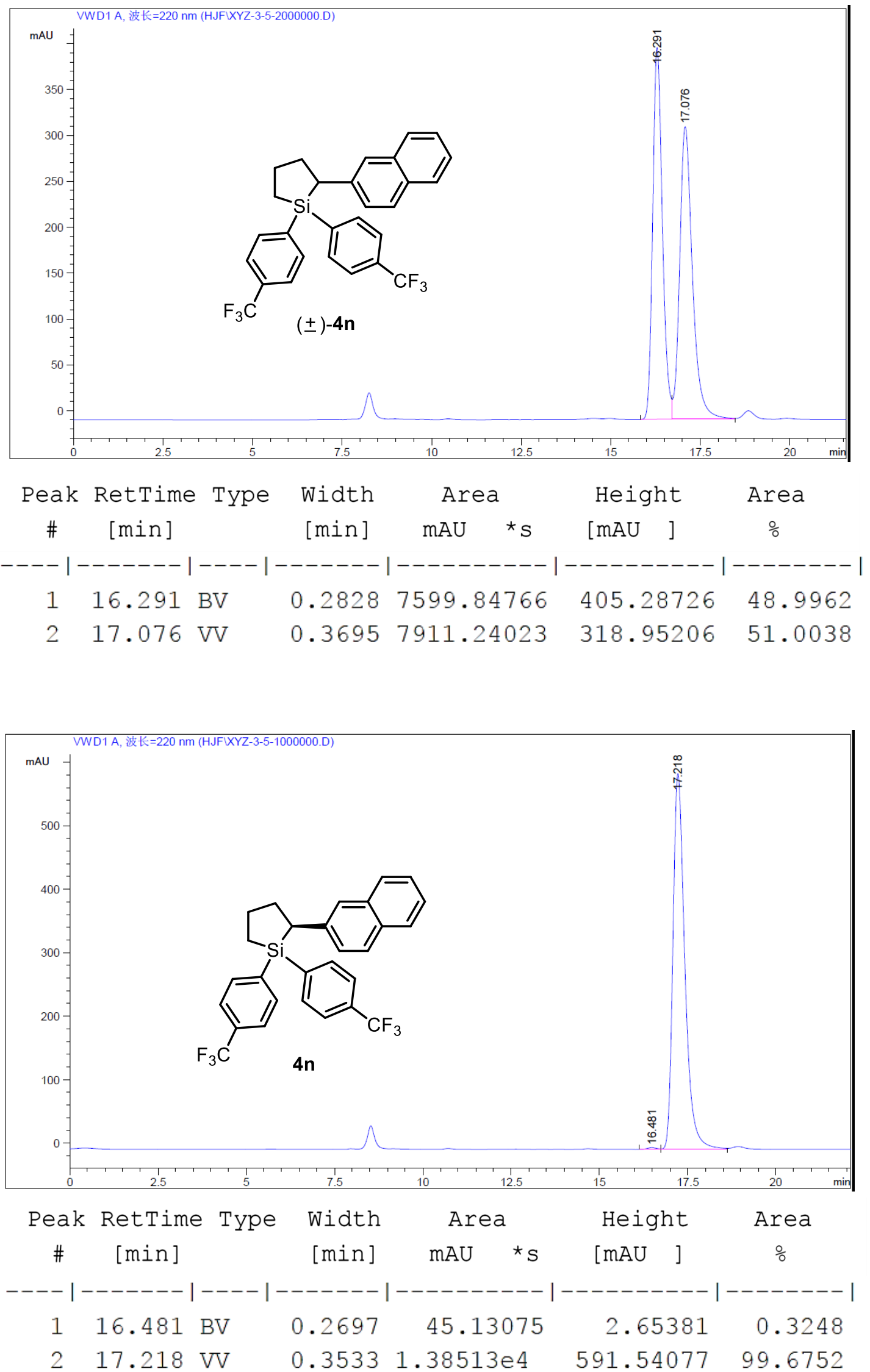

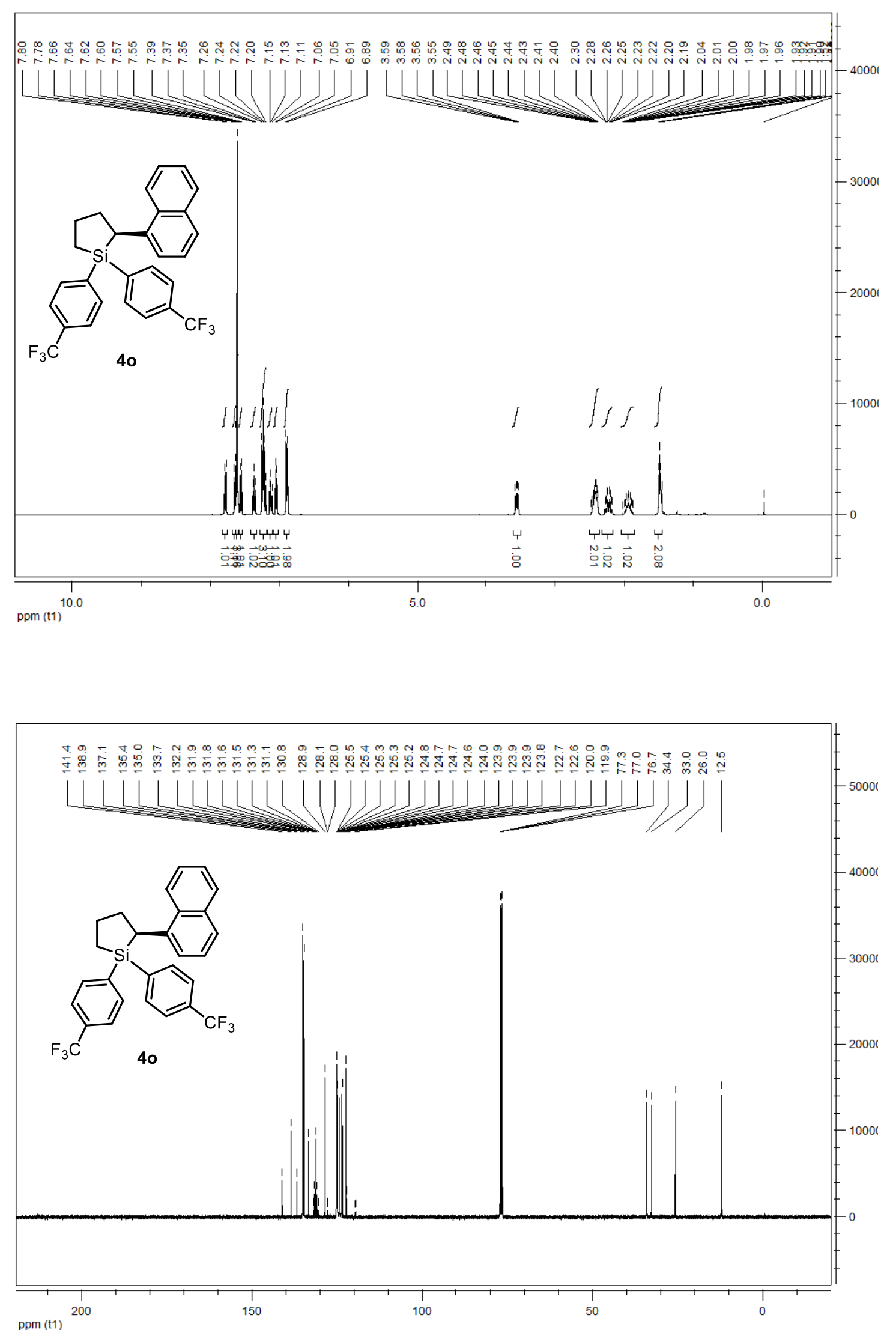


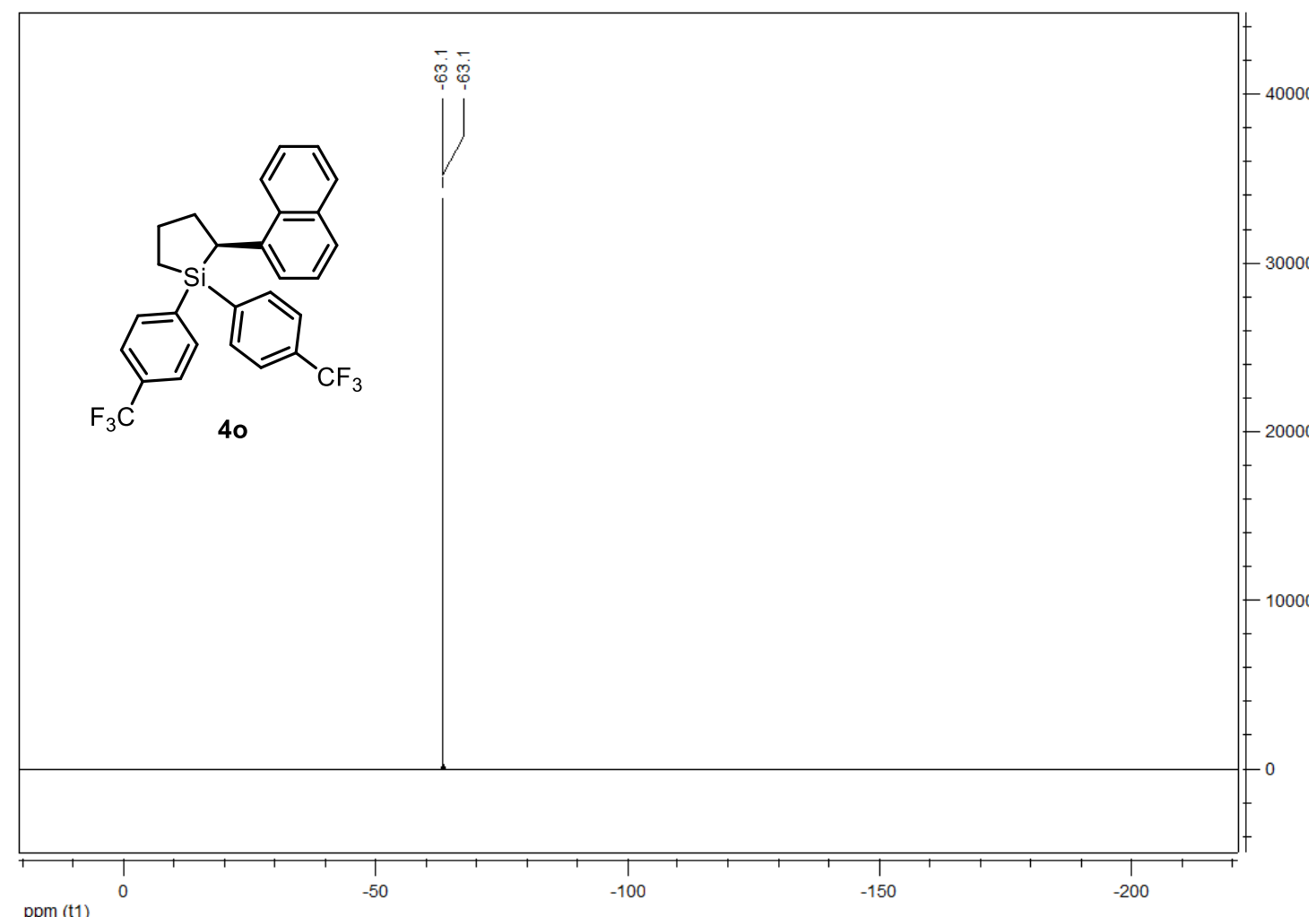

HPLC conditions: Daicel chiral column AD-3, hexane: ${ }^{i} \mathrm{PrOH}=100: 0,0.25 \mathrm{~mL} / \mathrm{min}, 25^{\circ} \mathrm{C}$, wavelength $=$ $210 \mathrm{~nm}$

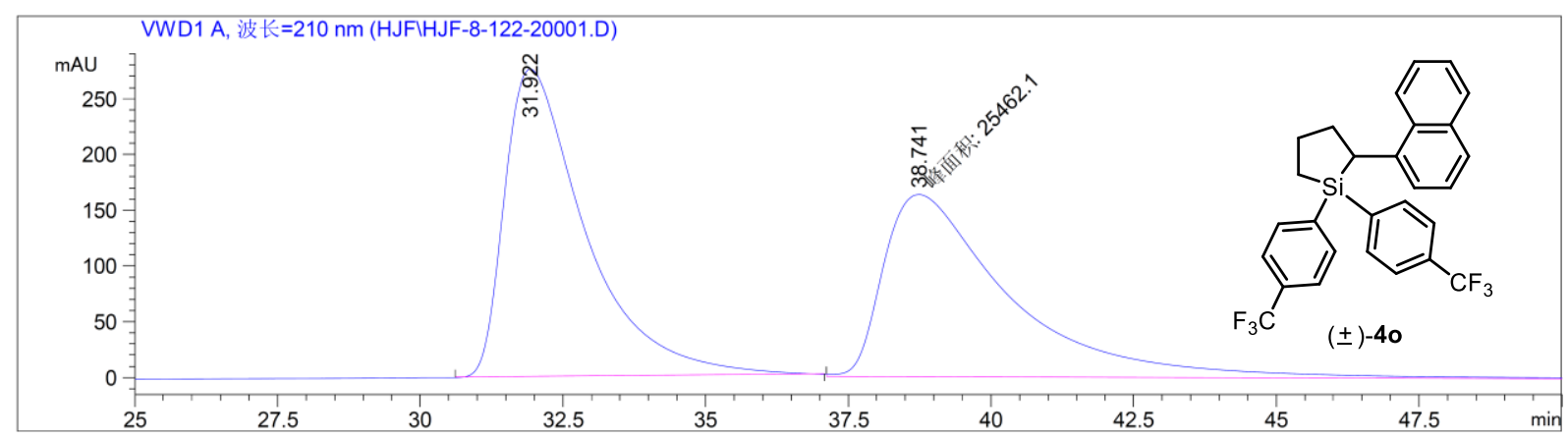

\begin{tabular}{|c|c|c|c|c|c|}
\hline Peak & RetTime Type & Width & Area & Height & Area \\
\hline \# & {$[\mathrm{min}]$} & [min] & $\mathrm{mAU}$ & {$[\mathrm{mAU} \quad]$} & $\%$ \\
\hline & & -1 & -------- & ---------- & --- \\
\hline 1 & $31.922 \mathrm{BB}$ & 1.3 & $2.73231 \mathrm{e} 4$ & 276.31866 & 51.7628 \\
\hline 2 & $38.741 \mathrm{MM}$ & 2.5967 & $2.54621 e 4$ & 163.42889 & 48.2372 \\
\hline
\end{tabular}



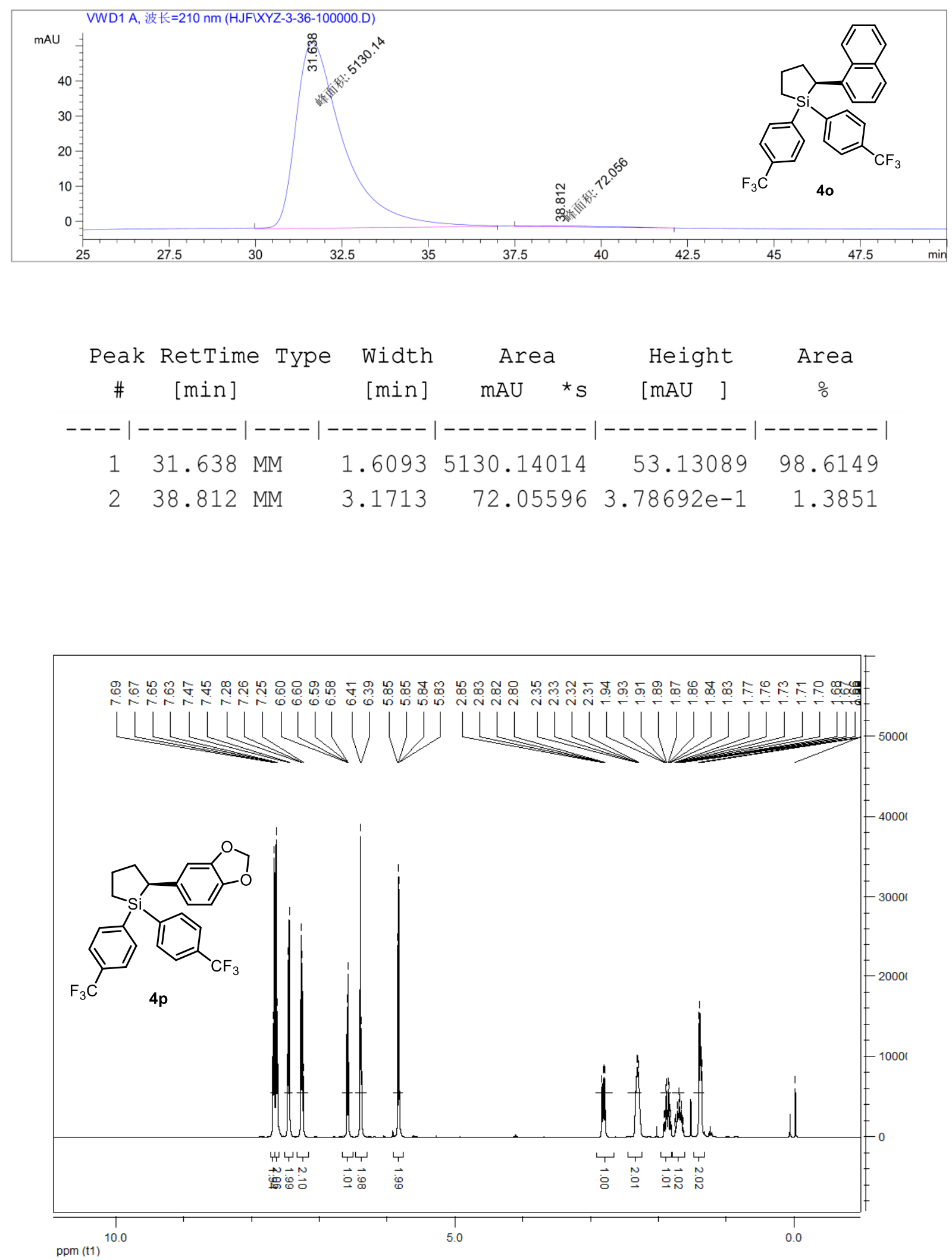

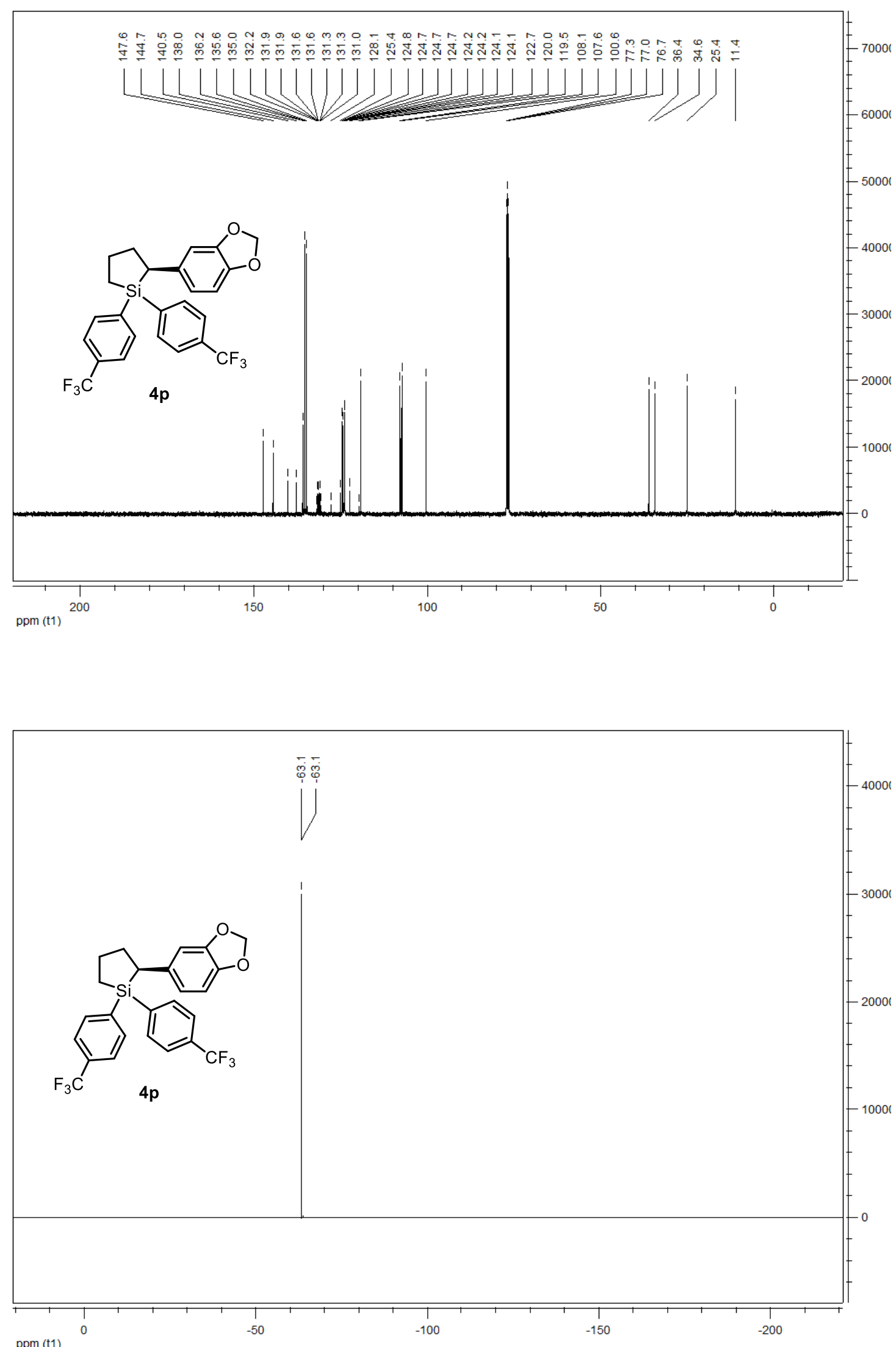
HPLC conditions: Daicel chiral column OD-3, hexane $:{ }^{i} \mathrm{PrOH}=300: 1,0.5 \mathrm{~mL} / \mathrm{min}, 25^{\circ} \mathrm{C}$, wavelength $=$ $220 \mathrm{~nm}$
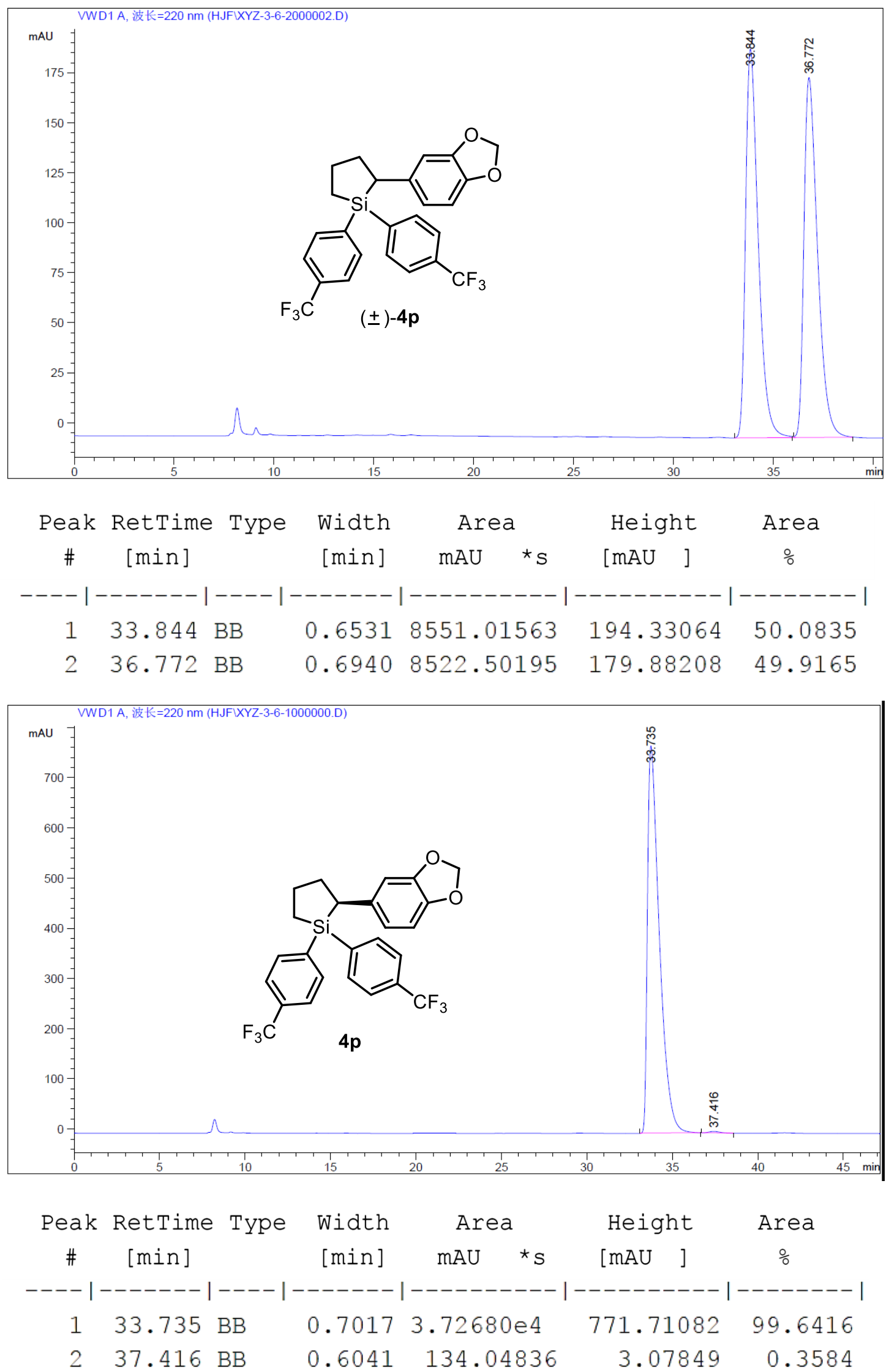


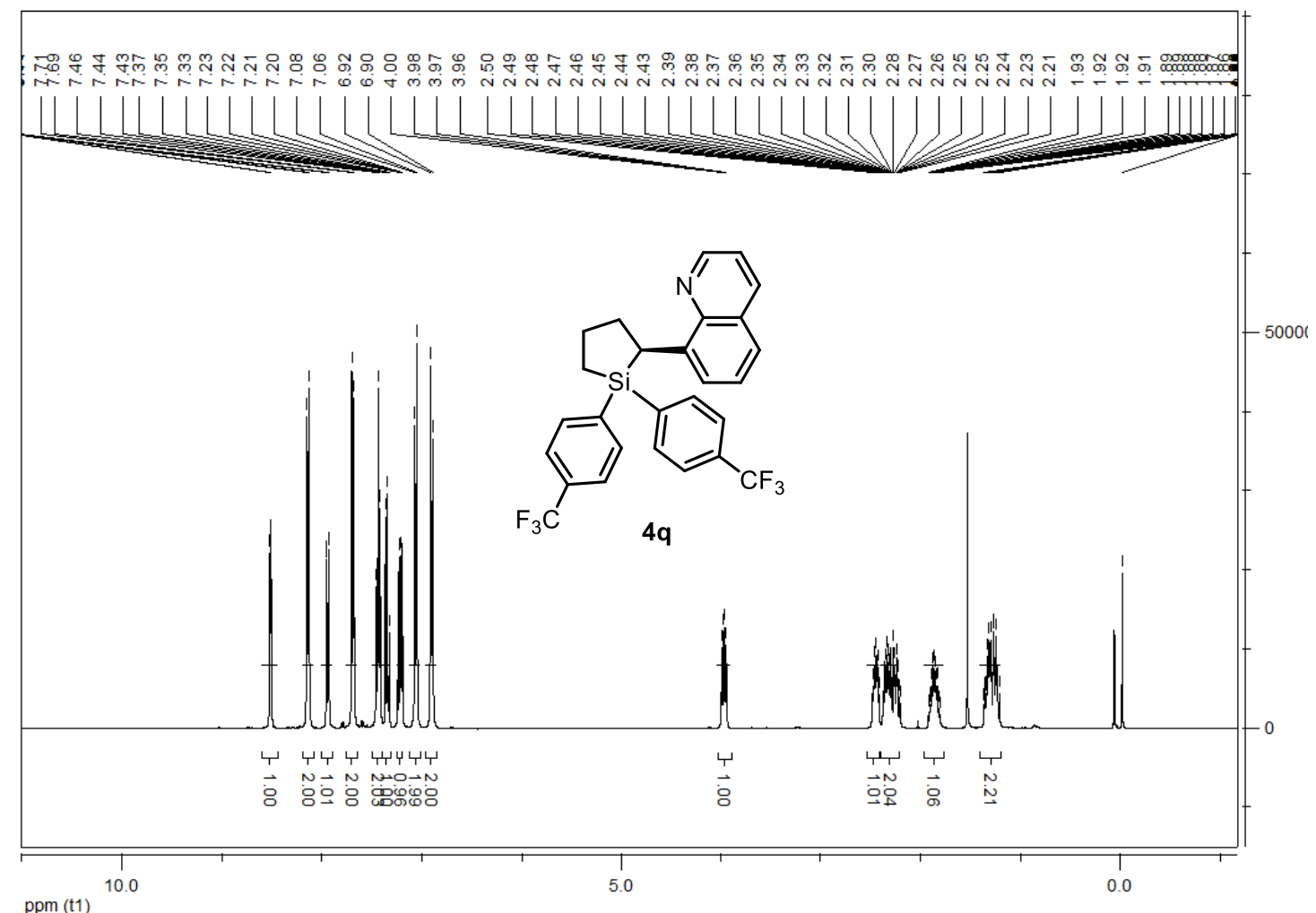

ppm (t1)

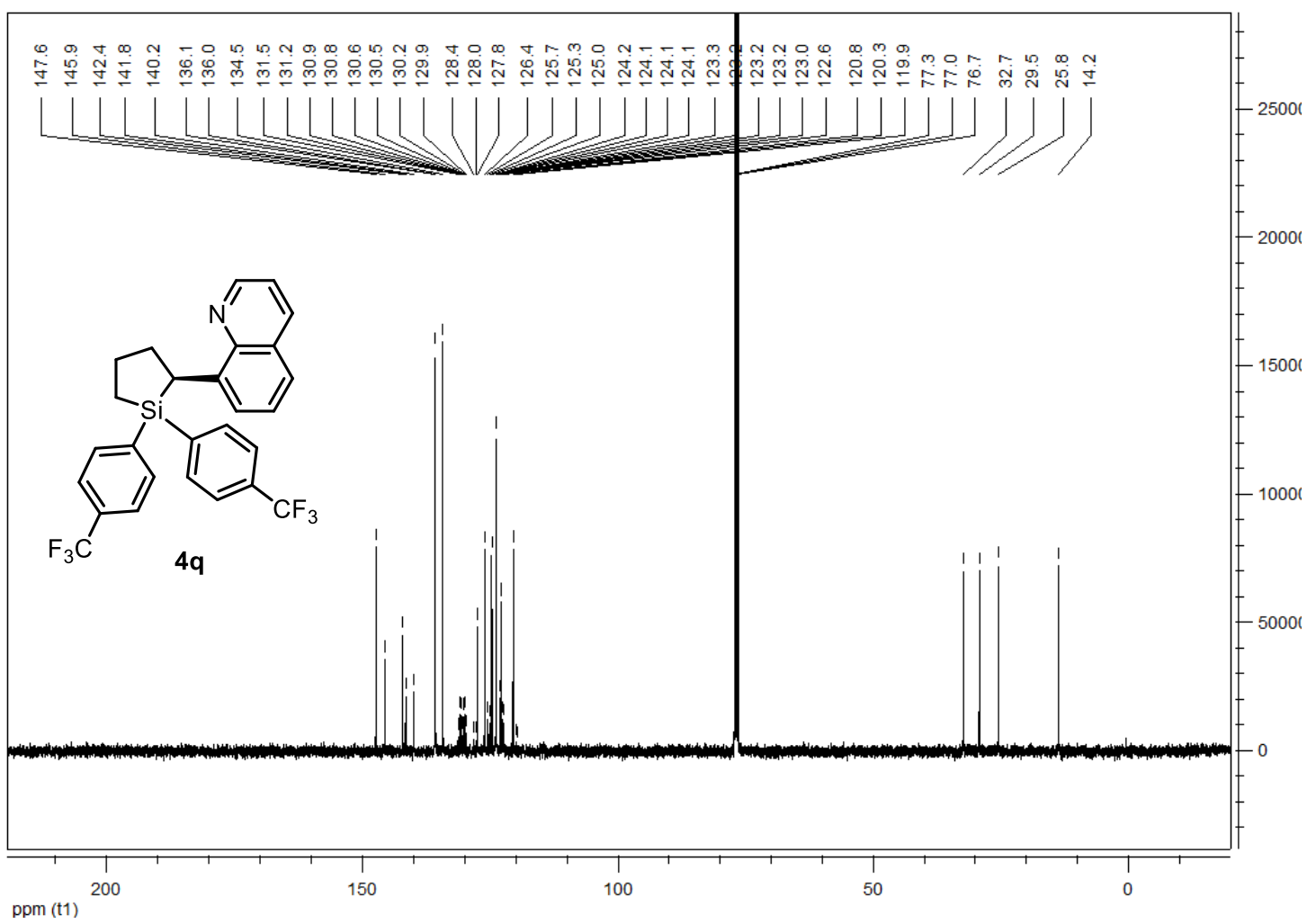




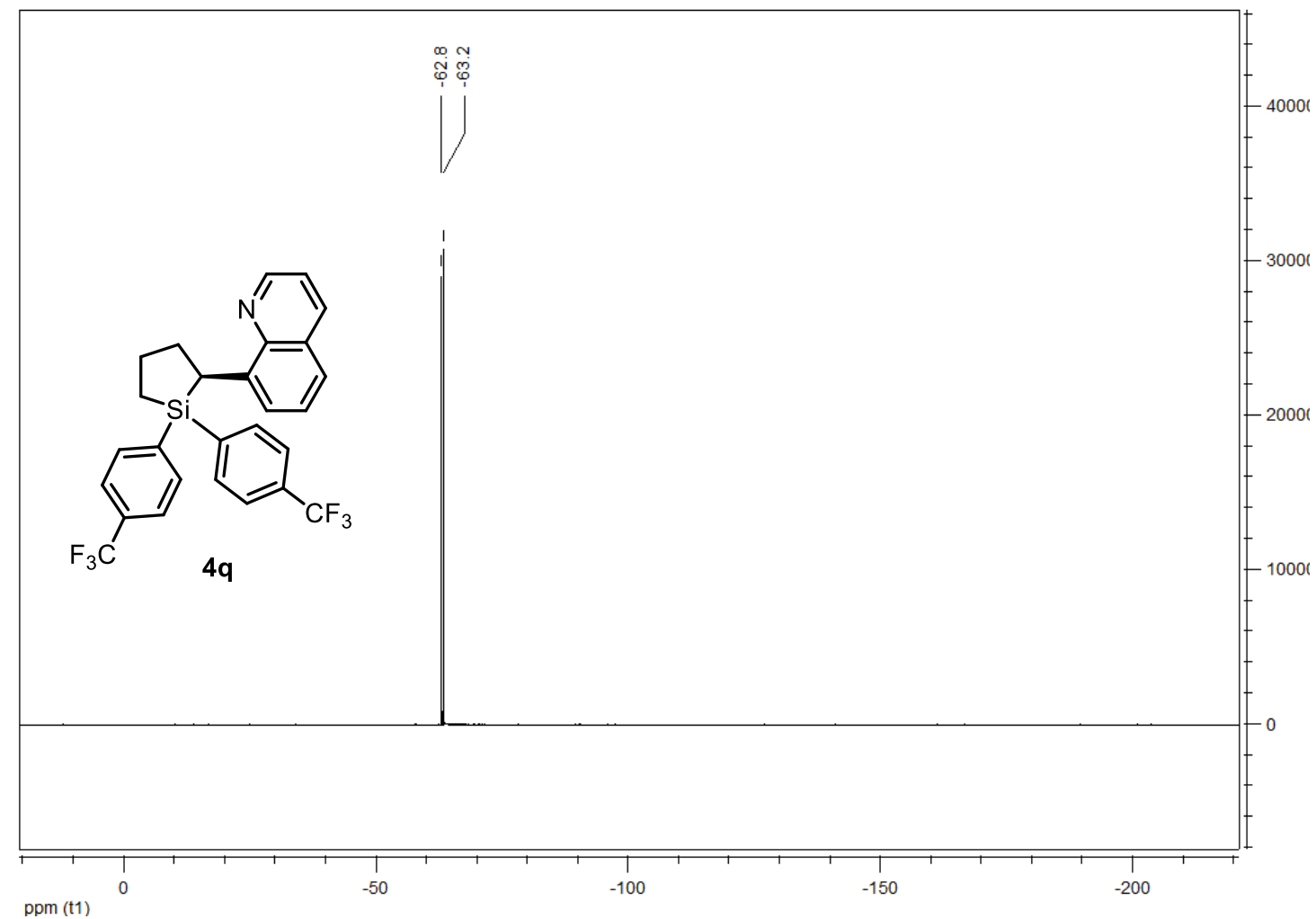

HPLC conditions: Daicel chiral column OD-3, hexane $:{ }^{i} \mathrm{PrOH}=200: 1,0.5 \mathrm{~mL} / \mathrm{min}, 25{ }^{\circ} \mathrm{C}$, wavelength $=$ $220 \mathrm{~nm}$

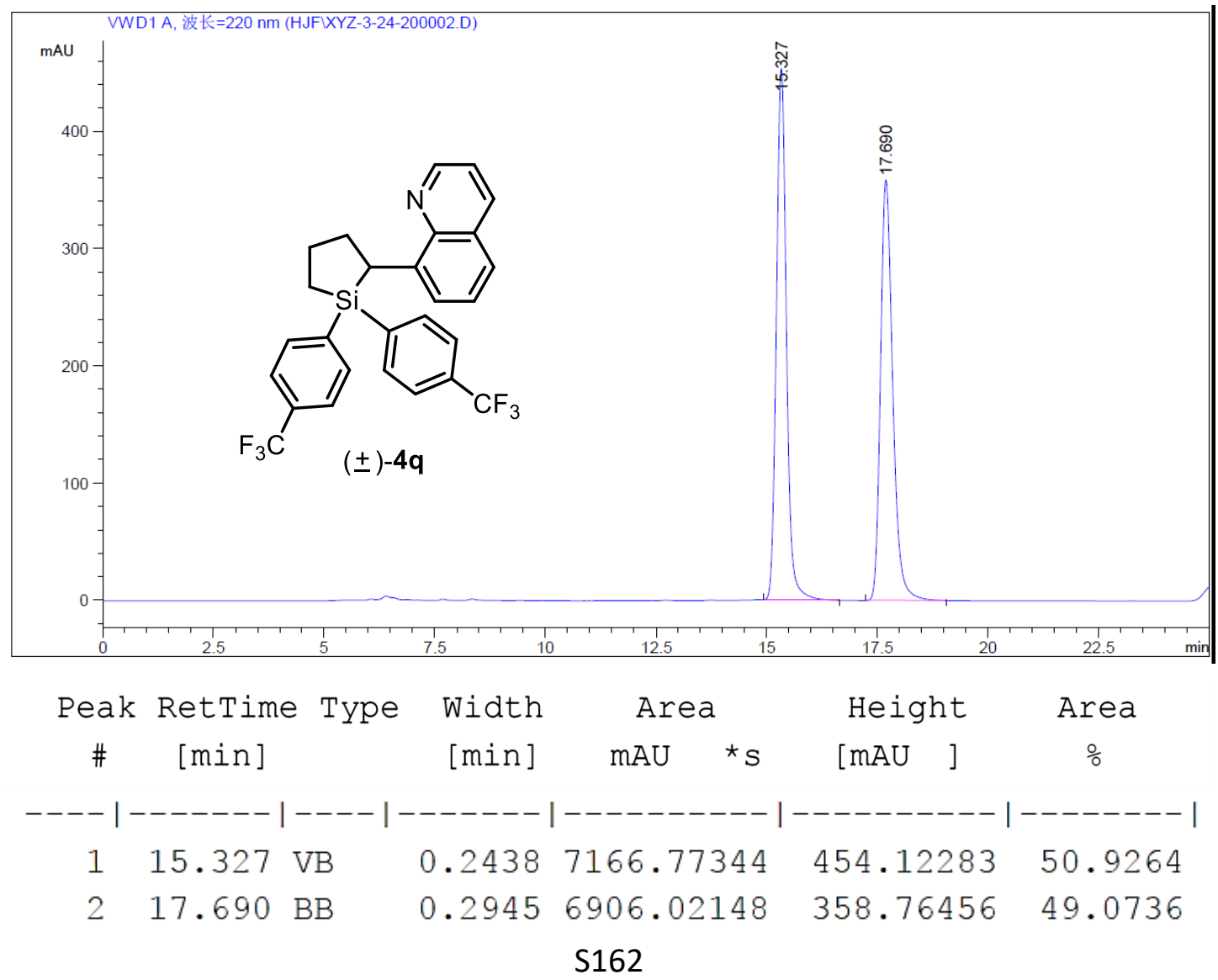



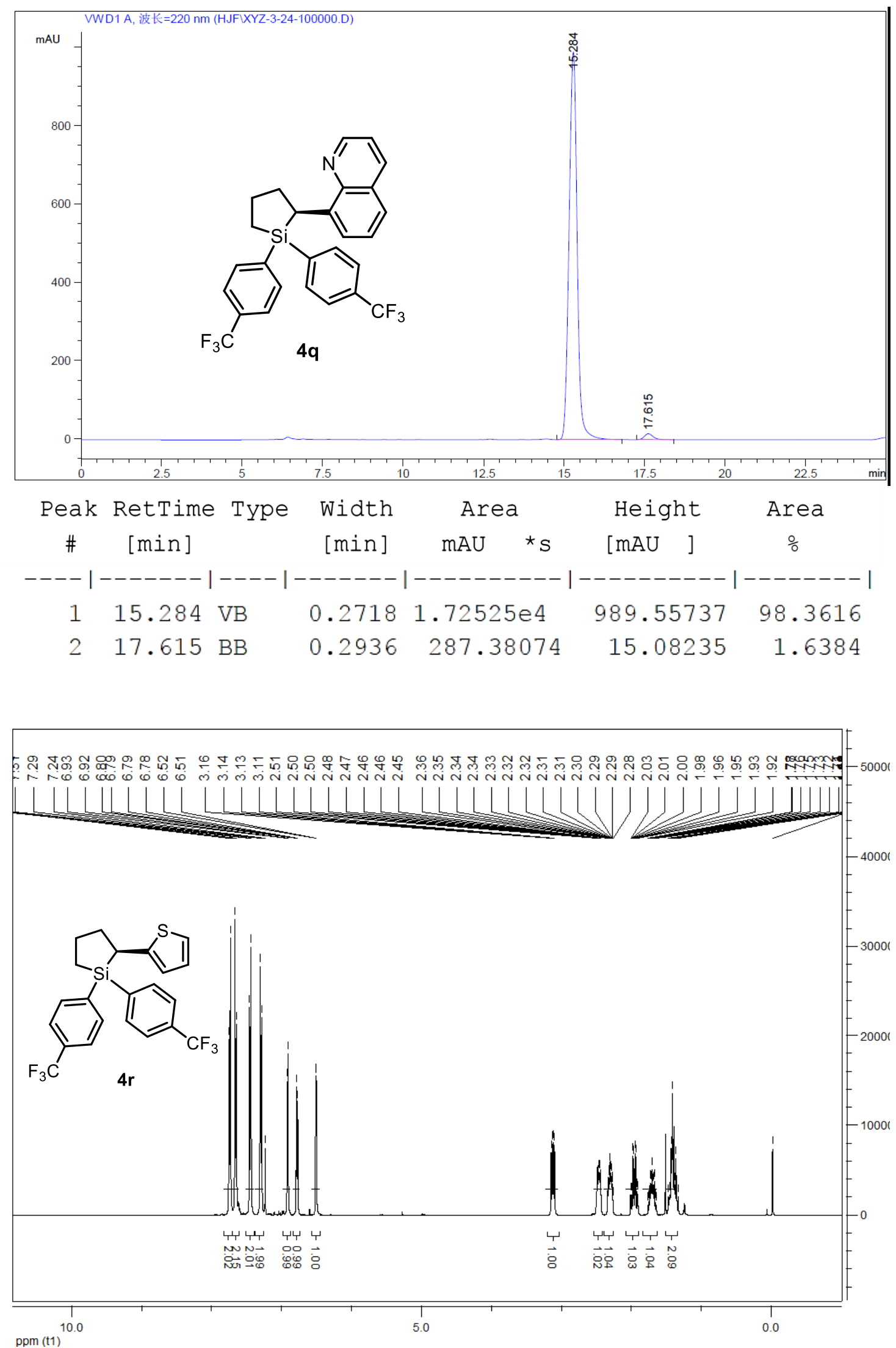

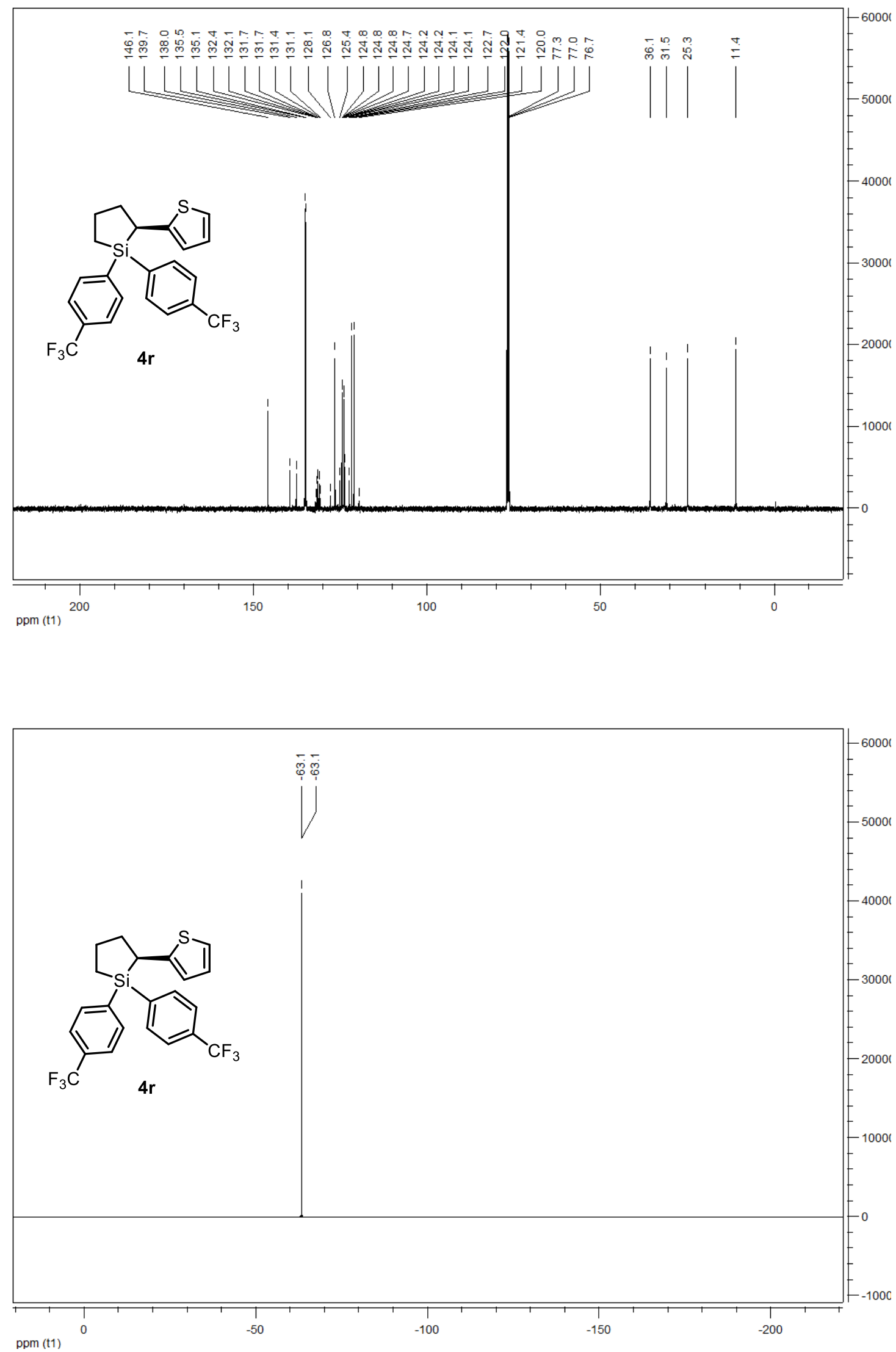
HPLC conditions: Daicel chiral column OD-3, hexane $:{ }^{i} \mathrm{PrOH}=100: 0,0.5 \mathrm{~mL} / \mathrm{min}, 25^{\circ} \mathrm{C}$, wavelength $=$ $220 \mathrm{~nm}$
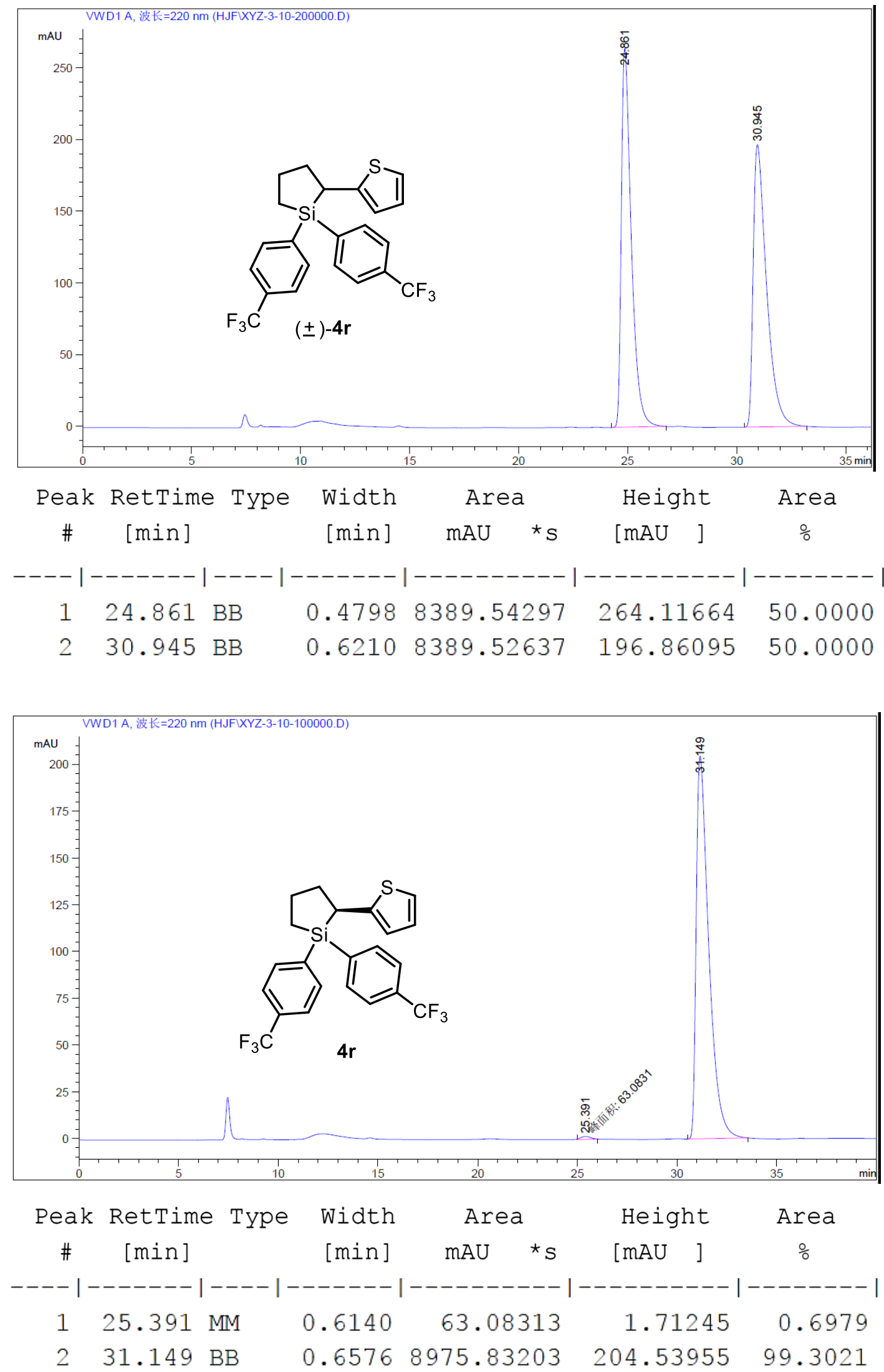


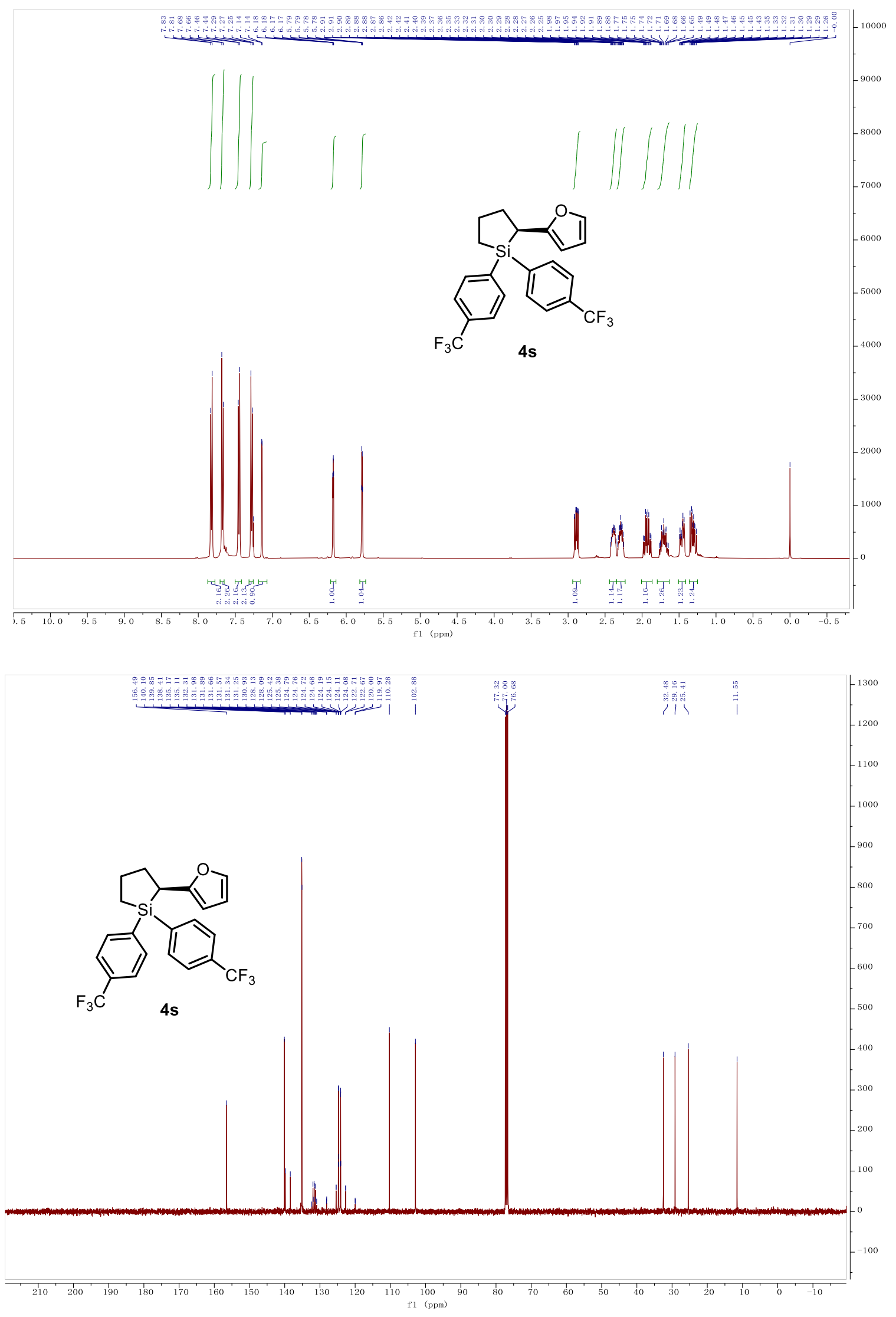




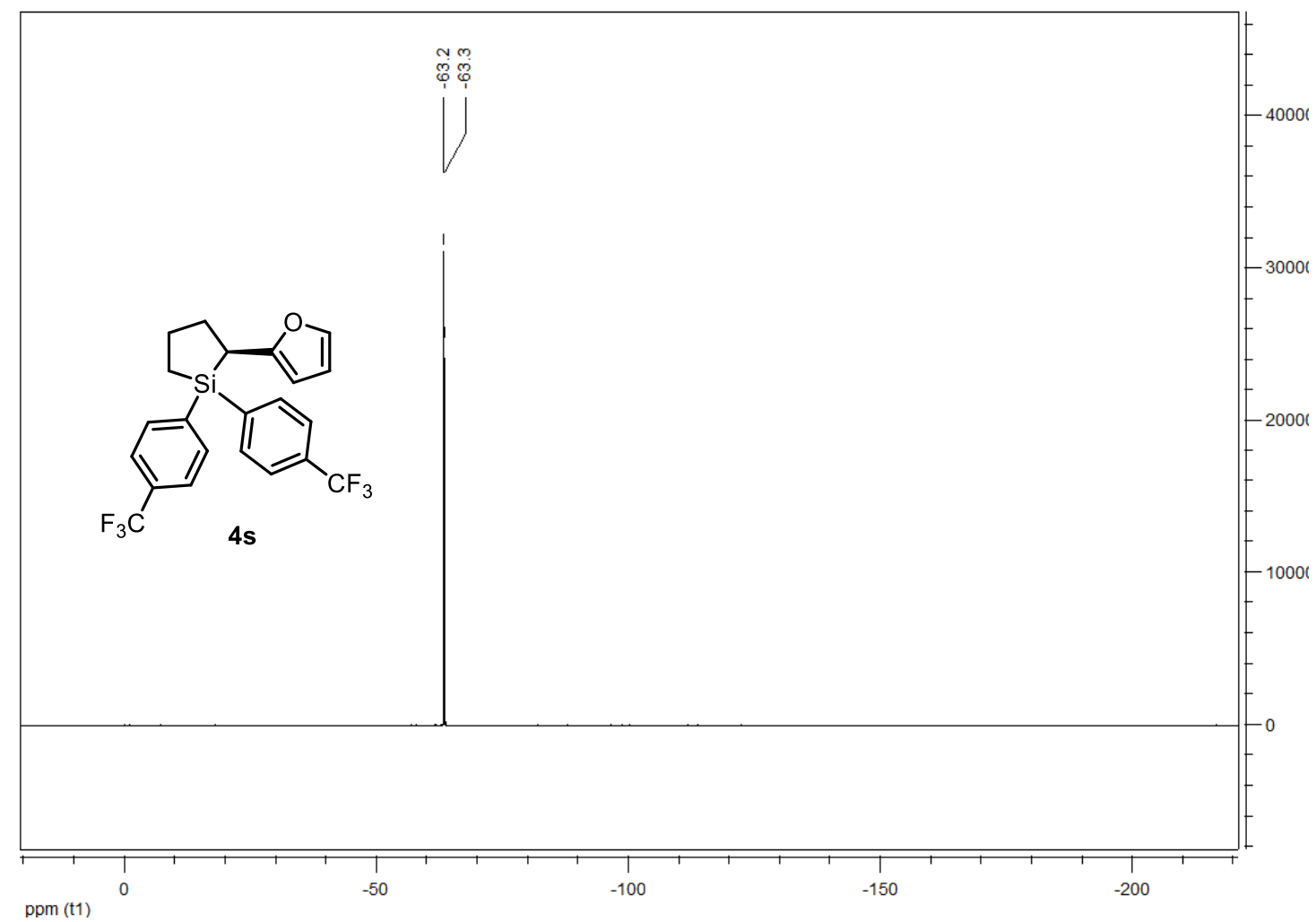

HPLC conditions: Daicel chiral column OD-3, hexane: ${ }^{i} \mathrm{PrOH}=100: 0,0.5 \mathrm{~mL} / \mathrm{min}, 25{ }^{\circ} \mathrm{C}$, wavelength $=$ $220 \mathrm{~nm}$

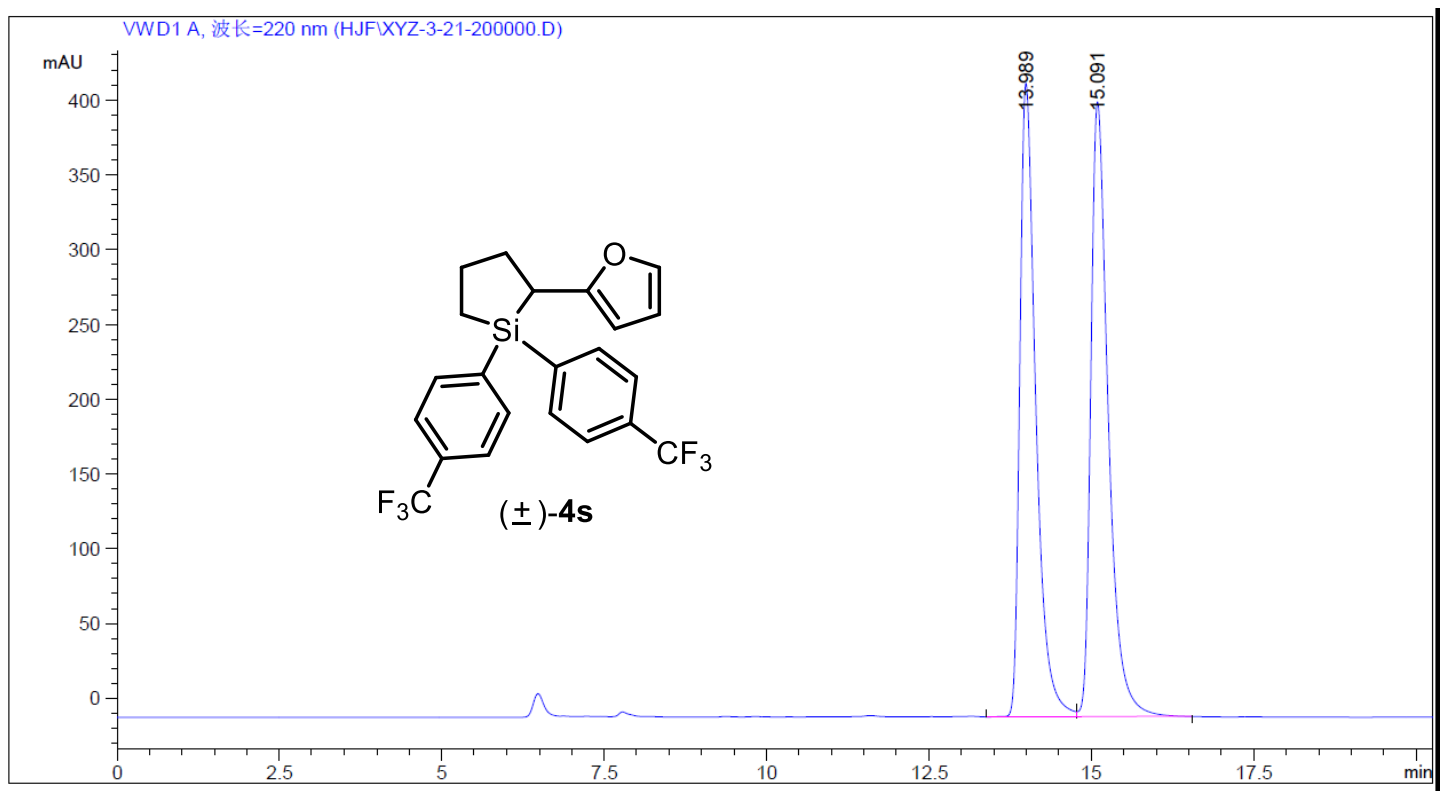

\begin{tabular}{cccccc}
$\begin{array}{c}\text { Peak } \\
\text { \# }\end{array}$ & [min] & Width & Area & Height & Area \\
[min] & mAU ${ }_{S}$ & {$[\mathrm{mAU}]$} & $\%$ \\
\hline 1 & 13.989 VV & 0.2578 & 7251.45264 & 423.85611 & 48.5901 \\
2 & 15.091 VB & 0.2803 & 7672.27930 & 411.15561 & 51.4099
\end{tabular}



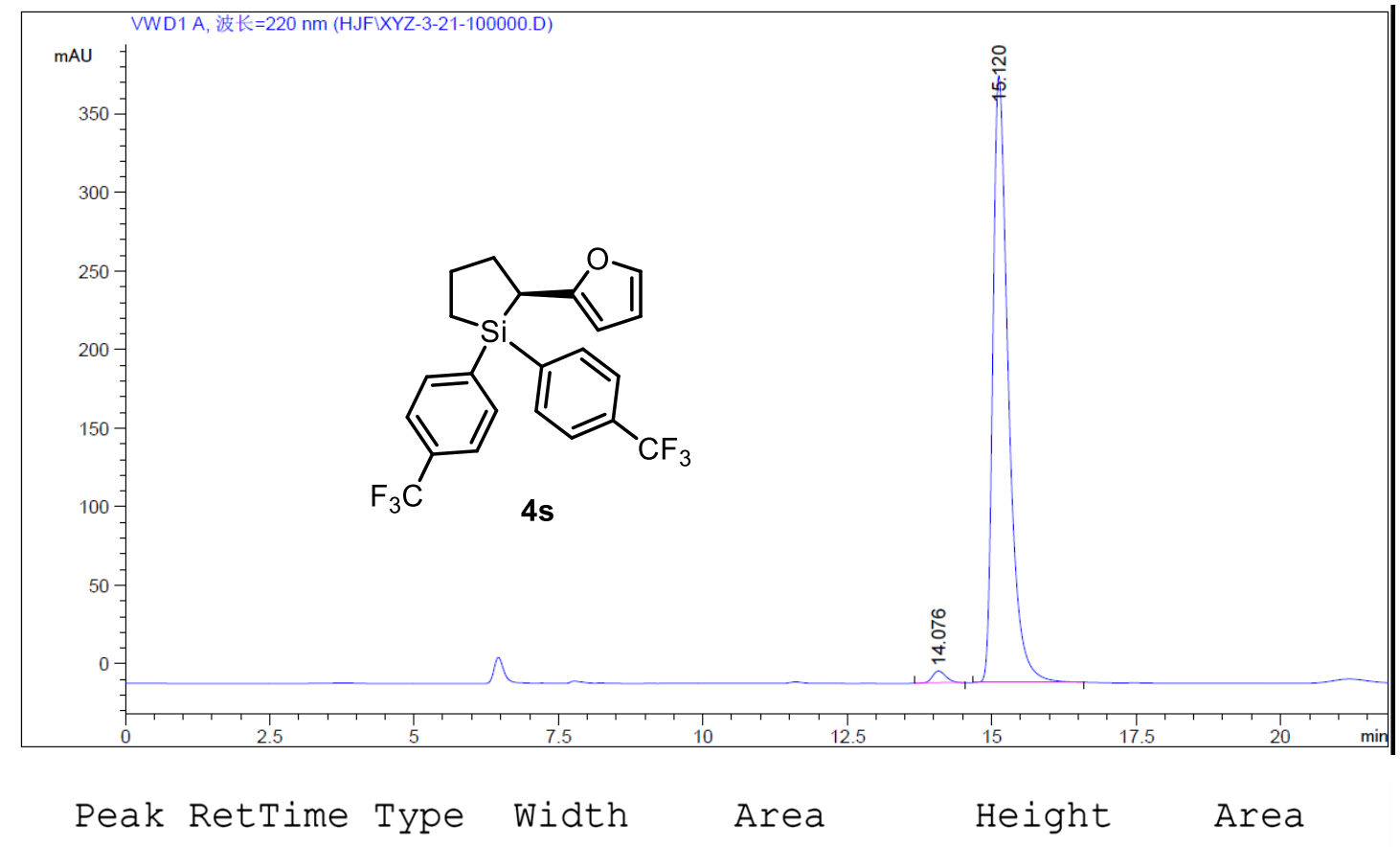

\# [min] [min] mAU *s [mAU ]

$----|-------|----|-------|----------|----------|--------\mid$

$\begin{array}{lllllll}1 & 14.076 & \mathrm{BB} & 0.2527 & 127.49263 & 7.70741 & 1.7290\end{array}$

$\begin{array}{lllllll}2 & 15.120 & \text { BB } & 0.2843 & 7246.09033 & 386.36313 & 98.2710\end{array}$

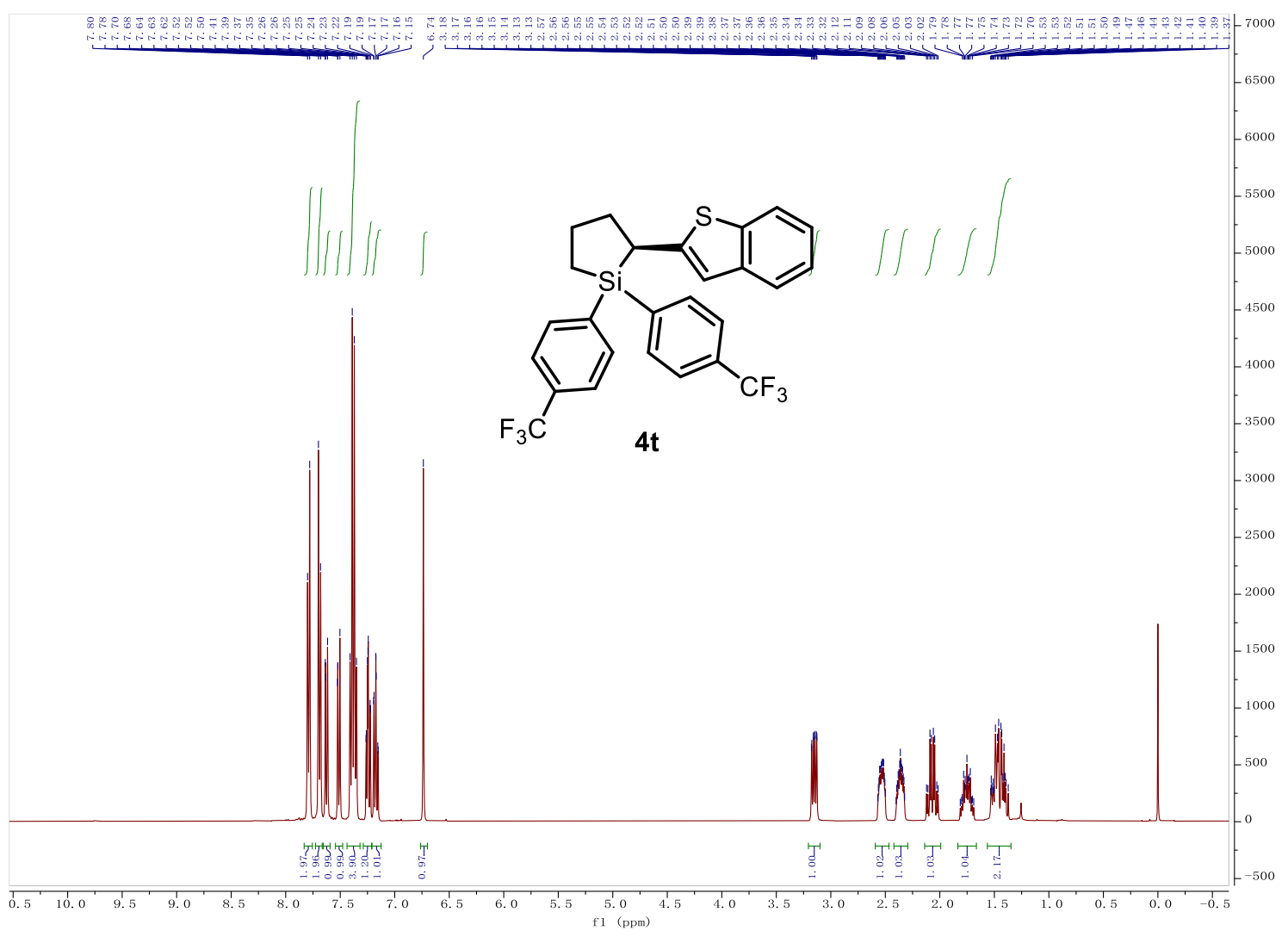



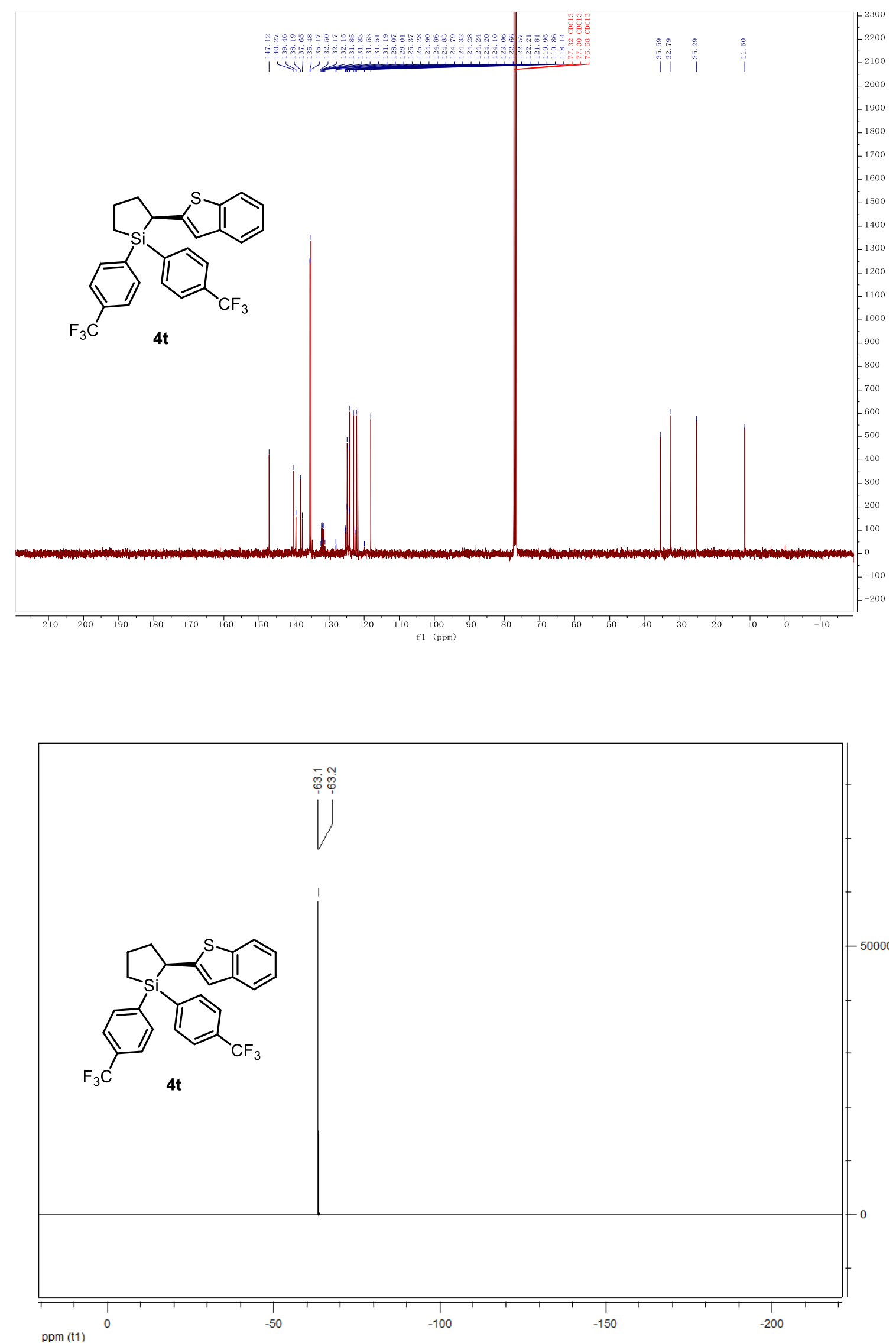
HPLC conditions: Daicel chiral column AD-H, hexane $:{ }^{i} \mathrm{PrOH}=500: 1,0.5 \mathrm{~mL} / \mathrm{min}, 25^{\circ} \mathrm{C}$, wavelength $=$ $220 \mathrm{~nm}$
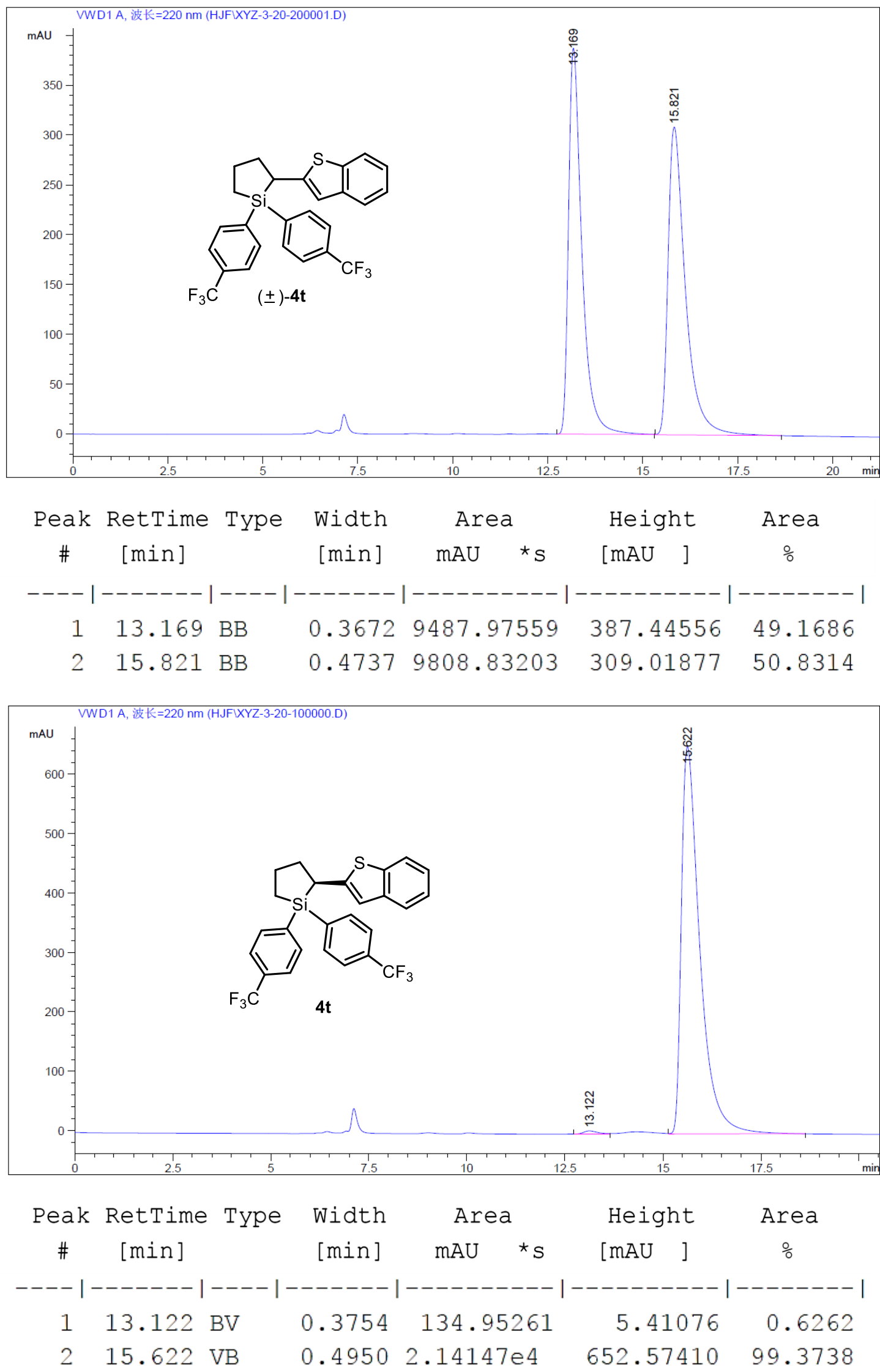

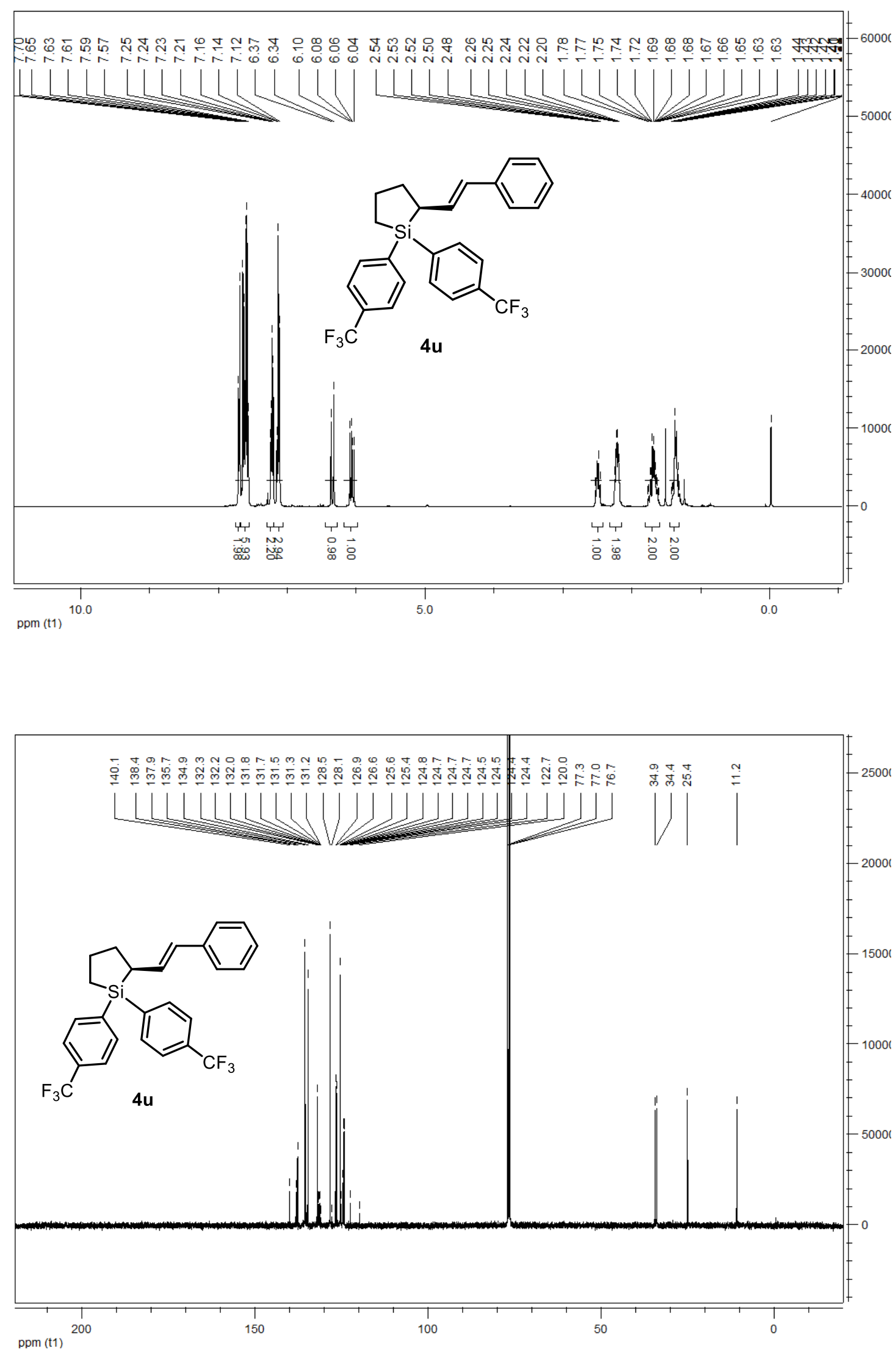


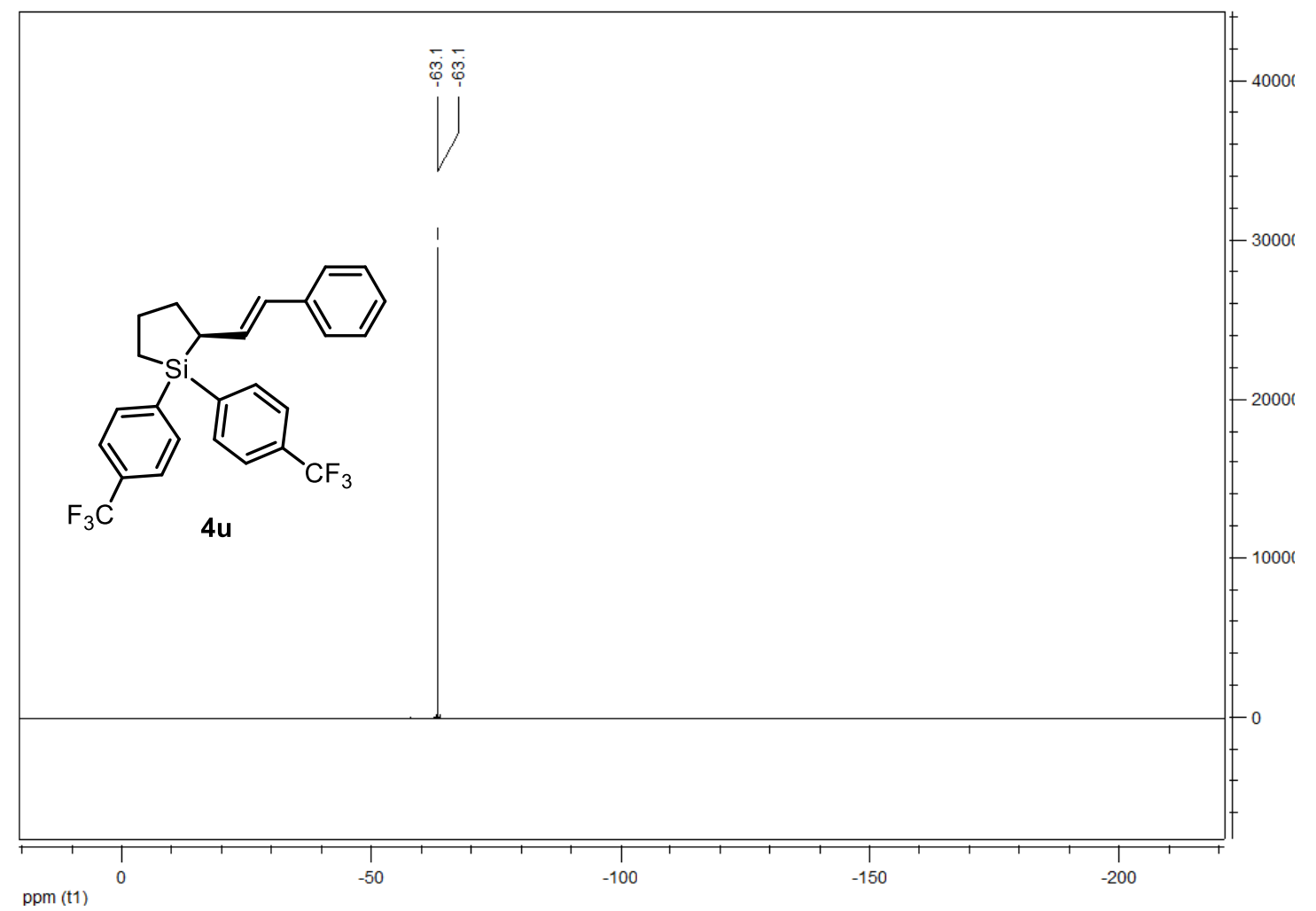

HPLC conditions: Daicel chiral column OD-3, hexane $:{ }^{i} \mathrm{PrOH}=100: 0,0.5 \mathrm{~mL} / \mathrm{min}, 25{ }^{\circ} \mathrm{C}$, wavelength $=$ $220 \mathrm{~nm}$

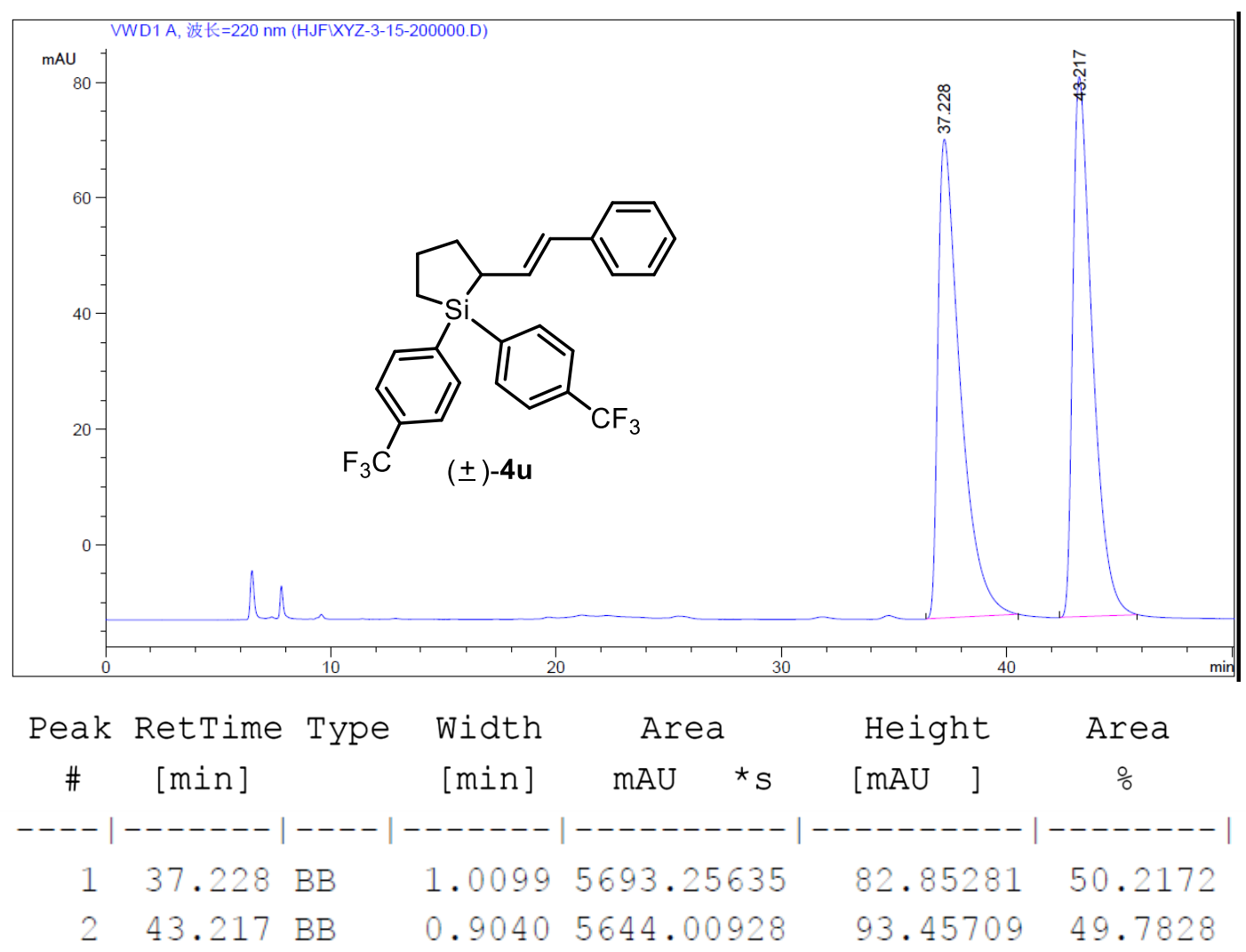



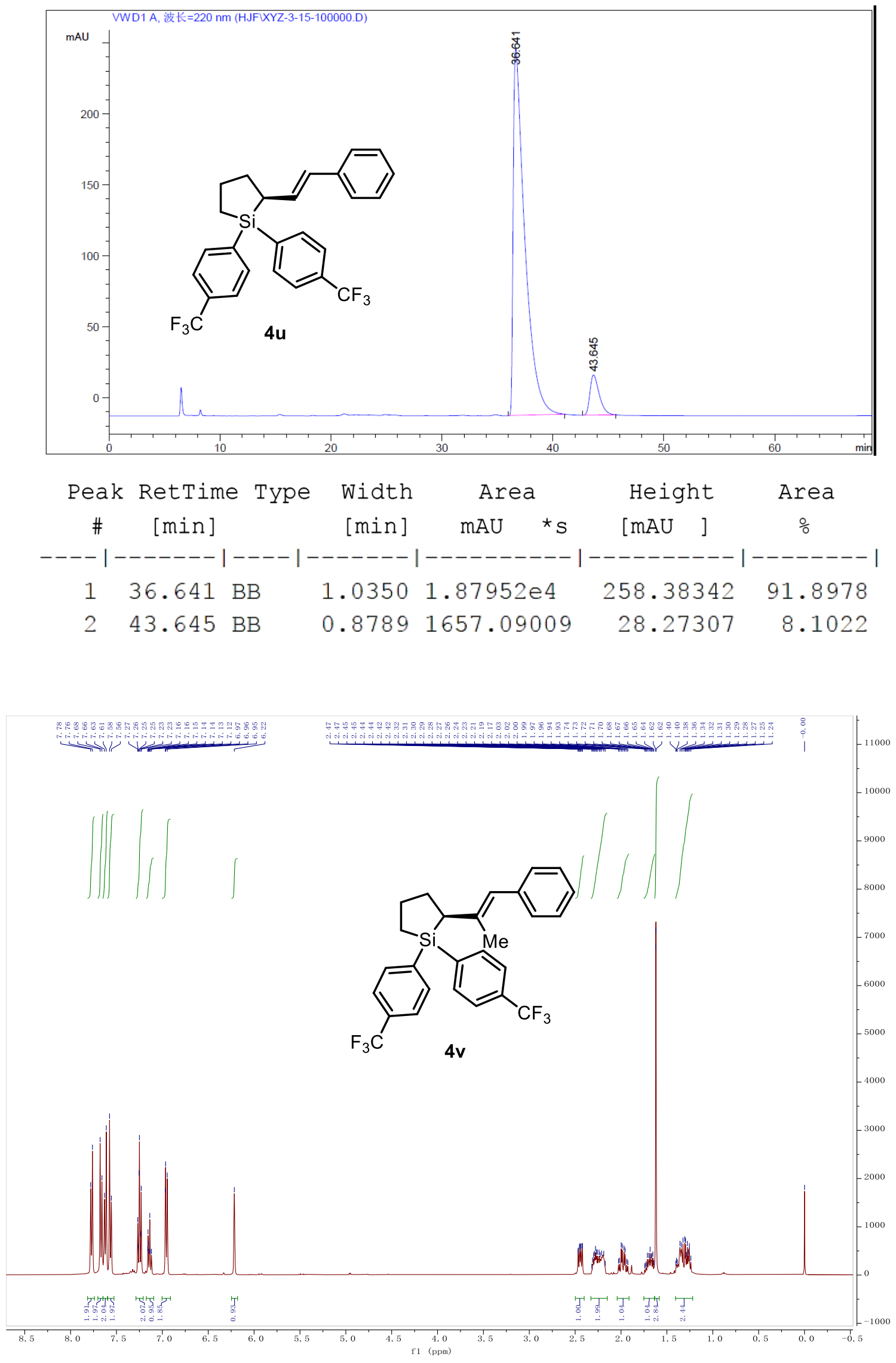

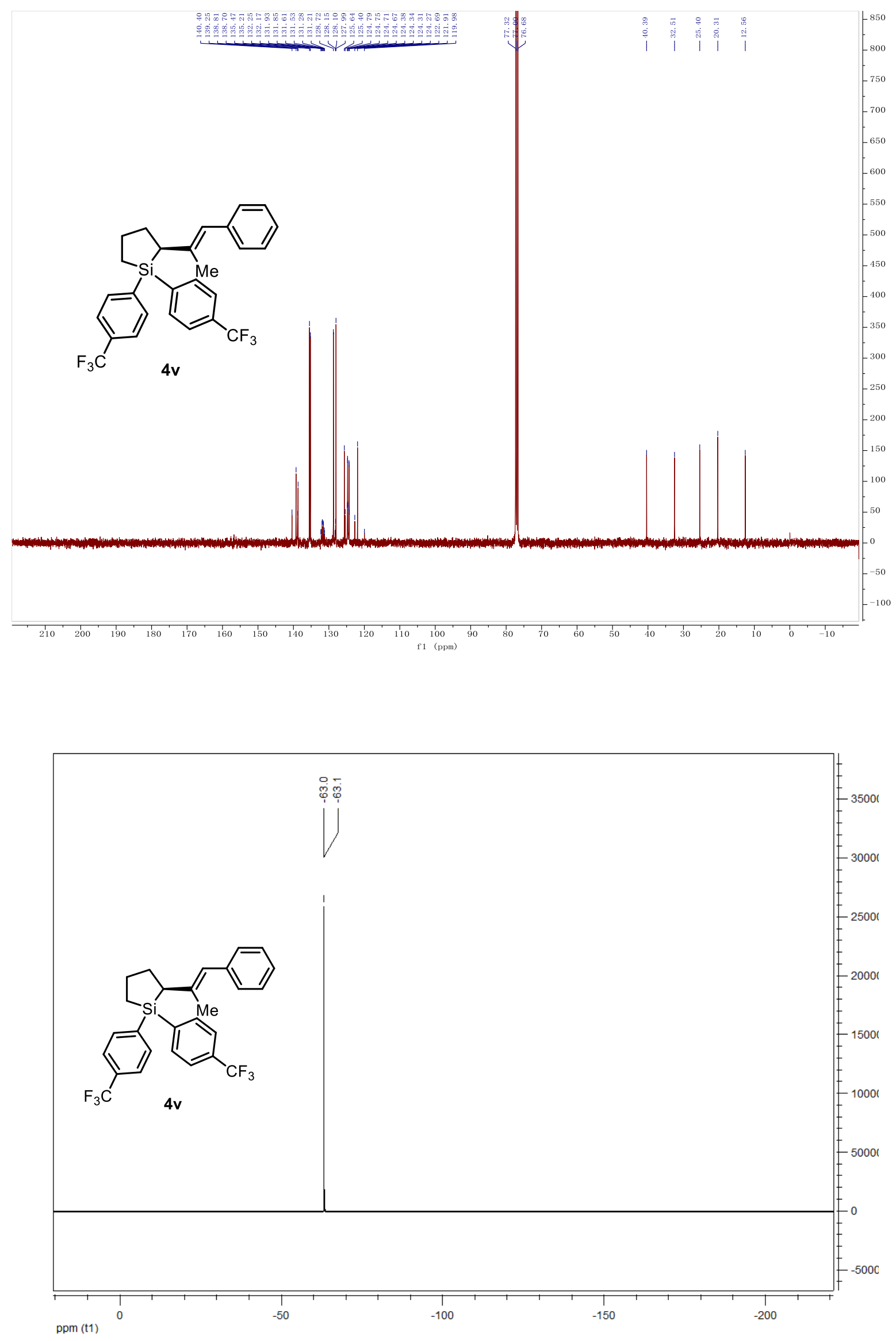
HPLC conditions: Daicel chiral column OD-3, hexane: ${ }^{i} \mathrm{PrOH}=100: 0,0.2 \mathrm{~mL} / \mathrm{min}, 25^{\circ} \mathrm{C}$, wavelength $=$ $210 \mathrm{~nm}$
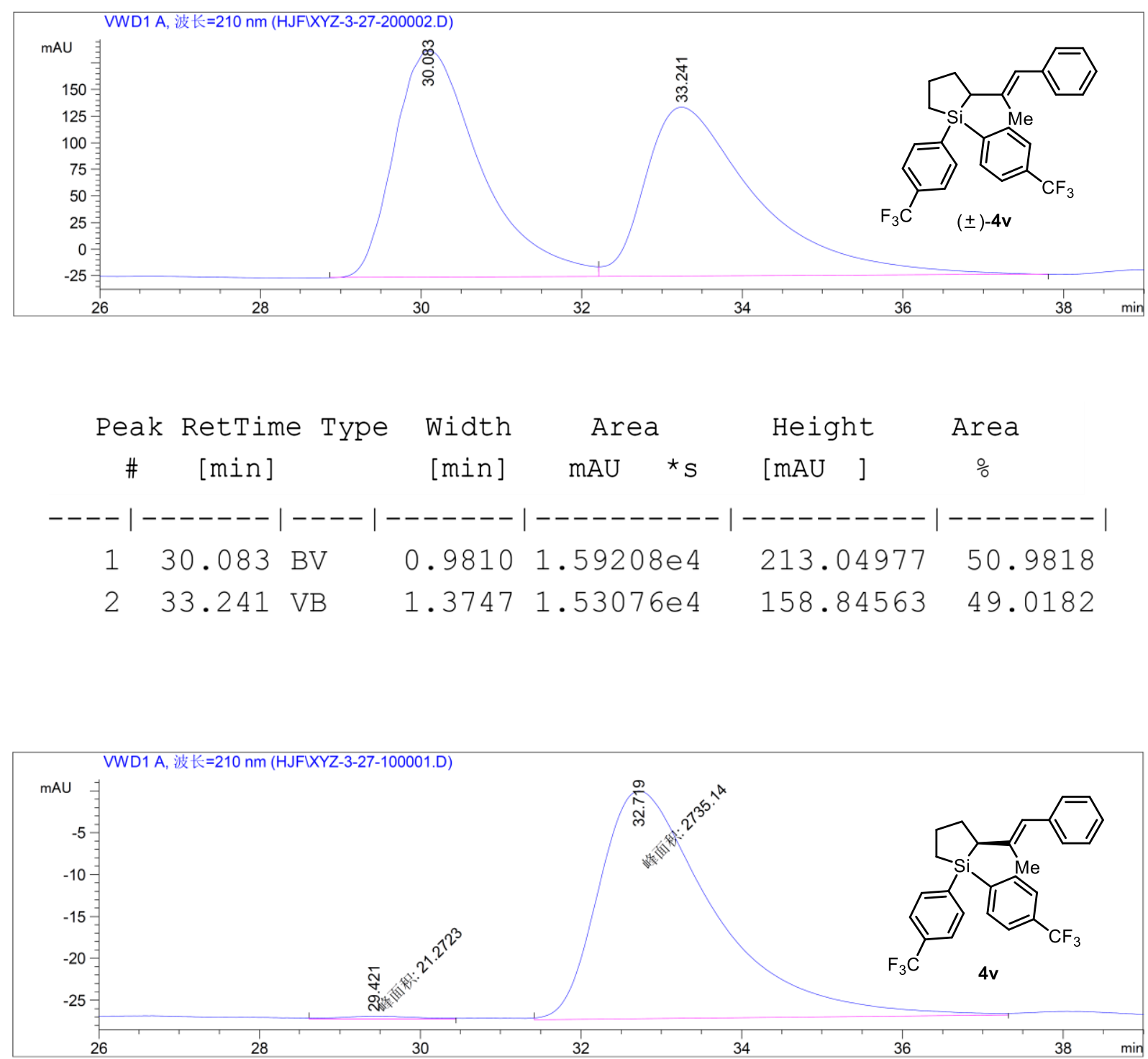

\begin{tabular}{|c|c|c|c|c|c|c|}
\hline \multirow{2}{*}{$\begin{array}{c}\text { Peak } \\
\#\end{array}$} & RetTime & Type & Width & & Height & \multirow{2}{*}{$\begin{array}{c}\text { Area } \\
\frac{\circ}{0}\end{array}$} \\
\hline & [min] & & [min] & mAU & {$[\mathrm{mAU} \quad]$} & \\
\hline & & & & 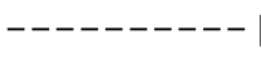 & & \\
\hline 1 & 29.421 & $\mathrm{MM}$ & 1.0894 & 21.27235 & $3.25437 e-1$ & 0.7717 \\
\hline 2 & 32.719 & $\mathrm{MM}$ & 1.6736 & 2735.14453 & 27.23884 & 99.2283 \\
\hline
\end{tabular}



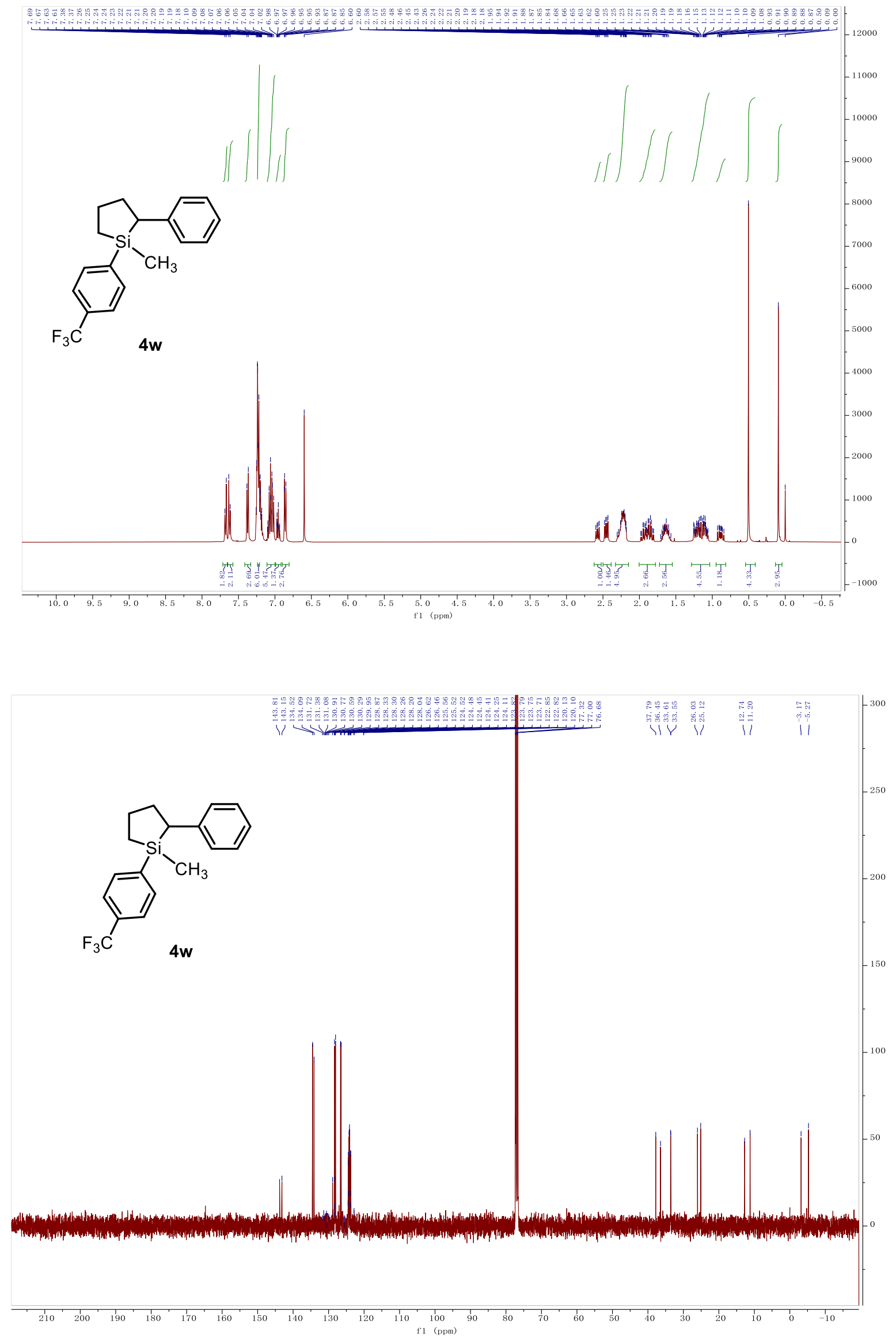
HPLC conditions: Daicel chiral column OD-3*OD-H, hexane: ${ }^{i} \mathrm{PrOH}=100: 0,0.5 \mathrm{~mL} / \mathrm{min}, 25^{\circ} \mathrm{C}$, wavelength $=210 \mathrm{~nm}$

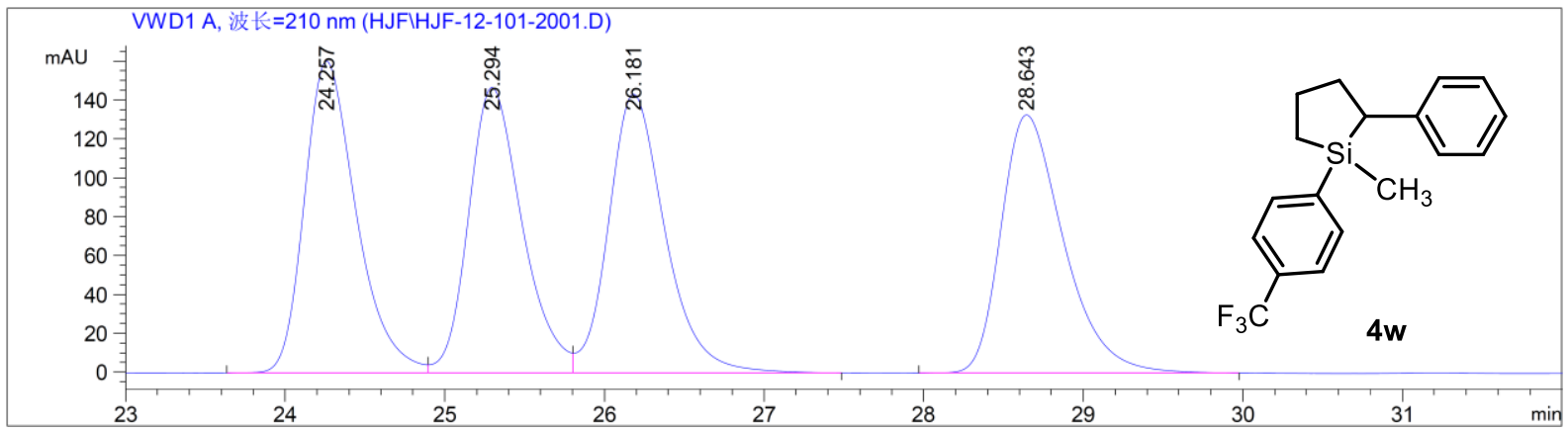

\begin{tabular}{|c|c|c|c|c|c|c|}
\hline \multirow{2}{*}{$\begin{array}{c}\text { Peak } \\
\#\end{array}$} & \multirow{2}{*}{$\begin{array}{c}\mathrm{k} \text { RetTime } \\
\text { [min] }\end{array}$} & \multirow{2}{*}{ e Type } & \multirow{2}{*}{$\begin{array}{l}\text { Width } \\
\text { [min] }\end{array}$} & Area & Height & \multirow{2}{*}{$\begin{array}{c}\text { Area } \\
\therefore\end{array}$} \\
\hline & & & & $\mathrm{mAU}$ & {$[\mathrm{mAU} \quad]$} & \\
\hline & & & & & & \\
\hline 1 & & BV & & 3627.825 & 160 & \\
\hline 2 & & VV & & 29. & & \\
\hline 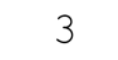 & 81 & & & 534.42 & 143 & \\
\hline & 43 & & & 3622.02417 & 132 & 8 \\
\hline
\end{tabular}

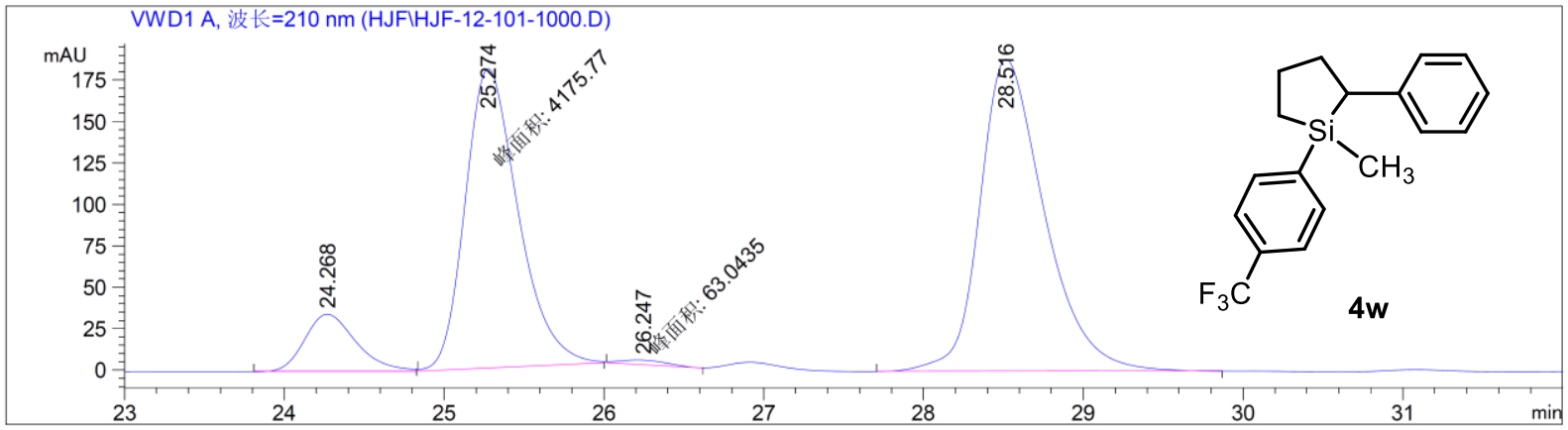

\begin{tabular}{|c|c|c|c|c|c|c|}
\hline Peak & RetTime & e Type & Width & Area & Height & Area \\
\hline \# & {$[\mathrm{min}]$} & & [min $]$ & $\mathrm{mAU}$ & {$[\mathrm{mAU} \quad]$} & $\%$ \\
\hline & 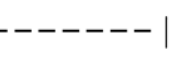 & & 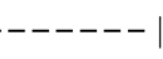 & ---- & 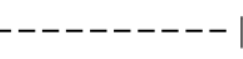 & -------- \\
\hline 1 & 24.268 & BV & 0.3457 & 782.58521 & 34.74934 & 7.5877 \\
\hline 2 & 25.274 & MM & 0.3848 & 4175.77051 & 180.86349 & 40.4868 \\
\hline 3 & 26.247 & MM & 0.3462 & 63.04351 & 3.03494 & 0.6112 \\
\hline 4 & 28.516 & BB & 0.4254 & 5292.50488 & 188.33424 & 51.3143 \\
\hline
\end{tabular}



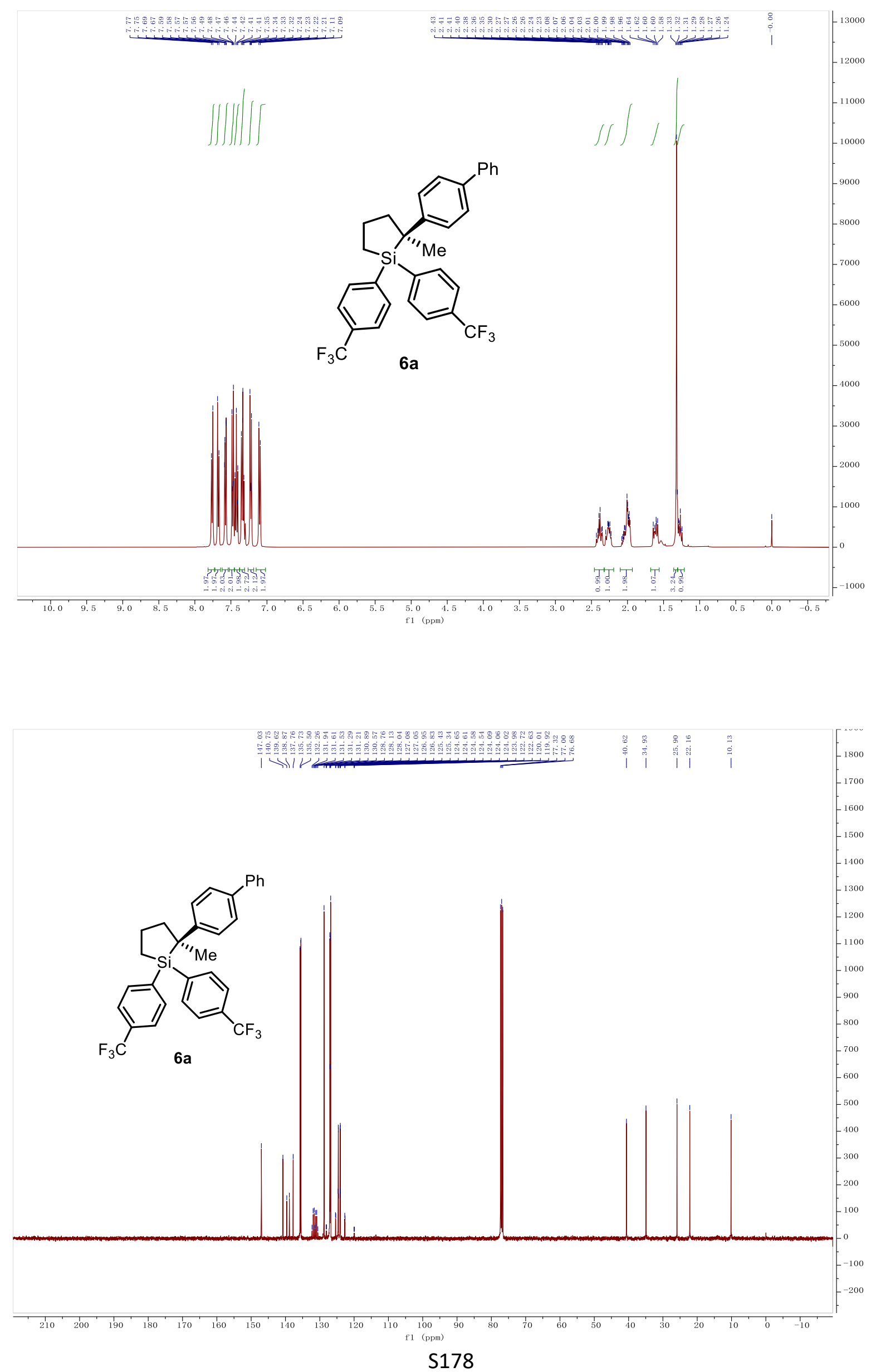


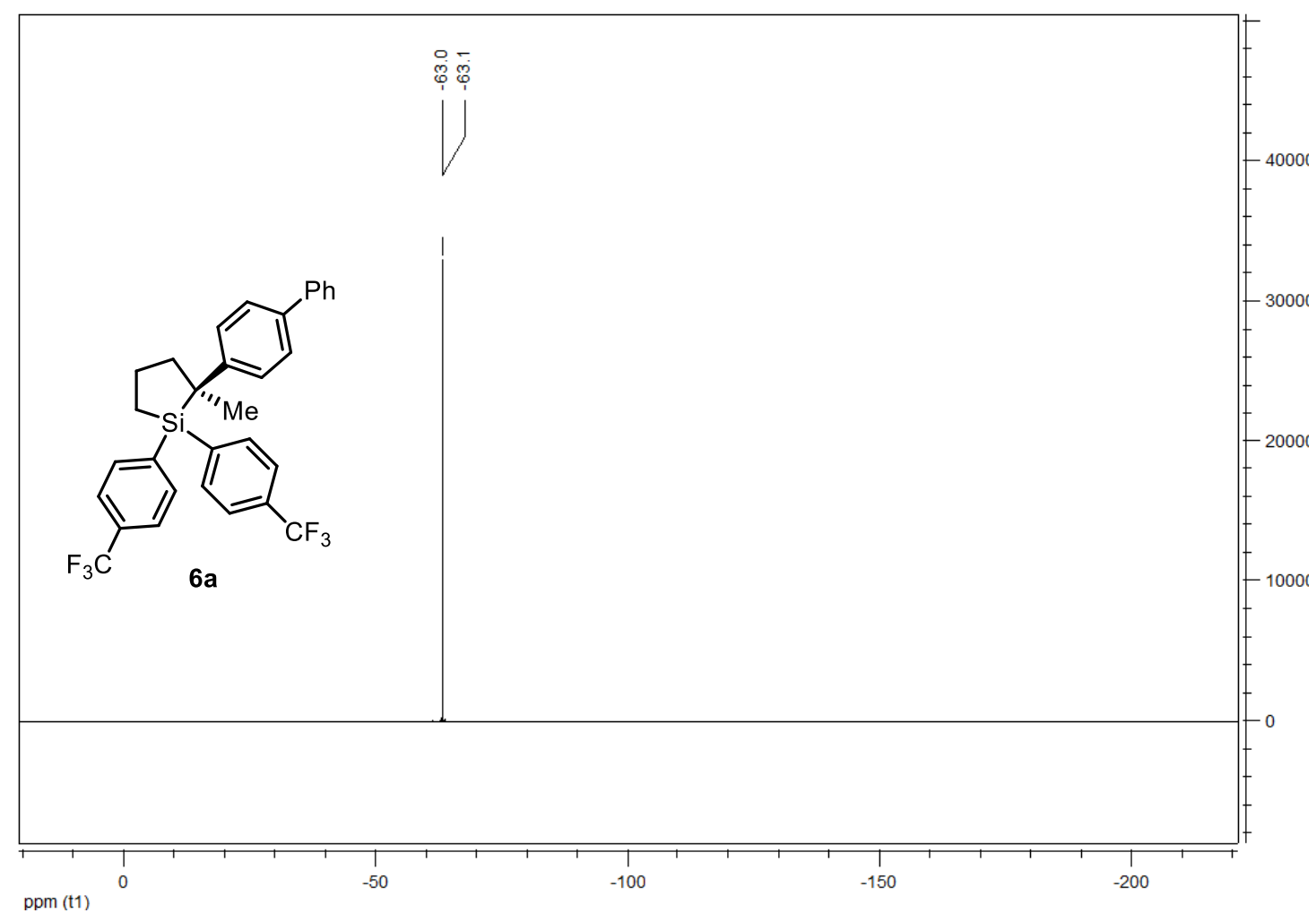

HPLC conditions: Daicel chiral column AD-H, hexane $:{ }^{i} \mathrm{PrOH}=100: 0,0.5 \mathrm{~mL} / \mathrm{min}, 25{ }^{\circ} \mathrm{C}$, wavelength $=$ $220 \mathrm{~nm}$

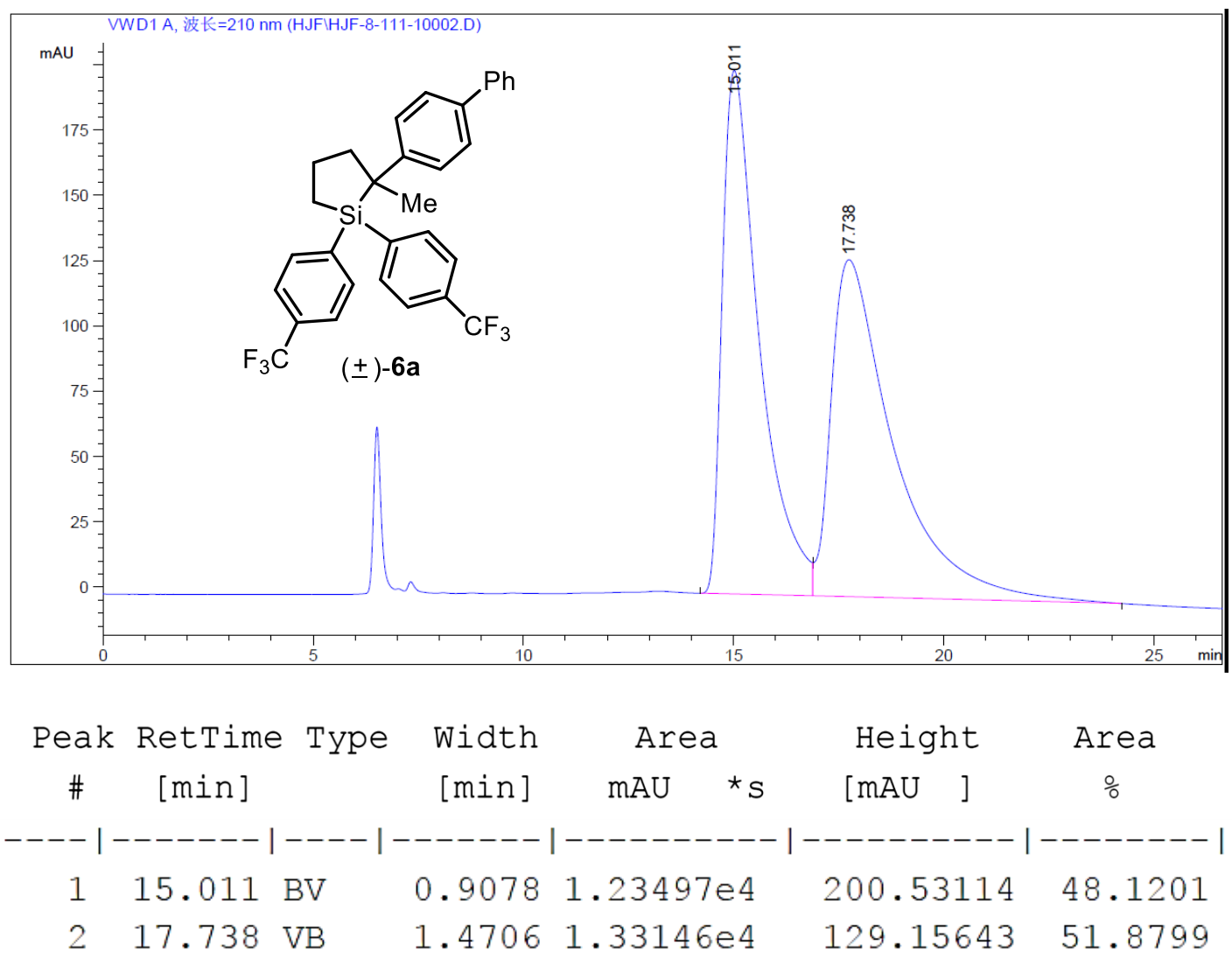



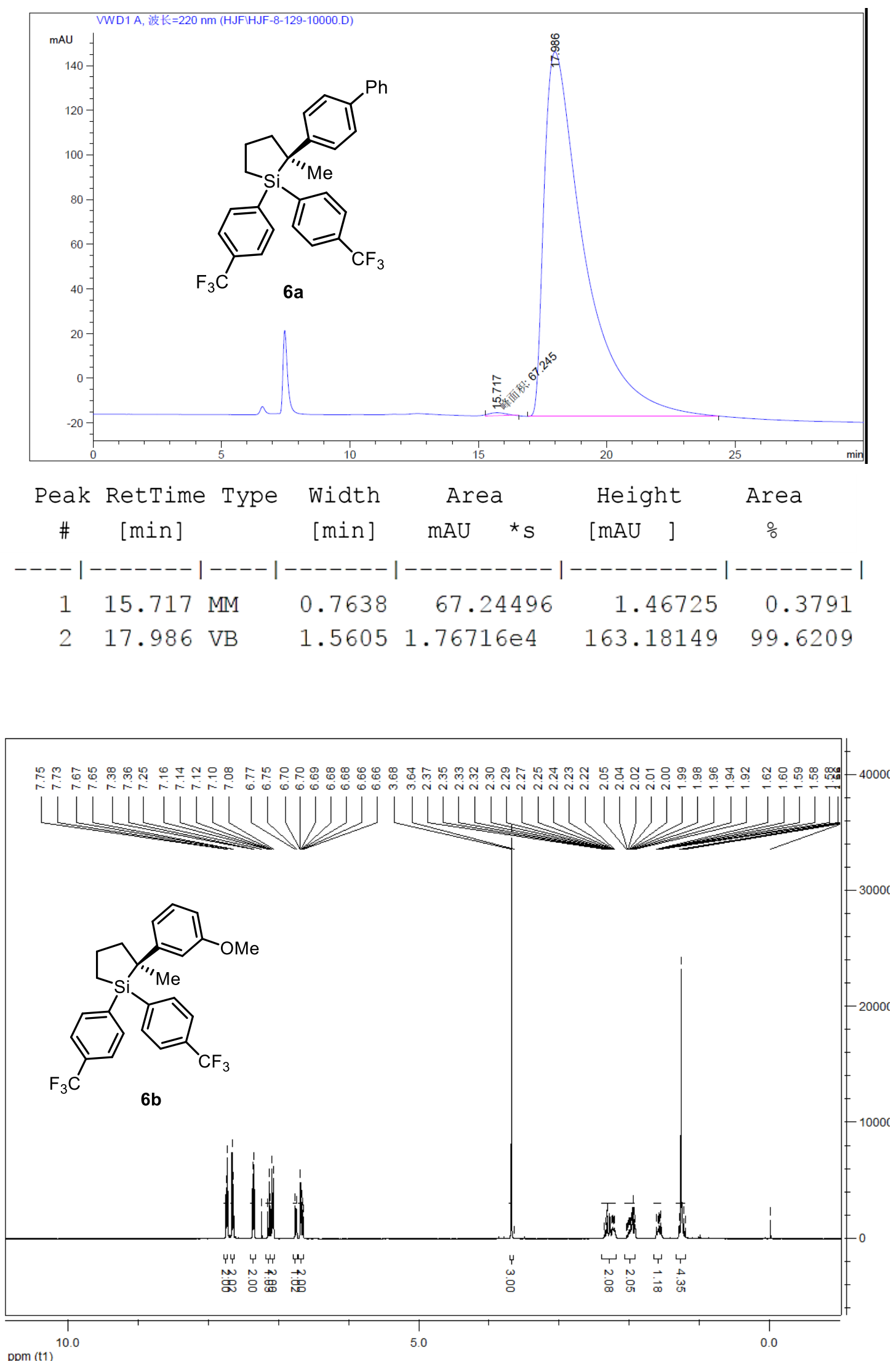

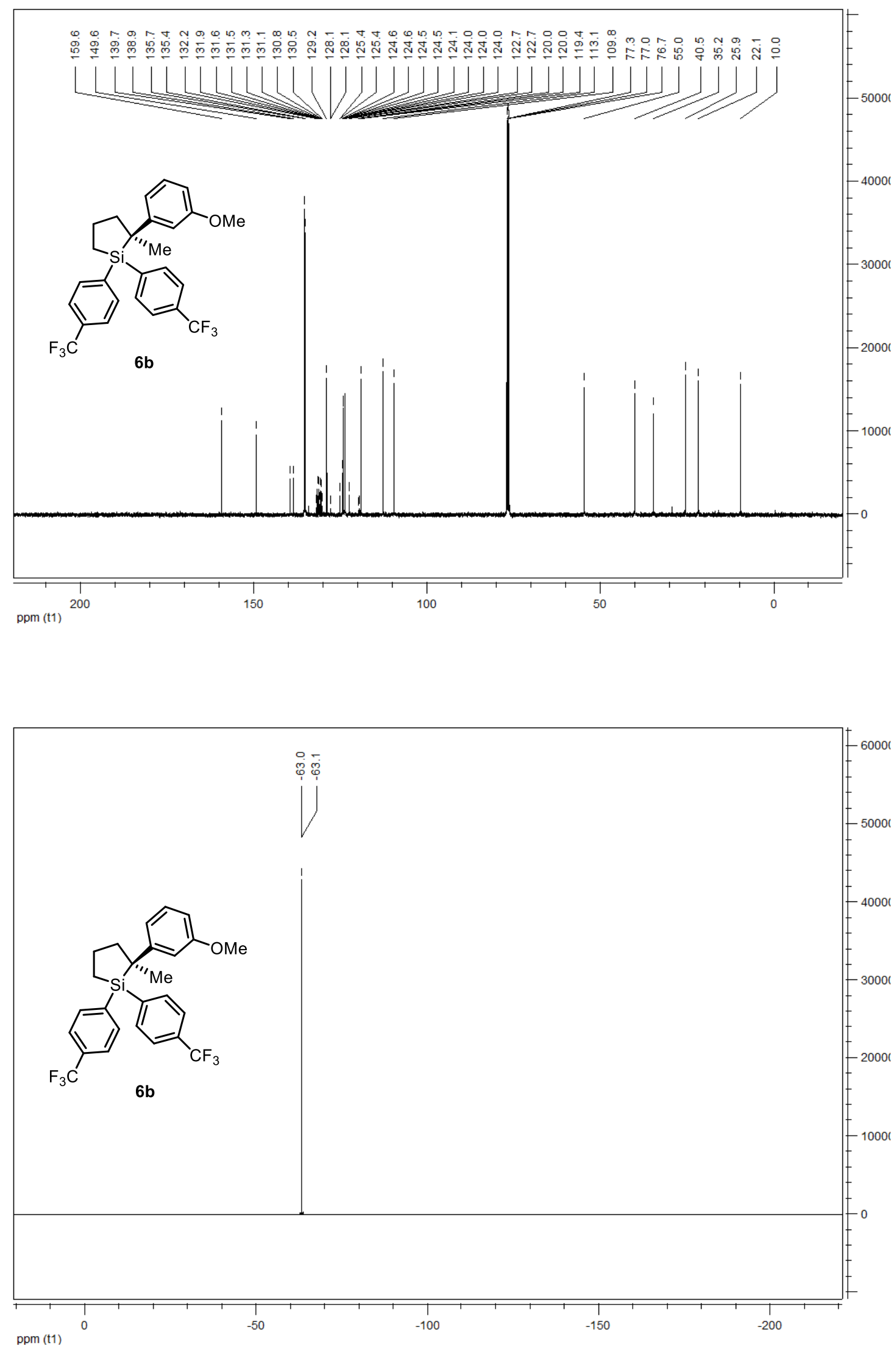
HPLC conditions: Daicel chiral column OD-3, hexane $:{ }^{i} \mathrm{PrOH}=100: 0,0.8 \mathrm{~mL} / \mathrm{min}, 25^{\circ} \mathrm{C}$, wavelength $=$ $220 \mathrm{~nm}$
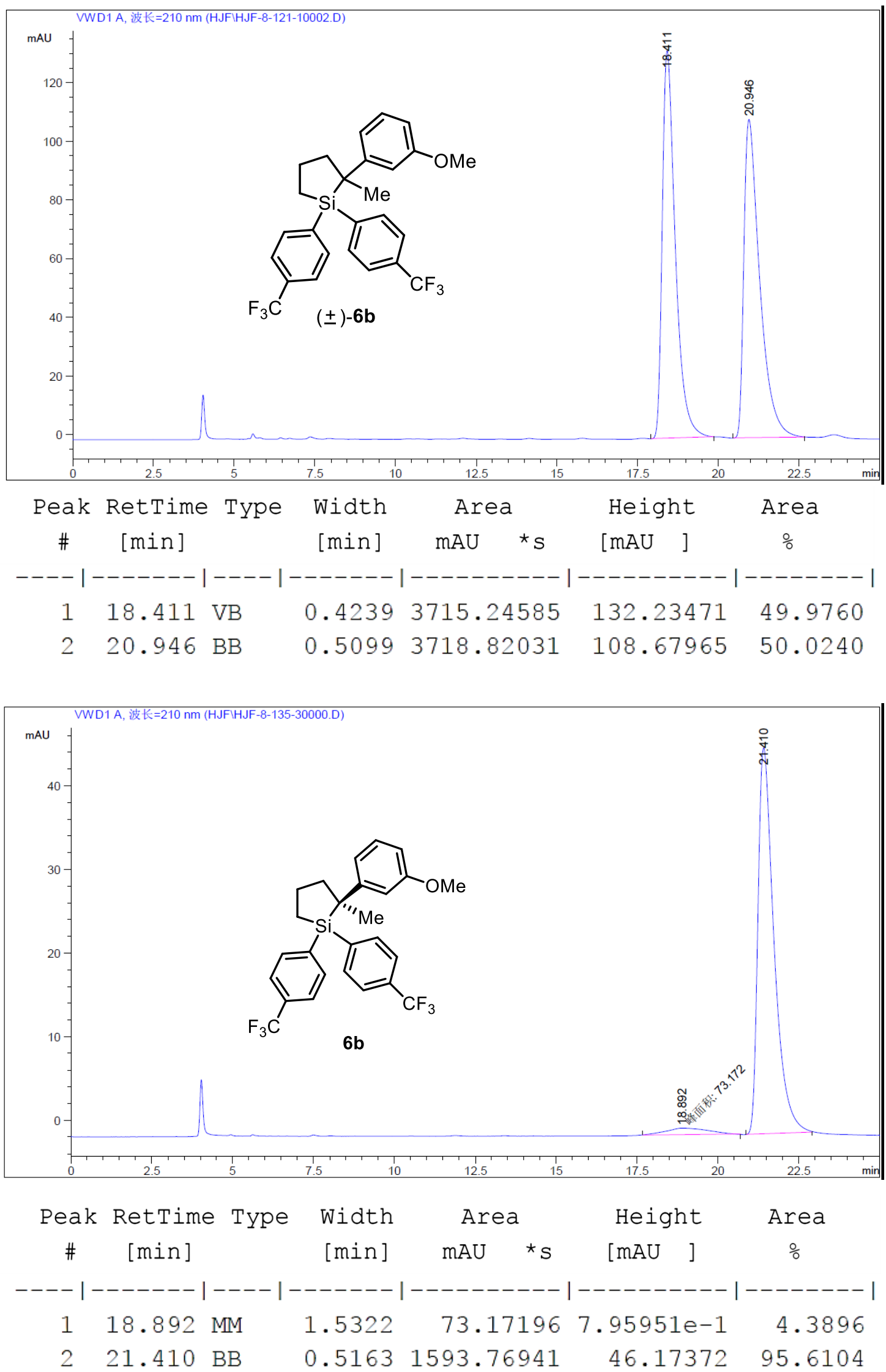

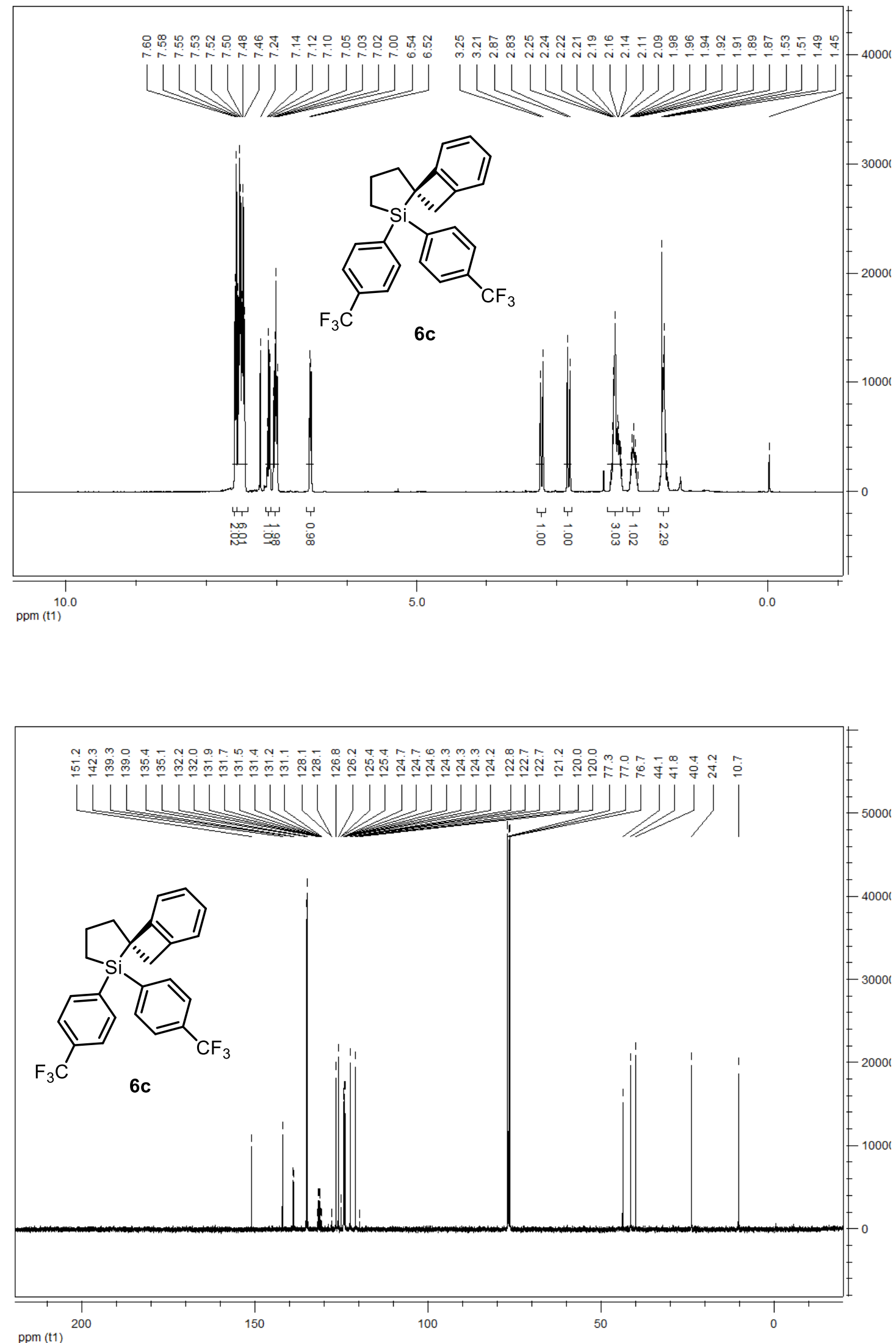


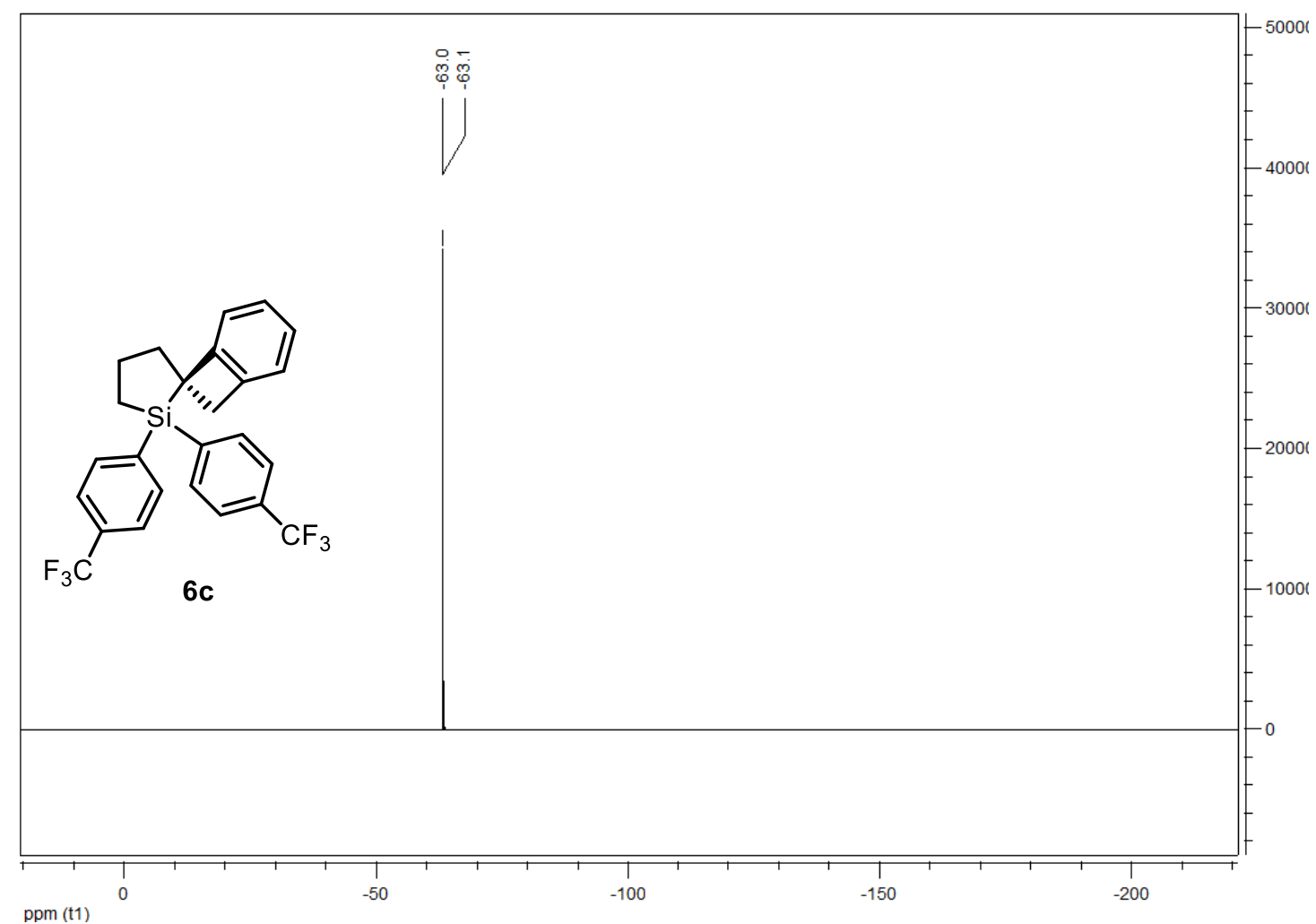

HPLC conditions: Daicel chiral column OD-3, hexane: ${ }^{i} \mathrm{PrOH}$ : Trifluoroacetic acid $=95: 5: 0.1,0.7 \mathrm{~mL} / \mathrm{min}$, $25^{\circ} \mathrm{C}$, wavelength $=220 \mathrm{~nm}$

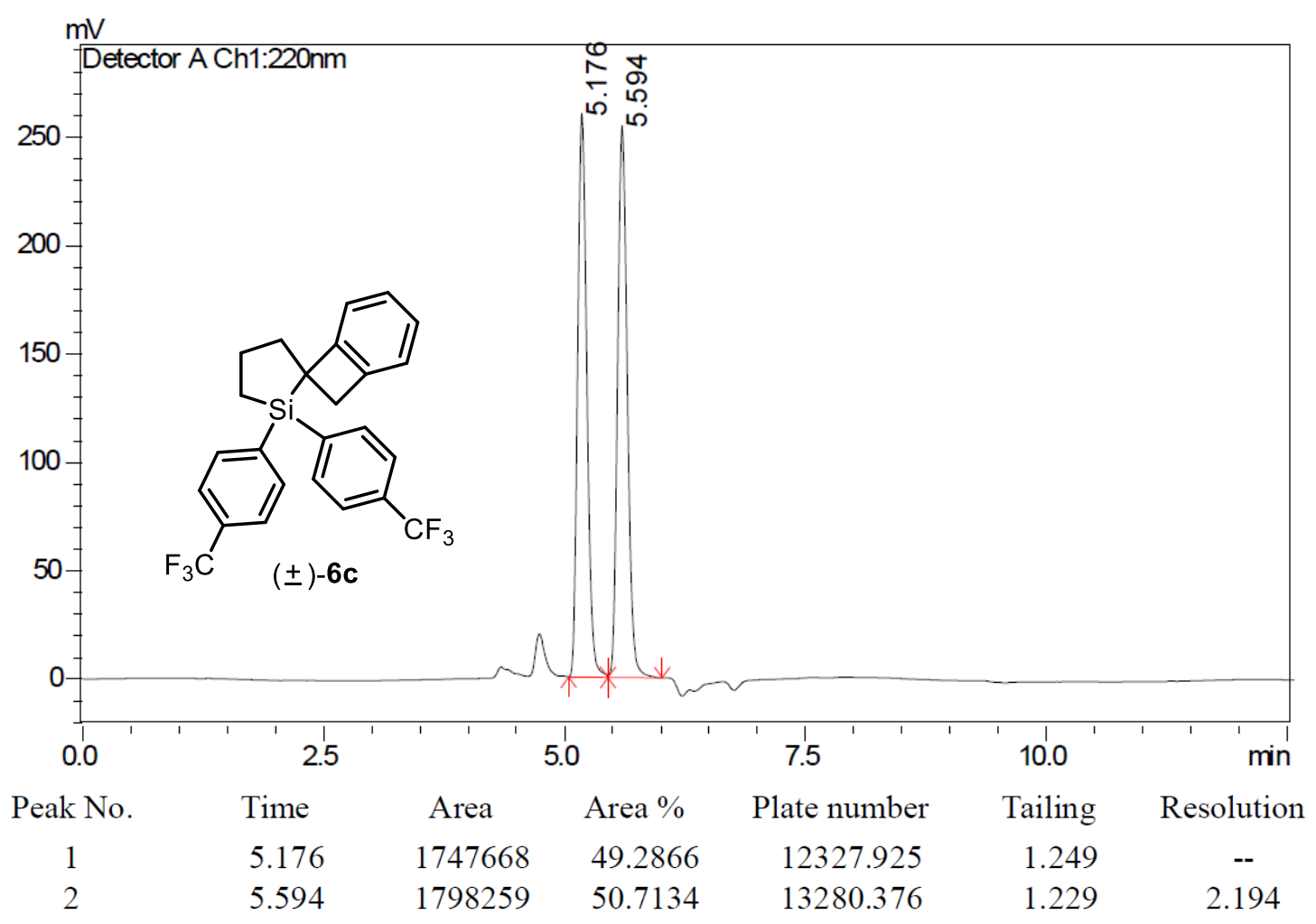



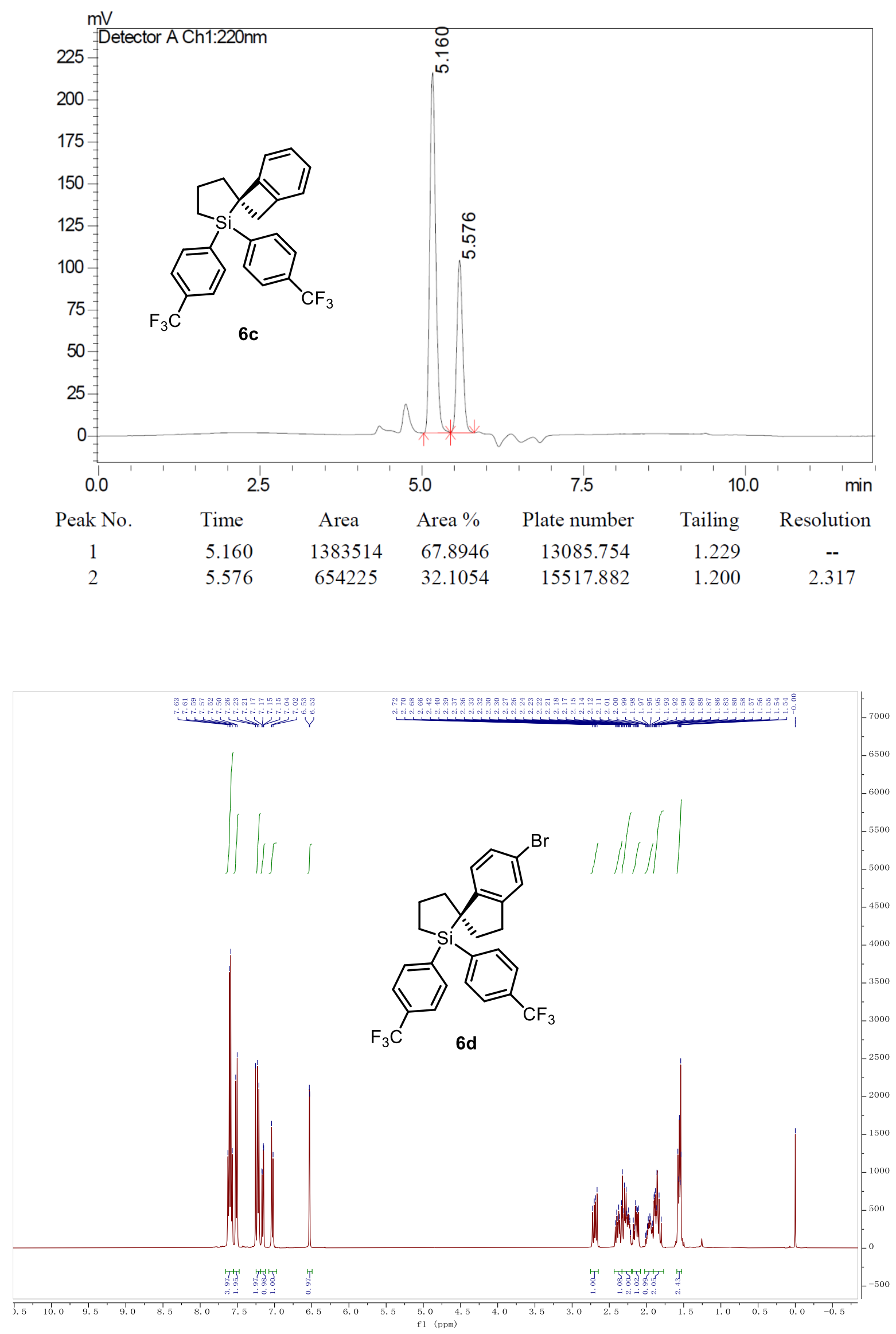

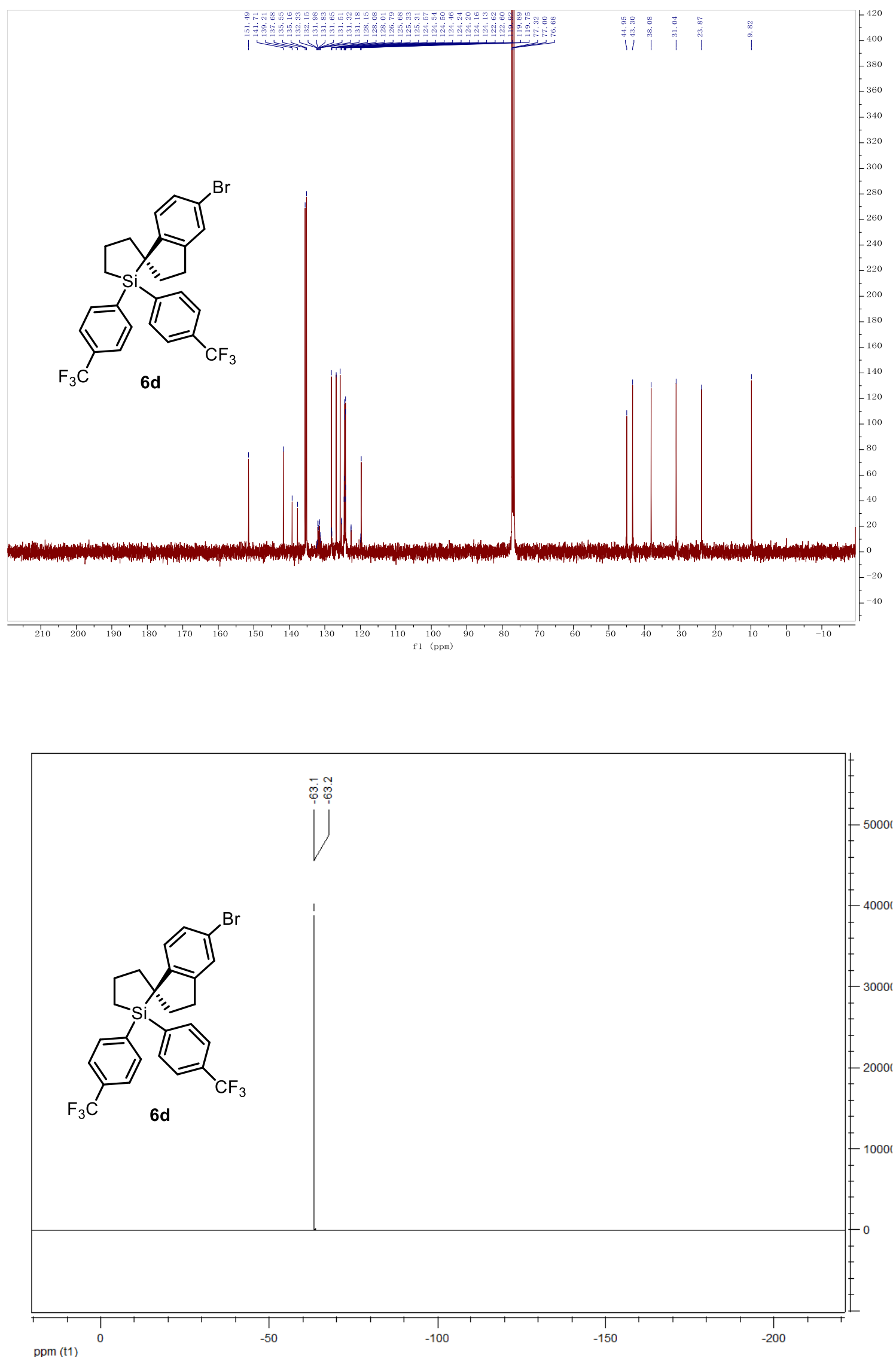
HPLC conditions: Daicel chiral column $O D-H+O D-3$, hexane: ${ }^{i} \mathrm{PrOH}=100: 0,0.25 \mathrm{~mL} / \mathrm{min}, 25^{\circ} \mathrm{C}$, wavelength $=210 \mathrm{~nm}$
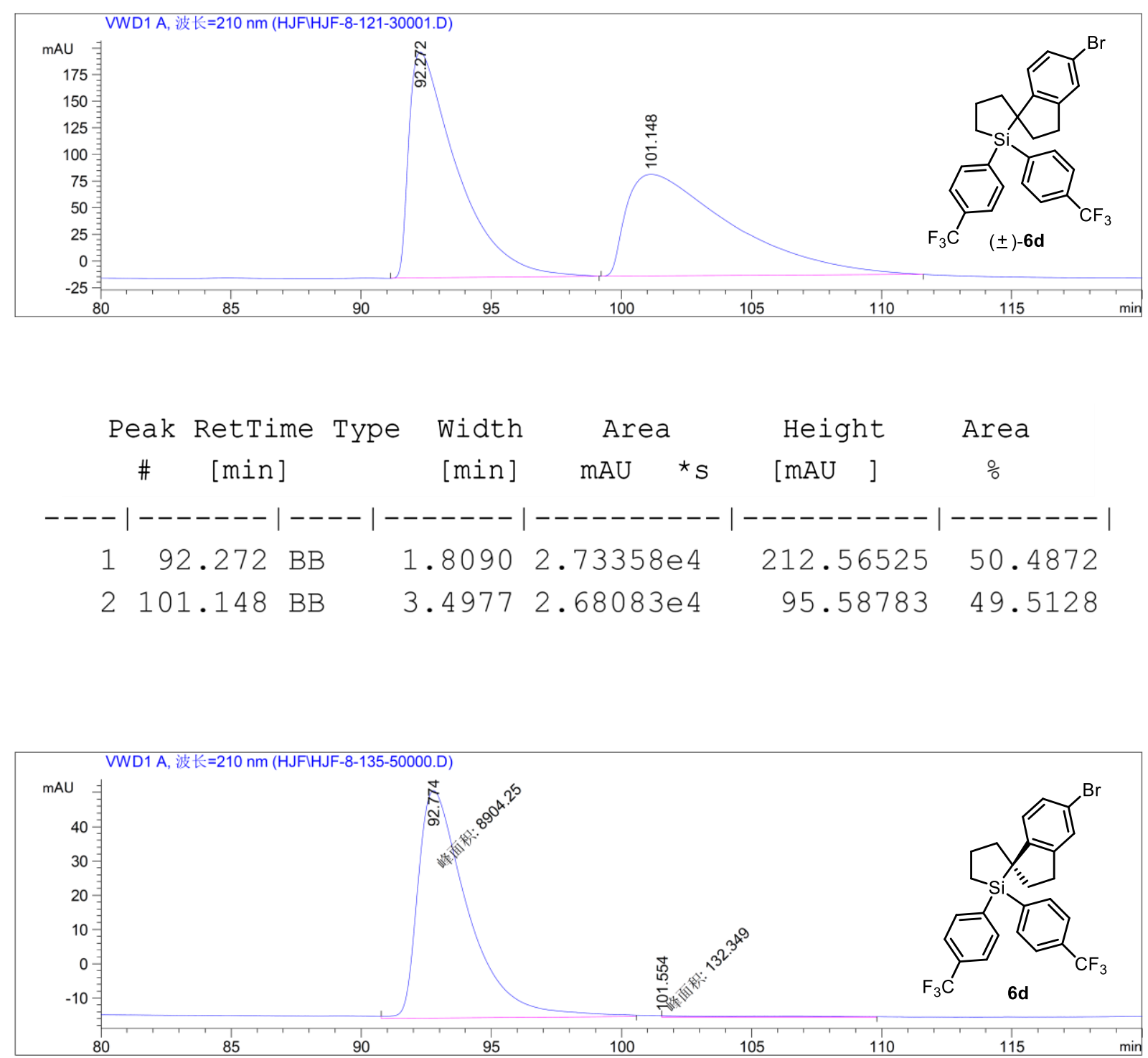

\begin{tabular}{|c|c|c|c|c|c|c|c|}
\hline \multirow{2}{*}{$\begin{array}{c}\text { Peak } \\
\quad \#\end{array}$} & RetTime & Type & Width & \multicolumn{2}{|c|}{ Area } & Height & \multirow{2}{*}{$\begin{array}{c}\text { Area } \\
\frac{\circ}{0}\end{array}$} \\
\hline & [min] & & {$[\mathrm{min}]$} & mAU & $*_{S}$ & {$[\mathrm{mAU} \quad]$} & \\
\hline & - & & --- & - & $=--$ & -- & $--------\mid$ \\
\hline 1 & 92.774 & MM & 54 & 8904 & 5000 & 704 & 98.5354 \\
\hline 2 & 101.554 & MM & 5.4871 & 132 & 4883 & $4.01999 e-1$ & 1.4646 \\
\hline
\end{tabular}



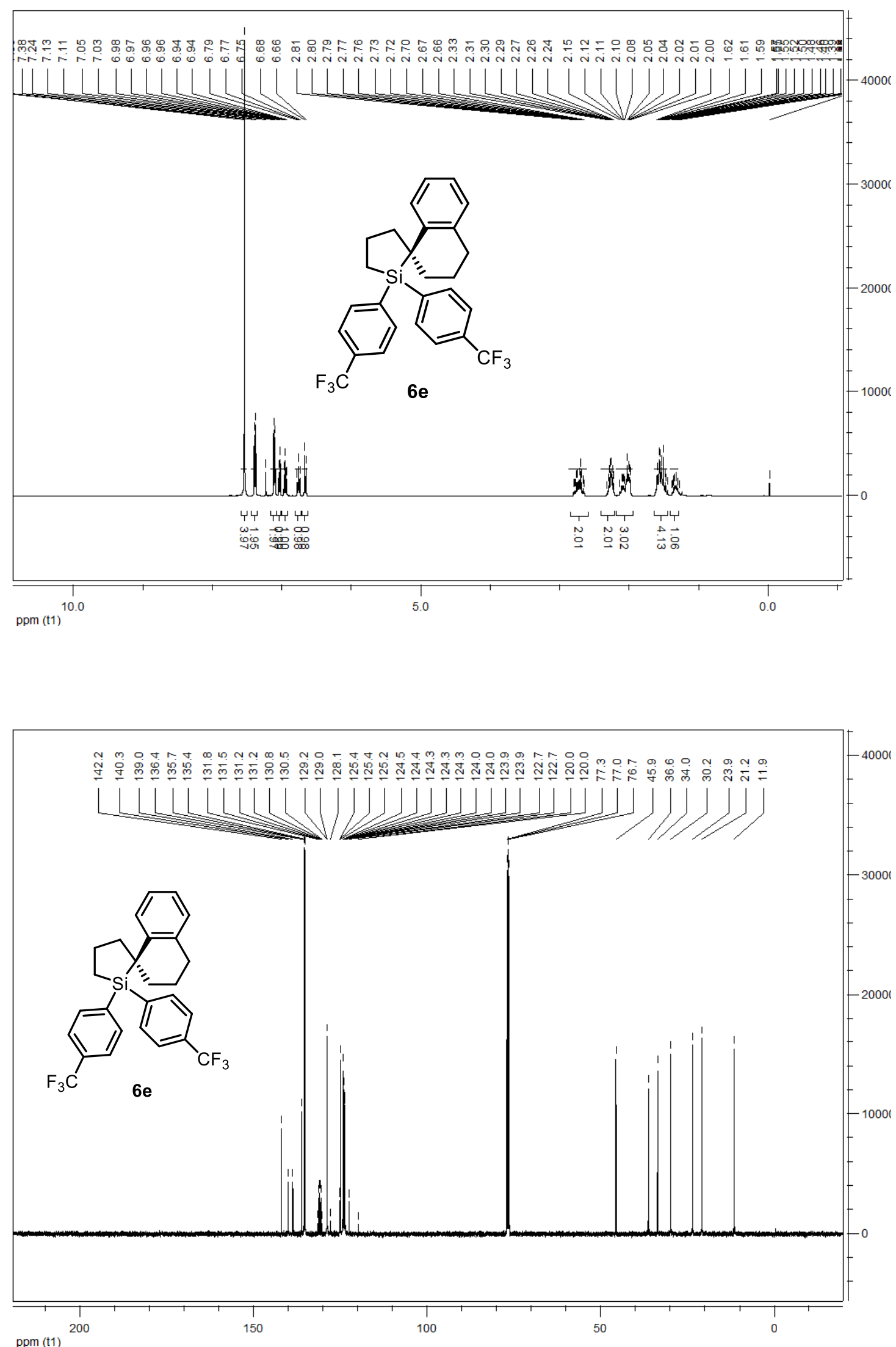


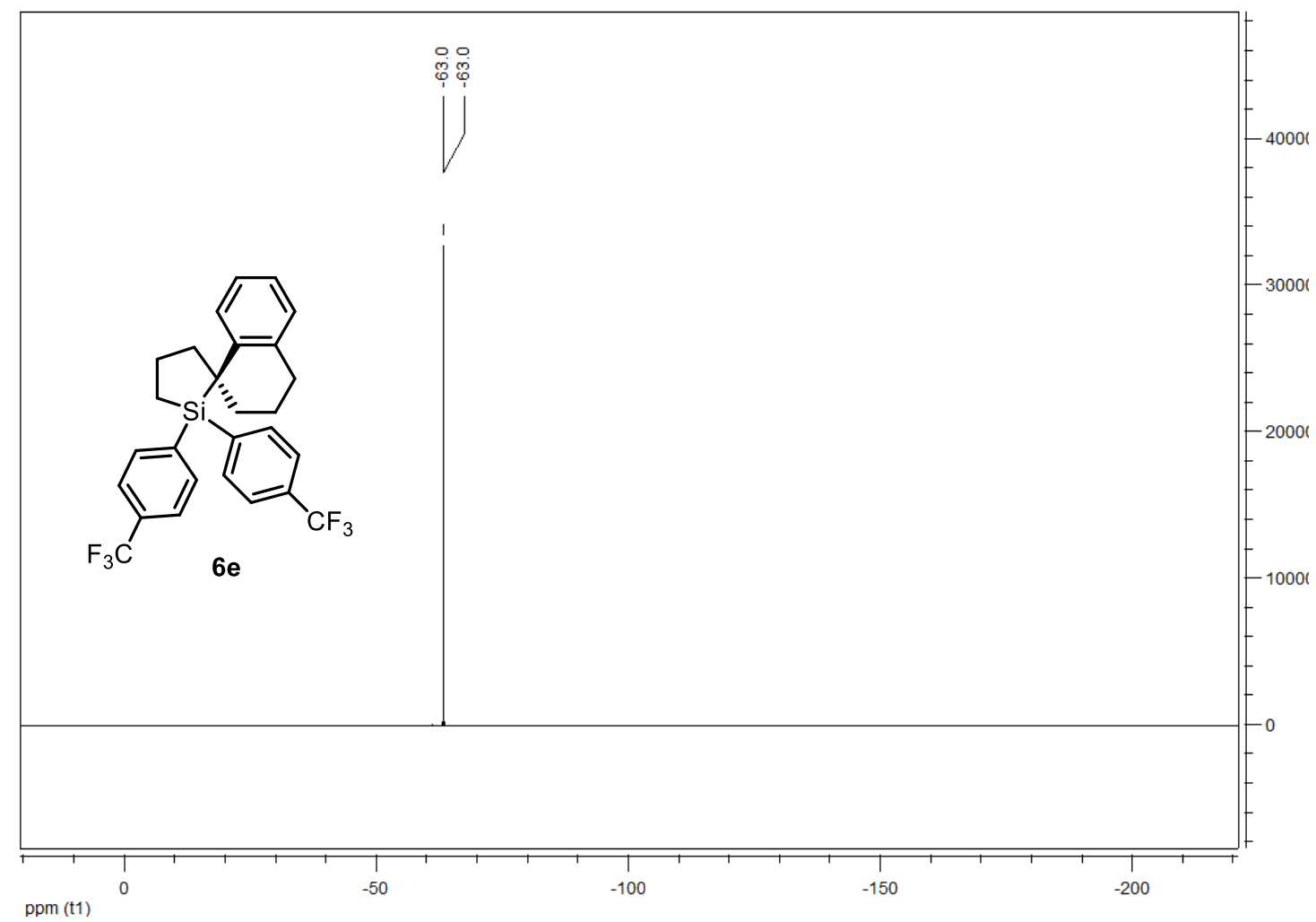

HPLC conditions: Daicel chiral column $O D-3 * O D-H$, hexane: ${ }^{i} \mathrm{PrOH}=100: 0,0.3 \mathrm{~mL} / \mathrm{min}, 25{ }^{\circ} \mathrm{C}$, wavelength $=210 \mathrm{~nm}$
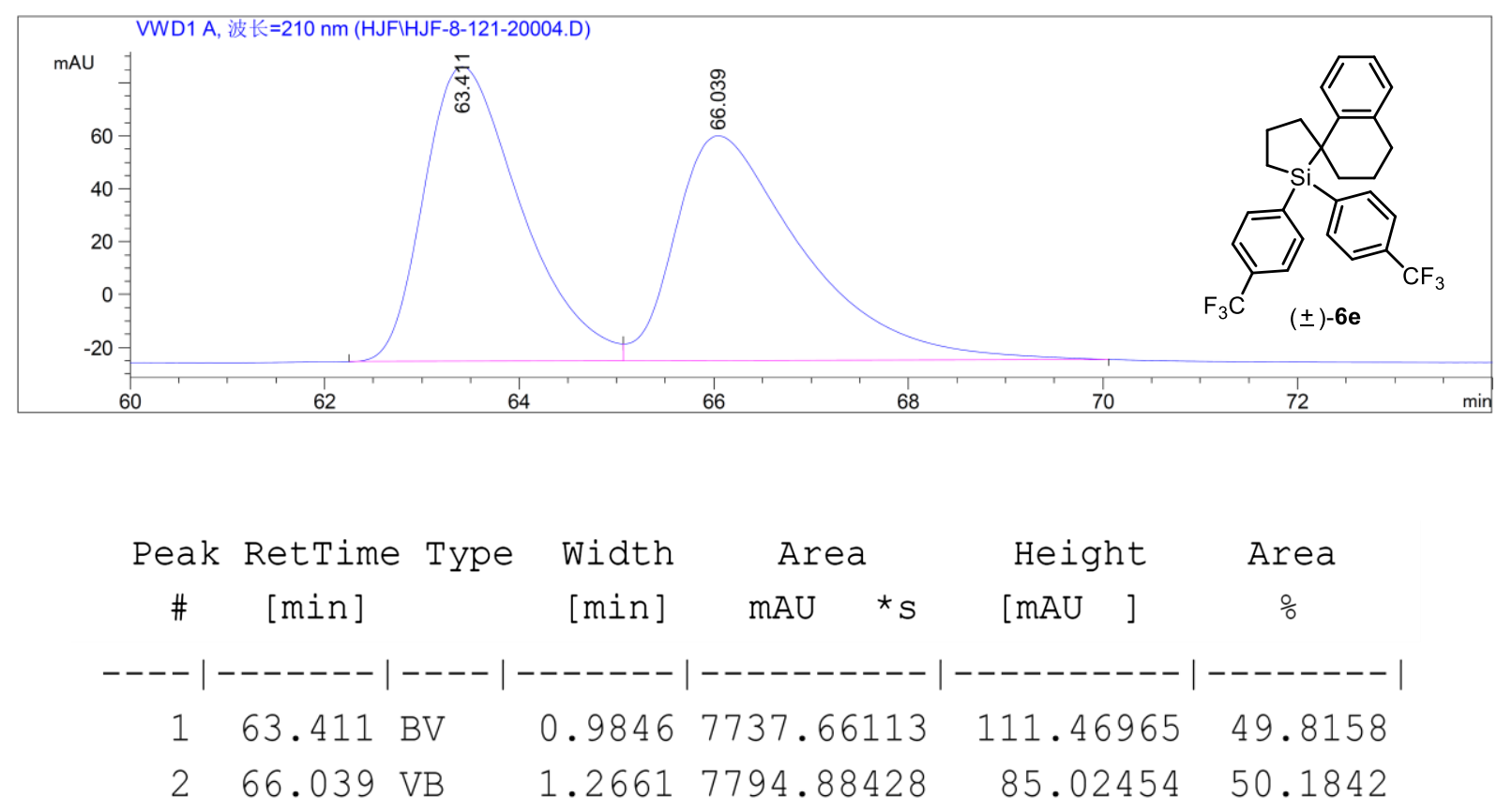


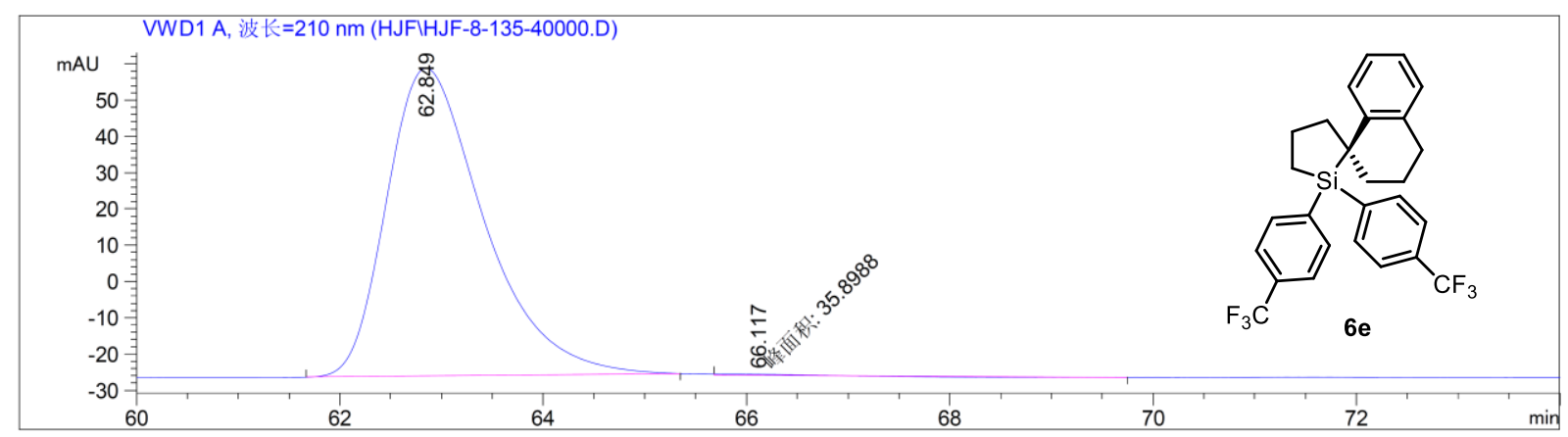

\begin{tabular}{|c|c|c|c|c|c|c|c|}
\hline Peak & RetTime & Type & Width & Ar & & Height & Area \\
\hline \# & [min] & & [min $]$ & $\mathrm{mAU}$ & $\star_{S}$ & {$[\mathrm{mAU} \quad]$} & $\frac{\circ}{0}$ \\
\hline & & & & & & & \\
\hline 1 & 49 & $\mathrm{BB}$ & 779 & 5656.2 & 02 & 84.93235 & 99 \\
\hline 2 & $66.117 \mathrm{I}$ & MM & 1.4941 & 35.8 & 75 & $4.00437 e-1$ & 0.6307 \\
\hline
\end{tabular}

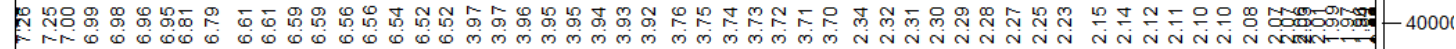

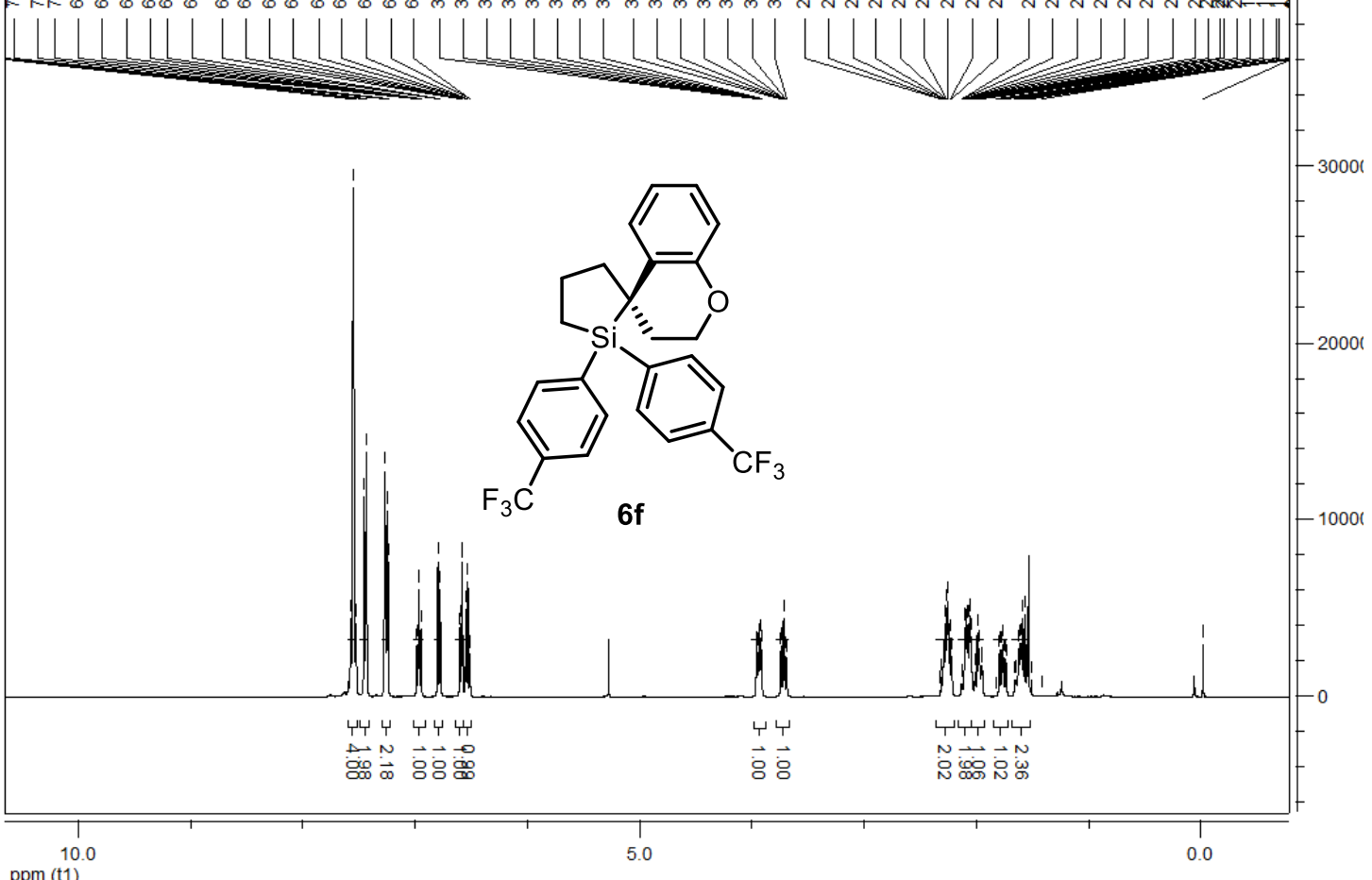



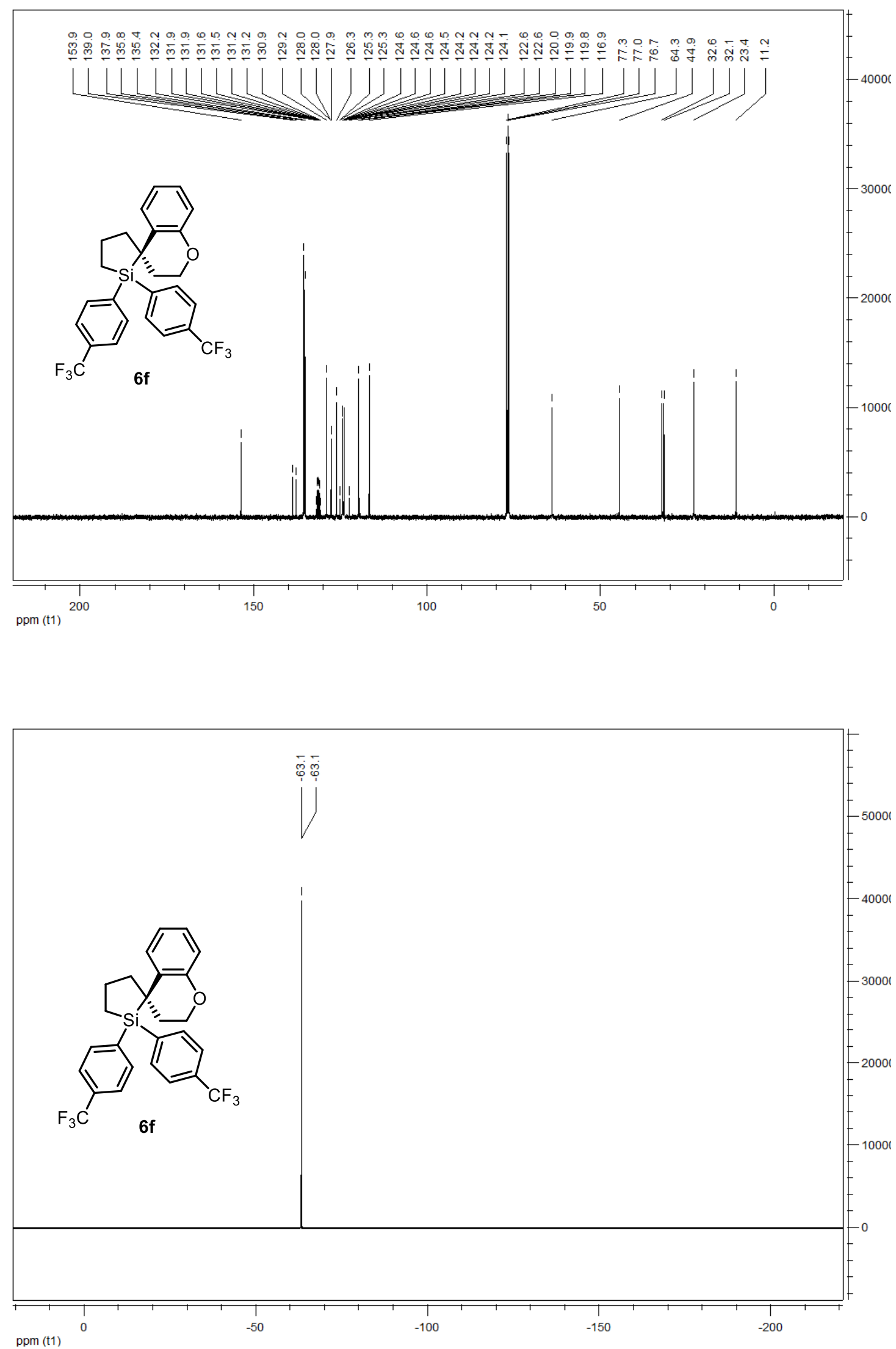
HPLC conditions: Daicel chiral column OD-3, hexane $:{ }^{i} \mathrm{PrOH}=100: 0,0.5 \mathrm{~mL} / \mathrm{min}, 25^{\circ} \mathrm{C}$, wavelength $=$ $220 \mathrm{~nm}$
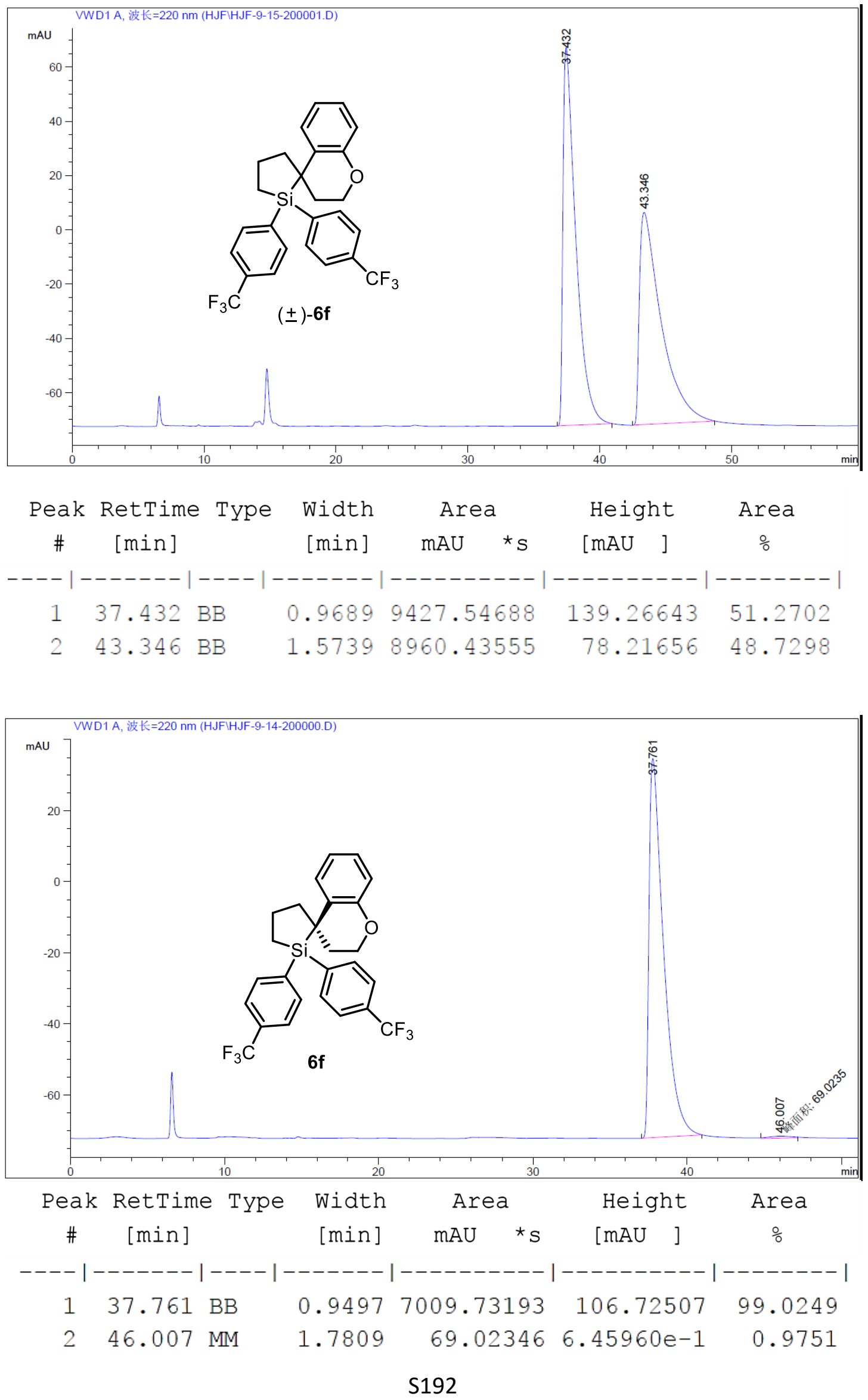

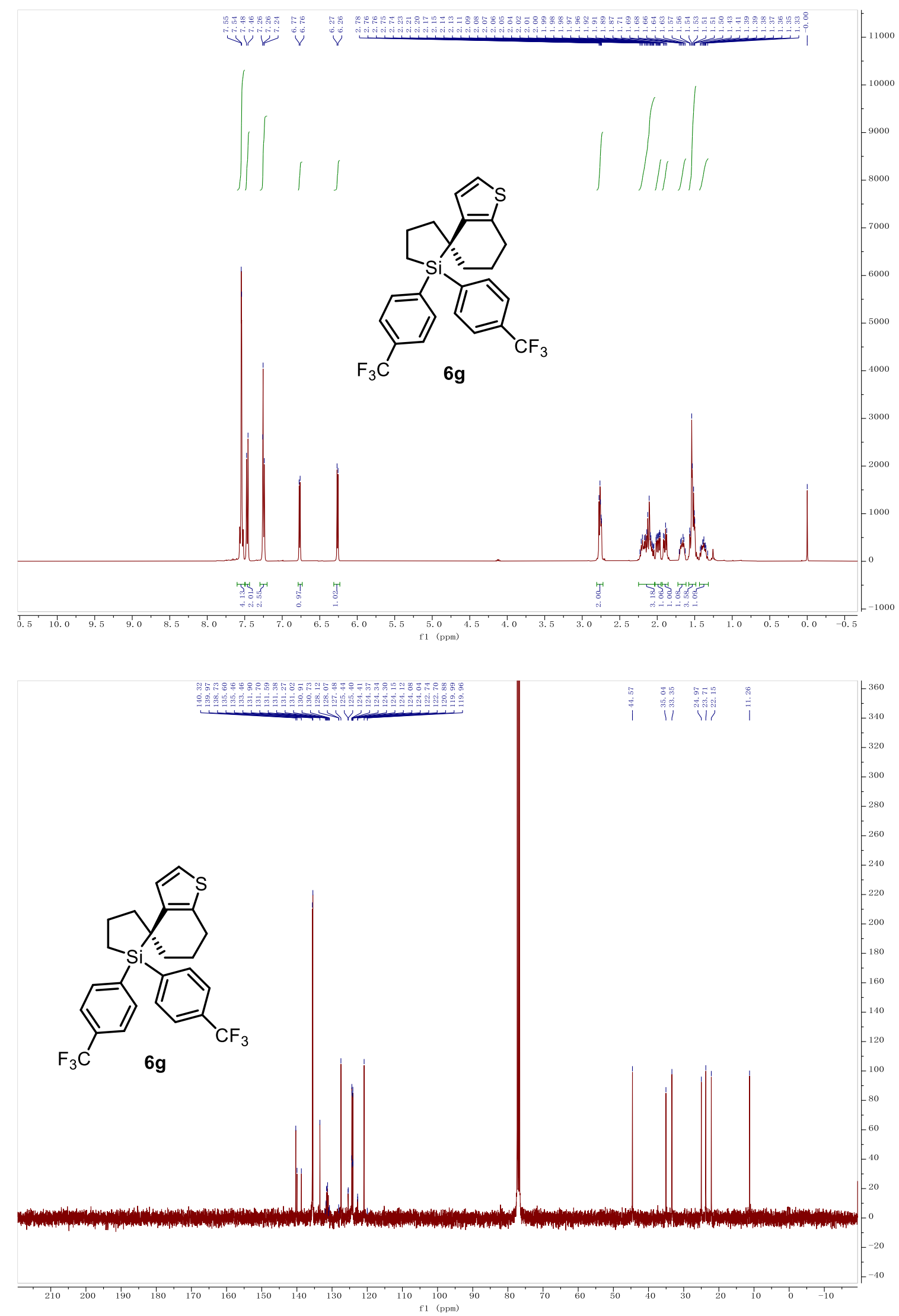


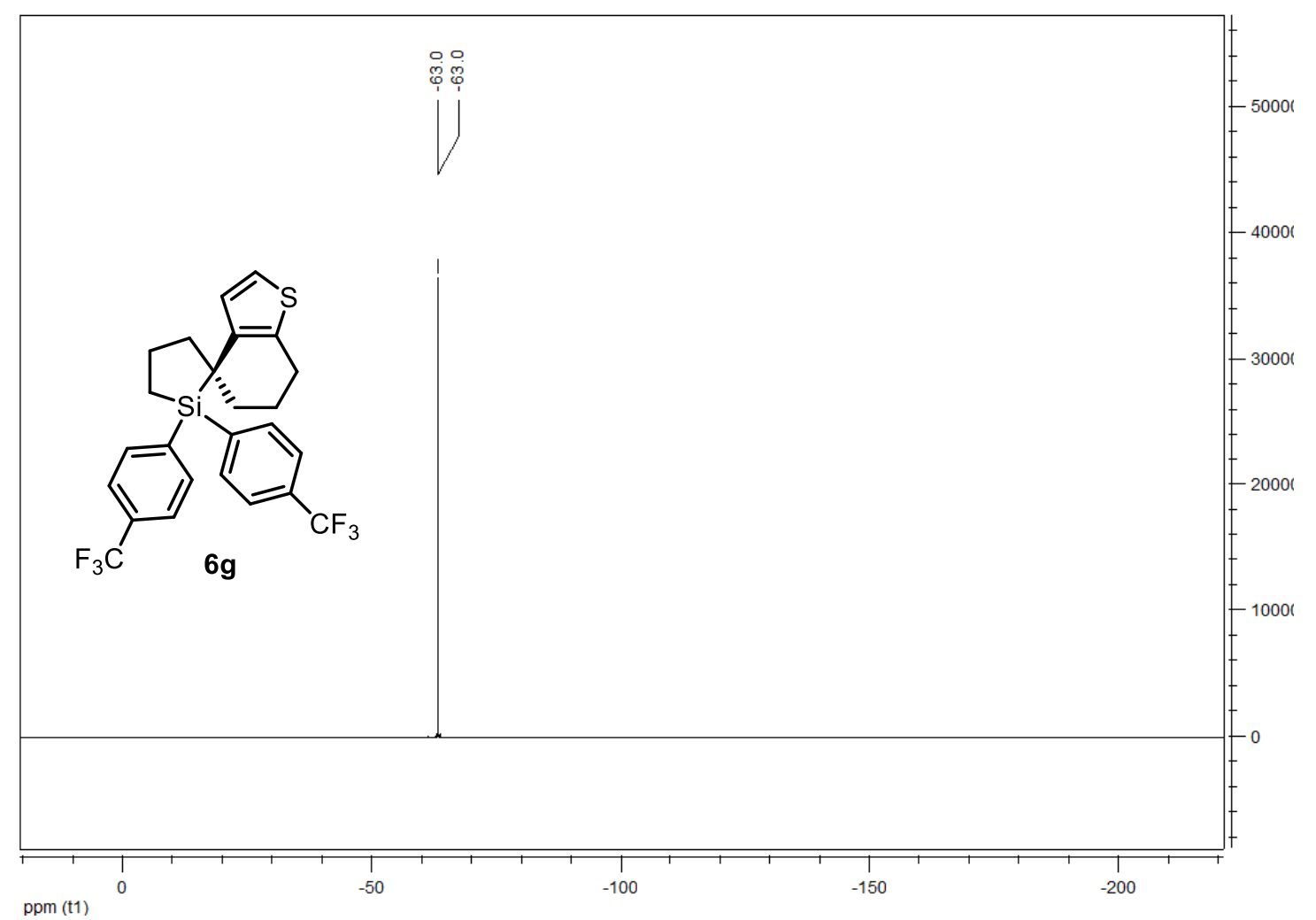

HPLC conditions: Daicel chiral column OD-3, hexane $:{ }^{i} \mathrm{PrOH}=100: 0,0.5 \mathrm{~mL} / \mathrm{min}, 25{ }^{\circ} \mathrm{C}$, wavelength $=$ $220 \mathrm{~nm}$
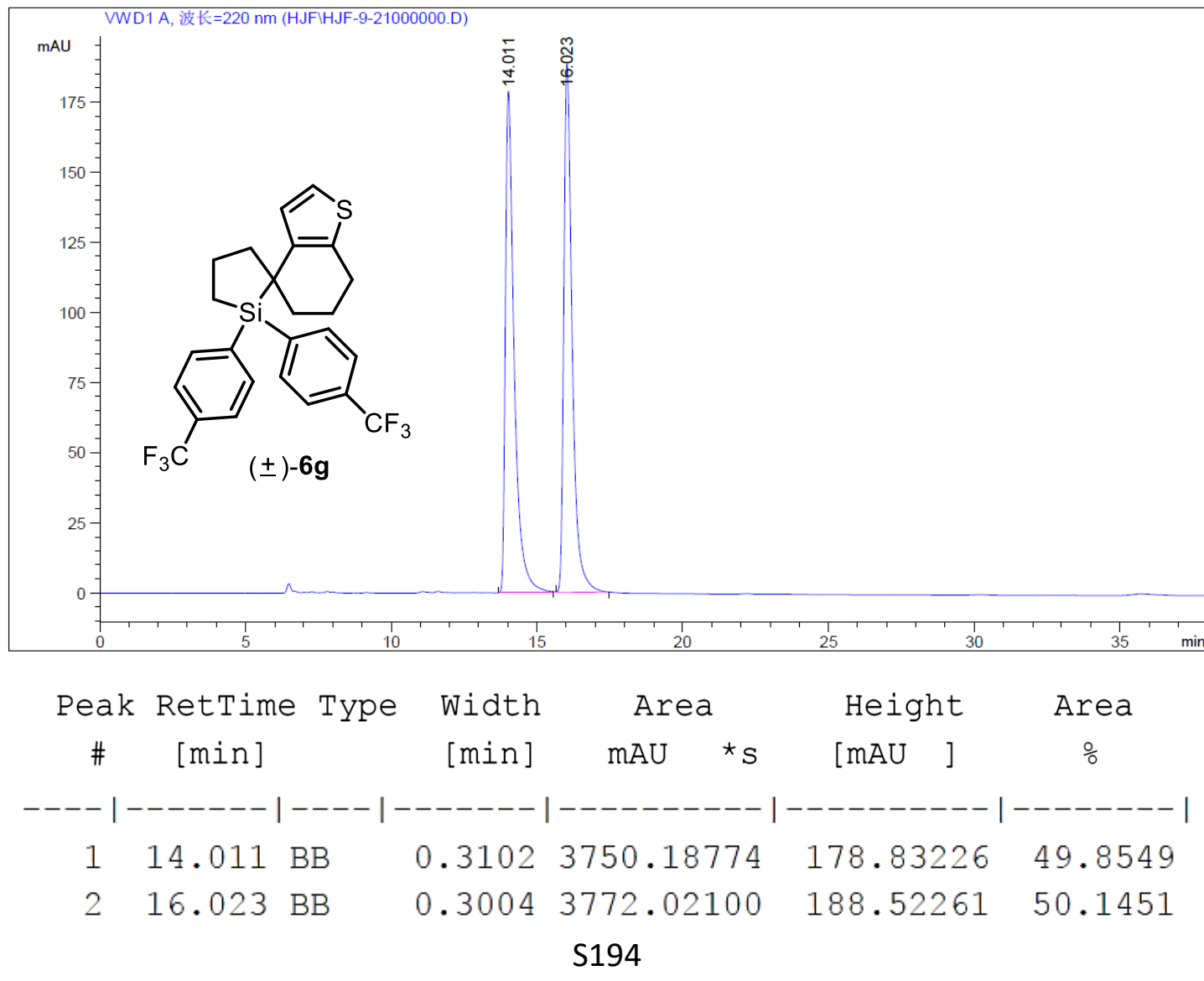

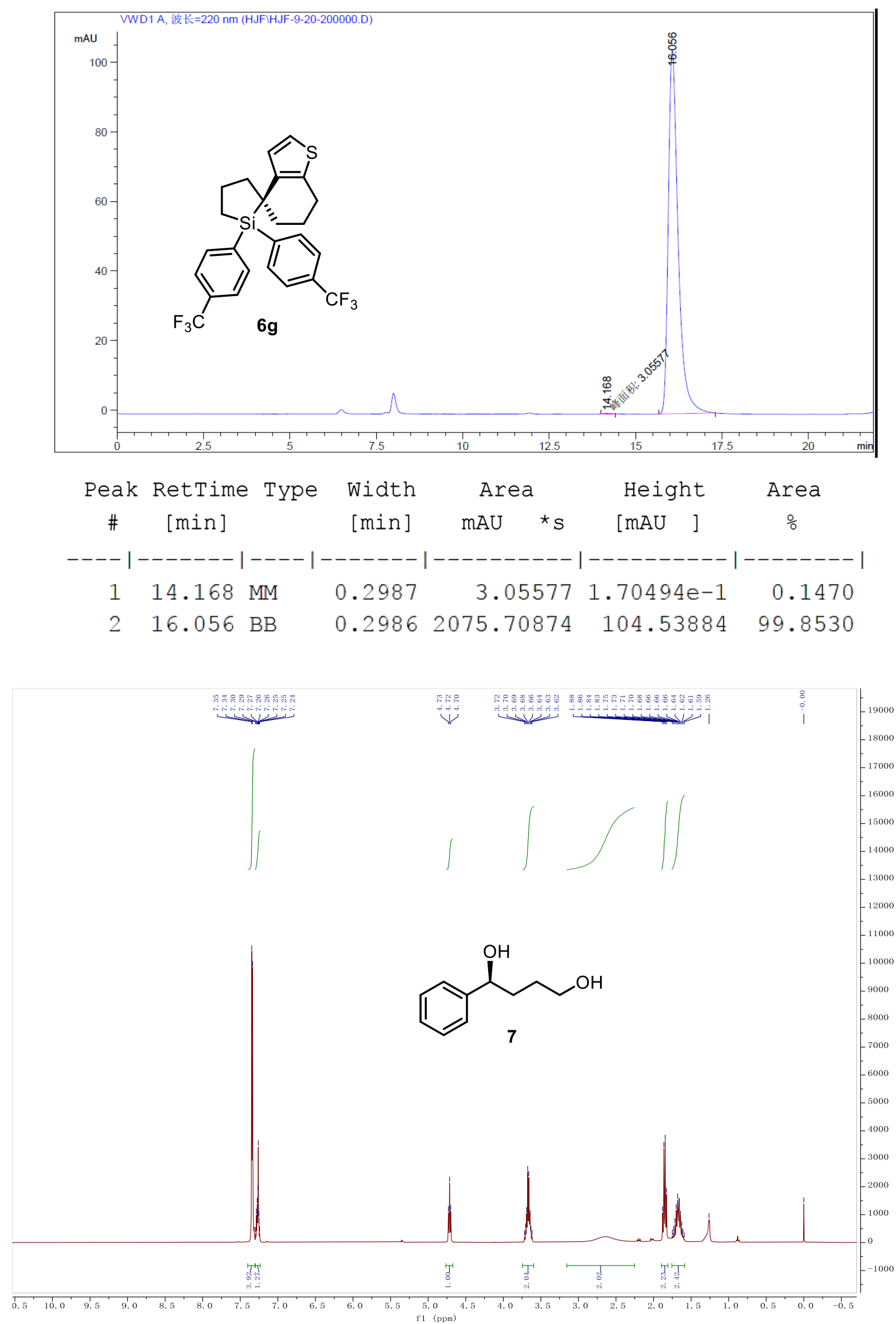


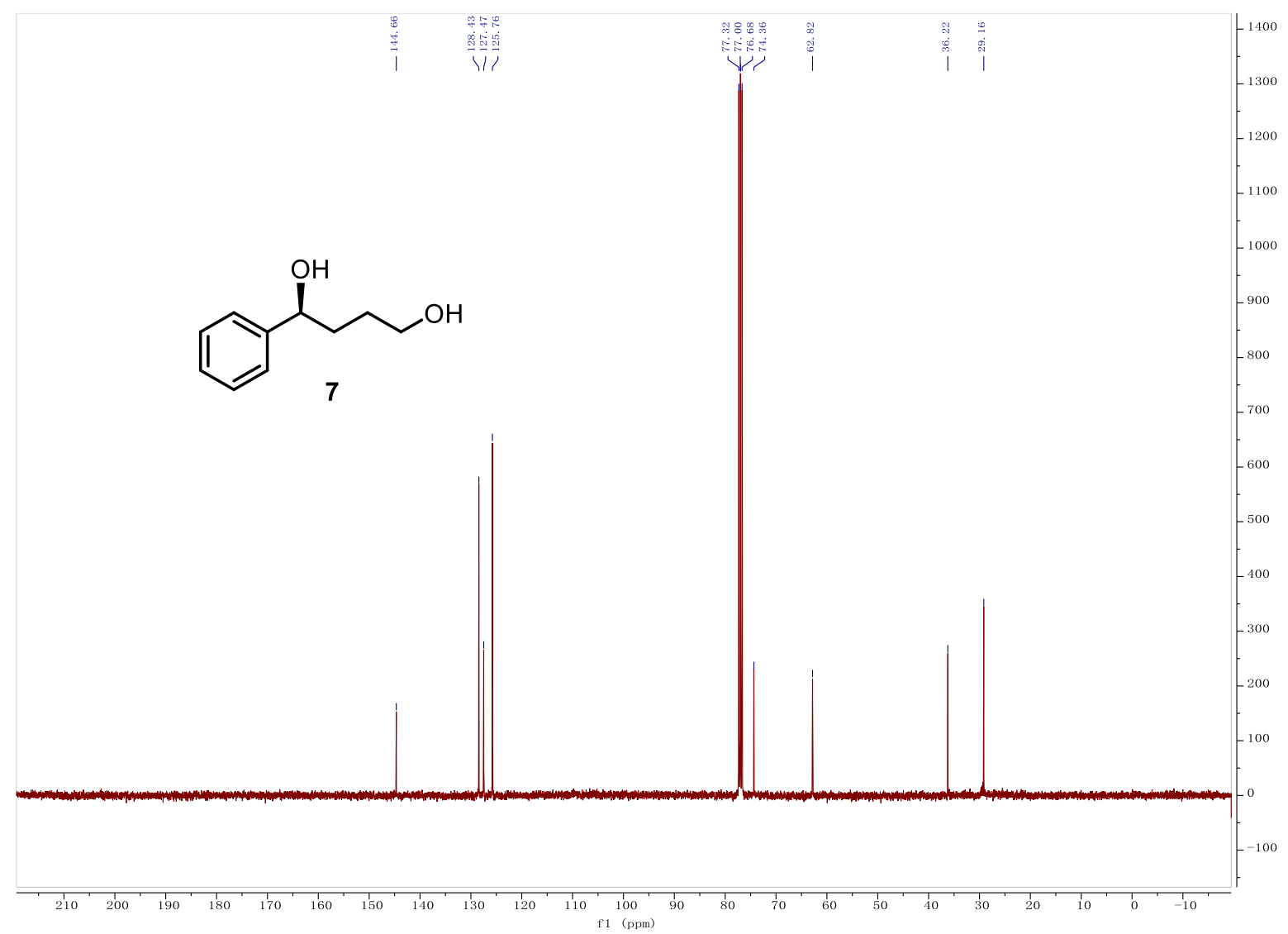

HPLC conditions: Daicel chiral column OD-H, hexane: ${ }^{i} \mathrm{PrOH}=90: 10,0.5 \mathrm{~mL} / \mathrm{min}, 25^{\circ} \mathrm{C}$, wavelength $=$ $220 \mathrm{~nm}$

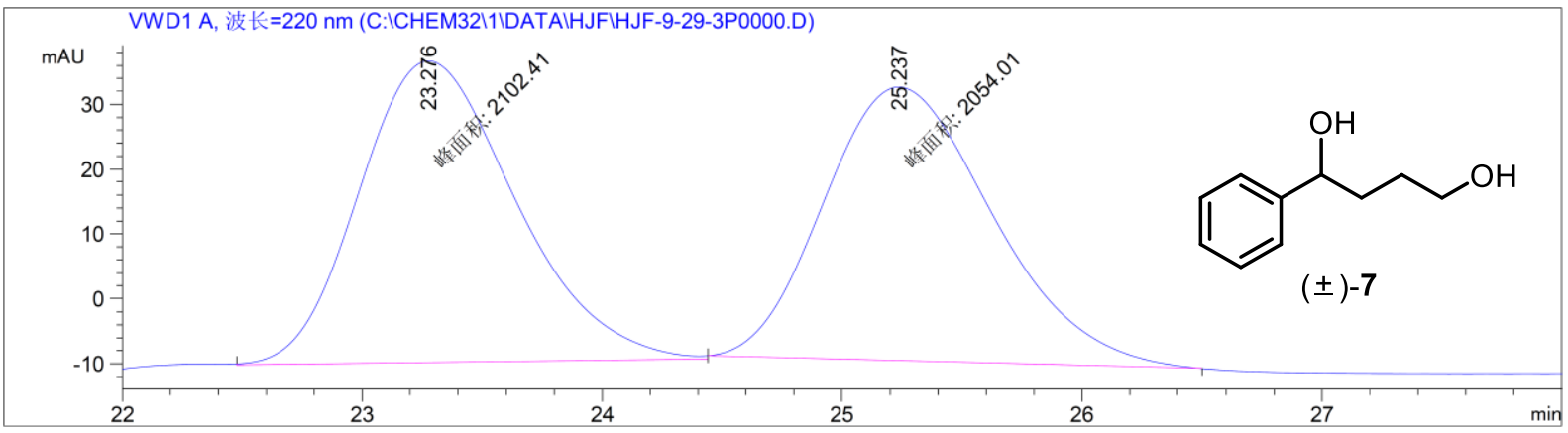

\begin{tabular}{|c|c|c|c|c|c|c|c|}
\hline Peak & RetTime & Type & Width & Ar & & Height & Area \\
\hline \# & [min ] & & [min ] & $\mathrm{mAU}$ & $\star_{S}$ & {$[\mathrm{mAU} \quad]$} & $\%$ \\
\hline & & & & 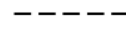 & 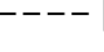 & 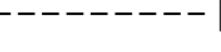 & \\
\hline 1 & & & & 210 & 0649 & 18 & 22 \\
\hline 2 & 25.237 & & 12 & 2054 & 0879 & 0219 & 178 \\
\hline
\end{tabular}




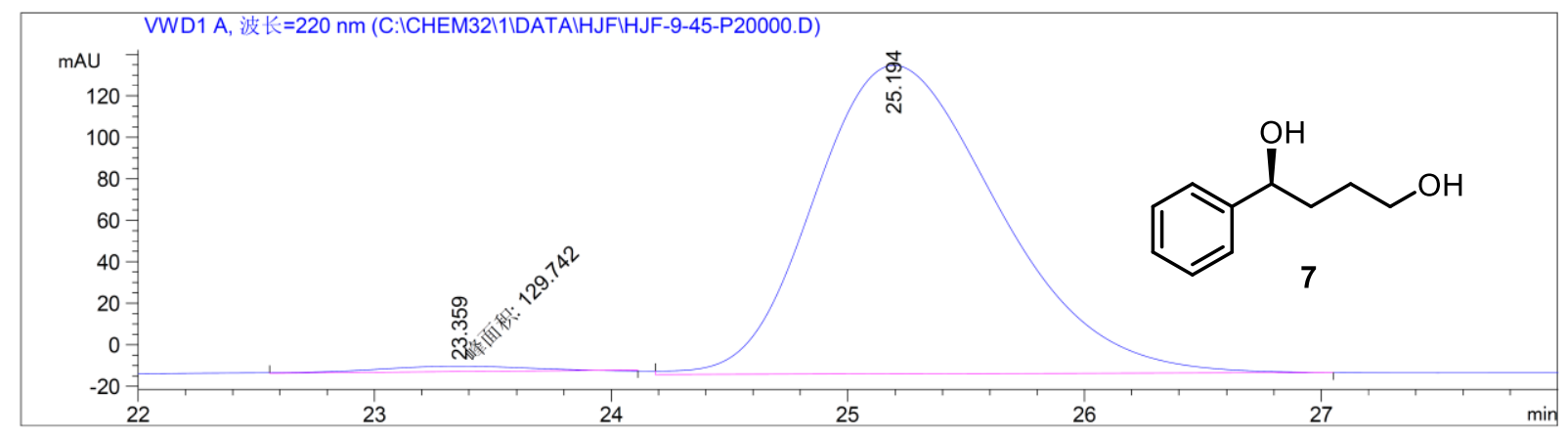

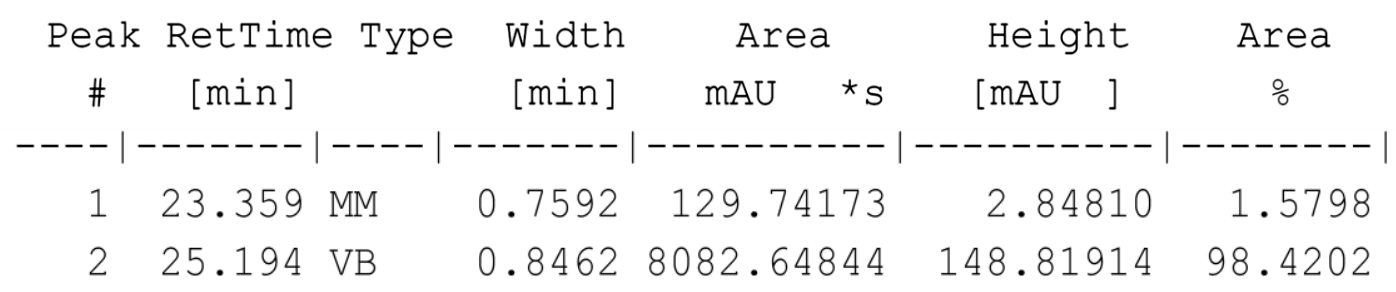

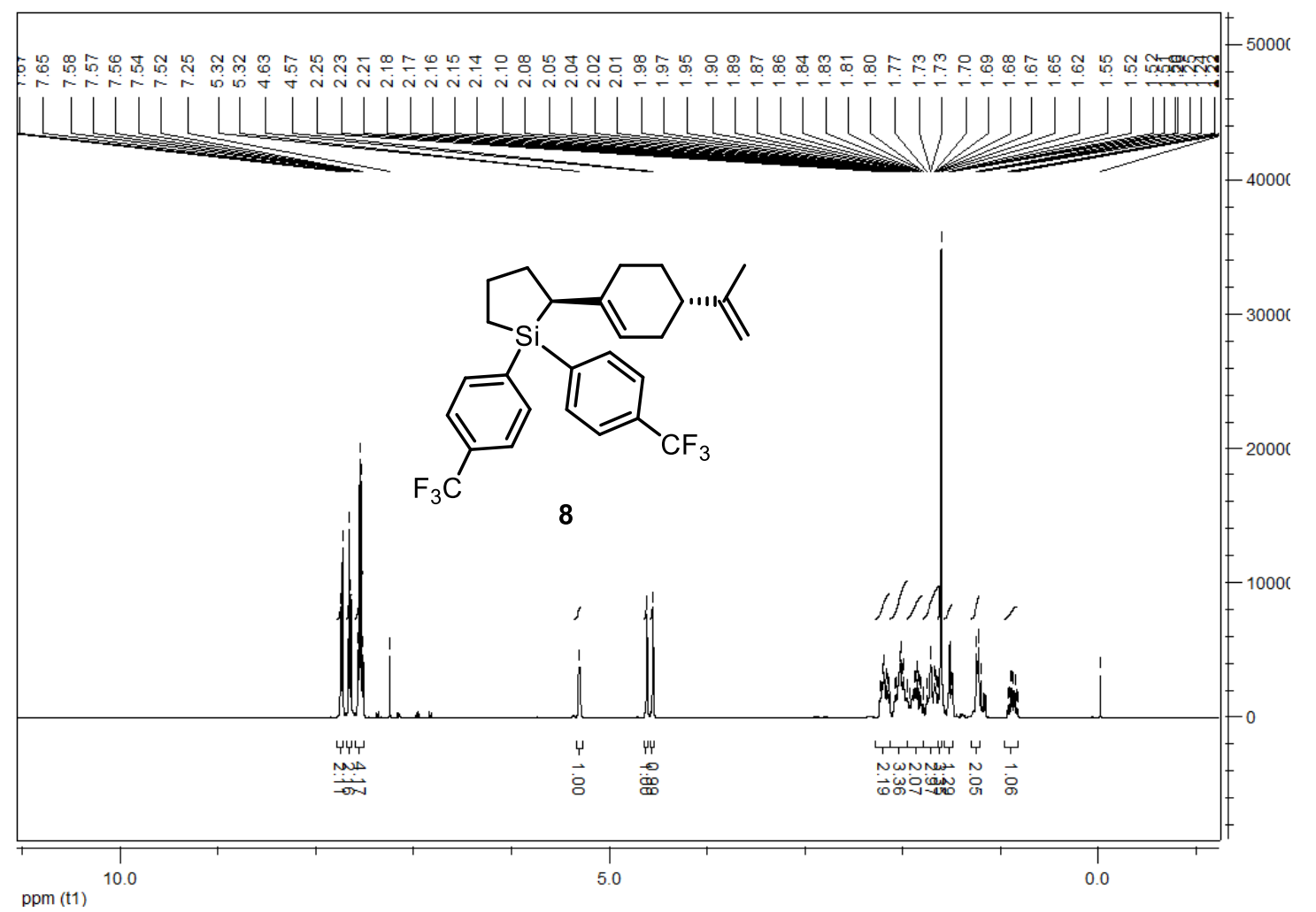



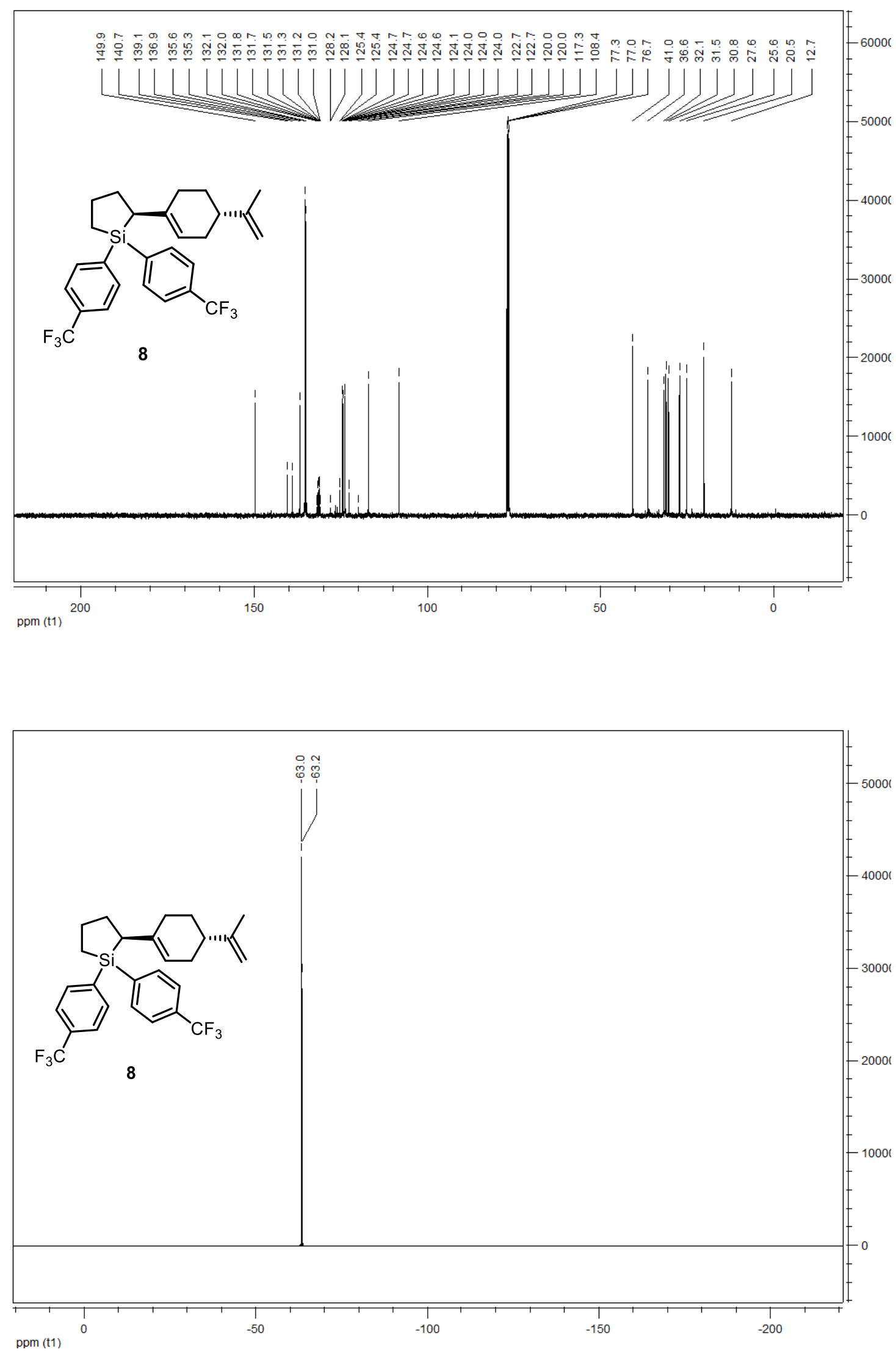

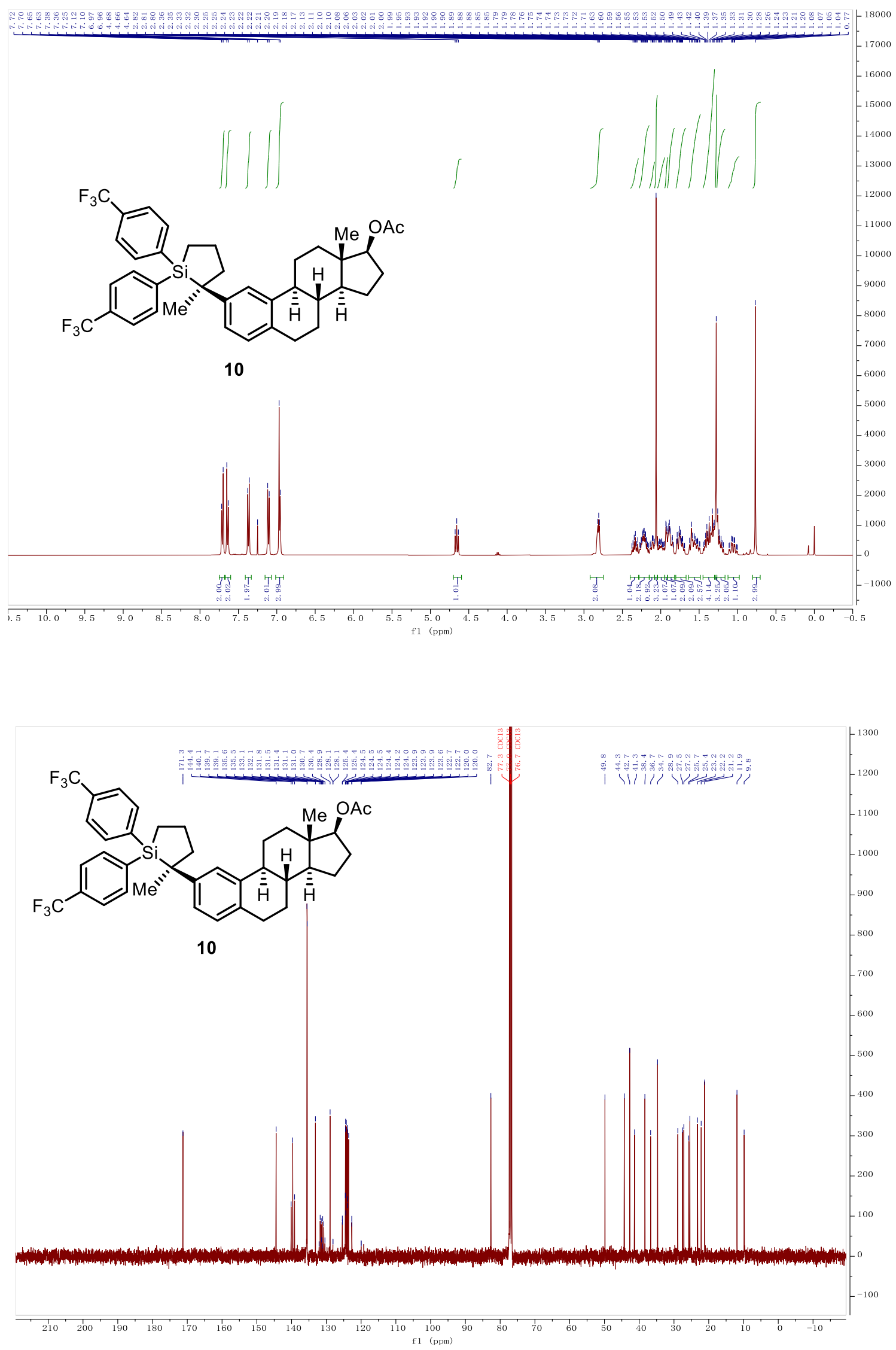

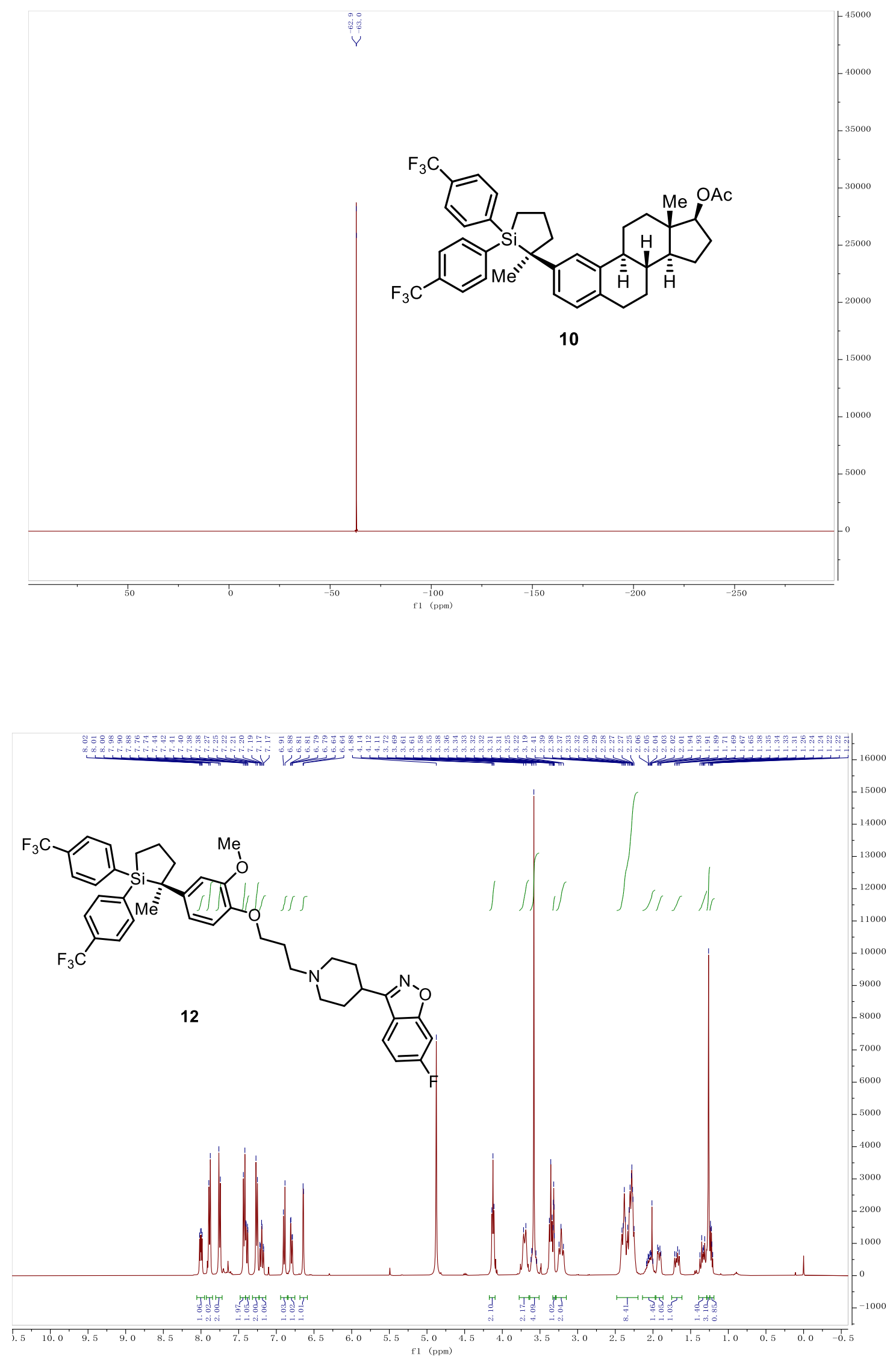


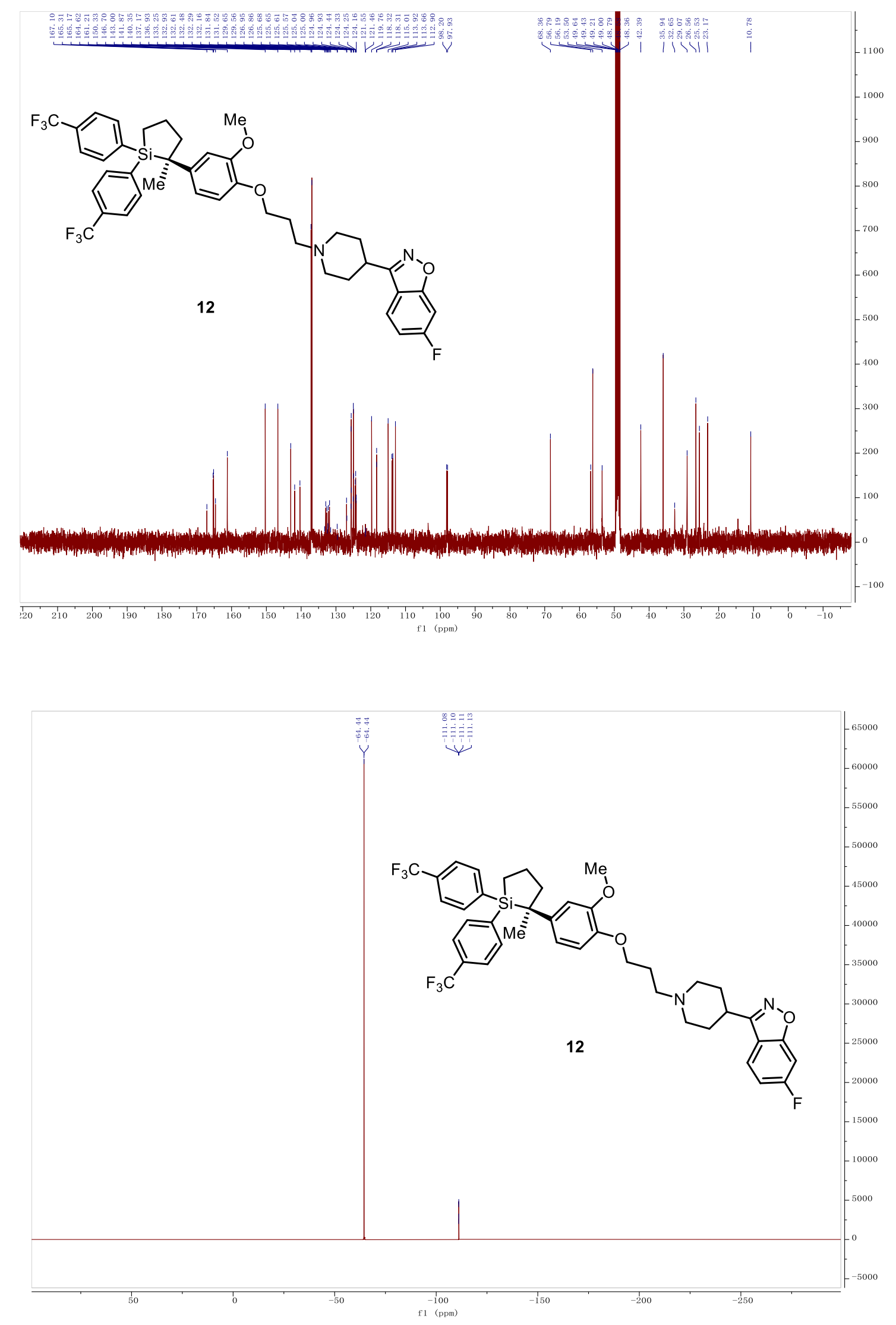


HPLC conditions: Daicel chiral column AD-H, hexane: ${ }^{i} \mathrm{PrOH}=90: 10,1.0 \mathrm{~mL} / \mathrm{min}, 25^{\circ} \mathrm{C}$, wavelength $=$ $210 \mathrm{~nm}$

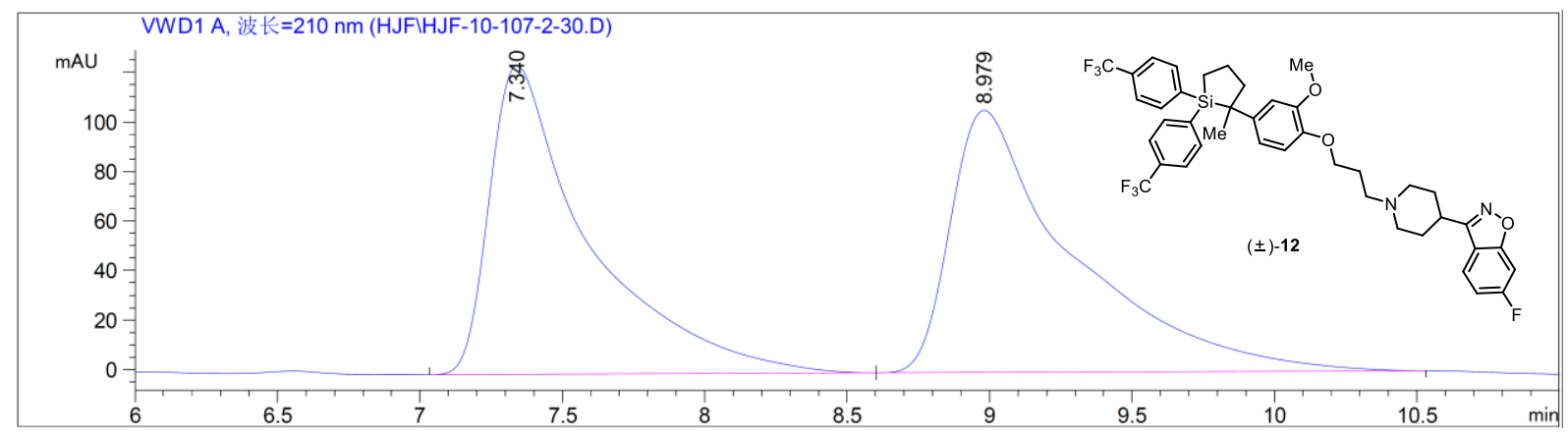

\begin{tabular}{|c|c|c|c|c|c|c|}
\hline \multirow{2}{*}{$\begin{array}{c}\text { Peak } \\
\#\end{array}$} & RetTime & Type & Width & Area & Height & \multirow{2}{*}{$\begin{array}{c}\text { Area } \\
\frac{\circ}{0}\end{array}$} \\
\hline & [min] & & [min] & mAU & {$[\mathrm{mAU}$} & \\
\hline & & & & -- & & \\
\hline 1 & 3401 & BV & & 3027.42944 & 124.78896 & \\
\hline$?$ & $8.979 \mathrm{~T}$ & VB & 0.4 & 3203.61206 & 106 & 51 \\
\hline
\end{tabular}

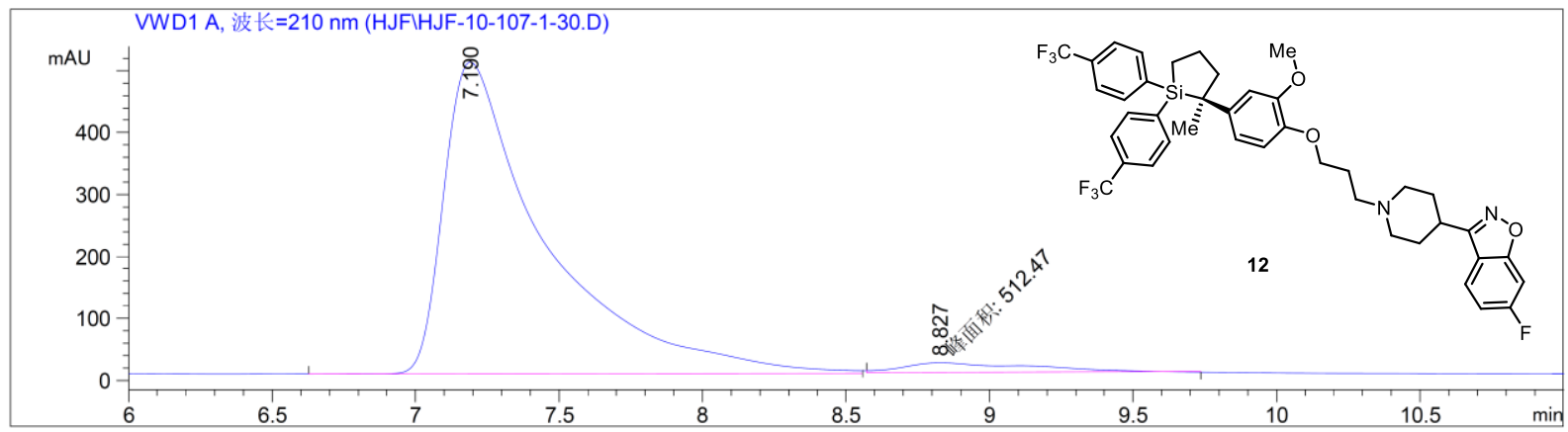

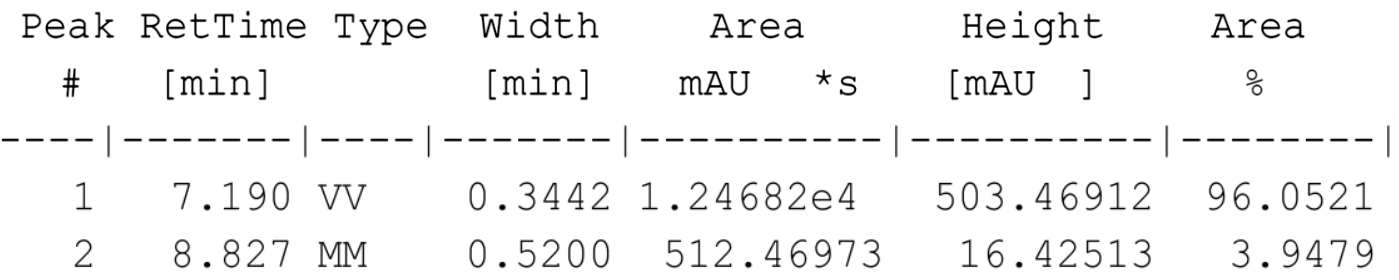




\section{References}

1. Tatsuno, Y.; Yoshida, T. \& Otsuka, S. “[( $\eta^{3}$-allyl)palladium(II) complexes]” in Inorganic Syntheses, R. J. Angelici, Eds. (Wiley, 1990), vol. 28, chap. 8, pp. 342-345.

2. Aggarwal, V. K.; Alonso, E.; Bae, I.; Hynd, G.; Lydon, K. M.; Palmer, M. J.; Patel, M.; Porcelloni, M.; Richardson, J.; Stenson, R. A.; Studley, J. R.; Vasse, J.-L.; Winn, C. L. J. Am. Chem. Soc. 2003, 125, 10926-10940.

3. Denmark, S. E.; Griedel, B. D.; Coe, D. M.; Schnute, M. E. J. Am. Chem. Soc. 1994, 116, 7026-7043.

4. Auner, N.; Grobe, J. J. Organomet. Chem. 1980, 188, 25-52.

5. Bradaric, C. J.; Leigh, W. J. Can. J. Chem. 1997, 75, 1393-1402.

6. Chen, H.; Chen, Y.; Tang, X.; Liu, S.; Wang, R.; Hu, T.; Song, Z. Angew. Chem. Int. Ed. 2019, 58, 46954699.

7. Li, H.; Ma, B.; Liu, Q.-S.; Wang, M.-L.; Wang, Z.-Y.; Xu, H.; Li, L.-J.; Wang, X.; Dai, H.-X. Angew. Chem. Int. Ed. 2020, 59, 14388-14393.

8. Becke, A. D. J. Chem. Phys. 1993, 98, 5648-5652.

9. Lee, C.; Yang, W.; Parr, R. G. Phys. Rev. B. 1988, 37, 785-789.

10. Zhao, Y.; Truhlar, D. G. Theor. Chem. Acc. 2008, 119, 525.

11. Cancès, E.; Mennucci, B.; Tomasi, J. J. Chem. Phys. 1997, 107, 3032-3041.

12. Cossi, M.; Barone, V.; Cammi, R.; Tomasi, J. Chem. Phys. Lett. 1996, 255, 327-335.

13. Johnson, E. R.; Keinan, S.; Mori-Sanchez, P.; Contreras-Garcia, J.; Cohen, A. J.; Yang, W. J. Am. Chem. Soc. 2010, 132, 6498-6506.

14. Lu, T.; Chen, F. J. Comput. Chem. 2012, 33, 580-592.

15. Humphrey, W.; Dalke, A.; Schulten, K. J. Mol. Graphics. 1996, 14, 33-38.

16. Legault, C. Y. CYLview, 1.0b; Universitéde Sherbrooke: 2009 (http://www.cylview.org).

17. Ahlquist, M.; Fristrup, P.; Tanner, D.; Norrby, P.-O. Organometallics 2006, 25, 2066-2073.

18. Barder, T. E.; Biscoe, M. R.; Buchwald, S. L. Organometallics 2007, 26, 2183-2192.

19. Ryu, H.; Park, J.; Kim, H. K.; Park, J. Y.; Kim, S.-T.; Baik, M.-H. Organometallics 2018, 37, 3228-3239.

20. Chen, W.-J.; Lin, Z. Dalton Trans. 2014, 43, 11138-11144.

21. Garlets, Z. J.; Hicks, E. F.; Fu, J.; Voight, E. A.; Davies, H. M. L. Org. Lett. 2019, 21, 4910-4914. 